\title{
Hydroalkylation of Alkenes with 1,3-Diketones via Gold(III) or Silver(I) Catalysis: Divergent Mechanistic Pathways Revealed by a DFT-based Investigation
}

\author{
Mona Jalali, ${ }^{\dagger}$ Christopher J. T. Hyland, ${ }^{\star}$ Alex C. Bissember,${ }^{\dagger}$ Brian F. Yates,${ }^{\dagger}$ and Alireza \\ Ariafard, ${ }^{* \dagger}$ \\ † School of Natural Sciences - Chemistry, University of Tasmania, Hobart, Tasmania, Australia \\ \$ School of Chemistry and Molecular Bioscience, University of Wollongong, Wollongong, NSW, \\ Australia
}

Correspondence to: alireza.ariafard@utas.edu.au

\section{Supporting Information}

\section{Table of Contents}

Figure S1. Calculated free energy (potential energy) for the mechanism of the oxidative addition of 1,3-diketone to $\left[\mathrm{Au}(\mathrm{Cl})_{2}\right]^{-}$(the first step of mechanism i). (Page S3)

Figure S2. Calculated energy profile for the $\mathrm{AuCl}_{3}$-catalyzed hydroalkylation process via cycle ii, occurring through inner or outer addition pathway. (Page S3)

Figure S3. Calculated energy profiles for the $\mathrm{AuCl}_{3}$-catalyzed hydroalkylation reaction via cycle iii. (Page S4)

Figure S4. Calculated energy profiles for the $\mathrm{AuCl}_{3}$-catalyzed hydroalkylation reaction via cycle iv. (Page S5)

Figure S5. Calculated energy profile for the AgOTf-catalyzed hydroalkylation process via cycle ii, occurring through inner or outer addition pathway. (Page S6)

Figure S6. Calculated energy profile for the AgOTf-catalyzed hydroalkylation process via cycle iii. (Page S7)

Figure S7. Calculated energy profiles for the AgOTf-catalyzed hydroalkylation reaction via cycle iv. (Page S8)

$\mathrm{p} K_{\mathrm{a}}$ values for the enol coordinated to different metal complexes. (page S9)

Figure S8. A plot of the correlation between $\mathrm{p} K_{\mathrm{a}}$ and $\Delta \mathrm{G}$ and a plot of the correlation between $\mathrm{p} K_{\mathrm{a}}$ and $\Delta \mathrm{G}^{\ddagger}$. (Page S9)

Figure S9. Calculated reaction free energy for hydroalkylation of 2_Cl with 1_Ph leading to formation of 3_Ph. (Page S10)

Calculated mechanism for the C-C coupling from $24^{\prime}$. (page S10)

Figure S10. Calculated energy profiles for competition between $\mathbf{9}$ and $\mathbf{2 4}$ ' for trapping carbocation $\mathbf{2 3}$. (Page S11) 
Figure S11. Calculated energy profile for competition between THF and enol $\mathbf{9}$ for trapping carbocation 23. (Page S12)

Figure S12. Calculated energy profile for competition between water and enol 9 for trapping carbocation 23. (Page S13)

A comparison of different levels of theory. (Page S14)

Table S1. Relative free energy of key intermediates and transition structures at different levels of theory. (Page S14)

Figure S13. Calculated energy profile for hydroalkylation via mechanisms v and vi. (Page S15)

Figure S14. Calculated energy profile comparing the activation barrier to the protodemetallation step via two transition structures $\mathbf{T S}_{\mathbf{1 8 - 1 7}}$ and $\mathbf{T S} \mathbf{1}_{\mathbf{1 8 - 1 7 - H 2 O}}$. (Page S16)

Coordination of the acac anion as a bidentate ligand to the silver(I) center. (Page S16)

Figure S15. Calculated mechanism for competition between formation of 32' and the hydroalkylation reaction via mechanism $\mathbf{v}$ starting from ion pair 32. (Page S16)

Figure S16. Calculated reaction free energy for ligand exchange between $\left[\mathrm{Au}(\mathrm{OTf})_{2}\right]^{-}$and styrene 2 and calculated energy profile for the AuOTf-catalysed hydroalkylation reaction via mechanism ii. (Page S17)

Impact of the ligands of $\mathrm{AuX}_{3}$ on mechanisms v and vi. (Page S18)

Table S2. NPA charge on the enol ligand in 1_M', q(enol), activation energy for the protonation step via transition structure TS-M' from 1_M', $\mathbf{M}^{\prime}, \mathrm{G}_{1}^{\ddagger}$, and activation energy for the demetallation via transition structure TS_M" from ion pair 2_M', $\Delta \mathrm{G}_{2}{ }^{\star}$, where $\mathrm{CH}_{2} \mathrm{Cl}_{2}$ is employed as the solvent. (Page S18)

Figure S17. A plot of the correlation between $\mathrm{q}\left(\right.$ enol) and $\Delta \mathrm{G}_{1}^{\ddagger}$. (Page S18)

Table S3. Total potential (E), enthalpy $(\mathrm{H})$ and Gibbs free energies $(\mathrm{G})$ of all optimized structures. (Page S19) 


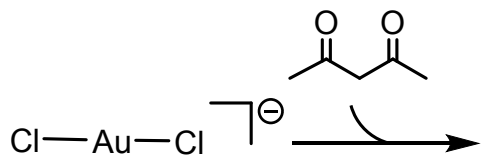

$A^{\prime}$

$0.0(0.0)$<smiles>CC(=O)C1(C(=O)[Hg])[Al](C)[Al]1Cl</smiles>

$\mathrm{TS}_{\mathrm{A}^{\prime}-\mathrm{B}^{\prime}}$

$50.4(45.4)$<smiles>CC(=O)C(C(C)=O)[AlH](Cl)Cl</smiles>

$40.1(31.8)$

Figure S1. Calculated mechanism for the oxidative addition of 1,3-diketone to $\left[\mathrm{Au}(\mathrm{Cl})_{2}\right]^{-}$(the first step of mechanism i). Free energies (potential energies) are given in $\mathrm{kcal} / \mathrm{mol}$ where $\mathrm{CH}_{2} \mathrm{Cl}_{2}$ is employed as the solvent.<smiles>CC(=O)C(C(C)=O)C(c1ccccc1)C([14CH3])[AlH2]</smiles>

TS $_{18 '-17}$

$31.7(21.3)$
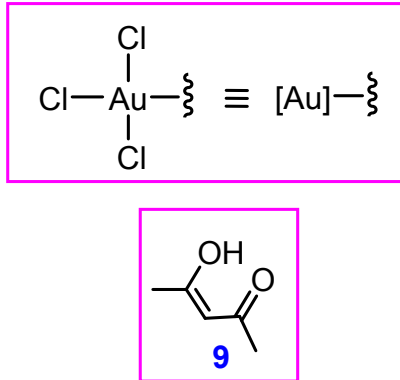

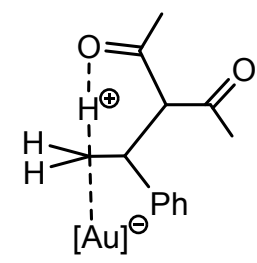

$\mathrm{TS}_{19}{ }^{\prime}-17^{\prime}$ $38.1(27.7)$

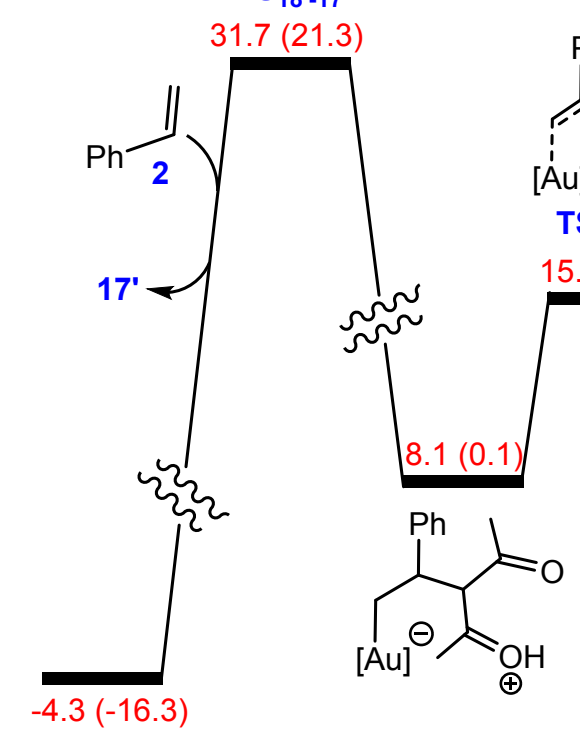

$-4.3(-16.3)$<smiles>CC(=O)C(C(C)=O)C(C)c1ccccc1</smiles>

3

inner addition pathway

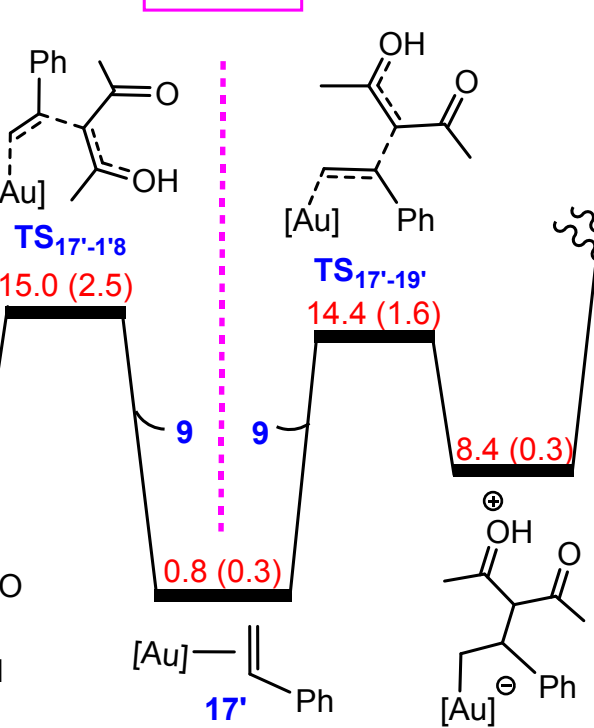

19

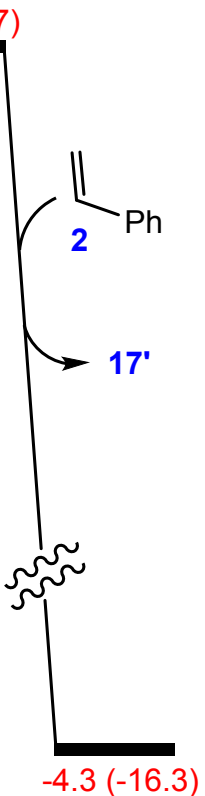

年

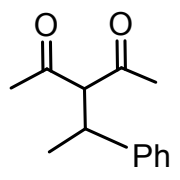

3

outer addition pathway

Figure S2. Calculated energy profile for the $\mathrm{AuCl}_{3}$-catalyzed hydroalkylation process via cycle ii, occurring through inner or outer addition pathway. Free energies (potential energies) are given in $\mathrm{kcal} / \mathrm{mol}$ where $\mathrm{CH}_{2} \mathrm{Cl}_{2}$ is employed as the solvent. 


$$
\mathrm{Cl}-\underset{\substack{\mathrm{Au} \\ \mathrm{Cl}}}{\mathrm{Cl}} \xi \equiv[\mathrm{Au}]-\xi
$$
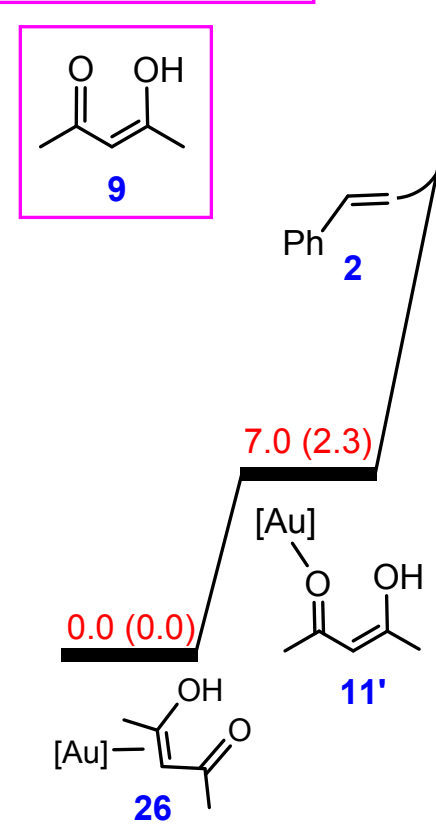<smiles>CC(=O)/C(=C(\C)O[AlH2])C(C)c1ccccc1</smiles>

$\mathrm{TS}_{20^{\prime}-21^{\prime}}$ $35.9(24.8)$
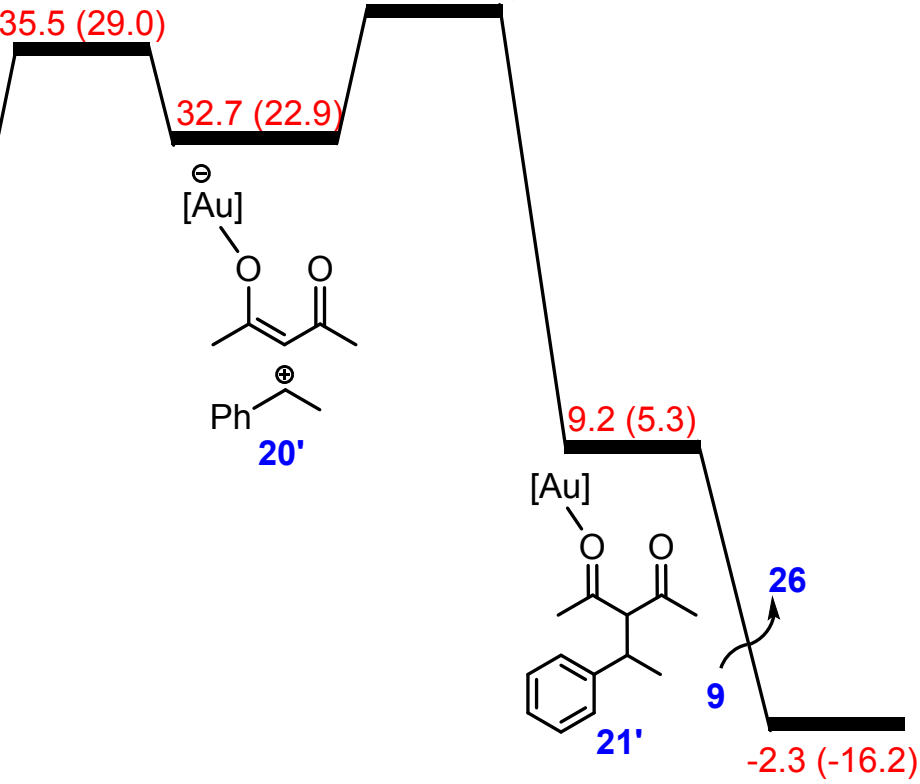<smiles>CC(=O)C(C(C)=O)C(C)c1ccccc1</smiles>

3

Figure S3. Calculated energy profiles for the $\mathrm{AuCl}_{3}$-catalyzed hydroalkylation reaction via our proposed hidden Brønsted acid mechanisms (cycle iii) where adduct 11' is the active catalyst. Free energies (potential energies) are given in $\mathrm{kcal} / \mathrm{mol}$ where $\mathrm{CH}_{2} \mathrm{Cl}_{2}$ is employed as the solvent. 


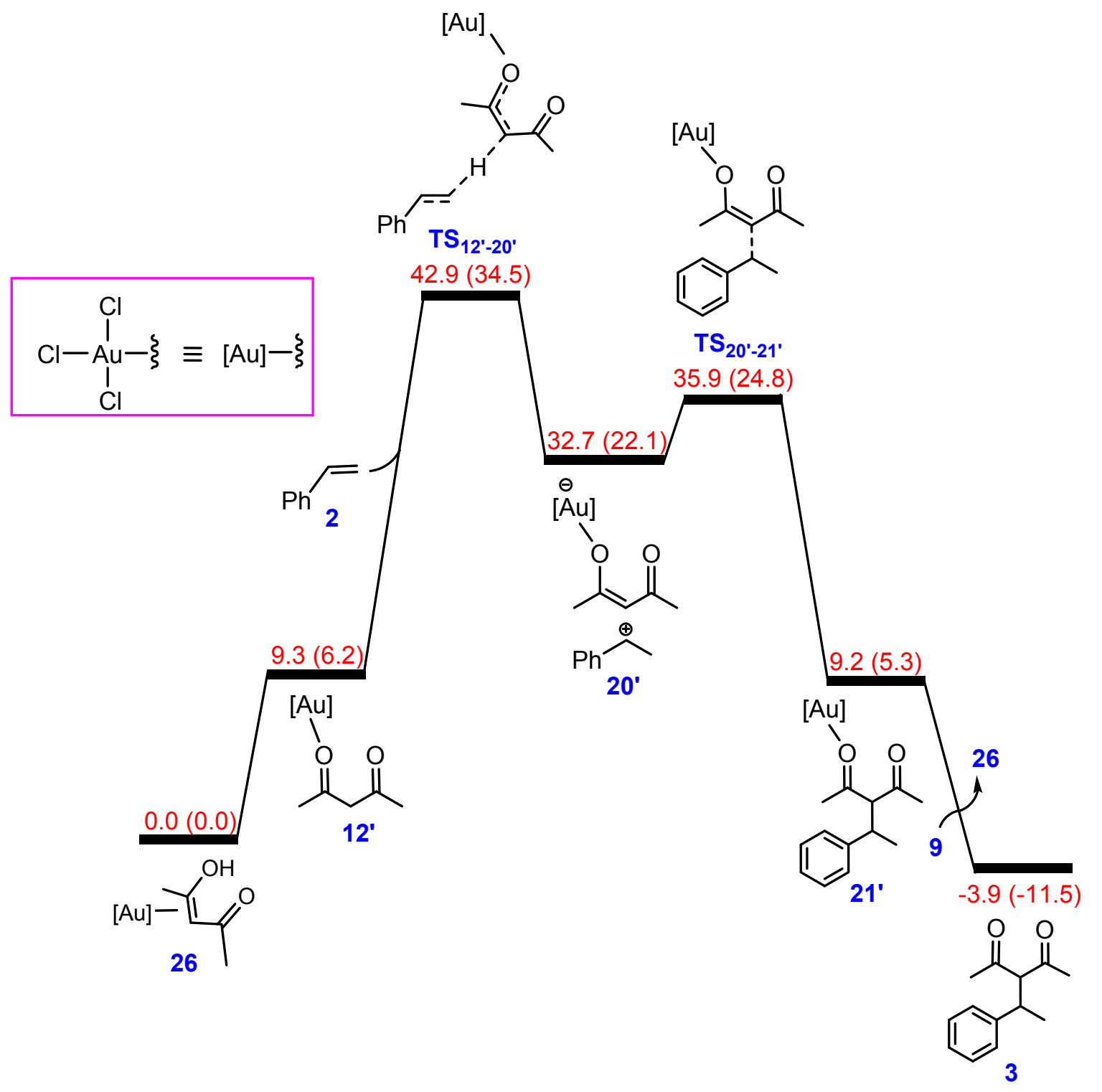

Figure S4. Calculated energy profiles for the $\mathrm{AuCl}_{3}$-catalyzed hydroalkylation reaction via our proposed hidden Brønsted acid mechanisms (cycle iv) where adduct 12' is the active catalyst. Free energies (potential energies) are given in $\mathrm{kcal} / \mathrm{mol}$ where $\mathrm{CH}_{2} \mathrm{Cl}_{2}$ is employed as the solvent. 


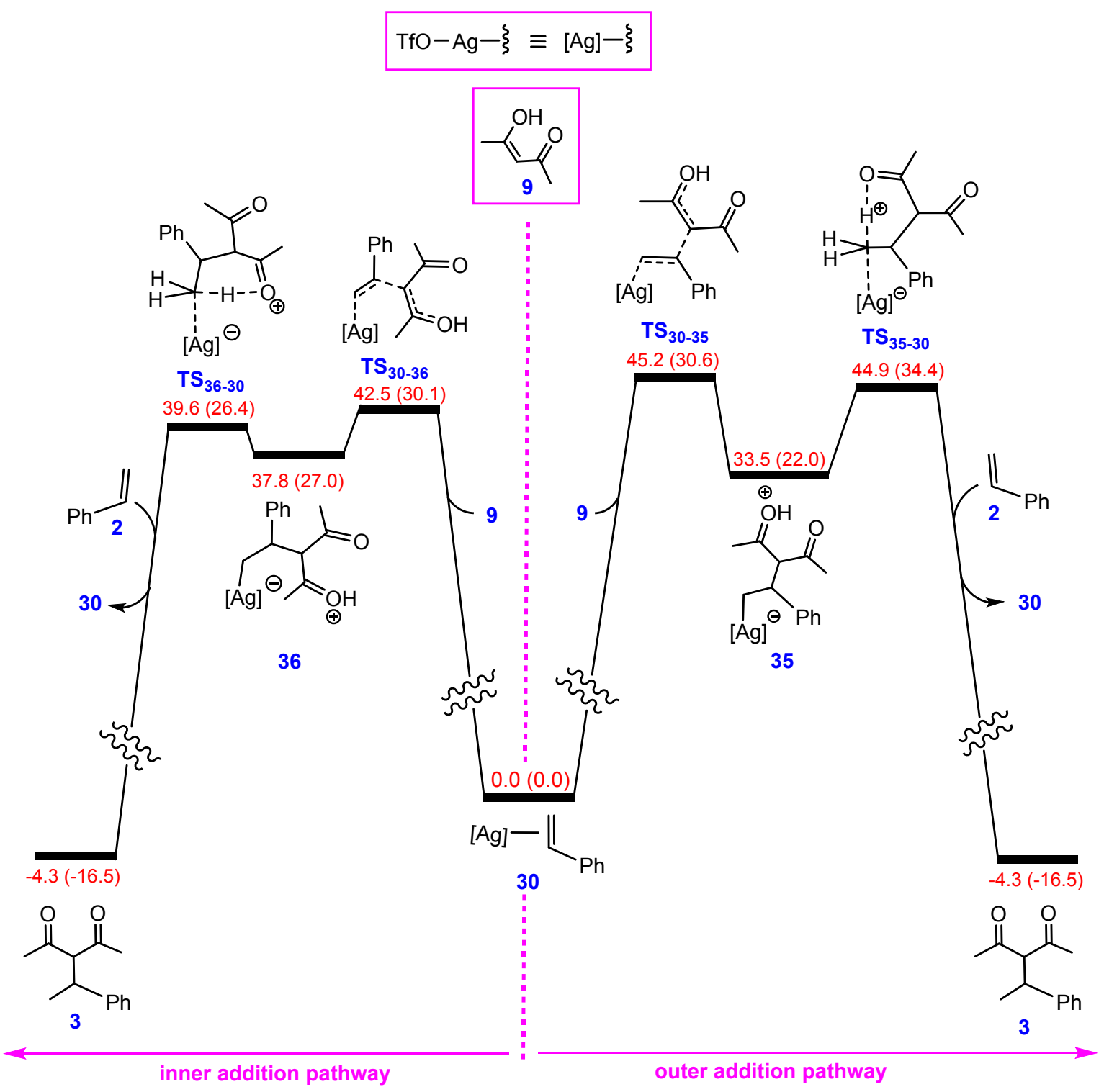

Figure S5. Calculated energy profile for the AgOTf-catalyzed hydroalkylation process via cycle ii, occurring through inner or outer addition pathway. Free energies (potential energies) are given in $\mathrm{kcal} / \mathrm{mol}$ where $\mathrm{CH}_{3} \mathrm{NO}_{2}$ is employed as the solvent. 


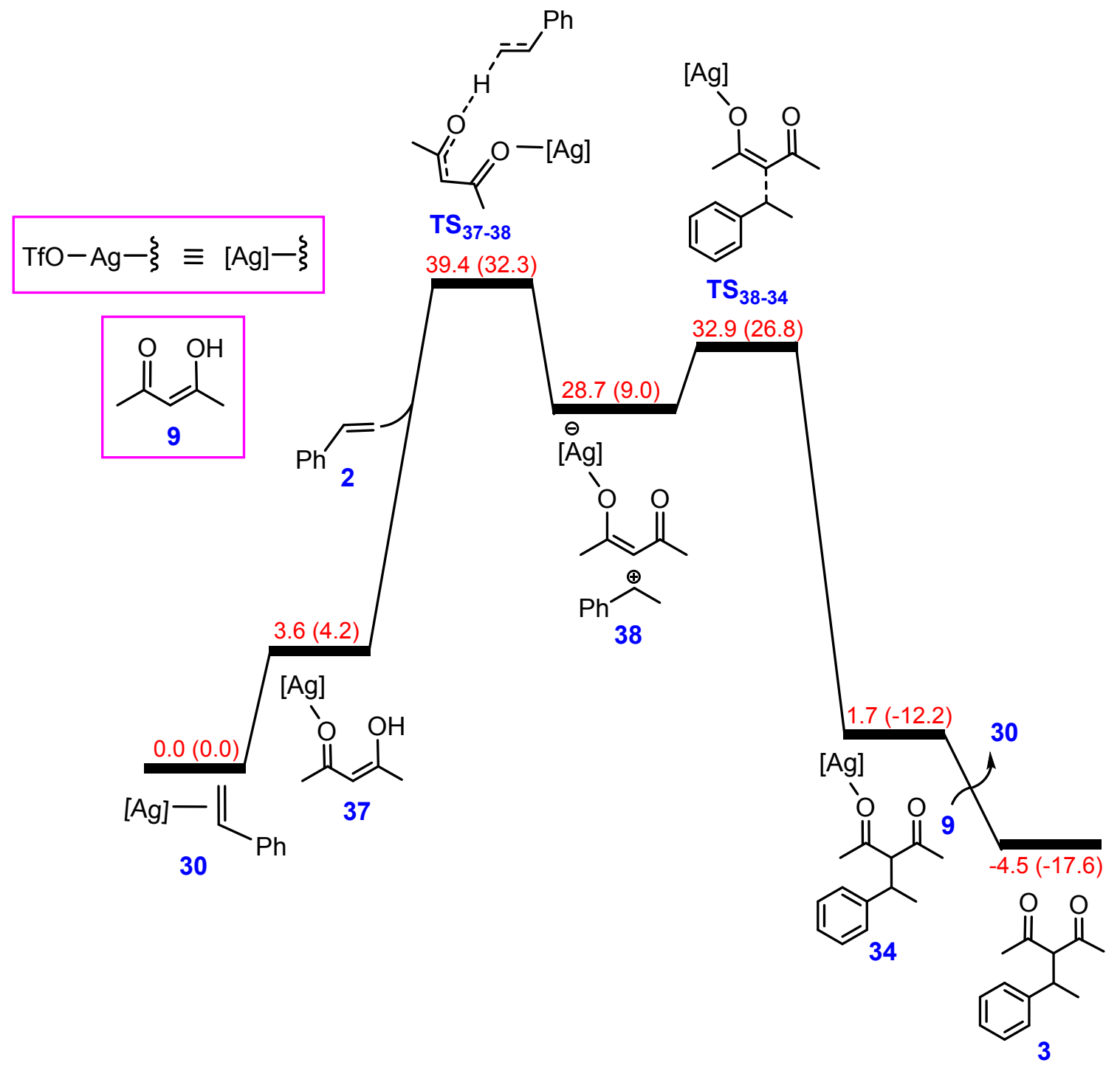

Figure S6. Calculated energy profiles for the hydroalkylation reaction via our proposed hidden Brønsted acid mechanism (cycle iii) where adduct $\mathbf{3 7}$ is the active catalyst. Free energies (potential energies) are given in $\mathrm{kcal} / \mathrm{mol}$ where $\mathrm{CH}_{3} \mathrm{NO}_{2}$ is employed as the solvent. 


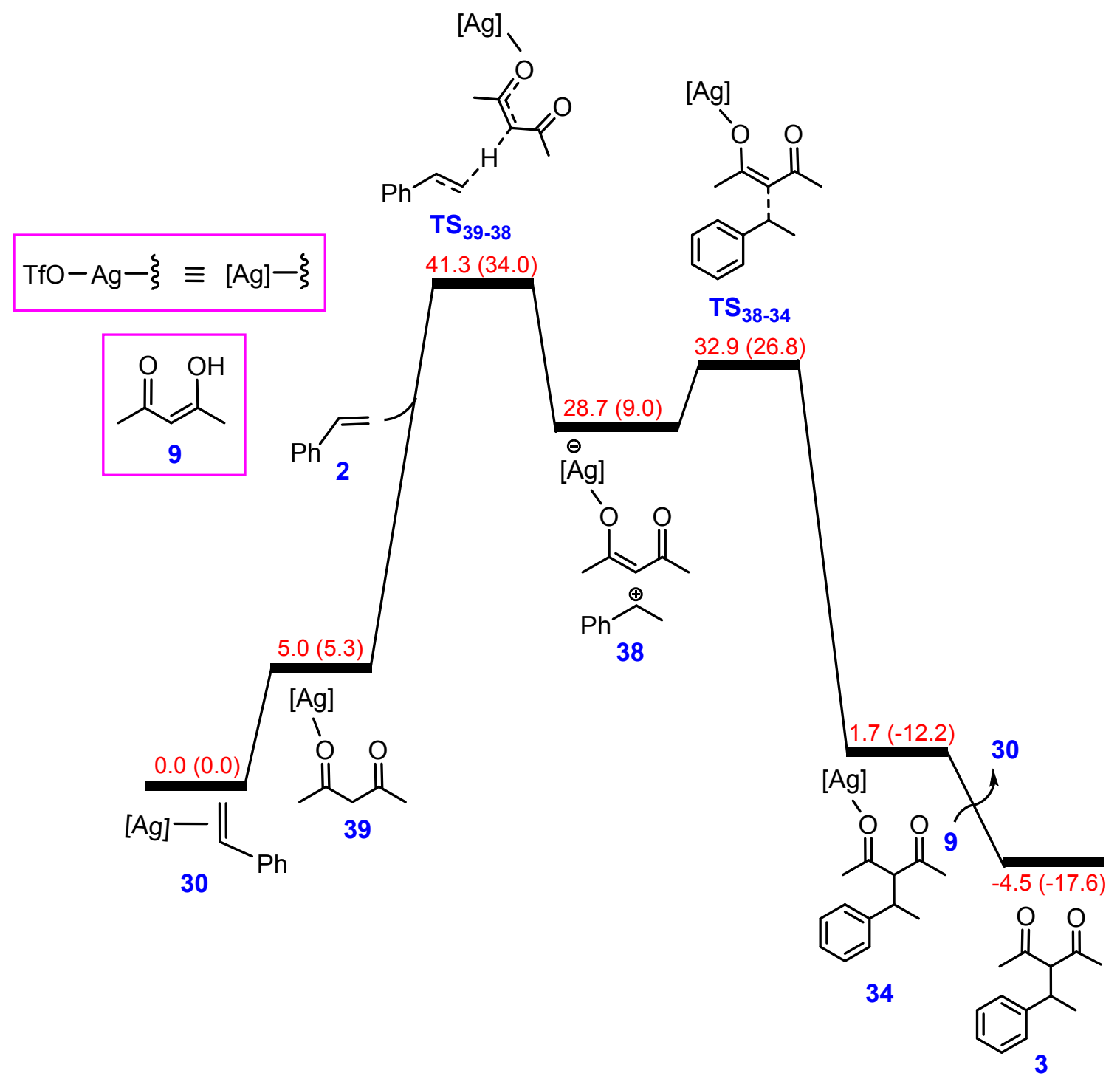

Figure S7. Calculated energy profiles for the hydroalkylation reaction via our proposed hidden Brønsted acid mechanisms (cycle iv) where adduct $\mathbf{3 8}$ is the active catalyst. Free energies (potential energies) are given in $\mathrm{kcal} / \mathrm{mol}$ where $\mathrm{CH}_{3} \mathrm{NO}_{2}$ is employed as the solvent. 
pKa values for the enol coordinated to different metal complexes. To investigate this point, we first benchmarked our method by calculating $\mathrm{p} K_{\mathrm{a}}$ of HOTf. We found that our method estimates this $\mathrm{pK}_{\mathrm{a}}$ value in water with an excellent accuracy $(-13.9)$ by considering that this acid has an experimental $\mathrm{p} K_{\mathrm{a}}$ of -14.7 in the same solvent (see: Trummal, A.; Lipping, L.; Kaljurand, I.; Koppel, I. A.; Leito, I. Acidity of Strong Acids in Water and Dimethyl Sulfoxide. J. Phys. Chem. A 2016, 120, 3663-3669). This result implies that our method (SMD/M06/def2-TZVP//SMD/B3LYP/6-31G(d),SDD) is adequately reliable for estimation of this parameter. Accordingly, the $\mathrm{p} K_{\mathrm{a}}$ values of the coordinated enol in different adducts are calculated based on the following equation (eq 2). For these calculations a number of $-270.3 \mathrm{kcal} / \mathrm{mol}$ was used as the free energy of $\mathrm{H}^{+}$(the solvation free energy and gas phase free energy of a proton are reported as -264.0 and $-6.3 \mathrm{kcal} / \mathrm{mol}$, respectively; Tissandier, M. D.; Cowen, K. A.; Feng, W. Y.; Gundlanch, E.; Cohen, M. H.; Earhart, A. D.; Coe, J. V.; Tuttle, T. R. the Proton's Absolute Aqueous Enthalpy and Gibbs Free energy of Solvation from Cluster-Ion Solvation Data. $J$. Phys. Chem. A 1998, 102, 40, 7787-7794). As expected, when the enol coordinates to the highly electron deficient complex $\left[\mathrm{Au}(\mathrm{OTf})_{3}\right]$, its proton becomes extremely acidic ( $K_{\mathrm{a}}$ of -11.6$)$. In comparison, enol coordination to the slightly electron deficient complex [AgOTf] does not significantly increase the acidity of the corresponding proton ( $\mathrm{p} K_{\mathrm{a}}$ of 6.2 , Table 1) compared to the free enol with an experimental $\mathrm{p} K_{\mathrm{a}}$ of 8.93. We found a clear correlation between the $\mathrm{p} K_{\mathrm{a}}$ value and the reaction free energy for formation of ion-pair 2_M and the activation free energy for demetallation via TS_M (Figure S8). These correlations reveal that the more electron-deficient the metal center is, the lower the $\mathrm{p} K_{\mathrm{a}}$ value, the lower the energy of ion pair 2_M and the higher the activation barrier to the demetallation via TS_M.
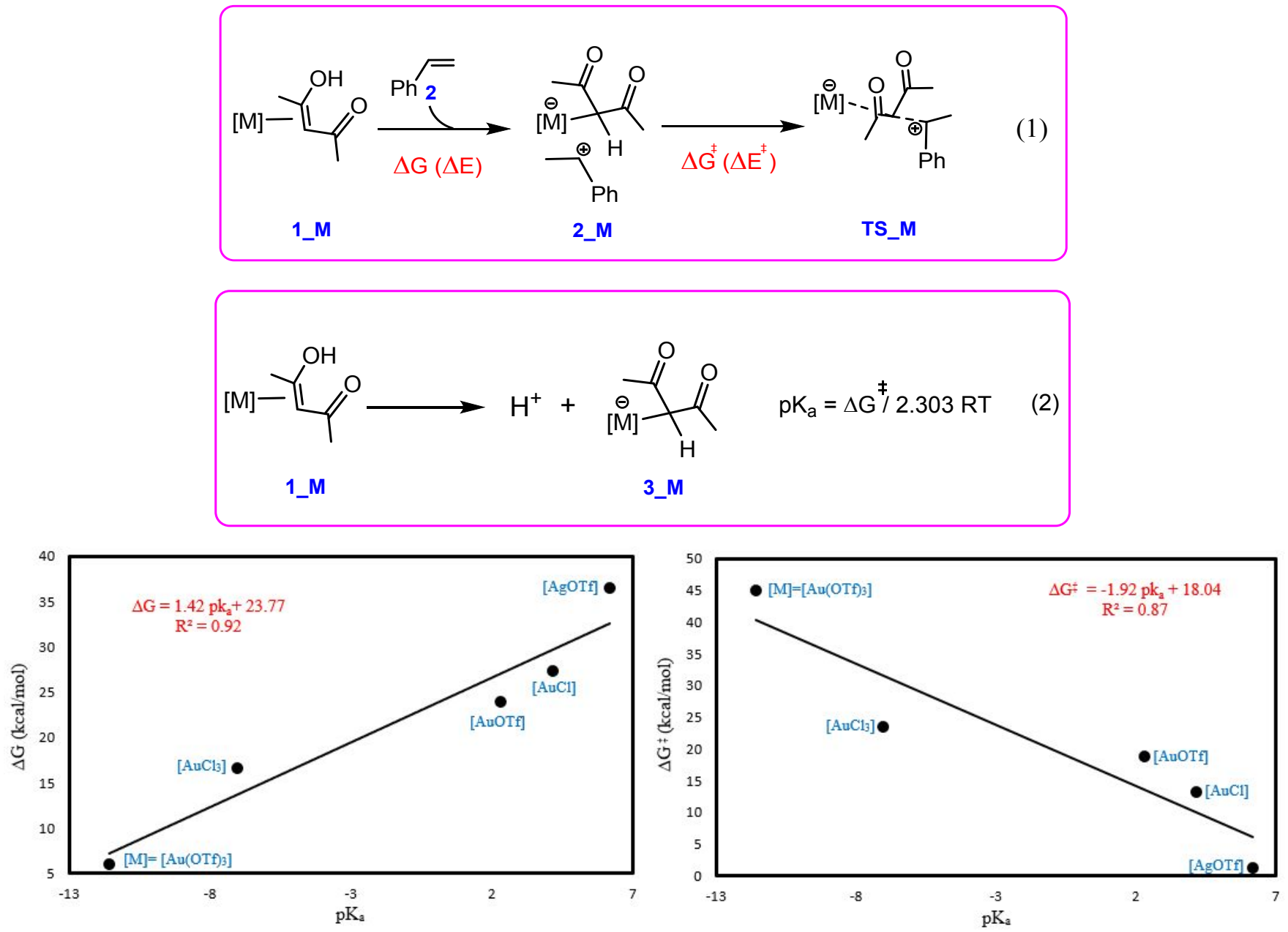

Figure S8. A plot of the correlation between $\mathrm{pK}_{\mathrm{a}}$ and $\Delta \mathrm{G}$. (b) A plot of the correlation between $\mathrm{p} K_{\mathrm{a}}$ and $\Delta \mathrm{G}^{\ddagger}$. 
<smiles></smiles>

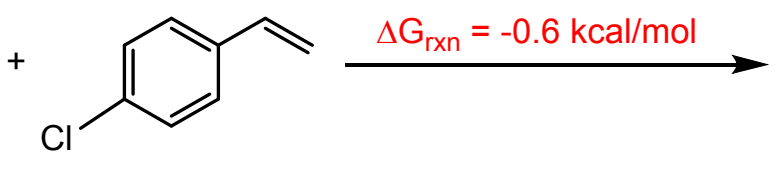

1_Ph<smiles>CC(c1ccc(Cl)cc1)C(C(=O)c1ccccc1)C(=O)c1ccccc1</smiles>

3_Ph

Figure S9. Calculated reaction free energy for hydroalkylation of 2_Cl with 1_Ph leading to formation of 3_Ph. A careful exploration of the Li's et al work (ref 6) indicates that the hydroalkylation reactions in some instances are reversible. This observation was clearly highlighted for transformation 2_Cl + 1_Ph $\rightarrow$ 3_Ph in ref 6. The reaction free energy for this transformation is calculated to be only -0.6 $\mathrm{kcal} / \mathrm{mol}$. This specific example reveals that the observed reversibility could be related to the thermoneutral nature of these reactions.

Calculated mechanism for the $\mathbf{C}-\mathbf{C}$ coupling from $\mathbf{2 4}^{\prime}$. It should be noted that anionic complex $\mathbf{2 4}$ may be in equilibrium with $\mathbf{2 4 ^ { \prime }}$ in which the acac anion coordinates to the gold(III) center as a bidentate ligand (Figure S10). To evaluate whether intermediate 24' drives the $\mathrm{C}-\mathrm{C}$ bond coupling step, we calculated the mechanism shown in Figure S10. Accordingly, although the stability of $\mathbf{2 4}$ and $\mathbf{2 4}^{\prime}$ is almost comparable, $\mathbf{2 4}^{\prime}$ is computed to be much less reactive than enol substrate 9 toward trapping the benzylic carbocation. The low reactivity of $\mathbf{2 4}^{\prime}$ can be explained based on the large endergonicity of transformation $\mathbf{2 4}^{\prime}+\mathbf{2 3} \rightarrow \mathbf{2 5}^{\prime}$ with $\Delta \mathrm{G}=21.6 \mathrm{kcal} / \mathrm{mol}$. 
<smiles>C=C[PH+](CC)C1C[C@@H]1P(CC)c1ccccc1</smiles>

$0.0(0.0)$<smiles>CC(=O)C(C)C(C)=O</smiles>

24

$4.3(5.3)$<smiles>CC1=CC(C)O[Al]C1</smiles>

24

$6.8(19.1)$<smiles>CC(=O)C(C)(C(=O)O)C(C)c1ccccc1</smiles>

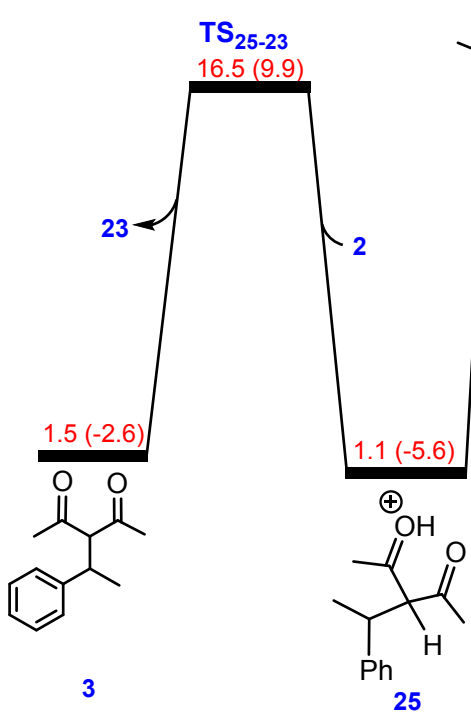

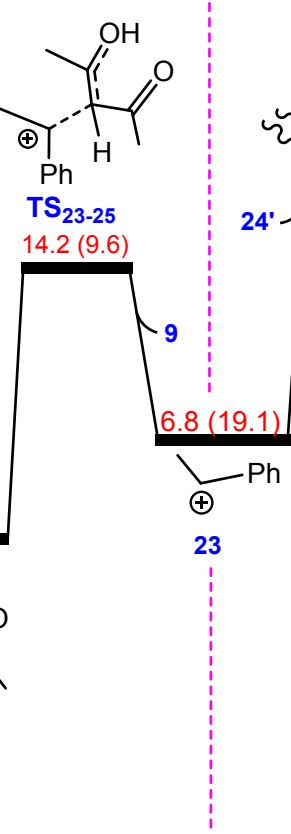

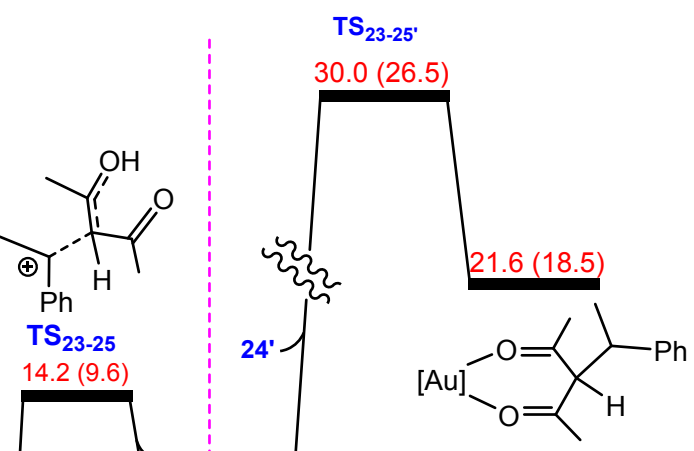

25'

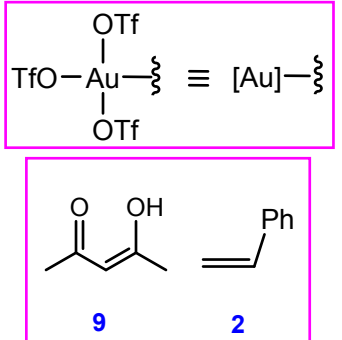

Figure S10. Calculated energy profiles for competition between 9 and 24' for trapping carbocation 23. Free energies (potential energies) obtained from the SMD/M06/def2-TZVP//SMD/B3LYP/6-31G(d), SDD calculation in $\mathrm{CH}_{2} \mathrm{Cl}_{2}$ are given in $\mathrm{kcal} / \mathrm{mol}$.

Deactivating effect of water and THF on the hydroalkylation reaction. $\mathrm{Li}$ et al reported that the $\mathrm{AuCl}_{3} / \mathrm{AgOTf}$-catalysed hydroalkylation process does not operate in solvents such as water and THF. ${ }^{3}$ Specifically, they showed that when the reaction is conducted in THF, decomposition of the solvent takes place. To understand why this is the case, we recalculated the most favourable pathway (mechanism vi) by considering either THF or water as the solvent. Figures S11 and S12 reveal that the in-situ-generated benzylic carbocation is trapped by water/THF much faster than that by enol 9. This feature results in the diketone substrate remaining intact, leading to no reaction occurring. The calculations also show that when cationic intermediate 25_THF is formed (Figure S11), it can react with a second THF molecule via a $\mathrm{S}_{\mathrm{N}} 2$ mechanism by crossing transition structure $\mathbf{T S}_{3-\mathrm{THF}}$, bringing about decomposition of THF. These additional calculations also allowed us to state that the stability of the ion pair 22 toward fragmentation depends on the nature of the solvent. A more polar solvent such as water results in the fragmentation being more exergonic due to the fact that the separated anion and cation are stabilized more significantly under these conditions. 


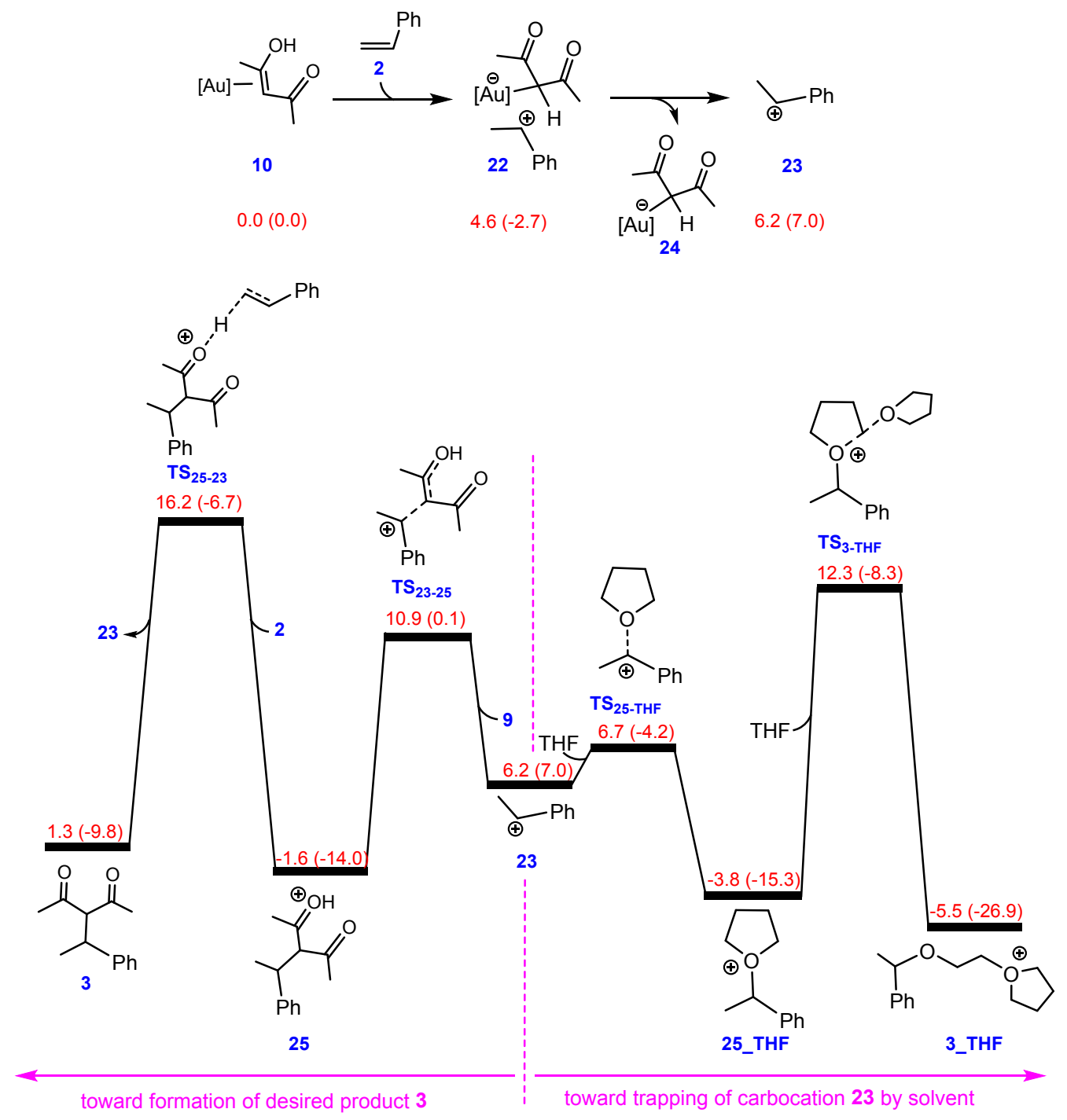

Figure S11. Calculated energy profile for competition between THF and enol 9 for trapping carbocation 23. Free energies (potential energies) obtained from SMD/M06/def2TZVP//SMD/B3LYP/6-31G(d), SDD in THF are given in $\mathrm{kcal} / \mathrm{mol}$. 
(b)
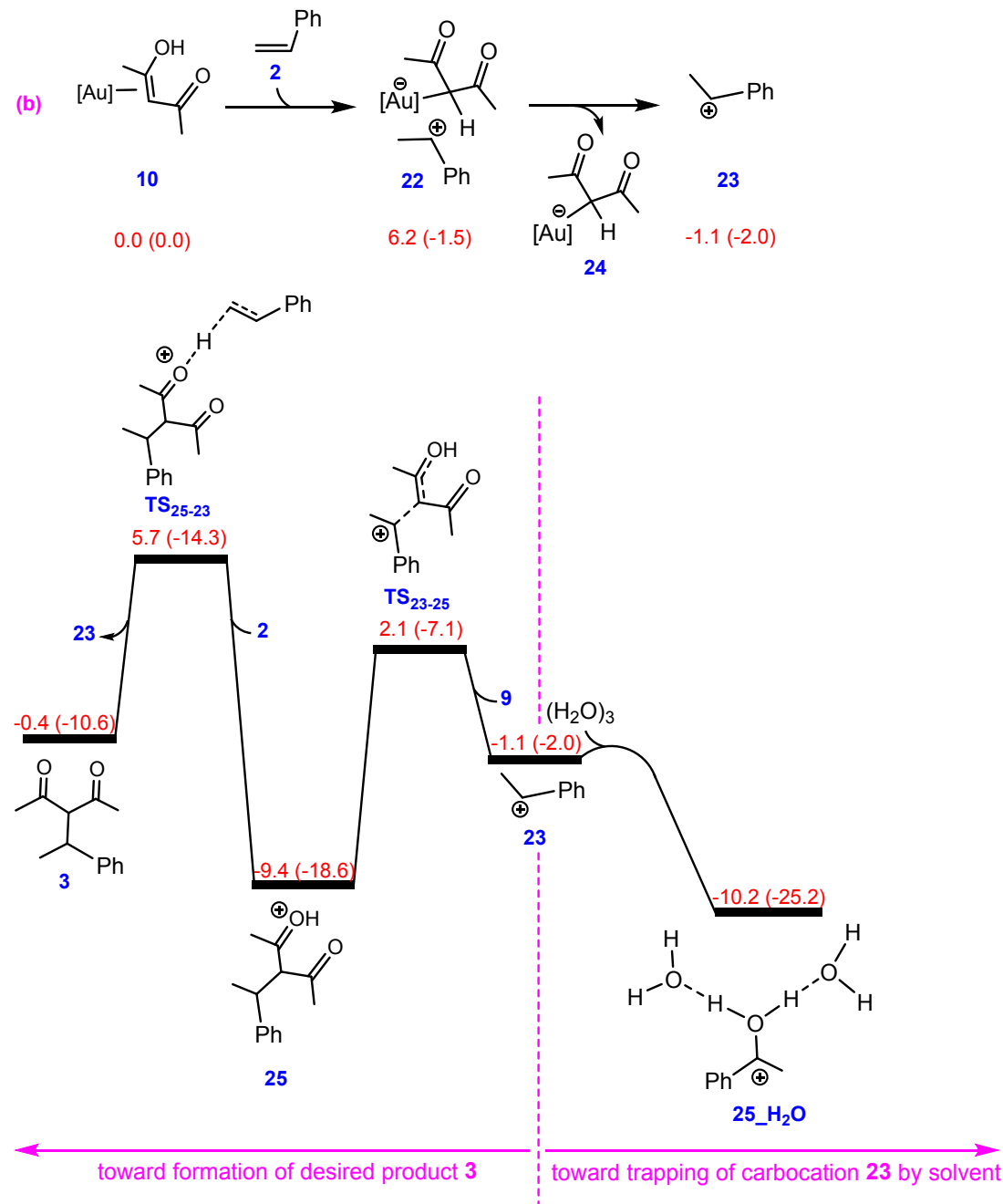

Figure S12. Calculated energy profile for competition between water and enol 9 for trapping carbocation 23. Free energies (potential energies) obtained from SMD/M06/def2TZVP//SMD/B3LYP/6-31G(d), SDD in water are given in $\mathrm{kcal} / \mathrm{mol}$. 
A comparison of different levels of theory. To evaluate whether the relative free energy of the key intermediates and transition structures is influenced by the D3 dispersion and the size of the basis set, we tested different levels of theory as shown in Table S1. These different levels of theory are also utilized to compute the energy of all intermediates and transition structures formed on the most favourable mechanism vi for the gold system (Figure S13). Accordingly, we found that the effect of the dispersion and basis set is not significant on the relative free energies. These additional calculations also support the favourability of mechanism vi over the other mechanisms under $\mathrm{Au}(\mathrm{OTf})_{3}$ catalysis.

Table S1. Relative free energy of key intermediates and transition structures at different levels of theory. Free energies are given in $\mathrm{kcal} / \mathrm{mol}$.

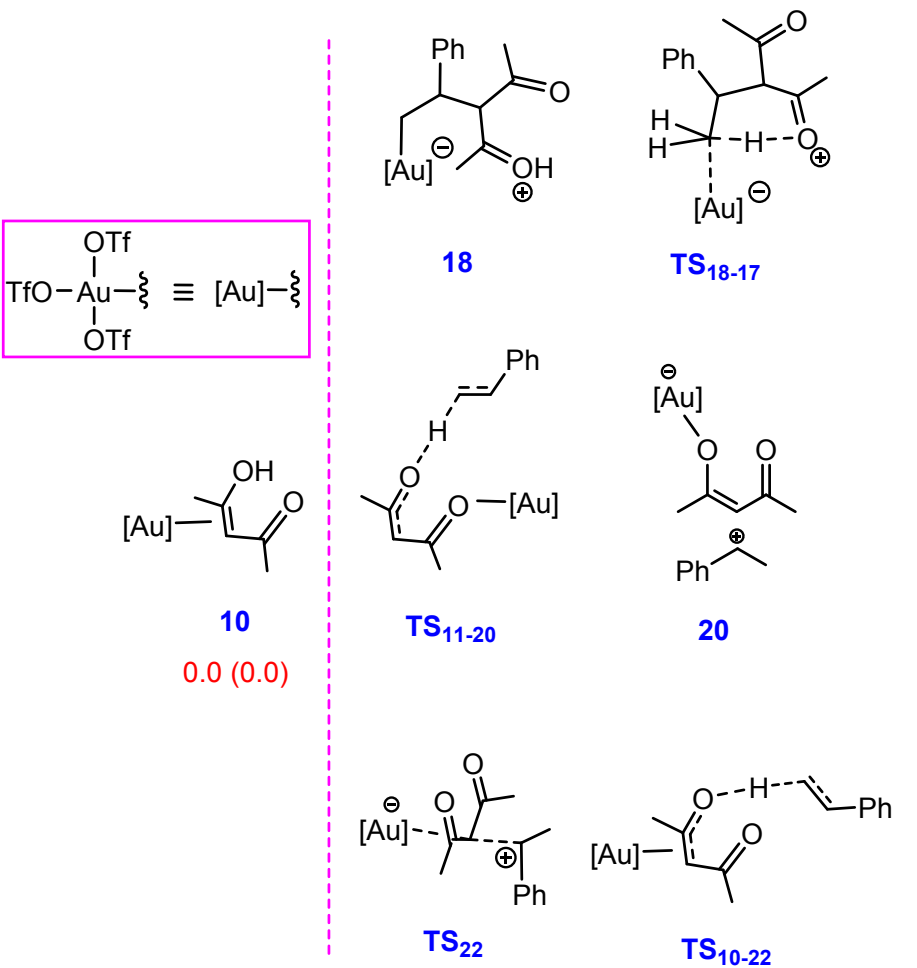<smiles>CCC(c1ccccc1)C(C(C)=O)C(C)=O</smiles>

19<smiles>CC(=O)C(=C(C)O[AlH2])C(C)(c1ccccc1)c1ccccc1</smiles><smiles>CC(=O)C(C)(C(C)=O)C(I)(I)c1ccccc1</smiles>

\begin{tabular}{|c|c|c|c|c|c|c|c|c|c|c|c|c|}
\hline mechanism & \multicolumn{5}{|c|}{ ii } & \multicolumn{3}{c|}{ iii and iv } & \multicolumn{2}{c|}{ v } & \multicolumn{3}{c|}{ vi } \\
\cline { 2 - 13 } method $^{\text {a }}$ & $\mathbf{1 8}$ & $\mathbf{T S}_{18-17}$ & $\mathbf{1 9}$ & $\mathbf{T S}_{19-17}$ & $\mathbf{T S}_{11-20}$ & $\mathbf{2 0}$ & $\mathbf{T S}_{20-21}$ & $\mathbf{T S}_{12-20}$ & $\mathbf{T S}_{22}$ & TS $_{10-22}$ & $\mathbf{2 2}_{2}$ & TS $_{25-23}$ \\
\hline 1 & -7.9 & 33.2 & -7.9 & 45.9 & 36.1 & 31.0 & 34.3 & 40.6 & 50.9 & 16.9 & 6.0 & 14.0 \\
\hline 2 & -7.7 & 33.5 & -8.2 & 45.9 & 36.6 & 33.7 & 34.7 & 41.0 & 50.9 & 17.1 & 4.9 & 13.6 \\
\hline 3 & -7.9 & 35.4 & -7.3 & 46.0 & 38.7 & 29.7 & 35.9 & 41.2 & 51.5 & 16.9 & 4.6 & 11.9 \\
\hline 4 & -9.5 & 32.9 & -8.9 & 42.5 & 36.4 & 27.3 & 33.4 & 38.9 & 48.4 & 15.5 & 4.1 & 11.0 \\
\hline
\end{tabular}

${ }^{a}$ method 1: SMD/M06/def2-TZVP//SMD/B3LYP/6-31G(d), SDD

method 2: SMD/M06/def2-TZVP//SMD/B3LYP-D3/6-31G(d), SDD

method 3: SMD/M06/def2-TZVP//SMD/B3LYP-D3/6-31+G(d,p), SDD.

method 4: SMD/M06-D3/def2-TZVP//SMD/B3LYP-D3/6-31+G(d,p), SDD. 


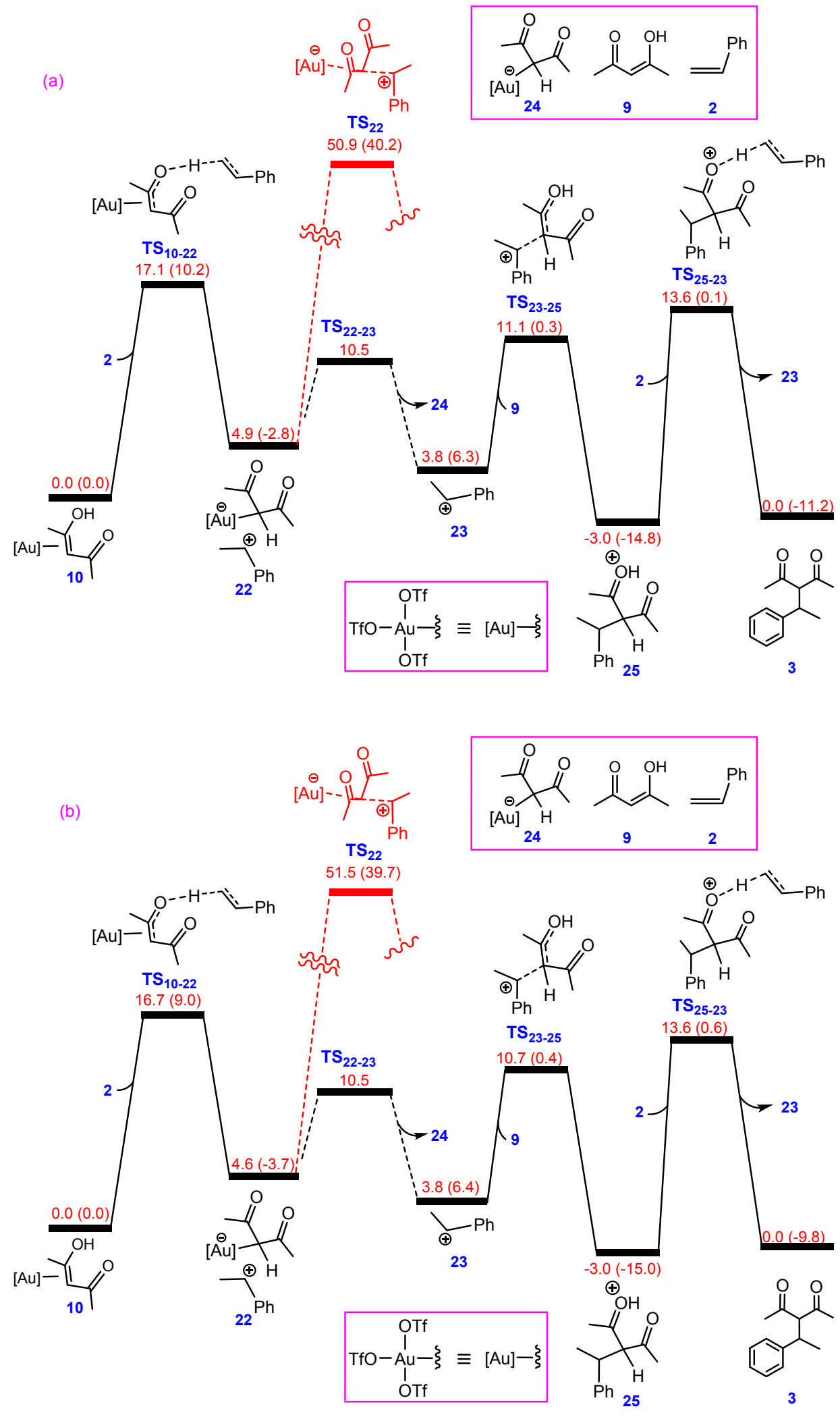

Figure S13. Calculated energy profile for the hydroalkylation via mechanisms $\mathbf{v}$ and vi. Free energies (potential energies) obtained from (a) SMD/M06/def2-TZVP//SMD/B3LYP-D3/6-31G(d), SDD and (b) $\mathrm{SMD} / \mathrm{M} 06 / \mathrm{def} 2-\mathrm{TZVP} / \mathrm{SMD} / \mathrm{B} 3 \mathrm{LYP}-\mathrm{D} 3 / 6-31+\mathrm{G}(\mathrm{d}, \mathrm{p})$, SDD are given in $\mathrm{kcal} / \mathrm{mol}$ where $\mathrm{CH}_{2} \mathrm{Cl}_{2}$ is employed as the solvent. 


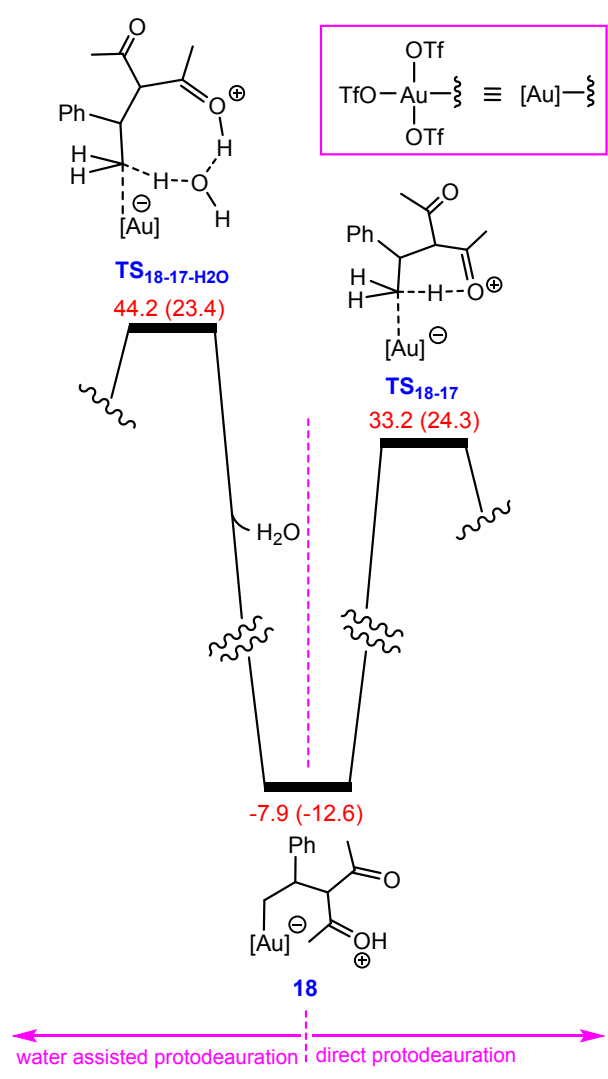

Figure S14. Calculated energy profile comparing the activation barrier to the protodemetallation step via transition structures $\mathbf{T S}_{18-17}$ and $\mathbf{T S} \mathbf{1 8}_{18-17-\mathrm{H} 2 \mathrm{O}}$. Free energies (potential energies) in $\mathrm{CH}_{2} \mathrm{Cl}_{2}$ are given in $\mathrm{kcal} / \mathrm{mol}$.

Coordination of the acac anion as a bidentate ligand to the silver(I) center. The coordination of the acac anion to the silver(I) center as a bidentate ligand leads to formation of 32' lying 3.4 $\mathrm{kcal} / \mathrm{mol}$ higher in energy than transition structure $\mathbf{T S}_{\mathbf{3 2 - 3 4}}$ which indicates that formation of $\mathbf{3 2}^{\prime}$ is unlikely (Figure S15). The relative instability of this trigonal complex (32') can be related to the $\mathrm{d}^{10}$ electron configuration of the silver(I) atom which led it to adopt a linear, two-coordinate geometry.

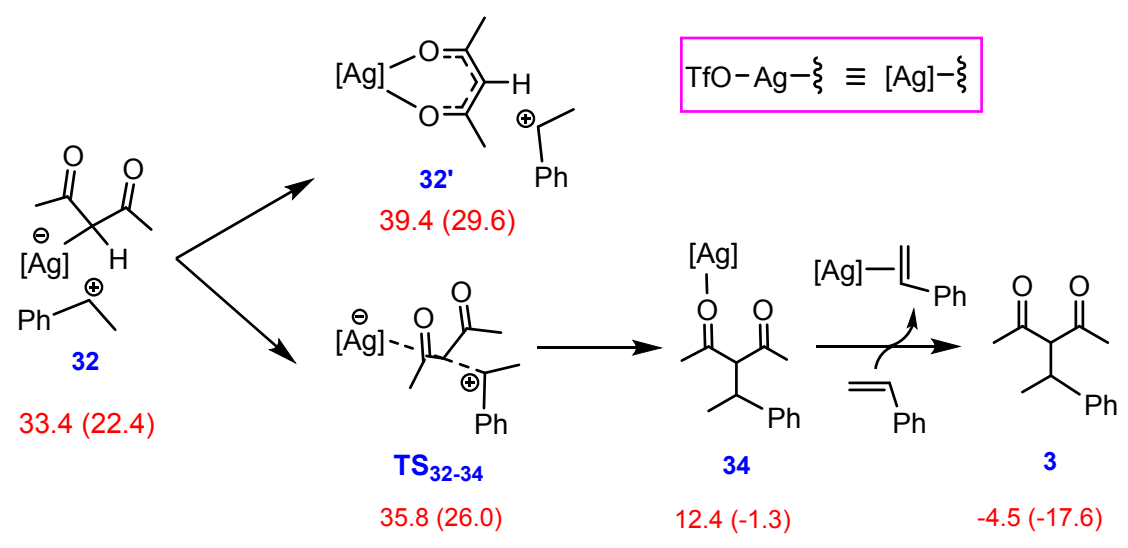

Figure S15. Calculated mechanism for competition between formation of $\mathbf{3 2}^{\prime}$ and the hydroalkylation reaction via mechanism $\mathbf{v}$ starting from ion pair 32. Free energies (potential 
energies) obtained from the SMD/M06/def2-TZVP//SMD/B3LYP/6-31G(d), SDD calculation in nitromethane are given in $\mathrm{kcal} / \mathrm{mol}$.

(a)

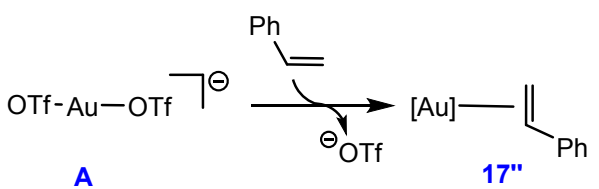

$0.0(0.0)$

$-9.3(-10.4)$

(b)
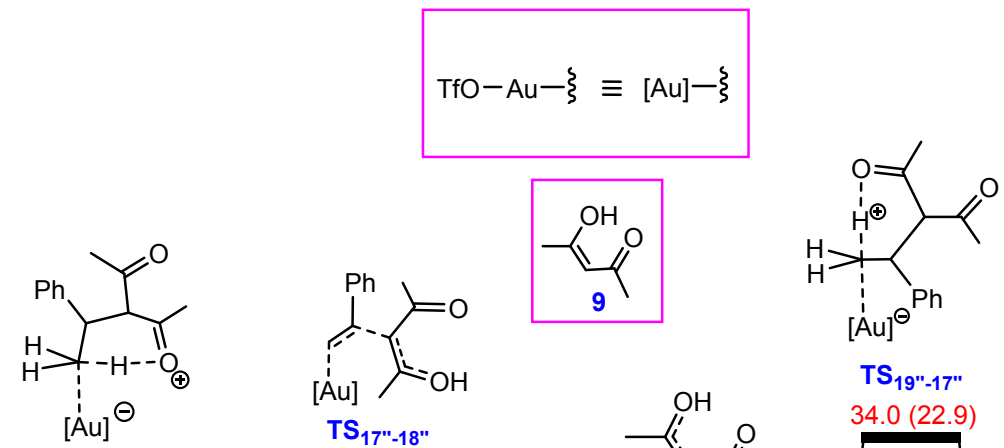

$\mathrm{TS}_{18 "-17 "}$

$26.1(16.5)$
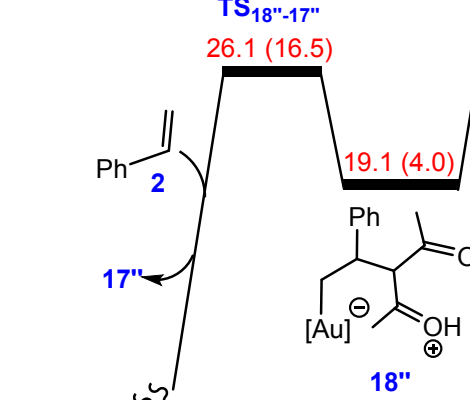

$30.7(15.1)$
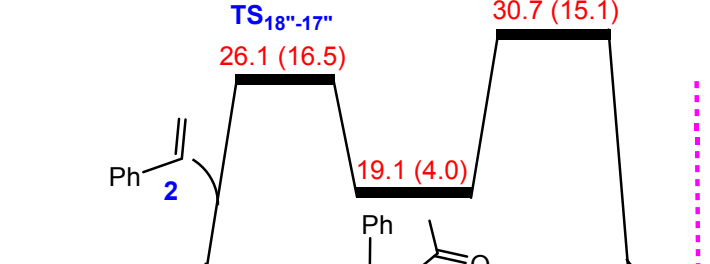

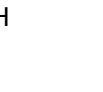

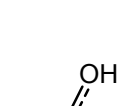

$\mathrm{TS}_{19 "-17 "}$ $34.0(22.9)$

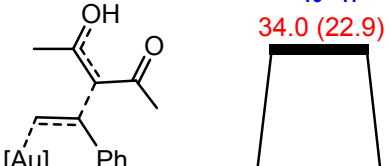
$19.2(6.1)$

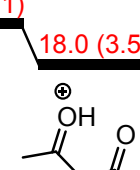<smiles>CC(=O)C(C(C)=O)C(CC(C)C)c1ccccc1</smiles>

[Au]

$19 "$

Figure S16. (a) Calculated reaction free energy for ligand exchange between $\left[\mathrm{Au}(\mathrm{OTf})_{2}\right]^{-}$and styrene 2. (b) Calculated energy profile for the AuOTf-catalysed hydroalkylation reaction via mechanism ii. Free energies (potential energies) are given in $\mathrm{kcal} / \mathrm{mol}$ where $\mathrm{CH}_{2} \mathrm{Cl}_{2}$ is employed as the solvent. 
Impact of the ligands of $\mathrm{AuX}_{3}$ on mechanisms $\mathrm{v}$ and vi. We have considered the effect of these anions on the mechanisms $\mathbf{v}$ and vi by considering $\mathrm{AuX}_{3}$ as the catalyst where $\mathrm{X}=\mathrm{OTf}, \mathrm{SbF}_{6}, \mathrm{TFA}$, and $\mathrm{Cl}$. We found that the activation barrier to the styrene protonation hinges on the identity of the $\mathrm{X}$ ligands (Table S2). The more weakly the coordinating anionic X ligand, the more electron deficient the gold(III) centre, the lower the activation barrier to the protonation process. This is supported by a good correlation between the NPA charge on the enol ligand in 1_. $\mathbf{M}^{\prime}(\mathrm{q}(\mathrm{enol}))$ and the activation energy of the protonation via TS-M' $\left(\mathrm{R}^{2}=0.92\right.$, Figure S17). These findings are consistent with the experimental observations which have indicated that while catalysts such as $\mathrm{AuCl}_{3} / \mathrm{AgOTf}$ and $\mathrm{AuCl}_{3} / \mathrm{AgSbF}_{6}$ give the desired product with good yields, $\mathrm{AuCl}_{3}$ and $\mathrm{AuCl}_{3} / \mathrm{Ag}(\mathrm{TFA})_{3}$ do not. ${ }^{3}$ Finally, it is worth noting that none of these catalysts are capable of mediating the hydroalkylation reaction via mechanism $\mathbf{v}$ due to the high electron deficiency of the gold(III) centre. This is corroborated by the high activation barrier calculated for the demetallation step via transition structure TS-M" (Table S2).

Table S2. NPA charge on the enol ligand in 1_M', q(enol), the activation energy for the protonation step via transition structure TS- $\mathbf{M}^{\prime}$ from $\mathbf{1}_{-} \mathbf{M}^{\prime}, \Delta \mathrm{G}_{1}{ }^{\ddagger}$, and the overall activation energy for the demetallation via transition structure TS_M", $\Delta \mathrm{G}_{2}^{\ddagger}$, where $\mathrm{CH}_{2} \mathrm{Cl}_{2}$ is employed as the solvent.

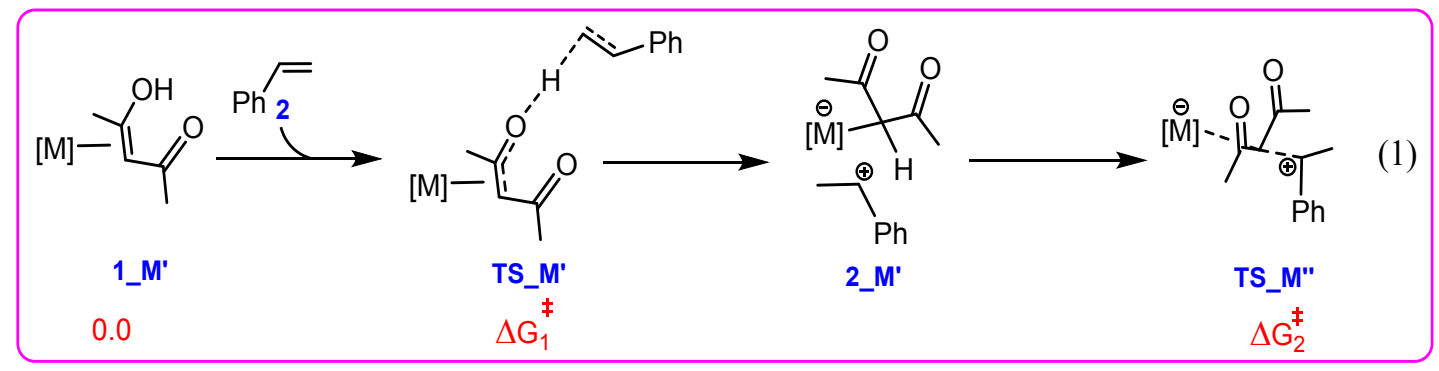

\begin{tabular}{|c|c|c|c|}
\hline$\left[\mathrm{M}^{\prime}\right]$ & $\mathrm{q}(\mathrm{enol})$ & $\Delta \mathrm{G}_{1}^{*}$ & $\Delta \mathrm{G}_{2}{ }^{*}$ \\
\hline$\left[\mathrm{AuCl}_{3}\right]$ & +0.486 & 26.3 & 40.2 \\
\hline$\left[\mathrm{Au}(\mathrm{TFA})_{3}\right]$ & +0.509 & 23.6 & 50.9 \\
\hline$\left[\mathrm{Au}(\mathrm{OTf})_{3}\right]$ & +0.589 & 16.9 & 50.9 \\
\hline$\left[\mathrm{Au}\left(\mathrm{SbF}_{6}\right)_{3}\right]$ & +0.791 & 11.1 & 54.1 \\
\hline
\end{tabular}

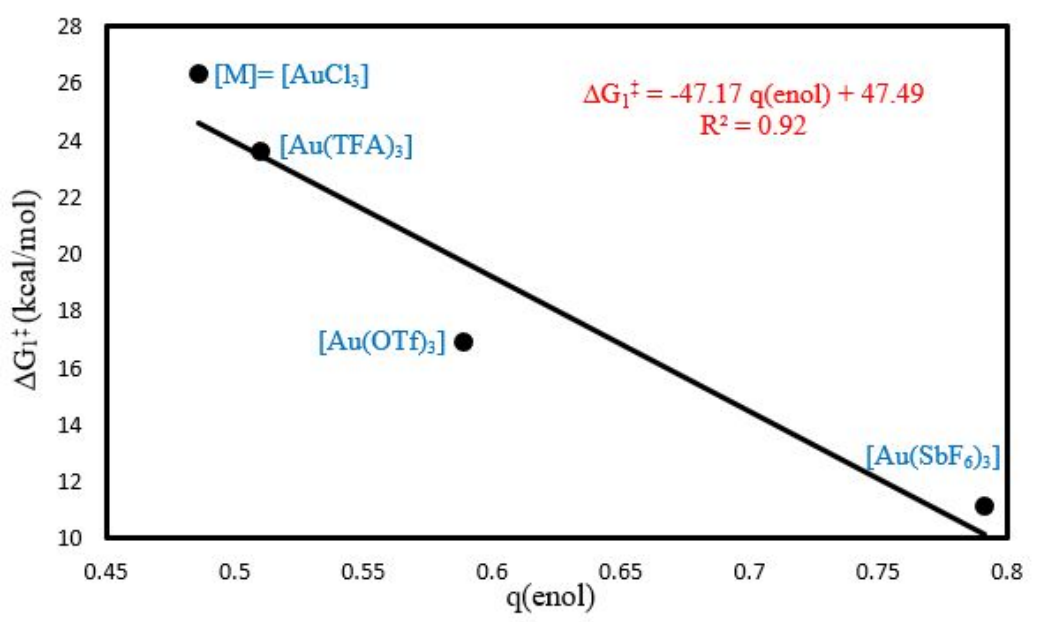

Figure S17. A plot of the correlation between $\mathrm{q}(\mathrm{enol})$ and $\Delta \mathrm{G}_{1}^{\ddagger}$. 
Table S3. Total potential (E), enthalpy $(\mathrm{H})$ and Gibbs free energies $(\mathrm{G})$ of all structures optimized at the SMD/B3LYP/BS1, SMD/B3LYP-D3/BS1, and SMD/B3LYP-D3/BS3 level of theory along with the total potential energies calculated by SMD/M06/BS2//SMD/B3LYP/BS1, SMD/M06/BS2//SMD/B3LYP-D3/BS1, SMD/M06/BS2//SMD/B3LYP/BS3, and SMD/M06$\mathrm{D} 3 / \mathrm{BS} 2 / / \mathrm{SMD} / \mathrm{B} 3 \mathrm{LYP} / \mathrm{BS} 3$ for all the calculated structures.

$$
\begin{aligned}
& \mathrm{BS} 1=6-31 \mathrm{G}(\mathrm{d}), \text { SDD } \\
& \mathrm{BS} 2=\mathrm{def} 2-\mathrm{TZVP} \\
& \mathrm{BS} 3=6-31+\mathrm{G}(\mathrm{d}, \mathrm{p}), \text { SDD }
\end{aligned}
$$

\section{1}

$\mathrm{E}(\mathrm{SMD} / \mathrm{B} 3 \mathrm{LYP} / \mathrm{BS} 1)=-345.800017596 \mathrm{au}$

$\mathrm{H}(\mathrm{SMD} / \mathrm{B} 3 \mathrm{LYP} / \mathrm{BS} 1)=-345.668657 \mathrm{au}$

$\mathrm{G}(\mathrm{SMD} / \mathrm{B} 3 \mathrm{LYP} / \mathrm{BS} 1)=-345.712852 \mathrm{au}$

$\mathrm{E}(\mathrm{SMD} / \mathrm{M} 06 / \mathrm{BS} 2 / / \mathrm{SMD} / \mathrm{B} 3 \mathrm{LYP} / \mathrm{BS} 1)=-345.721697796 \mathrm{au}$

C $\quad-1.96187900 \quad-0.95985700 \quad 0.47667500$

$\mathrm{H} \quad-1.30838800 \quad-1.80853300 \quad 0.24397500$

$\mathrm{H} \quad-2.92690000 \quad-1.08304400 \quad-0.02111300$

$\mathrm{H} \quad-2.10690600 \quad-0.94829400 \quad 1.56486200$

$\begin{array}{llll}\text { C } & -1.29923300 & 0.32071900 & 0.03054400\end{array}$

$\begin{array}{lllll}\mathrm{O} & -1.82752300 & 1.09505900 & -0.74991300\end{array}$

$\begin{array}{lllll}\mathrm{C} & 0.08323100 & 0.62375600 & 0.59985700\end{array}$

$\begin{array}{llll}\mathrm{H} & 0.29208600 & 1.69318600 & 0.49047900\end{array}$

$\begin{array}{llll}\mathrm{H} & 0.11757700 & 0.38884600 & 1.67362900\end{array}$

C $\quad \begin{array}{llll}1.20727900 & -0.18023700 & -0.06140600\end{array}$

$\begin{array}{llll}\mathrm{O} & 0.98904900 & -1.18645200 & -0.71513600\end{array}$

$\begin{array}{lllll}\mathrm{C} & 2.60391700 & 0.35712400 & 0.14966300\end{array}$

$\mathrm{H} \quad 3.34370100 \quad-0.36072900 \quad-0.21292200$

$\mathrm{H} \quad 2.71582300 \quad 1.30146700 \quad-0.39935600$

$\begin{array}{llll}\mathrm{H} & 2.78089900 & 0.57921100 & 1.20883700\end{array}$

\section{$1-\mathrm{CH}_{3} \mathrm{NO}_{2}$}

$\mathrm{E}(\mathrm{SMD} / \mathrm{B} 3 \mathrm{LYP} / \mathrm{BS} 1)=-345.800066184 \mathrm{au}$

$\mathrm{H}(\mathrm{SMD} / \mathrm{B} 3 \mathrm{LYP} / \mathrm{BS} 1)=-345.668781 \mathrm{au}$

$\mathrm{G}(\mathrm{SMD} / \mathrm{B} 3 \mathrm{LYP} / \mathrm{BS} 1)=-345.71294 \mathrm{au}$

$\mathrm{E}(\mathrm{SMD} / \mathrm{M} 06 / \mathrm{BS} 2 / / \mathrm{SMD} / \mathrm{B} 3 \mathrm{LYP} / \mathrm{BS} 1)=-345.722316962 \mathrm{au}$

$\begin{array}{llll}\text { C } & -1.96187900 & -0.95985700 & 0.47667500\end{array}$

$\mathrm{H} \quad \begin{array}{llll}\mathrm{H} & -1.30838800 & -1.80853300 & 0.24397500\end{array}$

$\mathrm{H} \quad-2.92690000 \quad-1.08304400 \quad-0.02111300$

$\mathrm{H} \quad \begin{array}{llll}-2.10690600 & -0.94829400 & 1.56486200\end{array}$

$\begin{array}{llll}\text { C } & -1.29923300 & 0.32071900 & 0.03054400\end{array}$

$\begin{array}{lllll}\mathrm{O} & -1.82752300 & 1.09505900 & -0.74991300\end{array}$

$\begin{array}{llll}\mathrm{C} & 0.08323100 & 0.62375600 & 0.59985700\end{array}$

$\begin{array}{llll}\mathrm{H} & 0.29208600 & 1.69318600 & 0.49047900\end{array}$

$\mathrm{H} \quad \begin{array}{llll}\mathrm{H} & 0.11757700 & 0.38884600 & 1.67362900\end{array}$

C $\quad \begin{array}{llll}1.20727900 & -0.18023700 & -0.06140600\end{array}$

O $\quad 0.98904900 \quad-1.18645200 \quad-0.71513600$

$\begin{array}{llll}\mathrm{C} & 2.60391700 & 0.35712400 & 0.14966300\end{array}$

$\mathrm{H} \quad 3.34370100 \quad-0.36072900 \quad-0.21292200$

$\mathrm{H} \quad 2.71582300 \quad 1.30146700 \quad-0.39935600$

$\begin{array}{llll}\mathrm{H} & 2.78089900 & 0.57921100 & 1.20883700\end{array}$ 


\section{2}

$\mathrm{E}(\mathrm{SMD} / \mathrm{B} 3 \mathrm{LYP} / \mathrm{BS} 1)=-309.652807817 \mathrm{au}$

$\mathrm{H}(\mathrm{SMD} / \mathrm{B} 3 \mathrm{LYP} / \mathrm{BS} 1)=-309.511343 \mathrm{au}$

$\mathrm{G}(\mathrm{SMD} / \mathrm{B} 3 \mathrm{LYP} / \mathrm{BS} 1)=-309.550088 \mathrm{au}$

$\mathrm{E}(\mathrm{SMD} / \mathrm{M} 06 / \mathrm{BS} 2 / / \mathrm{SMD} / \mathrm{B} 3 \mathrm{LYP} / \mathrm{BS} 1)=-309.512867747 \mathrm{au}$

$\begin{array}{llll}\mathrm{C} & -1.36228900 & 1.33134400 & 0.00000000\end{array}$

$\begin{array}{llll}\mathrm{C} & 0.01036300 & 1.09373600 & 0.00000000\end{array}$

$\begin{array}{llll}\mathrm{C} & 0.51553400 & -0.22126600 & 0.00000000\end{array}$

$\begin{array}{llll}\text { C } & -0.40696500 & -1.28367600 & 0.00000000\end{array}$

$\begin{array}{llll}\text { C } & -1.78241700 & -1.04689800 & 0.00000000\end{array}$

$\begin{array}{llll}\mathrm{C} & -2.26658200 & 0.26290100 & 0.00000000\end{array}$

$\begin{array}{llll}\mathrm{H} & -1.73001800 & 2.35434500 & 0.00000000\end{array}$

$\mathrm{H} \quad \begin{array}{llll}\mathrm{H} & 0.69577900 & 1.93658900 & 0.00000000\end{array}$

$\mathrm{H} \quad-0.03584500 \quad-2.30617700 \quad 0.00000000$

$\mathrm{H} \quad-2.47415400 \quad-1.88541100 \quad 0.00000000$

$\mathrm{H} \quad-3.33672100 \quad 0.45240000 \quad 0.00000000$

$\begin{array}{llll}\mathrm{C} & 1.95643000 & -0.53168000 & 0.00000000\end{array}$

$\begin{array}{llll}\mathrm{H} & 2.18748300 & -1.59724900 & 0.00000000\end{array}$

$\begin{array}{llll}\mathrm{C} & 2.97753700 & 0.33656800 & 0.00000000\end{array}$

$\begin{array}{llll}\mathrm{H} & 2.83827000 & 1.41513400 & 0.00000000\end{array}$

$\mathrm{H} \quad 4.00553900 \quad-0.01580600 \quad 0.00000000$

\section{$2-\mathrm{CH}_{3} \mathrm{NO}_{2}$}

$\mathrm{E}(\mathrm{SMD} / \mathrm{B} 3 \mathrm{LYP} / \mathrm{BS} 1)=-309.650530969 \mathrm{au}$

$\mathrm{H}(\mathrm{SMD} / \mathrm{B} 3 \mathrm{LYP} / \mathrm{BS} 1)=-309.509073 \mathrm{au}$

$\mathrm{G}(\mathrm{SMD} / \mathrm{B} 3 \mathrm{LYP} / \mathrm{BS} 1)=-309.547956 \mathrm{au}$

$\mathrm{E}(\mathrm{SMD} / \mathrm{M} 06 / \mathrm{BS} 2 / / \mathrm{SMD} / \mathrm{B} 3 \mathrm{LYP} / \mathrm{BS} 1)=-309.510531382 \mathrm{au}$

$\begin{array}{llll}\mathrm{C} & -1.36228900 & 1.33134400 & 0.00000000\end{array}$

$\begin{array}{llll}\text { C } & 0.01036300 & 1.09373600 & 0.00000000\end{array}$

$\begin{array}{llll}\text { C } & 0.51553400 & -0.22126600 & 0.00000000\end{array}$

$\begin{array}{llll}\mathrm{C} & -0.40696500 & -1.28367600 & 0.00000000\end{array}$

$\begin{array}{llll}\mathrm{C} & -1.78241700 & -1.04689800 & 0.00000000\end{array}$

$\begin{array}{llll}\text { C } & -2.26658200 & 0.26290100 & 0.00000000\end{array}$

$\begin{array}{llll}\mathrm{H} & -1.73001800 & 2.35434500 & 0.00000000\end{array}$

$\mathrm{H} \quad \begin{array}{llll}\mathrm{H} & 0.69577900 & 1.93658900 & 0.00000000\end{array}$

$\mathrm{H} \quad-0.03584500 \quad-2.30617700 \quad 0.00000000$

$\mathrm{H} \quad-2.47415400 \quad-1.88541100 \quad 0.00000000$

$\begin{array}{llll}\mathrm{H} & -3.33672100 & 0.45240000 & 0.00000000\end{array}$

$\begin{array}{llll}\mathrm{C} & 1.95643000 & -0.53168000 & 0.00000000\end{array}$

$\begin{array}{llll}\mathrm{H} & 2.18748300 & -1.59724900 & 0.00000000\end{array}$

$\begin{array}{llll}\mathrm{C} & 2.97753700 & 0.33656800 & 0.00000000\end{array}$

$\begin{array}{llll}\mathrm{H} & 2.83827000 & 1.41513400 & 0.00000000\end{array}$

$\mathrm{H} \quad 4.00553900 \quad-0.01580600 \quad 0.00000000$

3

$\mathrm{E}(\mathrm{SMD} / \mathrm{B} 3 \mathrm{LYP} / \mathrm{BS} 1)=-655.472434823 \mathrm{au}$

$\mathrm{H}(\mathrm{SMD} / \mathrm{B} 3 \mathrm{LYP} / \mathrm{BS} 1)=-655.195385 \mathrm{au}$

$\mathrm{G}(\mathrm{SMD} / \mathrm{B} 3 \mathrm{LYP} / \mathrm{BS} 1)=-655.257317 \mathrm{au}$

$\mathrm{E}(\mathrm{SMD} / \mathrm{M} 06 / \mathrm{BS} 2 / / \mathrm{SMD} / \mathrm{B} 3 \mathrm{LYP} / \mathrm{BS} 1)=-655.261793843 \mathrm{au}$

C $\quad-2.97460800 \quad-1.91470800 \quad-0.73539000$ 


$\begin{array}{lrrr}\mathrm{H} & -2.24074300 & -2.68923800 & -0.48717300 \\ \mathrm{H} & -2.99174800 & -1.82931100 & -1.83018600 \\ \mathrm{H} & -3.96500500 & -2.21203000 & -0.38206000 \\ \mathrm{C} & -2.59465900 & -0.57597000 & -0.14574200 \\ \mathrm{O} & -3.39886500 & 0.11459500 & 0.46017100 \\ \mathrm{C} & -1.14489000 & -0.10442000 & -0.36319500 \\ \mathrm{H} & -0.87940600 & -0.37584400 & -1.39340300 \\ \mathrm{C} & -1.11096700 & 1.42584300 & -0.26376300 \\ \mathrm{C} & -1.55934800 & 2.16561300 & -1.50615900 \\ \mathrm{H} & -2.49065600 & 1.74734300 & -1.90486800 \\ \mathrm{H} & -1.68946000 & 3.22871300 & -1.28878100 \\ \mathrm{H} & -0.79471300 & 2.04866800 & -2.28623000 \\ \mathrm{O} & -0.74263400 & 2.02130300 & 0.73422100 \\ \mathrm{C} & 3.96580100 & 0.06200500 & -0.47002600 \\ \mathrm{C} & 3.35564800 & 0.72245200 & 0.59885400 \\ \mathrm{C} & 2.03305600 & 0.43317000 & 0.94794500 \\ \mathrm{C} & 1.29427400 & -0.52472200 & 0.23742500 \\ \mathrm{C} & 1.92281800 & -1.18356800 & -0.83222100 \\ \mathrm{C} & 3.24205700 & -0.89506600 & -1.18691000 \\ \mathrm{H} & 4.99455600 & 0.28710300 & -0.73915100 \\ \mathrm{H} & 3.90904500 & 1.46693400 & 1.16606100 \\ \mathrm{H} & 1.57523900 & 0.96213300 & 1.77673100 \\ \mathrm{H} & 1.37115100 & -1.93801600 & -1.38978900 \\ \mathrm{H} & 3.70589300 & -1.42258500 & -2.01669900 \\ \mathrm{C} & -0.15309500 & -0.87744400 & 0.57712000 \\ \mathrm{H} & -0.27459600 & -1.93169100 & 0.30037900 \\ \mathrm{C} & -0.49800600 & -0.76413900 & 2.07056100 \\ \mathrm{H} & -0.49132900 & 0.27096400 & 2.41597300 \\ \mathrm{H} & -1.49455400 & -1.17584000 & 2.26595100 \\ \mathrm{H} & 0.21983100 & -1.33876000 & 2.66712200\end{array}$

\begin{tabular}{|c|c|c|c|}
\hline & \\
\hline \\
\hline \multicolumn{4}{|c|}{$\mathrm{E}(\mathrm{SMD} / \mathrm{B} 3 \mathrm{LYP} / \mathrm{BS} 1)=-655.470911734 \mathrm{au}$} \\
\hline \multicolumn{4}{|c|}{$\mathrm{H}(\mathrm{SMD} / \mathrm{B} 3 \mathrm{LYP} / \mathrm{BS} 1)=-655.193896 \mathrm{au}$} \\
\hline \multicolumn{4}{|c|}{$\mathrm{G}(\mathrm{SMD} / \mathrm{B} 3 \mathrm{LYP} / \mathrm{BS} 1)=-655.255167 \mathrm{au}$} \\
\hline \multicolumn{4}{|c|}{$\mathrm{E}(\mathrm{SMD} / \mathrm{M} 06 / \mathrm{BS} 2 / / \mathrm{SMD} / \mathrm{B} 3 \mathrm{LYP} / \mathrm{BS} 1)=-655.260875$} \\
\hline $\mathrm{C}$ & -2.97460800 & -1.91470800 & -0.73539000 \\
\hline $\mathrm{H}$ & -2.24074300 & -2.68923800 & -0.48717300 \\
\hline $\mathrm{H}$ & -2.99174800 & -1.82931100 & -1.83018600 \\
\hline $\mathrm{H}$ & -3.96500500 & -2.21203000 & -0.38206000 \\
\hline $\mathrm{C}$ & -2.59465900 & -0.57597000 & -0.14574200 \\
\hline $\mathrm{O}$ & -3.39886500 & 0.11459500 & 0.46017100 \\
\hline $\mathrm{C}$ & -1.14489000 & -0.10442000 & -0.36319500 \\
\hline $\mathrm{H}$ & -0.87940600 & -0.37584400 & -1.39340300 \\
\hline $\mathrm{C}$ & -1.11096700 & 1.42584300 & -0.26376300 \\
\hline $\mathrm{C}$ & -1.55934800 & 2.16561300 & -1.50615900 \\
\hline $\mathrm{H}$ & -2.49065600 & 1.74734300 & -1.90486800 \\
\hline $\mathrm{H}$ & -1.68946000 & 3.22871300 & -1.28878100 \\
\hline $\mathrm{H}$ & -0.79471300 & 2.04866800 & -2.28623000 \\
\hline $\mathrm{O}$ & -0.74263400 & 2.02130300 & 0.73422100 \\
\hline $\mathrm{C}$ & 3.96580100 & 0.06200500 & -0.47002600 \\
\hline
\end{tabular}




$\begin{array}{lrrr}\mathrm{C} & 3.35564800 & 0.72245200 & 0.59885400 \\ \mathrm{C} & 2.03305600 & 0.43317000 & 0.94794500 \\ \mathrm{C} & 1.29427400 & -0.52472200 & 0.23742500 \\ \mathrm{C} & 1.92281800 & -1.18356800 & -0.83222100 \\ \mathrm{C} & 3.24205700 & -0.89506600 & -1.18691000 \\ \mathrm{H} & 4.99455600 & 0.28710300 & -0.73915100 \\ \mathrm{H} & 3.90904500 & 1.46693400 & 1.16606100 \\ \mathrm{H} & 1.57523900 & 0.96213300 & 1.77673100 \\ \mathrm{H} & 1.37115100 & -1.93801600 & -1.38978900 \\ \mathrm{H} & 3.70589300 & -1.42258500 & -2.01669900 \\ \mathrm{C} & -0.15309500 & -0.87744400 & 0.57712000 \\ \mathrm{H} & -0.27459600 & -1.93169100 & 0.30037900 \\ \mathrm{C} & -0.49800600 & -0.76413900 & 2.07056100 \\ \mathrm{H} & -0.49132900 & 0.27096400 & 2.41597300 \\ \mathrm{H} & -1.49455400 & -1.17584000 & 2.26595100 \\ \mathrm{H} & 0.21983100 & -1.33876000 & 2.66712200\end{array}$

\section{A}

$\mathrm{E}(\mathrm{SMD} / \mathrm{B} 3 \mathrm{LYP} / \mathrm{BS} 1)=-2058.80842897 \mathrm{au}$

$\mathrm{H}(\mathrm{SMD} / \mathrm{B} 3 \mathrm{LYP} / \mathrm{BS} 1)=-2058.732956 \mathrm{au}$

$\mathrm{G}(\mathrm{SMD} / \mathrm{B} 3 \mathrm{LYP} / \mathrm{BS} 1)=-2058.804013 \mathrm{au}$

$\mathrm{E}(\mathrm{SMD} / \mathrm{M} 06 / \mathrm{BS} 2 / / \mathrm{SMD} / \mathrm{B} 3 \mathrm{LYP} / \mathrm{BS} 1)=-2059.00647544 \mathrm{au}$

$\mathrm{Au} \quad-0.00005100 \quad-0.31742900 \quad-0.00003200$

$\begin{array}{llll}\mathrm{O} & 1.76745100 & -0.27781500 & 1.09583000\end{array}$

S $\quad 3.13304500 \quad-0.59791500 \quad 0.45811300$

$\begin{array}{llll}\mathrm{O} & 4.15984800 & -0.68752300 & 1.49942700\end{array}$

$\begin{array}{llll}\mathrm{O} & 3.07820200 & -1.63676800 & -0.57688800\end{array}$

$\begin{array}{lllll}\text { C } & 3.51033800 & 0.98176300 & -0.45686800\end{array}$

F $\quad 3.44204000 \quad 2.02812100 \quad 0.37343500$

F $\quad 2.63777300 \quad 1.17184700 \quad-1.45827600$

F $\quad 4.74311000 \quad 0.91458800 \quad-0.97158800$

$\mathrm{O} \quad-1.76747100 \quad-0.27747800 \quad-1.09581000$

S $\quad-3.13298800 \quad-0.59793400 \quad-0.45807000$

$\begin{array}{lllll}\mathrm{O} & -4.15982400 & -0.68784900 & -1.49932000\end{array}$

$\begin{array}{llll}\mathrm{O} & -3.07779500 & -1.63666600 & 0.57699800\end{array}$

$\begin{array}{llll}\text { C } & -3.51035900 & 0.98177700 & 0.45684200\end{array}$

F $\quad \begin{array}{llll}\text { C } & -3.44416800 & 2.02789400 & -0.37388100\end{array}$

F $\quad \begin{array}{llll}-2.63645400 & 1.17298900 & 1.45691900\end{array}$

$\begin{array}{lllll}\mathrm{F} & -4.74230300 & 0.91367600 & 0.97340400\end{array}$

\footnotetext{
TS $_{\text {A-B }}$

$\mathrm{E}(\mathrm{SMD} / \mathrm{B} 3 \mathrm{LYP} / \mathrm{BS} 1)=-2404.55524411 \mathrm{au}$

$\mathrm{H}(\mathrm{SMD} / \mathrm{B} 3 \mathrm{LYP} / \mathrm{BS} 1)=-2404.352152 \mathrm{au}$

$\mathrm{G}(\mathrm{SMD} / \mathrm{B} 3 \mathrm{LYP} / \mathrm{BS} 1)=-2404.448664 \mathrm{au}$

$\mathrm{E}(\mathrm{SMD} / \mathrm{M} 06 / \mathrm{BS} 2 / / \mathrm{SMD} / \mathrm{B} 3 \mathrm{LYP} / \mathrm{BS} 1)=-2404.6855326 \mathrm{au}$

$\begin{array}{llll}\mathrm{O} & 1.52330500 & -1.05315700 & -0.92181100\end{array}$

S $\quad 3.00709100-0.71089700 \quad-0.99488100$

O $\quad 3.76847600 \quad-1.75421100 \quad-1.69256500$

$\begin{array}{lllll}\mathrm{O} & 3.28365700 & 0.69304200 & -1.34140300\end{array}$

$\begin{array}{llll}\mathrm{C} & 3.51658800 & -0.85948100 & 0.78925300\end{array}$

F $\quad 3.25212300 \quad-2.08487800 \quad 1.25512000$
} 


$\begin{array}{lccc}\mathrm{F} & 4.82833700 & -0.62255700 & 0.91336100 \\ \mathrm{~F} & 2.85167500 & 0.03423100 & 1.54572200 \\ \mathrm{Au} & 0.03312100 & 0.42885800 & -0.20734300 \\ \mathrm{H} & 0.09116200 & 1.93125000 & -0.75222500 \\ \mathrm{C} & -1.15975000 & 2.25015000 & 0.15052600 \\ \mathrm{H} & -1.96311500 & 1.59963000 & 0.52724000 \\ \mathrm{C} & -1.76859200 & 3.09900100 & -0.96830100 \\ \mathrm{C} & -2.36448900 & 2.36266100 & -2.14322500 \\ \mathrm{H} & -1.55982800 & 1.96722600 & -2.77642900 \\ \mathrm{H} & -2.96396000 & 1.50886300 & -1.80857400 \\ \mathrm{H} & -2.97936100 & 3.04654800 & -2.73327000 \\ \mathrm{O} & -1.79750400 & 4.31338500 & -0.86252000 \\ \mathrm{C} & -0.56925700 & 3.05415600 & 1.30736600 \\ \mathrm{O} & -1.11332700 & 3.00612000 & 2.39688700 \\ \mathrm{C} & 0.68520200 & 3.85886900 & 1.06550800 \\ \mathrm{H} & 1.54928800 & 3.18101800 & 1.05491700 \\ \mathrm{H} & 0.65323300 & 4.37032700 & 0.09900200 \\ \mathrm{H} & 0.81884200 & 4.58454000 & 1.87151800 \\ \mathrm{O} & -0.74096400 & -1.42470800 & 0.94563400 \\ \mathrm{~S} & -2.22803500 & -1.65211000 & 1.10654300 \\ \mathrm{O} & -2.54318400 & -2.72699600 & 2.05681700 \\ \mathrm{O} & -3.01683100 & -0.40725600 & 1.20496400 \\ \mathrm{C} & -2.69316300 & -2.34511700 & -0.55580300 \\ \mathrm{~F} & -2.42341500 & -1.45468700 & -1.53277300 \\ \mathrm{~F} & -4.00283900 & -2.62331600 & -0.59587000 \\ \mathrm{~F} & -2.00765100 & -3.46570700 & -0.81318500\end{array}$

\section{B}

$\mathrm{E}(\mathrm{SMD} / \mathrm{B} 3 \mathrm{LYP} / \mathrm{BS} 1)=-2404.57585999 \mathrm{au}$

$\mathrm{H}(\mathrm{SMD} / \mathrm{B} 3 \mathrm{LYP} / \mathrm{BS} 1)=-2404.369803 \mathrm{au}$

$\mathrm{G}(\mathrm{SMD} / \mathrm{B} 3 \mathrm{LYP} / \mathrm{BS} 1)=-2404.464772 \mathrm{au}$

$\mathrm{E}(\mathrm{SMD} / \mathrm{M} 06 / \mathrm{BS} 2 / / \mathrm{SMD} / \mathrm{B} 3 \mathrm{LYP} / \mathrm{BS} 1)=-2404.69850957 \mathrm{au}$

$\begin{array}{lccc}\mathrm{O} & 1.33665000 & 1.14316700 & 0.54967400 \\ \mathrm{~S} & 2.82118600 & 0.98205600 & 0.88998500 \\ \mathrm{O} & 3.40797700 & 2.26421400 & 1.29239600 \\ \mathrm{O} & 3.12309400 & -0.20982900 & 1.69574200 \\ \mathrm{C} & 3.54794700 & 0.61967700 & -0.78538500 \\ \mathrm{~F} & 3.27706400 & 1.61284300 & -1.63823700 \\ \mathrm{~F} & 4.87453800 & 0.47992900 & -0.68700600 \\ \mathrm{~F} & 3.03423300 & -0.52008400 & -1.28301200 \\ \mathrm{Au} & -0.00724400 & -0.54114400 & 0.19233200 \\ \mathrm{H} & 0.76651500 & -1.53203000 & 1.09225400 \\ \mathrm{C} & -1.25882800 & -2.17882000 & -0.15905100 \\ \mathrm{H} & -2.12150100 & -1.68350700 & -0.61479600 \\ \mathrm{C} & -1.70166500 & -2.84279200 & 1.13680600 \\ \mathrm{C} & -2.51598800 & -1.99611800 & 2.09267000 \\ \mathrm{H} & -1.83192400 & -1.42760000 & 2.73683300 \\ \mathrm{H} & -3.14364000 & -1.27479600 & 1.56084100 \\ \mathrm{H} & -3.12738800 & -2.64333500 & 2.72777300 \\ \mathrm{O} & -1.44256400 & -4.01240900 & 1.38096500 \\ \mathrm{C} & -0.60770400 & -3.08897100 & -1.19002300\end{array}$




$\begin{array}{lrrr}\mathrm{O} & -1.11844500 & -3.18153200 & -2.29716400 \\ \mathrm{C} & 0.67218300 & -3.82338300 & -0.85484500 \\ \mathrm{H} & 1.52350000 & -3.16770500 & -1.08110400 \\ \mathrm{H} & 0.72356300 & -4.09777400 & 0.20037200 \\ \mathrm{H} & 0.75347500 & -4.71522000 & -1.48272900 \\ \mathrm{O} & -1.05594300 & 0.83735800 & -1.17020900 \\ \mathrm{~S} & -2.46267400 & 1.39727200 & -0.99530900 \\ \mathrm{O} & -2.87157500 & 2.19063400 & -2.15867000 \\ \mathrm{O} & -3.42358700 & 0.42547800 & -0.44703800 \\ \mathrm{C} & -2.22292300 & 2.63640500 & 0.37209100 \\ \mathrm{~F} & -1.79644500 & 2.02413100 & 1.49197900 \\ \mathrm{~F} & -3.38356300 & 3.24664100 & 0.64081100 \\ \mathrm{~F} & -1.32021700 & 3.55770300 & 0.02128400\end{array}$

\section{9}

$\mathrm{E}(\mathrm{SMD} / \mathrm{B} 3 \mathrm{LYP} / \mathrm{BS} 1)=-345.803099103 \mathrm{au}$

$\mathrm{H}(\mathrm{SMD} / \mathrm{B} 3 \mathrm{LYP} / \mathrm{BS} 1)=-345.671075 \mathrm{au}$

$\mathrm{G}(\mathrm{SMD} / \mathrm{B} 3 \mathrm{LYP} / \mathrm{BS} 1)=-345.712497 \mathrm{au}$

$\mathrm{E}(\mathrm{SMD} / \mathrm{M} 06 / \mathrm{BS} 2 / / \mathrm{SMD} / \mathrm{B} 3 \mathrm{LYP} / \mathrm{BS} 1)=-345.724814539 \mathrm{au}$

$\begin{array}{llll}\mathrm{C} & 2.54839500 & -0.76098600 & 0.00000100\end{array}$

$\mathrm{H} \quad 2.60781400 \quad-1.41066500 \quad 0.88220800$

$\mathrm{H} \quad 3.39382400 \quad-0.06889800 \quad-0.00003100$

$\mathrm{H} \quad 2.60778800 \quad-1.41072400 \quad-0.88216300$

C $\quad 1.24683600 \quad 0.00590200 \quad-0.00000200$

$\begin{array}{llll}\mathrm{O} & 1.26530100 & 1.26130700 & 0.00000500\end{array}$

C $\quad 0.00879700 \quad-0.73474300 \quad-0.00000600$

$\begin{array}{llll}\text { C } & -1.20119100 & -0.08427900 & -0.00000300\end{array}$

$\begin{array}{llll}\mathrm{C} & -2.52684100 & -0.77540300 & 0.00000600\end{array}$

$\mathrm{H} \quad-3.10502300 \quad-0.47488500 \quad-0.88258300$

$\mathrm{H} \quad-2.41176000 \quad-1.86159700 \quad 0.00003100$

$\mathrm{H} \quad-3.10502900 \quad-0.47484500 \quad 0.88257800$

$\begin{array}{lllll}\mathrm{O} & -1.28365800 & 1.24432200 & -0.00000300\end{array}$

$\mathrm{H} \quad-0.32709400 \quad 1.57182200 \quad-0.00001600$

$\mathrm{H} \quad 0.03036600 \quad-1.81818400 \quad-0.00002000$

\section{9- $\mathrm{CH}_{3} \mathrm{NO}_{2}$}

$\mathrm{E}(\mathrm{SMD} / \mathrm{B} 3 \mathrm{LYP} / \mathrm{BS} 1)=-345.80226287 \mathrm{au}$

$\mathrm{H}(\mathrm{SMD} / \mathrm{B} 3 \mathrm{LYP} / \mathrm{BS} 1)=-345.670276 \mathrm{au}$

$\mathrm{G}(\mathrm{SMD} / \mathrm{B} 3 \mathrm{LYP} / \mathrm{BS} 1)=-345.711551 \mathrm{au}$

$\mathrm{E}(\mathrm{SMD} / \mathrm{M} 06 / \mathrm{BS} 2 / / \mathrm{SMD} / \mathrm{B} 3 \mathrm{LYP} / \mathrm{BS} 1)=-345.724069995 \mathrm{au}$

$\begin{array}{lrrr}\mathrm{C} & 2.54839500 & -0.76098600 & 0.00000100 \\ \mathrm{H} & 2.60781400 & -1.41066500 & 0.88220800 \\ \mathrm{H} & 3.39382400 & -0.06889800 & -0.00003100 \\ \mathrm{H} & 2.60778800 & -1.41072400 & -0.88216300 \\ \mathrm{C} & 1.24683600 & 0.00590200 & -0.00000200 \\ \mathrm{O} & 1.26530100 & 1.26130700 & 0.00000500 \\ \mathrm{C} & 0.00879700 & -0.73474300 & -0.00000600 \\ \mathrm{C} & -1.20119100 & -0.08427900 & -0.00000300 \\ \mathrm{C} & -2.52684100 & -0.77540300 & 0.00000600 \\ \mathrm{H} & -3.10502300 & -0.47488500 & -0.88258300 \\ \mathrm{H} & -2.41176000 & -1.86159700 & 0.00003100\end{array}$




$\begin{array}{lrrr}\mathrm{H} & -3.10502900 & -0.47484500 & 0.88257800 \\ \mathrm{O} & -1.28365800 & 1.24432200 & -0.00000300 \\ \mathrm{H} & -0.32709400 & 1.57182200 & -0.00001600 \\ \mathrm{H} & 0.03036600 & -1.81818400 & -0.00002000\end{array}$

10

$\mathrm{E}(\mathrm{SMD} / \mathrm{B} 3 \mathrm{LYP} / \mathrm{BS} 1)=-3365.80153675 \mathrm{au}$
$\mathrm{H}(\mathrm{SMD} / \mathrm{B} 3 \mathrm{LYP} / \mathrm{BS} 1)=-3365.554924 \mathrm{au}$
$\mathrm{G}(\mathrm{SMD} / \mathrm{B} 3 \mathrm{LYP} / \mathrm{BS} 1)=-3365.661903 \mathrm{au}$

$\mathrm{E}(\mathrm{SMD} / \mathrm{M} 06 / \mathrm{BS} 2 / / \mathrm{SMD} / \mathrm{B} 3 \mathrm{LYP} / \mathrm{BS} 1)=-3366.0222285 \mathrm{au}$

$\begin{array}{llll}\mathrm{C} & -1.85485700 & 3.52008200 & 1.02935000\end{array}$

$\begin{array}{llll}\mathrm{H} & -2.75041200 & 4.03806200 & 0.64882600\end{array}$

$\mathrm{H} \quad-1.35260900 \quad 4.16241000 \quad 1.75425300$

$\begin{array}{llll}\mathrm{H} & -2.20322400 & 2.58744400 & 1.47922400\end{array}$

C $\quad-0.98974300 \quad 3.25535400 \quad-0.13320100$

$\begin{array}{llll}\mathrm{O} & 0.01685500 & 4.01603900 & -0.32041400\end{array}$

$\begin{array}{llll}\mathrm{C} & -1.27368800 & 2.17595800 & -1.10539400\end{array}$

$\begin{array}{llll}\mathrm{C} & -0.65970000 & 2.36617500 & -2.47932000\end{array}$

$\begin{array}{llll}\mathrm{O} & 0.32875000 & 3.10160000 & -2.60090300\end{array}$

C $\quad-1.28242800 \quad 1.67485400 \quad-3.64678300$

$\mathrm{H} \quad-2.22610400 \quad 2.18557700 \quad-3.88628800$

$\mathrm{H} \quad-0.61703700 \quad 1.72064600 \quad-4.51106000$

$\mathrm{H} \quad-1.53310300 \quad 0.63638500 \quad-3.40531100$

$\mathrm{H} \quad-2.33135200 \quad 1.91808600 \quad-1.12768100$

$\mathrm{H} \quad \begin{array}{llll}0.43919900 & 3.77151800 & -1.24233700\end{array}$

$\mathrm{Au} \quad-0.39193200 \quad 0.35296100 \quad-0.39090700$

$\begin{array}{lllll}\mathrm{O} & 0.45493400 & -1.49135700 & 0.19721700\end{array}$

S $\quad 0.70528200 \quad-2.61505600 \quad-0.85275500$

O $\quad 0.50461700 \quad-3.92332000 \quad-0.23357500$

$\begin{array}{lllll}\mathrm{O} & 0.09669300 & -2.31579500 & -2.15229100\end{array}$

C $\quad 2.54387700 \quad-2.44983300 \quad-1.11250600$

F $\quad 3.19125400 \quad-2.60793200 \quad 0.04217000$

F $\quad 2.94529200 \quad-3.38376500 \quad-1.97745500$

F $\quad \begin{array}{llll}2.82238100 & -1.24056800 & -1.61434400\end{array}$

$\begin{array}{llll}\mathrm{O} & 2.85092800 & 2.93341400 & 0.90253700\end{array}$

$\begin{array}{llll}\mathrm{S} & 1.97788600 & 1.79526400 & 1.16858300\end{array}$

$\begin{array}{lllll}\mathrm{O} & 0.91092400 & 1.88174500 & 2.16808600\end{array}$

$\begin{array}{llll}\text { O } & 1.42476200 & 1.28518600 & -0.22485400\end{array}$

$\begin{array}{llll}\mathrm{C} & 3.11166700 & 0.40846900 & 1.71318400\end{array}$

$\begin{array}{llll}\mathrm{F} & 3.82942600 & 0.86078100 & 2.74166800\end{array}$

F $\quad \begin{array}{llll}2.40020500 & -0.64621600 & 2.09881400\end{array}$

$\begin{array}{lllll}\mathrm{F} & 3.92304500 & 0.07136000 & 0.71405700\end{array}$

$\begin{array}{llll}\mathrm{O} & -3.52085200 & 0.72224300 & 0.95202600\end{array}$

S $\quad-3.35319700 \quad-0.59957000 \quad 0.34365500$

$\begin{array}{llll}\mathrm{O} & -4.48442100 & -1.27639200 & -0.27934000\end{array}$

O $\quad-2.16322700 \quad-0.63297400 \quad-0.68891600$

$\begin{array}{llll}\text { C } & -2.74055100 & -1.72157500 & 1.70808000\end{array}$

F $\quad-3.72759200 \quad-1.88656700 \quad 2.58668500$

F $\quad \begin{array}{llll}-2.38251300 & -2.89736800 & 1.20315500\end{array}$

F $\quad-1.69366000 \quad-1.15575600 \quad 2.31894900$ 


\section{1}

$\mathrm{E}(\mathrm{SMD} / \mathrm{B} 3 \mathrm{LYP} / \mathrm{BS} 1)=-3365.78850536 \mathrm{au}$

$\mathrm{H}(\mathrm{SMD} / \mathrm{B} 3 \mathrm{LYP} / \mathrm{BS} 1)=-3365.540819 \mathrm{au}$

$\mathrm{G}(\mathrm{SMD} / \mathrm{B} 3 \mathrm{LYP} / \mathrm{BS} 1)=-3365.651882 \mathrm{au}$

$\mathrm{E}(\mathrm{SMD} / \mathrm{M} 06 / \mathrm{BS} 2 / / \mathrm{SMD} / \mathrm{B} 3 \mathrm{LYP} / \mathrm{BS} 1)=-3365.99972695 \mathrm{au}$

$\begin{array}{llll}\mathrm{C} & 2.76579400 & 1.43768700 & -1.80146000\end{array}$

$\mathrm{H} \quad 3.40092600 \quad 2.12779900 \quad-2.35917500$

$\mathrm{H} \quad \begin{array}{llll}\mathrm{H} & 1.72167000 & 1.57483400 & -2.10019800\end{array}$

$\mathrm{H} \quad 3.04304100 \quad 0.40652200 \quad-2.05331800$

$\begin{array}{llll}\mathrm{C} & 2.93421300 & 1.62448700 & -0.33473500\end{array}$

$\begin{array}{llll}\text { O } & 2.14371200 & 1.02550400 & 0.51786500\end{array}$

$\begin{array}{llll}\text { C } & 3.97343600 & 2.41145800 & 0.18605700\end{array}$

$\begin{array}{llll}\text { C } & 4.19437900 & 2.63596100 & 1.54326800\end{array}$

$\begin{array}{llll}\mathrm{O} & 3.45412800 & 2.11157500 & 2.49775400\end{array}$

$\begin{array}{llll}\mathrm{C} & 5.29230200 & 3.50645400 & 2.04550900\end{array}$

$\mathrm{H} \quad 5.93382500 \quad 2.93137200 \quad 2.72419200$

$\mathrm{H} \quad \begin{array}{llll}\mathrm{H} & 5.89153100 & 3.90858100 & 1.22779200\end{array}$

$\mathrm{H} \quad 4.86401500 \quad 4.33247400 \quad 2.62675500$

$\mathrm{H} \quad 4.64479500 \quad 2.88191600 \quad-0.51998800$

$\begin{array}{llll}\mathrm{H} & 2.75086100 & 1.54486900 & 2.09868900\end{array}$

$\begin{array}{llll}\mathrm{Au} & 0.38203600 & 0.04531300 & 0.16528400\end{array}$

$\begin{array}{llll}\mathrm{O} & -1.40123400 & -0.91413800 & -0.03560300\end{array}$

S $\quad-2.09777600 \quad-1.64314700 \quad 1.18926100$

$\begin{array}{llll}\mathrm{O} & -2.97121900 & -2.67016700 & 0.63457000\end{array}$

$\begin{array}{llll}\mathrm{O} & -1.16399500 & -1.93980700 & 2.27082900\end{array}$

$\begin{array}{llll}\mathrm{C} & -3.20979500 & -0.29353700 & 1.84895900\end{array}$

F $\quad-4.13871200 \quad 0.02515600 \quad 0.95287000$

F $\quad \begin{array}{llll}-3.79299300 & -0.74267300 & 2.95933300\end{array}$

F $\quad-2.47581100 \quad 0.78588000 \quad 2.13984900$

$\begin{array}{llll}\mathrm{O} & -0.86620700 & 3.92211000 & -1.31421200\end{array}$

S $\quad-1.04440000 \quad 2.47545200 \quad-1.38820800$

$\begin{array}{lllll}\mathrm{O} & -0.55857000 & 1.72020000 & -2.54445000\end{array}$

$\begin{array}{lllll}\mathrm{O} & -0.54071600 & 1.86159700 & -0.02278100\end{array}$

C $\quad-2.89053200 \quad 2.18184500 \quad-1.30184700$

F $\quad-3.46289300 \quad 2.91060000 \quad-2.25985300$

F $\quad-3.15782800 \quad 0.89479200 \quad-1.50382600$

F $\quad-3.35319300 \quad 2.56168900 \quad-0.11273300$

O $\quad 2.88952000 \quad-1.76405800 \quad-1.33673000$

S $\quad 2.14097300 \quad-2.58013400 \quad-0.38276900$

$\begin{array}{llll}\mathrm{O} & 2.80926300 & -3.61425100 & 0.39843400\end{array}$

O $\quad \begin{array}{llll}\text { O } & 1.29667900 & -1.71720900 & 0.63271800\end{array}$

C $\quad \begin{array}{llll}0.79963400 & -3.42718700 & -1.37031900\end{array}$

F $\quad 1.36648600 \quad-4.32527000 \quad-2.17240600$

F $\quad-0.05733300 \quad-4.03094800 \quad-0.55038300$

F $\quad 0.15008300 \quad-2.51885800 \quad-2.10557000$

12

$\mathrm{E}(\mathrm{SMD} / \mathrm{B} 3 \mathrm{LYP} / \mathrm{BS} 1)=-3365.78220633 \mathrm{au}$

$\mathrm{H}(\mathrm{SMD} / \mathrm{B} 3 \mathrm{LYP} / \mathrm{BS} 1)=-3365.536365 \mathrm{au}$

$\mathrm{G}(\mathrm{SMD} / \mathrm{B} 3 \mathrm{LYP} / \mathrm{BS} 1)=-3365.646596 \mathrm{au}$

$\mathrm{E}(\mathrm{SMD} / \mathrm{M} 06 / \mathrm{BS} 2 / / \mathrm{SMD} / \mathrm{B} 3 \mathrm{LYP} / \mathrm{BS} 1)=-3365.99306922 \mathrm{au}$ 


$\begin{array}{lrrr}\mathrm{C} & 2.95393500 & 1.23719500 & -1.47319500 \\ \mathrm{H} & 3.83358100 & 0.78379200 & -1.94448100 \\ \mathrm{H} & 2.99407100 & 2.31152900 & -1.70800600 \\ \mathrm{H} & 2.04357900 & 0.79905800 & -1.88001900 \\ \mathrm{C} & 3.07001600 & 1.09677300 & -0.00413800 \\ \mathrm{O} & 2.17474400 & 0.64593300 & 0.75266300 \\ \mathrm{C} & 4.30626700 & 1.56894400 & 0.69234000 \\ \mathrm{C} & 4.06913700 & 2.89527300 & 1.47045400 \\ \mathrm{O} & 2.95905900 & 3.26303300 & 1.78817700 \\ \mathrm{C} & 5.33165500 & 3.64730400 & 1.80113100 \\ \mathrm{H} & 5.10999600 & 4.45912200 & 2.49721700 \\ \mathrm{H} & 6.08132300 & 2.97175000 & 2.22938800 \\ \mathrm{H} & 5.75782100 & 4.06204900 & 0.87839800 \\ \mathrm{Au} & 0.30276200 & -0.07127600 & 0.23936700 \\ \mathrm{O} & -1.56275600 & -0.78187400 & -0.13851600 \\ \mathrm{~S} & -2.45875000 & -1.47137400 & 0.97569800 \\ \mathrm{O} & -3.57465300 & -2.08988400 & 0.26996600 \\ \mathrm{O} & -1.67782800 & -2.21083500 & 1.95967600 \\ \mathrm{C} & -3.14811800 & 0.00655200 & 1.89172600 \\ \mathrm{~F} & -3.88370600 & 0.75746100 & 1.07824100 \\ \mathrm{~F} & -3.89955600 & -0.43866800 & 2.89637900 \\ \mathrm{~F} & -2.13908100 & 0.73720000 & 2.38375200 \\ \mathrm{H} & 4.60417300 & 0.82905000 & 1.44691200 \\ \mathrm{O} & -0.21988300 & 3.75073700 & -1.58915200 \\ \mathrm{~S} & -0.70703700 & 2.37801200 & -1.50007900 \\ \mathrm{O} & -0.36064000 & 1.39277100 & -2.52663100 \\ \mathrm{O} & -0.39135100 & 1.83133300 & -0.04741000 \\ \mathrm{C} & -2.57535900 & 2.50010200 & -1.45522100 \\ \mathrm{~F} & -2.95741100 & 3.25225500 & -2.48685100 \\ \mathrm{~F} & -3.11530300 & 1.29025500 & -1.56249400 \\ \mathrm{~F} & -2.96019500 & 3.07106700 & -0.31638800 \\ \mathrm{O} & 2.76002100 & -1.87043800 & -1.06079800 \\ \mathrm{~S} & 1.96215400 & -2.74114300 & -0.19606900 \\ \mathrm{O} & 2.59679300 & -3.76866200 & 0.62037200 \\ \mathrm{O} & 0.99403000 & -1.92409800 & 0.74443700 \\ \mathrm{C} & 0.74081600 & -3.60990200 & -1.31180800 \\ \mathrm{~F} & 1.41005200 & -4.42896200 & -2.12022500 \\ \mathrm{~F} & -0.12593100 & -4.30368000 & -0.58016300 \\ \mathrm{~F} & 0.08406800 & -2.70161400 & -2.04067000 \\ \mathrm{H} & 5.12862000 & 1.69117200 & -0.01646700\end{array}$

13

$\mathrm{E}(\mathrm{SMD} / \mathrm{B} 3 \mathrm{LYP} / \mathrm{BS} 1)=-3019.943277 \mathrm{au}$

$\mathrm{H}(\mathrm{SMD} / \mathrm{B} 3 \mathrm{LYP} / \mathrm{BS} 1)=-3019.832443 \mathrm{au}$

$\mathrm{G}(\mathrm{SMD} / \mathrm{B} 3 \mathrm{LYP} / \mathrm{BS} 1)=-3019.903307 \mathrm{au}$

$\mathrm{E}(\mathrm{SMD} / \mathrm{M} 06 / \mathrm{BS} 2 / / \mathrm{SMD} / \mathrm{B} 3 \mathrm{LYP} / \mathrm{BS} 1)=-3020.228244 \mathrm{au}$

$\begin{array}{llcc}\mathrm{Au} & 2.28283300 & 0.15495400 & 0.37135300 \\ \mathrm{Au} & -2.62375700 & -0.04774600 & -0.12700500 \\ \mathrm{O} & -4.18572400 & -1.09214300 & 0.57410500 \\ \mathrm{~S} & -5.38139700 & -1.58721500 & -0.38586400 \\ \mathrm{O} & -6.44971100 & -1.95544200 & 0.53291100\end{array}$




\begin{tabular}{lccc}
$\mathrm{O}$ & -5.59316000 & -0.69316700 & -1.51399900 \\
$\mathrm{C}$ & -4.65832300 & -3.17561600 & -1.07088200 \\
$\mathrm{~F}$ & -5.60716700 & -3.79822100 & -1.75910200 \\
$\mathrm{~F}$ & -4.23151500 & -3.94130500 & -0.07591700 \\
$\mathrm{~F}$ & -3.63945900 & -2.87069300 & -1.87924100 \\
$\mathrm{O}$ & -3.78698000 & 1.58439500 & -0.30965200 \\
$\mathrm{~S}$ & -3.85155000 & 2.66995600 & 0.86758600 \\
$\mathrm{O}$ & -2.63991900 & 2.65429200 & 1.68106300 \\
$\mathrm{O}$ & -4.34571000 & 3.88886000 & 0.24218900 \\
$\mathrm{C}$ & -5.23897500 & 1.99148200 & 1.93464600 \\
$\mathrm{~F}$ & -4.79348400 & 0.93248500 & 2.60821800 \\
$\mathrm{~F}$ & -5.60851500 & 2.94442200 & 2.78272000 \\
$\mathrm{~F}$ & -6.26390100 & 1.64069300 & 1.16447500 \\
$\mathrm{O}$ & 3.03984800 & -0.78459900 & 1.98562100 \\
$\mathrm{~S}$ & 3.99253300 & -2.06354800 & 1.86842700 \\
$\mathrm{O}$ & 3.72235600 & -2.87364000 & 3.04922600 \\
$\mathrm{O}$ & 3.96500600 & -2.62845000 & 0.52197400 \\
$\mathrm{C}$ & 5.69789400 & -1.31417900 & 2.13004400 \\
$\mathrm{~F}$ & 5.63381800 & -0.36309700 & 3.05530900 \\
$\mathrm{~F}$ & 6.14975900 & -0.81081200 & 0.98677100 \\
$\mathrm{~F}$ & 6.50198300 & -2.29210000 & 2.53794300 \\
$\mathrm{O}$ & 4.08381600 & 1.05437400 & 0.26873700 \\
$\mathrm{~S}$ & 4.67121800 & 1.89779000 & -0.95456000 \\
$\mathrm{O}$ & 3.71466700 & 2.83086000 & -1.52852900 \\
$\mathrm{O}$ & 5.98189900 & 2.34117100 & -0.49583000 \\
$\mathrm{C}$ & 4.93981600 & 0.53624900 & -2.21092800 \\
$\mathrm{~F}$ & 3.77635800 & -0.09266400 & -2.42298700 \\
$\mathrm{~F}$ & 5.83494400 & -0.32975500 & -1.75153800 \\
$\mathrm{~F}$ & 5.36862800 & 1.08343600 & -3.34181600 \\
$\mathrm{O}$ & 0.19710200 & -3.27596800 & 1.02126300 \\
$\mathrm{O}$ & 1.46081900 & 1.12282200 & -1.27575800 \\
$\mathrm{~S}$ & -0.12599600 & -2.10872100 & 0.22597700 \\
$\mathrm{~F}$ & -1.63314200 & -1.88252400 & 0.06444500 \\
$\mathrm{~F}$ & 0.44082000 & -2.41203000 & -1.55802600 \\
$\mathrm{C}$ & 1.33410100 & 4.08357500 & -0.50457400 \\
$\mathrm{~F}$ & -0.16031600 & -3.51528400 & -1.96695500 \\
$\mathrm{~F}$ & 1.75881400 & -2.54812100 & -1.58224400 \\
$\mathrm{~F}$ & 0.06970000 & -1.37194400 & -2.29848700 \\
$\mathrm{O}$ & 0.43727200 & -0.76326700 & 0.69258500 \\
$\mathrm{~S}$ & 0.13733100 & 1.91455800 & -1.38302800 \\
$\mathrm{O}$ & -1.02935700 & 1.13590800 & -0.86363100 \\
$\mathrm{O}$ & -0.00159100 & 2.44860200 & -2.72291700 \\
\hline & 0.28596700 & 3.35451500 & -0.16924200 \\
$\mathrm{~F}$ & -0.82820200 & 4.06234600 & -0.26507400 \\
$\mathrm{~F}$ & 0.43285900 & 2.87439700 & 1.06315100 \\
$\mathrm{~F}$ & & & \\
$\mathrm{~F}$ & & &
\end{tabular}

14

$\mathrm{E}(\mathrm{SMD} / \mathrm{B} 3 \mathrm{LYP} / \mathrm{BS} 1)=-3019.9408117 \mathrm{au}$

$\mathrm{H}(\mathrm{SMD} / \mathrm{B} 3 \mathrm{LYP} / \mathrm{BS} 1)=-3019.828316 \mathrm{au}$

$\mathrm{G}(\mathrm{SMD} / \mathrm{B} 3 \mathrm{LYP} / \mathrm{BS} 1)=-3019.918483 \mathrm{au}$

$\mathrm{E}(\mathrm{SMD} / \mathrm{M} 06 / \mathrm{BS} 2 / / \mathrm{SMD} / \mathrm{B} 3 \mathrm{LYP} / \mathrm{BS} 1)=-3020.22493012 \mathrm{au}$ 


$\begin{array}{lccc}\mathrm{Au} & 0.20598400 & 0.25031700 & -0.20801300 \\ \mathrm{O} & -1.36952800 & 0.63415600 & 1.00369300 \\ \mathrm{~S} & -2.13034900 & 2.03022800 & 0.89802400 \\ \mathrm{O} & -2.94571400 & 2.13779900 & 2.10025800 \\ \mathrm{O} & -1.23308700 & 3.10085300 & 0.47734600 \\ \mathrm{C} & -3.28887200 & 1.70105700 & -0.53494300 \\ \mathrm{~F} & -4.04397700 & 0.64176800 & -0.26760300 \\ \mathrm{~F} & -4.05112200 & 2.77457200 & -0.71497200 \\ \mathrm{~F} & -2.56858400 & 1.47179800 & -1.63886800 \\ \mathrm{O} & -1.35226900 & -3.48769100 & -1.75893800 \\ \mathrm{~S} & -0.52058200 & -2.73584200 & -0.82914700 \\ \mathrm{O} & 0.93301900 & -2.85998900 & -0.82025900 \\ \mathrm{O} & -0.97659200 & -1.21542500 & -0.95288400 \\ \mathrm{C} & -1.11012400 & -3.14207700 & 0.89940500 \\ \mathrm{~F} & -0.89215700 & -4.43226100 & 1.13072600 \\ \mathrm{~F} & -0.42152700 & -2.40525500 & 1.77881400 \\ \mathrm{~F} & -2.40562800 & -2.86992400 & 1.00742900 \\ \mathrm{O} & 2.01942900 & 0.01608600 & -1.27374100 \\ \mathrm{~S} & 2.75690300 & 1.18226100 & -0.56544800 \\ \mathrm{O} & 3.50447300 & 2.09950500 & -1.39373200 \\ \mathrm{O} & 1.60173300 & 1.76798900 & 0.28879700 \\ \mathrm{C} & 3.92867000 & 0.35938500 & 0.66627400 \\ \mathrm{~F} & 4.84434700 & -0.29478500 & -0.02456800 \\ \mathrm{~F} & 4.47268200 & 1.31274600 & 1.40110600 \\ \mathrm{~F} & 3.22173500 & -0.47174900 & 1.42009100\end{array}$

\section{5}

$\mathrm{E}(\mathrm{SMD} / \mathrm{B} 3 \mathrm{LYP} / \mathrm{BS} 1)=-2404.1826797 \mathrm{au}$

$\mathrm{H}(\mathrm{SMD} / \mathrm{B} 3 \mathrm{LYP} / \mathrm{BS} 1)=-2403.973171 \mathrm{au}$

$\mathrm{G}(\mathrm{SMD} / \mathrm{B} 3 \mathrm{LYP} / \mathrm{BS} 1)=-2404.060485 \mathrm{au}$

$\mathrm{E}(\mathrm{SMD} / \mathrm{M} 06 / \mathrm{BS} 2 / / \mathrm{SMD} / \mathrm{B} 3 \mathrm{LYP} / \mathrm{BS} 1)=-2404.28020938 \mathrm{au}$

$\mathrm{Au} \quad-0.72783400 \quad-0.18076200 \quad-0.12223700$

$\begin{array}{llll}\mathrm{O} & 0.79437300 & -1.07411400 & 0.87111000\end{array}$

$\mathrm{S} \quad 1.42288900 \quad-2.42201300 \quad 0.29218300$

$\begin{array}{llll}\mathrm{O} & 2.08828600 & -3.08073500 & 1.40769600\end{array}$

$\begin{array}{llll}\mathrm{O} & 0.47434900 & -3.12938600 & -0.56425200\end{array}$

C $\quad 2.76062200 \quad-1.74124900 \quad-0.82665800$

F $\quad 3.52700200 \quad-0.89587700 \quad-0.14665100$

F $\quad 3.48761200 \quad-2.75861000 \quad-1.27695500$

F $\quad 2.18902300 \quad-1.10684200 \quad-1.85290200$

$\begin{array}{llll}\mathrm{O} & 1.08337600 & 3.55077300 & -1.28198100\end{array}$

S $\quad 0.61435000 \quad 2.64621600 \quad-0.24183200$

$\begin{array}{llll}\mathrm{O} & -0.59108000 & 2.93221300 & 0.53609100\end{array}$

$\begin{array}{lllll}\mathrm{O} & 0.55886900 & 1.18743500 & -0.88205100\end{array}$

$\begin{array}{llll}\mathrm{C} & 2.01315700 & 2.43935600 & 0.98376100\end{array}$

$\begin{array}{llll}\mathrm{F} & 2.29256600 & 3.63016000 & 1.50453100\end{array}$

F $\quad \begin{array}{llll}\text { F } & 1.62505100 & 1.60421000 & 1.94959400\end{array}$

F $\quad 3.08063000 \quad 1.94824400 \quad 0.36513700$

$\begin{array}{lllll}\mathrm{O} & -2.27331600 & 0.74592300 & -1.12470500\end{array}$

$\begin{array}{lllll}\mathrm{O} & -2.03093100 & -1.59407400 & 0.62587800\end{array}$

$\begin{array}{llll}\text { C } & -3.45299600 & 0.71959200 & -0.70485600\end{array}$ 


$\begin{array}{llll}\mathrm{C} & -4.48130800 & 1.46156700 & -1.46941900 \\ \mathrm{H} & -5.20969300 & 0.73359200 & -1.85461400 \\ \mathrm{H} & -5.02673700 & 2.11978000 & -0.78007400 \\ \mathrm{H} & -4.03687900 & 2.03055400 & -2.28637900 \\ \mathrm{C} & -3.87488600 & 0.00054500 & 0.55221900 \\ \mathrm{H} & -3.61946200 & 0.66964700 & 1.39704800 \\ \mathrm{H} & -4.96069300 & -0.10523600 & 0.56927000 \\ \mathrm{C} & -3.23334100 & -1.33102400 & 0.85659300 \\ \mathrm{C} & -4.06494700 & -2.37909300 & 1.49345600 \\ \mathrm{H} & -4.81217000 & -2.70799600 & 0.75638200 \\ \mathrm{H} & -3.45834400 & -3.22423600 & 1.82057600 \\ \mathrm{H} & -4.61912800 & -1.93717800 & 2.33128300\end{array}$

\section{6}

$\mathrm{E}(\mathrm{SMD} / \mathrm{B} 3 \mathrm{LYP} / \mathrm{BS} 1)=-2404.15813456 \mathrm{au}$

$\mathrm{H}(\mathrm{SMD} / \mathrm{B} 3 \mathrm{LYP} / \mathrm{BS} 1)=-2403.948161 \mathrm{au}$

$\mathrm{G}(\mathrm{SMD} / \mathrm{B} 3 \mathrm{LYP} / \mathrm{BS} 1)=-2404.035624 \mathrm{au}$

$\mathrm{E}(\mathrm{SMD} / \mathrm{M} 06 / \mathrm{BS} 2 / / \mathrm{SMD} / \mathrm{B} 3 \mathrm{LYP} / \mathrm{BS} 1)=-2404.26827935 \mathrm{au}$

$\begin{array}{lllll}\mathrm{C} & -3.35668700 & 2.63815000 & -0.14590900\end{array}$

$\mathrm{H} \quad-3.35892400 \quad 3.54519100 \quad-0.77286700$

$\mathrm{H} \quad-4.09524800 \quad 2.73087400 \quad 0.65113100$

$\mathrm{H} \quad-3.58732700 \quad 1.80599800 \quad-0.82253800$

$\begin{array}{llll}\text { C } & -1.99760900 & 2.47704200 & 0.38437800\end{array}$

$\begin{array}{llll}\mathrm{O} & -1.86195000 & 2.64340900 & 1.65201600\end{array}$

$\begin{array}{llll}\text { C } & -0.88109000 & 2.13677900 & -0.50749600\end{array}$

$\begin{array}{llll}\mathrm{C} & 0.44608800 & 2.87066500 & -0.43673700\end{array}$

$\begin{array}{llll}\mathrm{O} & 1.37008900 & 2.02171600 & -0.33633100\end{array}$

C $\quad 0.68581800 \quad 4.31094000 \quad-0.55801900$

$\mathrm{H} \quad 0.10115700 \quad 4.85171200 \quad 0.19914200$

$\mathrm{H} \quad 1.74928600 \quad 4.54454100 \quad-0.47424200$

$\mathrm{H} \quad 0.29801300 \quad 4.63091700 \quad-1.53745500$

$\mathrm{H} \quad-1.19954500 \quad 2.06146600 \quad-1.55152000$

$\mathrm{H} \quad-0.94422700 \quad 2.51574400 \quad 1.99780200$

$\mathrm{Au} \quad 0.17906700 \quad 0.31307400 \quad-0.13185300$

$\begin{array}{llll}\mathrm{O} & 1.54336700 & -1.26003500 & 0.19185000\end{array}$

S $\quad 2.92776400 \quad-1.24269500 \quad-0.53176900$

$\begin{array}{llll}\mathrm{O} & 3.37307100 & -2.60988600 & -0.77953800\end{array}$

$\begin{array}{llll}\text { O } & 2.95628400 & -0.24528100 & -1.60595500\end{array}$

$\begin{array}{llll}\mathrm{C} & 4.01474200 & -0.57448100 & 0.82796200\end{array}$

F $\quad \begin{array}{llll}\text { F } & 4.01298600 & -1.40987700 & 1.86482800\end{array}$

F $\quad \begin{array}{llll}5.25613600 & -0.42865300 & 0.36927000\end{array}$

$\begin{array}{lllll}\mathrm{F} & 3.54703000 & 0.61800900 & 1.22536700\end{array}$

O $\quad-2.73309400 \quad-0.38376400 \quad-1.58237700$

S $\quad-2.41824200-1.50424400 \quad-0.69176700$

$\begin{array}{llll}\mathrm{O} & -2.38662500 & -2.86789300 & -1.20204000\end{array}$

$\begin{array}{llll}\mathrm{O} & -1.12251700 & -1.22315100 & 0.16667800\end{array}$

$\begin{array}{llll}\text { C } & -3.67186300 & -1.47341800 & 0.69665500\end{array}$

F $\quad \begin{array}{llll}\text { C } & -4.88653700 & -1.62875200 & 0.17820200\end{array}$

F $\quad-3.41344800 \quad-2.45305800 \quad 1.55504100$

F $\quad-3.60585500 \quad-0.29581000 \quad 1.32997100$ 


\begin{tabular}{|c|c|c|c|}
\hline \multicolumn{4}{|c|}{$\mathbf{T S}_{10-17}$} \\
\hline \multicolumn{4}{|c|}{$\mathrm{E}(\mathrm{SMD} / \mathrm{B} 3 \mathrm{LYP} / \mathrm{BS} 1)=-3675.42798857 \mathrm{au}$} \\
\hline \multicolumn{4}{|c|}{$\mathrm{H}(\mathrm{SMD} / \mathrm{B} 3 \mathrm{LYP} / \mathrm{BS} 1)=-3675.039016 \mathrm{au}$} \\
\hline \multicolumn{4}{|c|}{$\mathrm{G}(\mathrm{SMD} / \mathrm{B} 3 \mathrm{LYP} / \mathrm{BS} 1)=-3675.164752 \mathrm{au}$} \\
\hline \multicolumn{4}{|c|}{$\mathrm{E}(\mathrm{SMD} / \mathrm{M} 06 / \mathrm{BS} 2 / / \mathrm{SMD} / \mathrm{B} 3 \mathrm{LYP} / \mathrm{BS} 1)=-3675.51447103 \mathrm{au}$} \\
\hline $\mathrm{C}$ & 1.85185900 & 4.18471700 & 1.36062100 \\
\hline $\mathrm{H}$ & 1.64243900 & 5.22615900 & 1.64680600 \\
\hline $\mathrm{H}$ & 2.90143900 & 4.12734400 & 1.06364700 \\
\hline $\mathrm{H}$ & 1.65898600 & 3.54747100 & 2.22470500 \\
\hline $\mathrm{C}$ & 0.95751100 & 3.84867100 & 0.22180500 \\
\hline $\mathrm{O}$ & 1.37062400 & 4.21856400 & -0.95729400 \\
\hline $\mathrm{C}$ & -0.32083400 & 3.25967600 & 0.38976300 \\
\hline $\mathrm{C}$ & -1.31954700 & 3.47676100 & -0.66579600 \\
\hline $\mathrm{O}$ & -0.95212100 & 3.71378900 & -1.83658100 \\
\hline $\mathrm{C}$ & -2.78237700 & 3.49763900 & -0.31607800 \\
\hline $\mathrm{H}$ & -2.97288800 & 3.28760300 & 0.73670300 \\
\hline $\mathrm{H}$ & -3.16931200 & 4.49543900 & -0.55806600 \\
\hline $\mathrm{H}$ & -3.32938800 & 2.77827200 & -0.93286300 \\
\hline $\mathrm{H}$ & -0.65628400 & 3.11500400 & 1.40932000 \\
\hline $\mathrm{C}$ & -3.46034400 & -1.86683900 & -2.04020200 \\
\hline $\mathrm{C}$ & -4.84616000 & -1.86324200 & -2.06550100 \\
\hline $\mathrm{C}$ & -5.53105100 & -0.68699500 & -2.40121700 \\
\hline $\mathrm{C}$ & -4.82707200 & 0.48988500 & -2.71168200 \\
\hline $\mathrm{C}$ & -3.44375800 & 0.49908100 & -2.67791100 \\
\hline $\mathrm{C}$ & -2.72940900 & -0.68312400 & -2.33751400 \\
\hline $\mathrm{H}$ & -2.91364600 & -2.76609300 & -1.77435700 \\
\hline $\mathrm{H}$ & -5.39918000 & -2.76643800 & -1.82773600 \\
\hline $\mathrm{H}$ & -6.61705800 & -0.68427200 & -2.42589300 \\
\hline $\mathrm{H}$ & -5.37123800 & 1.38973300 & -2.98119400 \\
\hline $\mathrm{H}$ & -2.90479500 & 1.40618700 & -2.92967000 \\
\hline $\mathrm{C}$ & -1.30069200 & -0.75626000 & -2.31465700 \\
\hline $\mathrm{H}$ & -0.88255600 & -1.74780200 & -2.17768500 \\
\hline $\mathrm{C}$ & -0.40123300 & 0.29781300 & -2.43551700 \\
\hline $\mathrm{H}$ & -0.72403400 & 1.31067100 & -2.65928600 \\
\hline $\mathrm{H}$ & 0.61282100 & 4.03410300 & -1.61091800 \\
\hline $\mathrm{H}$ & 0.64228000 & 0.06787400 & -2.62473900 \\
\hline $\mathrm{Au}$ & 0.16824700 & 0.65543700 & -0.01699200 \\
\hline $\mathrm{O}$ & 1.04015600 & -1.47507600 & 0.80902200 \\
\hline S & 0.54271400 & -2.87312100 & 0.50913100 \\
\hline $\mathrm{O}$ & 0.87727000 & -3.82968800 & 1.57371400 \\
\hline $\mathrm{O}$ & -0.82379500 & -2.91930900 & -0.04308400 \\
\hline $\mathrm{C}$ & 1.59774500 & -3.40626100 & -0.93168200 \\
\hline $\mathrm{F}$ & 2.88070300 & -3.53570400 & -0.58313900 \\
\hline $\mathrm{F}$ & 1.15975200 & -4.58423900 & -1.39552600 \\
\hline $\mathrm{F}$ & 1.51595900 & -2.50590100 & -1.93279700 \\
\hline $\mathrm{O}$ & 4.15738500 & 2.17012500 & -0.52625700 \\
\hline S & 3.30965700 & 1.13069900 & 0.05659400 \\
\hline $\mathrm{O}$ & 2.98216300 & 1.16201800 & 1.48281300 \\
\hline $\mathrm{O}$ & 2.02372300 & 0.99041400 & -0.83704800 \\
\hline $\mathrm{C}$ & 4.23394900 & -0.48082100 & -0.25946600 \\
\hline $\mathrm{F}$ & 5.54062500 & -0.21303300 & -0.21766600 \\
\hline
\end{tabular}




$\begin{array}{llcc}\text { F } & 3.94020500 & -1.37015400 & 0.67956100 \\ \text { F } & 3.92194100 & -0.96295400 & -1.46282300 \\ \text { O } & -1.23215100 & 1.17014100 & 2.87901400 \\ \text { S } & -2.25036300 & 0.51651900 & 2.05103200 \\ \text { O } & -3.61842200 & 1.03220400 & 2.03180100 \\ \text { O } & -1.76997500 & 0.32098200 & 0.56880800 \\ \text { C } & -2.40436800 & -1.23631200 & 2.68370700 \\ \text { F } & -3.06164200 & -1.18510000 & 3.84554800 \\ \text { F } & -3.08993200 & -1.97574600 & 1.81813400 \\ \text { F } & -1.19932100 & -1.76338800 & 2.88694800\end{array}$

17

$\mathrm{E}(\mathrm{SMD} / \mathrm{B} 3 \mathrm{LYP} / \mathrm{BS} 1)=-3329.66064938 \mathrm{au}$

$\mathrm{H}(\mathrm{SMD} / \mathrm{B} 3 \mathrm{LYP} / \mathrm{BS} 1)=-3329.403837 \mathrm{au}$

$\mathrm{G}(\mathrm{SMD} / \mathrm{B} 3 \mathrm{LYP} / \mathrm{BS} 1)=-3329.510365 \mathrm{au}$

$\mathrm{E}(\mathrm{SMD} / \mathrm{M} 06 / \mathrm{BS} 2 / / \mathrm{SMD} / \mathrm{B} 3 \mathrm{LYP} / \mathrm{BS} 1)=-3329.81650101 \mathrm{au}$

$\begin{array}{llll}\mathrm{Au} & -0.09562400 & -0.40342600 & 0.28685600\end{array}$

$\begin{array}{llll}\mathrm{O} & 1.22982800 & 0.72617700 & -0.99557700\end{array}$

S $\quad 0.86919000 \quad 0.81365700 \quad-2.49575300$

$\begin{array}{llll}\mathrm{O} & 2.06843800 & 0.74643600 & -3.33199900\end{array}$

O $\quad-0.29469400 \quad-0.01663200 \quad-2.83919700$

$\begin{array}{llll}\mathrm{C} & 0.27360000 & 2.57421600 & -2.62415000\end{array}$

F $\quad \begin{array}{llll}\text { F } & 1.21580600 & 3.41554800 & -2.19276000\end{array}$

F $\quad-0.00874600 \quad 2.84735400 \quad-3.90223300$

F $\quad-0.83294600 \quad 2.74186800 \quad-1.89087800$

$\begin{array}{llll}\mathrm{O} & -1.60080500 & 3.08025400 & 2.49275600\end{array}$

S $\quad-0.59297400 \quad 2.05807400 \quad 2.22469700$

$\begin{array}{llll}\mathrm{O} & -0.20253600 & 1.10762300 & 3.26690200\end{array}$

$\begin{array}{lllll}\mathrm{O} & -0.98734100 & 1.34498800 & 0.88050200\end{array}$

$\begin{array}{llll}\mathrm{C} & 0.96558800 & 2.97242500 & 1.74881300\end{array}$

F $\quad \begin{array}{llll}\text { C } & 1.30117500 & 3.78271300 & 2.75318100\end{array}$

$\begin{array}{llll}\text { F } & 1.95380400 & 2.09893800 & 1.53673800\end{array}$

F $\quad \begin{array}{llll}0.75800200 & 3.68854500 & 0.64618000\end{array}$

$\begin{array}{llll}\mathrm{O} & 1.46883100 & -3.09471300 & 1.76162400\end{array}$

S $\quad 1.75729000 \quad-3.02167000 \quad 0.32744800$

$\begin{array}{lllll}\mathrm{O} & 1.96332600 & -4.24288400 & -0.44461900\end{array}$

$\begin{array}{llll}\mathrm{O} & 0.72148500 & -2.13668200 & -0.45660100\end{array}$

$\begin{array}{llll}\mathrm{C} & 3.32714500 & -2.02307100 & 0.17203800\end{array}$

F $\quad 4.32258700 \quad-2.70980400 \quad 0.73258600$

F $\quad \begin{array}{llll}3.60193900 & -1.79873200 & -1.10959500\end{array}$

F $\quad 3.18209900 \quad-0.85592900 \quad 0.80937400$

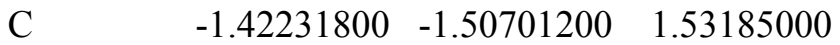

$\mathrm{H} \quad-0.73136800 \quad-2.18454200 \quad 2.03556000$

$\mathrm{H} \quad-1.88062300 \quad-0.77381900 \quad 2.18879700$

$\begin{array}{llll}\mathrm{C} & -2.26063100 & -2.14118500 & 0.54222600\end{array}$

$\begin{array}{llll}\mathrm{H} & -1.83406400 & -3.00982800 & 0.04400900\end{array}$

$\begin{array}{llll}\mathrm{C} & -3.54438400 & -1.76014500 & 0.12555000\end{array}$

$\begin{array}{llll}\mathrm{C} & -4.25632300 & -0.65192600 & 0.68570300\end{array}$

$\begin{array}{llll}\text { C } & -4.15384700 & -2.53022300 & -0.91598400\end{array}$

$\begin{array}{llll}\text { C } & -5.51094900 & -0.33338000 & 0.20865200\end{array}$

$\mathrm{H} \quad-3.81158100 \quad-0.06336800 \quad 1.47959300$ 


$\begin{array}{llrc}\mathrm{C} & -5.41206300 & -2.20040300 & -1.38054100 \\ \mathrm{H} & -3.60701400 & -3.37086000 & -1.33359700 \\ \mathrm{C} & -6.08646700 & -1.10332500 & -0.82027600 \\ \mathrm{H} & -6.05707900 & 0.50698500 & 0.62385600 \\ \mathrm{H} & -5.87670300 & -2.77883700 & -2.17186100 \\ \mathrm{H} & -7.07521600 & -0.84293700 & -1.18723800\end{array}$

18

$\mathrm{E}(\mathrm{SMD} / \mathrm{B} 3 \mathrm{LYP} / \mathrm{BS} 1)=-3675.47768954 \mathrm{au}$

$\mathrm{H}(\mathrm{SMD} / \mathrm{B} 3 \mathrm{LYP} / \mathrm{BS} 1)=-3675.085973 \mathrm{au}$

$\mathrm{G}(\mathrm{SMD} / \mathrm{B} 3 \mathrm{LYP} / \mathrm{BS} 1)=-3675.207588 \mathrm{au}$

$\mathrm{E}(\mathrm{SMD} / \mathrm{M} 06 / \mathrm{BS} 2 / / \mathrm{SMD} / \mathrm{B} 3 \mathrm{LYP} / \mathrm{BS} 1)=-3675.57247834 \mathrm{au}$

$\begin{array}{llll}\mathrm{C} & -3.76227200 & 0.90886400 & 3.19410200\end{array}$

$\mathrm{H} \quad-4.37824900 \quad 1.19437500 \quad 2.33696700$

$\mathrm{H} \quad-3.45245300 \quad 1.78730700 \quad 3.76390700$

$\mathrm{H} \quad-4.38601300 \quad 0.26568900 \quad 3.83197500$

$\begin{array}{llll}\mathrm{C} & -2.56753800 & 0.11658300 & 2.77974700\end{array}$

$\begin{array}{llll}\mathrm{O} & -1.46105400 & 0.30530600 & 3.29347500\end{array}$

$\begin{array}{llll}\mathrm{C} & -2.74249200 & -0.99726000 & 1.74109200\end{array}$

$\begin{array}{llll}\text { C } & -1.73731600 & -2.10892200 & 1.91415600\end{array}$

$\begin{array}{llll}\text { C } & -2.01327200 & -3.47729500 & 1.43481600\end{array}$

$\mathrm{H} \quad-1.08857400 \quad-4.04254900 \quad 1.30587000$

$\mathrm{H} \quad-2.61560500 \quad-3.96273400 \quad 2.22016500$

$\mathrm{H} \quad-2.61376400 \quad-3.47743400 \quad 0.52224500$

$\begin{array}{llll}\mathrm{O} & -0.65029600 & -1.92581100 & 2.54306100\end{array}$

$\mathrm{H} \quad-3.71896800 \quad-1.46925700 \quad 1.89599900$

$\begin{array}{llll}\mathrm{C} & -2.78706500 & -0.48717400 & 0.20748200\end{array}$

$\begin{array}{llll}\text { C } & -4.22682600 & -0.17420900 & -0.22627700\end{array}$

$\begin{array}{llll}\text { C } & -5.03281000 & -1.23840600 & -0.66136300\end{array}$

$\begin{array}{lllll}\text { C } & & -4.76293700 & 1.12272900 & -0.23122000\end{array}$

C $\quad-6.34771900 \quad-1.01730600 \quad-1.07047200$

$\mathrm{H} \quad-4.62635300 \quad-2.24678300 \quad-0.68583400$

$\begin{array}{llll}\mathrm{C} & -6.07877300 & 1.34405400 & -0.64818900\end{array}$

$\mathrm{H} \quad-4.16234000 \quad 1.97246400 \quad 0.07738600$

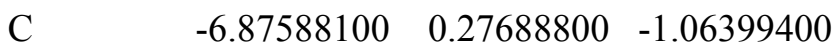

$\mathrm{H} \quad-6.95491100 \quad-1.85435800 \quad-1.40409200$

$\mathrm{H} \quad-6.47505100 \quad 2.35565700 \quad-0.64906700$

$\begin{array}{llll}\mathrm{H} & -7.89808600 & 0.45163700 & -1.38791700\end{array}$

$\begin{array}{llll}\mathrm{C} & -1.86913700 & 0.67825400 & -0.14691300\end{array}$

$\mathrm{H} \quad-2.00013700 \quad 1.55679400 \quad 0.48314200$

$\mathrm{H} \quad-1.98182500 \quad 0.94089700 \quad-1.20006300$

$\mathrm{H} \quad-2.48958200 \quad-1.35183300 \quad-0.38493000$

$\mathrm{Au} \quad 0.18966100 \quad 0.34669900 \quad-0.01451500$

$\begin{array}{lllll}\mathrm{O} & 2.38345400 & 0.02559000 & 0.22147500\end{array}$

$\begin{array}{llll}\mathrm{S} & 2.90244900 & -0.35524000 & 1.60722400\end{array}$

$\begin{array}{llll}\mathrm{O} & 4.16476000 & 0.31352700 & 1.93612400\end{array}$

$\begin{array}{llll}\text { O } & 1.83963400 & -0.37555200 & 2.62994600\end{array}$

$\begin{array}{llll}\mathrm{C} & 3.35703800 & -2.14309200 & 1.35629700\end{array}$

F $\quad 4.23482800 \quad-2.26625300 \quad 0.35516300$

F $\quad 3.90690800 \quad-2.62549300 \quad 2.47803900$

F $\quad \begin{array}{llll}2.26857400 & -2.86919500 & 1.06780800\end{array}$ 


$\begin{array}{lccc}\mathrm{O} & -0.37154500 & -3.49663100 & -2.12116700 \\ \mathrm{~S} & -0.14207800 & -2.05373700 & -2.10432200 \\ \mathrm{O} & -1.00525600 & -1.16332100 & -2.88182700 \\ \mathrm{O} & -0.01705000 & -1.61223400 & -0.60480800 \\ \mathrm{C} & 1.58814800 & -1.79372200 & -2.75924700 \\ \mathrm{~F} & 1.64333900 & -2.28189900 & -3.99921600 \\ \mathrm{~F} & 1.87269000 & -0.48748200 & -2.78819800 \\ \mathrm{~F} & 2.47201700 & -2.42607300 & -1.99127400 \\ \mathrm{O} & -0.52539000 & 3.39822300 & -1.28692800 \\ \mathrm{~S} & 0.39700100 & 3.51510700 & -0.15550100 \\ \mathrm{O} & 0.34821000 & 4.68865000 & 0.71255000 \\ \mathrm{O} & 0.40868700 & 2.24048100 & 0.75397600 \\ \mathrm{C} & 2.11002400 & 3.48926500 & -0.89691500 \\ \mathrm{~F} & 2.27792600 & 4.60025500 & -1.61723000 \\ \mathrm{~F} & 3.02916000 & 3.43878300 & 0.06446500 \\ \mathrm{~F} & 2.24374900 & 2.42467600 & -1.69661100 \\ \mathrm{H} & -0.62224700 & -0.95442100 & 2.90034600\end{array}$

19

$\begin{array}{lrrl}\mathrm{E}(\mathrm{SMD} / \mathrm{B} 3 \mathrm{LYP} / \mathrm{BS} 1)= & -3675.47389615 \mathrm{au} \\ \mathrm{H}(\mathrm{SMD} / \mathrm{B} 3 \mathrm{LYP} / \mathrm{BS} 1)= & -3675.081418 \mathrm{au} \\ \mathrm{G}(\mathrm{SMD} / \mathrm{B} 3 \mathrm{~L} \text { YP/BS1 })= & -3675.20776 \mathrm{au} & \\ \mathrm{E}(\mathrm{SMD} / \mathrm{M} 06 / \mathrm{BS} 2 / / \mathrm{SMD} / \mathrm{B} 3 \mathrm{LYP} / \mathrm{BS} 1) & -3675.56851538 \mathrm{au} \\ \mathrm{C} & 4.51831000 & -3.36008500 & 0.08814700 \\ \mathrm{H} & 3.47468400 & -3.46836000 & 0.40552200 \\ \mathrm{H} & 5.19513100 & -3.65657300 & 0.89145500 \\ \mathrm{H} & 4.66022200 & -4.02118000 & -0.77801500 \\ \mathrm{C} & 4.80607500 & -1.95967300 & -0.33625600 \\ \mathrm{O} & 5.78542600 & -1.33287100 & 0.07909300 \\ \mathrm{C} & 3.84778700 & -1.27901800 & -1.31905700 \\ \mathrm{C} & 4.56504100 & -0.32361800 & -2.21218900 \\ \mathrm{C} & 4.15815300 & -0.06632700 & -3.60573400 \\ \mathrm{H} & 4.40392900 & -0.96961200 & -4.18614400 \\ \mathrm{H} & 3.07461800 & 0.06554800 & -3.68719800 \\ \mathrm{H} & 4.69258800 & 0.78981400 & -4.02095100 \\ \mathrm{O} & 5.58807000 & 0.29039800 & -1.76897700 \\ \mathrm{H} & 5.84257500 & -0.13176700 & -0.84824600 \\ \mathrm{H} & 3.33064900 & -2.02403900 & -1.92762700 \\ \mathrm{C} & 2.67337200 & -0.54044300 & -0.43736400 \\ \mathrm{C} & 3.19922800 & 0.54005600 & 0.48648800 \\ \mathrm{C} & 3.38094300 & 0.23374100 & 1.84528100 \\ \mathrm{C} & 3.51379600 & 1.83389600 & 0.04009700 \\ \mathrm{C} & 3.86105200 & 1.19472000 & 2.73565900 \\ \mathrm{H} & 3.13208100 & -0.76016300 & 2.20865300 \\ \mathrm{C} & 3.99896400 & 2.79382200 & 0.93008400 \\ \mathrm{H} & 3.37299200 & 2.10737300 & -1.00249700 \\ \mathrm{C} & 4.17482800 & 2.47670800 & 2.27882900 \\ \mathrm{H} & 3.98715800 & 0.94125800 & 3.78455600 \\ \mathrm{H} & 4.23087800 & 3.79146800 & 0.56844700 \\ \mathrm{H} & 4.54913900 & 3.22641700 & 2.97040600 \\ \mathrm{C} & 1.54759900 & -0.13652600 & -1.38025800 \\ & & & \\ \mathrm{H} & & & \end{array}$




$\begin{array}{lrrc}\mathrm{H} & 1.34180100 & -0.89792600 & -2.13655700 \\ \mathrm{H} & 1.66092200 & 0.84820200 & -1.83366700 \\ \mathrm{H} & 2.31738800 & -1.37177500 & 0.17469000 \\ \mathrm{Au} & -0.34406700 & 0.00698200 & -0.49890800 \\ \mathrm{O} & -2.46721700 & 0.16120800 & 0.16789600 \\ \mathrm{~S} & -3.53067100 & 0.30445300 & -0.92360800 \\ \mathrm{O} & -4.57618500 & 1.26779300 & -0.56542700 \\ \mathrm{O} & -2.94474800 & 0.37569700 & -2.27399200 \\ \mathrm{C} & -4.37702100 & -1.35247700 & -0.85968300 \\ \mathrm{~F} & -4.88735300 & -1.57309500 & 0.35650600 \\ \mathrm{~F} & -5.37256400 & -1.37187900 & -1.75600900 \\ \mathrm{~F} & -3.51724500 & -2.33571000 & -1.15469100 \\ \mathrm{O} & -0.56533000 & -4.36930200 & 0.28853800 \\ \mathrm{~S} & -0.19237100 & -3.01719200 & 0.69498100 \\ \mathrm{O} & 1.13860200 & -2.78181800 & 1.27083600 \\ \mathrm{O} & -0.52622500 & -2.05188900 & -0.48122400 \\ \mathrm{C} & -1.38255300 & -2.52975700 & 2.05027000 \\ \mathrm{~F} & -1.17825500 & -3.34344600 & 3.08842100 \\ \mathrm{~F} & -1.15296000 & -1.26834000 & 2.43237200 \\ \mathrm{~F} & -2.63910900 & -2.65030900 & 1.63330900 \\ \mathrm{O} & 0.13454500 & 2.35843200 & 1.73844200 \\ \mathrm{~S} & -0.12259900 & 3.03545100 & 0.46664600 \\ \mathrm{O} & 0.68723700 & 4.18185300 & 0.05706900 \\ \mathrm{O} & -0.21660800 & 2.04028300 & -0.74306100 \\ \mathrm{C} & -1.87715700 & 3.69134200 & 0.57005800 \\ \mathrm{~F} & -1.83302100 & 4.89874100 & 1.14211800 \\ \mathrm{~F} & -2.40161200 & 3.80323100 & -0.65138700 \\ \mathrm{~F} & -2.62968800 & 2.88524100 & 1.31189600\end{array}$

\begin{tabular}{|c|c|c|c|}
\hline \multicolumn{4}{|c|}{$\mathbf{T S}_{17-19}$} \\
\hline \multicolumn{4}{|c|}{$\mathrm{E}(\mathrm{SMD} / \mathrm{B} 3 \mathrm{LYP} / \mathrm{BS} 1)=-3675.46749678 \mathrm{au}$} \\
\hline \multicolumn{4}{|c|}{$\mathrm{H}(\mathrm{SMD} / \mathrm{B} 3 \mathrm{LYP} / \mathrm{BS} 1)=-3675.076178 \mathrm{au}$} \\
\hline \multicolumn{4}{|c|}{$\mathrm{G}(\mathrm{SMD} / \mathrm{B} 3 \mathrm{LYP} / \mathrm{BS} 1)=-3675.204793 \mathrm{au}$} \\
\hline \multicolumn{4}{|c|}{$\mathrm{E}(\mathrm{SMD} / \mathrm{M} 06 / \mathrm{BS} 2 / / \mathrm{SMD} / \mathrm{B} 3 \mathrm{LYP} / \mathrm{BS} 1)=-3675.55304242 \mathrm{au}$} \\
\hline $\mathrm{C}$ & 5.15537600 & 2.45366600 & -1.05675600 \\
\hline $\mathrm{H}$ & 4.11503100 & 2.78127100 & -1.17299300 \\
\hline $\mathrm{H}$ & 5.66045900 & 2.46191700 & -2.02474700 \\
\hline $\mathrm{H}$ & 5.64246500 & 3.17176900 & -0.38433200 \\
\hline $\mathrm{C}$ & 5.21373000 & 1.08156100 & -0.45113300 \\
\hline $\mathrm{O}$ & 5.86780000 & 0.16544900 & -0.98948700 \\
\hline $\mathrm{C}$ & 4.47763300 & 0.82935000 & 0.79032500 \\
\hline $\mathrm{C}$ & 4.77040200 & -0.32115300 & 1.54592500 \\
\hline $\mathrm{C}$ & 4.34228500 & -0.51998500 & 2.95661200 \\
\hline $\mathrm{H}$ & 5.22944500 & -0.46134200 & 3.60172000 \\
\hline $\mathrm{H}$ & 3.62828600 & 0.24131500 & 3.27429200 \\
\hline $\mathrm{H}$ & 3.91570500 & -1.52055300 & 3.08777800 \\
\hline $\mathrm{O}$ & 5.44848800 & -1.30529600 & 1.01828000 \\
\hline $\mathrm{H}$ & 5.74815900 & -0.99394300 & 0.09318500 \\
\hline $\mathrm{H}$ & 4.00611100 & 1.66395200 & 1.29508500 \\
\hline $\mathrm{C}$ & 2.47007700 & 0.02450900 & -0.22152800 \\
\hline $\mathrm{C}$ & 2.80271700 & -1.19244600 & -0.90500100 \\
\hline
\end{tabular}




$\begin{array}{lccc}\mathrm{C} & 3.26672600 & -1.10003300 & -2.24485100 \\ \mathrm{C} & 2.65966000 & -2.47750200 & -0.31514600 \\ \mathrm{C} & 3.54249900 & -2.24706700 & -2.97582200 \\ \mathrm{H} & 3.37683000 & -0.12052200 & -2.70016800 \\ \mathrm{C} & 2.94313600 & -3.61651500 & -1.05293400 \\ \mathrm{H} & 2.31970700 & -2.57754000 & 0.70959000 \\ \mathrm{C} & 3.38090500 & -3.50403400 & -2.38094600 \\ \mathrm{H} & 3.88093400 & -2.16861100 & -4.00404000 \\ \mathrm{H} & 2.82511400 & -4.59652100 & -0.60144900 \\ \mathrm{H} & 3.59785900 & -4.40214000 & -2.95223300 \\ \mathrm{C} & 1.59656600 & 0.12515400 & 0.95269100 \\ \mathrm{H} & 1.64778000 & 1.07939000 & 1.47339800 \\ \mathrm{H} & 1.58149400 & -0.72269700 & 1.63075400 \\ \mathrm{H} & 2.54174000 & 0.92851600 & -0.82000600 \\ \mathrm{Au} & -0.34412900 & 0.18314700 & 0.12927200 \\ \mathrm{O} & -2.36422700 & 0.25259500 & -0.71964400 \\ \mathrm{~S} & -2.56165300 & -0.02143000 & -2.21816600 \\ \mathrm{O} & -3.71800000 & 0.70833600 & -2.74498500 \\ \mathrm{O} & -1.30125800 & 0.00189200 & -2.97665400 \\ \mathrm{C} & -3.07563300 & -1.81118900 & -2.20699300 \\ \mathrm{~F} & -4.12869200 & -1.99364300 & -1.40505400 \\ \mathrm{~F} & -3.40277400 & -2.18757500 & -3.44883600 \\ \mathrm{~F} & -2.06518000 & -2.58004600 & -1.77768000 \\ \mathrm{O} & -1.10442300 & -3.48823200 & 2.45848800 \\ \mathrm{~S} & -1.08262400 & -2.03637200 & 2.30108800 \\ \mathrm{O} & -0.33897900 & -1.20602700 & 3.25199500 \\ \mathrm{O} & -0.72283400 & -1.72055000 & 0.81088100 \\ \mathrm{C} & -2.85913100 & -1.47316800 & 2.41423500 \\ \mathrm{~F} & -3.33863300 & -1.81964700 & 3.60976400 \\ \mathrm{~F} & -2.92351400 & -0.14302800 & 2.28431400 \\ \mathrm{~F} & -3.58459500 & -2.04819000 & 1.45786500 \\ \mathrm{O} & 0.57359800 & 3.19313100 & 1.47612900 \\ \mathrm{~S} & 0.08151700 & 3.35726500 & 0.10528900 \\ \mathrm{O} & 0.64077600 & 4.40149200 & -0.74905400 \\ \mathrm{O} & 0.08794500 & 2.01814600 & -0.70498900 \\ \mathrm{C} & -1.74301700 & 3.72083700 & 0.25676500 \\ \mathrm{~F} & -1.89204500 & 4.93139900 & 0.79616900 \\ \mathrm{~F} & -2.31506800 & 3.69296000 & -0.94450800 \\ \mathrm{~F} & -2.32076700 & 2.81180300 & 1.04907000\end{array}$

\section{TS $_{\text {17-18 }}$}

\begin{tabular}{|c|c|c|c|}
\hline \\
\hline \multicolumn{4}{|c|}{$\begin{array}{l}\mathrm{E}(\mathrm{SMD} / \mathrm{B} 3 \mathrm{~L} / \mathrm{P} / \mathrm{BS} 1)=-36 / 5.45325569 \mathrm{au} \\
\mathrm{H}(\mathrm{SMD} / \mathrm{B} 3 \mathrm{LYP} / \mathrm{BS} 1)=-3675.063045 \mathrm{au}\end{array}$} \\
\hline \multicolumn{4}{|c|}{$\mathrm{G}(\mathrm{SMD} / \mathrm{B} 3 \mathrm{LYP} / \mathrm{BS} 1)=-3675.189981 \mathrm{au}$} \\
\hline \multicolumn{4}{|c|}{$\mathrm{E}(\mathrm{SMD} / \mathrm{M} 06 / \mathrm{BS} 2 / / \mathrm{SMD} / \mathrm{B} 3 \mathrm{LYP} / \mathrm{BS} 1)=-3675.53938448 \mathrm{au}$} \\
\hline $\mathrm{C}$ & -3.72480400 & 1.92451500 & 2.80217100 \\
\hline $\mathrm{H}$ & -4.34291100 & 1.87801300 & 1.89782600 \\
\hline $\mathrm{H}$ & -3.30875500 & 2.92715800 & 2.92416100 \\
\hline $\mathrm{H}$ & -4.38562700 & 1.70476600 & 3.65149200 \\
\hline $\mathrm{C}$ & -2.62067200 & 0.90346100 & 2.75649800 \\
\hline $\mathrm{O}$ & -1.45287700 & 1.21125500 & 3.06612800 \\
\hline
\end{tabular}




\begin{tabular}{|c|c|c|c|}
\hline $\mathrm{C}$ & -2.95483100 & -0.46521500 & 2.37352500 \\
\hline $\mathrm{C}$ & -2.04190200 & -1.49948500 & 2.59134900 \\
\hline $\mathrm{C}$ & -2.38655000 & -2.94503100 & 2.47519400 \\
\hline $\mathrm{H}$ & -1.72321000 & -3.43280500 & 1.75096500 \\
\hline $\mathrm{H}$ & -2.21751600 & -3.43251900 & 3.44364300 \\
\hline $\mathrm{H}$ & -3.42732600 & -3.08908500 & 2.18052200 \\
\hline $\mathrm{O}$ & -0.79308900 & -1.26104500 & 2.92042200 \\
\hline $\mathrm{H}$ & -3.99436800 & -0.73120200 & 2.23975700 \\
\hline $\mathrm{C}$ & -2.79812400 & -0.45016600 & -0.21232100 \\
\hline $\mathrm{C}$ & -4.18998200 & -0.32884300 & -0.48882800 \\
\hline $\mathrm{C}$ & -4.97564800 & -1.51499400 & -0.49359000 \\
\hline $\mathrm{C}$ & -4.80670800 & 0.91163300 & -0.81229700 \\
\hline $\mathrm{C}$ & -6.32229400 & -1.46214700 & -0.81429500 \\
\hline $\mathrm{H}$ & -4.50248400 & -2.46254400 & -0.25200100 \\
\hline $\mathrm{C}$ & -6.15459200 & 0.95179200 & -1.13032800 \\
\hline $\mathrm{H}$ & -4.22338400 & 1.82613300 & -0.81722800 \\
\hline $\mathrm{C}$ & -6.91202000 & -0.22980800 & -1.12935300 \\
\hline $\mathrm{H}$ & -6.91746300 & -2.36961100 & -0.82322400 \\
\hline $\mathrm{H}$ & -6.62528300 & 1.89698800 & -1.38113300 \\
\hline $\mathrm{H}$ & -7.96865800 & -0.18853200 & -1.37772900 \\
\hline $\mathrm{C}$ & -1.82856800 & 0.61992700 & -0.53884100 \\
\hline $\mathrm{H}$ & -2.07028000 & 1.59250100 & -0.10994100 \\
\hline $\mathrm{H}$ & -1.79252100 & 0.71471900 & -1.63412800 \\
\hline $\mathrm{H}$ & -2.42532400 & -1.46350100 & -0.10455100 \\
\hline $\mathrm{Au}$ & 0.16562700 & 0.27079300 & -0.10219200 \\
\hline $\mathrm{O}$ & 2.29142400 & -0.04642300 & 0.26919700 \\
\hline S & 2.87960500 & -0.16435300 & 1.68192000 \\
\hline $\mathrm{O}$ & 4.21873800 & 0.42664800 & 1.75442800 \\
\hline $\mathrm{O}$ & 1.91209400 & 0.14755400 & 2.74283600 \\
\hline $\mathrm{C}$ & 3.15539200 & -2.00142300 & 1.79781500 \\
\hline $\mathrm{F}$ & 3.98397700 & -2.41183700 & 0.83184900 \\
\hline $\mathrm{F}$ & 3.69388900 & -2.29823400 & 2.98696000 \\
\hline $\mathrm{F}$ & 1.99337500 & -2.65761700 & 1.67727500 \\
\hline $\mathrm{O}$ & -0.46613500 & -3.55762500 & -2.17094100 \\
\hline S & -0.00198100 & -2.17316300 & -2.13419400 \\
\hline $\mathrm{O}$ & -0.56706600 & -1.18088800 & -3.05171700 \\
\hline $\mathrm{O}$ & -0.03988500 & -1.70093900 & -0.63649400 \\
\hline $\mathrm{C}$ & 1.82840100 & -2.22823200 & -2.50240700 \\
\hline $\mathrm{F}$ & 1.99640600 & -2.80694800 & -3.69278300 \\
\hline $\mathrm{F}$ & 2.32172200 & -0.98855800 & -2.54157900 \\
\hline $\mathrm{F}$ & 2.46298200 & -2.93476000 & -1.56991700 \\
\hline $\mathrm{O}$ & -0.44301700 & 3.25230000 & -1.64284900 \\
\hline S & 0.44712700 & 3.41398900 & -0.49105100 \\
\hline $\mathrm{O}$ & 0.42092300 & 4.64945700 & 0.28657700 \\
\hline $\mathrm{O}$ & 0.36561500 & 2.21511600 & 0.51412800 \\
\hline $\mathrm{C}$ & 2.18081000 & 3.26224700 & -1.16694800 \\
\hline $\mathrm{F}$ & 2.42138400 & 4.31192600 & -1.95508300 \\
\hline $\mathrm{F}$ & 3.06389700 & 3.23981500 & -0.17187300 \\
\hline $\mathrm{F}$ & 2.29187300 & 2.13972500 & -1.88691000 \\
\hline $\mathrm{H}$ & -0.68220400 & -0.26098300 & 3.01871900 \\
\hline
\end{tabular}




\begin{tabular}{|c|c|c|c|}
\hline \multicolumn{4}{|c|}{$\mathbf{T S}_{19}$} \\
\hline \multicolumn{4}{|c|}{$\mathrm{E}(\mathrm{SMD} / \mathrm{B} 3 \mathrm{LYP} / \mathrm{BS} 1)=-3675.38752015 \mathrm{au}$} \\
\hline \multicolumn{4}{|c|}{$\mathrm{H}(\mathrm{SMD} / \mathrm{B} 3 \mathrm{LYP} / \mathrm{BS} 1)=-3675.002199 \mathrm{au}$} \\
\hline \multicolumn{4}{|c|}{$\mathrm{G}(\mathrm{SMD} / \mathrm{B} 3 \mathrm{~L} Y \mathrm{P} / \mathrm{BS} 1)=-3675.124718 \mathrm{au}$} \\
\hline \multicolumn{4}{|c|}{$\mathrm{E}(\mathrm{SMD} / \mathrm{M} 06 / \mathrm{BS} 2 / / \mathrm{SMD} / \mathrm{B} 3 \mathrm{LYP} / \mathrm{BS} 1)=-3675.47940032 \mathrm{au}$} \\
\hline $\mathrm{C}$ & 2.86368400 & -1.14308200 & 0.72588400 \\
\hline $\mathrm{C}$ & 2.90361000 & -0.53707200 & 1.99057000 \\
\hline $\mathrm{C}$ & 2.78973400 & -2.54277500 & 0.65095100 \\
\hline $\mathrm{C}$ & 2.86852200 & -1.31333200 & 3.15297600 \\
\hline $\mathrm{H}$ & 2.95532800 & 0.54403900 & 2.06630000 \\
\hline $\mathrm{C}$ & 2.76112400 & -3.31722000 & 1.81119600 \\
\hline $\mathrm{H}$ & 2.75889300 & -3.03812400 & -0.31634000 \\
\hline $\mathrm{C}$ & 2.80320400 & -2.70462200 & 3.06685100 \\
\hline $\mathrm{H}$ & 2.89912200 & -0.82588200 & 4.12379400 \\
\hline $\mathrm{H}$ & 2.70230500 & -4.39910700 & 1.73296900 \\
\hline $\mathrm{H}$ & 2.78286200 & -3.30865100 & 3.96982600 \\
\hline $\mathrm{C}$ & 2.95228100 & -0.28893800 & -0.54021600 \\
\hline $\mathrm{H}$ & 3.00892400 & 0.76195900 & -0.24967200 \\
\hline $\mathrm{C}$ & 1.75872100 & -0.44256500 & -1.48431300 \\
\hline $\mathrm{H}$ & 1.31153600 & -1.42732000 & -1.60899500 \\
\hline $\mathrm{C}$ & 4.30612800 & -0.64228900 & -1.27599700 \\
\hline $\mathrm{H}$ & 4.36907800 & -1.73275100 & -1.40028200 \\
\hline $\mathrm{C}$ & 5.53786600 & -0.26386300 & -0.41455200 \\
\hline $\mathrm{C}$ & 5.63758500 & 1.14985100 & 0.11058600 \\
\hline $\mathrm{H}$ & 5.23115700 & 1.88287100 & -0.59438500 \\
\hline $\mathrm{H}$ & 5.05843100 & 1.23322400 & 1.03887400 \\
\hline $\mathrm{H}$ & 6.68141400 & 1.37916800 & 0.33806500 \\
\hline $\mathrm{C}$ & 4.45696300 & -0.13054100 & -2.71327400 \\
\hline $\mathrm{C}$ & 5.83628200 & 0.06293400 & -3.27445800 \\
\hline $\mathrm{H}$ & 6.31050800 & 0.92757800 & -2.79186100 \\
\hline $\mathrm{H}$ & 6.45735100 & -0.81309400 & -3.05704700 \\
\hline $\mathrm{H}$ & 5.78120100 & 0.23487000 & -4.35067600 \\
\hline $\mathrm{H}$ & 2.18909300 & -0.23621200 & -2.55958000 \\
\hline $\mathrm{H}$ & 1.17048600 & 0.47353100 & -1.78054100 \\
\hline $\mathrm{O}$ & 3.47715000 & 0.045555000 & -3.44047600 \\
\hline $\mathrm{O}$ & 6.39363400 & -1.10041100 & -0.19691800 \\
\hline $\mathrm{Au}$ & -0.34823400 & 0.21398400 & -0.45800600 \\
\hline $\mathrm{O}$ & -2.28285600 & 0.64869000 & 0.27045500 \\
\hline S & -3.25106400 & 1.50220200 & -0.62725600 \\
\hline $\mathrm{O}$ & -4.01793000 & 2.40956300 & 0.22276700 \\
\hline $\mathrm{O}$ & -2.59893400 & 1.99306200 & -1.84211600 \\
\hline $\mathrm{C}$ & -4.46420700 & 0.21007200 & -1.21644600 \\
\hline $\mathrm{F}$ & -5.19707800 & -0.24253700 & -0.20242900 \\
\hline $\mathrm{F}$ & -5.26371600 & 0.77527600 & -2.12313600 \\
\hline $\mathrm{F}$ & -3.80646200 & -0.80616000 & -1.78529300 \\
\hline $\mathrm{O}$ & -0.35364900 & -4.00138100 & 0.19417500 \\
\hline S & -1.02580100 & -2.74554000 & 0.51602400 \\
\hline $\mathrm{O}$ & -0.80575900 & -2.07353700 & 1.79511000 \\
\hline $\mathrm{O}$ & -0.85944700 & -1.75781900 & -0.70639200 \\
\hline $\mathrm{C}$ & -2.85264700 & -3.13265400 & 0.42043700 \\
\hline $\mathrm{F}$ & -3.08683800 & -4.18610800 & 1.20445200 \\
\hline
\end{tabular}




$\begin{array}{lrrc}\text { F } & -3.56361500 & -2.09477400 & 0.85341100 \\ \text { F } & -3.19489400 & -3.42570500 & -0.83292900 \\ \text { O } & 2.07807200 & 2.51902000 & 0.98489900 \\ \text { S } & 0.79934400 & 3.10577200 & 0.58277600 \\ \text { O } & 0.74689200 & 4.48877100 & 0.11946600 \\ \text { O } & 0.02860400 & 2.24328400 & -0.47882700 \\ \text { C } & -0.30488500 & 3.00110200 & 2.08643300 \\ \text { F } & 0.23122600 & 3.74476800 & 3.05256900 \\ \text { F } & -1.51932200 & 3.45265900 & 1.79099100 \\ \text { F } & -0.38672600 & 1.73057000 & 2.50018600\end{array}$

$\mathrm{TS}_{18-17}$

$\mathrm{E}(\mathrm{SMD} / \mathrm{B} 3 \mathrm{LYP} / \mathrm{BS} 1)=-3675.40978005 \mathrm{au}$

$\mathrm{H}(\mathrm{SMD} / \mathrm{B} 3 \mathrm{LYP} / \mathrm{BS} 1)=-3675.025684 \mathrm{au}$

$\mathrm{G}(\mathrm{SMD} / \mathrm{B} 3 \mathrm{LYP} / \mathrm{BS} 1)=-3675.150221 \mathrm{au}$

$\mathrm{E}(\mathrm{SMD} / \mathrm{M} 06 / \mathrm{BS} 2 / / \mathrm{SMD} / \mathrm{B} 3 \mathrm{LYP} / \mathrm{BS} 1)=-3675.49631102 \mathrm{au}$

$\begin{array}{llll}\mathrm{C} & 5.63771700 & -0.08644000 & -2.77450100\end{array}$

$\mathrm{H} \quad \begin{array}{llll}\mathrm{H} & 6.21349600 & -0.27169000 & -1.86124600\end{array}$

$\mathrm{H} \quad \begin{array}{llll}\mathrm{H} & 5.97630900 & 0.83138500 & -3.26018700\end{array}$

$\mathrm{H} \quad 5.81473300 \quad-0.94047400 \quad-3.44195300$

C $\quad 4.16943400 \quad 0.00324500 \quad-2.46625100$

$\begin{array}{lllll}\mathrm{O} & 3.45155200 & 0.90592500 & -2.85303000\end{array}$

C $\quad 3.55686900 \quad-1.15920600 \quad-1.61989300$

C $\quad 2.42652300 \quad-1.73392300 \quad-2.44507200$

C $\quad 2.70387500 \quad-2.76563700 \quad-3.48588600$

$\mathrm{H} \quad 1.90875900 \quad-2.78610700 \quad-4.23427200$

$\mathrm{H} \quad 3.67960300 \quad-2.59656300 \quad-3.95280500$

$\mathrm{H} \quad 2.74372800 \quad-3.74365000 \quad-2.98428100$

O $\quad \begin{array}{llll}1.27123500 & -1.32433100 & -2.25686700\end{array}$

$\mathrm{H} \quad \begin{array}{llll}1.25251400 & -0.41835100 & -1.10946300\end{array}$

$\mathrm{H} \quad 4.31730800 \quad-1.93424700 \quad-1.49200400$

$\begin{array}{llll}\mathrm{C} & 3.06848800 & -0.71324000 & -0.20875000\end{array}$

$\begin{array}{llll}\mathrm{C} & 4.16982800 & -0.13660200 & 0.67611800\end{array}$

$\begin{array}{llll}\text { C } & 4.62757700 & -0.90316300 & 1.75982400\end{array}$

$\begin{array}{llll}\mathrm{C} & 4.72413200 & 1.13775600 & 0.46341400\end{array}$

$\begin{array}{llll}\mathrm{C} & 5.63842600 & -0.42322600 & 2.59293200\end{array}$

$\mathrm{H} \quad 4.18837700 \quad-1.87844600 \quad 1.94996000$

$\begin{array}{llll}\mathrm{C} & 5.73083400 & 1.61803800 & 1.30262000\end{array}$

$\mathrm{H} \quad 4.37415000 \quad 1.76668000 \quad-0.34927600$

$\begin{array}{llll}\mathrm{C} & 6.19310900 & 0.83938600 & 2.36662200\end{array}$

$\mathrm{H} \quad 5.98803100 \quad-1.03432800 \quad 3.42045200$

$\begin{array}{llll}\mathrm{H} & 6.14933000 & 2.60481600 & 1.12556900\end{array}$

$\begin{array}{llll}\mathrm{H} & 6.97571400 & 1.21664000 & 3.01899900\end{array}$

$\begin{array}{llll}\mathrm{C} & 1.88027800 & 0.31023300 & -0.23365300\end{array}$

$\mathrm{H} \quad 2.05911600 \quad 1.20600100 \quad-0.82755900$

$\begin{array}{llll}\mathrm{H} & 1.68813600 & 0.58837400 & 0.80349000\end{array}$

$\mathrm{H} \quad 2.71192100 \quad-1.62157300 \quad 0.28391100$

$\mathrm{Au} \quad-0.44341600 \quad 0.10998400 \quad-0.19435300$

$\begin{array}{llll}\mathrm{O} & -2.52598800 & 0.09412100 & 0.06590200\end{array}$

S $\quad \begin{array}{llll}\text { S } & -3.50687400 & 0.05047900 & -1.15283500\end{array}$

$\begin{array}{lllll}\mathrm{O} & -4.84430500 & 0.38387500 & -0.66750900\end{array}$ 


$\begin{array}{lrrr}\mathrm{O} & -2.97177000 & 0.69635200 & -2.35017000 \\ \mathrm{C} & -3.53467600 & -1.77510600 & -1.54130800 \\ \mathrm{~F} & -3.73980100 & -2.48576400 & -0.43302900 \\ \mathrm{~F} & -4.51628600 & -2.01527700 & -2.41217400 \\ \mathrm{~F} & -2.36575500 & -2.13700500 & -2.08625500 \\ \mathrm{O} & -0.20587400 & -3.88726400 & 1.67572100 \\ \mathrm{~S} & -0.11383500 & -2.43209200 & 1.73903300 \\ \mathrm{O} & 1.08350900 & -1.78725800 & 2.28146900 \\ \mathrm{O} & -0.50205100 & -1.88259700 & 0.31802300 \\ \mathrm{C} & -1.53016600 & -1.84465600 & 2.80837400 \\ \mathrm{~F} & -1.33056800 & -2.28610200 & 4.04944800 \\ \mathrm{~F} & -1.55946200 & -0.50566600 & 2.81634600 \\ \mathrm{~F} & -2.68356100 & -2.31381700 & 2.34404500 \\ \mathrm{O} & 0.65785800 & 3.12567200 & 1.05227600 \\ \mathrm{~S} & -0.33068600 & 3.31315300 & -0.00997100 \\ \mathrm{O} & -0.25322600 & 4.46154300 & -0.90680500 \\ \mathrm{O} & -0.49652700 & 2.03328900 & -0.91141800 \\ \mathrm{C} & -1.98836100 & 3.43489100 & 0.85136100 \\ \mathrm{~F} & -2.04848600 & 4.61383800 & 1.46979000 \\ \mathrm{~F} & -2.97616500 & 3.34457300 & -0.03683200 \\ \mathrm{~F} & -2.10743700 & 2.45738000 & 1.75404400\end{array}$

$\mathrm{TS}_{11-20}$

$\mathrm{E}(\mathrm{SMD} / \mathrm{B} 3 \mathrm{LYP} / \mathrm{BS} 1)=-3675.41648451 \mathrm{au}$

$\mathrm{H}(\mathrm{SMD} / \mathrm{B} 3 \mathrm{LYP} / \mathrm{BS} 1)=-3675.031163 \mathrm{au}$

$\mathrm{G}(\mathrm{SMD} / \mathrm{B} 3 \mathrm{LYP} / \mathrm{BS} 1)=-3675.161257 \mathrm{au}$

$\mathrm{E}(\mathrm{SMD} / \mathrm{M} 06 / \mathrm{BS} 2 / / \mathrm{SMD} / \mathrm{B} 3 \mathrm{LYP} / \mathrm{BS} 1)=-3675.48747974 \mathrm{au}$

C $\quad-1.26887400 \quad-1.29256500 \quad-2.62632200$

$\mathrm{H} \quad-1.58641900 \quad-1.96431900 \quad-3.42620800$

$\mathrm{H} \quad-0.19705000 \quad-1.09749800 \quad-2.73045600$

$\mathrm{H} \quad-1.80324100 \quad-0.34118800 \quad-2.74124000$

C $\quad-1.57775900 \quad-1.87354800 \quad-1.27781600$

O $\quad-1.09535400 \quad-1.30718400 \quad-0.19304000$

C $\quad-2.41379800 \quad-2.97057300 \quad-1.15537100$

$\begin{array}{llll}\text { C } & -2.71047800 & -3.68779500 & 0.04315100\end{array}$

$\begin{array}{llll}\mathrm{O} & -2.54143000 & -3.27123100 & 1.22850700\end{array}$

C $\quad-3.21590500 \quad-5.10292400 \quad-0.09954600$

$\mathrm{H} \quad-2.35410500 \quad-5.78379500 \quad-0.12283100$

$\mathrm{H} \quad-3.83797900 \quad-5.37166100 \quad 0.75810200$

$\mathrm{H} \quad-3.77267100 \quad-5.24134900 \quad-1.03077100$

$\mathrm{H} \quad \begin{array}{llll}-2.66618200 & -1.96465500 & 1.57173100\end{array}$

$\begin{array}{llll}\mathrm{C} & -3.53664000 & -0.04184000 & 1.42764800\end{array}$

$\begin{array}{llll}\mathrm{C} & -2.91721700 & -0.91888600 & 2.31597500\end{array}$

$\mathrm{H} \quad-2.88207900 \quad 0.56531200 \quad 0.80391000$

$\mathrm{H} \quad-1.89029000 \quad-0.67396000 \quad 2.59439800$

$\mathrm{H} \quad-\quad-3.51014700 \quad-1.37858300 \quad 3.10771100$

$\begin{array}{lllll}\mathrm{C} & & -4.93885600 & 0.10686000 & 1.15378600\end{array}$

$\begin{array}{llll}\text { C } & -5.32069000 & 0.97274700 & 0.10108900\end{array}$

$\begin{array}{llll}\text { C } & -5.94450500 & -0.56649300 & 1.88926700\end{array}$

$\begin{array}{llll}\mathrm{C} & -6.66274900 & 1.15641400 & -0.21124400\end{array}$

$\begin{array}{lllll}\mathrm{H} & -4.54976200 & 1.49188200 & -0.46209600\end{array}$ 


$\begin{array}{lrrr}\mathrm{C} & -7.28187900 & -0.38109000 & 1.57012600 \\ \mathrm{H} & -5.67124300 & -1.22545200 & 2.70700700 \\ \mathrm{C} & -7.64231600 & 0.47889300 & 0.52195300 \\ \mathrm{H} & -6.94788300 & 1.82212800 & -1.02004700 \\ \mathrm{H} & -8.05143100 & -0.89780700 & 2.13567900 \\ \mathrm{H} & -8.69211100 & 0.62168200 & 0.28100700 \\ \mathrm{H} & -2.78844600 & -3.39226700 & -2.08047900 \\ \mathrm{Au} & 0.52574600 & -0.09783500 & -0.13074200 \\ \mathrm{O} & 2.15765300 & 1.12953200 & 0.16058900 \\ \mathrm{~S} & 2.32272100 & 1.93586200 & 1.49870900 \\ \mathrm{O} & 3.29256000 & 3.00152300 & 1.26686600 \\ \mathrm{O} & 1.04031600 & 2.21710200 & 2.14528200 \\ \mathrm{C} & 3.18020500 & 0.69752500 & 2.60358000 \\ \mathrm{~F} & 4.23970000 & 0.17991300 & 1.98474100 \\ \mathrm{~F} & 3.57371200 & 1.32486800 & 3.71340900 \\ \mathrm{~F} & 2.33241300 & -0.28286000 & 2.93193600 \\ \mathrm{O} & 2.72913800 & -3.87070700 & 0.30081800 \\ \mathrm{~S} & 2.23359800 & -2.76718000 & -0.51480500 \\ \mathrm{O} & 1.33525700 & -3.01639400 & -1.64105300 \\ \mathrm{O} & 1.68975400 & -1.66566300 & 0.46886500 \\ \mathrm{C} & 3.73358600 & -1.91726300 & -1.23135600 \\ \mathrm{~F} & 4.34049300 & -2.75564400 & -2.07066700 \\ \mathrm{~F} & 3.34974500 & -0.82277700 & -1.89890600 \\ \mathrm{~F} & 4.57131600 & -1.57106700 & -0.25760000 \\ \mathrm{O} & 0.49206300 & 1.93454400 & -2.65971200 \\ \mathrm{~S} & -0.63627900 & 2.33617100 & -1.82059500 \\ \mathrm{O} & -1.97329200 & 2.47715500 & -2.39673600 \\ \mathrm{O} & -0.73595600 & 1.47398900 & -0.50635900 \\ \mathrm{C} & -0.22194100 & 4.00799800 & -1.09564500 \\ \mathrm{~F} & -0.31221600 & 4.91604500 & -2.06791000 \\ \mathrm{~F} & -1.08438600 & 4.30445600 & -0.12498000 \\ \mathrm{~F} & 1.01807900 & 4.00226200 & -0.60976300\end{array}$

\section{0}

\begin{tabular}{|c|c|c|c|}
\hline \\
\hline \multicolumn{4}{|c|}{$\begin{array}{l}\mathrm{E}(\mathrm{SMD} / \mathrm{B} 3 L Y \mathrm{~L} / \mathrm{BS} 1)=-3675.42837498 \mathrm{au} \\
\mathrm{H}(\mathrm{SMD} / \mathrm{B} 3 \mathrm{LYP} / \mathrm{BS} 1)=-3675.039294 \mathrm{au}\end{array}$} \\
\hline \multicolumn{4}{|c|}{$\mathrm{G}(\mathrm{SMD} / \mathrm{B} 3 \mathrm{LYP} / \mathrm{BS} 1)=-3675.172442 \mathrm{au}$} \\
\hline \multicolumn{4}{|c|}{$\mathrm{E}(\mathrm{SMD} / \mathrm{M} 06 / \mathrm{BS} 2 / / \mathrm{SMD} / \mathrm{B} 3 \mathrm{LYP} / \mathrm{BS} 1)=-3675.49620668 \mathrm{au}$} \\
\hline $\mathrm{C}$ & 0.25174500 & 2.12838300 & -3.18701600 \\
\hline $\mathrm{H}$ & 0.01913600 & 3.00134300 & -3.80217200 \\
\hline $\mathrm{H}$ & -0.57138700 & 1.40918600 & -3.28117300 \\
\hline $\mathrm{H}$ & 1.15287600 & 1.63810400 & -3.57197200 \\
\hline $\mathrm{C}$ & 0.45248500 & 2.50364800 & -1.74446000 \\
\hline $\mathrm{O}$ & 0.87891300 & 1.55288600 & -0.92140400 \\
\hline $\mathrm{C}$ & 0.29748300 & 3.79179300 & -1.29528700 \\
\hline $\mathrm{H}$ & 0.08313300 & 4.54809200 & -2.04505100 \\
\hline $\mathrm{C}$ & 0.33438300 & 4.25850400 & 0.10356800 \\
\hline $\mathrm{C}$ & -0.18962800 & 5.66560700 & 0.31707200 \\
\hline $\mathrm{H}$ & -1.22756300 & 5.74359400 & -0.03093800 \\
\hline $\mathrm{H}$ & -0.13900800 & 5.92618000 & 1.37669000 \\
\hline $\mathrm{H}$ & 0.39956100 & 6.38328400 & -0.26844600 \\
\hline
\end{tabular}




$\begin{array}{lccc}\mathrm{O} & 0.73295300 & 3.58659100 & 1.05516900 \\ \mathrm{C} & 7.81819300 & 0.63153400 & 0.53117200 \\ \mathrm{C} & 7.32662600 & 1.57164800 & 1.45590400 \\ \mathrm{C} & 5.97827800 & 1.60567000 & 1.74945600 \\ \mathrm{C} & 5.08840300 & 0.68779300 & 1.10890800 \\ \mathrm{C} & 5.61337800 & -0.25946100 & 0.17378300 \\ \mathrm{C} & 6.96713900 & -0.28205200 & -0.10801600 \\ \mathrm{H} & 8.88164000 & 0.61333100 & 0.30933400 \\ \mathrm{H} & 8.00984800 & 2.26457700 & 1.93573100 \\ \mathrm{H} & 5.59429100 & 2.32548900 & 2.46311500 \\ \mathrm{H} & 4.93154300 & -0.95517700 & -0.30773900 \\ \mathrm{H} & 7.36989600 & -0.99821400 & -0.81648700 \\ \mathrm{C} & 3.71142100 & 0.67083200 & 1.34911400 \\ \mathrm{H} & 3.13746900 & -0.07411900 & 0.79790400 \\ \mathrm{C} & 2.92568400 & 1.53388700 & 2.22542500 \\ \mathrm{H} & 3.49671700 & 2.22627400 & 2.84442100 \\ \mathrm{H} & 2.21281300 & 2.10054200 & 1.59321600 \\ \mathrm{H} & 2.27577400 & 0.90250000 & 2.85123200 \\ \mathrm{Au} & -0.35426100 & 0.05062100 & -0.30592200 \\ \mathrm{O} & -1.51411400 & -1.50138700 & 0.53671300 \\ \mathrm{~S} & -1.26400600 & -2.00599100 & 1.98683600 \\ \mathrm{O} & -1.86069200 & -3.33174600 & 2.14218600 \\ \mathrm{O} & 0.10038700 & -1.75991000 & 2.46086000 \\ \mathrm{C} & -2.32719900 & -0.84602300 & 2.98593500 \\ \mathrm{~F} & -3.60776700 & -0.95225900 & 2.63225700 \\ \mathrm{~F} & -2.20088700 & -1.15033900 & 4.28055800 \\ \mathrm{~F} & -1.92188500 & 0.41827700 & 2.79539700 \\ \mathrm{O} & -3.58011100 & 2.64531800 & -1.54457600 \\ \mathrm{~S} & -3.12568900 & 1.28432900 & -1.26599400 \\ \mathrm{O} & -2.83235900 & 0.36433800 & -2.36857900 \\ \mathrm{O} & -1.95981000 & 1.34790900 & -0.21744300 \\ \mathrm{C} & -4.50534900 & 0.49871500 & -0.27940300 \\ \mathrm{~F} & -5.64160300 & 0.65148800 & -0.96479000 \\ \mathrm{~F} & -4.27240500 & -0.79911700 & -0.10066000 \\ \mathrm{~F} & -4.61855500 & 1.10294800 & 0.90404500 \\ \mathrm{O} & 1.51762900 & -1.20025500 & -2.85049000 \\ \mathrm{~S} & 1.81972500 & -1.90176400 & -1.60369600 \\ \mathrm{O} & 3.18216900 & -2.36978500 & -1.33830300 \\ \mathrm{O} & 1.32386100 & -1.15751100 & -0.32152200 \\ \mathrm{C} & 0.74008800 & -3.42554300 & -1.60218100 \\ \mathrm{~F} & 1.19404000 & -4.27451900 & -2.52565500 \\ \mathrm{~F} & 0.77289800 & -4.01122400 & -0.40499800 \\ \mathrm{~F} & -0.51827700 & -3.08530000 & -1.89602600\end{array}$

$\mathrm{TS}_{20-21}$

$\mathrm{E}(\mathrm{SMD} / \mathrm{B} 3 \mathrm{LYP} / \mathrm{BS} 1)=-3675.42042724 \mathrm{au}$

$\mathrm{H}(\mathrm{SMD} / \mathrm{B} 3 \mathrm{LYP} / \mathrm{BS} 1)=-3675.030974 \mathrm{au}$

$\mathrm{G}(\mathrm{SMD} / \mathrm{B} 3 \mathrm{LYP} / \mathrm{BS} 1)=-3675.159936 \mathrm{au}$

$\mathrm{E}(\mathrm{SMD} / \mathrm{M} 06 / \mathrm{BS} 2 / / \mathrm{SMD} / \mathrm{B} 3 \mathrm{LYP} / \mathrm{BS} 1)=-3675.49548516 \mathrm{au}$

$\begin{array}{llll}\mathrm{C} & 1.86760500 & -1.09967400 & 1.68488000\end{array}$

$\mathrm{H} \quad 2.73353900 \quad-1.48421100 \quad 2.22830400$ 


\begin{tabular}{|c|c|c|c|}
\hline $\mathrm{H}$ & 0.98396800 & -1.65391600 & 2.01844200 \\
\hline $\mathrm{H}$ & 1.71283600 & -0.04647500 & 1.93962500 \\
\hline $\mathrm{C}$ & 2.05611900 & -1.25337200 & 0.20310100 \\
\hline $\mathrm{O}$ & 1.20025900 & -0.70786900 & -0.63540500 \\
\hline $\mathrm{C}$ & 3.15723700 & -1.92579000 & -0.30658000 \\
\hline $\mathrm{H}$ & 3.84307400 & -2.34643100 & 0.42032400 \\
\hline $\mathrm{C}$ & 3.32809700 & -2.29898800 & -1.71019300 \\
\hline $\mathrm{C}$ & 4.42081900 & -3.31686200 & -1.99468000 \\
\hline $\mathrm{H}$ & 4.19994800 & -4.26385900 & -1.48619900 \\
\hline $\mathrm{H}$ & 4.49253400 & -3.49252400 & -3.07060000 \\
\hline $\mathrm{H}$ & 5.38806200 & -2.96587000 & -1.61381300 \\
\hline $\mathrm{O}$ & 2.63952400 & -1.83979600 & -2.62403500 \\
\hline $\mathrm{C}$ & 8.39763600 & -0.64397900 & 0.71759600 \\
\hline $\mathrm{C}$ & 8.10381100 & -0.56732500 & -0.65333700 \\
\hline $\mathrm{C}$ & 6.83775800 & -0.19182700 & -1.06950000 \\
\hline $\mathrm{C}$ & 5.83444500 & 0.11644400 & -0.10678300 \\
\hline $\mathrm{C}$ & 6.15530900 & 0.02856800 & 1.27899900 \\
\hline $\mathrm{C}$ & 7.42574500 & -0.34462700 & 1.68328000 \\
\hline $\mathrm{H}$ & 9.39404700 & -0.93921700 & 1.03420300 \\
\hline $\mathrm{H}$ & 8.87063300 & -0.80104300 & -1.38478500 \\
\hline $\mathrm{H}$ & 6.61186400 & -0.13257700 & -2.12824900 \\
\hline $\mathrm{H}$ & 5.38875500 & 0.26622500 & 2.01091200 \\
\hline $\mathrm{H}$ & 7.66972200 & -0.40526600 & 2.73898800 \\
\hline $\mathrm{C}$ & 4.53547800 & 0.54462300 & -0.46314200 \\
\hline $\mathrm{H}$ & 3.87840400 & 0.80813900 & 0.36226400 \\
\hline $\mathrm{C}$ & 4.05090700 & 0.91709000 & -1.79974200 \\
\hline $\mathrm{H}$ & 4.69419100 & 0.61303800 & -2.62562600 \\
\hline $\mathrm{H}$ & 3.03143200 & 0.54479200 & -1.96152900 \\
\hline $\mathrm{H}$ & 3.96289100 & 2.01787500 & -1.80094800 \\
\hline $\mathrm{Au}$ & -0.63247100 & 0.01402300 & -0.15961400 \\
\hline $\mathrm{O}$ & -2.54891200 & 0.75737700 & 0.09523700 \\
\hline S & -3.34096300 & 1.41074300 & -1.09420500 \\
\hline $\mathrm{O}$ & -4.32507200 & 2.33316500 & -0.53437400 \\
\hline $\mathrm{O}$ & -2.47578700 & 1.82226500 & -2.19814900 \\
\hline $\mathrm{C}$ & -4.30826800 & -0.04066400 & -1.75923300 \\
\hline $\mathrm{F}$ & -5.19347200 & -0.47043400 & -0.86204800 \\
\hline $\mathrm{F}$ & -4.95134500 & 0.35213900 & -2.86076500 \\
\hline $\mathrm{F}$ & -3.47093100 & -1.03656500 & -2.07216900 \\
\hline $\mathrm{O}$ & -1.37245100 & -3.94264900 & 1.42740700 \\
\hline S & -1.76486200 & -2.53555600 & 1.42262500 \\
\hline $\mathrm{O}$ & -1.43885200 & -1.67043400 & 2.55836800 \\
\hline $\mathrm{O}$ & -1.32340200 & -1.90720400 & 0.04940000 \\
\hline $\mathrm{C}$ & -3.63097600 & -2.53570800 & 1.28347800 \\
\hline $\mathrm{F}$ & -4.11009200 & -3.36870700 & 2.20929400 \\
\hline $\mathrm{F}$ & -4.10553400 & -1.31273100 & 1.50414200 \\
\hline $\mathrm{F}$ & -4.00073300 & -2.95477500 & 0.07440900 \\
\hline $\mathrm{O}$ & 1.83207500 & 2.13790900 & 1.13932100 \\
\hline S & 0.85409900 & 2.84374300 & 0.30690800 \\
\hline $\mathrm{O}$ & 1.27242600 & 3.99946400 & -0.48074300 \\
\hline $\mathrm{O}$ & 0.04133500 & 1.89796900 & -0.63661400 \\
\hline $\mathrm{C}$ & -0.46608600 & 3.44343700 & 1.48199700 \\
\hline
\end{tabular}




$\begin{array}{lrrr}\text { F } & 0.06782300 & 4.34450900 & 2.30575600 \\ F & -1.46743400 & 3.99509000 & 0.80019400 \\ \text { F } & -0.93117500 & 2.41158100 & 2.19661300\end{array}$

\section{1}

$\mathrm{E}(\mathrm{SMD} / \mathrm{B} 3 \mathrm{LYP} / \mathrm{BS} 1)=-3675.45405956 \mathrm{au}$

$\mathrm{H}(\mathrm{SMD} / \mathrm{B} 3 \mathrm{LYP} / \mathrm{BS} 1)=-3675.061271 \mathrm{au}$

$\mathrm{G}(\mathrm{SMD} / \mathrm{B} 3 \mathrm{LYP} / \mathrm{BS} 1)=-3675.188097 \mathrm{au}$

$\mathrm{E}(\mathrm{SMD} / \mathrm{M} 06 / \mathrm{BS} 2 / / \mathrm{SMD} / \mathrm{B} 3 \mathrm{LYP} / \mathrm{BS} 1)=-3675.53653057 \mathrm{au}$

C $\quad-1.76712700 \quad-0.21054900 \quad-2.02878600$

$\mathrm{H} \quad-2.43365000 \quad 0.56109300 \quad-2.43190800$

$\mathrm{H} \quad-1.99721000 \quad-1.14204100 \quad-2.55878200$

$\mathrm{H} \quad-0.72740100 \quad 0.07148000 \quad-2.19326900$

C $\quad-2.08754600 \quad-0.40192800 \quad-0.59255600$

$\begin{array}{llll}\mathrm{O} & -1.26013200 & -0.30232100 & 0.34817200\end{array}$

$\begin{array}{llll}\mathrm{C} & -3.50652500 & -0.72609000 & -0.21267000\end{array}$

$\mathrm{H} \quad-4.03762700 \quad-0.93307100 \quad-1.14468500$

$\begin{array}{llll}\mathrm{C} & -3.57199500 & -1.98551300 & 0.69631600\end{array}$

$\begin{array}{llll}\text { C } & -4.47283600 & -3.09165700 & 0.20829200\end{array}$

$\mathrm{H} \quad-4.05368500 \quad-3.50855200 \quad-0.71762200$

$\mathrm{H} \quad \begin{array}{llll}-4.54312800 & -3.87739800 & 0.96367800\end{array}$

$\mathrm{H} \quad-5.46922600 \quad-2.70586800 \quad-0.03299500$

$\begin{array}{llll}\mathrm{O} & -2.92316100 & -2.05782600 & 1.71988100\end{array}$

$\begin{array}{llll}\text { C } & -8.51974400 & -0.11759700 & 0.42108300\end{array}$

$\begin{array}{llll}\text { C } & -7.76544900 & -0.48370700 & 1.53836000\end{array}$

$\begin{array}{llll}\text { C } & -6.38360200 & -0.27641400 & 1.55580800\end{array}$

$\begin{array}{llll}\mathrm{C} & -5.73335100 & 0.30275400 & 0.45553800\end{array}$

C $\quad \begin{array}{lllll}\text { C } & -6.50282600 & 0.66786800 & -0.65987900\end{array}$

$\begin{array}{lllll}\text { C } & -7.88323800 & 0.46045600 & -0.68036700\end{array}$

$\mathrm{H} \quad-9.59460300 \quad-0.27737100 \quad 0.41064500$

$\mathrm{H} \quad-8.25155600 \quad-0.92933800 \quad 2.40230200$

$\mathrm{H} \quad-5.81602900 \quad-0.56475400 \quad 2.43577200$

$\mathrm{H} \quad-6.01472400 \quad 1.12733600 \quad-1.51692700$

$\mathrm{H} \quad \begin{array}{llll}-8.46054000 & 0.75607000 & -1.55253500\end{array}$

$\begin{array}{llll}\mathrm{C} & -4.22621700 & 0.54466000 & 0.43542000\end{array}$

$\mathrm{H} \quad-4.04593300 \quad 1.34857300 \quad-0.28701100$

$\begin{array}{llll}\mathrm{C} & -3.65424800 & 0.98910400 & 1.78617400\end{array}$

$\mathrm{H} \quad-3.71186300 \quad 0.20852800 \quad 2.54819700$

$\begin{array}{llll}\mathrm{H} & -2.60755500 & 1.29152300 & 1.69731400\end{array}$

$\mathrm{H} \quad-4.22114800 \quad 1.85709100 \quad 2.14001500$

$\begin{array}{llll}\mathrm{Au} & 0.75546000 & 0.10064700 & 0.21907600\end{array}$

$\begin{array}{lllll}\text { O } & 2.74923100 & 0.49894700 & 0.14710800\end{array}$

$\begin{array}{lllll}\mathrm{S} & 3.56227600 & 0.83848900 & 1.46563400\end{array}$

$\begin{array}{lllll}\mathrm{O} & 4.64899400 & 1.73565800 & 1.08953200\end{array}$

$\begin{array}{llll}\mathrm{O} & 2.68227400 & 1.13836400 & 2.59197900\end{array}$

$\begin{array}{llll}\mathrm{C} & 4.35190100 & -0.81693000 & 1.83139900\end{array}$

F $\quad \begin{array}{llll}\text { C } & 4.94803100 & -1.28670600 & 0.73777400\end{array}$

F $\quad \begin{array}{llll}5.25450800 & -0.64335500 & 2.79656500\end{array}$

F $\quad 3.42130800 \quad-1.67805600 \quad 2.24164600$

O $\quad 1.36484600 \quad-4.25531000 \quad-0.27047800$

S $\quad 0.99530900 \quad-2.96175000 \quad-0.83171900$ 


$\begin{array}{lccc}\mathrm{O} & -0.28474600 & -2.78363200 & -1.51492800 \\ \mathrm{O} & 1.23671800 & -1.88801900 & 0.29236400 \\ \mathrm{C} & 2.31458100 & -2.51288600 & -2.07554600 \\ \mathrm{~F} & 2.29521800 & -3.40584200 & -3.06175300 \\ \mathrm{~F} & 2.05850600 & -1.29502500 & -2.57466400 \\ \mathrm{~F} & 3.50830800 & -2.50817200 & -1.49060200 \\ \mathrm{O} & -1.22686300 & 2.63895600 & -1.23085800 \\ \mathrm{~S} & -0.14725900 & 3.16461900 & -0.39664600 \\ \mathrm{O} & -0.33265200 & 4.39069900 & 0.37024500 \\ \mathrm{O} & 0.43438300 & 2.08873600 & 0.59323400 \\ \mathrm{C} & 1.30187000 & 3.45525100 & -1.53893000 \\ \mathrm{~F} & 0.97524200 & 4.40665400 & -2.41032700 \\ \mathrm{~F} & 2.36638000 & 3.82946100 & -0.83674700 \\ \mathrm{~F} & 1.57986300 & 2.32425700 & -2.20116500\end{array}$

TS $_{12-20}$

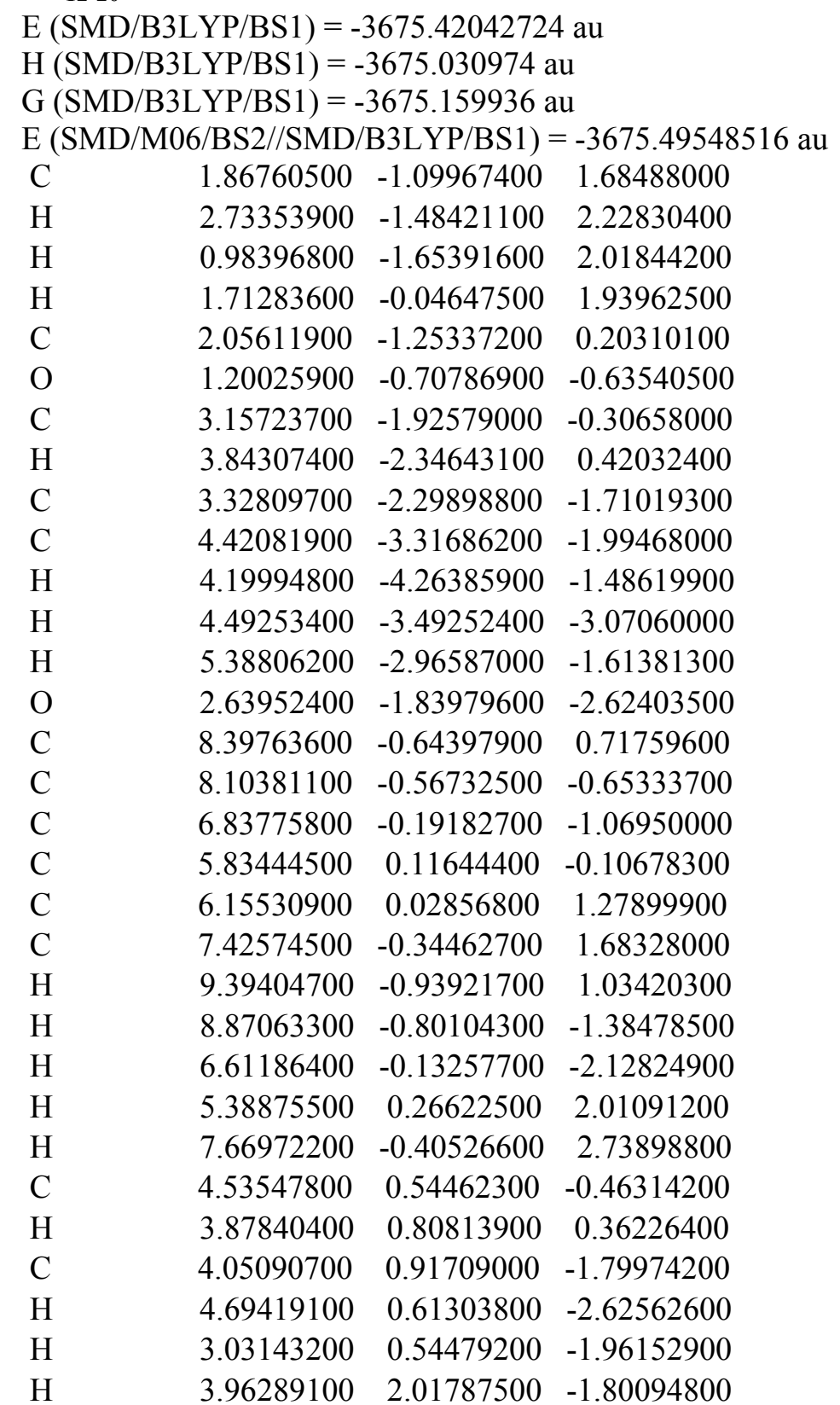




$\begin{array}{lccc}\mathrm{Au} & -0.63247100 & 0.01402300 & -0.15961400 \\ \mathrm{O} & -2.54891200 & 0.75737700 & 0.09523700 \\ \mathrm{~S} & -3.34096300 & 1.41074300 & -1.09420500 \\ \mathrm{O} & -4.32507200 & 2.33316500 & -0.53437400 \\ \mathrm{O} & -2.47578700 & 1.82226500 & -2.19814900 \\ \mathrm{C} & -4.30826800 & -0.04066400 & -1.75923300 \\ \mathrm{~F} & -5.19347200 & -0.47043400 & -0.86204800 \\ \mathrm{~F} & -4.95134500 & 0.35213900 & -2.86076500 \\ \mathrm{~F} & -3.47093100 & -1.03656500 & -2.07216900 \\ \mathrm{O} & -1.37245100 & -3.94264900 & 1.42740700 \\ \mathrm{~S} & -1.76486200 & -2.53555600 & 1.42262500 \\ \mathrm{O} & -1.43885200 & -1.67043400 & 2.55836800 \\ \mathrm{O} & -1.32340200 & -1.90720400 & 0.04940000 \\ \mathrm{C} & -3.63097600 & -2.53570800 & 1.28347800 \\ \mathrm{~F} & -4.11009200 & -3.36870700 & 2.20929400 \\ \mathrm{~F} & -4.10553400 & -1.31273100 & 1.50414200 \\ \mathrm{~F} & -4.00073300 & -2.95477500 & 0.07440900 \\ \mathrm{O} & 1.83207500 & 2.13790900 & 1.13932100 \\ \mathrm{~S} & 0.85409900 & 2.84374300 & 0.30690800 \\ \mathrm{O} & 1.27242600 & 3.99946400 & -0.48074300 \\ \mathrm{O} & 0.04133500 & 1.89796900 & -0.63661400 \\ \mathrm{C} & -0.46608600 & 3.44343700 & 1.48199700 \\ \mathrm{~F} & 0.06782300 & 4.34450900 & 2.30575600 \\ \mathrm{~F} & -1.46743400 & 3.99509000 & 0.80019400 \\ \mathrm{~F} & -0.93117500 & 2.41158100 & 2.19661300\end{array}$

$\mathrm{TS}_{10-22}$

$\mathrm{E}(\mathrm{SMD} / \mathrm{B} 3 \mathrm{LYP} / \mathrm{BS} 1)=-3675.43757199 \mathrm{au}$

$\mathrm{H}(\mathrm{SMD} / \mathrm{B} 3 \mathrm{LYP} / \mathrm{BS} 1)=-3675.052632 \mathrm{au}$

$\mathrm{G}(\mathrm{SMD} / \mathrm{B} 3 \mathrm{LYP} / \mathrm{BS} 1)=-3675.181038 \mathrm{au}$

$\mathrm{E}(\mathrm{SMD} / \mathrm{M} 06 / \mathrm{BS} 2 / / \mathrm{SMD} / \mathrm{B} 3 \mathrm{LYP} / \mathrm{BS} 1)=-3675.51929508 \mathrm{au}$

$\begin{array}{lrrr}\mathrm{C} & 1.34330000 & 3.05418800 & 2.78349400 \\ \mathrm{H} & 0.87723600 & 4.04702100 & 2.85417900 \\ \mathrm{H} & 2.40213900 & 3.11873300 & 3.03907000 \\ \mathrm{H} & 0.81021100 & 2.40819600 & 3.49072800 \\ \mathrm{C} & 1.16101300 & 2.53974300 & 1.39542800 \\ \mathrm{O} & 2.18277500 & 2.34620500 & 0.70278700 \\ \mathrm{C} & -0.24454800 & 2.40383700 & 0.88452400 \\ \mathrm{C} & -0.49333800 & 3.45704800 & -0.23159300 \\ \mathrm{O} & 0.25167900 & 3.52716900 & -1.18864600 \\ \mathrm{C} & -1.62215100 & 4.42003100 & 0.01826600 \\ \mathrm{H} & -2.50200000 & 3.92532700 & 0.43774700 \\ \mathrm{H} & -1.28081300 & 5.15089500 & 0.76560900 \\ \mathrm{H} & -1.87475700 & 4.94768200 & -0.90412500 \\ \mathrm{H} & -0.95558000 & 2.51757500 & 1.70359700 \\ \mathrm{C} & 6.21008800 & 1.72678500 & -1.29446200 \\ \mathrm{C} & 7.38667400 & 1.11462700 & -0.87289700 \\ \mathrm{C} & 7.36957000 & -0.22996400 & -0.49091400 \\ \mathrm{C} & 6.17606500 & -0.96355100 & -0.53528100 \\ \mathrm{C} & 4.99849600 & -0.35756100 & -0.95490000 \\ \mathrm{C} & 4.99751000 & 1.00413900 & -1.33448000\end{array}$




$\begin{array}{lccc}\mathrm{H} & 6.21425300 & 2.77204000 & -1.59202200 \\ \mathrm{H} & 8.31324800 & 1.68013500 & -0.84221500 \\ \mathrm{H} & 8.28670500 & -0.71175800 & -0.16334400 \\ \mathrm{H} & 6.17182900 & -2.01045800 & -0.24665600 \\ \mathrm{H} & 4.08350300 & -0.93894500 & -1.00224600 \\ \mathrm{C} & 3.80329000 & 1.69574300 & -1.77125600 \\ \mathrm{H} & 3.96649800 & 2.71432500 & -2.12472800 \\ \mathrm{C} & 2.49720100 & 1.26201500 & -1.70337400 \\ \mathrm{H} & 2.27697900 & 0.21908500 & -1.48729200 \\ \mathrm{H} & 2.23664100 & 1.88096400 & -0.44384800 \\ \mathrm{H} & 1.74651400 & 1.80948100 & -2.26912900 \\ \mathrm{Au} & -0.65435700 & 0.46886500 & 0.16788500 \\ \mathrm{O} & -1.06818900 & -1.52955600 & -0.54094500 \\ \mathrm{~S} & -0.57035100 & -1.98778400 & -1.92813700 \\ \mathrm{O} & -1.49039000 & -2.95824400 & -2.52134900 \\ \mathrm{O} & -0.07187300 & -0.87388200 & -2.74363300 \\ \mathrm{C} & 0.94244900 & -2.97463500 & -1.46912900 \\ \mathrm{~F} & 0.60877300 & -4.01641700 & -0.70640000 \\ \mathrm{~F} & 1.53751500 & -3.41493600 & -2.58111300 \\ \mathrm{~F} & 1.81163100 & -2.20357800 & -0.79239400 \\ \mathrm{O} & 1.73313600 & -1.07590900 & 3.53016700 \\ \mathrm{~S} & 0.46408800 & -0.72213500 & 2.90243200 \\ \mathrm{O} & -0.44826100 & 0.22390800 & 3.55007200 \\ \mathrm{O} & 0.77646800 & -0.31834200 & 1.41313300 \\ \mathrm{C} & -0.49846200 & -2.31725100 & 2.73416500 \\ \mathrm{~F} & -0.61851600 & -2.85211600 & 3.94960300 \\ \mathrm{~F} & -1.71307200 & -2.06579200 & 2.24115500 \\ \mathrm{~F} & 0.14773900 & -3.16211500 & 1.93597000 \\ \mathrm{O} & -3.74351000 & 1.64063400 & 0.68014600 \\ \mathrm{~S} & -3.55891200 & 1.39099900 & -0.75172400 \\ \mathrm{O} & -4.16926700 & 2.27409400 & -1.74078500 \\ \mathrm{O} & -2.04079500 & 1.22832600 & -1.13949400 \\ \mathrm{C} & -4.27104300 & -0.31024400 & -1.07387200 \\ \mathrm{~F} & -5.59917900 & -0.19405300 & -1.12037500 \\ \mathrm{~F} & -3.82392300 & -0.78355300 & -2.23464700 \\ \mathrm{~F} & -3.93612500 & -1.13998000 & -0.08660800 \\ & & & \\ & & & \end{array}$

22

$\mathrm{E}(\mathrm{SMD} / \mathrm{B} 3 \mathrm{LYP} / \mathrm{BS} 1)=-3675.46023945 \mathrm{au}$

$\mathrm{H}(\mathrm{SMD} / \mathrm{B} 3 \mathrm{LYP} / \mathrm{BS} 1)=-3675.070827 \mathrm{au}$

$\mathrm{G}(\mathrm{SMD} / \mathrm{B} 3 \mathrm{LYP} / \mathrm{BS} 1)=-3675.202256 \mathrm{au}$

$\mathrm{E}(\mathrm{SMD} / \mathrm{M} 06 / \mathrm{BS} 2 / / \mathrm{SMD} / \mathrm{B} 3 \mathrm{LYP} / \mathrm{BS} 1)=-3675.53812659 \mathrm{au}$

$\begin{array}{llll}\mathrm{C} & -0.96323100 & -3.21247300 & 1.44049100 \\ \mathrm{H} & -0.75198200 & -4.25565300 & 1.16635600 \\ \mathrm{H} & -1.81221800 & -3.18435300 & 2.12560200 \\ \mathrm{H} & -0.06477900 & -2.82098500 & 1.92517000 \\ \mathrm{C} & -1.28404700 & -2.44678800 & 0.18530300 \\ \mathrm{O} & -2.42063400 & -2.11180500 & -0.11606200 \\ \mathrm{C} & -0.12817600 & -2.17663700 & -0.77355500 \\ \mathrm{C} & -0.56879400 & -2.00851500 & -2.22543400 \\ \mathrm{O} & -0.50756800 & -0.93211100 & -2.79568600\end{array}$


$\mathrm{H} \quad-1.83405000 \quad-3.75669600 \quad-2.33937100$

$\mathrm{H} \quad-1.39615300 \quad-3.02735600 \quad-3.91662800$

$\mathrm{H} \quad-0.20239700 \quad-3.97619400 \quad-2.98234000$

$\mathrm{H} \quad 0.62156500 \quad-2.96580100 \quad-0.68534500$

$\mathrm{Au} \quad 0.96907800 \quad-0.45226700 \quad-0.26865600$

$\begin{array}{lllll}\text { O } & 2.17665700 & 1.34851700 & 0.07631900\end{array}$

S $\quad 2.94195800 \quad 2.06334100 \quad-1.04724400$

$\begin{array}{llll}\mathrm{O} & 4.03878300 & 2.86601600 & -0.49789900\end{array}$

$\begin{array}{llll}\text { O } & 3.19984500 & 1.21808500 & -2.21932500\end{array}$

$\begin{array}{lllll}\text { C } & 1.68371200 & 3.29972500 & -1.64220600\end{array}$

F $\quad \begin{array}{llll}\text { C } & 1.37923800 & 4.17387600 & -0.67820300\end{array}$

F $\quad 2.17908400 \quad 3.96836900 \quad-2.69059400$

F $\quad \begin{array}{llll}0.56071300 & 2.67224000 & -2.02666600\end{array}$

$\begin{array}{llll}\mathrm{O} & -2.71682700 & 1.48499500 & 1.22122500\end{array}$

S $\quad \begin{array}{llll}-1.39727100 & 0.89126200 & 1.45238900\end{array}$

$\begin{array}{llll}\text { O } & -1.25786000 & -0.24575600 & 2.36292600\end{array}$

$\begin{array}{llll}\mathrm{O} & -0.73056500 & 0.66459100 & 0.06054900\end{array}$

$\begin{array}{llll}\text { C } & -0.37518100 & 2.25548200 & 2.21530100\end{array}$

F $\quad-1.01805400 \quad 2.69642400 \quad 3.30084100$

F $\quad \begin{array}{llll}\text { F } & 0.81881000 & 1.78342000 & 2.57703900\end{array}$

F $\quad \begin{array}{llll}-0.22170200 & 3.25996300 & 1.35523700\end{array}$

$\begin{array}{llll}\mathrm{O} & 2.45552700 & -3.26799100 & 1.03215000\end{array}$

S $\quad 3.38947500 \quad-2.47244100 \quad 0.23101000$

$\begin{array}{llll}\mathrm{O} & 4.47043000 & -3.12947000 & -0.49821100\end{array}$

$\begin{array}{llll}\mathrm{O} & 2.66527800 & -1.50051900 & -0.75870500\end{array}$

$\begin{array}{llll}\mathrm{C} & 4.21181100 & -1.29934300 & 1.42951800\end{array}$

F $\quad \begin{array}{llll}\text { F } & 5.00321200 & -2.00680400 & 2.23768700\end{array}$

F $\quad 4.93852800 \quad-0.39956400 \quad 0.77067600$

F $\quad 3.28526900 \quad-0.67260300 \quad 2.16459400$

$\begin{array}{llll}\mathrm{C} & -5.23430100 & 0.35151200 & -0.52906100\end{array}$

$\begin{array}{llll}\text { C } & -6.57763700 & 0.06821100 & -0.27111800\end{array}$

$\begin{array}{llll}\text { C } & -7.43442200 & -0.57182800 & -1.22073400\end{array}$

$\begin{array}{llll}\text { C } & -7.10309700 & 0.43484600 & 1.00816700\end{array}$

$\begin{array}{llll}\text { C } & -8.75123600 & -0.82649200 & -0.89423900\end{array}$

$\mathrm{H} \quad-7.04876100 \quad-0.85488600 \quad-2.19373600$

$\begin{array}{llll}\mathrm{C} & -8.42401000 & 0.17433400 & 1.32084700\end{array}$

$\mathrm{H} \quad \begin{array}{llll}\mathrm{H} & -6.44538000 & 0.91707600 & 1.72507400\end{array}$

$\begin{array}{llll}\text { C } & -9.24314000 & -0.45421100 & 0.37053500\end{array}$

$\mathrm{H} \quad-9.40941900 \quad-1.31101400 \quad-1.60774300$

$\mathrm{H} \quad-8.82660400 \quad 0.45055300 \quad 2.28958100$

$\mathrm{H} \quad-10.28167100 \quad-0.65838000 \quad 0.61571400$

$\begin{array}{llll}\mathrm{H} & -4.67624400 & 0.83894500 & 0.27093000\end{array}$

$\begin{array}{lllll}\mathrm{C} & -4.45385900 & 0.05300500 & -1.72809700\end{array}$

$\mathrm{H} \quad-3.91440300 \quad 0.96065400 \quad-2.03802100$

$\mathrm{H} \quad-3.65331400 \quad-0.64486300 \quad-1.42024000$

$\mathrm{H} \quad-5.01386600 \quad-0.36687500 \quad-2.56323100$

$\mathbf{T S}_{22}$

$\mathrm{E}(\mathrm{SMD} / \mathrm{B} 3 \mathrm{LYP} / \mathrm{BS} 1)=-3675.38497651 \mathrm{au}$

$\mathrm{H}(\mathrm{SMD} / \mathrm{B} 3 \mathrm{LYP} / \mathrm{BS} 1)=-3674.994767 \mathrm{au}$ 


\begin{tabular}{|c|c|c|c|}
\hline & $\begin{array}{l}\mathrm{LYP} / \mathrm{BS} 1)=- \\
6 / \mathrm{BS} 2 / / \mathrm{SMD} /\end{array}$ & $\begin{array}{l}3675.121759 \text { a } \\
\text { B3LYP/BS1) }\end{array}$ & $=-3675.47184358$ \\
\hline $\mathrm{C}$ & -2.13840000 & 0.86200800 & -2.33741700 \\
\hline $\mathrm{H}$ & -2.20257900 & 1.94379700 & -2.18153400 \\
\hline $\mathrm{H}$ & -2.63418300 & 0.58677500 & -3.27102600 \\
\hline $\mathrm{H}$ & -1.07680200 & 0.59650600 & -2.40294500 \\
\hline $\mathrm{C}$ & -2.77554200 & 0.10638000 & -1.18834000 \\
\hline $\mathrm{O}$ & -3.59184700 & -0.77482600 & -1.37208300 \\
\hline $\mathrm{C}$ & -2.37342300 & 0.52211300 & 0.22414400 \\
\hline $\mathrm{C}$ & -2.26456300 & -0.50644800 & 1.33963500 \\
\hline $\mathrm{O}$ & -2.68020800 & -1.64257900 & 1.20634800 \\
\hline $\mathrm{C}$ & -1.65594200 & -0.03196300 & 2.64975900 \\
\hline $\mathrm{H}$ & -0.82179600 & -0.69178600 & 2.91239900 \\
\hline $\mathrm{H}$ & -1.29811100 & 1.00031500 & 2.61647900 \\
\hline $\mathrm{H}$ & -2.40375600 & -0.12902700 & 3.44557100 \\
\hline $\mathrm{H}$ & -1.73228600 & 1.39585300 & 0.32828800 \\
\hline $\mathrm{C}$ & -5.36809800 & -0.03512400 & 1.97616500 \\
\hline $\mathrm{C}$ & -6.51059000 & -0.81036600 & 2.04863200 \\
\hline $\mathrm{C}$ & -7.37876500 & -0.87231300 & 0.94573700 \\
\hline $\mathrm{C}$ & -7.10444100 & -0.14666700 & -0.22688200 \\
\hline $\mathrm{C}$ & -5.96461300 & 0.63244500 & -0.30669400 \\
\hline $\mathrm{C}$ & -5.07606500 & 0.72192700 & 0.80327100 \\
\hline $\mathrm{H}$ & -4.69812400 & 0.03141000 & 2.82741400 \\
\hline $\mathrm{H}$ & -6.73729400 & -1.36813000 & 2.95149300 \\
\hline $\mathrm{H}$ & -8.27019200 & -1.49065000 & 0.99749700 \\
\hline $\mathrm{H}$ & -7.78427200 & -0.20555300 & -1.07070300 \\
\hline $\mathrm{H}$ & -5.75276900 & 1.18385500 & -1.21560300 \\
\hline $\mathrm{C}$ & -3.92645000 & 1.60077700 & 0.80750600 \\
\hline $\mathrm{H}$ & -3.51476000 & 1.75942600 & 1.80431300 \\
\hline $\mathrm{C}$ & -3.90067000 & 2.84913000 & -0.03827500 \\
\hline $\mathrm{H}$ & -4.18605400 & 2.68161100 & -1.07911100 \\
\hline $\mathrm{H}$ & -4.63065500 & 3.54715800 & 0.39509900 \\
\hline $\mathrm{H}$ & -2.92150300 & 3.33287300 & -0.00649600 \\
\hline $\mathrm{Au}$ & 0.42224700 & 0.05526100 & -0.08795100 \\
\hline $\mathrm{O}$ & 2.57557900 & -0.19896600 & 0.15742800 \\
\hline S & 3.24214800 & -0.01793700 & 1.54891500 \\
\hline $\mathrm{O}$ & 4.68566000 & 0.13349900 & 1.35397200 \\
\hline $\mathrm{O}$ & 2.52397400 & 0.90825400 & 2.42631000 \\
\hline $\mathrm{C}$ & 3.00846200 & -1.70156700 & 2.31601900 \\
\hline $\mathrm{F}$ & 3.50057100 & -2.64820100 & 1.51779400 \\
\hline $\mathrm{F}$ & 3.64304600 & -1.73545600 & 3.48998800 \\
\hline $\mathrm{F}$ & 1.70388400 & -1.93239300 & 2.51996600 \\
\hline $\mathrm{O}$ & -0.46741600 & -4.12417700 & -1.09419400 \\
\hline S & 0.02446400 & -2.79171400 & -1.43245800 \\
\hline $\mathrm{O}$ & -0.62965700 & -1.99854800 & -2.47411700 \\
\hline $\mathrm{O}$ & 0.21705900 & -1.98880300 & -0.10186900 \\
\hline $\mathrm{C}$ & 1.78738500 & -3.01665500 & -2.00303900 \\
\hline $\mathrm{F}$ & 1.79193700 & -3.81650900 & -3.07068200 \\
\hline $\mathrm{F}$ & 2.30784500 & -1.83132000 & -2.33880800 \\
\hline $\mathrm{F}$ & 2.51872000 & -3.56339700 & -1.03326500 \\
\hline $\mathrm{O}$ & 1.28722600 & 2.18906400 & -2.25707100 \\
\hline
\end{tabular}




$\begin{array}{lllr}\text { S } & 0.95341300 & 3.00051000 & -1.08371500 \\ \mathrm{O} & 0.03815100 & 4.13465300 & -1.21350600 \\ \mathrm{O} & 0.52113100 & 2.11232200 & 0.13102900 \\ \mathrm{C} & 2.55560800 & 3.70936200 & -0.43887600 \\ \mathrm{~F} & 2.99063200 & 4.62359400 & -1.30739800 \\ \mathrm{~F} & 2.34966600 & 4.28178600 & 0.74634800 \\ \mathrm{~F} & 3.46777100 & 2.74510200 & -0.31870900\end{array}$

23

$\mathrm{E}(\mathrm{SMD} / \mathrm{B} 3 \mathrm{LYP} / \mathrm{BS} 1)=-310.072874511 \mathrm{au}$

$\mathrm{H}(\mathrm{SMD} / \mathrm{B} 3 \mathrm{LYP} / \mathrm{BS} 1)=-309.920048 \mathrm{au}$

$\mathrm{G}(\mathrm{SMD} / \mathrm{B} 3 \mathrm{LYP} / \mathrm{BS} 1)=-309.958267 \mathrm{au}$

$\mathrm{E}(\mathrm{SMD} / \mathrm{M} 06 / \mathrm{BS} 2 / / \mathrm{SMD} / \mathrm{B} 3 \mathrm{LYP} / \mathrm{BS} 1)=-309.924174617 \mathrm{au}$

$\begin{array}{llll}\mathrm{C} & 1.33944200 & 1.37831300 & -0.00012500\end{array}$

$\begin{array}{llll}\text { C } & -0.00997500 & 1.09670500 & -0.00046000\end{array}$

$\begin{array}{llll}\text { C } & -0.45297800 & -0.26573800 & -0.00032400\end{array}$

$\begin{array}{llll}\mathrm{C} & 0.51928800 & -1.31776500 & 0.00003200\end{array}$

$\begin{array}{llll}\mathrm{C} & 1.86833800 & -1.01951800 & 0.00035900\end{array}$

$\begin{array}{llll}\mathrm{C} & 2.27407500 & 0.32393800 & 0.00028600\end{array}$

$\mathrm{H} \quad \begin{array}{llll}\mathrm{H} & 1.68688000 & 2.40588600 & -0.00020700\end{array}$

$\mathrm{H} \quad \begin{array}{llll}0.73877600 & 1.90042800 & -0.00082300\end{array}$

$\mathrm{H} \quad 0.17857000 \quad-2.34872600 \quad 0.00011900$

$\mathrm{H} \quad 2.60907100 \quad-1.81208300 \quad 0.00067400$

$\begin{array}{llll}\mathrm{H} & 3.33494800 & 0.55815300 & 0.00053300\end{array}$

C $\quad-1.80118600 \quad-0.61577900 \quad-0.00028200$

$\mathrm{H} \quad-2.01889700 \quad-1.68344700 \quad-0.00029100$

$\begin{array}{llll}\text { C } & -2.95604900 & 0.30170500 & 0.00018000\end{array}$

$\mathrm{H} \quad-3.90068300 \quad-0.24246600 \quad-0.00198400$

$\mathrm{H} \quad \begin{array}{llll}\mathrm{H} & -2.91936600 & 0.96326600 & 0.87817300\end{array}$

$\mathrm{H} \quad-2.91747500 \quad 0.96782400 \quad-0.87418700$

24

$\mathrm{E}(\mathrm{SMD} / \mathrm{B} 3 \mathrm{LYP} / \mathrm{BS} 1)=-3365.37308759 \mathrm{au}$

$\mathrm{H}(\mathrm{SMD} / \mathrm{B} 3 \mathrm{LYP} / \mathrm{BS} 1)=-3365.138615 \mathrm{au}$

$\mathrm{G}(\mathrm{SMD} / \mathrm{B} 3 \mathrm{LYP} / \mathrm{BS} 1)=-3365.249673 \mathrm{au}$

$\mathrm{E}(\mathrm{SMD} / \mathrm{M} 06 / \mathrm{BS} 2 / / \mathrm{SMD} / \mathrm{B} 3 \mathrm{LYP} / \mathrm{BS} 1)=-3365.60055709 \mathrm{au}$

$\begin{array}{llll}\mathrm{C} & 2.56065100 & 3.03264600 & -1.02772200\end{array}$

$\mathrm{H} \quad 3.62173300 \quad 3.09606700 \quad-0.74833400$

$\mathrm{H} \quad 2.29422300 \quad 3.90578100 \quad-1.62636000$

$\mathrm{H} \quad 2.43627800 \quad 2.11581600 \quad-1.60905100$

$\begin{array}{llll}\mathrm{C} & 1.73842200 & 3.00373100 & 0.23437000\end{array}$

$\begin{array}{llll}\mathrm{O} & 1.12276600 & 3.97189900 & 0.65029000\end{array}$

$\begin{array}{llll}\mathrm{C} & 1.77700500 & 1.71859600 & 1.06196600\end{array}$

$\begin{array}{llll}\text { C } & 1.46190100 & 1.94349500 & 2.53918500\end{array}$

$\begin{array}{llll}\mathrm{O} & 0.43880000 & 1.52013100 & 3.05027200\end{array}$

$\begin{array}{llll}\text { C } & 2.52016300 & 2.67946000 & 3.32574700\end{array}$

$\begin{array}{llll}\mathrm{H} & 2.75961400 & 3.64024300 & 2.85768500\end{array}$

$\begin{array}{llll}\mathrm{H} & 2.17028600 & 2.84141200 & 4.34836400\end{array}$

$\mathrm{H} \quad 3.44129500 \quad 2.08243200 \quad 3.34389400$

$\begin{array}{llll}\mathrm{H} & 2.74345000 & 1.22259800 & 0.94269700\end{array}$

$\begin{array}{llll}\mathrm{Au} & 0.43042700 & 0.25520700 & 0.37483000\end{array}$ 


$\begin{array}{llcc}\text { O } & -0.96720300 & -1.35846100 & -0.16544200 \\ \text { S } & -1.43206400 & -2.38558300 & 0.87457500 \\ \text { O } & -1.69875300 & -3.68766900 & 0.25445100 \\ \text { O } & -0.66270300 & -2.34462900 & 2.12574100 \\ \text { C } & -3.10751600 & -1.71946600 & 1.33397000 \\ \text { F } & -3.91653700 & -1.69955700 & 0.26940300 \\ \text { F } & -3.65330700 & -2.50076500 & 2.27532300 \\ \text { F } & -2.99861000 & -0.47534500 & 1.82010200 \\ \text { O } & -2.31410200 & 3.50240600 & -0.77094600 \\ \text { S } & -1.49450400 & 2.34083600 & -1.10889900 \\ \text { O } & -0.37522400 & 2.47018600 & -2.04509200 \\ \text { O } & -1.10249500 & 1.61631500 & 0.21994600 \\ \text { C } & -2.66708200 & 1.13634800 & -1.92283700 \\ \text { F } & -3.27582400 & 1.76837100 & -2.93256400 \\ \text { F } & -1.99666800 & 0.09046200 & -2.40952200 \\ \text { F } & -3.58511700 & 0.71591700 & -1.05284800 \\ \text { O } & 3.56765300 & -0.22823700 & -1.00737200 \\ \text { S } & 3.02513000 & -1.44446600 & -0.39673200 \\ \text { O } & 3.92980600 & -2.43887900 & 0.17446500 \\ \text { O } & 1.90524000 & -1.15310500 & 0.65332600 \\ \text { C } & 2.09502900 & -2.31747600 & -1.76025000 \\ \text { F } & 2.97717700 & -2.75126700 & -2.66356600 \\ \text { F } & 1.41756200 & -3.35217200 & -1.26867000 \\ \text { F } & 1.24585000 & -1.46919700 & -2.35401600\end{array}$

\section{4'}

$\mathrm{E}(\mathrm{SMD} / \mathrm{B} 3 \mathrm{LYP} / \mathrm{BS} 1)=-2403.79060198 \mathrm{au}$

$\mathrm{H}(\mathrm{SMD} / \mathrm{B} 3 \mathrm{LYP} / \mathrm{BS} 1)=-2403.592392 \mathrm{au}$

$\mathrm{G}(\mathrm{SMD} / \mathrm{B} 3 \mathrm{LYP} / \mathrm{BS} 1)=-2403.684711 \mathrm{au}$

$\mathrm{E}(\mathrm{SMD} / \mathrm{M} 06 / \mathrm{BS} 2 / / \mathrm{SMD} / \mathrm{B} 3 \mathrm{LYP} / \mathrm{BS} 1)=-2403.89307343 \mathrm{au}$

$\begin{array}{lllll}\mathrm{C} & -0.32222300 & 4.75727400 & -0.98044900\end{array}$

$\mathrm{H} \quad 0.32026500 \quad 5.62133200 \quad-0.80398700$

$\mathrm{H} \quad-1.29464500 \quad 4.91803200 \quad-0.50012400$

$\mathrm{H} \quad-0.49957200 \quad 4.63919000 \quad-2.05519000$

C $\quad 0.29345900 \quad 3.49986100 \quad-0.43304400$

$\begin{array}{llll}\mathrm{O} & -0.42585200 & 2.44988700 & -0.67768500\end{array}$

$\begin{array}{llll}\mathrm{C} & 1.50888200 & 3.54074700 & 0.25553800\end{array}$

$\begin{array}{llll}\mathrm{H} & 1.97647800 & 4.51084300 & 0.35891500\end{array}$

$\begin{array}{llll}\text { C } & 2.17850600 & 2.45944300 & 0.83084200\end{array}$

$\begin{array}{llll}\mathrm{C} & 3.48404700 & 2.65838100 & 1.54898700\end{array}$

$\begin{array}{llll}\mathrm{H} & 3.37378400 & 2.34975100 & 2.59493800\end{array}$

$\begin{array}{llll}\mathrm{H} & 4.24852800 & 2.01842800 & 1.09385700\end{array}$

$\begin{array}{llll}\mathrm{H} & 3.80310600 & 3.70074000 & 1.50543900\end{array}$

$\begin{array}{lllll}\mathrm{O} & 1.79649800 & 1.21901800 & 0.83357800\end{array}$

$\mathrm{Au} \quad 0.10092300 \quad 0.61371500 \quad-0.05706500$

$\begin{array}{lllll}\mathrm{O} & 0.56865100 & -1.27222500 & 0.61062700\end{array}$

S $\quad 1.14603900 \quad-2.37160300 \quad-0.35345600$

$\begin{array}{lllll}\mathrm{O} & 0.93145600 & -3.67360500 & 0.26983300\end{array}$

$\begin{array}{lllll}\mathrm{O} & 0.78733500 & -2.14059900 & -1.75124900\end{array}$

$\begin{array}{llll}\text { C } & 2.97797200 & -2.03481500 & -0.23387500\end{array}$

F $\quad 3.36184600 \quad-2.00854800 \quad 1.04149100$ 


$\begin{array}{llcc}\text { F } & 3.63486400 & -2.99952300 & -0.87838300 \\ \text { F } & 3.25905600 & -0.85647100 & -0.80423200 \\ \text { O } & -3.08731300 & 1.23008100 & 0.56594200 \\ \text { S } & -3.01020500 & 0.07383400 & -0.32714100 \\ \text { O } & -4.00841700 & -0.12831600 & -1.37248000 \\ \text { O } & -1.59001600 & -0.10145600 & -0.97777700 \\ \text { C } & -3.09733600 & -1.42663500 & 0.77985900 \\ \text { F } & -4.33625800 & -1.53247500 & 1.26165400 \\ \text { F } & -2.78948000 & -2.52539700 & 0.09276600 \\ \text { F } & -2.24033600 & -1.28484500 & 1.79674200\end{array}$

$\mathbf{T S}_{23-25}$

$\mathrm{E}(\mathrm{SMD} / \mathrm{B} 3 \mathrm{LYP} / \mathrm{BS} 1)=-655.879418646 \mathrm{au}$

$\mathrm{H}(\mathrm{SMD} / \mathrm{B} 3 \mathrm{LYP} / \mathrm{BS} 1)=-655.59353 \mathrm{au}$

$\mathrm{G}(\mathrm{SMD} / \mathrm{B} 3 \mathrm{LYP} / \mathrm{BS} 1)=-655.651006 \mathrm{au}$

$\mathrm{E}(\mathrm{SMD} / \mathrm{M} 06 / \mathrm{BS} 2 / / \mathrm{SMD} / \mathrm{B} 3 \mathrm{LYP} / \mathrm{BS} 1)=-655.659382417 \mathrm{au}$

$\begin{array}{llll}\mathrm{C} & 2.77818400 & -2.13740200 & 0.68043100\end{array}$

$\mathrm{H} \quad 3.83201900 \quad-2.02242400 \quad 0.39360500$

$\mathrm{H} \quad 2.48965800 \quad-3.18388400 \quad 0.55982900$

C $\quad 1.92794100 \quad-1.25803000 \quad-0.19472700$

O $\quad 1.18923100 \quad-1.75530300 \quad-1.07187900$

$\begin{array}{llll}\mathrm{C} & 1.98321100 & 0.18603800 & -0.00193400\end{array}$

C $\quad 1.42961200 \quad 1.03842100 \quad-0.96037900$

$\begin{array}{llll}\mathrm{O} & 0.64396900 & 0.57272600 & -1.90262000\end{array}$

C $\quad 1.65436000 \quad 2.51037900 \quad-0.99881800$

$\begin{array}{llll}\mathrm{H} & 0.69318900 & 3.03700800 & -1.02854900\end{array}$

$\mathrm{H} \quad 2.24153100 \quad 2.85225600 \quad-0.14521300$

$\mathrm{H} \quad 2.18729100 \quad 2.76575600 \quad-1.92384500$

$\begin{array}{llll}\mathrm{H} & 2.69170100 & 0.59729800 & 0.70659500\end{array}$

$\mathrm{H} \quad 0.63658400 \quad-0.43965200 \quad-1.79793100$

$\begin{array}{llll}\mathrm{C} & 0.04223300 & 0.35297200 & 1.59566400\end{array}$

$\begin{array}{llll}\mathrm{H} & 0.53088600 & -0.50675900 & 2.04890800\end{array}$

$\begin{array}{llll}\mathrm{C} & -1.10301700 & 0.06268500 & 0.80556200\end{array}$

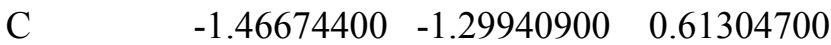

$\begin{array}{llll}\mathrm{C} & -1.92125200 & 1.07948800 & 0.23919100\end{array}$

$\begin{array}{llll}\text { C } & -2.61597600 & -1.62785800 & -0.09103000\end{array}$

$\mathrm{H} \quad-0.84799000 \quad-2.07766500 \quad 1.04855900$

$\begin{array}{llll}\text { C } & -3.06395000 & 0.73985000 & -0.46582100\end{array}$

$\begin{array}{llll}\mathrm{H} & -1.65536700 & 2.12313900 & 0.36610300\end{array}$

C $\quad-3.41219700 \quad-0.61013400 \quad-0.62997700$

$\mathrm{H} \quad-2.89664600 \quad-2.66795600 \quad-0.22252100$

$\mathrm{H} \quad-3.69056200 \quad 1.51667900 \quad-0.89249500$

$\mathrm{H} \quad-4.31105300 \quad-0.86749700 \quad-1.18316700$

$\begin{array}{llll}\mathrm{C} & 0.35176100 & 1.66412000 & 2.21893500\end{array}$

$\mathrm{H} \quad-0.25401400 \quad 1.71433400 \quad 3.13998900$

$\begin{array}{llll}\mathrm{H} & 1.39793800 & 1.72659100 & 2.52549100\end{array}$

$\begin{array}{llll}\mathrm{H} & 0.08535400 & 2.52838000 & 1.60696500\end{array}$

$\mathrm{H} \quad 2.69888900 \quad-1.84169700 \quad 1.73280800$

25

$\mathrm{E}(\mathrm{SMD} / \mathrm{B} 3 \mathrm{LYP} / \mathrm{BS} 1)=-655.897269704 \mathrm{au}$ 


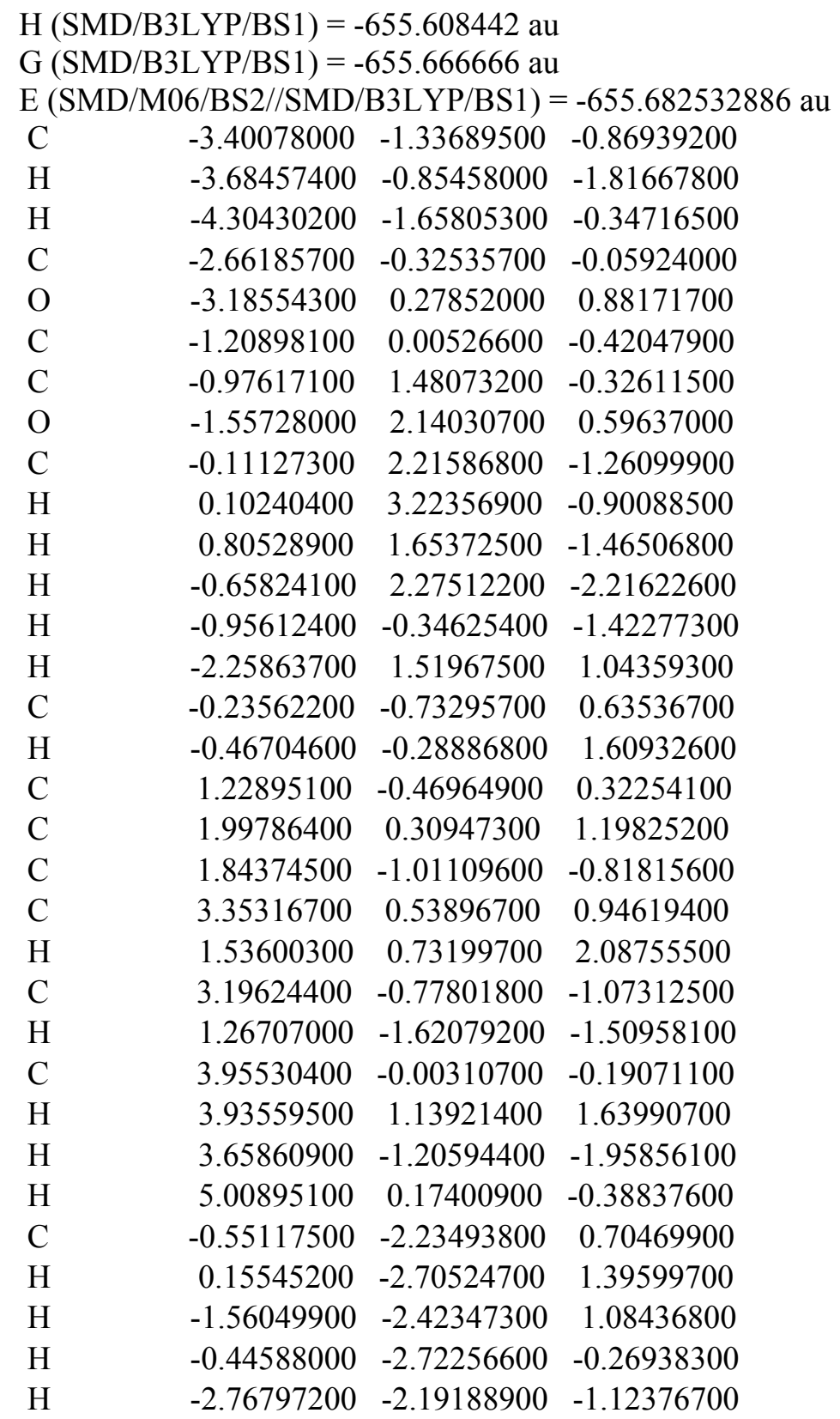

\section{$\mathbf{T S}_{25-23}$}

\begin{tabular}{|c|c|c|c|}
\hline \multirow{2}{*}{\multicolumn{4}{|c|}{$\mathrm{E}(\mathrm{SMD} / \mathrm{B} 3 \mathrm{LYP} / \mathrm{BS} 1)=-965.537891089 \mathrm{au}$}} \\
\hline & & & \\
\hline \multicolumn{4}{|c|}{$\mathrm{G}(\mathrm{SMD} / \mathrm{B} 3 \mathrm{LYP} / \mathrm{BS} 1)=-965.189716 \mathrm{au}$} \\
\hline \multicolumn{4}{|c|}{$\mathrm{E}(\mathrm{SMD} / \mathrm{M} 06 / \mathrm{BS} 2 / / \mathrm{SMD} / \mathrm{B} 3 \mathrm{LYP} / \mathrm{BS} 1)=-965.182545786 \mathrm{au}$} \\
\hline $\mathrm{C}$ & 2.09639700 & 0.24008600 & -2.70605500 \\
\hline $\mathrm{H}$ & 3.04384500 & 0.74895000 & -2.92821000 \\
\hline $\mathrm{H}$ & 1.38225000 & 0.39386400 & -3.51646600 \\
\hline $\mathrm{H}$ & 2.33181800 & -0.82702600 & -2.60030500 \\
\hline $\mathrm{C}$ & 1.56610600 & 0.74694100 & -1.40944900 \\
\hline $\mathrm{O}$ & 0.42227000 & 1.25370500 & -1.40998800 \\
\hline $\mathrm{C}$ & 2.43627800 & 0.63631400 & -0.18337700 \\
\hline $\mathrm{C}$ & 2.81803200 & 2.06227200 & 0.31371500 \\
\hline $\mathrm{O}$ & 1.95204000 & 2.85205100 & 0.63366700 \\
\hline $\mathrm{C}$ & 4.28920000 & 2.37352200 & 0.36747000 \\
\hline
\end{tabular}




$\begin{array}{lrrr}\mathrm{H} & 4.81589000 & 1.61655800 & 0.96087700 \\ \mathrm{H} & 4.70764100 & 2.33070400 & -0.64689000 \\ \mathrm{H} & 4.45149600 & 3.36630000 & 0.79282200 \\ \mathrm{H} & 3.34921500 & 0.11333900 & -0.47694400 \\ \mathrm{C} & -4.23830600 & 0.99592000 & -0.96259300 \\ \mathrm{C} & -5.20747700 & 0.01721400 & -0.76345500 \\ \mathrm{C} & -5.18356400 & -0.75547000 & 0.40121000 \\ \mathrm{C} & -4.19200900 & -0.54612300 & 1.36976300 \\ \mathrm{C} & -3.22147800 & 0.42905200 & 1.17518100 \\ \mathrm{C} & -3.22578100 & 1.21246100 & -0.00218000 \\ \mathrm{H} & -4.25049800 & 1.59994800 & -1.86604000 \\ \mathrm{H} & -5.98008700 & -0.14247200 & -1.50956200 \\ \mathrm{H} & -5.94153600 & -1.51722400 & 0.56105800 \\ \mathrm{H} & -4.18712600 & -1.14064300 & 2.27850200 \\ \mathrm{H} & -2.47129800 & 0.60012100 & 1.94058700 \\ \mathrm{C} & -2.24381800 & 2.24233700 & -0.26318300 \\ \mathrm{H} & -2.43941100 & 2.84373300 & -1.15150000 \\ \mathrm{C} & -1.06546700 & 2.49327600 & 0.40766300 \\ \mathrm{H} & -0.86167500 & 2.02866600 & 1.37114700 \\ \mathrm{H} & -0.21074100 & 1.77098400 & -0.46785400 \\ \mathrm{H} & -0.57445300 & 3.44891500 & 0.23121000 \\ \mathrm{C} & 1.73895600 & -0.19032200 & 0.95910500 \\ \mathrm{H} & 0.81907800 & 0.34049400 & 1.22201300 \\ \mathrm{C} & 1.36305200 & -1.58082500 & 0.45712700 \\ \mathrm{C} & 0.01621300 & -1.94049000 & 0.31130800 \\ \mathrm{C} & 2.34759800 & -2.52807000 & 0.13005900 \\ \mathrm{C} & -0.34237000 & -3.21209700 & -0.14502800 \\ \mathrm{H} & -0.76075100 & -1.22204600 & 0.56029500 \\ \mathrm{C} & 1.99228000 & -3.79791600 & -0.32842500 \\ \mathrm{H} & 3.39970500 & -2.27673000 & 0.23949100 \\ \mathrm{C} & 0.64488800 & -4.14477900 & -0.46736200 \\ \mathrm{H} & -1.39324000 & -3.47038800 & -0.24648100 \\ \mathrm{H} & 2.76849900 & -4.51795200 & -0.57396700 \\ \mathrm{H} & 0.36893800 & -5.13412700 & -0.82223100 \\ \mathrm{C} & 2.62816700 & -0.25078800 & 2.21280200 \\ \mathrm{H} & 3.59799300 & -0.71390100 & 2.00146700 \\ \mathrm{H} & 2.80644600 & 0.74751000 & 2.62714200 \\ \mathrm{H} & 2.13213500 & -0.84870100 & 2.98463700 \\ & & & \\ & & & \end{array}$

26

$\mathrm{E}(\mathrm{SMD} / \mathrm{B} 3 \mathrm{LYP} / \mathrm{BS} 1)=-941.857868748 \mathrm{au}$

$\mathrm{H}(\mathrm{SMD} / \mathrm{B} 3 \mathrm{LYP} / \mathrm{BS} 1)=-941.720155 \mathrm{au}$

$\mathrm{G}(\mathrm{SMD} / \mathrm{B} 3 \mathrm{LYP} / \mathrm{BS} 1)=-941.773202 \mathrm{au}$

$\mathrm{E}(\mathrm{SMD} / \mathrm{M} 06 / \mathrm{BS} 2 / / \mathrm{SMD} / \mathrm{B} 3 \mathrm{LYP} / \mathrm{BS} 1)=-941.764248991 \mathrm{au}$

$\begin{array}{llll}\mathrm{C} & 1.81907900 & 0.89348300 & 2.56106800 \\ \mathrm{H} & 2.60639900 & 1.64857800 & 2.71027300 \\ \mathrm{H} & 1.90640500 & 0.15313100 & 3.36056600 \\ \mathrm{H} & 0.85369900 & 1.40127500 & 2.59735400 \\ \mathrm{C} & 2.05798700 & 0.26724100 & 1.24008800 \\ \mathrm{O} & 2.86719900 & -0.72472000 & 1.20020500 \\ \mathrm{C} & 1.44721700 & 0.76643500 & 0.00958800\end{array}$




$\begin{array}{lccc}\mathrm{C} & 2.10875100 & 0.35406700 & -1.27597200 \\ \mathrm{O} & 2.90319100 & -0.59742300 & -1.28220500 \\ \mathrm{C} & 1.79646000 & 1.12284300 & -2.52032000 \\ \mathrm{H} & 0.72369800 & 1.05931600 & -2.73999400 \\ \mathrm{H} & 2.03112400 & 2.18405800 & -2.37054100 \\ \mathrm{H} & 2.37048300 & 0.72675600 & -3.36012600 \\ \mathrm{H} & 1.21724400 & 1.82846900 & 0.06821800 \\ \mathrm{H} & 3.01312000 & -0.96375500 & 0.20023000 \\ \mathrm{Au} & -0.60131700 & -0.03367300 & 0.00747700 \\ \mathrm{Cl} & -1.39791200 & 2.19406700 & 0.09748200 \\ \mathrm{Cl} & -2.84543800 & -0.83393900 & -0.07146300 \\ \mathrm{Cl} & 0.19875100 & -2.25571300 & -0.05468600\end{array}$

TS $_{26-27}$

$\mathrm{E}(\mathrm{SMD} / \mathrm{B} 3 \mathrm{LYP} / \mathrm{BS} 1)=-2171.87841621 \mathrm{au}$

$\mathrm{H}(\mathrm{SMD} / \mathrm{B} 3 \mathrm{LYP} / \mathrm{BS} 1)=-2171.591362 \mathrm{au}$

$\mathrm{G}(\mathrm{SMD} / \mathrm{B} 3 \mathrm{LYP} / \mathrm{BS} 1)=-2171.671885 \mathrm{au}$

$\mathrm{E}(\mathrm{SMD} / \mathrm{M} 06 / \mathrm{BS} 2 / / \mathrm{SMD} / \mathrm{B} 3 \mathrm{LYP} / \mathrm{BS} 1)=-2171.65393844 \mathrm{au}$

$\begin{array}{llll}\mathrm{C} & 3.70563300 & -1.32279500 & 1.42567700\end{array}$

$\mathrm{H} \quad 4.76349500 \quad-1.28817000 \quad 1.71526800$

$\mathrm{H} \quad 3.53206700 \quad-2.29027300 \quad 0.93978500$

$\mathrm{H} \quad 3.07962700 \quad-1.24350300 \quad 2.31579200$

C $\quad 3.43167500 \quad-0.22101700 \quad 0.46295700$

$\begin{array}{llll}\mathrm{O} & 4.11852600 & -0.25375700 & -0.64286900\end{array}$

$\begin{array}{llll}\text { C } & 2.50915600 & 0.82879500 & 0.71153800\end{array}$

$\begin{array}{llll}\text { C } & 2.55979700 & 2.03264600 & -0.13181800\end{array}$

$\begin{array}{llll}\mathrm{O} & 3.16317600 & 2.01711300 & -1.22205500\end{array}$

$\begin{array}{llll}\mathrm{C} & 1.93147900 & 3.29806200 & 0.38142700\end{array}$

$\begin{array}{llll}\mathrm{H} & 1.03738400 & 3.09725300 & 0.97733400\end{array}$

$\mathrm{H} \quad 2.65879000 \quad 3.79705200 \quad 1.03737200$

$\mathrm{H} \quad \begin{array}{llll}\mathrm{H} & 1.70051000 & 3.97140000 & -0.44772500\end{array}$

$\begin{array}{llll}\mathrm{H} & 2.17304800 & 0.94286900 & 1.73477700\end{array}$

C $\quad-4.07652100 \quad-0.32582900 \quad-1.24940000$

$\begin{array}{llll}\text { C } & -5.33006600 & -0.54348100 & -0.68577600\end{array}$

$\begin{array}{llll}\text { C } & -5.73151400 & 0.20250100 & 0.42691800\end{array}$

$\begin{array}{llll}\text { C } & -4.87438500 & 1.16777500 & 0.97208000\end{array}$

$\begin{array}{llll}\text { C } & -3.62062400 & 1.38659600 & 0.41395400\end{array}$

$\begin{array}{llll}\text { C } & -3.19696800 & 0.63734000 & -0.70752200\end{array}$

$\mathrm{H} \quad-3.75934100 \quad-0.90186300 \quad-2.11482500$

$\mathrm{H} \quad-\quad-5.99386300 \quad-1.29067300 \quad-1.11094200$

$\begin{array}{llll}\mathrm{H} & -6.71063400 & 0.03687100 & 0.86787000\end{array}$

$\begin{array}{llll}\mathrm{H} & -5.19058400 & 1.75017800 & 1.83278000\end{array}$

$\mathrm{H} \quad-2.97120900 \quad 2.14455700 \quad 0.83940000$

C $\quad-1.90696400 \quad 0.82256900 \quad-1.34965400$

$\mathrm{H} \quad \begin{array}{llll}\mathrm{H} & -1.76056800 & 0.24726500 & -2.26206300\end{array}$

$\begin{array}{lllll}\mathrm{C} & -0.87871800 & 1.64369800 & -0.95628500\end{array}$

$\mathrm{H} \quad-0.96478700 \quad 2.30169000 \quad-0.09814500$

$\mathrm{H} \quad 3.86663000 \quad 0.58179400 \quad-1.17186700$

$\mathrm{H} \quad-0.05674900 \quad 1.83115900 \quad-1.63953700$

$\mathrm{Au} \quad 0.40936900 \quad-0.35981300 \quad 0.06768600$

$\begin{array}{llll}\mathrm{Cl} & -0.41796100 & 0.21239900 & 2.20461000\end{array}$ 


$\begin{array}{lrrr}\text { Cl } & -0.37949300 & -2.70002600 & 0.35136600 \\ \mathrm{Cl} & 1.20160300 & -0.86592400 & -2.09289900\end{array}$

27

$\mathrm{E}(\mathrm{SMD} / \mathrm{B} 3 \mathrm{LYP} / \mathrm{BS} 1)=-1826.09761117 \mathrm{au}$

$\mathrm{H}(\mathrm{SMD} / \mathrm{B} 3 \mathrm{LYP} / \mathrm{BS} 1)=-1825.942844 \mathrm{au}$

$\mathrm{G}(\mathrm{SMD} / \mathrm{B} 3 \mathrm{LYP} / \mathrm{BS} 1)=-1826.001876 \mathrm{au}$

$\mathrm{E}(\mathrm{SMD} / \mathrm{M} 06 / \mathrm{BS} 2 / / \mathrm{SMD} / \mathrm{B} 3 \mathrm{LYP} / \mathrm{BS} 1)=-1825.94263658 \mathrm{au}$

$\begin{array}{llll}\mathrm{Au} & 1.06834100 & 0.04628200 & 0.13326000\end{array}$

$\begin{array}{llll}\mathrm{Cl} & 0.20540300 & 2.23846300 & 0.30444800\end{array}$

$\begin{array}{llll}\mathrm{Cl} & 1.88683700 & -2.17341000 & 0.01129000\end{array}$

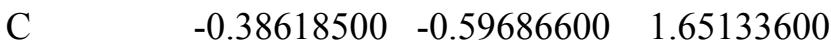

$\begin{array}{llll}\mathrm{H} & -0.65722900 & 0.30349900 & 2.19400800\end{array}$

$\begin{array}{llll}\mathrm{C} & -1.31700900 & -1.17237300 & 0.74768800\end{array}$

$\mathrm{H} \quad-1.06405300 \quad-2.16180000 \quad 0.37025100$

$\begin{array}{llll}\mathrm{C} & -2.53188800 & -0.62083400 & 0.25655300\end{array}$

$\begin{array}{llll}\mathrm{C} & -3.07075400 & 0.61387100 & 0.71435700\end{array}$

C $\quad-3.23850600 \quad-1.36144200 \quad-0.73142200$

$\begin{array}{llll}\text { C } & -4.26271900 & 1.08330600 & 0.18962400\end{array}$

$\mathrm{H} \quad \begin{array}{llll}\mathrm{H} & -2.55533800 & 1.18361600 & 1.47868200\end{array}$

C $\quad-4.42881000 \quad-0.88009700 \quad-1.25235300$

$\mathrm{H} \quad-2.82771400 \quad-2.30601800 \quad-1.07630300$

$\begin{array}{llll}\mathrm{C} & -4.93862500 & 0.34196300 & -0.79321500\end{array}$

$\mathrm{H} \quad-4.67718600 \quad 2.02370400 \quad 0.53843700$

$\mathrm{H} \quad-4.96251900-1.44387800 \quad-2.01066000$

$\mathrm{H} \quad-5.87228500 \quad 0.72008400 \quad-1.19986800$

$\mathrm{H} \quad 0.23210100 \quad-1.30026500 \quad 2.20598500$

$\mathrm{Cl} \quad 2.79201200 \quad 0.81021700 \quad-1.35829800$

$\mathbf{T S}_{26-28}$

$\mathrm{E}(\mathrm{SMD} / \mathrm{B} 3 \mathrm{LYP} / \mathrm{BS} 1)=-2171.87228881 \mathrm{au}$

$\mathrm{H}(\mathrm{SMD} / \mathrm{B} 3 \mathrm{LYP} / \mathrm{BS} 1)=-2171.589787 \mathrm{au}$

$\mathrm{G}(\mathrm{SMD} / \mathrm{B} 3 \mathrm{LYP} / \mathrm{BS} 1)=-2171.670954 \mathrm{au}$

$\mathrm{E}(\mathrm{SMD} / \mathrm{M} 06 / \mathrm{BS} 2 / / \mathrm{SMD} / \mathrm{B} 3 \mathrm{LYP} / \mathrm{BS} 1)=-2171.64172963 \mathrm{au}$

$\begin{array}{llll}\mathrm{C} & 1.59517700 & 0.18594000 & -3.15163400\end{array}$

$\mathrm{H} \quad 0.56892800 \quad-0.02942200 \quad-3.46440700$

$\mathrm{H} \quad 2.27989100 \quad-0.55899700 \quad-3.56339400$

$\mathrm{H} \quad \begin{array}{llll}\mathrm{H} & 1.85592200 & 1.17708400 & -3.54700000\end{array}$

$\begin{array}{lllll}\mathrm{C} & 1.70771400 & 0.21776000 & -1.64722000\end{array}$

$\begin{array}{llll}\mathrm{O} & 2.62147300 & -0.32444800 & -1.04602800\end{array}$

$\begin{array}{llll}\mathrm{C} & 0.65933700 & 1.06976600 & -0.92575500\end{array}$

$\begin{array}{llll}\mathrm{C} & 1.30060100 & 1.92463500 & 0.12406600\end{array}$

$\begin{array}{llll}\mathrm{O} & 1.93055100 & 1.46914800 & 1.09623500\end{array}$

$\begin{array}{llll}\text { C } & 1.29762700 & 3.40962100 & -0.08656800\end{array}$

$\begin{array}{llll}\mathrm{H} & 1.65996600 & 3.92569200 & 0.80507200\end{array}$

$\mathrm{H} \quad \begin{array}{llll}\mathrm{H} & 1.96224600 & 3.63779700 & -0.93203000\end{array}$

$\begin{array}{llll}\mathrm{H} & 0.29707500 & 3.76103500 & -0.35285800\end{array}$

$\begin{array}{llll}\mathrm{H} & 1.91443200 & 0.13391100 & 1.44705500\end{array}$

$\begin{array}{llll}\text { C } & 2.75474300 & -1.64207400 & 1.47607800\end{array}$

$\begin{array}{llll}\mathrm{C} & 1.82582800 & -0.93570200 & 2.20994000\end{array}$

$\mathrm{H} \quad 2.39942500 \quad-2.25412900 \quad 0.65013800$ 


$\begin{array}{llll}\mathrm{H} & 0.77104100 & -1.19011300 & 2.10665400 \\ \mathrm{H} & 2.13161600 & -0.48853800 & 3.15784100 \\ \mathrm{H} & 0.12039500 & 1.69799400 & -1.63673100 \\ \mathrm{Au} & -0.96051500 & -0.07772000 & -0.10026500 \\ \mathrm{C} & 4.21658200 & -1.57742500 & 1.66761700 \\ \mathrm{H} & 4.58863100 & -2.60180400 & 1.81990200 \\ \mathrm{H} & 4.69142600 & -1.22500200 & 0.74224800 \\ \mathrm{H} & 4.51158600 & -0.94904800 & 2.51127400 \\ \mathrm{Cl} & -0.31427100 & -2.04613200 & -1.24088300 \\ \mathrm{Cl} & -2.87132800 & -1.26497000 & 0.81042500 \\ \mathrm{Cl} & -1.66344300 & 1.90114200 & 1.00553800\end{array}$

\section{8}

$\mathrm{E}(\mathrm{SMD} / \mathrm{B} 3 \mathrm{LYP} / \mathrm{BS} 1)=-2171.8882887 \mathrm{au}$

$\mathrm{H}(\mathrm{SMD} / \mathrm{B} 3 \mathrm{LYP} / \mathrm{BS} 1)=-2171.601004 \mathrm{au}$

$\mathrm{G}(\mathrm{SMD} / \mathrm{B} 3 \mathrm{LYP} / \mathrm{BS} 1)=-2171.688213 \mathrm{au}$

$\mathrm{E}(\mathrm{SMD} / \mathrm{M} 06 / \mathrm{BS} 2 / / \mathrm{SMD} / \mathrm{B} 3 \mathrm{LYP} / \mathrm{BS} 1)=-2171.65299658 \mathrm{au}$

$\begin{array}{llll}\mathrm{C} & -4.59565600 & 0.02115500 & -0.25566000\end{array}$

$\mathrm{H} \quad-4.19110200 \quad-0.97025300 \quad-0.03354900$

$\mathrm{H} \quad-5.18928400 \quad-0.00907300 \quad-1.17272800$

$\mathrm{H} \quad-5.25536400 \quad 0.30498900 \quad 0.57660200$

C $\quad-3.51052100 \quad 1.06820100 \quad-0.37568100$

$\begin{array}{llll}\mathrm{O} & -3.61312900 & 2.02692400 & -1.12638800\end{array}$

$\begin{array}{llll}\mathrm{C} & -2.32954100 & 0.94873900 & 0.57450600\end{array}$

$\begin{array}{llll}\mathrm{C} & -1.49230100 & 2.21990700 & 0.62042200\end{array}$

$\begin{array}{llll}\mathrm{O} & -0.42856000 & 2.33653000 & 0.03177600\end{array}$

$\begin{array}{llll}\text { C } & -2.05501600 & 3.33798500 & 1.46907000\end{array}$

$\mathrm{H} \quad-1.46345200 \quad 4.24688500 \quad 1.33275100$

$\mathrm{H} \quad-3.10283300 \quad 3.53113500 \quad 1.21498800$

$\mathrm{H} \quad-2.02391000 \quad 3.03793700 \quad 2.52499300$

$\begin{array}{llll}\mathrm{C} & 3.23900700 & 1.77712800 & -1.18914600\end{array}$

$\begin{array}{llll}\mathrm{C} & 2.08793300 & 1.35181300 & -1.98183700\end{array}$

$\mathrm{H} \quad 3.32284300 \quad 2.85208600 \quad-1.02775900$

$\mathrm{H} \quad 2.02377100 \quad 1.97219800 \quad-2.88793200$

$\mathrm{H} \quad 2.05818600 \quad 0.29247200 \quad-2.23459200$

$\mathrm{H} \quad-2.71429800 \quad 0.71284500 \quad 1.57158300$

$\mathrm{Au} \quad-1.08897200 \quad-0.72185000 \quad 0.15766200$

$\mathrm{Cl} \quad-1.64454200 \quad-0.65924800 \quad-2.14297100$

$\mathrm{Cl} \quad 0.37828800 \quad-2.66327100 \quad-0.22948200$

$\mathrm{Cl} \quad-0.56436500 \quad-0.72851500 \quad 2.47422400$

$\begin{array}{llll}\mathrm{C} & 4.22620500 & 0.99200500 & -0.59006600\end{array}$

$\begin{array}{llll}\mathrm{C} & 4.25195700 & -0.43466800 & -0.69462600\end{array}$

$\begin{array}{llll}\mathrm{C} & 5.24576500 & 1.65671800 & 0.16359700\end{array}$

C $\quad \begin{array}{llll}\text { C } & 5.25130700 & -1.15026900 & -0.06571900\end{array}$

$\mathrm{H} \quad 3.48589200 \quad-0.95080800 \quad-1.26156200$

$\begin{array}{lllll}\mathrm{C} & 6.24191400 & 0.92719900 & 0.78307300\end{array}$

$\begin{array}{llll}\mathrm{H} & 5.21907200 & 2.73985500 & 0.23901600\end{array}$

$\begin{array}{llll}\mathrm{C} & 6.24128600 & -0.47220200 & 0.66798800\end{array}$

$\mathrm{H} \quad \begin{array}{llll}\mathrm{H} & 5.27607900 & -2.23256800 & -0.13541400\end{array}$

$\begin{array}{llll}\mathrm{H} & 7.01714500 & 1.42580500 & 1.35515500\end{array}$

H $\quad \begin{array}{llll}7.02381200 & -1.04565800 & 1.15700300\end{array}$ 


\section{$\mathbf{T S}_{22}$}

$\mathrm{E}(\mathrm{SMD} / \mathrm{B} 3 \mathrm{LYP} / \mathrm{BS} 1)=-2171.83118378 \mathrm{au}$
$\mathrm{H}(\mathrm{SMD} / \mathrm{B} 3 \mathrm{LYP} / \mathrm{BS} 1)=-2171.546782 \mathrm{au}$
$\mathrm{G}(\mathrm{SMD} / \mathrm{B} 3 \mathrm{LYP} / \mathrm{BS} 1)=-2171.624321 \mathrm{au}$

$\mathrm{E}(\mathrm{SMD} / \mathrm{M} 06 / \mathrm{BS} 2 / / \mathrm{SMD} / \mathrm{B} 3 \mathrm{LYP} / \mathrm{BS} 1)=-2171.61236299 \mathrm{au}$

C $\quad-0.31689600 \quad-0.21313600 \quad-2.57462500$

$\mathrm{H} \quad-0.90349800 \quad-0.55797700 \quad-3.43091800$

$\mathrm{H} \quad 0.65835400 \quad-0.71617700 \quad-2.61780800$

$\mathrm{H} \quad-0.13478400 \quad 0.86265500 \quad-2.64877300$

C $\quad-1.02993900 \quad-0.61831800 \quad-1.29300600$

$\begin{array}{llll}\mathrm{O} & -1.80636100 & -1.55582400 & -1.28498200\end{array}$

$\begin{array}{llll}\text { C } & -0.77962600 & 0.20980900 & -0.04477600\end{array}$

$\begin{array}{llll}\text { C } & -0.90100400 & -0.38529200 & 1.35175900\end{array}$

$\begin{array}{llll}\mathrm{O} & -1.44117800 & -1.45758300 & 1.54706300\end{array}$

$\begin{array}{llll}\mathrm{C} & -0.33220400 & 0.42356300 & 2.50834400\end{array}$

$\mathrm{H} \quad-0.15259300 \quad 1.47514500 \quad 2.26965700$

$\mathrm{H} \quad \begin{array}{llll}-0.99909900 & 0.33525800 & 3.37057700\end{array}$

$\mathrm{H} \quad \begin{array}{llll}0.63150500 & -0.02144000 & 2.78867500\end{array}$

$\begin{array}{llll}\mathrm{H} & -0.18446200 & 1.11941300 & -0.16586200\end{array}$

$\begin{array}{llll}\text { C } & -4.12151400 & 0.31463100 & 1.32230600\end{array}$

$\begin{array}{llll}\text { C } & -5.34663800 & -0.33269400 & 1.41220900\end{array}$

$\begin{array}{llll}\text { C } & -6.03004500 & -0.69199600 & 0.24412800\end{array}$

$\begin{array}{llll}\text { C } & -5.48604600 & -0.39866800 & -1.01458400\end{array}$

$\begin{array}{llll}\text { C } & -4.26447500 & 0.25240200 & -1.10889700\end{array}$

$\begin{array}{llll}\mathrm{C} & -3.56584300 & 0.63861100 & 0.06037800\end{array}$

$\begin{array}{llll}\mathrm{H} & -3.59430600 & 0.60676500 & 2.22563800\end{array}$

$\mathrm{H} \quad-5.77321900 \quad-0.55652900 \quad 2.38519400$

$\mathrm{H} \quad-6.98716600 \quad-1.20133000 \quad 0.31272800$

$\mathrm{H} \quad-6.01959100 \quad-0.68312100 \quad-1.91640800$

$\mathrm{H} \quad \begin{array}{llll}\mathrm{H} & -3.84983000 & 0.47620800 & -2.08574800\end{array}$

$\begin{array}{llll}\mathrm{C} & -2.34889100 & 1.43922800 & 0.00931600\end{array}$

$\begin{array}{llll}\mathrm{H} & -2.08386500 & 1.85508700 & 0.97965600\end{array}$

C $\quad \begin{array}{llll}-2.14975800 & 2.42923200 & -1.11434700\end{array}$

$\mathrm{H} \quad \begin{array}{llll}\mathrm{H} & -2.16323600 & 1.97859100 & -2.10975300\end{array}$

$\mathrm{H} \quad-2.98338700 \quad 3.14306300 \quad-1.07040400$

$\mathrm{H} \quad-1.21936600 \quad 2.98955400 \quad-0.99115100$

$\mathrm{Au} \quad 1.86066000 \quad-0.01682900 \quad 0.00742200$

$\begin{array}{llll}\mathrm{Cl} & 2.06355800 & 2.34507200 & 0.13302700\end{array}$

$\begin{array}{llll}\mathrm{Cl} & 4.21889400 & -0.23379200 & 0.08665300\end{array}$

$\mathrm{Cl} \quad 1.64601700 \quad-2.35084800 \quad-0.13306400$

29

$\mathrm{E}(\mathrm{SMD} / \mathrm{B} 3 \mathrm{LYP} / \mathrm{BS} 1)=-1861.80071947 \mathrm{au}$

$\mathrm{H}(\mathrm{SMD} / \mathrm{B} 3 \mathrm{LYP} / \mathrm{BS} 1)=-1861.669332 \mathrm{au}$

$\mathrm{G}(\mathrm{SMD} / \mathrm{B} 3 \mathrm{LYP} / \mathrm{BS} 1)=-1861.732248 \mathrm{au}$

$\mathrm{E}(\mathrm{SMD} / \mathrm{M} 06 / \mathrm{BS} 2 / / \mathrm{SMD} / \mathrm{B} 3 \mathrm{LYP} / \mathrm{BS} 1)=-1861.71166947 \mathrm{au}$

$\begin{array}{llll}\mathrm{C} & 3.35038300 & 1.75774300 & -0.69692700\end{array}$

$\mathrm{H} \quad 3.15747500 \quad 2.63291900-0.06226600$

$\mathrm{H} \quad 3.75115500 \quad 2.08980200 \quad-1.65821200$ 


$\begin{array}{lccc}\mathrm{H} & 4.08605100 & 1.13216800 & -0.18067900 \\ \mathrm{C} & 2.05652500 & 1.00198400 & -0.91180700 \\ \mathrm{O} & 1.51941800 & 0.94601600 & -2.00545800 \\ \mathrm{C} & 1.45626700 & 0.35686500 & 0.33145600 \\ \mathrm{C} & 2.19555800 & -0.89217300 & 0.77809300 \\ \mathrm{O} & 3.08464400 & -1.37518200 & 0.09287700 \\ \mathrm{C} & 1.85643200 & -1.43565700 & 2.14955900 \\ \mathrm{H} & 2.20477200 & -2.46773800 & 2.23892700 \\ \mathrm{H} & 2.37459900 & -0.82485800 & 2.90232000 \\ \mathrm{H} & 0.78461600 & -1.37871800 & 2.36088000 \\ \mathrm{H} & 1.45985000 & 1.07101500 & 1.16122800 \\ \mathrm{Au} & -0.61596200 & 0.01274800 & 0.02671300 \\ \mathrm{Cl} & -0.19406700 & -2.16527900 & -0.80427300 \\ \mathrm{Cl} & -3.03763400 & -0.29518200 & -0.30219000 \\ \mathrm{Cl} & -0.97306500 & 2.19217300 & 0.90210300\end{array}$

\section{$\mathbf{T S}_{22}$}

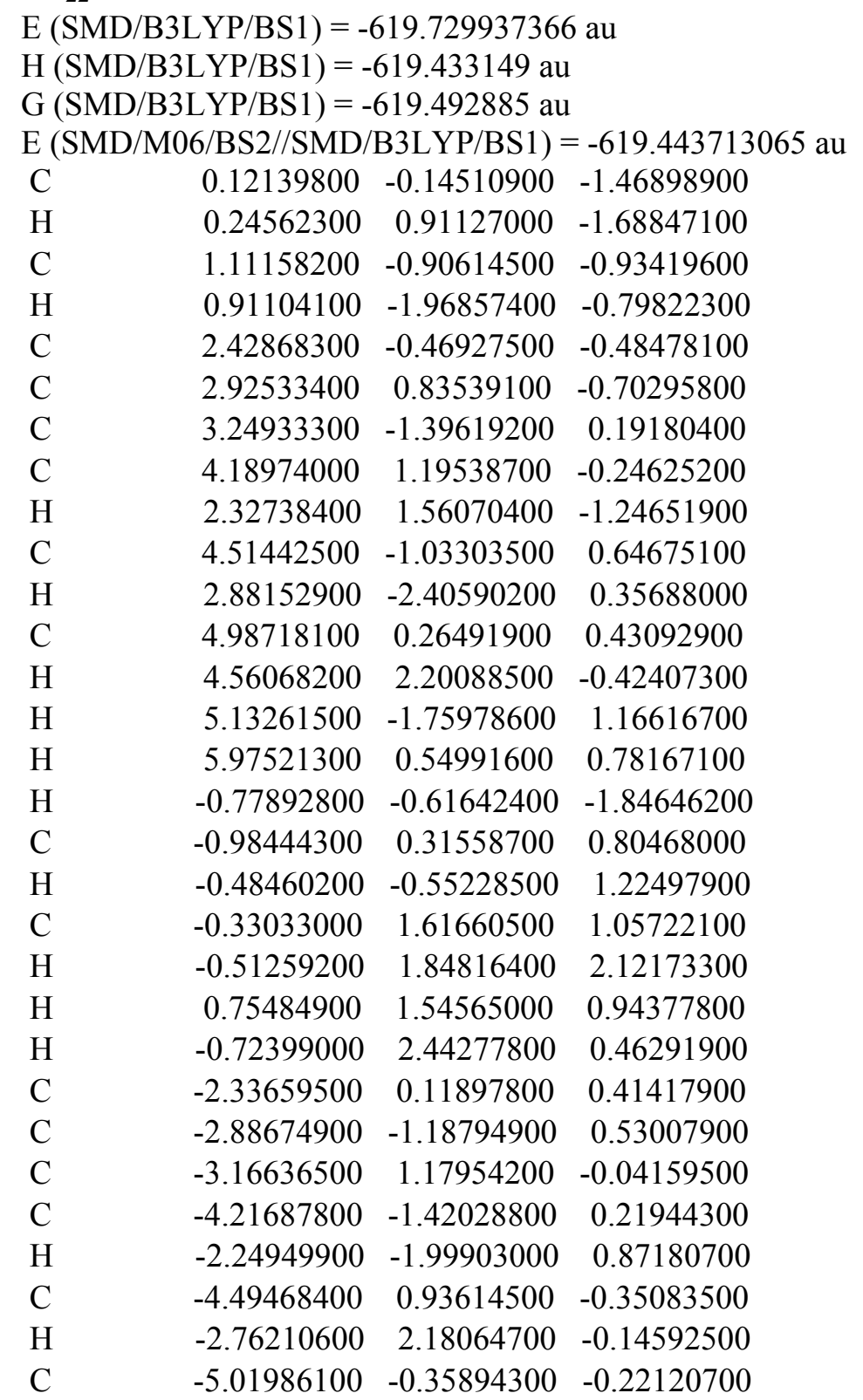




$\begin{array}{rrrr}H & -4.63552000 & -2.41693900 & 0.31632200 \\ H & -5.13019100 & 1.74612400 & -0.69465100 \\ H & -6.06212100 & -0.54090600 & -0.46756600\end{array}$

\section{0}

$\mathrm{E}(\mathrm{SMD} / \mathrm{B} 3 \mathrm{LYP} / \mathrm{BS} 1)=-1418.14267327 \mathrm{au}$

$\mathrm{H}(\mathrm{SMD} / \mathrm{B} 3 \mathrm{LYP} / \mathrm{BS} 1)=-1417.960714 \mathrm{au}$

$\mathrm{G}(\mathrm{SMD} / \mathrm{B} 3 \mathrm{LYP} / \mathrm{BS} 1)=-1418.031171 \mathrm{au}$

$\mathrm{E}(\mathrm{SMD} / \mathrm{M} 06 / \mathrm{BS} 2 / / \mathrm{SMD} / \mathrm{B} 3 \mathrm{LYP} / \mathrm{BS} 1)=-1418.13454358 \mathrm{au}$

$\begin{array}{llll}\mathrm{O} & -1.71167300 & -0.61074600 & -1.11496900\end{array}$

S $\quad-2.92559500 \quad-0.24138400 \quad-0.27781100$

$\begin{array}{llll}\mathrm{O} & -4.15417300 & -0.16251600 & -1.07878800\end{array}$

$\begin{array}{llll}\mathrm{O} & -2.98703900 & -0.95189100 & 1.01197000\end{array}$

$\begin{array}{llll}\text { C } & -2.53781100 & 1.51961800 & 0.18077700\end{array}$

F $\quad-2.38049800 \quad 2.26888100 \quad-0.91713600$

F $\quad-3.52802200 \quad 2.03547700 \quad 0.91819800$

F $\quad \begin{array}{llll}-1.39969600 & 1.57603200 & 0.89822100\end{array}$

$\begin{array}{llll}\mathrm{C} & 1.92138800 & -2.28215900 & 0.83837700\end{array}$

$\mathrm{H} \quad \begin{array}{llll}\mathrm{H} & 1.38995800 & -2.91746900 & 1.54340800\end{array}$

$\begin{array}{llll}\mathrm{H} & 2.40846500 & -2.77890800 & 0.00257600\end{array}$

$\begin{array}{llll}\text { C } & 2.17809000 & -0.97343200 & 1.16486700\end{array}$

$\begin{array}{llll}\mathrm{H} & 1.75596200 & -0.59831900 & 2.09716100\end{array}$

$\begin{array}{llll}\text { C } & 3.07335900 & -0.02798100 & 0.47431900\end{array}$

$\begin{array}{llll}\text { C } & 3.79934100 & -0.36175100 & -0.68604000\end{array}$

$\begin{array}{llll}\mathrm{C} & 3.22383900 & 1.25984700 & 1.02028500\end{array}$

$\begin{array}{llll}\mathrm{C} & 4.64586500 & 0.57112100 & -1.27863200\end{array}$

$\mathrm{H} \quad 3.70517700 \quad-1.35143600 \quad-1.12343100$

$\begin{array}{llll}\mathrm{C} & 4.07311700 & 2.19276800 & 0.42471100\end{array}$

$\begin{array}{llll}\mathrm{H} & 2.67151800 & 1.52287200 & 1.91922500\end{array}$

$\begin{array}{lllll}\mathrm{C} & 4.78530600 & 1.85099300 & -0.72700000\end{array}$

$\mathrm{H} \quad 5.20218900 \quad 0.30107800 \quad-2.17198200$

$\mathrm{H} \quad 4.17883000 \quad 3.18248700 \quad 0.86005200$

$\mathrm{H} \quad \begin{array}{llll}5.44823700 & 2.57455000 & -1.19335300\end{array}$

Ag $\quad 0.12066500 \quad-1.22742900 \quad-0.09782800$

\section{1}

$\mathrm{E}(\mathrm{SMD} / \mathrm{B} 3 \mathrm{LYP} / \mathrm{BS} 1)=-1454.29019075 \mathrm{au}$

$\mathrm{H}(\mathrm{SMD} / \mathrm{B} 3 \mathrm{LYP} / \mathrm{BS} 1)=-1454.119274 \mathrm{au}$

$\mathrm{G}(\mathrm{SMD} / \mathrm{B} 3 \mathrm{LYP} / \mathrm{BS} 1)=-1454.188243 \mathrm{au}$

$\mathrm{E}(\mathrm{SMD} / \mathrm{M} 06 / \mathrm{BS} 2 / / \mathrm{SMD} / \mathrm{B} 3 \mathrm{LYP} / \mathrm{BS} 1)=-1454.34472105 \mathrm{au}$

$\begin{array}{llll}\mathrm{C} & 2.33825300 & 2.65530300 & -0.90328800\end{array}$

$\mathrm{H} \quad 3.19010600 \quad 3.34479400 \quad-0.83950300$

$\mathrm{H} \quad \begin{array}{llll}1.44657100 & 3.20672000 & -0.58620600\end{array}$

$\mathrm{H} \quad 2.22032800 \quad 2.32362300 \quad-1.93628300$

$\begin{array}{llll}\mathrm{C} & 2.58852100 & 1.50508800 & 0.01358800\end{array}$

$\begin{array}{llll}\mathrm{O} & 2.63705000 & 1.81000200 & 1.28928100\end{array}$

$\begin{array}{llll}\text { C } & 2.81330700 & 0.18550100 & -0.42895300\end{array}$

$\begin{array}{llll}\mathrm{C} & 3.35335900 & -0.80467300 & 0.51564900\end{array}$

$\begin{array}{llll}\mathrm{O} & 3.38998500 & -0.56640300 & 1.74062800\end{array}$

C $\quad 3.90239100 \quad-2.09296200 \quad-0.03292300$

$\mathrm{H} \quad 3.23318300 \quad-2.51621700 \quad-0.78911900$ 


$\begin{array}{crcc}\mathrm{H} & 4.86292500 & -1.88788300 & -0.52490400 \\ \mathrm{H} & 4.06324400 & -2.81033600 & 0.77498800 \\ \mathrm{H} & 3.01855900 & 0.04738800 & -1.48858400 \\ \mathrm{H} & 2.90692900 & 0.95675900 & 1.77559900 \\ \mathrm{O} & -1.44209300 & -0.94542100 & -0.99004500 \\ \mathrm{~S} & -2.42005200 & -0.92966200 & 0.17289600 \\ \mathrm{O} & -3.66737100 & -1.64179800 & -0.13517700 \\ \mathrm{O} & -1.78365300 & -1.18488900 & 1.47746600 \\ \mathrm{C} & -2.90028000 & 0.86720800 & 0.23386700 \\ \mathrm{~F} & -3.43606300 & 1.25146800 & -0.93122200 \\ \mathrm{~F} & -3.79193800 & 1.07604800 & 1.20953200 \\ \mathrm{~F} & -1.81970500 & 1.63348800 & 0.47487600 \\ \mathrm{Ag} & 0.62901500 & -0.36391500 & -0.62501900\end{array}$

TS $_{\text {31-32 }}$

\begin{tabular}{lrrr} 
E $(\mathrm{SMD} / \mathrm{B} 3 \mathrm{LYP} / \mathrm{BS} 1)=-1763.90256396 \mathrm{au}$ \\
$\mathrm{H}(\mathrm{SMD} / \mathrm{B} 3 \mathrm{~L}$ YP/BS1 $)=-1763.592794 \mathrm{au}$ \\
$\mathrm{G}(\mathrm{SMD} / \mathrm{B} 3 \mathrm{~L}$ /BS1) $=-1763.686813 \mathrm{au}$ \\
$\mathrm{E}(\mathrm{SMD} / \mathrm{M} 06 / \mathrm{BS} 2 / / \mathrm{SMD} / \mathrm{B} 3 \mathrm{LYP} / \mathrm{BS} 1)=-1763.81399544 \mathrm{au}$ \\
$\mathrm{C}$ & -0.07972300 & 3.10147700 & 1.99107300 \\
$\mathrm{H}$ & 1.00351900 & 2.95440700 & 1.92516400 \\
$\mathrm{H}$ & -0.42456300 & 2.88193800 & 3.00515600 \\
$\mathrm{H}$ & -0.28078400 & 4.16001700 & 1.77666200 \\
$\mathrm{C}$ & -0.83387900 & 2.23724400 & 0.98896200 \\
$\mathrm{O}$ & -1.90896500 & 1.72458900 & 1.30357600 \\
$\mathrm{C}$ & -0.21025400 & 2.13663000 & -0.34983500 \\
$\mathrm{C}$ & -0.97632000 & 1.85507900 & -1.54067700 \\
$\mathrm{O}$ & -2.04240900 & 1.17629400 & -1.60250000 \\
$\mathrm{C}$ & -0.50592600 & 2.47113800 & -2.84275000 \\
$\mathrm{H}$ & -0.73497000 & 1.80802100 & -3.68183300 \\
$\mathrm{H}$ & -1.05132100 & 3.41194600 & -2.99834400 \\
$\mathrm{H}$ & 0.56312200 & 2.69987400 & -2.82776200 \\
$\mathrm{H}$ & -2.31022800 & 0.16239900 & -0.64655500 \\
$\mathrm{C}$ & -3.53166000 & -0.74054400 & 0.72877100 \\
$\mathrm{C}$ & -2.44133400 & -0.97652400 & -0.11614000 \\
$\mathrm{H}$ & -3.31283900 & -0.24075300 & 1.66943700 \\
$\mathrm{H}$ & -1.46706900 & -1.04623000 & 0.37551700 \\
$\mathrm{H}$ & -2.56922200 & -1.64497400 & -0.96836900 \\
$\mathrm{C}$ & -4.92314100 & -0.96703100 & 0.46554800 \\
$\mathrm{C}$ & -5.86104800 & -0.51122100 & 1.42380300 \\
$\mathrm{C}$ & -5.39472700 & -1.62252500 & -0.69904100 \\
$\mathrm{C}$ & -7.22371600 & -0.69922300 & 1.22547600 \\
$\mathrm{H}$ & -5.50006700 & -0.00904200 & 2.31714700 \\
$\mathrm{C}$ & -6.75646400 & -1.80431500 & -0.89192600 \\
$\mathrm{H}$ & -4.69271400 & -1.98710900 & -1.44157600 \\
$\mathrm{C}$ & -7.67086400 & -1.34427500 & 0.06752800 \\
$\mathrm{H}$ & -7.93654000 & -0.34604200 & 1.96423900 \\
$\mathrm{H}$ & -7.11543000 & -2.30692800 & -1.78490500 \\
$\mathrm{H}$ & -8.73545800 & -1.49328100 & -0.09024800 \\
$\mathrm{O}$ & 2.33485800 & -1.43487300 & -0.08994800 \\
$\mathrm{~S}$ & 3.78197700 & -1.36461000 & -0.54150400 \\
& & & \\
\hline
\end{tabular}




$\begin{array}{cccc}\mathrm{O} & 4.41060600 & -2.69089600 & -0.61458900 \\ \mathrm{O} & 4.00798900 & -0.43574300 & -1.66321200 \\ \mathrm{C} & 4.59419500 & -0.53729600 & 0.91433000 \\ \mathrm{~F} & 4.39891500 & -1.25041600 & 2.03097500 \\ \mathrm{~F} & 5.91041300 & -0.41538400 & 0.70429200 \\ \mathrm{~F} & 4.08028400 & 0.69287100 & 1.10569600 \\ \mathrm{Ag} & 1.07340700 & 0.34888900 & -0.23063700 \\ \mathrm{H} & 0.56884100 & 2.88143800 & -0.51575200\end{array}$

32

$\mathrm{E}(\mathrm{SMD} / \mathrm{B} 3 \mathrm{LYP} / \mathrm{BS} 1)=-1763.90256396 \mathrm{au}$

$\mathrm{H}(\mathrm{SMD} / \mathrm{B} 3 \mathrm{LYP} / \mathrm{BS} 1)=-1763.592794 \mathrm{au}$

$\mathrm{G}(\mathrm{SMD} / \mathrm{B} 3 \mathrm{LYP} / \mathrm{BS} 1)=-1763.686813 \mathrm{au}$

$\mathrm{E}(\mathrm{SMD} / \mathrm{M} 06 / \mathrm{BS} 2 / / \mathrm{SMD} / \mathrm{B} 3 \mathrm{LYP} / \mathrm{BS} 1)=-1763.81399544 \mathrm{au}$

$\begin{array}{llll}\mathrm{C} & -1.67271300 & 1.24743700 & 2.99460200\end{array}$

$\mathrm{H} \quad-0.64446200 \quad 1.08290400 \quad 3.33319200$

$\mathrm{H} \quad-2.30258100 \quad 0.40360000 \quad 3.28908700$

$\mathrm{H} \quad-2.04282200 \quad 2.15300400 \quad 3.49358400$

$\begin{array}{llll}\mathrm{C} & -1.73323400 & 1.46261200 & 1.49171900\end{array}$

$\begin{array}{llll}\mathrm{O} & -2.73264200 & 1.00152700 & 0.89676400\end{array}$

$\begin{array}{llll}\mathrm{C} & -0.65849500 & 2.26298100 & 0.91127000\end{array}$

C $\quad-0.91891200 \quad 3.31129200 \quad-0.09497800$

$\begin{array}{llll}\mathrm{O} & -1.92807700 & 3.40072100 & -0.79644900\end{array}$

C $\quad 0.15701000 \quad 4.39237100 \quad-0.19709800$

$\mathrm{H} \quad 0.10687500 \quad 4.86881900 \quad-1.18006600$

$\mathrm{H} \quad-0.03227200 \quad 5.16109000 \quad 0.56466200$

$\mathrm{H} \quad \begin{array}{llll}\mathrm{H} & 1.16413800 & 3.99876300 & -0.02624800\end{array}$

$\mathrm{H} \quad-3.30643500 \quad 1.99483900 \quad-1.45501400$

C $\quad-2.91105100 \quad-0.01719200 \quad-1.09910900$

$\begin{array}{lllll}\mathrm{C} & & -3.69993900 & 1.02293200 & -1.77897800\end{array}$

$\mathrm{H} \quad-1.83626400 \quad 0.01803500 \quad-1.26273300$

$\mathrm{H} \quad-3.47403900 \quad 0.95804100 \quad-2.85634400$

$\mathrm{H} \quad-4.77766100 \quad 0.95064000 \quad-1.63839200$

C $\quad-3.41990400 \quad-1.24668800 \quad-0.58279100$

$\begin{array}{llll}\text { C } & -2.49858900 & -2.23715200 & -0.15821400\end{array}$

C $\quad-4.80903000 \quad-1.52264600 \quad-0.50845100$

$\begin{array}{llll}\text { C } & -2.94651100 & -3.46145000 & 0.31542600\end{array}$

$\mathrm{H} \quad-1.43483500 \quad-2.02404500 \quad-0.21343200$

C $\quad-5.25025600 \quad-2.75173800 \quad-0.03765700$

$\mathrm{H} \quad-5.53164100 \quad-0.77947800 \quad-0.82542300$

$\begin{array}{llll}\text { C } & -4.32279900 & -3.71824900 & 0.37342900\end{array}$

$\mathrm{H} \quad-2.23620800 \quad-4.21757200 \quad 0.63458100$

$\mathrm{H} \quad-6.31364700 \quad-2.96420100 \quad 0.01367700$

$\mathrm{H} \quad-4.67618000 \quad-4.67772300 \quad 0.74124800$

$\begin{array}{llll}\mathrm{O} & 1.80721500 & -0.78702900 & -1.02600400\end{array}$

S $\quad 3.32237000 \quad-0.73281200 \quad-0.96754000$

$\begin{array}{llll}\mathrm{O} & 3.95449000 & -1.71926700 & -1.85445700\end{array}$

$\begin{array}{lllll}\mathrm{O} & 3.86626000 & 0.63734100 & -0.96820100\end{array}$

C $\quad 3.65585200 \quad-1.35803200 \quad 0.75248300$

F $\quad 3.16755100 \quad-2.59542500 \quad 0.90822800$

F $\quad 4.97180000 \quad-1.37976600 \quad 0.99346500$ 


$\begin{array}{ccrc}\mathrm{F} & 3.07077300 & -0.55725200 & 1.66453100 \\ \mathrm{Ag} & 0.63122100 & 0.81707400 & -0.11054000 \\ \mathrm{H} & 0.05507000 & 2.60260400 & 1.66450000\end{array}$

TS $_{\mathbf{3 2 - 3 4}}$
$\mathrm{E}(\mathrm{SMD} / \mathrm{B} 3 \mathrm{LYP} / \mathrm{BS} 1)=-1763.89588504 \mathrm{au}$
$\mathrm{H}(\mathrm{SMD} / \mathrm{B} 3 \mathrm{LYP} / \mathrm{BS} 1)=-1763.581948 \mathrm{au}$
$\mathrm{G}(\mathrm{SMD} / \mathrm{B} 3 \mathrm{LYP} / \mathrm{BS} 1)=-1763.675009 \mathrm{au}$

$\mathrm{E}(\mathrm{SMD} / \mathrm{M} 06 / \mathrm{BS} 2 / / \mathrm{SMD} / \mathrm{B} 3 \mathrm{LYP} / \mathrm{BS} 1)=-1763.81724739 \mathrm{au}$

$\begin{array}{llll}\mathrm{C} & -1.29939000 & 2.51546300 & 0.61085300\end{array}$

$\begin{array}{llll}\mathrm{H} & -2.19644800 & 3.13121500 & 0.74383900\end{array}$

$\begin{array}{llll}\mathrm{H} & -0.58858500 & 3.10378900 & 0.01715000\end{array}$

$\begin{array}{llll}\mathrm{H} & -0.85106600 & 2.31242800 & 1.58709400\end{array}$

C $\quad-1.66782700 \quad 1.25246100 \quad-0.16844800$

$\begin{array}{llll}\mathrm{O} & -2.28491300 & 1.35919600 & -1.22969300\end{array}$

$\begin{array}{llll}\text { C } & -1.26012800 & -0.03666900 & 0.42911800\end{array}$

C $\quad-1.37248000 \quad-1.36519700 \quad-0.20672800$

$\begin{array}{llll}\mathrm{O} & -1.88385400 & -1.56987700 & -1.31052100\end{array}$

$\begin{array}{llll}\text { C } & -0.83419300 & -2.54980400 & 0.59762800\end{array}$

$\mathrm{H} \quad-1.62872200 \quad-3.29695700 \quad 0.71129900$

$\mathrm{H} \quad-0.02192600 \quad-3.02607500 \quad 0.03553200$

$\mathrm{H} \quad-0.46026300 \quad-2.27056100 \quad 1.58695300$

$\begin{array}{llll}\mathrm{O} & 2.91637100 & 0.42488700 & -1.26215800\end{array}$

S $\quad 4.03521000 \quad 0.79929600 \quad-0.30657300$

O $\quad \begin{array}{llll}5.24131100 & 1.26477900 & -1.00431000\end{array}$

$\begin{array}{lllll}\mathrm{O} & 3.57356100 & 1.57230300 & 0.86135600\end{array}$

$\begin{array}{llll}\mathrm{C} & 4.49892700 & -0.86092200 & 0.39331700\end{array}$

F $\quad 4.88741500 \quad-1.69205500 \quad-0.58139800$

F $\quad \begin{array}{llll}5.49693600 & -0.73199800 & 1.27539000\end{array}$

F $\quad 3.44409600 \quad-1.41328900 \quad 1.02315500$

$\mathrm{H} \quad \begin{array}{llll}-0.81907700 & -0.00431500 & 1.42385000\end{array}$

C $\quad-4.71568800 \quad-1.39344000 \quad-0.07819400$

C $\quad-5.73229800 \quad-1.33330700 \quad-1.02156500$

C $\quad-6.39762300 \quad-0.12292200 \quad-1.24944500$

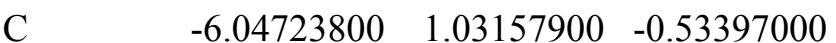

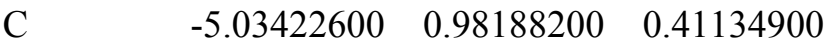

$\begin{array}{llll}\text { C } & -4.35651300 & -0.24002600 & 0.67099700\end{array}$

$\mathrm{H} \quad-4.20643300 \quad-2.33048600 \quad 0.11918400$

$\mathrm{H} \quad-6.01199600 \quad-2.22248300 \quad-1.57819300$

$\mathrm{H} \quad-7.19434300 \quad-0.07665000 \quad-1.98679500$

$\mathrm{H} \quad-6.56876800 \quad 1.96510300 \quad-0.72210600$

$\mathrm{H} \quad-4.76808900 \quad 1.87720300 \quad 0.96214300$

$\begin{array}{llll}\text { C } & -3.36003900 & -0.36482200 & 1.68043200\end{array}$

$\mathrm{H} \quad \begin{array}{llll}\mathrm{H} & -2.99707700 & -1.37271300 & 1.86023400\end{array}$

$\begin{array}{llll}\mathrm{C} & -3.16629800 & 0.58597900 & 2.80807900\end{array}$

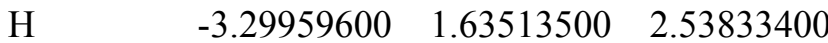

$\mathrm{H} \quad-3.93805600 \quad 0.33890800 \quad 3.55650900$

$\mathrm{H} \quad-2.19706300 \quad 0.44412600 \quad 3.29153200$

Ag $\quad 0.90847700 \quad 0.18144300 \quad-0.40837500$ 


$\begin{array}{lrrr}\text { E }(\mathrm{SMD} / \mathrm{B} 3 \mathrm{LYP} / \mathrm{BS} 1)=-1763.95928963 \mathrm{au} \\ \mathrm{H}(\mathrm{SMD} / \mathrm{B} 3 \mathrm{~L} Y \mathrm{~B} / \mathrm{BS} 1)=-1763.64207 \mathrm{au} \\ \mathrm{G}(\mathrm{SMD} / \mathrm{B} 3 \mathrm{LYP} / \mathrm{BS} 1)=-1763.735432 \mathrm{au} \\ \mathrm{E}(\mathrm{SMD} / \mathrm{M} 06 / \mathrm{BS} 2 / \mathrm{SMD} / \mathrm{B} 3 \mathrm{LYP} / \mathrm{BS} 1)=-1763.87637414 \mathrm{au} \\ \mathrm{C} & 2.87336600 & 2.71159400 & 0.54441700 \\ \mathrm{H} & 1.92713500 & 2.73643900 & 1.09585800 \\ \mathrm{H} & 2.90576200 & 3.53915500 & -0.17050700 \\ \mathrm{H} & 3.68194500 & 2.85610500 & 1.27453700 \\ \mathrm{C} & 3.08811000 & 1.38333100 & -0.18481600 \\ \mathrm{O} & 3.65422400 & 1.38306300 & -1.28020100 \\ \mathrm{C} & 2.61282100 & 0.18500100 & 0.53855800 \\ \mathrm{C} & 3.25888100 & -1.13386100 & 0.37051800 \\ \mathrm{O} & 3.87342700 & -1.51072500 & -0.62972500 \\ \mathrm{C} & 3.16830000 & -2.06188100 & 1.58429700 \\ \mathrm{H} & 3.30681900 & -3.10073000 & 1.27002100 \\ \mathrm{H} & 3.97095700 & -1.80806500 & 2.29079500 \\ \mathrm{H} & 2.21736200 & -1.96125200 & 2.11843700 \\ \mathrm{O} & -1.34799100 & -0.40626800 & -1.20517500 \\ \mathrm{~S} & -2.52846000 & -0.81477000 & -0.34611500 \\ \mathrm{O} & -3.70604100 & -1.17885000 & -1.14737200 \\ \mathrm{O} & -2.16949200 & -1.69492800 & 0.78028500 \\ \mathrm{C} & -2.99037600 & 0.79769900 & 0.45921600 \\ \mathrm{~F} & -3.27190100 & 1.72397900 & -0.46678500 \\ \mathrm{~F} & -4.06318900 & 0.63408700 & 1.24397200 \\ \mathrm{~F} & -1.97733800 & 1.25584200 & 1.21841900 \\ \mathrm{Ag} & 0.62523700 & -0.13145100 & -0.29575100 \\ \mathrm{H} & 2.37148400 & 0.39805200 & 1.58290700\end{array}$

\section{4}

$\begin{array}{llll}\text { E }(\mathrm{SMD} / \mathrm{B} 3 L Y P / B S 1)=-1453.81724306 \mathrm{au} \\ \mathrm{H}(\mathrm{SMD} / \mathrm{B} 3 L Y P / B S 1)=-1453.658183 \mathrm{au} \\ \mathrm{G}(\mathrm{SMD} / \mathrm{B} 3 L Y P / B S 1)=-1453.731357 \mathrm{au} \\ \mathrm{E}(\mathrm{SMD} / \mathrm{M} 06 / \mathrm{BS} 2 / / \mathrm{SMD} / \mathrm{B} 3 \mathrm{LYP} / \mathrm{BS} 1)=-1453.87693379 \mathrm{au} \\ \mathrm{C} & 1.16201700 & 1.92082200 & -1.84739700 \\ \mathrm{H} & 1.33829400 & 1.35867700 & -2.77622800 \\ \mathrm{H} & 1.83242200 & 2.78509800 & -1.86175000 \\ \mathrm{H} & 0.11802600 & 2.24893200 & -1.83032200 \\ \mathrm{C} & 1.46165600 & 1.00001800 & -0.70405100 \\ \mathrm{O} & 0.57584700 & 0.35992100 & -0.12359400 \\ \mathrm{C} & 2.91521800 & 0.83927700 & -0.29417500 \\ \mathrm{H} & 3.50306900 & 0.82959800 & -1.21932100 \\ \mathrm{C} & 3.30507600 & 2.11271400 & 0.49294400 \\ \mathrm{C} & 4.73860100 & 2.56612200 & 0.40781500 \\ \mathrm{H} & 4.95057700 & 2.89014800 & -0.61978800 \\ \mathrm{H} & 4.91021500 & 3.39708700 & 1.09618100 \\ \mathrm{H} & 5.42529700 & 1.74182500 & 0.62879100 \\ \mathrm{O} & 2.46226600 & 2.71577600 & 1.13805300 \\ \mathrm{C} & 7.16837200 & -2.03265100 & -0.41305300 \\ \mathrm{C} & 6.91870100 & -1.46748000 & 0.83826100 \\ \mathrm{C} & 5.64932400 & -0.96600200 & 1.14809100 \\ \mathrm{C} & 4.60673800 & -1.01772300 & 0.21178500\end{array}$




$\begin{array}{lrrr}\mathrm{C} & 4.87232700 & -1.59642400 & -1.04137300 \\ \mathrm{C} & 6.13617600 & -2.09681400 & -1.35464300 \\ \mathrm{H} & 8.15362000 & -2.42430000 & -0.65191300 \\ \mathrm{H} & 7.71033100 & -1.41685700 & 1.58159200 \\ \mathrm{H} & 5.47886700 & -0.53863100 & 2.13125400 \\ \mathrm{H} & 4.07348300 & -1.65929600 & -1.77778000 \\ \mathrm{H} & 6.31332300 & -2.54313900 & -2.32992000 \\ \mathrm{C} & 3.20007600 & -0.49296900 & 0.49852400 \\ \mathrm{H} & 2.50976300 & -1.22140800 & 0.05779300 \\ \mathrm{C} & 2.85648800 & -0.39745000 & 1.99198700 \\ \mathrm{H} & 3.47908300 & 0.32929300 & 2.52419400 \\ \mathrm{H} & 1.81218600 & -0.10248300 & 2.12473700 \\ \mathrm{H} & 2.99767900 & -1.37252900 & 2.47105400 \\ \mathrm{O} & -3.69842500 & 0.55720700 & -0.92050300 \\ \mathrm{~S} & -4.55161700 & -0.66989700 & -0.64120900 \\ \mathrm{O} & -5.85458000 & -0.60956600 & -1.31630200 \\ \mathrm{O} & -3.79650000 & -1.93385000 & -0.70776700 \\ \mathrm{C} & -4.93700400 & -0.45148600 & 1.16549400 \\ \mathrm{~F} & -5.58395400 & 0.70177500 & 1.37265500 \\ \mathrm{~F} & -5.70128200 & -1.46033100 & 1.60048600 \\ \mathrm{~F} & -3.80148100 & -0.43594400 & 1.88721100 \\ \mathrm{Ag} & -1.57088000 & 0.44534500 & -0.51473900\end{array}$

\begin{tabular}{|c|c|c|c|}
\hline \multicolumn{4}{|c|}{ 1_AuOTf } \\
\hline \multicolumn{4}{|c|}{$\overline{\mathrm{E}}(\mathrm{SMD} / \mathrm{B} 3 \mathrm{LYP} / \mathrm{BS} 1)=-1443.04688103 \mathrm{au}$} \\
\hline \multicolumn{4}{|c|}{$\mathrm{H}(\mathrm{SMD} / \mathrm{B} 3 \mathrm{LYP} / \mathrm{BS} 1)=-1442.874911 \mathrm{au}$} \\
\hline \multicolumn{4}{|c|}{$\mathrm{G}(\mathrm{SMD} / \mathrm{B} 3 \mathrm{LYP} / \mathrm{BS} 1)=-1442.946207 \mathrm{au}$} \\
\hline \multicolumn{4}{|c|}{$\mathrm{E}(\mathrm{SMD} / \mathrm{M} 06 / \mathrm{BS} 2 / / \mathrm{SMD} / \mathrm{B} 3 \mathrm{LYP} / \mathrm{BS} 1)=-1443.06019184 \mathrm{au}$} \\
\hline $\mathrm{C}$ & -2.04811700 & 2.76802800 & 0.70934500 \\
\hline $\mathrm{H}$ & -2.89277300 & 3.37653000 & 1.06009500 \\
\hline $\mathrm{H}$ & -1.51368900 & 3.35408000 & -0.04565300 \\
\hline $\mathrm{H}$ & -1.38756200 & 2.54789400 & 1.54904400 \\
\hline $\mathrm{C}$ & -2.59698300 & 1.52374300 & 0.10230600 \\
\hline $\mathrm{O}$ & -3.28765000 & 1.68518600 & -0.98685900 \\
\hline $\mathrm{C}$ & -2.44563200 & 0.22214400 & 0.69291500 \\
\hline $\mathrm{C}$ & -3.36672500 & -0.85412600 & 0.22944700 \\
\hline $\mathrm{O}$ & -4.01321800 & -0.72175100 & -0.82457800 \\
\hline $\mathrm{C}$ & -3.52111700 & -2.07680600 & 1.08534700 \\
\hline $\mathrm{H}$ & -2.54895400 & -2.43345200 & 1.44036600 \\
\hline $\mathrm{H}$ & -4.11548000 & -1.81228100 & 1.97092300 \\
\hline $\mathrm{H}$ & -4.03791200 & -2.86391700 & 0.53202700 \\
\hline $\mathrm{H}$ & -2.21554000 & 0.22633400 & 1.75856300 \\
\hline $\mathrm{H}$ & -3.69770000 & 0.77422800 & -1.21467200 \\
\hline $\mathrm{Au}$ & -0.53143600 & -0.21289000 & -0.14390800 \\
\hline $\mathrm{O}$ & 1.35617700 & -0.72637600 & -0.97985700 \\
\hline S & 2.58658500 & -0.90497600 & -0.07586700 \\
\hline $\mathrm{C}$ & 3.13126300 & 0.86014800 & 0.16712300 \\
\hline $\mathrm{F}$ & 4.25763800 & 0.89073300 & 0.88589400 \\
\hline $\mathrm{F}$ & 3.34999600 & 1.44813100 & -1.01348600 \\
\hline $\mathrm{F}$ & 2.18614200 & 1.55712700 & 0.82211200 \\
\hline $\mathrm{O}$ & 3.68004300 & -1.54654600 & -0.81030800 \\
\hline
\end{tabular}




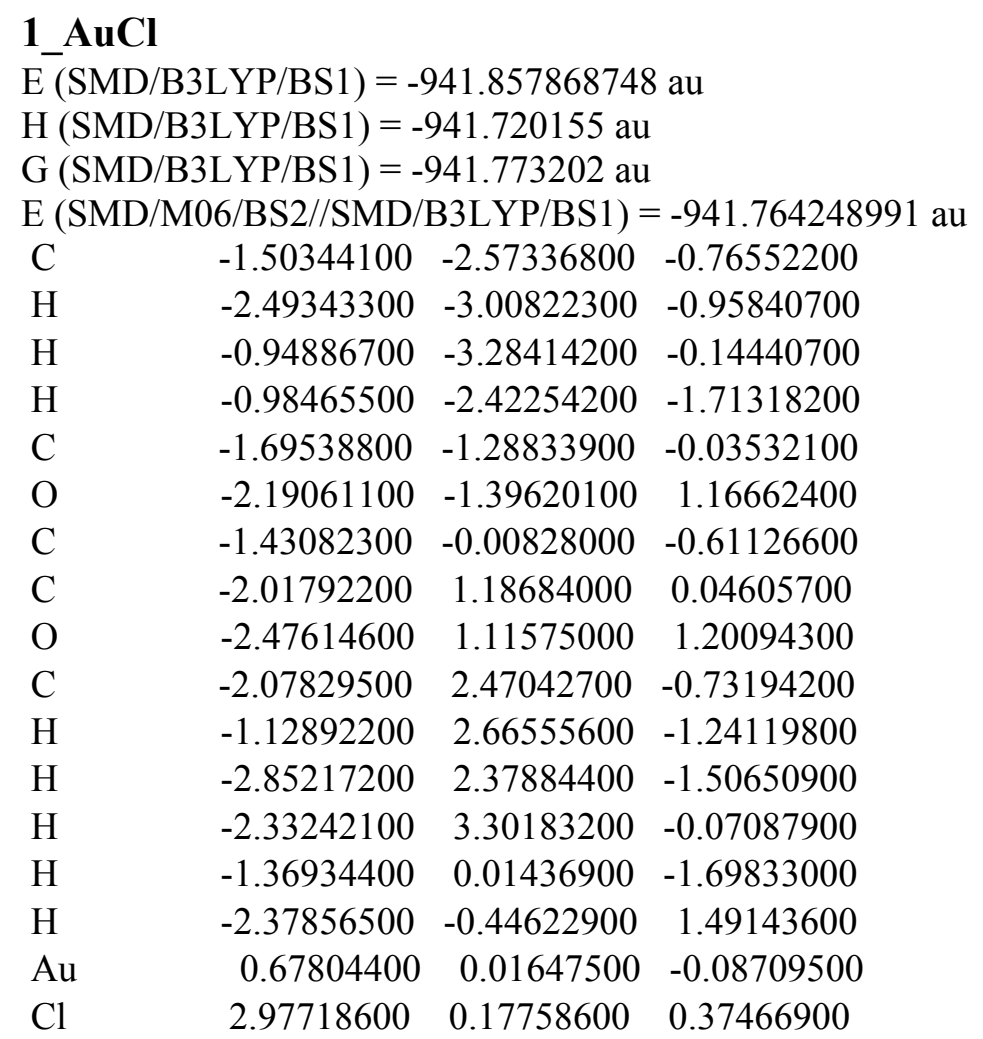

\section{2_AuOTf}

$\mathrm{E}(\mathrm{SMD} / \mathrm{B} 3 \mathrm{LYP} / \mathrm{BS} 1)=-1752.67732246 \mathrm{au}$

$\mathrm{H}(\mathrm{SMD} / \mathrm{B} 3 \mathrm{LYP} / \mathrm{BS} 1)=-1752.361978 \mathrm{au}$

$\mathrm{G}(\mathrm{SMD} / \mathrm{B} 3 \mathrm{LYP} / \mathrm{BS} 1)=-1752.455689 \mathrm{au}$

$\mathrm{E}(\mathrm{SMD} / \mathrm{M} 06 / \mathrm{BS} 2 / / \mathrm{SMD} / \mathrm{B} 3 \mathrm{LYP} / \mathrm{BS} 1)=-1752.55017646 \mathrm{au}$

$\begin{array}{llll}\mathrm{C} & -2.92930000 & 3.55958400 & 0.74945000\end{array}$

$\begin{array}{llll}\mathrm{H} & -2.08968000 & 4.00901500 & 0.20774000\end{array}$

$\begin{array}{llll}\mathrm{H} & -2.97155500 & 3.95728200 & 1.76655700\end{array}$

$\mathrm{H} \quad \begin{array}{llll}\mathrm{H} & -3.84855500 & 3.84026700 & 0.21765700\end{array}$

$\begin{array}{llll}\mathrm{C} & -2.81128400 & 2.04447100 & 0.78417400\end{array}$

$\begin{array}{llll}\mathrm{O} & -3.01802100 & 1.44084400 & 1.83572200\end{array}$

$\begin{array}{llll}\mathrm{C} & -2.47495600 & 1.39840700 & -0.52663100\end{array}$

C $\quad-3.12102000 \quad 0.08931500 \quad-0.86574800$

$\begin{array}{lllll}\mathrm{O} & -3.39348900 & -0.79726100 & -0.05727000\end{array}$

C $\quad-3.48839700 \quad-0.09038600 \quad-2.33122800$

$\mathrm{H} \quad-3.72947700 \quad-1.13749400 \quad-2.53191200$

$\mathrm{H} \quad-4.36784300 \quad 0.52635700 \quad-2.56159100$

$\mathrm{H} \quad-2.67810100 \quad 0.23978900 \quad-2.99029900$

$\begin{array}{llll}\text { C } & -2.13336300 & -2.30463600 & 1.75370000\end{array}$

$\begin{array}{llll}\text { C } & -1.81846700 & -1.26685100 & 2.72435000\end{array}$

$\mathrm{H} \quad-3.17049100 \quad-2.62985100 \quad 1.73071600$

$\mathrm{H} \quad \begin{array}{llll}-2.31492800 & -1.48685500 & 3.67958600\end{array}$

$\mathrm{H} \quad \begin{array}{llll}-0.75631300 & -1.06938500 & 2.87122500\end{array}$

$\mathrm{H} \quad \begin{array}{llll}\mathrm{H} & -2.62888300 & 2.11091800 & -1.34303300\end{array}$

$\mathrm{Au} \quad-0.40286900 \quad 1.09803100 \quad-0.53690000$

$\begin{array}{llll}\text { C } & -1.26444200 & -2.96046800 & 0.86322800\end{array}$ 


$\begin{array}{lrrr}\mathrm{C} & 0.12291500 & -2.64713500 & 0.77090500 \\ \mathrm{C} & -1.80981000 & -3.96782800 & 0.01402100 \\ \mathrm{C} & 0.92157000 & -3.32250700 & -0.13627800 \\ \mathrm{H} & 0.55707500 & -1.87491800 & 1.39603400 \\ \mathrm{C} & -0.99896800 & -4.64049800 & -0.88323400 \\ \mathrm{H} & -2.86929500 & -4.19628200 & 0.08433700 \\ \mathrm{C} & 0.36425800 & -4.31536600 & -0.95763800 \\ \mathrm{H} & 1.97496500 & -3.07640000 & -0.21662600 \\ \mathrm{H} & -1.41128100 & -5.41022900 & -1.52755700 \\ \mathrm{H} & 1.00044100 & -4.84076800 & -1.66463600 \\ \mathrm{H} & -2.31638900 & -0.34151000 & 2.35289600 \\ \mathrm{O} & 1.73491500 & 0.82362500 & -0.68306000 \\ \mathrm{~S} & 2.61727100 & 0.82347700 & 0.56331200 \\ \mathrm{C} & 4.11560700 & -0.02129400 & -0.14304800 \\ \mathrm{~F} & 5.04809200 & -0.14533400 & 0.80928300 \\ \mathrm{~F} & 4.62331700 & 0.68968900 & -1.15527200 \\ \mathrm{~F} & 3.79940400 & -1.24478200 & -0.59410800 \\ \mathrm{O} & 3.07573700 & 2.16093700 & 0.96260800 \\ \mathrm{O} & 2.13676700 & -0.07377000 & 1.62896500\end{array}$

\section{2_AuCl}

$\mathrm{E}(\mathrm{SMD} / \mathrm{B} 3 \mathrm{LYP} / \mathrm{BS} 1)=-1752.67732246 \mathrm{au}$

$\mathrm{H}(\mathrm{SMD} / \mathrm{B} 3 \mathrm{LYP} / \mathrm{BS} 1)=-1752.361978 \mathrm{au}$

$\mathrm{G}(\mathrm{SMD} / \mathrm{B} 3 \mathrm{LYP} / \mathrm{BS} 1)=-1752.455689 \mathrm{au}$

$\mathrm{E}(\mathrm{SMD} / \mathrm{M} 06 / \mathrm{BS} 2 / / \mathrm{SMD} / \mathrm{B} 3 \mathrm{LYP} / \mathrm{BS} 1)=-1752.55017646 \mathrm{au}$

$\begin{array}{lccc}\mathrm{C} & 3.74143500 & 1.78307200 & 0.61347700 \\ \mathrm{H} & 4.15045700 & 0.80800900 & 0.32779400 \\ \mathrm{H} & 4.06358500 & 2.03997100 & 1.62592400 \\ \mathrm{H} & 4.14746100 & 2.52629000 & -0.08629500 \\ \mathrm{C} & 2.22171500 & 1.79424500 & 0.52870100 \\ \mathrm{O} & 1.57330600 & 2.33960300 & 1.42404300 \\ \mathrm{C} & 1.64829300 & 1.15485100 & -0.69020200 \\ \mathrm{C} & 0.42466900 & 1.73129400 & -1.31521000 \\ \mathrm{O} & -0.50653100 & 2.26948000 & -0.71219700 \\ \mathrm{C} & 0.38281700 & 1.66635500 & -2.83628700 \\ \mathrm{H} & -0.63102900 & 1.86750500 & -3.19219300 \\ \mathrm{H} & 1.05928800 & 2.42762900 & -3.24839000 \\ \mathrm{H} & 0.72233400 & 0.69324300 & -3.20678800 \\ \mathrm{C} & -2.19837200 & 1.87448900 & 1.25902400 \\ \mathrm{C} & -1.28339000 & 1.79188900 & 2.38674100 \\ \mathrm{H} & -2.35480000 & 2.86931100 & 0.85086300 \\ \mathrm{H} & -1.47857100 & 2.61403900 & 3.08891900 \\ \mathrm{H} & -1.26017700 & 0.83196600 & 2.90372700 \\ \mathrm{H} & 2.42732200 & 1.01550300 & -1.44545300 \\ \mathrm{Au} & 1.16910300 & -0.84193200 & -0.14274800 \\ \mathrm{Cl} & 0.68139700 & -3.10361100 & 0.43918700 \\ \mathrm{C} & -2.93467900 & 0.83936500 & 0.65111600 \\ \mathrm{C} & -2.87027100 & -0.51456800 & 1.08888400 \\ \mathrm{C} & -3.77180100 & 1.17391100 & -0.45232700 \\ \mathrm{C} & -3.61723800 & -1.48490000 & 0.44344700 \\ \mathrm{H} & -2.23845200 & -0.78582600 & 1.92679400\end{array}$




$\begin{array}{lrrr}\mathrm{C} & -4.51607200 & 0.19539200 & -1.08886100 \\ \mathrm{H} & -3.81326700 & 2.20661200 & -0.78542100 \\ \mathrm{C} & -4.43630700 & -1.13141400 & -0.64103900 \\ \mathrm{H} & -3.56970300 & -2.51777800 & 0.77257200 \\ \mathrm{H} & -5.15467400 & 0.44952800 & -1.92865900 \\ \mathrm{H} & -5.01924000 & -1.90019100 & -1.14061900 \\ \mathrm{H} & -0.27242300 & 2.01169000 & 1.96855500\end{array}$

\section{TS_AuOTf}

$\mathrm{E}(\overline{\mathrm{SMD}} / \mathrm{B} 3 \mathrm{LYP} / \mathrm{BS} 1)=-1752.64087787 \mathrm{au}$

$\mathrm{H}(\mathrm{SMD} / \mathrm{B} 3 \mathrm{LYP} / \mathrm{BS} 1)=-1752.327504 \mathrm{au}$

$\mathrm{G}(\mathrm{SMD} / \mathrm{B} 3 \mathrm{LYP} / \mathrm{BS} 1)=-1752.415259 \mathrm{au}$

$\mathrm{E}(\mathrm{SMD} / \mathrm{M} 06 / \mathrm{BS} 2 / / \mathrm{SMD} / \mathrm{B} 3 \mathrm{LYP} / \mathrm{BS} 1)=-1752.52413771999 \mathrm{au}$

$\begin{array}{llll}\mathrm{C} & -0.89232500 & 2.48456400 & -0.07069700\end{array}$

$\mathrm{H} \quad-1.62315300 \quad 3.29191100 \quad-0.19252500$

$\mathrm{H} \quad-0.05656300 \quad 2.69965000 \quad-0.74786700$

$\mathrm{H} \quad-0.51535400 \quad 2.48163000 \quad 0.95477700$

$\begin{array}{lllll}\mathrm{C} & & -1.52970300 & 1.16335800 & -0.49320900\end{array}$

$\begin{array}{lllll}\mathrm{O} & -2.11896500 & 1.07896900 & -1.56196500\end{array}$

$\begin{array}{llll}\mathrm{C} & -1.43626200 & 0.01254200 & 0.47852600\end{array}$

$\begin{array}{llll}\mathrm{C} & -1.70972000 & -1.43180900 & 0.12534000\end{array}$

$\begin{array}{llll}\mathrm{O} & -2.22887300 & -1.77814300 & -0.92693100\end{array}$

$\begin{array}{llll}\mathrm{C} & -1.37263100 & -2.46627300 & 1.19573900\end{array}$

$\mathrm{H} \quad-0.61162700 \quad-3.14878400 \quad 0.79979800$

$\mathrm{H} \quad-0.99913800 \quad-2.02895400 \quad 2.12581100$

$\mathrm{H} \quad-2.26575700 \quad-3.06520700 \quad 1.40823600$

$\begin{array}{lllll}\mathrm{H} & -0.90809000 & 0.19640900 & 1.41919500\end{array}$

$\begin{array}{lllll}\mathrm{C} & & -4.94149100 & -0.99617900 & 0.38989000\end{array}$

C $\quad \begin{array}{llll}\text { C } & -6.04641900 & -1.08486600 & -0.44976300\end{array}$

$\begin{array}{lllll}\text { C } & & -6.52928100 & 0.06228900 & -1.08742200\end{array}$

$\begin{array}{llll}\text { C } & -5.90874400 & 1.29986200 & -0.87908800\end{array}$

$\begin{array}{lllll}\mathrm{C} & & -4.80721500 & 1.39489000 & -0.03674300\end{array}$

$\begin{array}{lllll}\mathrm{C} & -4.30818700 & 0.24705400 & 0.62121400\end{array}$

$\mathrm{H} \quad-4.57468700 \quad-1.88247200 \quad 0.89819000$

$\mathrm{H} \quad-6.53191700 \quad-2.04334000 \quad-0.60768700$

$\mathrm{H} \quad-7.39074000 \quad-0.00629800 \quad-1.74606400$

$\mathrm{H} \quad-6.28654600 \quad 2.18810500 \quad-1.37671500$

$\begin{array}{llll}\mathrm{H} & -4.33540900 & 2.35911200 & 0.11730000\end{array}$

$\begin{array}{llll}\text { C } & -3.20363600 & 0.30903000 & 1.56439400\end{array}$

$\mathrm{H} \quad-3.06124800 \quad-0.60981400 \quad 2.12836000$

$\begin{array}{llll}\mathrm{C} & -2.95548400 & 1.54232600 & 2.38733500\end{array}$

$\begin{array}{llll}\mathrm{H} & -2.94044500 & 2.46648000 & 1.80709700\end{array}$

$\mathrm{H} \quad-3.79024500 \quad 1.61645800 \quad 3.10003600$

$\mathrm{H} \quad-2.03334300 \quad 1.46287600 \quad 2.96855800$

$\mathrm{Au} \quad 0.75281500 \quad-0.37420100 \quad-0.21397600$

$\begin{array}{llll}\mathrm{O} & 2.71748400 & -0.78469800 & -0.91117300\end{array}$

$\begin{array}{llll}\mathrm{S} & 3.91186100 & -0.68936500 & 0.05580200\end{array}$

$\begin{array}{llll}\mathrm{C} & 4.27843900 & 1.13637600 & 0.01205500\end{array}$

$\begin{array}{llll}\mathrm{F} & 3.21260500 & 1.83557800 & 0.44026400\end{array}$

F $\quad \begin{array}{llll}\text { F } & 5.31506900 & 1.40846200 & 0.81005500\end{array}$

F $\quad \begin{array}{llll}4.56935900 & 1.52465800 & -1.23261200\end{array}$ 


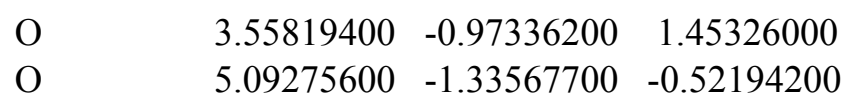

\section{TS_AuCl}

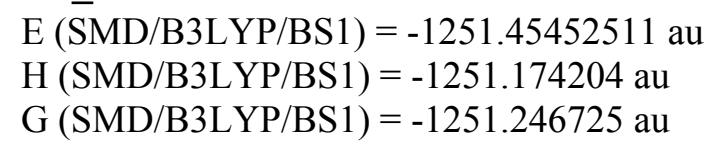

$\mathrm{E}(\mathrm{SMD} / \mathrm{M} 06 / \mathrm{BS} 2 / / \mathrm{SMD} / \mathrm{B} 3 \mathrm{LYP} / \mathrm{BS} 1)=-1251.23000709 \mathrm{au}$

$\begin{array}{llll}\mathrm{C} & -0.02636200 & -2.63295100 & -0.05488000\end{array}$

$\mathrm{H} \quad 0.77529500 \quad-3.35729800 \quad-0.23876700$

$\mathrm{H} \quad \begin{array}{llll}0.86813900 & -2.91005900 & -0.70100400\end{array}$

$\begin{array}{llll}\mathrm{H} & -0.35258100 & -2.70389300 & 0.98560400\end{array}$

C $\quad \begin{array}{llll}0.45543100 & -1.23901200 & -0.45263600\end{array}$

$\begin{array}{lllll}\mathrm{O} & 0.99578700 & -1.06786300 & -1.53942500\end{array}$

$\begin{array}{llll}\text { C } & 0.26744600 & -0.14189400 & 0.55047700\end{array}$

$\begin{array}{lllll}\text { C } & 0.39276800 & 1.32345200 & 0.25685000\end{array}$

$\begin{array}{lllll}\mathrm{O} & 0.83826500 & 1.76930600 & -0.79501600\end{array}$

$\begin{array}{llll}\text { C } & -0.00704300 & 2.27790400 & 1.38108200\end{array}$

$\begin{array}{llll}\mathrm{H} & -0.86329700 & 2.87698900 & 1.05033600\end{array}$

$\mathrm{H} \quad \begin{array}{llll}\mathrm{H} & -0.27796000 & 1.76908000 & 2.31049400\end{array}$

$\begin{array}{llll}\mathrm{H} & 0.81916800 & 2.97229200 & 1.57315900\end{array}$

$\mathrm{H} \quad \begin{array}{llll}0.15245300 & -0.41836900 & 1.51928500\end{array}$

$\begin{array}{llll}\mathrm{C} & 3.65457700 & 1.28919400 & 0.31726500\end{array}$

$\begin{array}{lllll}\mathrm{C} & 4.66777000 & 1.56217900 & -0.59378400\end{array}$

$\begin{array}{llll}\mathrm{C} & 5.25362900 & 0.51667100 & -1.31497900\end{array}$

C $\quad 4.82878800 \quad-0.80372600 \quad-1.11985900$

$\begin{array}{llll}\text { C } & 3.82043400 & -1.08382100 & -0.20679400\end{array}$

$\begin{array}{llll}\mathrm{C} & 3.22084800 & -0.04089800 & 0.54062300\end{array}$

$\begin{array}{llll}\mathrm{H} & 3.20766400 & 2.09548900 & 0.88983700\end{array}$

$\mathrm{H} \quad 5.00114500 \quad 2.58453800 \quad-0.74458700$

$\mathrm{H} \quad \begin{array}{lllll}6.04384700 & 0.72919600 & -2.02977700\end{array}$

$\mathrm{H} \quad 5.28686900 \quad-1.61055500 \quad-1.68408900$

$\mathrm{H} \quad 3.50220300 \quad-2.10978800 \quad-0.06105900$

$\begin{array}{llll}\mathrm{C} & 2.22185200 & -0.28795200 & 1.55303000\end{array}$

$\begin{array}{llll}\mathrm{H} & 1.97576300 & 0.57737800 & 2.16306300\end{array}$

$\begin{array}{llll}\text { C } & 2.13455200 & -1.58705900 & 2.29534000\end{array}$

$\begin{array}{llll}\mathrm{H} & 2.19554600 & -2.47084200 & 1.65708100\end{array}$

$\mathrm{H} \quad 2.99777700 \quad-1.61640300 \quad 2.97824800$

$\mathrm{H} \quad 1.23193200 \quad-1.64030100 \quad 2.90866000$

$\mathrm{Au} \quad-1.99729600 \quad 0.02406500 \quad-0.04107600$

$\mathrm{Cl} \quad-4.27746900 \quad 0.16513900 \quad-0.56272500$
$\mathbf{A}^{\prime}$
$\mathrm{E}(\mathrm{SMD} / \mathrm{B} 3 \mathrm{LYP} / \mathrm{BS} 1)=-1056.43105504 \mathrm{au}$
$\mathrm{H}(\mathrm{SMD} / \mathrm{B} 3 \mathrm{LYP} / \mathrm{BS} 1)=-1056.424105 \mathrm{au}$
$\mathrm{G}(\mathrm{SMD} / \mathrm{B} 3 \mathrm{LYP} / \mathrm{BS} 1)=-1056.457016 \mathrm{au}$
$\mathrm{E}(\mathrm{SMD} / \mathrm{M} 06 / \mathrm{BS} 2 / / \mathrm{SMD} / \mathrm{B} 3 \mathrm{LYP} / \mathrm{BS} 1)=-1056.41407854 \mathrm{au}$
$\mathrm{Au} \quad 0.00000000 \quad 0.00000000 \quad 0.01675500$
$\begin{array}{llll}\mathrm{Cl} & 0.00000000 & 2.34679600 & -0.03893000\end{array}$
$\mathrm{Cl} \quad 0.00000000 \quad-2.34679600 \quad-0.03893000$ 


\begin{tabular}{|c|c|c|c|}
\hline \multicolumn{4}{|c|}{$\mathbf{T S}_{\mathbf{A}^{\prime}-\mathbf{B}^{\prime}}$} \\
\hline \multicolumn{4}{|c|}{$\mathrm{E}(\mathrm{SMD} / \mathrm{B} 3 \mathrm{LYP} / \mathrm{BS} 1)=-1402.15490299 \mathrm{au}$} \\
\hline \multicolumn{4}{|c|}{$\mathrm{H}(\mathrm{SMD} / \mathrm{B} 3 \mathrm{LYP} / \mathrm{BS} 1)=-1402.020412 \mathrm{au}$} \\
\hline \multicolumn{4}{|c|}{$\mathrm{G}(\mathrm{SMD} / \mathrm{B} 3 \mathrm{LYP} / \mathrm{BS} 1)=-1402.082775 \mathrm{au}$} \\
\hline \multicolumn{4}{|c|}{$\mathrm{E}(\mathrm{SMD} / \mathrm{M} 06 / \mathrm{BS} 2 / / \mathrm{SMD} / \mathrm{B} 3 \mathrm{LYP} / \mathrm{BS} 1)=-1402.06344128 \mathrm{au}$} \\
\hline $\mathrm{Au}$ & -0.64190900 & -0.11417700 & 0.02759300 \\
\hline $\mathrm{H}$ & 0.69299800 & -0.88525500 & 0.47265000 \\
\hline $\mathrm{C}$ & 1.59109500 & 0.19082000 & -0.34643700 \\
\hline $\mathrm{H}$ & 1.33973700 & 0.66850000 & -1.29736900 \\
\hline $\mathrm{C}$ & 2.53385300 & -0.97245700 & -0.63378800 \\
\hline $\mathrm{C}$ & 2.27552000 & -1.70856900 & -1.93048000 \\
\hline $\mathrm{H}$ & 1.21563500 & -1.96491500 & -2.03681600 \\
\hline $\mathrm{H}$ & 2.53808300 & -1.05680100 & -2.77430100 \\
\hline $\mathrm{H}$ & 2.88707500 & -2.61301600 & -1.97249100 \\
\hline $\mathrm{O}$ & 3.46410800 & -1.26639400 & 0.10179000 \\
\hline $\mathrm{C}$ & 2.05827000 & 1.24531500 & 0.64199700 \\
\hline $\mathrm{O}$ & 2.17167300 & 2.40116300 & 0.26006200 \\
\hline $\mathrm{C}$ & 2.35867300 & 0.84089400 & 2.06456400 \\
\hline $\mathrm{H}$ & 1.44254400 & 0.48342800 & 2.55165700 \\
\hline $\mathrm{H}$ & 3.07665200 & 0.01493800 & 2.07274900 \\
\hline $\mathrm{H}$ & 2.75285800 & 1.69820100 & 2.61589000 \\
\hline $\mathrm{Cl}$ & -1.89052800 & 1.71487300 & -1.21004900 \\
\hline $\mathrm{Cl}$ & -2.53450000 & -1.36071100 & 1.00524000 \\
\hline \multicolumn{4}{|l|}{ B' } \\
\hline \multicolumn{4}{|c|}{$\mathrm{E}(\mathrm{SMD} / \mathrm{B} 3 \mathrm{LYP} / \mathrm{BS} 1)=-1402.18040851 \mathrm{au}$} \\
\hline \multicolumn{4}{|c|}{$\mathrm{H}(\mathrm{SMD} / \mathrm{B} 3 \mathrm{LYP} / \mathrm{BS} 1)=-1402.043289 \mathrm{au}$} \\
\hline \multicolumn{4}{|c|}{$\mathrm{G}(\mathrm{SMD} / \mathrm{B} 3 \mathrm{LYP} / \mathrm{BS} 1)=-1402.1029 \mathrm{au}$} \\
\hline \multicolumn{4}{|c|}{$\mathrm{E}(\mathrm{SMD} / \mathrm{M} 06 / \mathrm{BS} 2 / / \mathrm{SMD} / \mathrm{B} 3 \mathrm{LYP} / \mathrm{BS} 1)=-1402.08509181 \mathrm{au}$} \\
\hline $\mathrm{Cl}$ & -1.42758500 & -1.59218900 & -1.41250500 \\
\hline $\mathrm{Au}$ & -0.56231400 & 0.16558300 & 0.10987500 \\
\hline $\mathrm{H}$ & 0.03512300 & 1.25818800 & 1.05357400 \\
\hline $\mathrm{C}$ & 1.45775000 & -0.31394400 & -0.37182000 \\
\hline $\mathrm{H}$ & 1.31239300 & -1.03659800 & -1.17544600 \\
\hline $\mathrm{C}$ & 2.05881400 & -0.97622800 & 0.84600300 \\
\hline $\mathrm{C}$ & 1.93251100 & -2.48747500 & 0.89054900 \\
\hline $\mathrm{H}$ & 0.90769800 & -2.80562000 & 0.66923500 \\
\hline $\mathrm{H}$ & 2.57934700 & -2.92957600 & 0.12063900 \\
\hline $\mathrm{H}$ & 2.23749000 & -2.86010500 & 1.87181200 \\
\hline $\mathrm{O}$ & 2.63404300 & -0.36429500 & 1.73804600 \\
\hline $\mathrm{C}$ & 2.10663600 & 0.92087300 & -0.96145000 \\
\hline $\mathrm{O}$ & 2.05984900 & 1.08377100 & -2.17655000 \\
\hline $\mathrm{C}$ & 2.80808400 & 1.93282800 & -0.08107900 \\
\hline $\mathrm{H}$ & 2.15448600 & 2.28544600 & 0.72243400 \\
\hline $\mathrm{H}$ & 3.67715200 & 1.47416500 & 0.40192800 \\
\hline $\mathrm{H}$ & 3.12756000 & 2.77552900 & -0.70002900 \\
\hline $\mathrm{Cl}$ & -2.76902600 & 0.91838900 & 0.82018300 \\
\hline $17^{\prime}$ & & & \\
\hline & $\begin{array}{l}\mathrm{YP} / \mathrm{BS} 1)= \\
\mathrm{YP} / \mathrm{BS} 1)=\end{array}$ & $\begin{array}{l}1826.09761117 \\
1825.942844 \mathrm{a}\end{array}$ & \\
\hline
\end{tabular}




$\begin{array}{lrrr}\mathrm{G}(\mathrm{SMD} / \mathrm{B} 3 \mathrm{LYP} / \mathrm{BS} 1)=-1826.001876 \mathrm{au} \\ \mathrm{E}(\mathrm{SMD} / \mathrm{M} 06 / \mathrm{BS} 2 / / \mathrm{SMD} / \mathrm{B} 3 \mathrm{LYP} / \mathrm{BS} 1)= & -1825.94263658 \mathrm{au} \\ \mathrm{Au} & 1.06834100 & 0.04628200 & 0.13326000 \\ \mathrm{Cl} & 0.20540300 & 2.23846300 & 0.30444800 \\ \mathrm{Cl} & 1.88683700 & -2.17341000 & 0.01129000 \\ \mathrm{C} & -0.38618500 & -0.59686600 & 1.65133600 \\ \mathrm{H} & -0.65722900 & 0.30349900 & 2.19400800 \\ \mathrm{C} & -1.31700900 & -1.17237300 & 0.74768800 \\ \mathrm{H} & -1.06405300 & -2.16180000 & 0.37025100 \\ \mathrm{C} & -2.53188800 & -0.62083400 & 0.25655300 \\ \mathrm{C} & -3.07075400 & 0.61387100 & 0.71435700 \\ \mathrm{C} & -3.23850600 & -1.36144200 & -0.73142200 \\ \mathrm{C} & -4.26271900 & 1.08330600 & 0.18962400 \\ \mathrm{H} & -2.55533800 & 1.18361600 & 1.47868200 \\ \mathrm{C} & -4.42881000 & -0.88009700 & -1.25235300 \\ \mathrm{H} & -2.82771400 & -2.30601800 & -1.07630300 \\ \mathrm{C} & -4.93862500 & 0.34196300 & -0.79321500 \\ \mathrm{H} & -4.67718600 & 2.02370400 & 0.53843700 \\ \mathrm{H} & -4.96251900 & -1.44387800 & -2.01066000 \\ \mathrm{H} & -5.87228500 & 0.72008400 & -1.19986800 \\ \mathrm{H} & 0.23210100 & -1.30026500 & 2.20598500 \\ \mathrm{Cl} & 2.79201200 & 0.81021700 & -1.35829800\end{array}$

\begin{tabular}{|c|c|c|c|}
\hline \multicolumn{4}{|c|}{$\mathbf{T S}_{17^{\prime}-19^{\prime}}$} \\
\hline \multicolumn{4}{|c|}{$\mathrm{E}(\mathrm{SMD} / \mathrm{B} 3 \mathrm{LYP} / \mathrm{BS} 1)=-2171.89448339 \mathrm{au}$} \\
\hline \multicolumn{4}{|c|}{$\mathrm{H}(\mathrm{SMD} / \mathrm{B} 3 \mathrm{LYP} / \mathrm{BS} 1)=-2171.606406 \mathrm{au}$} \\
\hline \multicolumn{4}{|c|}{$\mathrm{G}(\mathrm{SMD} / \mathrm{B} 3 \mathrm{LYP} / \mathrm{BS} 1)=-2171.683789 \mathrm{au}$} \\
\hline \multicolumn{4}{|c|}{$\mathrm{E}(\mathrm{SMD} / \mathrm{M} 06 / \mathrm{BS} 2 / / \mathrm{SMD} / \mathrm{B} 3 \mathrm{LYP} / \mathrm{BS} 1)=-2171.66707095 \mathrm{au}$} \\
\hline $\mathrm{C}$ & -3.32297400 & -2.65345300 & -1.75426800 \\
\hline $\mathrm{H}$ & -2.25919800 & -2.73486900 & -2.00817500 \\
\hline $\mathrm{H}$ & -3.90827900 & -2.42465200 & -2.64718100 \\
\hline $\mathrm{H}$ & -3.63164800 & -3.62901100 & -1.35590800 \\
\hline $\mathrm{C}$ & -3.53884100 & -1.60124500 & -0.70767100 \\
\hline $\mathrm{O}$ & -4.40252500 & -0.71604400 & -0.84832300 \\
\hline $\mathrm{C}$ & -2.69562200 & -1.62631300 & 0.50093800 \\
\hline $\mathrm{C}$ & -3.15677000 & -0.93696200 & 1.64989900 \\
\hline $\mathrm{C}$ & -2.66780000 & -1.20677900 & 3.02641000 \\
\hline $\mathrm{H}$ & -2.49047500 & -0.26766000 & 3.56097600 \\
\hline $\mathrm{H}$ & -3.45605100 & -1.74678800 & 3.56976100 \\
\hline $\mathrm{H}$ & -1.76737700 & -1.82263400 & 3.02292700 \\
\hline $\mathrm{O}$ & -4.04739500 & 0.00402700 & 1.53677300 \\
\hline $\mathrm{H}$ & -4.36712000 & -0.00399000 & 0.56423500 \\
\hline $\mathrm{H}$ & -2.09236500 & -2.51044500 & 0.67255100 \\
\hline $\mathrm{C}$ & -1.03730100 & -0.36311800 & -0.12453600 \\
\hline $\mathrm{C}$ & -1.60314300 & 0.93374900 & -0.47721800 \\
\hline $\mathrm{C}$ & -2.00279600 & 1.15295400 & -1.81529700 \\
\hline $\mathrm{C}$ & -1.75600900 & 1.98167100 & 0.45880700 \\
\hline $\mathrm{C}$ & -2.50388400 & 2.38830700 & -2.21230500 \\
\hline $\mathrm{H}$ & -1.89360800 & 0.35156800 & -2.54079100 \\
\hline $\mathrm{C}$ & -2.26803900 & 3.21119200 & 0.05859700 \\
\hline $\mathrm{H}$ & -1.46868200 & 1.83463400 & 1.49377100 \\
\hline
\end{tabular}




$\begin{array}{lrcc}\mathrm{C} & -2.63846700 & 3.41801100 & -1.27528100 \\ \mathrm{H} & -2.79170600 & 2.54929900 & -3.24683400 \\ \mathrm{H} & -2.37743400 & 4.01148300 & 0.78439200 \\ \mathrm{H} & -3.03407300 & 4.38191700 & -1.58300400 \\ \mathrm{C} & -0.05682400 & -0.55787000 & 0.95626700 \\ \mathrm{H} & 0.05320500 & -1.59731100 & 1.26026600 \\ \mathrm{H} & -0.15454800 & 0.10737100 & 1.81057400 \\ \mathrm{Au} & 1.86808500 & -0.15655800 & 0.12533400 \\ \mathrm{Cl} & 1.91140900 & 1.89876600 & 1.29228600 \\ \mathrm{Cl} & 1.73655000 & -2.26184400 & -0.97024000 \\ \mathrm{H} & -0.90624100 & -1.03858800 & -0.96546900 \\ \mathrm{Cl} & 4.12010400 & 0.23149300 & -0.76364400\end{array}$

\section{$19^{\prime}$}

$\mathrm{E}(\mathrm{SMD} / \mathrm{B} 3 \mathrm{LYP} / \mathrm{BS} 1)=-2171.90377125 \mathrm{au}$ $\mathrm{H}(\mathrm{SMD} / \mathrm{B} 3 \mathrm{LYP} / \mathrm{BS} 1)=-2171.61472599 \mathrm{au}$ $\mathrm{G}(\mathrm{SMD} / \mathrm{B} 3 \mathrm{LYP} / \mathrm{BS} 1)=-2171.692006 \mathrm{au}$ $\mathrm{E}(\mathrm{SMD} / \mathrm{M} 06 / \mathrm{BS} 2 / / \mathrm{SMD} / \mathrm{B} 3 \mathrm{LYP} / \mathrm{BS} 1)=-2171.6805356 \mathrm{au}$

$\begin{array}{llrr}\mathrm{C} & -3.38536400 & -2.65159200 & -1.57027800 \\ \mathrm{H} & -2.33338000 & -2.83906000 & -1.81161300 \\ \mathrm{H} & -3.97243900 & -2.52063000 & -2.48121900 \\ \mathrm{H} & -3.75032800 & -3.53255200 & -1.02378400 \\ \mathrm{C} & -3.53062500 & -1.45909600 & -0.68414700 \\ \mathrm{O} & -4.38732200 & -0.59169000 & -0.88212100 \\ \mathrm{C} & -2.57383900 & -1.31147400 & 0.49645500 \\ \mathrm{C} & -3.20483500 & -0.60648100 & 1.64240000 \\ \mathrm{C} & -2.86033300 & -0.89377300 & 3.04711600 \\ \mathrm{H} & -3.36252300 & -1.83898100 & 3.30975700 \\ \mathrm{H} & -1.78728500 & -1.06818200 & 3.16620200 \\ \mathrm{H} & -3.21633100 & -0.10480800 & 3.71233600 \\ \mathrm{O} & -4.09657500 & 0.27651200 & 1.41890600 \\ \mathrm{H} & -4.34643000 & 0.22903700 & 0.40887000 \\ \mathrm{H} & -2.20869200 & -2.28636200 & 0.82614500 \\ \mathrm{C} & -1.23349700 & -0.52203500 & -0.05261700 \\ \mathrm{C} & -1.52365300 & 0.88361400 & -0.54200600 \\ \mathrm{C} & -1.63021600 & 1.11895000 & -1.92230200 \\ \mathrm{C} & -1.67393700 & 1.96866100 & 0.33699600 \\ \mathrm{C} & -1.87279400 & 2.40330000 & -2.41265400 \\ \mathrm{H} & -1.50960700 & 0.29191000 & -2.61773500 \\ \mathrm{C} & -1.92102500 & 3.25255700 & -0.15132700 \\ \mathrm{H} & -1.58299400 & 1.81864900 & 1.40861500 \\ \mathrm{C} & -2.02070700 & 3.47350700 & -1.52743500 \\ \mathrm{H} & -1.94356700 & 2.56617900 & -3.48453400 \\ \mathrm{H} & -2.02971600 & 4.08002300 & 0.54435600 \\ \mathrm{H} & -2.20907700 & 4.47419900 & -1.90660100 \\ \mathrm{C} & -0.11887600 & -0.64965100 & 0.98054700 \\ \mathrm{H} & -0.01261200 & -1.67748400 & 1.33482900 \\ \mathrm{H} & -0.22434400 & 0.03256300 & 1.82221200 \\ \mathrm{Au} & 1.78072500 & -0.23048400 & 0.16561600 \\ \mathrm{Cl} & 1.98025100 & 1.57900200 & 1.67834300 \\ \mathrm{Cl} & 1.51209000 & -2.12869000 & -1.24005900\end{array}$




$\begin{array}{lrrr}\mathrm{H} & -0.96671100 & -1.13942000 & -0.91257800 \\ \mathrm{Cl} & 4.03402100 & 0.20884400 & -0.76285500\end{array}$

\section{$\mathrm{TS}_{19^{\prime}-17^{\prime}}$ \\ $\mathrm{E}(\mathrm{SMD} / \mathrm{B} 3 \mathrm{LYP} / \mathrm{BS} 1)=-2171.8418495 \mathrm{au}$ \\ $\mathrm{H}(\mathrm{SMD} / \mathrm{B} 3 \mathrm{LYP} / \mathrm{BS} 1)=-2171.558487 \mathrm{au}$ \\ $\mathrm{G}(\mathrm{SMD} / \mathrm{B} 3 \mathrm{LYP} / \mathrm{BS} 1)=-2171.635994 \mathrm{au}$}

$\mathrm{E}(\mathrm{SMD} / \mathrm{M} 06 / \mathrm{BS} 2 / / \mathrm{SMD} / \mathrm{B} 3 \mathrm{LYP} / \mathrm{BS} 1)=-2171.62457496 \mathrm{au}$

$\begin{array}{llll}\mathrm{C} & -1.18660900 & 1.29856200 & -0.03219700\end{array}$

$\begin{array}{llll}\mathrm{C} & -0.91224700 & 2.08011500 & 1.09903800\end{array}$

C $\quad \begin{array}{llll}\text { C } & -1.16569500 & 1.91093500 & -1.29477700\end{array}$

$\begin{array}{llll}\mathrm{C} & -0.62025600 & 3.44152400 & 0.97318000\end{array}$

$\mathrm{H} \quad-0.91984000 \quad 1.62030600 \quad 2.08345500$

C $\quad-0.87420400 \quad 3.26994400 \quad-1.42193100$

$\mathrm{H} \quad-1.37419900 \quad 1.32858000 \quad-2.18902000$

C $\quad-0.60057000 \quad 4.04015600 \quad-0.28772500$

$\mathrm{H} \quad-\quad-4.41077400 \quad 4.03023900 \quad 1.86227500$

$\mathrm{H} \quad-0.86242700 \quad 3.72678600 \quad-2.40784000$

$\mathrm{H} \quad-0.37598500 \quad 5.09860900 \quad-0.38761300$

$\begin{array}{llll}\mathrm{C} & -1.50570300 & -0.18956800 & 0.12424600\end{array}$

$\begin{array}{llll}\mathrm{H} & -1.49761200 & -0.43069700 & 1.19031000\end{array}$

C $\quad-0.48237600 \quad-1.11554500 \quad-0.55782300$

$\mathrm{H} \quad-0.27312400 \quad-0.97446700 \quad-1.61651600$

C $\quad-2.95687300 \quad-0.46992900 \quad-0.41530200$

$\mathrm{H} \quad-3.02554800 \quad-0.12012600 \quad-1.45595100$

$\begin{array}{llll}\mathrm{C} & -4.03914300 & 0.33216200 & 0.34854500\end{array}$

$\begin{array}{llll}\mathrm{C} & -4.06845700 & 0.23965900 & 1.85691100\end{array}$

$\mathrm{H} \quad \begin{array}{llll}\mathrm{H} & -3.81959200 & -0.76533300 & 2.21469600\end{array}$

$\mathrm{H} \quad-3.32433100 \quad 0.92988500 \quad 2.27399900$

$\mathrm{H} \quad-5.05464000 \quad 0.53837100 \quad 2.22025700$

$\begin{array}{llll}\mathrm{C} & -3.33638900 & -1.94608200 & -0.52728300\end{array}$

C $\quad-4.77661600 \quad-2.35059400 \quad-0.51669100$

$\mathrm{H} \quad-5.18839300 \quad-2.20639200 \quad 0.49108400$

$\mathrm{H} \quad-\quad-5.35367400 \quad-1.71197400 \quad-1.19489800$

$\mathrm{H} \quad-4.87596500 \quad-3.39955200 \quad-0.80139200$

$\mathrm{Au} \quad 1.67297100 \quad-0.48242800 \quad 0.00723700$

$\mathrm{Cl} \quad 1.22302900 \quad-0.90903900 \quad 2.30410400$

$\mathrm{Cl} \quad 2.15606800 \quad-0.23361500 \quad-2.29823300$

$\mathrm{H} \quad-1.18430000 \quad-2.12044000 \quad-0.69448200$

$\mathrm{H} \quad 0.05063300 \quad-1.91450100 \quad 0.00677000$

$\begin{array}{llll}\mathrm{O} & -2.46450800 & -2.80942100 & -0.69878600\end{array}$

$\begin{array}{lllll}\mathrm{O} & -4.83776500 & 1.00446100 & -0.27630000\end{array}$

$\begin{array}{llll}\mathrm{Cl} & 3.84996700 & 0.29995800 & 0.55557900\end{array}$

18

$\mathrm{E}(\mathrm{SMD} / \mathrm{B} 3 \mathrm{LYP} / \mathrm{BS} 1)=-2171.90091049 \mathrm{au}$

$\mathrm{H}(\mathrm{SMD} / \mathrm{B} 3 \mathrm{LYP} / \mathrm{BS} 1)=-2171.611626 \mathrm{au}$

$\mathrm{G}(\mathrm{SMD} / \mathrm{B} 3 \mathrm{LYP} / \mathrm{BS} 1)=-2171.689187 \mathrm{au}$

$\mathrm{E}(\mathrm{SMD} / \mathrm{M} 06 / \mathrm{BS} 2 / / \mathrm{SMD} / \mathrm{B} 3 \mathrm{LYP} / \mathrm{BS} 1)=-2171.67813473 \mathrm{au}$

$\begin{array}{llll}\mathrm{C} & 3.42460400 & 2.69347900 & 0.16421300\end{array}$

$\mathrm{H} \quad 4.03858600 \quad 1.79025300 \quad 0.18191500$ 


$\begin{array}{lrrr}\mathrm{H} & 3.55981100 & 3.27935600 & 1.07594200 \\ \mathrm{H} & 3.75582700 & 3.29364000 & -0.69673100 \\ \mathrm{C} & 1.97863200 & 2.38527100 & -0.04430200 \\ \mathrm{O} & 1.09114000 & 3.03000000 & 0.52462200 \\ \mathrm{C} & 1.58688800 & 1.28816700 & -1.03346600 \\ \mathrm{C} & 0.31887100 & 1.62246500 & -1.76074900 \\ \mathrm{C} & 0.04605700 & 1.10241000 & -3.11310400 \\ \mathrm{H} & -1.01949000 & 1.14303200 & -3.34687900 \\ \mathrm{H} & 0.58954300 & 1.75461100 & -3.81645800 \\ \mathrm{H} & 0.44599700 & 0.09293600 & -3.23828500 \\ \mathrm{O} & -0.53956800 & 2.40019500 & -1.23752800 \\ \mathrm{H} & 2.36909400 & 1.17075200 & -1.78966200 \\ \mathrm{C} & 1.45918100 & -0.17823500 & -0.35681000 \\ \mathrm{C} & 2.84118000 & -0.70397800 & 0.03867400 \\ \mathrm{C} & 3.60684700 & -1.37540500 & -0.92711900 \\ \mathrm{C} & 3.35589000 & -0.57655900 & 1.33800800 \\ \mathrm{C} & 4.85901500 & -1.90172500 & -0.60698300 \\ \mathrm{H} & 3.21346800 & -1.49469200 & -1.93421800 \\ \mathrm{C} & 4.60734400 & -1.10772800 & 1.66025900 \\ \mathrm{H} & 2.78238200 & -0.06609800 & 2.10639000 \\ \mathrm{C} & 5.36275200 & -1.76988600 & 0.68991900 \\ \mathrm{H} & 5.43435000 & -2.42343900 & -1.36703700 \\ \mathrm{H} & 4.98837000 & -1.00479600 & 2.67266500 \\ \mathrm{H} & 6.33412000 & -2.18549600 & 0.94358600 \\ \mathrm{C} & 0.45952500 & -0.28550900 & 0.79617900 \\ \mathrm{H} & 0.53407500 & 0.52509900 & 1.52029100 \\ \mathrm{H} & 0.59983100 & -1.23982200 & 1.30807800 \\ \mathrm{Au} & -1.59240600 & -0.40922800 & 0.30601500 \\ \mathrm{Cl} & -1.09907700 & -2.29228600 & -1.04893900 \\ \mathrm{Cl} & -1.97952100 & 1.46100100 & 1.70612600 \\ \mathrm{H} & 1.12061300 & -0.81161600 & -1.17870200 \\ \mathrm{Cl} & -4.01793800 & -0.62996600 & -0.16578600 \\ \mathrm{H} & -0.15870800 & 2.74838800 & -0.33495100\end{array}$

TS $_{18 '-17^{\prime}}$

$\mathrm{E}(\mathrm{SMD} / \mathrm{B} 3 \mathrm{LYP} / \mathrm{BS} 1)=-2171.85631706 \mathrm{au}$

$\mathrm{H}(\mathrm{SMD} / \mathrm{B} 3 \mathrm{LYP} / \mathrm{BS} 1)=-2171.572953 \mathrm{au}$

$\mathrm{G}(\mathrm{SMD} / \mathrm{B} 3 \mathrm{LYP} / \mathrm{BS} 1)=-2171.650348 \mathrm{au}$

$\mathrm{E}(\mathrm{SMD} / \mathrm{M} 06 / \mathrm{BS} 2 / / \mathrm{SMD} / \mathrm{B} 3 \mathrm{LYP} / \mathrm{BS} 1)=-2171.63482855 \mathrm{au}$

$\begin{array}{llll}\mathrm{C} & 4.38155100 & 2.13370200 & 0.54456400\end{array}$

$\mathrm{H} \quad 4.88141600 \quad 1.23328200 \quad 0.16848600$

$\mathrm{H} \quad 4.79504500 \quad 2.41819600 \quad 1.51456000$

$\mathrm{H} \quad 4.56628900 \quad 2.93063700 \quad-0.18782900$

$\begin{array}{llll}\mathrm{C} & 2.90212000 & 1.89155900 & 0.66057000\end{array}$

$\begin{array}{llll}\mathrm{O} & 2.27182200 & 2.04990500 & 1.68871400\end{array}$

$\begin{array}{llll}\text { C } & 2.16846900 & 1.41660300 & -0.63564200\end{array}$

$\begin{array}{llll}\mathrm{C} & 1.08748000 & 2.42767300 & -0.93376200\end{array}$

C $\quad 1.41464500 \quad 3.67757200 \quad-1.67498200$

$\mathrm{H} \quad 0.58304200 \quad 4.38371800 \quad-1.63776000$

$\mathrm{H} \quad 2.32720700 \quad 4.13231400 \quad-1.27222200$

$\mathrm{H} \quad \begin{array}{llll}1.62448200 & 3.41198000 & -2.72057000\end{array}$ 


$\begin{array}{lccc}\mathrm{O} & -0.07515200 & 2.21724200 & -0.54463700 \\ \mathrm{H} & -0.19972400 & 0.95838000 & -0.01877500 \\ \mathrm{H} & 2.88611500 & 1.43264300 & -1.46036900 \\ \mathrm{C} & 1.59203900 & -0.03089900 & -0.50387100 \\ \mathrm{C} & 2.68860600 & -1.04825900 & -0.18785100 \\ \mathrm{C} & 3.31276400 & -1.72181600 & -1.24872600 \\ \mathrm{C} & 3.10452900 & -1.32409000 & 1.12444300 \\ \mathrm{C} & 4.33378400 & -2.64312000 & -1.00778000 \\ \mathrm{H} & 2.99227500 & -1.52606100 & -2.26944800 \\ \mathrm{C} & 4.12311600 & -2.24952900 & 1.36530900 \\ \mathrm{H} & 2.63525700 & -0.82430400 & 1.96656700 \\ \mathrm{C} & 4.74209700 & -2.90984800 & 0.30150600 \\ \mathrm{H} & 4.80359700 & -3.15642000 & -1.84251200 \\ \mathrm{H} & 4.42938800 & -2.45415400 & 2.38767800 \\ \mathrm{H} & 5.53252200 & -3.63087400 & 0.49141300 \\ \mathrm{C} & 0.41795500 & -0.13731500 & 0.50199700 \\ \mathrm{H} & 0.59203300 & 0.32979100 & 1.47311100 \\ \mathrm{H} & 0.22279600 & -1.19894800 & 0.67751700 \\ \mathrm{Au} & -1.85665000 & -0.24564400 & 0.07376200 \\ \mathrm{Cl} & -1.44576100 & -0.82067400 & -2.18245100 \\ \mathrm{Cl} & -2.17606500 & 0.39296300 & 2.32381700 \\ \mathrm{H} & 1.20519300 & -0.28234700 & -1.49641700 \\ \mathrm{Cl} & -4.16581500 & -0.73596500 & -0.17594700\end{array}$

11'

$\mathrm{E}(\mathrm{SMD} / \mathrm{B} 3 \mathrm{LYP} / \mathrm{BS} 1)=-1862.24058067 \mathrm{au}$

$\mathrm{H}(\mathrm{SMD} / \mathrm{B} 3 \mathrm{LYP} / \mathrm{BS} 1)=-1862.096637 \mathrm{au}$

$\mathrm{G}(\mathrm{SMD} / \mathrm{B} 3 \mathrm{LYP} / \mathrm{BS} 1)=-1862.156183 \mathrm{au}$

$\mathrm{E}(\mathrm{SMD} / \mathrm{M} 06 / \mathrm{BS} 2 / / \mathrm{SMD} / \mathrm{B} 3 \mathrm{LYP} / \mathrm{BS} 1)=-1862.14553855 \mathrm{au}$

$\begin{array}{llll}\mathrm{C} & -1.28893400 & -0.66273800 & 2.26242400\end{array}$

$\mathrm{H} \quad-2.04586800 \quad-0.82166000 \quad 3.03240800$

$\mathrm{H} \quad-0.60653700 \quad 0.13372400 \quad 2.58358800$

$\begin{array}{llll}\mathrm{H} & -0.69789000 & -1.57960300 & 2.14483300\end{array}$

C $\quad-1.90859700 \quad-0.30168900 \quad 0.95269700$

$\begin{array}{llll}\mathrm{O} & -1.16016100 & -0.03082500 & -0.07247900\end{array}$

$\begin{array}{llll}\text { C } & -3.30720300 & -0.25162800 & 0.79065500\end{array}$

$\begin{array}{llll}\text { C } & -3.93642700 & 0.09044200 & -0.40073000\end{array}$

$\begin{array}{llll}\mathrm{O} & -3.28054900 & 0.39790200 & -1.50308600\end{array}$

C $\quad-5.41756700 \quad 0.15089900 \quad-0.54698500$

$\mathrm{H} \quad-5.73270200 \quad-0.50149500 \quad-1.37014100$

$\mathrm{H} \quad \begin{array}{rrrr}-5.92385000 & -0.14785800 & 0.37210700\end{array}$

$\mathrm{H} \quad-5.71545000 \quad 1.17323000 \quad-0.81135300$

$\begin{array}{llll}\mathrm{Au} & 0.92111700 & 0.02389700 & -0.07489800\end{array}$

$\mathrm{Cl} \quad 0.96518700 \quad-2.31515800 \quad-0.38205400$

$\mathrm{Cl} \quad 0.79557800 \quad 2.35695400 \quad 0.25235200$

$\mathrm{H} \quad-3.92680500 \quad-0.48744000 \quad 1.64573600$

$\mathrm{H} \quad \begin{array}{llll}-2.30806700 & 0.34081000 & -1.32589600\end{array}$

$\mathrm{Cl} \quad 3.23141000 \quad 0.12962100 \quad-0.22901500$

$\mathrm{TS}_{11^{\prime}-20^{\prime}}$

$\mathrm{E}(\mathrm{SMD} / \mathrm{B} 3 \mathrm{LYP} / \mathrm{BS} 1)=-2171.85984489 \mathrm{au}$ 


$\begin{array}{lrrr}\text { H }(\mathrm{SMD} / \mathrm{B} 3 \mathrm{~L} Y \mathrm{P} / \mathrm{BS} 1)= & -2171.577488 \mathrm{au} \\ \mathrm{G}(\mathrm{SMD} / \mathrm{B} 3 \mathrm{~L} \text { P/BS1 })=-2171.661381 \mathrm{au} & \\ \mathrm{E}(\mathrm{SMD} / \mathrm{M} 06 / \mathrm{BS} 2 / / \mathrm{SMD} / \mathrm{B} 3 \mathrm{LYP} / \mathrm{BS} 1) & -2171.62130079 \mathrm{au} \\ \mathrm{C} & 0.55759000 & 0.99337200 & 2.49306000 \\ \mathrm{H} & 0.08453900 & 1.50710900 & 3.33250400 \\ \mathrm{H} & 1.61168500 & 1.29517800 & 2.44682100 \\ \mathrm{H} & 0.53157600 & -0.08810700 & 2.67138800 \\ \mathrm{C} & -0.12366600 & 1.31703500 & 1.19159500 \\ \mathrm{O} & 0.26013500 & 0.71708600 & 0.09945400 \\ \mathrm{C} & -1.14778900 & 2.26155600 & 1.16578900 \\ \mathrm{C} & -1.76413000 & 2.82365200 & 0.01401700 \\ \mathrm{O} & -1.70614800 & 2.36766000 & -1.16921200 \\ \mathrm{C} & -2.53138800 & 4.11770600 & 0.18071300 \\ \mathrm{H} & -1.99886400 & 4.91654300 & -0.35079300 \\ \mathrm{H} & -3.51924600 & 4.02210500 & -0.28350200 \\ \mathrm{H} & -2.64282200 & 4.40964400 & 1.22776000 \\ \mathrm{Au} & 2.05076400 & -0.29603900 & -0.04814300 \\ \mathrm{Cl} & 0.90713000 & -2.32297100 & 0.39684500 \\ \mathrm{Cl} & 3.12605500 & 1.76095100 & -0.48008600 \\ \mathrm{H} & -1.74637000 & 0.98104400 & -1.38612400 \\ \mathrm{C} & -2.52065500 & -0.87838700 & -0.83592800 \\ \mathrm{C} & -2.00986000 & -0.14612700 & -1.91308300 \\ \mathrm{H} & -1.79000400 & -1.35290000 & -0.18108800 \\ \mathrm{H} & -1.00909400 & -0.42923100 & -2.24427700 \\ \mathrm{H} & -2.69083200 & 0.14483000 & -2.71447400 \\ \mathrm{C} & -3.88856300 & -1.00775100 & -0.42726200 \\ \mathrm{C} & -4.16423100 & -1.69452600 & 0.78016200 \\ \mathrm{C} & -4.96711700 & -0.48202200 & -1.18103300 \\ \mathrm{C} & -5.47246000 & -1.84624400 & 1.22343300 \\ \mathrm{H} & -3.33788100 & -2.10163000 & 1.35644500 \\ \mathrm{C} & -6.27016800 & -0.63293100 & -0.73038000 \\ \mathrm{H} & -4.77345200 & 0.03619500 & -2.11454300 \\ \mathrm{C} & -6.52407200 & -1.31391600 & 0.46973100 \\ \mathrm{H} & -5.67710100 & -2.37448500 & 2.14963300 \\ \mathrm{H} & -7.09520200 & -0.22882300 & -1.30911000 \\ \mathrm{H} & -7.54776100 & -1.43156900 & 0.81424600 \\ \mathrm{H} & -1.40169500 & 2.71029800 & 2.11864600 \\ \mathrm{Cl} & 4.05618600 & -1.46007500 & -0.37305600 \\ & & & \\ & & \end{array}$

\section{0'}

$\mathrm{E}(\mathrm{SMD} / \mathrm{B} 3 \mathrm{LYP} / \mathrm{BS} 1)=-2171.8637281 \mathrm{au}$

$\mathrm{H}(\mathrm{SMD} / \mathrm{B} 3 \mathrm{LYP} / \mathrm{BS} 1)=-2171.576412 \mathrm{au}$

$\mathrm{G}(\mathrm{SMD} / \mathrm{B} 3 \mathrm{LYP} / \mathrm{BS} 1)=-2171.660429 \mathrm{au}$

$\mathrm{E}(\mathrm{SMD} / \mathrm{M} 06 / \mathrm{BS} 2 / / \mathrm{SMD} / \mathrm{B} 3 \mathrm{LYP} / \mathrm{BS} 1)=-2171.62782521 \mathrm{au}$

$\begin{array}{llll}\mathrm{C} & -0.07174900 & 0.02160400 & 2.16775500\end{array}$

$\begin{array}{llll}\mathrm{H} & -0.86284600 & 0.13834200 & 2.91191600\end{array}$

$\begin{array}{llll}\mathrm{H} & 0.84937200 & 0.46358900 & 2.56858900\end{array}$

$\begin{array}{llll}\mathrm{H} & 0.11969800 & -1.04577800 & 2.00755400\end{array}$

$\begin{array}{lllll}\mathrm{C} & -0.43609600 & 0.69181300 & 0.86844300\end{array}$

$\begin{array}{lllll}\mathrm{O} & 0.35914900 & 0.57635600 & -0.16323600\end{array}$

$\mathrm{Au} \quad 2.28033700 \quad-0.15127900 \quad-0.03342800$ 


$\begin{array}{lrrr}\mathrm{Cl} & 1.45642900 & -2.34151300 & -0.41239100 \\ \mathrm{Cl} & 3.02684500 & 2.05202600 & 0.37546400 \\ \mathrm{C} & -1.61601900 & 1.42111400 & 0.77284000 \\ \mathrm{H} & -2.22273400 & 1.46873800 & 1.67122100 \\ \mathrm{C} & -1.98908800 & 2.28619300 & -0.33196500 \\ \mathrm{C} & -3.09499400 & 3.29199200 & -0.04552300 \\ \mathrm{H} & -2.75269500 & 4.02837100 & 0.69298700 \\ \mathrm{H} & -3.37137400 & 3.81225900 & -0.96597100 \\ \mathrm{H} & -3.97822800 & 2.79877000 & 0.37823800 \\ \mathrm{O} & -1.46244900 & 2.24651700 & -1.45373600 \\ \mathrm{C} & -6.83609400 & -0.79793700 & 0.68841100 \\ \mathrm{C} & -6.53484400 & -0.32441000 & -0.59906800 \\ \mathrm{C} & -5.23322300 & -0.37316100 & -1.06644800 \\ \mathrm{C} & -4.19850400 & -0.89618600 & -0.23787100 \\ \mathrm{C} & -4.52787500 & -1.36447800 & 1.06796900 \\ \mathrm{C} & -5.83493100 & -1.31944200 & 1.52098600 \\ \mathrm{H} & -7.86184200 & -0.76017000 & 1.04405300 \\ \mathrm{H} & -7.32472400 & 0.07521400 & -1.22692200 \\ \mathrm{H} & -5.00130900 & -0.00970300 & -2.06121100 \\ \mathrm{H} & -3.73617500 & -1.75951900 & 1.69813100 \\ \mathrm{H} & -6.08524700 & -1.68225600 & 2.51260000 \\ \mathrm{C} & -2.85563400 & -0.99181000 & -0.65955100 \\ \mathrm{H} & -2.16534100 & -1.42997300 & 0.05741900 \\ \mathrm{C} & -2.30389200 & -0.67158500 & -1.97488600 \\ \mathrm{H} & -3.05048600 & -0.49570700 & -2.75070900 \\ \mathrm{H} & -1.68100000 & 0.24569900 & -1.88095700 \\ \mathrm{H} & -1.60438200 & -1.45982500 & -2.28113900 \\ \mathrm{Cl} & 4.49359300 & -0.93770400 & -0.07047100\end{array}$

\begin{tabular}{|c|c|c|c|}
\hline \multicolumn{4}{|c|}{$\mathbf{T S}_{20^{\prime}-21^{\prime}}$} \\
\hline \multicolumn{4}{|c|}{$\mathrm{E}(\mathrm{SMD} / \mathrm{B} 3 \mathrm{LYP} / \mathrm{BS} 1)=-2171.86367681 \mathrm{au}$} \\
\hline \multicolumn{4}{|c|}{$\mathrm{H}(\mathrm{SMD} / \mathrm{B} 3 \mathrm{LYP} / \mathrm{BS} 1)=-2171.577567 \mathrm{au}$} \\
\hline \multicolumn{4}{|c|}{$\mathrm{G}(\mathrm{SMD} / \mathrm{B} 3 \mathrm{LYP} / \mathrm{BS} 1)=-2171.660094 \mathrm{au}$} \\
\hline \multicolumn{4}{|c|}{$\mathrm{E}(\mathrm{SMD} / \mathrm{M} 06 / \mathrm{BS} 2 / / \mathrm{SMD} / \mathrm{B} 3 \mathrm{LYP} / \mathrm{BS} 1)=-2171.62603235 \mathrm{au}$} \\
\hline $\mathrm{C}$ & 0.03621400 & 0.30381900 & 2.23975900 \\
\hline $\mathrm{H}$ & -0.67031400 & 0.59734600 & 3.01970100 \\
\hline $\mathrm{H}$ & 1.03400300 & 0.66221100 & 2.52315900 \\
\hline $\mathrm{H}$ & 0.08370400 & -0.79067700 & 2.18977800 \\
\hline $\mathrm{C}$ & -0.35529500 & 0.87219900 & 0.89936100 \\
\hline $\mathrm{O}$ & 0.33822400 & 0.53689100 & -0.16388300 \\
\hline $\mathrm{Au}$ & 2.25436700 & -0.20080800 & -0.03577400 \\
\hline $\mathrm{Cl}$ & 1.39176500 & -2.38617400 & -0.35439500 \\
\hline $\mathrm{Cl}$ & 3.03663000 & 2.00502900 & 0.28874200 \\
\hline $\mathrm{C}$ & -1.44852200 & 1.71587700 & 0.79789100 \\
\hline $\mathrm{H}$ & -1.98199300 & 1.92357500 & 1.72007600 \\
\hline $\mathrm{C}$ & -1.84918500 & 2.45834900 & -0.38119200 \\
\hline $\mathrm{C}$ & -2.83777300 & 3.59264500 & -0.14691200 \\
\hline $\mathrm{H}$ & -2.38443100 & 4.37042100 & 0.48073300 \\
\hline $\mathrm{H}$ & -3.13277200 & 4.03109800 & -1.10346400 \\
\hline $\mathrm{H}$ & -3.72895700 & 3.23167200 & 0.38151400 \\
\hline $\mathrm{O}$ & -1.43724200 & 2.23175500 & -1.53031000 \\
\hline
\end{tabular}




$\begin{array}{lrrr}\mathrm{C} & -6.85592200 & -0.86739100 & 0.75636100 \\ \mathrm{C} & -6.60988600 & -0.49294200 & -0.57582000 \\ \mathrm{C} & -5.32138000 & -0.53010100 & -1.07555500 \\ \mathrm{C} & -4.24476800 & -0.94388600 & -0.23569000 \\ \mathrm{C} & -4.52093500 & -1.32120400 & 1.11314300 \\ \mathrm{C} & -5.81500600 & -1.28244800 & 1.60031000 \\ \mathrm{H} & -7.87253200 & -0.83685700 & 1.13812900 \\ \mathrm{H} & -7.43293600 & -0.17916300 & -1.20956800 \\ \mathrm{H} & -5.12918700 & -0.24884900 & -2.10484100 \\ \mathrm{H} & -3.69719600 & -1.63573600 & 1.74734900 \\ \mathrm{H} & -6.02587300 & -1.56910800 & 2.62532600 \\ \mathrm{C} & -2.91234300 & -1.00410000 & -0.67900100 \\ \mathrm{H} & -2.18622000 & -1.36074400 & 0.04920800 \\ \mathrm{C} & -2.38026400 & -0.65547000 & -1.98774100 \\ \mathrm{H} & -3.12798400 & -0.50542800 & -2.76751100 \\ \mathrm{H} & -1.80545000 & 0.29532300 & -1.86255900 \\ \mathrm{H} & -1.62519800 & -1.39018700 & -2.29533200 \\ \mathrm{Cl} & 4.45812900 & -1.02781700 & -0.05796400\end{array}$

\section{1'}

$\mathrm{E}(\mathrm{SMD} / \mathrm{B} 3 \mathrm{LYP} / \mathrm{BS} 1)=-2171.90424965 \mathrm{au}$

$\mathrm{H}(\mathrm{SMD} / \mathrm{B} 3 \mathrm{LYP} / \mathrm{BS} 1)=-2171.615464 \mathrm{au}$

$\mathrm{G}(\mathrm{SMD} / \mathrm{B} 3 \mathrm{LYP} / \mathrm{BS} 1)=-2171.692817 \mathrm{au}$

$\mathrm{E}(\mathrm{SMD} / \mathrm{M} 06 / \mathrm{BS} 2 / / \mathrm{SMD} / \mathrm{B} 3 \mathrm{LYP} / \mathrm{BS} 1)=-2171.67642389 \mathrm{au}$

$\begin{array}{llll}\text { C } & -0.16908700 & 0.22646500 & 2.12010200\end{array}$

$\mathrm{H} \quad-0.94050100 \quad-0.30576300 \quad 2.68573000$

$\begin{array}{llll}\mathrm{H} & -0.14387000 & 1.25738600 & 2.50230200\end{array}$

$\mathrm{H} \quad 0.80875200 \quad-0.23520000 \quad 2.27516100$

$\begin{array}{llll}\text { C } & -0.54319300 & 0.29138100 & 0.67950900\end{array}$

$\begin{array}{llll}\mathrm{O} & 0.27773000 & 0.17378300 & -0.25634300\end{array}$

$\mathrm{Au} \quad 2.36188300 \quad-0.06234000 \quad-0.03577900$

$\mathrm{Cl} \quad 2.08309500 \quad-2.37583500 \quad-0.39023400$

$\begin{array}{llll}\mathrm{Cl} & 2.53807700 & 2.26156200 & 0.33449900\end{array}$

$\begin{array}{llll}\text { C } & -1.99873600 & 0.51825700 & 0.34484800\end{array}$

$\begin{array}{llll}\mathrm{H} & -2.43504000 & 1.00807000 & 1.22049700\end{array}$

C $\quad \begin{array}{llll}\text { C } & -2.19585900 & 1.42727200 & -0.89015300\end{array}$

$\begin{array}{llll}\text { C } & -2.96812600 & 2.70122600 & -0.64277700\end{array}$

$\mathrm{H} \quad-2.42292800 \quad 3.32784500 \quad 0.07589700$

$\mathrm{H} \quad-3.10166800 \quad 3.24723100 \quad-1.57934800$

$\mathrm{H} \quad-3.94419100 \quad 2.47790700 \quad-0.19593500$

$\begin{array}{lllll}\mathrm{O} & -1.74402400 & 1.12325400 & -1.99475500\end{array}$

$\begin{array}{lllll}\text { C } & & -7.03943700 & -0.19120100 & 0.28398100\end{array}$

$\begin{array}{lllll}\text { C } & -6.35683300 & -0.20315100 & -0.93460700\end{array}$

$\begin{array}{llll}\text { C } & -4.97791000 & -0.42766200 & -0.96935700\end{array}$

$\begin{array}{llll}\text { C } & -4.25785600 & -0.64381100 & 0.21571500\end{array}$

$\begin{array}{lllll}\text { C } & & -4.95579900 & -0.62980000 & 1.43321400\end{array}$

$\begin{array}{llll}\text { C } & -6.33353600 & -0.40669700 & 1.47041300\end{array}$

$\mathrm{H} \quad-8.11227900 \quad-0.01971300 \quad 0.30861800$

$\mathrm{H} \quad-6.89693100 \quad-0.04079000 \quad-1.86375200$

$\mathrm{H} \quad-4.46765000 \quad-0.43772400 \quad-1.92783700$

$\mathrm{H} \quad \begin{array}{llll}-4.41490100 & -0.80517700 & 2.36086100\end{array}$ 


$\begin{array}{lrrr}\mathrm{H} & -6.85488100 & -0.40753800 & 2.42412900 \\ \mathrm{C} & -2.75065700 & -0.88383800 & 0.21958900 \\ \mathrm{H} & -2.51611800 & -1.40433400 & 1.15618300 \\ \mathrm{C} & -2.25624700 & -1.75881200 & -0.93862400 \\ \mathrm{H} & -2.35873400 & -1.26942600 & -1.90948000 \\ \mathrm{H} & -1.20262300 & -2.02494300 & -0.81065000 \\ \mathrm{H} & -2.83536100 & -2.68855300 & -0.95712300 \\ \mathrm{Cl} & 4.66119000 & -0.31211000 & 0.09802900\end{array}$

$12 '$

$\mathrm{E}(\mathrm{SMD} / \mathrm{B} 3 \mathrm{LYP} / \mathrm{BS} 1)=-1862.23437301 \mathrm{au}$

$\mathrm{H}(\mathrm{SMD} / \mathrm{B} 3 \mathrm{LYP} / \mathrm{BS} 1)=-1862.090187 \mathrm{au}$

$\mathrm{G}(\mathrm{SMD} / \mathrm{B} 3 \mathrm{LYP} / \mathrm{BS} 1)=-1862.153489 \mathrm{au}$

$\mathrm{E}(\mathrm{SMD} / \mathrm{M} 06 / \mathrm{BS} 2 / / \mathrm{SMD} / \mathrm{B} 3 \mathrm{LYP} / \mathrm{BS} 1)=-1862.13862236 \mathrm{au}$

$\begin{array}{llll}\mathrm{C} & -1.66091600 & -0.66552900 & 1.84219200\end{array}$

$\mathrm{H} \quad-2.48091500 \quad-0.13902300 \quad 2.34230600$

$\begin{array}{llll}\mathrm{H} & -0.70042400 & -0.22116300 & 2.11184100\end{array}$

$\mathrm{H} \quad \quad \quad-1.69156500 \quad-1.70918600 \quad 2.18716300$

$\begin{array}{llll}\mathrm{C} & -1.89600400 & -0.66889500 & 0.37675900\end{array}$

$\begin{array}{llll}\mathrm{O} & -1.02320800 & -0.41343500 & -0.47978600\end{array}$

C $\quad-3.26223000 \quad-1.00508300 \quad-0.15391400$

$\mathrm{H} \quad-3.18843200 \quad-1.36675600 \quad-1.18463200$

$\mathrm{H} \quad-3.71217500 \quad-1.79652600 \quad 0.45857900$

C $\quad-4.19133900 \quad 0.22494200 \quad-0.12187400$

$\begin{array}{llll}\mathrm{O} & -3.81010900 & 1.29108500 & 0.32361000\end{array}$

C $\quad-5.57131600 \quad 0.00144700 \quad-0.68354400$

$\mathrm{H} \quad-6.16174100 \quad 0.91590600 \quad-0.59375900$

$\mathrm{H} \quad-5.50024200 \quad-0.29139200 \quad-1.73855500$

$\mathrm{H} \quad-6.06929100 \quad-0.81966000 \quad-0.15365800$

$\mathrm{Au} \quad 1.00509600 \quad 0.02756300 \quad-0.10212400$

$\begin{array}{llll}\mathrm{Cl} & 1.44244100 & -2.28972400 & -0.19422900\end{array}$

$\begin{array}{llll}\mathrm{Cl} & 3.25985600 & 0.48740400 & 0.13776600\end{array}$

$\begin{array}{llll}\mathrm{Cl} & 0.48944300 & 2.32631200 & -0.04175800\end{array}$

\section{$\mathrm{TS}_{12^{\prime}-20^{\prime}}$}

$\mathrm{E}(\mathrm{SMD} / \mathrm{B} 3 \mathrm{LYP} / \mathrm{BS} 1)=-2171.85060944 \mathrm{au}$

$\mathrm{H}(\mathrm{SMD} / \mathrm{B} 3 \mathrm{LYP} / \mathrm{BS} 1)=-2171.568338 \mathrm{au}$

$\mathrm{G}(\mathrm{SMD} / \mathrm{B} 3 \mathrm{LYP} / \mathrm{BS} 1)=-2171.651502 \mathrm{au}$

$\mathrm{E}(\mathrm{SMD} / \mathrm{M} 06 / \mathrm{BS} 2 / / \mathrm{SMD} / \mathrm{B} 3 \mathrm{LYP} / \mathrm{BS} 1)=-2171.61048141 \mathrm{au}$

$\begin{array}{lrrr}\mathrm{C} & 0.03955800 & -0.10831400 & 1.93534200 \\ \mathrm{H} & -0.80485600 & 0.27762800 & 2.52057100 \\ \mathrm{H} & 0.93646300 & -0.09015300 & 2.55732900 \\ \mathrm{H} & -0.19438900 & -1.14472900 & 1.66358300 \\ \mathrm{C} & 0.20568400 & 0.72481600 & 0.69761600 \\ \mathrm{C} & 1.41704300 & 1.43525400 & 0.48639500 \\ \mathrm{H} & 2.24385400 & 0.39317100 & -0.34467400 \\ \mathrm{O} & -0.72137600 & 0.76695200 & -0.19138700 \\ \mathrm{C} & 6.27701700 & -1.81837500 & 1.34516200 \\ \mathrm{C} & 5.14786300 & -1.49581700 & 0.60648600 \\ \mathrm{C} & 5.23191400 & -0.55706700 & -0.45246900 \\ \mathrm{C} & 6.48635300 & 0.03459800 & -0.74169500\end{array}$




$\begin{array}{lccc}\mathrm{C} & 7.61395900 & -0.29554100 & 0.00047600 \\ \mathrm{C} & 7.51016600 & -1.22093000 & 1.04456100 \\ \mathrm{H} & 6.20732600 & -2.53917500 & 2.15439800 \\ \mathrm{H} & 4.19939600 & -1.96938500 & 0.83888700 \\ \mathrm{H} & 6.55573900 & 0.75334400 & -1.55397600 \\ \mathrm{H} & 8.57079200 & 0.16344600 & -0.22928100 \\ \mathrm{H} & 8.39073100 & -1.48221700 & 1.62481100 \\ \mathrm{C} & 4.10346200 & -0.17976100 & -1.25328800 \\ \mathrm{H} & 4.31859800 & 0.53220000 & -2.04964400 \\ \mathrm{C} & 2.76207000 & -0.53900000 & -1.07458300 \\ \mathrm{H} & 2.53327600 & -1.41501100 & -0.46580600 \\ \mathrm{H} & 2.11872700 & -0.42131100 & -1.94913100 \\ \mathrm{H} & 2.08171600 & 1.45328300 & 1.34730200 \\ \mathrm{C} & 1.54035300 & 2.57921400 & -0.44981500 \\ \mathrm{O} & 0.76288800 & 2.79451300 & -1.37257100 \\ \mathrm{C} & 2.73887300 & 3.47838000 & -0.19941800 \\ \mathrm{H} & 2.63519900 & 3.98362700 & 0.76952200 \\ \mathrm{H} & 3.66570000 & 2.89168800 & -0.15572400 \\ \mathrm{H} & 2.81441600 & 4.22737600 & -0.99127000 \\ \mathrm{Au} & -2.52983200 & -0.22437100 & -0.03955700 \\ \mathrm{Cl} & -3.43047100 & 1.67734100 & 1.03327000 \\ \mathrm{Cl} & -1.54692400 & -2.09545000 & -1.09930300 \\ \mathrm{Cl} & -4.62224900 & -1.26453500 & -0.03801600\end{array}$

TS $_{\text {30-35 }}$

$\begin{array}{lccc}\mathrm{E}(\mathrm{SMD} / \mathrm{B} 3 L Y P / \mathrm{BS} 1)=-1763.89465887 \mathrm{au} \\ \mathrm{H}(\mathrm{SMD} / \mathrm{B} 3 \mathrm{LYP} / \mathrm{BS} 1)=-1763.579138 \mathrm{au} \\ \mathrm{G}(\mathrm{SMD} / \mathrm{B} 3 \mathrm{~L} \text { YP/BS1 }=-1763.667653 \mathrm{au} \\ \mathrm{E}(\mathrm{SMD} / \mathrm{M} 06 / \mathrm{BS} 2 / \mathrm{SMD} / \mathrm{B} 3 \mathrm{LYP} / \mathrm{BS} 1)=-1763.81719492 \mathrm{au} \\ \mathrm{C} & 4.28990900 & -1.15578300 & 2.69477000 \\ \mathrm{H} & 3.30257300 & -1.60498000 & 2.85414700 \\ \mathrm{H} & 4.55134200 & -0.51114500 & 3.53652200 \\ \mathrm{H} & 5.01002600 & -1.98222800 & 2.63060600 \\ \mathrm{C} & 4.32259500 & -0.37781500 & 1.41216300 \\ \mathrm{O} & 4.76526800 & 0.78594800 & 1.37973100 \\ \mathrm{C} & 3.81181500 & -1.02355700 & 0.18285000 \\ \mathrm{C} & 4.34901600 & -0.53771400 & -1.04211200 \\ \mathrm{C} & 4.34466500 & -1.33958700 & -2.28843000 \\ \mathrm{H} & 5.08310000 & -2.14731200 & -2.19778300 \\ \mathrm{H} & 3.36737800 & -1.82064900 & -2.41748700 \\ \mathrm{H} & 4.58719600 & -0.72281500 & -3.15620700 \\ \mathrm{O} & 4.82732700 & 0.66958500 & -1.12562900 \\ \mathrm{H} & 4.85083300 & 1.03896900 & -0.17031300 \\ \mathrm{H} & 3.72771200 & -2.10440700 & 0.23512200 \\ \mathrm{C} & 1.85755900 & -0.66415900 & 0.16045200 \\ \mathrm{C} & 1.69917600 & 0.81694200 & 0.04233000 \\ \mathrm{C} & 1.56309800 & 1.58431600 & 1.21169900 \\ \mathrm{C} & 1.65678000 & 1.47184400 & -1.20172500 \\ \mathrm{C} & 1.36227000 & 2.96412700 & 1.14273600 \\ \mathrm{H} & 1.60035600 & 1.09090100 & 2.17985700 \\ \mathrm{C} & 1.46314600 & 2.85116400 & -1.27054900\end{array}$




$\begin{array}{cccc}\mathrm{H} & 1.77288100 & 0.90275200 & -2.11979500 \\ \mathrm{C} & 1.31160500 & 3.60137900 & -0.09932200 \\ \mathrm{H} & 1.24773600 & 3.53877100 & 2.05778300 \\ \mathrm{H} & 1.43115000 & 3.34212400 & -2.23944300 \\ \mathrm{H} & 1.15851800 & 4.67565500 & -0.15617000 \\ \mathrm{C} & 1.20018100 & -1.55421200 & -0.77867700 \\ \mathrm{H} & 1.45730600 & -2.60618000 & -0.62978100 \\ \mathrm{H} & 1.28759200 & -1.27982000 & -1.83256500 \\ \mathrm{H} & 1.76283000 & -0.99374100 & 1.19343500 \\ \mathrm{O} & -3.05464000 & -1.49214900 & 0.07566200 \\ \mathrm{~S} & -3.74217800 & -0.37014400 & 0.82740600 \\ \mathrm{O} & -5.11377500 & -0.71886300 & 1.22549500 \\ \mathrm{O} & -2.88603700 & 0.28684800 & 1.83129800 \\ \mathrm{C} & -3.95297000 & 0.90817600 & -0.50738600 \\ \mathrm{~F} & -4.67726100 & 0.41633900 & -1.52156600 \\ \mathrm{~F} & -4.57466100 & 1.98992600 & -0.02050200 \\ \mathrm{~F} & -2.75636600 & 1.28993400 & -0.99122800 \\ \mathrm{Ag} & -0.89845300 & -1.44002200 & -0.32451100\end{array}$

\section{5}

$\mathrm{E}(\mathrm{SMD} / \mathrm{B} 3 \mathrm{LYP} / \mathrm{BS} 1)=-1763.90159431 \mathrm{au}$

$\mathrm{H}(\mathrm{SMD} / \mathrm{B} 3 \mathrm{LYP} / \mathrm{BS} 1)=-1763.586988 \mathrm{au}$

$\mathrm{G}(\mathrm{SMD} / \mathrm{B} 3 \mathrm{LYP} / \mathrm{BS} 1)=-1763.678012 \mathrm{au}$

$\mathrm{E}(\mathrm{SMD} / \mathrm{M} 06 / \mathrm{BS} 2 / / \mathrm{SMD} / \mathrm{B} 3 \mathrm{LYP} / \mathrm{BS} 1)=-1763.82364307 \mathrm{au}$

$\begin{array}{llll}\mathrm{C} & -3.89190400 & 1.65518900 & -2.05721900\end{array}$

$\mathrm{H} \quad-4.31423500 \quad 0.65339000 \quad-1.92620800$

$\mathrm{H} \quad-3.71335000 \quad 1.86490600 \quad-3.11368200$

$\mathrm{H} \quad \begin{array}{llll}-4.62984900 & 2.36453300 & -1.65770700\end{array}$

C $\quad-2.63067600 \quad 1.80309800 \quad-1.27633200$

$\begin{array}{llll}\mathrm{O} & -1.60424600 & 2.27306600 & -1.78905700\end{array}$

$\begin{array}{lllll}\text { C } & -2.61466900 & 1.39347200 & 0.18920000\end{array}$

$\begin{array}{llll}\text { C } & -1.64003800 & 2.18102600 & 0.99109000\end{array}$

$\begin{array}{llll}\mathrm{C} & -1.81946600 & 2.41224600 & 2.43750900\end{array}$

$\begin{array}{llll}\mathrm{H} & -0.87215800 & 2.66207000 & 2.92022200\end{array}$

$\mathrm{H} \quad-2.50449900 \quad 3.26941700 \quad 2.53710600$

$\mathrm{H} \quad-2.30252900 \quad 1.55742200 \quad 2.91729300$

$\begin{array}{llll}\mathrm{O} & -0.61095500 & 2.68066300 & 0.42996200\end{array}$

$\begin{array}{llll}\mathrm{H} & -3.60201700 & 1.53091600 & 0.63402000\end{array}$

$\begin{array}{llll}\mathrm{C} & -2.25500000 & -0.20103600 & 0.37828100\end{array}$

$\begin{array}{llll}\text { C } & -3.57501600 & -0.98476200 & 0.37338700\end{array}$

$\begin{array}{llll}\text { C } & -4.36419700 & -0.99923800 & 1.53562400\end{array}$

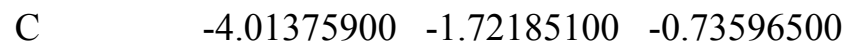

$\begin{array}{llll}\text { C } & -5.55904200 & -1.71819200 & 1.58726600\end{array}$

$\mathrm{H} \quad-4.03409500 \quad-0.44460200 \quad 2.41173900$

C $\quad-5.21212600 \quad-2.44150400 \quad-0.68821200$

$\mathrm{H} \quad \begin{array}{llll}\mathrm{H} & -3.41487400 & -1.74777100 & -1.64070600\end{array}$

$\begin{array}{llll}\text { C } & -5.98975500 & -2.44219000 & 0.47130600\end{array}$

$\mathrm{H} \quad-6.14904400 \quad-1.71889800 \quad 2.50018600$

$\mathrm{H} \quad \quad-5.53219100 \quad-3.00634000 \quad-1.56006100$

$\begin{array}{llll}\mathrm{H} & -6.91807500 & -3.00594100 & 0.50853900\end{array}$

$\begin{array}{llll}\text { C } & -1.17347300 & -0.71702000 & -0.57035500\end{array}$ 


$\begin{array}{lccc}\mathrm{H} & -1.51294100 & -0.66757700 & -1.61234300 \\ \mathrm{H} & -1.04858900 & -1.78358100 & -0.33711200 \\ \mathrm{H} & -1.88117000 & -0.26286100 & 1.40563100 \\ \mathrm{O} & 2.87665400 & 0.77400800 & -0.51806700 \\ \mathrm{~S} & 3.90153500 & 0.34316000 & 0.50983500 \\ \mathrm{O} & 5.13848400 & 1.13547200 & 0.43832300 \\ \mathrm{O} & 3.33733900 & 0.08228900 & 1.84643000 \\ \mathrm{C} & 4.39193200 & -1.33822300 & -0.11776900 \\ \mathrm{~F} & 4.85883900 & -1.25672400 & -1.37126600 \\ \mathrm{~F} & 5.34540600 & -1.86516800 & 0.66198000 \\ \mathrm{~F} & 3.33658100 & -2.17397000 & -0.11652600 \\ \mathrm{H} & -0.72581200 & 2.53683800 & -0.60276200 \\ \mathrm{Ag} & 0.80528400 & 0.03234500 & -0.47823000\end{array}$

\section{6}

$\mathrm{E}(\mathrm{SMD} / \mathrm{B} 3 \mathrm{LYP} / \mathrm{BS} 1)=-1763.89465887 \mathrm{au}$

$\mathrm{H}(\mathrm{SMD} / \mathrm{B} 3 \mathrm{LYP} / \mathrm{BS} 1)=-1763.579138 \mathrm{au}$

$\mathrm{G}(\mathrm{SMD} / \mathrm{B} 3 \mathrm{LYP} / \mathrm{BS} 1)=-1763.667653 \mathrm{au}$

$\mathrm{E}(\mathrm{SMD} / \mathrm{M} 06 / \mathrm{BS} 2 / / \mathrm{SMD} / \mathrm{B} 3 \mathrm{LYP} / \mathrm{BS} 1)=-1763.81999492 \mathrm{au}$

$\begin{array}{llrr}\mathrm{C} & -3.89190400 & 1.65518900 & -2.05721900 \\ \mathrm{H} & -4.31423500 & 0.65339000 & -1.92620800 \\ \mathrm{H} & -3.71335000 & 1.86490600 & -3.11368200 \\ \mathrm{H} & -4.62984900 & 2.36453300 & -1.65770700 \\ \mathrm{C} & -2.63067600 & 1.80309800 & -1.27633200 \\ \mathrm{O} & -1.60424600 & 2.27306600 & -1.78905700 \\ \mathrm{C} & -2.61466900 & 1.39347200 & 0.18920000 \\ \mathrm{C} & -1.64003800 & 2.18102600 & 0.99109000 \\ \mathrm{C} & -1.81946600 & 2.41224600 & 2.43750900 \\ \mathrm{H} & -0.87215800 & 2.66207000 & 2.92022200 \\ \mathrm{H} & -2.50449900 & 3.26941700 & 2.53710600 \\ \mathrm{H} & -2.30252900 & 1.55742200 & 2.91729300 \\ \mathrm{O} & -0.61095500 & 2.68066300 & 0.42996200 \\ \mathrm{H} & -3.60201700 & 1.53091600 & 0.63402000 \\ \mathrm{C} & -2.25500000 & -0.20103600 & 0.37828100 \\ \mathrm{C} & -3.57501600 & -0.98476200 & 0.37338700 \\ \mathrm{C} & -4.36419700 & -0.99923800 & 1.53562400 \\ \mathrm{C} & -4.01375900 & -1.72185100 & -0.73596500 \\ \mathrm{C} & -5.55904200 & -1.71819200 & 1.58726600 \\ \mathrm{H} & -4.03409500 & -0.44460200 & 2.41173900 \\ \mathrm{C} & -5.21212600 & -2.44150400 & -0.68821200 \\ \mathrm{H} & -3.41487400 & -1.74777100 & -1.64070600 \\ \mathrm{C} & -5.98975500 & -2.44219000 & 0.47130600 \\ \mathrm{H} & -6.14904400 & -1.71889800 & 2.50018600 \\ \mathrm{H} & -5.53219100 & -3.00634000 & -1.56006100 \\ \mathrm{H} & -6.91807500 & -3.00594100 & 0.50853900 \\ \mathrm{C} & -1.17347300 & -0.71702000 & -0.57035500 \\ \mathrm{H} & -1.51294100 & -0.66757700 & -1.61234300 \\ \mathrm{H} & -1.04858900 & -1.78358100 & -0.33711200 \\ \mathrm{H} & -1.88117000 & -0.26286100 & 1.40563100 \\ \mathrm{O} & 2.87665400 & 0.77400800 & -0.51806700 \\ \mathrm{~S} & 3.90153500 & 0.34316000 & 0.50983500\end{array}$




$\begin{array}{lccc}\mathrm{O} & 5.13848400 & 1.13547200 & 0.43832300 \\ \mathrm{O} & 3.33733900 & 0.08228900 & 1.84643000 \\ \mathrm{C} & 4.39193200 & -1.33822300 & -0.11776900 \\ \mathrm{~F} & 4.85883900 & -1.25672400 & -1.37126600 \\ \mathrm{~F} & 5.34540600 & -1.86516800 & 0.66198000 \\ \mathrm{~F} & 3.33658100 & -2.17397000 & -0.11652600 \\ \mathrm{H} & -0.72581200 & 2.53683800 & -0.60276200 \\ \mathrm{Ag} & 0.80528400 & 0.03234500 & -0.47823000\end{array}$

TS $_{\text {35-30 }}$

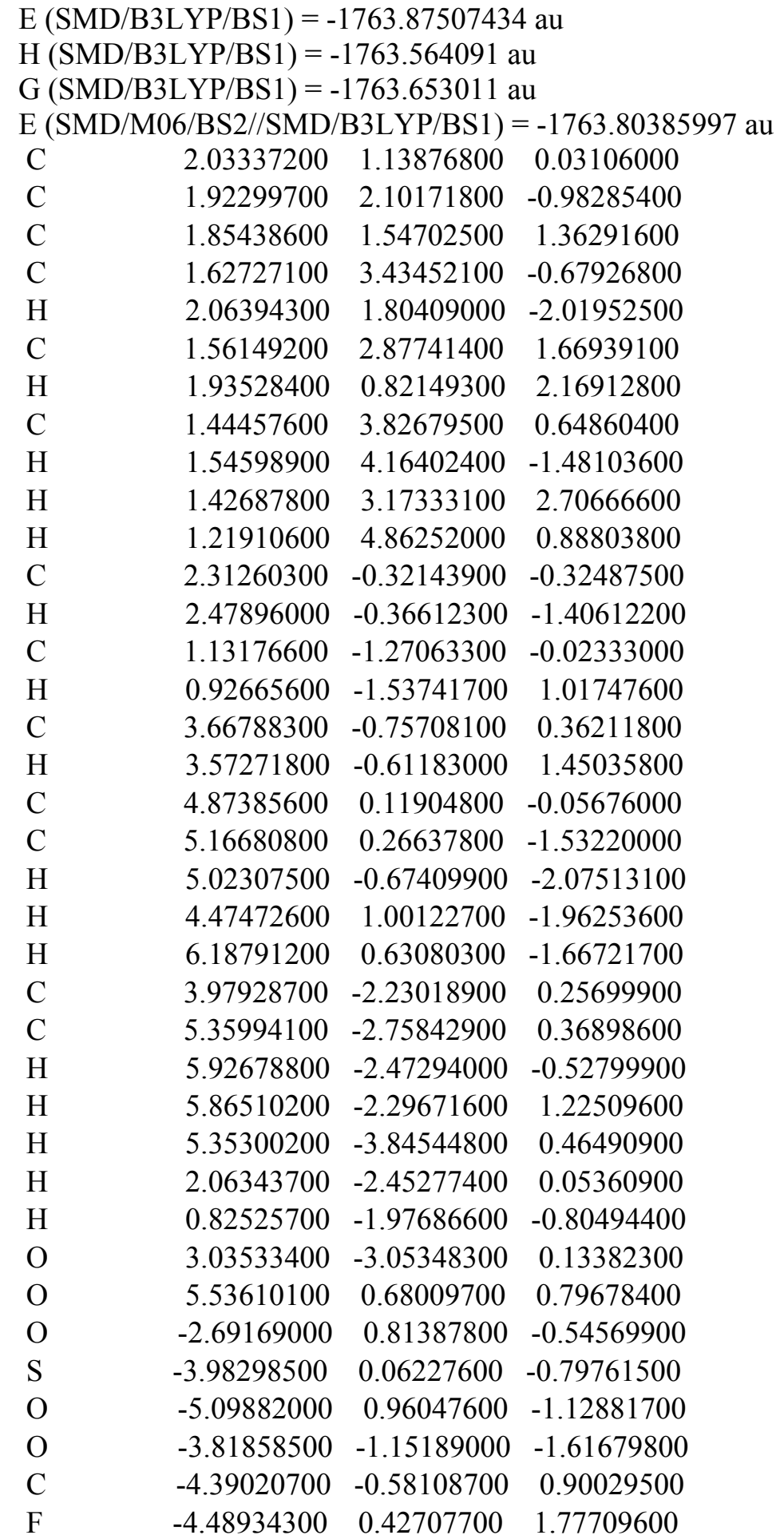




$\begin{array}{llll}\text { F } & -5.55204500 & -1.24690100 & 0.87780400 \\ \text { F } & -3.43076600 & -1.42097800 & 1.33287300 \\ \mathrm{Ag} & -0.78482100 & -0.24259500 & -0.30382400\end{array}$

TS $_{\mathbf{3 0 - 3 6}}$
$\mathrm{E}(\mathrm{SMD} / \mathrm{B} 3 \mathrm{LYP} / \mathrm{BS} 1)=-1763.89448342 \mathrm{au}$
$\mathrm{H}(\mathrm{SMD} / \mathrm{B} 3 \mathrm{LYP} / \mathrm{BS} 1)=-1763.580148 \mathrm{au}$
$\mathrm{G}(\mathrm{SMD} / \mathrm{B} 3 \mathrm{LYP} / \mathrm{BS} 1)=-1763.668703 \mathrm{au}$

$\mathrm{E}(\mathrm{SMD} / \mathrm{M} 06 / \mathrm{BS} 2 / / \mathrm{SMD} / \mathrm{B} 3 \mathrm{LYP} / \mathrm{BS} 1)=-1763.81133185 \mathrm{au}$

$\begin{array}{llll}\mathrm{C} & 3.30194000 & 0.54086200 & 2.45615300\end{array}$

$\mathrm{H} \quad 4.06660600 \quad-0.05760800 \quad 1.95493800$

$\mathrm{H} \quad 2.76676300 \quad-0.05611200 \quad 3.19848200$

$\begin{array}{llll}\mathrm{H} & 3.81795800 & 1.36217600 & 2.97444400\end{array}$

$\begin{array}{llll}\text { C } & 2.33974200 & 1.15272700 & 1.48415500\end{array}$

$\begin{array}{llll}\mathrm{O} & 1.13665600 & 1.30282900 & 1.77659700\end{array}$

$\begin{array}{llll}\text { C } & 2.83985100 & 1.61888000 & 0.16820500\end{array}$

$\begin{array}{llll}\mathrm{C} & 2.16419700 & 2.76185100 & -0.38302400\end{array}$

C $\quad 2.80604000 \quad 3.68101500 \quad-1.35148000$

$\mathrm{H} \quad 2.06655300 \quad 4.31739100 \quad-1.84167900$

$\mathrm{H} \quad 3.52627800 \quad 4.31175100 \quad-0.81214900$

$\mathrm{H} \quad 3.37614800 \quad 3.11018200 \quad-2.09346900$

$\begin{array}{llll}\mathrm{O} & 0.92984500 & 2.99752700 & -0.07308600\end{array}$

$\mathrm{H} \quad 3.92177800 \quad 1.68049600 \quad 0.09234600$

$\begin{array}{llll}\mathrm{C} & 2.53527400 & 0.25507800 & -1.14656100\end{array}$

C $\quad 3.19878000 \quad-0.97427000 \quad-0.60501000$

$\begin{array}{llll}\mathrm{C} & 4.56753800 & -1.17356500 & -0.85550900\end{array}$

$\begin{array}{llll}\mathrm{C} & 2.50041800 & -1.94884000 & 0.12806100\end{array}$

$\begin{array}{llll}\mathrm{C} & 5.21837200 & -2.32122500 & -0.40125800\end{array}$

$\mathrm{H} \quad 5.11995800 \quad-0.42513400 \quad-1.41914400$

$\begin{array}{llll}\mathrm{C} & 3.15008000 & -3.09781300 & 0.58084400\end{array}$

$\mathrm{H} \quad 1.44537900 \quad-1.80503700 \quad 0.34716900$

$\begin{array}{llll}\mathrm{C} & 4.51029500 & -3.28767800 & 0.31913900\end{array}$

$\mathrm{H} \quad 6.27522000 \quad-2.46172300 \quad-0.61139400$

$\begin{array}{llll}\mathrm{H} & 2.59458200 & -3.84323500 & 1.14389000\end{array}$

$\mathrm{H} \quad 5.01539800 \quad-4.18084100 \quad 0.67689100$

$\begin{array}{llll}\mathrm{C} & 1.13413400 & 0.16010700 & -1.60023500\end{array}$

$\mathrm{H} \quad 0.98242100 \quad-0.82106800 \quad-2.06785000$

$\mathrm{H} \quad 0.89882700 \quad 0.94976400 \quad-2.32033900$

$\mathrm{H} \quad 3.20030500 \quad 0.76191000 \quad-1.84693400$

$\begin{array}{llll}\mathrm{O} & -2.25716900 & -0.27791600 & 1.08263400\end{array}$

S $\quad \begin{array}{llll}\text { S } & -3.64463300 & 0.22427200 & 0.74541900\end{array}$

$\begin{array}{llll}\text { O } & -4.60828900 & -0.01845000 & 1.82946100\end{array}$

$\begin{array}{llll}\mathrm{O} & -3.66425900 & 1.55047600 & 0.10265200\end{array}$

C $\quad-4.16275300 \quad-0.94662300 \quad-0.60535100$

F $\quad-4.09387000 \quad-2.21666500 \quad-0.18255100$

F $\quad-5.42016700 \quad-0.68887500 \quad-0.98736400$

F $\quad \begin{array}{llll}-3.36120600 & -0.81947300 & -1.68060100\end{array}$

$\mathrm{H} \quad 0.67465000 \quad 2.33877500 \quad 0.66754000$

Ag $\quad-0.52075000 \quad 0.03821900 \quad-0.23807700$

$\mathbf{T S}_{36-30}$ 


$\begin{array}{cccc}\mathrm{H} & 5.58417200 & -0.10848000 & -0.17459900 \\ \mathrm{H} & 5.90321600 & -1.10586200 & -1.63575000 \\ \mathrm{H} & 5.85606000 & -1.84215400 & -0.00427400 \\ \mathrm{C} & 3.94612800 & -1.35278400 & -0.80312200 \\ \mathrm{O} & 3.42290700 & -1.71000200 & -1.83917400 \\ \mathrm{C} & 3.08343900 & -1.17670700 & 0.50047900 \\ \mathrm{C} & 2.34226700 & -2.45287100 & 0.68060200 \\ \mathrm{C} & 2.89526200 & -3.56936400 & 1.47695100 \\ \mathrm{H} & 2.33146800 & -4.49086200 & 1.32198100 \\ \mathrm{H} & 3.95670900 & -3.70986300 & 1.24346200 \\ \mathrm{H} & 2.84345200 & -3.27891000 & 2.53700700 \\ \mathrm{O} & 1.21930400 & -2.61466300 & 0.11735600 \\ \mathrm{H} & 0.83723500 & -1.67441300 & -0.28501900 \\ \mathrm{H} & 3.77176300 & -1.06114400 & 1.34077400 \\ \mathrm{C} & 2.13878500 & 0.08852400 & 0.42740400 \\ \mathrm{C} & 3.00394600 & 1.35124700 & 0.37077500 \\ \mathrm{C} & 3.46748100 & 1.92049200 & 1.56726500 \\ \mathrm{C} & 3.36499400 & 1.96024000 & -0.84072700 \\ \mathrm{C} & 4.27041900 & 3.06323100 & 1.55734800 \\ \mathrm{H} & 3.19082600 & 1.46488100 & 2.51592600 \\ \mathrm{C} & 4.16905300 & 3.10355900 & -0.85400600 \\ \mathrm{H} & 3.01292400 & 1.54717100 & -1.78142100 \\ \mathrm{C} & 4.62553400 & 3.65888500 & 0.34391400 \\ \mathrm{H} & 4.61261200 & 3.49043800 & 2.49647300 \\ \mathrm{H} & 4.43516500 & 3.56129600 & -1.80340800 \\ \mathrm{H} & 5.24707200 & 4.55033600 & 0.33250400 \\ \mathrm{C} & 1.05758700 & 0.01543200 & -0.67946800 \\ \mathrm{H} & 1.49596600 & -0.22862900 & -1.65100000 \\ \mathrm{H} & 0.66057500 & 1.03546800 & -0.78686500 \\ \mathrm{H} & 1.64243000 & 0.10326000 & 1.40461000 \\ \mathrm{O} & -3.16689500 & -1.04675900 & -0.42296100 \\ \mathrm{~S} & -4.07314800 & -0.35391300 & 0.57713000 \\ \mathrm{O} & -5.40300000 & -0.97497300 & 0.65271400 \\ \mathrm{O} & -3.40465300 & -0.01024200 & 1.84490900 \\ \mathrm{C} & -4.36471500 & 1.28656500 & -0.25045600 \\ \mathrm{~F} & -4.91624800 & 1.11447100 & -1.45880900 \\ \mathrm{~F} & -5.18268500 & 2.04275300 & 0.49235200 \\ \mathrm{~F} & -3.20286200 & 1.94787400 & -0.40777200 \\ \mathrm{Ag} & -1.02851200 & -0.60856100 & -0.46367000\end{array}$

$\mathrm{TS}_{\text {37-38 }}$

$\mathrm{E}(\mathrm{SMD} / \mathrm{B} 3 \mathrm{LYP} / \mathrm{BS} 1)=-1763.90087163 \mathrm{au}$

$\mathrm{H}(\mathrm{SMD} / \mathrm{B} 3 \mathrm{LYP} / \mathrm{BS} 1)=-1763.590368 \mathrm{au}$

$\mathrm{G}(\mathrm{SMD} / \mathrm{B} 3 \mathrm{LYP} / \mathrm{BS} 1)=-1763.684358 \mathrm{au}$

$\mathrm{E}(\mathrm{SMD} / \mathrm{M} 06 / \mathrm{BS} 2 / / \mathrm{SMD} / \mathrm{B} 3 \mathrm{LYP} / \mathrm{BS} 1)=-1763.80706425 \mathrm{au}$

$\begin{array}{lrrr}\mathrm{C} & 0.55972300 & -1.43398300 & 2.25140200 \\ \mathrm{H} & 1.25201700 & -1.49429200 & 3.09506800 \\ \mathrm{H} & -0.23637600 & -2.17705000 & 2.39306400 \\ \mathrm{H} & 0.08508700 & -0.44409900 & 2.24988500 \\ \mathrm{C} & 1.23294500 & -1.67007300 & 0.91758000 \\ \mathrm{O} & 0.52385900 & -1.58468200 & -0.14268100\end{array}$




$\begin{array}{lccc}\mathrm{C} & 2.60298400 & -2.01601500 & 0.90719100 \\ \mathrm{C} & 3.37459000 & -2.38057000 & -0.22080800 \\ \mathrm{O} & 3.04239700 & -2.18375500 & -1.43550300 \\ \mathrm{C} & 4.71741700 & -3.04794300 & 0.00314900 \\ \mathrm{H} & 4.72647700 & -4.02088900 & -0.50332900 \\ \mathrm{H} & 5.50929700 & -2.44106100 & -0.45290300 \\ \mathrm{H} & 4.94337900 & -3.19899900 & 1.06201500 \\ \mathrm{H} & 2.53419400 & -0.82618000 & -1.58556000 \\ \mathrm{C} & 2.68782300 & 1.01432100 & -0.62120200 \\ \mathrm{C} & 2.35736100 & 0.37257800 & -1.83211000 \\ \mathrm{H} & 1.89925000 & 1.09634600 & 0.12528900 \\ \mathrm{H} & 1.29428800 & 0.38465600 & -2.07893000 \\ \mathrm{H} & 3.01547100 & 0.54004900 & -2.68698100 \\ \mathrm{C} & 3.96030500 & 1.53473100 & -0.22033300 \\ \mathrm{C} & 4.07453900 & 2.09895500 & 1.07476400 \\ \mathrm{C} & 5.10024200 & 1.51512000 & -1.06325000 \\ \mathrm{C} & 5.28270000 & 2.62621900 & 1.51213700 \\ \mathrm{H} & 3.20187700 & 2.11321700 & 1.72190200 \\ \mathrm{C} & 6.30590800 & 2.03561400 & -0.61614700 \\ \mathrm{H} & 5.03200000 & 1.09240500 & -2.05999100 \\ \mathrm{C} & 6.39874000 & 2.59234000 & 0.66810700 \\ \mathrm{H} & 5.36179000 & 3.05902000 & 2.50461700 \\ \mathrm{H} & 7.17779700 & 2.01708600 & -1.26292100 \\ \mathrm{H} & 7.34528600 & 3.00230400 & 1.00899800 \\ \mathrm{H} & 3.07468900 & -2.13351400 & 1.87646500 \\ \mathrm{O} & -3.64842900 & -0.77989200 & -0.60641600 \\ \mathrm{~S} & -4.43811400 & 0.14287000 & 0.30725300 \\ \mathrm{O} & -5.87807900 & 0.10565500 & 0.01974700 \\ \mathrm{O} & -4.01566000 & 0.08525000 & 1.71711600 \\ \mathrm{C} & -3.88034700 & 1.81957100 & -0.27562500 \\ \mathrm{~F} & -4.14411200 & 1.97792600 & -1.57855600 \\ \mathrm{~F} & -4.50742700 & 2.78115900 & 0.41217500 \\ \mathrm{~F} & -2.55376500 & 1.96400700 & -0.09319100 \\ \mathrm{Ag} & -1.55747300 & -1.16741600 & -0.21944300 \\ & & & \\ & & & \\ \mathrm{H} & & & \end{array}$

\section{8}

$\mathrm{E}(\mathrm{SMD} / \mathrm{B} 3 \mathrm{LYP} / \mathrm{BS} 1)=-1763.90087163 \mathrm{au}$

$\mathrm{H}(\mathrm{SMD} / \mathrm{B} 3 \mathrm{LYP} / \mathrm{BS} 1)=-1763.590368 \mathrm{au}$

$\mathrm{G}(\mathrm{SMD} / \mathrm{B} 3 \mathrm{LYP} / \mathrm{BS} 1)=-1763.684358 \mathrm{au}$

$\mathrm{E}(\mathrm{SMD} / \mathrm{M} 06 / \mathrm{BS} 2 / / \mathrm{SMD} / \mathrm{B} 3 \mathrm{LYP} / \mathrm{BS} 1)=-1763.80706425 \mathrm{au}$

$\begin{array}{llll}\mathrm{C} & 1.36627100 & -1.32626700 & -1.57130800\end{array}$

$\mathrm{H} \quad 2.30403600 \quad-1.66594900 \quad-2.01808800$

$\mathrm{H} \quad 0.66320000 \quad-1.07748400 \quad-2.37615000$

$\mathrm{H} \quad 0.92300800 \quad-2.14166200 \quad-0.98883600$

C $\quad 1.54575900 \quad-0.11990900 \quad-0.69020300$

$\begin{array}{llll}\mathrm{O} & 0.55923100 & 0.31180600 & -0.02510900\end{array}$

C $\quad 2.82552900 \quad 0.54682800 \quad-0.64589000$

$\mathrm{H} \quad 3.50873900 \quad 0.23452100 \quad-1.42691200$

$\begin{array}{llll}\mathrm{C} & 2.92806300 & 1.97254700 & -0.28018200\end{array}$

C $\quad 4.05218200 \quad 2.74865700 \quad-0.93011500$

$\mathrm{H} \quad 3.99213400 \quad 2.67478900 \quad-2.02257200$ 


$\begin{array}{lrrr}\mathrm{H} & 4.00317700 & 3.79742600 & -0.62883600 \\ \mathrm{H} & 5.02419900 & 2.33252400 & -0.63493100 \\ \mathrm{O} & 2.15481100 & 2.51451200 & 0.51465700 \\ \mathrm{C} & 7.81359594 & -1.17053666 & 0.32790225 \\ \mathrm{C} & 7.37811594 & -0.25216666 & 1.28842725 \\ \mathrm{C} & 6.01933394 & -0.11627866 & 1.56477725 \\ \mathrm{C} & 5.06492594 & -0.89676466 & 0.87635525 \\ \mathrm{C} & 5.52448994 & -1.82371166 & -0.08481375 \\ \mathrm{C} & 6.88210294 & -1.95632266 & -0.35863775 \\ \mathrm{H} & 8.87423294 & -1.27845366 & 0.11921625 \\ \mathrm{H} & 8.09966194 & 0.35735634 & 1.82500625 \\ \mathrm{H} & 5.69698294 & 0.59933434 & 2.31426925 \\ \mathrm{H} & 4.80237494 & -2.44294066 & -0.61100075 \\ \mathrm{H} & 7.21557194 & -2.67858166 & -1.09836975 \\ \mathrm{C} & 3.62569094 & -0.82698866 & 1.16039025 \\ \mathrm{H} & 3.10383294 & -1.70803366 & 0.79111225 \\ \mathrm{C} & 3.09429194 & -0.31783266 & 2.47206725 \\ \mathrm{H} & 3.86203394 & 0.10462634 & 3.12173125 \\ \mathrm{H} & 2.39972494 & 0.46088234 & 2.17960425 \\ \mathrm{H} & 2.55154794 & -1.08970866 & 3.02974825 \\ \mathrm{O} & -3.46177000 & -1.18478200 & -0.23767200 \\ \mathrm{~S} & -4.52543900 & -0.58035300 & 0.67213900 \\ \mathrm{O} & -5.59705100 & -1.52842200 & 1.00055000 \\ \mathrm{O} & -3.95929000 & 0.21985700 & 1.77230900 \\ \mathrm{C} & -5.31065800 & 0.67139500 & -0.45880000 \\ \mathrm{~F} & -5.81975500 & 0.07634000 & -1.54312100 \\ \mathrm{~F} & -6.29553800 & 1.31868700 & 0.17516500 \\ \mathrm{~F} & -4.39935100 & 1.57218000 & -0.86277700 \\ \mathrm{Ag} & -1.44659500 & -0.44008200 & -0.09426900\end{array}$

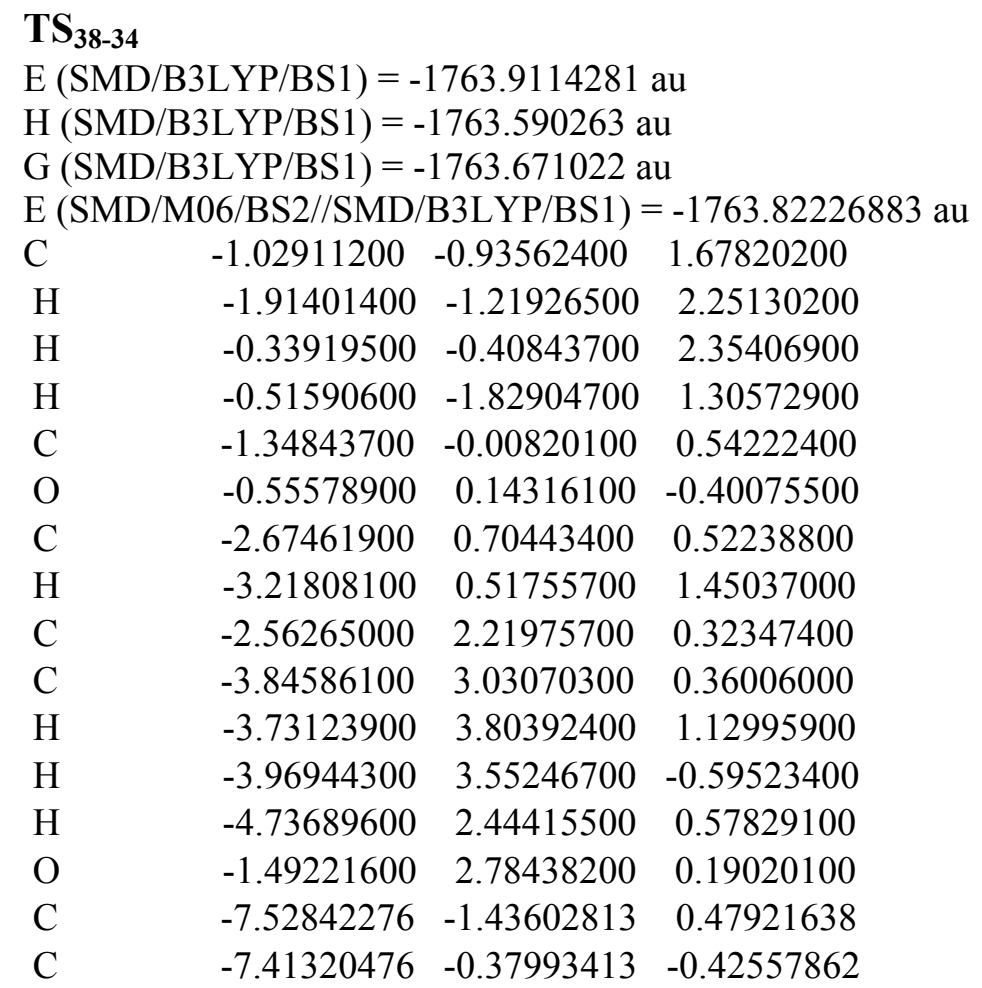




$\begin{array}{lrrr}\text { C } & -6.15226776 & 0.06538487 & -0.83727462 \\ \text { C } & -4.98294376 & -0.53383113 & -0.34687162 \\ \text { C } & -5.11595276 & -1.60398213 & 0.55573238 \\ \text { C } & -6.37082376 & -2.04854913 & 0.97001738 \\ \text { H } & -8.50855376 & -1.78315813 & 0.79529038 \\ \text { H } & -8.30508676 & 0.09990387 & -0.82027262 \\ \text { H } & -6.08908676 & 0.88549087 & -1.54506962 \\ \text { H } & -4.22179676 & -2.09472413 & 0.93469838 \\ \text { H } & -6.44520976 & -2.87857313 & 1.66782638 \\ \text { C } & -3.59124976 & -0.12842313 & -0.80344062 \\ \text { H } & -3.01909476 & -1.05181213 & -0.94726762 \\ \text { C } & -3.51036976 & 0.67045687 & -2.10527662 \\ \text { H } & -4.06783276 & 1.61202887 & -2.06735762 \\ \text { H } & -2.46745076 & 0.89914087 & -2.34373662 \\ \text { H } & -3.92526576 & 0.08064487 & -2.93101162 \\ \text { O } & 3.51970000 & -1.34838400 & -0.43783600 \\ \text { S } & 4.71190900 & -0.42949300 & -0.64927500 \\ \text { O } & 5.97802800 & -1.17308600 & -0.68840300 \\ \text { O } & 4.48957700 & 0.60652400 & -1.67140100 \\ \text { C } & 4.76022300 & 0.50570100 & 0.95890400 \\ \text { F } & 4.82188100 & -0.34078600 & 1.99476700 \\ \text { F } & 5.82778300 & 1.31130300 & 0.99503600 \\ \text { F } & 3.65360600 & 1.26076600 & 1.09688100 \\ \text { Ag } & 1.48652600 & -0.60549500 & -0.44259500 \\ & & & \end{array}$

\section{9}

$\mathrm{E}(\mathrm{SMD} / \mathrm{B} 3 \mathrm{LYP} / \mathrm{BS} 1)=-1454.29013656 \mathrm{au}$

$\mathrm{H}(\mathrm{SMD} / \mathrm{B} 3 \mathrm{LYP} / \mathrm{BS} 1)=-1454.118538 \mathrm{au}$

$\mathrm{G}(\mathrm{SMD} / \mathrm{B} 3 \mathrm{LYP} / \mathrm{BS} 1)=-1454.194524 \mathrm{au}$

$\mathrm{E}(\mathrm{SMD} / \mathrm{M} 06 / \mathrm{BS} 2 / / \mathrm{SMD} / \mathrm{B} 3 \mathrm{LYP} / \mathrm{BS} 1)=-1454.33791462 \mathrm{au}$

$\begin{array}{llll}\mathrm{C} & 3.04986000 & -1.87565200 & 0.32857600\end{array}$

$\mathrm{H} \quad 3.11330800 \quad-2.39691900 \quad 1.29442300$

$\mathrm{H} \quad 3.91309600 \quad-2.19788400 \quad-0.26345500$

$\mathrm{H} \quad 2.11864300 \quad-2.15558100 \quad-0.17225800$

$\begin{array}{llll}\text { C } & 3.11413500 & -0.40711400 & 0.59267100\end{array}$

$\begin{array}{llll}\mathrm{O} & 2.13158900 & 0.34213900 & 0.50225600\end{array}$

$\begin{array}{llll}\mathrm{C} & 4.44099500 & 0.19020200 & 0.98379700\end{array}$

$\begin{array}{llll}\mathrm{C} & 5.26797600 & 0.53566900 & -0.26503800\end{array}$

$\begin{array}{llll}\mathrm{O} & 4.79141000 & 0.44680500 & -1.38345500\end{array}$

$\begin{array}{lllll}\mathrm{C} & 6.68338200 & 0.98452700 & -0.00679100\end{array}$

$\begin{array}{llll}\mathrm{H} & 7.15006400 & 1.31123600 & -0.93899300\end{array}$

$\begin{array}{llll}\mathrm{H} & 6.69940100 & 1.79800400 & 0.72819000\end{array}$

$\begin{array}{llll}\mathrm{H} & 7.25827000 & 0.15287500 & 0.42038800\end{array}$

O $\quad-1.82944200 \quad-0.62564000 \quad-1.01054400$

S $\quad-3.01566400 \quad-0.74447200 \quad-0.06589600$

$\begin{array}{llll}\mathrm{O} & -4.17363200 & -1.38312000 & -0.70474500\end{array}$

$\begin{array}{llll}\mathrm{O} & -2.64347900 & -1.18538000 & 1.29004800\end{array}$

$\begin{array}{llll}\text { C } & -3.50577100 & 1.03775400 & 0.14315500\end{array}$

F $\quad-3.80442400 \quad 1.58635100 \quad-1.04051200$

$\begin{array}{lllll}\mathrm{F} & -4.57269500 & 1.13297100 & 0.94536400\end{array}$

$\begin{array}{llll}\text { F } & -2.49659600 & 1.73888200 & 0.69250000\end{array}$ 


$\begin{array}{lccl}\mathrm{H} & 4.28395000 & 1.11413800 & 1.55228800 \\ \mathrm{H} & 5.01425700 & -0.50350500 & 1.60990600 \\ \mathrm{Ag} & 0.12868200 & -0.18905100 & -0.18636200\end{array}$

\begin{tabular}{|c|c|c|c|}
\hline \multicolumn{4}{|c|}{$\mathbf{T S}_{39}$} \\
\hline \multicolumn{4}{|c|}{$\mathrm{E}(\mathrm{SMD} / \mathrm{B} 3 \mathrm{LYP} / \mathrm{BS} 1)=-1763.8966427 \mathrm{au}$} \\
\hline \multicolumn{4}{|c|}{$\mathrm{H}(\mathrm{SMD} / \mathrm{B} 3 \mathrm{LYP} / \mathrm{BS} 1)=-1763.586774 \mathrm{au}$} \\
\hline \multicolumn{4}{|c|}{ G (SMD/B3LYP/BS1) = -1763.683277 au } \\
\hline \multicolumn{4}{|c|}{$\mathrm{E}(\mathrm{SMD} / \mathrm{M} 06 / \mathrm{BS} 2 / / \mathrm{SMD} / \mathrm{B} 3 \mathrm{LYP} / \mathrm{BS} 1)=-1763.80275379 \mathrm{au}$} \\
\hline $\mathrm{C}$ & -0.13423600 & 1.98471400 & 1.47963400 \\
\hline $\mathrm{H}$ & -0.64308800 & 1.17272200 & 2.01390000 \\
\hline $\mathrm{H}$ & -0.90539900 & 2.57508400 & 0.96634300 \\
\hline $\mathrm{H}$ & 0.37544700 & 2.62988300 & 2.19939900 \\
\hline $\mathrm{C}$ & 0.80330200 & 1.40384000 & 0.44837700 \\
\hline $\mathrm{C}$ & 2.15109300 & 1.88663800 & 0.40448600 \\
\hline $\mathrm{H}$ & 2.80830100 & 0.64869100 & 1.17873300 \\
\hline $\mathrm{O}$ & 0.38251400 & 0.49846400 & -0.33111400 \\
\hline $\mathrm{C}$ & 7.62527100 & -0.19991100 & 0.78310000 \\
\hline $\mathrm{C}$ & 6.28159900 & -0.22998600 & 1.13312200 \\
\hline $\mathrm{C}$ & 5.36203200 & -0.99314400 & 0.37319700 \\
\hline $\mathrm{C}$ & 5.84219800 & -1.72500800 & -0.73945500 \\
\hline $\mathrm{C}$ & 7.18938400 & -1.69610500 & -1.08077400 \\
\hline $\mathrm{C}$ & 8.08256500 & -0.93189300 & -0.32156700 \\
\hline $\mathrm{H}$ & 8.32407500 & 0.38859400 & 1.37036700 \\
\hline $\mathrm{H}$ & 5.93930600 & 0.33240700 & 1.99573000 \\
\hline $\mathrm{H}$ & 5.14017600 & -2.31032200 & -1.32716800 \\
\hline $\mathrm{H}$ & 7.54608200 & -2.26272700 & -1.93579200 \\
\hline $\mathrm{H}$ & 9.13575500 & -0.90703600 & -0.58712200 \\
\hline $\mathrm{C}$ & 3.96183600 & -1.07244500 & 0.69068700 \\
\hline $\mathrm{H}$ & 3.37202400 & -1.71560100 & 0.04163700 \\
\hline $\mathrm{C}$ & 3.27800500 & -0.39397400 & 1.71547700 \\
\hline $\mathrm{H}$ & 3.86373900 & 0.02205400 & 2.53644200 \\
\hline $\mathrm{H}$ & 2.34310800 & -0.86111100 & 2.03330900 \\
\hline $\mathrm{H}$ & 2.34886000 & 2.75176000 & 1.03217000 \\
\hline $\mathrm{C}$ & 3.04936700 & 1.70353900 & -0.74159800 \\
\hline $\mathrm{O}$ & 3.03907300 & 0.69128400 & -1.44710100 \\
\hline $\mathrm{C}$ & 4.07239400 & 2.80398900 & -0.96858100 \\
\hline $\mathrm{H}$ & 3.57049600 & 3.74813400 & -1.21555900 \\
\hline $\mathrm{H}$ & 4.65709300 & 2.98031100 & -0.05689600 \\
\hline $\mathrm{H}$ & 4.74479300 & 2.52604100 & -1.78417600 \\
\hline $\mathrm{O}$ & -3.49687300 & -1.29361200 & -0.66460600 \\
\hline S & -4.59343700 & -1.07432500 & 0.36526400 \\
\hline $\mathrm{O}$ & -5.67624800 & -2.05922800 & 0.24342500 \\
\hline $\mathrm{O}$ & -4.08248300 & -0.77471800 & 1.71461100 \\
\hline $\mathrm{C}$ & -5.33652600 & 0.52817100 & -0.21720700 \\
\hline $\mathrm{F}$ & -5.80231200 & 0.40532900 & -1.46604100 \\
\hline $\mathrm{F}$ & -6.34427100 & 0.88975900 & 0.58601400 \\
\hline $\mathrm{F}$ & -4.41130800 & 1.50551300 & -0.20464200 \\
\hline $\mathrm{Ag}$ & -1.59153900 & -0.31155700 & -0.39188600 \\
\hline
\end{tabular}

\section{0-DCM}




$\begin{array}{lccc}\mathrm{E}(\mathrm{SMD} / \mathrm{B} 3 \mathrm{LYP} / \mathrm{BS} 1)=-1454.28734666 \mathrm{au} \\ \mathrm{H}(\mathrm{SMD} / \mathrm{B} 3 \mathrm{LYP} / \mathrm{BS} 1)=-1454.115474 \mathrm{au} \\ \mathrm{G}(\mathrm{SMD} / \mathrm{B} 3 \mathrm{~L} \text { PP/BS1 }=-1454.190481 \mathrm{au} \\ \mathrm{E}(\mathrm{SMD} / \mathrm{M} 06 / \mathrm{BS} 2 / / \mathrm{SMD} / \mathrm{B} 3 \mathrm{LYP} / \mathrm{BS} 1)=-1454.34153313 \mathrm{au} \\ \mathrm{C} & 2.47556700 & 2.67109800 & -0.83228000 \\ \mathrm{H} & 3.34557900 & 3.32707400 & -0.69965100 \\ \mathrm{H} & 1.58772700 & 3.24592100 & -0.54772200 \\ \mathrm{H} & 2.40459000 & 2.37633700 & -1.88083500 \\ \mathrm{C} & 2.63453000 & 1.48598600 & 0.06100700 \\ \mathrm{O} & 2.62832400 & 1.75497000 & 1.34494400 \\ \mathrm{C} & 2.83670600 & 0.17138200 & -0.40938500 \\ \mathrm{C} & 3.30170500 & -0.86229100 & 0.53153500 \\ \mathrm{O} & 3.28225400 & -0.65867600 & 1.76162400 \\ \mathrm{C} & 3.84529400 & -2.15002000 & -0.02488500 \\ \mathrm{H} & 3.21368500 & -2.53178800 & -0.83366100 \\ \mathrm{H} & 4.84113200 & -1.96132700 & -0.44868600 \\ \mathrm{H} & 3.93446800 & -2.89474900 & 0.76920500 \\ \mathrm{H} & 3.08879600 & 0.05639100 & -1.46144100 \\ \mathrm{H} & 2.84189000 & 0.88241600 & 1.82391300 \\ \mathrm{O} & -1.43109200 & -0.80561100 & -1.07617500 \\ \mathrm{~S} & -2.33622300 & -0.92319300 & 0.14191900 \\ \mathrm{O} & -3.50612900 & -1.77541000 & -0.10080100 \\ \mathrm{O} & -1.58439100 & -1.11422700 & 1.39662600 \\ \mathrm{C} & -3.01110800 & 0.80619500 & 0.26234900 \\ \mathrm{~F} & -3.68748900 & 1.12637300 & -0.84666900 \\ \mathrm{~F} & -3.82839700 & 0.91310300 & 1.31543400 \\ \mathrm{~F} & -2.00357100 & 1.68698200 & 0.41347000 \\ \mathrm{Ag} & 0.64231700 & -0.28105900 & -0.66110100 \\ & & & \end{array}$

\section{$\mathrm{TS}_{\text {32-34-DCM }}$}

$\begin{array}{lccc}\text { E }(\mathrm{SMD} / \mathrm{B} 3 L Y P / B S 1)= & -1763.89318057 \mathrm{au} \\ \mathrm{H}(\mathrm{SMD} / \mathrm{B} 3 L Y P / B S 1)= & -1763.578913 \mathrm{au} \\ \mathrm{G}(\mathrm{SMD} / \mathrm{B} 3 L Y P / B S 1)=-1763.670314 \mathrm{au} & \\ \mathrm{E}(\mathrm{SMD} / \mathrm{M} 06 / \mathrm{BS} 2 / / \mathrm{SMD} / \mathrm{B} 3 \mathrm{~L} Y \mathrm{P} / \mathrm{BS} 1)= & -1763.81449761 \mathrm{au} \\ \mathrm{C} & 1.56560400 & -2.71392900 & -0.14420900 \\ \mathrm{H} & 2.51499500 & -3.25124500 & -0.25043600 \\ \mathrm{H} & 0.86557000 & -3.15598800 & -0.86429900 \\ \mathrm{H} & 1.16973100 & -2.86991800 & 0.86306000 \\ \mathrm{C} & 1.77652500 & -1.24083700 & -0.49975400 \\ \mathrm{O} & 2.34438500 & -0.95061300 & -1.55336300 \\ \mathrm{C} & 1.28591400 & -0.24695900 & 0.47880700 \\ \mathrm{C} & 1.24051500 & 1.21734400 & 0.28735900 \\ \mathrm{O} & 1.67294300 & 1.80278300 & -0.70754200 \\ \mathrm{C} & 0.64170700 & 2.03458400 & 1.43466600 \\ \mathrm{H} & -0.23795100 & 2.57731000 & 1.06881800 \\ \mathrm{H} & 0.34558400 & 1.42871000 & 2.29619000 \\ \mathrm{H} & 1.36946500 & 2.78855600 & 1.75781200 \\ \mathrm{O} & -2.95257700 & -0.75705700 & -1.05552700 \\ \mathrm{~S} & -4.01078100 & -0.70483900 & 0.03357700 \\ \mathrm{O} & -5.21958000 & -1.46666200 & -0.30327600 \\ \mathrm{O} & -3.44547800 & -0.86719000 & 1.38825900\end{array}$




$\begin{array}{lrrr}\mathrm{C} & -4.52674600 & 1.08077500 & -0.03873600 \\ \mathrm{~F} & -5.02005400 & 1.38163100 & -1.24499300 \\ \mathrm{~F} & -5.46013900 & 1.33267700 & 0.88637100 \\ \mathrm{~F} & -3.46759200 & 1.87786200 & 0.19767500 \\ \mathrm{H} & 0.90905100 & -0.63024100 & 1.42548400 \\ \mathrm{C} & 4.56085100 & 1.57016600 & 0.26565200 \\ \mathrm{C} & 5.50507400 & 1.90529600 & -0.69471700 \\ \mathrm{C} & 6.25845100 & 0.89843300 & -1.30895000 \\ \mathrm{C} & 6.06966400 & -0.44795700 & -0.96373100 \\ \mathrm{C} & 5.12987100 & -0.79328200 & -0.00560300 \\ \mathrm{C} & 4.36396000 & 0.21347900 & 0.64398800 \\ \mathrm{H} & 3.98577500 & 2.34605300 & 0.75891000 \\ \mathrm{H} & 5.65834100 & 2.94477700 & -0.96766800 \\ \mathrm{H} & 6.99699600 & 1.16127000 & -2.06117200 \\ \mathrm{H} & 6.65690500 & -1.22035100 & -1.45084500 \\ \mathrm{H} & 4.98900800 & -1.83616300 & 0.25540500 \\ \mathrm{C} & 3.43453300 & -0.07947500 & 1.68048400 \\ \mathrm{H} & 2.99340700 & 0.78135800 & 2.17469500 \\ \mathrm{C} & 3.40565200 & -1.34175100 & 2.47037200 \\ \mathrm{H} & 3.59932400 & -2.24221600 & 1.88453000 \\ \mathrm{H} & 4.20900300 & -1.26183400 & 3.22187400 \\ \mathrm{H} & 2.46698100 & -1.45084700 & 3.01849000 \\ \mathrm{Ag} & -0.89907700 & -0.43801600 & -0.34046200 \\ & & & \end{array}$

\section{2-DCM}

$\mathrm{E}(\mathrm{SMD} / \mathrm{B} 3 \mathrm{LYP} / \mathrm{BS} 1)=-1763.90075144 \mathrm{au}$

$\mathrm{H}(\mathrm{SMD} / \mathrm{B} 3 \mathrm{LYP} / \mathrm{BS} 1)=-1763.58781 \mathrm{au}$

$\mathrm{G}(\mathrm{SMD} / \mathrm{B} 3 \mathrm{LYP} / \mathrm{BS} 1)=-1763.681692 \mathrm{au}$

$\mathrm{E}(\mathrm{SMD} / \mathrm{M} 06 / \mathrm{BS} 2 / / \mathrm{SMD} / \mathrm{B} 3 \mathrm{LYP} / \mathrm{BS} 1)=-1763.812659 \mathrm{au}$

$\begin{array}{llll}\mathrm{C} & 1.32691800 & -2.54407400 & 2.93156000\end{array}$

$\mathrm{H} \quad 0.23188500 \quad-2.57641800 \quad 2.92181000$

$\mathrm{H} \quad \begin{array}{llll}\mathrm{H} & 1.66948600 & -1.87436100 & 3.72601700\end{array}$

$\mathrm{H} \quad \begin{array}{llll}\mathrm{H} & 1.68298700 & -3.55827100 & 3.16038100\end{array}$

$\begin{array}{llll}\mathrm{C} & 1.91058600 & -2.10016000 & 1.58757900\end{array}$

$\begin{array}{llll}\mathrm{O} & 2.94399300 & -1.41130500 & 1.57203400\end{array}$

$\begin{array}{llll}\text { C } & 1.18286000 & -2.58520100 & 0.39389000\end{array}$

$\begin{array}{llll}\mathrm{C} & 1.87926700 & -2.83894400 & -0.88655000\end{array}$

$\begin{array}{llll}\mathrm{O} & 2.90797700 & -2.26354900 & -1.27408300\end{array}$

C $\quad 1.26625300 \quad-3.94088000 \quad-1.75419000$

$\mathrm{H} \quad 1.60127100 \quad-3.82939400 \quad-2.78974400$

$\mathrm{H} \quad 1.60563900 \quad-4.91937200 \quad-1.38674600$

$\mathrm{H} \quad \begin{array}{llll}0.17130400 & -3.93978100 & -1.71751200\end{array}$

$\mathrm{H} \quad \begin{array}{llll}1.02574000 & 0.57198400 & -3.15465100\end{array}$

$\begin{array}{lllll}\mathrm{C} & 1.46699000 & 2.20581900 & -1.92936400\end{array}$

C $\quad 1.85084200 \quad 1.28638000 \quad-3.00415000$

$\mathrm{H} \quad \begin{array}{llll}\mathrm{H} & 0.52860000 & 2.73626500 & -2.08553700\end{array}$

$\mathrm{H} \quad \begin{array}{llll}1.89201400 & 1.85932800 & -3.94464500\end{array}$

$\begin{array}{llll}\mathrm{H} & 2.78575200 & 0.74911000 & -2.84976800\end{array}$

$\begin{array}{llll}\text { C } & 2.14360100 & 2.49414300 & -0.74478600\end{array}$

$\begin{array}{llll}\text { C } & 1.55886400 & 3.45564500 & 0.14220000\end{array}$

$\begin{array}{lllll}\text { C } & 3.38496000 & 1.87679600 & -0.38825800\end{array}$ 


$\begin{array}{cccc}\mathrm{C} & 2.19003600 & 3.78527400 & 1.32544000 \\ \mathrm{H} & 0.61486600 & 3.91588800 & -0.13436800 \\ \mathrm{C} & 4.00337800 & 2.21778000 & 0.79773400 \\ \mathrm{H} & 3.83087800 & 1.13696300 & -1.04285900 \\ \mathrm{C} & 3.40901100 & 3.16686500 & 1.64915800 \\ \mathrm{H} & 1.75205400 & 4.51227800 & 2.00103900 \\ \mathrm{H} & 4.94146500 & 1.75152600 & 1.07918300 \\ \mathrm{H} & 3.90475800 & 3.42512100 & 2.58083000 \\ \mathrm{O} & -1.42646300 & 0.78576700 & -0.56129200 \\ \mathrm{~S} & -2.91119800 & 0.57550900 & -0.79759000 \\ \mathrm{O} & -3.56176300 & 1.77346000 & -1.34706800 \\ \mathrm{O} & -3.23522700 & -0.72463300 & -1.40960100 \\ \mathrm{C} & -3.55606600 & 0.44290800 & 0.94288400 \\ \mathrm{~F} & -3.25187700 & 1.54450900 & 1.64254500 \\ \mathrm{~F} & -4.88532500 & 0.29016600 & 0.93555400 \\ \mathrm{~F} & -3.01037500 & -0.61675400 & 1.56885400 \\ \mathrm{Ag} & -0.14740200 & -0.92704600 & -0.08043500 \\ \mathrm{H} & 0.46341800 & -3.37115600 & 0.63708400\end{array}$

\begin{tabular}{|c|c|c|c|}
\hline \multicolumn{4}{|c|}{ 1_AuOTf 3 (water) } \\
\hline \multicolumn{4}{|c|}{$\mathrm{E}(\mathrm{SMD} / \mathrm{B} 3 \mathrm{LYP} / \mathrm{BS} 1)=-3365.81933026 \mathrm{au}$} \\
\hline \multicolumn{4}{|c|}{$\mathrm{H}(\mathrm{SMD} / \mathrm{B} 3 \mathrm{LYP} / \mathrm{BS} 1)=-3365.573238 \mathrm{au}$} \\
\hline \multicolumn{4}{|c|}{$\mathrm{G}(\mathrm{SMD} / \mathrm{B} 3 \mathrm{LYP} / \mathrm{BS} 1)=-3365.682985 \mathrm{au}$} \\
\hline \multicolumn{4}{|c|}{$\mathrm{E}(\mathrm{SMD} / \mathrm{M} 06 / \mathrm{BS} 2 / / \mathrm{SMD} / \mathrm{B} 3 \mathrm{LYP} / \mathrm{BS} 1)=-3366.04076397 \mathrm{au}$} \\
\hline $\mathrm{C}$ & 1.72846500 & 3.38856900 & -1.16969900 \\
\hline $\mathrm{H}$ & 2.69615000 & 3.82802600 & -0.88082000 \\
\hline $\mathrm{H}$ & 1.22580600 & 4.05253200 & -1.87385700 \\
\hline $\mathrm{H}$ & 1.94480000 & 2.41238800 & -1.61103700 \\
\hline $\mathrm{C}$ & 0.94285200 & 3.23949900 & 0.06652300 \\
\hline $\mathrm{O}$ & 0.00533900 & 4.07219000 & 0.29247000 \\
\hline $\mathrm{C}$ & 1.23942200 & 2.18055800 & 1.06330000 \\
\hline $\mathrm{C}$ & 0.69458600 & 2.40667000 & 2.46173900 \\
\hline $\mathrm{O}$ & -0.22710200 & 3.21530700 & 2.62943600 \\
\hline $\mathrm{C}$ & 1.30903700 & 1.64348900 & 3.58566600 \\
\hline $\mathrm{H}$ & 2.35780300 & 1.95086000 & 3.69158000 \\
\hline $\mathrm{H}$ & 0.76830500 & 1.83643100 & 4.51366900 \\
\hline $\mathrm{H}$ & 1.31133000 & 0.57178300 & 3.35664000 \\
\hline $\mathrm{H}$ & 2.29682600 & 1.91935200 & 1.05163900 \\
\hline $\mathrm{H}$ & -0.36869600 & 3.89849600 & 1.24362700 \\
\hline $\mathrm{Au}$ & 0.37008100 & 0.35811500 & 0.36886000 \\
\hline $\mathrm{O}$ & -0.42078700 & -1.53623200 & -0.28169700 \\
\hline $\mathrm{S}$ & -0.61977900 & -2.72486200 & 0.68058500 \\
\hline $\mathrm{O}$ & -0.47736300 & -3.99074300 & -0.04689300 \\
\hline $\mathrm{O}$ & 0.08400800 & -2.56320400 & 1.96069500 \\
\hline $\mathrm{C}$ & -2.42945100 & -2.57848800 & 1.09469700 \\
\hline $\mathrm{F}$ & -3.16240100 & -2.67162000 & -0.01455400 \\
\hline $\mathrm{F}$ & -2.76649400 & -3.55923500 & 1.93383000 \\
\hline $\mathrm{F}$ & -2.66141400 & -1.39903600 & 1.68271500 \\
\hline $\mathrm{O}$ & -3.02668800 & 2.90582100 & -0.72160200 \\
\hline S & -2.14516900 & 1.79091800 & -1.06803300 \\
\hline $\mathrm{O}$ & -1.17913700 & 1.92918000 & -2.16187500 \\
\hline
\end{tabular}




$\begin{array}{lrrr}\mathrm{O} & -1.47200200 & 1.27868600 & 0.25600000 \\ \mathrm{C} & -3.28293200 & 0.38207500 & -1.53722100 \\ \mathrm{~F} & -4.10798700 & 0.82440900 & -2.48554600 \\ \mathrm{~F} & -2.57444400 & -0.64082700 & -2.00690200 \\ \mathrm{~F} & -3.98525700 & 0.00004600 & -0.47386600 \\ \mathrm{O} & 3.80736400 & 0.77034200 & -0.65227600 \\ \mathrm{~S} & 3.42993800 & -0.58124600 & -0.23240100 \\ \mathrm{O} & 4.42791100 & -1.45093100 & 0.38951300 \\ \mathrm{O} & 2.16108500 & -0.61007700 & 0.68494400 \\ \mathrm{C} & 2.85117600 & -1.45867100 & -1.77761500 \\ \mathrm{~F} & 3.89349400 & -1.60491600 & -2.59191100 \\ \mathrm{~F} & 2.35586500 & -2.65185900 & -1.45940200 \\ \mathrm{~F} & 1.90991300 & -0.73061100 & -2.38745900\end{array}$

\section{1_AuCl $\mathrm{Au}_{3}$ (water)}

E $(\mathrm{SMD} / \mathrm{B} 3 \mathrm{LYP} / \mathrm{BS} 1)=-1862.23742682 \mathrm{au}$

$\mathrm{H}(\mathrm{SMD} / \mathrm{B} 3 \mathrm{LYP} / \mathrm{BS} 1)=-1862.096176 \mathrm{au}$

$\mathrm{G}(\mathrm{SMD} / \mathrm{B} 3 \mathrm{LYP} / \mathrm{BS} 1)=-1862.153922 \mathrm{au}$

$\mathrm{E}(\mathrm{SMD} / \mathrm{M} 06 / \mathrm{BS} 2 / / \mathrm{SMD} / \mathrm{B} 3 \mathrm{LYP} / \mathrm{BS} 1)=-1862.14765122 \mathrm{au}$

$\begin{array}{llll}\mathrm{C} & -1.83588700 & 1.93022400 & -1.93406300\end{array}$

$\mathrm{H} \quad-2.58720400 \quad 2.69765800 \quad-1.69805100$

$\mathrm{H} \quad-1.97291300 \quad 1.64078100 \quad-2.97849500$

$\mathrm{H} \quad-0.85529400 \quad 2.37774300 \quad-1.76796100$

C $\quad \begin{array}{llll}\text { C } & -2.06694600 & 0.78814300 & -1.01479300\end{array}$

$\begin{array}{llll}\mathrm{O} & -2.88396300 & -0.12325700 & -1.38874300\end{array}$

$\begin{array}{llll}\text { C } & -1.44953400 & 0.73602000 & 0.32462000\end{array}$

$\begin{array}{llll}\text { C } & -2.10063600 & -0.16710200 & 1.34729000\end{array}$

$\begin{array}{llll}\text { O } & -3.03198600 & -0.91295200 & 1.00779600\end{array}$

$\begin{array}{llll}\mathrm{C} & -1.58322400 & -0.12693000 & 2.75275800\end{array}$

$\mathrm{H} \quad-0.51094600 \quad-0.33452800 \quad 2.73911300$

$\mathrm{H} \quad-1.71637400 \quad 0.87819400 \quad 3.17172400$

$\mathrm{H} \quad-2.10241700 \quad-0.85683200 \quad 3.37238200$

$\mathrm{H} \quad-1.22032700 \quad 1.71431400 \quad 0.74844300$

$\mathrm{H} \quad-3.07348900 \quad-0.71753300 \quad-0.56146700$

$\mathrm{Au} \quad 0.59658600 \quad-0.02082300 \quad-0.03157400$

$\begin{array}{llll}\mathrm{Cl} & 1.56494600 & 2.07853800 & 0.55747500\end{array}$

$\mathrm{Cl} \quad 2.78653400 \quad-0.95134200 \quad-0.23957500$

$\mathrm{Cl} \quad-0.32479400 \quad-2.09350500 \quad-0.69076000$

\section{1_AuCl(water)}

$\mathrm{E}(\mathrm{SMD} / \mathrm{B} 3 \mathrm{LYP} / \mathrm{BS} 1)=-941.854809792 \mathrm{au}$

$\mathrm{H}(\mathrm{SMD} / \mathrm{B} 3 \mathrm{LYP} / \mathrm{BS} 1)=-941.717205 \mathrm{au}$

$\mathrm{G}(\mathrm{SMD} / \mathrm{B} 3 \mathrm{LYP} / \mathrm{BS} 1)=-941.770383 \mathrm{au}$

$\mathrm{E}(\mathrm{SMD} / \mathrm{M} 06 / \mathrm{BS} 2 / / \mathrm{SMD} / \mathrm{B} 3 \mathrm{LYP} / \mathrm{BS} 1)=-941.760986834 \mathrm{au}$

$\begin{array}{llll}\mathrm{C} & -1.53884600 & -2.56355900 & -0.76801900\end{array}$

$\mathrm{H} \quad-2.53794700 \quad-2.97060500 \quad-0.97308700$

$\mathrm{H} \quad-1.00788000 \quad-3.29194300 \quad-0.14724700$

$\mathrm{H} \quad-1.01004700 \quad-2.41503200 \quad-1.71005700$

C $\quad-1.70859300 \quad-1.28190200 \quad-0.03012500$

$\begin{array}{lllll}\mathrm{O} & & -2.19469600 & -1.39198200 & 1.17965600\end{array}$

$\begin{array}{llll}\text { C } & -1.43418000 & -0.00412000 & -0.60382300\end{array}$ 


$\begin{array}{lrrr}\mathrm{C} & -1.99853100 & 1.19946100 & 0.05146200 \\ \mathrm{O} & -2.43641900 & 1.14762100 & 1.21834500 \\ \mathrm{C} & -2.06768700 & 2.47069100 & -0.74366800 \\ \mathrm{H} & -1.13186700 & 2.64687100 & -1.28296300 \\ \mathrm{H} & -2.86326300 & 2.37036200 & -1.49428700 \\ \mathrm{H} & -2.29724600 & 3.31558800 & -0.09098700 \\ \mathrm{H} & -1.37903200 & 0.01686600 & -1.69096300 \\ \mathrm{H} & -2.36830300 & -0.44984700 & 1.51664200 \\ \mathrm{Au} & 0.67625600 & 0.01198500 & -0.09030200 \\ \mathrm{Cl} & 2.98278300 & 0.16837400 & 0.37575400\end{array}$

\title{
1_AuOTf (water)
}

E $($ SMD/B3LYP/BS1) $=-1443.05167156$ au

$\mathrm{H}(\mathrm{SMD} / \mathrm{B} 3 \mathrm{LYP} / \mathrm{BS} 1)=-1442.880998 \mathrm{au}$

$\mathrm{G}(\mathrm{SMD} / \mathrm{B} 3 \mathrm{LYP} / \mathrm{BS} 1)=-1442.950562 \mathrm{au}$

$\mathrm{E}(\mathrm{SMD} / \mathrm{M} 06 / \mathrm{BS} 2 / / \mathrm{SMD} / \mathrm{B} 3 \mathrm{LYP} / \mathrm{BS} 1)=-1443.06482932 \mathrm{au}$

$\begin{array}{llll}\mathrm{C} & -1.72439600 & 2.62123200 & 1.17740500\end{array}$

$\mathrm{H} \quad-2.51990100 \quad 3.29660200 \quad 1.52005800$

$\mathrm{H} \quad-0.99374700 \quad 3.22417700 \quad 0.62915800$

$\mathrm{H} \quad-1.25293900 \quad 2.15083300 \quad 2.04062000$

$\begin{array}{llll}\mathrm{C} & -2.34098400 & 1.61163100 & 0.27469900\end{array}$

$\begin{array}{llll}\mathrm{O} & -2.80424000 & 2.07259900 & -0.85406800\end{array}$

$\begin{array}{llll}\text { C } & -2.49333700 & 0.22873500 & 0.62916100\end{array}$

C $\quad-3.46602800 \quad-0.58915500 \quad-0.14332600$

$\begin{array}{llll}\mathrm{O} & -3.89718700 & -0.19517600 & -1.24377300\end{array}$

C $\quad \begin{array}{llll}-3.94323800 & -1.87613800 & 0.46133500\end{array}$

$\mathrm{H} \quad-3.11230500 \quad-2.43253900 \quad 0.90531900$

$\mathrm{H} \quad \quad \quad-4.64709800 \quad-1.63811200 \quad 1.27048300$

$\mathrm{H} \quad-4.45474100 \quad-2.48260400 \quad-0.28903500$

$\mathrm{H} \quad \begin{array}{llll}-2.44223200 & 0.02271500 & 1.69810700\end{array}$

$\mathrm{H} \quad \begin{array}{llll}-3.29962500 & 1.30970400 & -1.30898100\end{array}$

$\mathrm{Au} \quad-0.55845000 \quad-0.38037200 \quad-0.01496000$

$\begin{array}{llll}\mathrm{O} & 1.36332900 & -1.10479800 & -0.66717700\end{array}$

S $\quad \begin{array}{llll}\text { S } & 2.62479500 & -0.85616000 & 0.15972900\end{array}$

$\begin{array}{lllll}\mathrm{C} & 3.01478700 & 0.92305000 & -0.22872700\end{array}$

F $\quad \begin{array}{llll}\text { C } & 4.17000200 & 1.26117900 & 0.34927500\end{array}$

F $\quad 3.11651400 \quad 1.09122200 \quad-1.54970000$

F $\quad \begin{array}{llll}2.04633100 & 1.72480600 & 0.23624600\end{array}$

$\begin{array}{llll}\mathrm{O} & 3.75954800 & -1.62404300 & -0.37320300\end{array}$

$\begin{array}{llll}\mathrm{O} & 2.39941800 & -0.88781200 & 1.61506800\end{array}$

\author{
1_AgOTf (water) \\ $\mathrm{E}(\mathrm{SMD} / \mathrm{B} 3 \mathrm{LYP} / \mathrm{BS} 1)=-1454.29451685 \mathrm{au}$ \\ $\mathrm{H}(\mathrm{SMD} / \mathrm{B} 3 \mathrm{LYP} / \mathrm{BS} 1)=-1454.122896 \mathrm{au}$ \\ $\mathrm{G}(\mathrm{SMD} / \mathrm{B} 3 \mathrm{LYP} / \mathrm{BS} 1)=-1454.195852 \mathrm{au}$ \\ $\mathrm{E}(\mathrm{SMD} / \mathrm{M} 06 / \mathrm{BS} 2 / / \mathrm{SMD} / \mathrm{B} 3 \mathrm{LYP} / \mathrm{BS} 1)=-1454.3478396 \mathrm{au}$ \\ $\begin{array}{llll}\mathrm{C} & 2.32978900 & 2.70706200 & -0.77149600\end{array}$ \\ $\mathrm{H} \quad 3.17392100 \quad 3.39891700 \quad-0.65650100$ \\ $\mathrm{H} \quad \begin{array}{llll}\mathrm{H} & 1.42892800 & 3.23061100 & -0.43488800\end{array}$ \\ $\begin{array}{llll}\mathrm{H} & 2.22963500 & 2.43263100 & -1.82271000\end{array}$ \\ $\begin{array}{lllll}\mathrm{C} & 2.57985900 & 1.51015800 & 0.08149100\end{array}$
}




$\begin{array}{crrr}\mathrm{O} & 2.59831100 & 1.74448600 & 1.37847800 \\ \mathrm{C} & 2.83720400 & 0.22551900 & -0.43244300 \\ \mathrm{C} & 3.37132900 & -0.81813200 & 0.45127600 \\ \mathrm{O} & 3.37723800 & -0.66857400 & 1.69369400 \\ \mathrm{C} & 3.95604400 & -2.05404100 & -0.17358900 \\ \mathrm{H} & 3.33557300 & -2.41048000 & -1.00153200 \\ \mathrm{H} & 4.94268900 & -1.80526400 & -0.58710600 \\ \mathrm{H} & 4.07557300 & -2.83796400 & 0.57755900 \\ \mathrm{H} & 3.04524000 & 0.14966400 & -1.49699800 \\ \mathrm{H} & 2.86555400 & 0.87600300 & 1.82712300 \\ \mathrm{O} & -1.42287800 & -1.03899700 & -0.90042600 \\ \mathrm{~S} & -2.42985600 & -0.93364000 & 0.22607000 \\ \mathrm{O} & -3.66712200 & -1.68094600 & -0.05777300 \\ \mathrm{O} & -1.83787900 & -1.10162900 & 1.56840600 \\ \mathrm{C} & -2.92127700 & 0.85889700 & 0.15128900 \\ \mathrm{~F} & -3.37754500 & 1.16159800 & -1.06819900 \\ \mathrm{~F} & -3.87614300 & 1.10953200 & 1.05118500 \\ \mathrm{~F} & -1.86135900 & 1.63899300 & 0.41861000 \\ \mathrm{Ag} & 0.64929700 & -0.33826600 & -0.61563100\end{array}$

\section{1_Ph}

$\mathrm{E}(\mathrm{SMD} / \mathrm{B} 3 \mathrm{LYP} / \mathrm{BS} 1)=-729.279705788 \mathrm{au}$

$\mathrm{H}(\mathrm{SMD} / \mathrm{B} 3 \mathrm{LYP} / \mathrm{BS} 1)=-729.034695 \mathrm{au}$

$\mathrm{G}(\mathrm{SMD} / \mathrm{B} 3 \mathrm{LYP} / \mathrm{BS} 1)=-729.093347 \mathrm{au}$

$\mathrm{E}(\mathrm{SMD} / \mathrm{M} 06 / \mathrm{BS} 2 / / \mathrm{SMD} / \mathrm{B} 3 \mathrm{LYP} / \mathrm{BS} 1)=-729.036903106 \mathrm{au}$

$\begin{array}{lrrr}\mathrm{C} & -1.36102700 & 1.41221600 & 0.17452200 \\ \mathrm{O} & -1.66703800 & 2.54707000 & -0.17075800 \\ \mathrm{C} & 0.06746300 & 1.12474200 & 0.62276600 \\ \mathrm{H} & 0.53658800 & 2.08522200 & 0.86462200 \\ \mathrm{H} & 0.09071600 & 0.51699800 & 1.53416000 \\ \mathrm{C} & 0.89185300 & 0.44699700 & -0.47137000 \\ \mathrm{O} & 0.40293300 & 0.23186200 & -1.57432000 \\ \mathrm{C} & 2.30721600 & 0.07241000 & -0.16786400 \\ \mathrm{C} & 2.91435900 & 0.34318000 & 1.06980600 \\ \mathrm{C} & 3.05234800 & -0.57293100 & -1.17059000 \\ \mathrm{C} & 4.24082500 & -0.02584800 & 1.29757000 \\ \mathrm{H} & 2.36088900 & 0.84309400 & 1.85802500 \\ \mathrm{C} & 4.37459500 & -0.94255000 & -0.94085000 \\ \mathrm{H} & 2.57610400 & -0.77721500 & -2.12413900 \\ \mathrm{C} & 4.97164600 & -0.66904100 & 0.29511100 \\ \mathrm{H} & 4.70311600 & 0.18867800 & 2.25699900 \\ \mathrm{H} & 4.94158800 & -1.44249000 & -1.72129600 \\ \mathrm{H} & 6.00404900 & -0.95649900 & 0.47527200 \\ \mathrm{C} & -2.37392700 & 0.31217800 & 0.17775100 \\ \mathrm{C} & -2.07122900 & -1.00120900 & 0.57413200 \\ \mathrm{C} & -3.68329100 & 0.61557400 & -0.23576600 \\ \mathrm{C} & -3.05845100 & -1.98777600 & 0.55609300 \\ \mathrm{H} & -1.06977100 & -1.26601100 & 0.89741800 \\ \mathrm{C} & -4.66749500 & -0.36849100 & -0.25241100 \\ \mathrm{H} & -3.90934300 & 1.63201800 & -0.54150700 \\ \mathrm{C} & -4.35620100 & -1.67419300 & 0.14421800\end{array}$




$\begin{array}{lllc}H & -2.81381900 & -3.00056100 & 0.86388500 \\ H & -5.67598300 & -0.12239800 & -0.57347700 \\ H & -5.12339600 & -2.44385000 & 0.13196100\end{array}$

\section{2_Cl}

$\mathrm{E}(\mathrm{SMD} / \mathrm{B} 3 \mathrm{LYP} / \mathrm{BS} 1)=-769.24450081 \mathrm{au}$

$\mathrm{H}(\mathrm{SMD} / \mathrm{B} 3 \mathrm{LYP} / \mathrm{BS} 1)=-769.111387 \mathrm{au}$

G $($ SMD/B3LYP/BS1) $=-769.153353$ au

$\mathrm{E}(\mathrm{SMD} / \mathrm{M} 06 / \mathrm{BS} 2 / / \mathrm{SMD} / \mathrm{B} 3 \mathrm{LYP} / \mathrm{BS} 1)=-769.108890578 \mathrm{au}$

$\begin{array}{llll}\mathrm{C} & 0.00000000 & 1.29834500 & 0.00000000\end{array}$

$\begin{array}{llll}\text { C } & 1.18961800 & 0.57543100 & 0.00000000\end{array}$

$\begin{array}{llll}\text { C } & 1.18952100 & -0.83294300 & 0.00000000\end{array}$

$\begin{array}{llll}\mathrm{C} & -0.05350600 & -1.49057300 & 0.00000000\end{array}$

$\begin{array}{llll}\text { C } & -1.25614800 & -0.78382000 & 0.00000000\end{array}$

$\begin{array}{llll}\mathrm{C} & -1.21440600 & 0.60853800 & 0.00000000\end{array}$

$\begin{array}{llll}\mathrm{H} & 0.01590500 & 2.38330600 & 0.00000000\end{array}$

$\mathrm{H} \quad \begin{array}{llll}\mathrm{H} & 2.12798300 & 1.12157700 & 0.00000000\end{array}$

$\mathrm{H} \quad-0.08122300 \quad-2.57729800 \quad 0.00000000$

$\mathrm{H} \quad \begin{array}{llll}\mathrm{H} & -2.20521100 & -1.30956600 & 0.00000000\end{array}$

$\begin{array}{llll}\mathrm{C} & 2.42122100 & -1.64083800 & 0.00000000\end{array}$

$\begin{array}{llll}\mathrm{H} & 2.25347800 & -2.71774800 & 0.00000000\end{array}$

C $\quad 3.68455600 \quad-1.19313800 \quad 0.00000000$

$\mathrm{H} \quad 3.93981500 \quad-0.13613200 \quad 0.00000000$

$\mathrm{H} \quad 4.51843000 \quad-1.89000900 \quad 0.00000000$

$\begin{array}{llll}\mathrm{Cl} & -2.72554700 & 1.52234500 & 0.00000000\end{array}$

\section{3_Ph}

$\mathrm{E}(\mathrm{SMD} / \mathrm{B} 3 \mathrm{LYP} / \mathrm{BS} 1)=-1498.53599646 \mathrm{au}$

$\mathrm{H}(\mathrm{SMD} / \mathrm{B} 3 \mathrm{LYP} / \mathrm{BS} 1)=-1498.154615 \mathrm{au}$

$\mathrm{G}(\mathrm{SMD} / \mathrm{B} 3 \mathrm{LYP} / \mathrm{BS} 1)=-1498.235207 \mathrm{au}$

$\mathrm{E}(\mathrm{SMD} / \mathrm{M} 06 / \mathrm{BS} 2 / / \mathrm{SMD} / \mathrm{B} 3 \mathrm{LYP} / \mathrm{BS} 1)=-1498.16856756 \mathrm{au}$

$\begin{array}{llll}\mathrm{C} & -0.86495300 & 2.98798100 & 2.25719700\end{array}$

$\begin{array}{llll}\mathrm{H} & 0.21359400 & 3.18226100 & 2.30280100\end{array}$

$\begin{array}{llll}\mathrm{H} & -1.11457900 & 2.33716800 & 3.10312800\end{array}$

$\begin{array}{llll}\mathrm{H} & -1.39906000 & 3.93734100 & 2.34419800\end{array}$

$\begin{array}{llll}\text { C } & -1.22104800 & 2.32635300 & 0.94545100\end{array}$

$\begin{array}{llll}\mathrm{O} & -1.87617000 & 2.89933700 & 0.09053800\end{array}$

$\begin{array}{llll}\text { C } & -0.73560800 & 0.87822900 & 0.73475500\end{array}$

$\begin{array}{llll}\mathrm{H} & -1.07931900 & 0.31661300 & 1.61057400\end{array}$

$\begin{array}{lllll}\mathrm{C} & & -1.41723900 & 0.27654700 & -0.49902900\end{array}$

$\begin{array}{lllll}\mathrm{O} & -0.83643500 & 0.20089100 & -1.57505600\end{array}$

$\begin{array}{llll}\text { C } & 2.11581600 & -3.31663300 & 0.15416600\end{array}$

$\begin{array}{llll}\text { C } & 2.27458900 & -2.39427500 & -0.87807200\end{array}$

$\begin{array}{llll}\text { C } & 1.86320100 & -1.07496900 & -0.67781600\end{array}$

$\begin{array}{llll}\mathrm{C} & 1.29953700 & -0.66667900 & 0.53775200\end{array}$

$\begin{array}{llll}\mathrm{C} & 1.16140800 & -1.62053100 & 1.55722400\end{array}$

$\begin{array}{llll}\mathrm{C} & 1.56149400 & -2.94538200 & 1.37766500\end{array}$

$\mathrm{H} \quad 2.71148500 \quad-2.69537700 \quad-1.82466500$

$\mathrm{H} \quad \begin{array}{llll}\mathrm{H} & 1.98880100 & -0.36041400 & -1.48361000\end{array}$

$\mathrm{H} \quad 0.73842500 \quad-1.32650200 \quad 2.51496800$

$\mathrm{H} \quad 1.45045700 \quad-3.66901500 \quad 2.17860800$ 


$\begin{array}{lrrr}\mathrm{C} & 0.83550900 & 0.76996300 & 0.80385600 \\ \mathrm{H} & 1.04548900 & 0.93950400 & 1.86547100 \\ \mathrm{Cl} & 2.63624000 & -4.98911500 & -0.08975500 \\ \mathrm{C} & 1.65577200 & 1.82999100 & 0.06653500 \\ \mathrm{C} & 2.83875300 & 2.27387100 & 0.68448000 \\ \mathrm{C} & 1.31475000 & 2.38174700 & -1.17751300 \\ \mathrm{C} & 3.65790800 & 3.22938800 & 0.08191500 \\ \mathrm{H} & 3.11948800 & 1.86293900 & 1.65201300 \\ \mathrm{C} & 2.13178800 & 3.34399200 & -1.78081600 \\ \mathrm{H} & 0.41452700 & 2.05088800 & -1.68022200 \\ \mathrm{C} & 3.30552500 & 3.77090600 & -1.15762000 \\ \mathrm{H} & 4.56607300 & 3.55436400 & 0.58323600 \\ \mathrm{H} & 1.84524000 & 3.75830800 & -2.74429400 \\ \mathrm{H} & 3.93747200 & 4.51878500 & -1.62947300 \\ \mathrm{C} & -2.82289000 & -0.23300300 & -0.37401000 \\ \mathrm{C} & -3.41564300 & -0.79464800 & -1.51997100 \\ \mathrm{C} & -3.57130000 & -0.17241500 & 0.81437000 \\ \mathrm{C} & -4.71754200 & -1.28490600 & -1.48045500 \\ \mathrm{H} & -2.83594100 & -0.83791800 & -2.43606400 \\ \mathrm{C} & -4.87821700 & -0.66100100 & 0.85224900 \\ \mathrm{H} & -3.15011600 & 0.25479800 & 1.71796200 \\ \mathrm{C} & -5.45332200 & -1.21882600 & -0.29195500 \\ \mathrm{H} & -5.16110600 & -1.71770000 & -2.37293100 \\ \mathrm{H} & -5.44573200 & -0.60651700 & 1.77697900 \\ \mathrm{H} & -6.47017300 & -1.60059700 & -0.25883600\end{array}$

\section{5'}

$\mathrm{E}(\mathrm{SMD} / \mathrm{B} 3 \mathrm{LYP} / \mathrm{BS} 1)=-2713.85471853 \mathrm{au}$

$\mathrm{H}(\mathrm{SMD} / \mathrm{B} 3 \mathrm{LYP} / \mathrm{BS} 1)=-2713.498995 \mathrm{au}$

$\mathrm{G}(\mathrm{SMD} / \mathrm{B} 3 \mathrm{LYP} / \mathrm{BS} 1)=-2713.602025 \mathrm{au}$

$\mathrm{E}(\mathrm{SMD} / \mathrm{M} 06 / \mathrm{BS} 2 / / \mathrm{SMD} / \mathrm{B} 3 \mathrm{LYP} / \mathrm{BS} 1)=-2713.82281602 \mathrm{au}$

$\begin{array}{llll}\mathrm{C} & 3.13192900 & -2.49222400 & -1.02461200 \\ \mathrm{H} & 4.13747100 & -2.25124100 & -0.66818500 \\ \mathrm{H} & 3.20979000 & -2.68675700 & -2.10644200 \\ \mathrm{H} & 2.72678700 & -3.37489400 & -0.52745500 \\ \mathrm{C} & 2.22519800 & -1.32568200 & -0.87202000 \\ \mathrm{O} & 1.04586600 & -1.57606500 & -0.51968800 \\ \mathrm{C} & 2.76438900 & 0.04851400 & -1.15428000 \\ \mathrm{H} & 3.62444900 & -0.06958100 & -1.81629200 \\ \mathrm{C} & 1.82747100 & 1.01336400 & -1.83246200 \\ \mathrm{C} & 2.39754300 & 1.95432700 & -2.83290200 \\ \mathrm{H} & 2.57219400 & 1.37307900 & -3.75174500 \\ \mathrm{H} & 1.70747900 & 2.77180000 & -3.04545600 \\ \mathrm{H} & 3.37058900 & 2.32705000 & -2.49616700 \\ \mathrm{O} & 0.59270500 & 1.09764200 & -1.62094000 \\ \mathrm{Au} & -0.44992100 & -0.17290700 & -0.38310600 \\ \mathrm{O} & -1.99573100 & 1.13598400 & -0.44260100 \\ \mathrm{~S} & -2.40116100 & 2.04519200 & 0.79823200 \\ \mathrm{O} & -3.81962200 & 2.34576100 & 0.66108100 \\ \mathrm{O} & -1.83981500 & 1.55361900 & 2.05196600 \\ \mathrm{C} & -1.47995600 & 3.61354500 & 0.37163700\end{array}$




$\begin{array}{lrrr}\text { F } & -1.85565200 & 4.04877800 & -0.82625900 \\ \text { F } & -1.74737200 & 4.53043200 & 1.29539700 \\ \text { F } & -0.16361400 & 3.36204000 & 0.36069900 \\ \text { O } & -1.71693500 & -2.98994100 & -1.09432300 \\ \text { S } & -2.20266000 & -2.68874600 & 0.25109100 \\ \text { O } & -2.23213700 & -3.70101800 & 1.29820200 \\ \text { O } & -1.47032500 & -1.40858100 & 0.85549900 \\ \text { C } & -3.95048400 & -2.04448400 & 0.06496600 \\ \text { F } & -4.73780700 & -3.06567300 & -0.26099100 \\ \text { F } & -4.35333000 & -1.50467000 & 1.21029000 \\ \text { F } & -3.97976300 & -1.12685000 & -0.90233600 \\ \text { C } & 3.38714800 & 0.75904200 & 0.19297800 \\ \text { C } & 2.36270000 & 0.92411300 & 1.31419200 \\ \text { H } & 1.51484400 & 1.54167300 & 1.00134300 \\ \text { H } & 1.97780700 & -0.02578500 & 1.69850500 \\ \text { H } & 2.84065400 & 1.44466400 & 2.15038700 \\ \text { C } & 4.68624100 & 0.07976500 & 0.60116800 \\ \text { C } & 4.76690300 & -0.78294200 & 1.70398800 \\ \text { C } & 5.85522100 & 0.35692500 & -0.12647700 \\ \text { C } & 5.98987900 & -1.35305700 & 2.06874600 \\ \text { H } & 3.88398300 & -1.01052800 & 2.29281900 \\ \text { C } & 7.07465700 & -0.21433300 & 0.23528700 \\ \text { H } & 5.81078300 & 1.03452000 & -0.97645700 \\ \text { C } & 7.14486900 & -1.07266400 & 1.33668700 \\ \text { H } & 6.03583600 & -2.01441700 & 2.92947300 \\ \text { H } & 7.96995600 & 0.01874400 & -0.33440100 \\ \text { H } & 8.09453200 & -1.51524900 & 1.62400600 \\ \text { H } & 3.65134000 & 1.75245200 & -0.18386800\end{array}$

TS $_{\text {23-25' }}$

$\begin{array}{lccc}\mathrm{E}(\mathrm{SMD} / \mathrm{B} 3 \mathrm{LYP} / \mathrm{BS} 1)=-2713.84666801 \mathrm{au} \\ \mathrm{H}(\mathrm{SMD} / \mathrm{B} 3 \mathrm{LYP} / \mathrm{BS} 1)=-2713.491842 \mathrm{au} \\ \mathrm{G}(\mathrm{SMD} / \mathrm{B} 3 \mathrm{LP} / \mathrm{BS} 1)=-2713.593412 \mathrm{au} \\ \mathrm{E}(\mathrm{SMD} / \mathrm{M} 06 / \mathrm{BS} 2 / / \mathrm{SMD} / \mathrm{B} 3 \mathrm{LYP} / \mathrm{BS} 1)=-2713.80997584 \mathrm{au} \\ \mathrm{C} & 2.78970300 & -2.86255200 & -0.66668500 \\ \mathrm{H} & 3.79216800 & -2.65755000 & -0.27673900 \\ \mathrm{H} & 2.90828200 & -3.21492800 & -1.70119500 \\ \mathrm{H} & 2.29048400 & -3.63375000 & -0.07790200 \\ \mathrm{C} & 1.98095700 & -1.60802100 & -0.70445700 \\ \mathrm{O} & 0.77536300 & -1.73108500 & -0.31170700 \\ \mathrm{C} & 2.59957100 & -0.38848200 & -1.15975300 \\ \mathrm{H} & 3.55074100 & -0.55027600 & -1.65316500 \\ \mathrm{C} & 1.84508600 & 0.69354600 & -1.74422200 \\ \mathrm{C} & 2.53865700 & 1.63857200 & -2.67189500 \\ \mathrm{H} & 2.68999000 & 1.12167900 & -3.62973700 \\ \mathrm{H} & 1.94007500 & 2.53590200 & -2.83611400 \\ \mathrm{H} & 3.52660300 & 1.89706100 & -2.27695800 \\ \mathrm{O} & 0.61253400 & 0.94098700 & -1.54195700 \\ \mathrm{Au} & -0.55701800 & -0.19499400 & -0.32274500 \\ \mathrm{O} & -1.96707800 & 1.26345400 & -0.49238700 \\ \mathrm{~S} & -2.28036700 & 2.28183900 & 0.67662600\end{array}$




$\begin{array}{lccc}\mathrm{O} & -3.59031800 & 2.85948700 & 0.40548100 \\ \mathrm{O} & -1.93981800 & 1.74578200 & 1.99093700 \\ \mathrm{C} & -1.02251900 & 3.61571600 & 0.32244500 \\ \mathrm{~F} & -1.13312700 & 4.03300900 & -0.93560300 \\ \mathrm{~F} & -1.22425400 & 4.63177500 & 1.15657800 \\ \mathrm{~F} & 0.20982900 & 3.12120900 & 0.51745800 \\ \mathrm{O} & -1.86584100 & -3.23027500 & -0.58181400 \\ \mathrm{~S} & -2.53284700 & -2.52779000 & 0.51118900 \\ \mathrm{O} & -2.92833700 & -3.22585900 & 1.72832000 \\ \mathrm{O} & -1.74791000 & -1.22863300 & 0.96165000 \\ \mathrm{C} & -4.08604900 & -1.78241500 & -0.21497100 \\ \mathrm{~F} & -4.93881200 & -2.76632600 & -0.49057200 \\ \mathrm{~F} & -4.63404900 & -0.93658700 & 0.65267400 \\ \mathrm{~F} & -3.77982600 & -1.13019300 & -1.34174900 \\ \mathrm{C} & 3.48227500 & 0.51004300 & 0.56043300 \\ \mathrm{C} & 2.51498700 & 0.33776500 & 1.69021900 \\ \mathrm{H} & 1.53066800 & 0.75585800 & 1.45787100 \\ \mathrm{H} & 2.40795400 & -0.69187400 & 2.03957300 \\ \mathrm{H} & 2.91711800 & 0.92863700 & 2.52740900 \\ \mathrm{C} & 4.82752500 & -0.04212400 & 0.60255000 \\ \mathrm{C} & 5.17731000 & -1.12398700 & 1.44187700 \\ \mathrm{C} & 5.83143500 & 0.56759100 & -0.18553900 \\ \mathrm{C} & 6.49461200 & -1.56814400 & 1.49663100 \\ \mathrm{H} & 4.42515700 & -1.60374700 & 2.05900000 \\ \mathrm{C} & 7.14452600 & 0.11845600 & -0.12737100 \\ \mathrm{H} & 5.56731000 & 1.40397200 & -0.82778100 \\ \mathrm{C} & 7.47686400 & -0.95125400 & 0.71334600 \\ \mathrm{H} & 6.75922800 & -2.39327400 & 2.15067700 \\ \mathrm{H} & 7.91133600 & 0.59964200 & -0.72669100 \\ \mathrm{H} & 8.50368300 & -1.30233400 & 0.76039000 \\ \mathrm{H} & 3.43875500 & 1.48959800 & 0.09025300\end{array}$

\section{0(THF)}

$\mathrm{E}(\mathrm{SMD} / \mathrm{B} 3 \mathrm{LYP} / \mathrm{BS} 1)=-3365.80280638 \mathrm{au}$

$\mathrm{H}(\mathrm{SMD} / \mathrm{B} 3 \mathrm{LYP} / \mathrm{BS} 1)=-3365.556097 \mathrm{au}$

G (SMD/B3LYP/BS1) $=-3365.664553$ au

$\mathrm{E}(\mathrm{SMD} / \mathrm{M} 06 / \mathrm{BS} 2 / / \mathrm{SMD} / \mathrm{B} 3 \mathrm{LYP} / \mathrm{BS} 1)=-3366.02319445 \mathrm{au}$

$\mathrm{C}$

$\begin{array}{lll}1.84516000 & 3.48916900 & -1.04816800\end{array}$

$\mathrm{H} \quad 2.73568100 \quad 4.04828400 \quad-0.71590800$

$\mathrm{H} \quad 1.28825000 \quad 4.10675200 \quad-1.75459600$

$\begin{array}{llll}\mathrm{H} & 2.20604100 & 2.56725500 & -1.50883100\end{array}$

$\begin{array}{llll}\mathrm{C} & 1.04687900 & 3.21039900 & 0.15963500\end{array}$

$\begin{array}{llll}\mathrm{O} & 0.07525000 & 3.99143500 & 0.43189700\end{array}$

$\begin{array}{llll}\mathrm{C} & 1.36607700 & 2.10187800 & 1.08411900\end{array}$

$\begin{array}{llll}\mathrm{C} & 0.85574000 & 2.27280700 & 2.50084200\end{array}$

$\begin{array}{llll}\mathrm{O} & -0.10306000 & 3.02678100 & 2.71148100\end{array}$

$\begin{array}{llll}\mathrm{C} & 1.55242100 & 1.53807900 & 3.59751700\end{array}$

$\begin{array}{llll}\mathrm{H} & 2.52461700 & 2.02178900 & 3.77083900\end{array}$

$\mathrm{H} \quad \begin{array}{llll}0.96253000 & 1.57506200 & 4.51523900\end{array}$

$\mathrm{H} \quad \begin{array}{llll}\mathrm{H} & 1.75932200 & 0.50067100 & 3.31382900\end{array}$

$\begin{array}{llll}\mathrm{H} & 2.41545500 & 1.81872800 & 1.02216000\end{array}$ 


$\begin{array}{lrrr}\mathrm{H} & -0.29130500 & 3.72910300 & 1.37075100 \\ \mathrm{Au} & 0.39333700 & 0.32219200 & 0.37009300 \\ \mathrm{O} & -0.53318000 & -1.46567500 & -0.24121000 \\ \mathrm{~S} & -0.76750700 & -2.62952100 & 0.77563700 \\ \mathrm{O} & -0.62899300 & -3.91267500 & 0.09185400 \\ \mathrm{O} & -0.09898600 & -2.39927900 & 2.05868200 \\ \mathrm{C} & -2.58831500 & -2.42456900 & 1.11889300 \\ \mathrm{~F} & -3.28938800 & -2.52999100 & -0.01004900 \\ \mathrm{~F} & -2.97773700 & -3.37356000 & 1.97271700 \\ \mathrm{~F} & -2.81140400 & -1.22405400 & 1.66786600 \\ \mathrm{O} & -2.80978400 & 3.02537800 & -0.80049900 \\ \mathrm{~S} & -1.97591100 & 1.87033500 & -1.11319000 \\ \mathrm{O} & -0.94188100 & 1.94271400 & -2.14790800 \\ \mathrm{O} & -1.38622800 & 1.32958000 & 0.25554700 \\ \mathrm{C} & -3.16805700 & 0.51877400 & -1.62502000 \\ \mathrm{~F} & -3.93352000 & 1.00951000 & -2.59992300 \\ \mathrm{~F} & -2.50507100 & -0.54212300 & -2.07350500 \\ \mathrm{~F} & -3.92854800 & 0.17659300 & -0.58800700 \\ \mathrm{O} & 3.63161500 & 0.65394100 & -0.78461800 \\ \mathrm{~S} & 3.34727600 & -0.70363000 & -0.31301000 \\ \mathrm{O} & 4.40105400 & -1.51539800 & 0.28211900 \\ \mathrm{O} & 2.11394600 & -0.74615800 & 0.66800800 \\ \mathrm{C} & 2.71060800 & -1.63779500 & -1.80116400 \\ \mathrm{~F} & 3.69538300 & -1.73376100 & -2.69175100 \\ \mathrm{~F} & 2.30451800 & -2.84987000 & -1.44172100 \\ \mathrm{~F} & 1.68831500 & -0.96311900 & -2.34280200 \\ & & & \end{array}$

\section{2(THF)}

$\mathrm{E}(\mathrm{SMD} / \mathrm{B} 3 \mathrm{LYP} / \mathrm{BS} 1)=-309.650252799 \mathrm{au}$

$\mathrm{H}(\mathrm{SMD} / \mathrm{B} 3 \mathrm{LYP} / \mathrm{BS} 1)=-309.508754 \mathrm{au}$

$\mathrm{G}(\mathrm{SMD} / \mathrm{B} 3 \mathrm{LYP} / \mathrm{BS} 1)=-309.54738 \mathrm{au}$

$\mathrm{E}(\mathrm{SMD} / \mathrm{M} 06 / \mathrm{BS} 2 / / \mathrm{SMD} / \mathrm{B} 3 \mathrm{LYP} / \mathrm{BS} 1)=-309.510317925 \mathrm{au}$

$\begin{array}{llll}\mathrm{C} & -0.68612700 & -1.77694600 & 0.00000000\end{array}$

$\begin{array}{llll}\text { C } & -1.00916100 & -0.42185200 & 0.00000000\end{array}$

$\begin{array}{llll}\mathrm{C} & 0.00000000 & 0.56101200 & 0.00000000\end{array}$

$\begin{array}{llll}\mathrm{C} & 1.34012600 & 0.13231400 & 0.00000000\end{array}$

C $\quad 1.66502800 \quad-1.22502600 \quad 0.00000000$

$\begin{array}{llll}\mathrm{C} & 0.65236300 & -2.18653500 & 0.00000000\end{array}$

$\mathrm{H} \quad-1.48116500 \quad-2.51834300 \quad 0.00000000$

$\mathrm{H} \quad-2.05402100 \quad-0.12442400 \quad 0.00000000$

$\begin{array}{llll}\mathrm{H} & 2.13336800 & 0.87662900 & 0.00000000\end{array}$

$\mathrm{H} \quad 2.70839300 \quad-1.52997400 \quad 0.00000000$

$\begin{array}{llll}\mathrm{H} & 0.90029400 & -3.24466400 & 0.00000000\end{array}$

$\begin{array}{llll}\mathrm{C} & -0.28304600 & 2.00753300 & 0.00000000\end{array}$

$\begin{array}{llll}\mathrm{H} & 0.60501500 & 2.64012200 & 0.00000000\end{array}$

$\begin{array}{llll}\mathrm{C} & -1.48364100 & 2.60342300 & 0.00000000\end{array}$

$\begin{array}{llll}\mathrm{H} & -2.41984700 & 2.05005400 & 0.00000000\end{array}$

$\mathrm{H} \quad-1.56528200 \quad 3.68707000 \quad 0.00000000$

\section{3(THF)}

$\mathrm{E}(\mathrm{SMD} / \mathrm{B} 3 \mathrm{LYP} / \mathrm{BS} 1)=-775.009540537 \mathrm{au}$ 


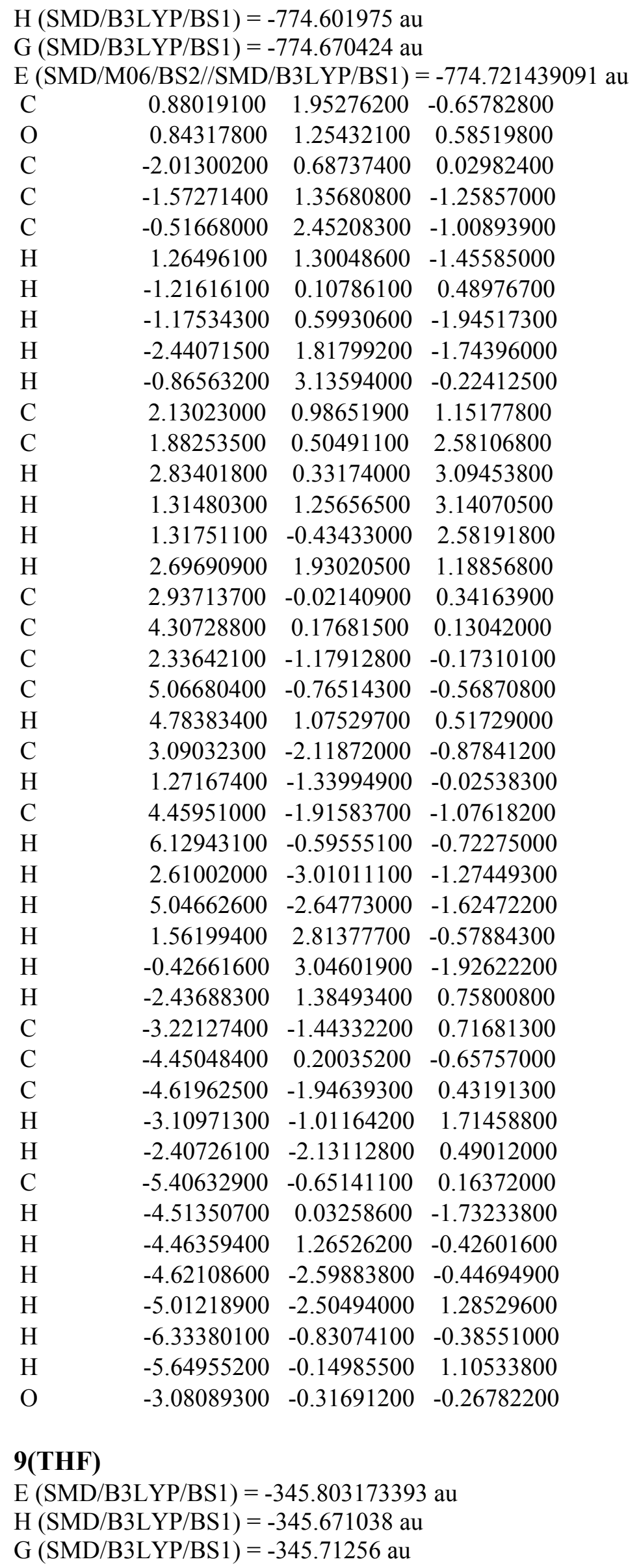




$\begin{array}{lrrr}\text { E }(\mathrm{SMD} / \mathrm{M} 06 / \mathrm{BS} 2 / / \mathrm{SMD} / \mathrm{B} 3 \mathrm{LYP} / \mathrm{BS} 1) & =-345.724853378 \mathrm{au} \\ \mathrm{C} & 2.54746600 & -0.76205600 & -0.00000700 \\ \mathrm{H} & 2.60634200 & -1.41195400 & 0.88213200 \\ \mathrm{H} & 3.39396900 & -0.07145600 & -0.00001200 \\ \mathrm{H} & 2.60633800 & -1.41196800 & -0.88213500 \\ \mathrm{C} & 1.24679400 & 0.00674200 & -0.00000500 \\ \mathrm{O} & 1.26599500 & 1.26153900 & 0.00005600 \\ \mathrm{C} & 0.00854200 & -0.73393700 & -0.00001400 \\ \mathrm{C} & -1.20128100 & -0.08326500 & -0.00000700 \\ \mathrm{C} & -2.52624200 & -0.77593800 & 0.00001000 \\ \mathrm{H} & -3.10513700 & -0.47662400 & -0.88243600 \\ \mathrm{H} & -2.41018300 & -1.86205900 & 0.00003700 \\ \mathrm{H} & -3.10513700 & -0.47658000 & 0.88244000 \\ \mathrm{O} & -1.28370600 & 1.24460500 & -0.00003000 \\ \mathrm{H} & -0.32621400 & 1.56966500 & -0.00007700 \\ \mathrm{H} & 0.03002900 & -1.81745000 & -0.00001700\end{array}$

\section{2(THF)}

$\mathrm{E}(\mathrm{SMD} / \mathrm{B} 3 \mathrm{LYP} / \mathrm{BS} 1)=-3675.45921224 \mathrm{au}$

$\mathrm{H}(\mathrm{SMD} / \mathrm{B} 3 \mathrm{LYP} / \mathrm{BS} 1)=-3675.068672 \mathrm{au}$

$\mathrm{G}(\mathrm{SMD} / \mathrm{B} 3 \mathrm{LYP} / \mathrm{BS} 1)=-3675.203445 \mathrm{au}$

$\mathrm{E}(\mathrm{SMD} / \mathrm{M} 06 / \mathrm{BS} 2 / / \mathrm{SMD} / \mathrm{B} 3 \mathrm{LYP} / \mathrm{BS} 1)=-3675.53777431 \mathrm{au}$

$\begin{array}{lccc}\mathrm{C} & 1.10727000 & -3.16096000 & -1.50543700 \\ \mathrm{H} & 0.96482300 & -4.21719000 & -1.23639300 \\ \mathrm{H} & 1.94444700 & -3.07807000 & -2.20075700 \\ \mathrm{H} & 0.18211300 & -2.82225800 & -1.97874300 \\ \mathrm{C} & 1.39689700 & -2.38864900 & -0.24598400 \\ \mathrm{O} & 2.51900100 & -2.01173700 & 0.06023800 \\ \mathrm{C} & 0.22999900 & -2.17799600 & 0.71574900 \\ \mathrm{C} & 0.68392200 & -2.03891500 & 2.16537000 \\ \mathrm{O} & 0.70498600 & -0.96077700 & 2.73425200 \\ \mathrm{C} & 1.07217800 & -3.32977900 & 2.84569800 \\ \mathrm{H} & 1.73758400 & -3.93259500 & 2.21774900 \\ \mathrm{H} & 1.55214400 & -3.11573000 & 3.80398000 \\ \mathrm{H} & 0.16463700 & -3.92288400 & 3.02202600 \\ \mathrm{H} & -0.49644500 & -2.98504900 & 0.60197900 \\ \mathrm{Au} & -0.91284700 & -0.46519700 & 0.28005500 \\ \mathrm{O} & -2.17244600 & 1.30797300 & 0.02797500 \\ \mathrm{~S} & -2.79667100 & 1.97160700 & 1.26782800 \\ \mathrm{O} & -4.13490000 & 2.49404500 & 0.97991800 \\ \mathrm{O} & -2.57993900 & 1.20301400 & 2.50079200 \\ \mathrm{C} & -1.72006900 & 3.47914400 & 1.45380400 \\ \mathrm{~F} & -1.80262400 & 4.25722500 & 0.36960400 \\ \mathrm{~F} & -2.12088000 & 4.18225900 & 2.52086500 \\ \mathrm{~F} & -0.44055500 & 3.12193500 & 1.63425500 \\ \mathrm{O} & 2.64782800 & 1.47024900 & -1.45917200 \\ \mathrm{~S} & 1.29314500 & 0.92279400 & -1.57799700 \\ \mathrm{O} & 1.03201100 & -0.19271900 & -2.48874500 \\ \mathrm{O} & 0.74437000 & 0.68521600 & -0.13572700 \\ \mathrm{C} & 0.25933200 & 2.34118900 & -2.21943800 \\ \mathrm{~F} & 0.82841800 & 2.79179600 & -3.34183400\end{array}$




$\begin{array}{lrrr}\text { F } & -0.97532700 & 1.92231800 & -2.49525900 \\ \text { F } & 0.21404200 & 3.32548400 & -1.32350600 \\ \text { O } & -2.35200000 & -3.41591600 & -0.85922000 \\ \text { S } & -3.29497200 & -2.55138000 & -0.14380000 \\ \text { O } & -4.41634500 & -3.13837900 & 0.58289000 \\ \text { O } & -2.58481700 & -1.53384500 & 0.81293800 \\ \text { C } & -4.03958200 & -1.43972500 & -1.44634600 \\ \text { F } & -4.76125000 & -2.19018200 & -2.28078100 \\ \text { F } & -4.81999100 & -0.52271000 & -0.88284600 \\ \text { F } & -3.06566000 & -0.83230900 & -2.13875300 \\ \text { C } & 4.95531800 & 0.38527000 & 0.63173500 \\ \text { C } & 6.28746900 & 0.08578100 & 0.33533000 \\ \text { C } & 7.20653300 & -0.41854200 & 1.30775800 \\ \text { C } & 6.73391500 & 0.28868600 & -1.00863900 \\ \text { C } & 8.50620100 & -0.70388200 & 0.94045600 \\ \text { H } & 6.88277300 & -0.57344600 & 2.33084300 \\ \text { C } & 8.03791300 & -0.00297500 & -1.36223900 \\ \text { H } & 6.02949200 & 0.67003600 & -1.74188900 \\ \text { C } & 8.91904000 & -0.49712300 & -0.38863600 \\ \text { H } & 9.21141800 & -1.08613400 & 1.67103700 \\ \text { H } & 8.37977400 & 0.14706700 & -2.38082800 \\ \text { H } & 9.94454800 & -0.72477600 & -0.66600800 \\ \text { H } & 4.34945200 & 0.77369700 & -0.18825400 \\ \text { C } & 4.24378900 & 0.21827300 & 1.89718800 \\ \mathrm{H} & 3.70589600 & 1.14903100 & 2.13256100 \\ \mathrm{H} & 3.44036300 & -0.51509500 & 1.70437700 \\ \mathrm{H} & 4.84958700 & -0.10250100 & 2.74443800\end{array}$

\section{3(THF)}

$\mathrm{E}(\mathrm{SMD} / \mathrm{B} 3 \mathrm{LYP} / \mathrm{BS} 1)=-310.068625934 \mathrm{au}$

$\mathrm{H}(\mathrm{SMD} / \mathrm{B} 3 \mathrm{LYP} / \mathrm{BS} 1)=-309.915757 \mathrm{au}$

$\mathrm{G}(\mathrm{SMD} / \mathrm{B} 3 \mathrm{LYP} / \mathrm{BS} 1)=-309.953989 \mathrm{au}$

$\mathrm{E}(\mathrm{SMD} / \mathrm{M} 06 / \mathrm{BS} 2 / / \mathrm{SMD} / \mathrm{B} 3 \mathrm{LYP} / \mathrm{BS} 1)=-309.919916039 \mathrm{au}$

$\begin{array}{lrrr}\mathrm{C} & 1.33951700 & 1.37827000 & -0.00020800 \\ \mathrm{C} & -0.00979900 & 1.09666300 & -0.00055000 \\ \mathrm{C} & -0.45282400 & -0.26576600 & -0.00034400 \\ \mathrm{C} & 0.51952600 & -1.31771300 & 0.00006800 \\ \mathrm{C} & 1.86844000 & -1.01942600 & 0.00041400 \\ \mathrm{C} & 2.27407700 & 0.32398900 & 0.00027900 \\ \mathrm{H} & 1.68688700 & 2.40587100 & -0.00034100 \\ \mathrm{H} & -0.73803700 & 1.90082800 & -0.00096600 \\ \mathrm{H} & 0.17950600 & -2.34888600 & 0.00021300 \\ \mathrm{H} & 2.60918400 & -1.81198900 & 0.00078700 \\ \mathrm{H} & 3.33493300 & 0.55821100 & 0.00053300 \\ \mathrm{C} & -1.80134700 & -0.61568200 & -0.00026400 \\ \mathrm{H} & -2.01873900 & -1.68347800 & -0.00021800 \\ \mathrm{C} & -2.95642100 & 0.30173200 & 0.00022600 \\ \mathrm{H} & -3.90109700 & -0.24253400 & -0.00241300 \\ \mathrm{H} & -2.92101100 & 0.96212200 & 0.87902900 \\ \mathrm{H} & -2.91863900 & 0.96745800 & -0.87434500\end{array}$




\section{4(THF)}

$\mathrm{E}(\mathrm{SMD} / \mathrm{B} 3 \mathrm{LYP} / \mathrm{BS} 1)=-3365.37479564 \mathrm{au}$

$\mathrm{H}(\mathrm{SMD} / \mathrm{B} 3 \mathrm{LYP} / \mathrm{BS} 1)=-3365.140195 \mathrm{au}$

$\mathrm{G}(\mathrm{SMD} / \mathrm{B} 3 \mathrm{LYP} / \mathrm{BS} 1)=-3365.249632 \mathrm{au}$

$\mathrm{E}(\mathrm{SMD} / \mathrm{M} 06 / \mathrm{BS} 2 / / \mathrm{SMD} / \mathrm{B} 3 \mathrm{LYP} / \mathrm{BS} 1)=-3365.6023284 \mathrm{au}$

$\begin{array}{lllll}\mathrm{C} & 2.56771400 & 3.04941600 & -0.88271800\end{array}$

$\mathrm{H} \quad 3.62186400 \quad 3.10033700 \quad-0.57584800$

$\mathrm{H} \quad 2.31848100 \quad 3.94246000 \quad-1.45861200$

$\mathrm{H} \quad 2.45519100 \quad 2.15362300 \quad-1.49808600$

$\begin{array}{llll}\mathrm{C} & 1.71415500 & 2.98224000 & 0.35789000\end{array}$

$\begin{array}{llll}\mathrm{O} & 1.09164200 & 3.93881800 & 0.78827600\end{array}$

$\begin{array}{llll}\mathrm{C} & 1.73237600 & 1.66796700 & 1.13831100\end{array}$

$\begin{array}{llll}\mathrm{C} & 1.39437100 & 1.80088100 & 2.62016800\end{array}$

$\begin{array}{llll}\mathrm{O} & 0.44690200 & 1.20656300 & 3.10817400\end{array}$

$\begin{array}{llll}\mathrm{C} & 2.32103200 & 2.66372000 & 3.44180900\end{array}$

$\mathrm{H} \quad 2.16731200 \quad 3.71640400 \quad 3.17847300$

$\mathrm{H} \quad 2.10739200 \quad 2.52081700 \quad 4.50405900$

$\begin{array}{llll}\mathrm{H} & 3.36978900 & 2.42024000 & 3.23407500\end{array}$

$\begin{array}{llll}\mathrm{H} & 2.70017600 & 1.17472400 & 1.01553200\end{array}$

$\begin{array}{llll}\mathrm{Au} & 0.39950500 & 0.22253300 & 0.38341300\end{array}$

$\begin{array}{llll}\mathrm{O} & -0.99119900 & -1.36814100 & -0.21689300\end{array}$

$\begin{array}{lllll}\mathrm{S} & -1.47077300 & -2.42407100 & 0.78991300\end{array}$

$\begin{array}{llll}\mathrm{O} & -1.81089800 & -3.68054600 & 0.11510000\end{array}$

$\begin{array}{llll}\mathrm{O} & -0.66702300 & -2.47154900 & 2.01764400\end{array}$

C $\quad-3.10328200 \quad-1.71224400 \quad 1.33176200$

F $\quad-3.93950700 \quad-1.59122600 \quad 0.29486200$

F $\quad-3.66160100 \quad-2.52292600 \quad 2.24107100$

F $\quad-2.92801200 \quad-0.50480600 \quad 1.88811000$

$\begin{array}{llll}\mathrm{O} & -2.32484800 & 3.49360200 & -0.74826000\end{array}$

$\mathrm{S} \quad-1.47230700 \quad 2.35769800 \quad-1.09038300$

$\begin{array}{llll}\mathrm{O} & -0.31176900 & 2.53583300 & -1.96649500\end{array}$

$\begin{array}{lllll}\mathrm{O} & -1.13316200 & 1.58513300 & 0.22730600\end{array}$

$\begin{array}{lllll}\mathrm{C} & -2.58610600 & 1.16911400 & -2.00368800\end{array}$

F $\quad-3.14149800 \quad 1.82517100 \quad-3.02945300$

F $\quad \begin{array}{llll}-1.88127100 & 0.14129600 & -2.48065100\end{array}$

F $\quad-3.55023300 \quad 0.71770800 \quad-1.20166600$

O $\quad 3.65124200 \quad-0.18668200 \quad-0.83741500$

S $\quad 3.04291400 \quad-1.42962900 \quad-0.35364200$

$\begin{array}{llll}\text { O } & 3.89038100 & -2.49195600 & 0.18100800\end{array}$

O $\quad 1.87420500 \quad-1.19017900 \quad 0.65530700$

C $\quad 2.16748700 \quad-2.16105800 \quad-1.83109400$

F $\quad 3.08275100 \quad-2.49873300 \quad-2.74289100$

F $\quad 1.47747500 \quad-3.24153400 \quad-1.47434300$

F $\quad 1.33426100 \quad-1.25829100 \quad-2.36704400$

TS $_{23-25}$ (THF)

$\mathrm{E}(\mathrm{SMD} / \mathrm{B} 3 \mathrm{LYP} / \mathrm{BS} 1)=-655.875870899 \mathrm{au}$

$\mathrm{H}(\mathrm{SMD} / \mathrm{B} 3 \mathrm{LYP} / \mathrm{BS} 1)=-655.588766 \mathrm{au}$

$\mathrm{G}(\mathrm{SMD} / \mathrm{B} 3 \mathrm{LYP} / \mathrm{BS} 1)=-655.649052 \mathrm{au}$

$\mathrm{E}(\mathrm{SMD} / \mathrm{M} 06 / \mathrm{BS} 2 / / \mathrm{SMD} / \mathrm{B} 3 \mathrm{LYP} / \mathrm{BS} 1)=-655.655945548 \mathrm{au}$

$\begin{array}{llll}\mathrm{C} & 2.35654500 & -2.49380000 & 0.71724900\end{array}$ 


$\begin{array}{lrrr}\mathrm{H} & 3.41594600 & -2.58941200 & 0.44397400 \\ \mathrm{H} & 1.86792700 & -3.46073400 & 0.57799300 \\ \mathrm{C} & 1.71094600 & -1.45274600 & -0.15783800 \\ \mathrm{O} & 0.91170200 & -1.79135700 & -1.05907500 \\ \mathrm{C} & 2.04515700 & -0.05396300 & 0.05246100 \\ \mathrm{C} & 1.67612400 & 0.90274000 & -0.88884400 \\ \mathrm{O} & 0.84315200 & 0.61057000 & -1.86482900 \\ \mathrm{C} & 2.17891900 & 2.30604800 & -0.89265100 \\ \mathrm{H} & 1.34648800 & 3.01015300 & -1.00197300 \\ \mathrm{H} & 2.74640400 & 2.53536200 & 0.01080700 \\ \mathrm{H} & 2.83254900 & 2.44701100 & -1.76347200 \\ \mathrm{H} & 2.77988900 & 0.21100300 & 0.80311400 \\ \mathrm{H} & 0.64460900 & -0.38248600 & -1.77767200 \\ \mathrm{C} & -0.03028100 & 0.49330100 & 1.61890600 \\ \mathrm{H} & 0.39625300 & -0.35238900 & 2.15359400 \\ \mathrm{C} & -1.14086400 & 0.20875900 & 0.79319500 \\ \mathrm{C} & -1.59030000 & -1.14119400 & 0.70174100 \\ \mathrm{C} & -1.85533900 & 1.22100400 & 0.08834300 \\ \mathrm{C} & -2.72256000 & -1.45663500 & -0.03186800 \\ \mathrm{H} & -1.04941400 & -1.91525400 & 1.23742700 \\ \mathrm{C} & -2.98096700 & 0.89252600 & -0.64570200 \\ \mathrm{H} & -1.52099600 & 2.25116300 & 0.13168000 \\ \mathrm{C} & -3.41547100 & -0.44183700 & -0.70422800 \\ \mathrm{H} & -3.06978100 & -2.48317000 & -0.08732000 \\ \mathrm{H} & -3.52805600 & 1.66416700 & -1.17771300 \\ \mathrm{H} & -4.30126200 & -0.69013500 & -1.28207500 \\ \mathrm{C} & 0.38122700 & 1.83126800 & 2.10264400 \\ \mathrm{H} & -0.20627200 & 2.01823100 & 3.01882200 \\ \mathrm{H} & 1.43196100 & 1.84444600 & 2.40095800 \\ \mathrm{H} & 0.17177900 & 2.64832000 & 1.40930800 \\ \mathrm{H} & 2.32432200 & -2.20280600 & 1.77332400\end{array}$

\section{5(THF)}

\begin{tabular}{|c|c|c|c|}
\hline \multicolumn{4}{|c|}{$\mathrm{E}(\mathrm{SMD} / \mathrm{B} 3 \mathrm{LYP} / \mathrm{BS} 1)=-655.893229777 \mathrm{au}$} \\
\hline \multicolumn{4}{|c|}{$\mathrm{H}(\mathrm{SMD} / \mathrm{B} 3 \mathrm{LYP} / \mathrm{BS} 1)=-655.604587 \mathrm{au}$} \\
\hline \multicolumn{4}{|c|}{$\mathrm{G}(\mathrm{SMD} / \mathrm{B} 3 \mathrm{LYP} / \mathrm{BS} 1)=-655.663895 \mathrm{au}$} \\
\hline \multicolumn{4}{|c|}{$\mathrm{E}(\mathrm{SMD} / \mathrm{M} 06 / \mathrm{BS} 2 / / \mathrm{SMD} / \mathrm{B} 3 \mathrm{LYP} / \mathrm{BS} 1)=-655.678429276 \mathrm{au}$} \\
\hline $\mathrm{C}$ & 3.38672900 & -1.35758900 & 0.86232000 \\
\hline $\mathrm{H}$ & 3.68433100 & -0.89423800 & 1.81496000 \\
\hline $\mathrm{H}$ & 4.28299100 & -1.68861800 & 0.33363800 \\
\hline $\mathrm{C}$ & 2.66549400 & -0.31983400 & 0.06994300 \\
\hline $\mathrm{O}$ & 3.20995700 & 0.30703800 & -0.84510800 \\
\hline $\mathrm{C}$ & 1.20911900 & 0.00889700 & 0.41729800 \\
\hline $\mathrm{C}$ & 0.96882100 & 1.48422000 & 0.32227100 \\
\hline $\mathrm{O}$ & 1.57368900 & 2.15178600 & -0.57934200 \\
\hline $\mathrm{C}$ & 0.06974100 & 2.20697300 & 1.23410800 \\
\hline $\mathrm{H}$ & -0.14549000 & 3.21326500 & 0.87137300 \\
\hline $\mathrm{H}$ & -0.84569900 & 1.63486000 & 1.41350900 \\
\hline $\mathrm{H}$ & 0.59030000 & 2.27140000 & 2.20393300 \\
\hline $\mathrm{H}$ & 0.95029300 & -0.34447200 & 1.41768500 \\
\hline $\mathrm{H}$ & 2.29527100 & 1.53087200 & -1.00238300 \\
\hline
\end{tabular}




$\begin{array}{lrrr}\mathrm{C} & 0.23883100 & -0.73183400 & -0.64327400 \\ \mathrm{H} & 0.46959300 & -0.28508400 & -1.61610000 \\ \mathrm{C} & -1.22653900 & -0.47316400 & -0.32878000 \\ \mathrm{C} & -2.00294500 & 0.29495300 & -1.20755800 \\ \mathrm{C} & -1.83335100 & -1.00610500 & 0.82013600 \\ \mathrm{C} & -3.35623600 & 0.52645500 & -0.94754500 \\ \mathrm{H} & -1.54802000 & 0.71052200 & -2.10370300 \\ \mathrm{C} & -3.18401900 & -0.77157500 & 1.08250600 \\ \mathrm{H} & -1.25127600 & -1.60930800 & 1.51293500 \\ \mathrm{C} & -3.94939800 & -0.00415100 & 0.19917400 \\ \mathrm{H} & -3.94369500 & 1.12068300 & -1.64211800 \\ \mathrm{H} & -3.63998100 & -1.19179100 & 1.97486400 \\ \mathrm{H} & -5.00112800 & 0.17611400 & 0.40384400 \\ \mathrm{C} & 0.55384700 & -2.23396300 & -0.71925400 \\ \mathrm{H} & -0.15480200 & -2.69793700 & -1.41276700 \\ \mathrm{H} & 1.56192400 & -2.42564700 & -1.10144600 \\ \mathrm{H} & 0.44616100 & -2.72679100 & 0.25185200 \\ \mathrm{H} & 2.73949000 & -2.20410500 & 1.10746400\end{array}$

\begin{tabular}{|c|c|c|c|}
\hline \multicolumn{4}{|c|}{$\mathrm{TS}_{25-23}$ (THF) } \\
\hline \multicolumn{4}{|c|}{$\mathrm{E}(\mathrm{SMD} / \mathrm{B} 3 \mathrm{LYP} / \mathrm{BS} 1)=-965.532407628 \mathrm{au}$} \\
\hline \multicolumn{4}{|c|}{$\mathrm{H}(\mathrm{SMD} / \mathrm{B} 3 \mathrm{LYP} / \mathrm{BS} 1)=-965.104373 \mathrm{au}$} \\
\hline \multicolumn{4}{|c|}{$\mathrm{G}(\mathrm{SMD} / \mathrm{B} 3 \mathrm{LYP} / \mathrm{BS} 1)=-965.183594 \mathrm{au}$} \\
\hline \multicolumn{4}{|c|}{$\mathrm{E}(\mathrm{SMD} / \mathrm{M} 06 / \mathrm{BS} 2 / / \mathrm{SMD} / \mathrm{B} 3 \mathrm{LYP} / \mathrm{BS} 1)=-965.176995$} \\
\hline $\mathrm{C}$ & 2.13861000 & -0.22431400 & 2.70216100 \\
\hline $\mathrm{H}$ & 3.10843300 & -0.70010900 & 2.89981700 \\
\hline $\mathrm{H}$ & 1.45459700 & -0.39360000 & 3.53502700 \\
\hline $\mathrm{H}$ & 2.33570500 & 0.84912100 & 2.58114700 \\
\hline $\mathrm{C}$ & 1.58671600 & -0.75721900 & 1.42529100 \\
\hline $\mathrm{O}$ & 0.45829500 & -1.29672700 & 1.46249400 \\
\hline $\mathrm{C}$ & 2.42030000 & -0.63187300 & 0.17566800 \\
\hline $\mathrm{C}$ & 2.81895900 & -2.04923100 & -0.33414100 \\
\hline $\mathrm{O}$ & 1.96711600 & -2.88426600 & -0.56324200 \\
\hline $\mathrm{C}$ & 4.29291800 & -2.29567800 & -0.51694800 \\
\hline $\mathrm{H}$ & 4.73209700 & -1.52778800 & -1.16468800 \\
\hline $\mathrm{H}$ & 4.79963400 & -2.22088300 & 0.45415800 \\
\hline $\mathrm{H}$ & 4.45918700 & -3.28688700 & -0.94415300 \\
\hline $\mathrm{H}$ & 3.33147900 & -0.09148200 & 0.44325900 \\
\hline $\mathrm{C}$ & -4.22969300 & -0.97588900 & 0.95924100 \\
\hline $\mathrm{C}$ & -5.18640600 & 0.00677500 & 0.72407900 \\
\hline $\mathrm{C}$ & -5.13534000 & 0.75610500 & -0.45473900 \\
\hline $\mathrm{C}$ & -4.12893500 & 0.51967700 & -1.40137500 \\
\hline $\mathrm{C}$ & -3.17092100 & -0.45973800 & -1.17102000 \\
\hline $\mathrm{C}$ & -3.20267600 & -1.22026000 & 0.02086100 \\
\hline $\mathrm{H}$ & -4.26244700 & -1.56164500 & 1.87401600 \\
\hline $\mathrm{H}$ & -5.97049800 & 0.18769200 & 1.45311900 \\
\hline $\mathrm{H}$ & -5.88363400 & 1.52093000 & -0.64278800 \\
\hline $\mathrm{H}$ & -4.10333000 & 1.09629300 & -2.32122300 \\
\hline $\mathrm{H}$ & -2.40941000 & -0.65224200 & -1.91994700 \\
\hline $\mathrm{C}$ & -2.23701000 & -2.25527200 & 0.31759800 \\
\hline $\mathrm{H}$ & -2.45097000 & -2.83298800 & 1.21755500 \\
\hline
\end{tabular}




$\begin{array}{lrrr}\mathrm{C} & -1.05442100 & -2.54068800 & -0.33252900 \\ \mathrm{H} & -0.83553300 & -2.10809200 & -1.30733500 \\ \mathrm{H} & -0.18776500 & -1.81637900 & 0.53251700 \\ \mathrm{H} & -0.58506000 & -3.50201500 & -0.12840400 \\ \mathrm{C} & 1.67712400 & 0.18767600 & -0.94522900 \\ \mathrm{H} & 0.74603700 & -0.34219100 & -1.16893900 \\ \mathrm{C} & 1.32127700 & 1.58201800 & -0.43925400 \\ \mathrm{C} & -0.01983700 & 1.95580000 & -0.28063400 \\ \mathrm{C} & 2.31895700 & 2.52210000 & -0.13296000 \\ \mathrm{C} & -0.36047000 & 3.23423400 & 0.16991800 \\ \mathrm{H} & -0.80627400 & 1.24300000 & -0.51615300 \\ \mathrm{C} & 1.98169200 & 3.79873400 & 0.31964800 \\ \mathrm{H} & 3.36703100 & 2.26043900 & -0.25609800 \\ \mathrm{C} & 0.63959400 & 4.15953400 & 0.47256400 \\ \mathrm{H} & -1.40742900 & 3.50370900 & 0.28184700 \\ \mathrm{H} & 2.76768900 & 4.51346300 & 0.54890500 \\ \mathrm{H} & 0.37770400 & 5.15439300 & 0.82253800 \\ \mathrm{C} & 2.51003200 & 0.24609100 & -2.23689900 \\ \mathrm{H} & 3.48902000 & 0.70795000 & -2.06951800 \\ \mathrm{H} & 2.66700700 & -0.75107500 & -2.66257700 \\ \mathrm{H} & 1.98062200 & 0.84683600 & -2.98390200\end{array}$

\begin{tabular}{|c|c|c|c|}
\hline \multicolumn{4}{|c|}{$\mathbf{T S}_{25-\mathrm{THF}}$} \\
\hline \multicolumn{4}{|c|}{$\mathrm{E}(\mathrm{SMD} / \mathrm{B} 3 \mathrm{LYP} / \mathrm{BS} 1)=-542.529950006 \mathrm{au}$} \\
\hline \multicolumn{4}{|c|}{$\mathrm{H}(\mathrm{SMD} / \mathrm{B} 3 \mathrm{LYP} / \mathrm{BS} 1)=-542.252016 \mathrm{au}$} \\
\hline \multicolumn{4}{|c|}{$\mathrm{G}(\mathrm{SMD} / \mathrm{B} 3 \mathrm{LYP} / \mathrm{BS} 1)=-542.303226 \mathrm{au}$} \\
\hline \multicolumn{4}{|c|}{$\mathrm{E}(\mathrm{SMD} / \mathrm{M} 06 / \mathrm{BS} 2 / / \mathrm{SMD} / \mathrm{B} 3 \mathrm{LYP} / \mathrm{BS} 1)=-542.311557537 \mathrm{au}$} \\
\hline $\mathrm{C}$ & -1.26814700 & -1.28394200 & -0.15960500 \\
\hline $\mathrm{O}$ & -1.53997200 & 0.03809800 & -0.71974700 \\
\hline $\mathrm{C}$ & -2.92173400 & 0.40584800 & -0.42758800 \\
\hline $\mathrm{C}$ & -3.32632000 & -0.48009300 & 0.73919600 \\
\hline $\mathrm{C}$ & -2.61061100 & -1.78804500 & 0.37343600 \\
\hline $\mathrm{H}$ & -0.84159300 & -1.90710600 & -0.94949800 \\
\hline $\mathrm{H}$ & -0.53355100 & -1.17351400 & 0.64512600 \\
\hline $\mathrm{H}$ & -2.96599900 & 1.47814200 & -0.23122200 \\
\hline $\mathrm{H}$ & -3.51529100 & 0.19068300 & -1.32482800 \\
\hline $\mathrm{H}$ & -2.93884500 & -0.07452600 & 1.68141800 \\
\hline $\mathrm{H}$ & -4.41165300 & -0.58322300 & 0.82477400 \\
\hline $\mathrm{H}$ & -2.48000900 & -2.46920000 & 1.21893100 \\
\hline $\mathrm{H}$ & -3.16320400 & -2.31466900 & -0.41267300 \\
\hline $\mathrm{C}$ & 0.10565800 & 1.46640600 & -0.55061300 \\
\hline $\mathrm{C}$ & -0.48278500 & 2.48850400 & 0.33996900 \\
\hline $\mathrm{H}$ & 0.14977900 & 3.38572000 & 0.23183800 \\
\hline $\mathrm{H}$ & -1.48224400 & 2.78260000 & 0.01533100 \\
\hline $\mathrm{H}$ & -0.49349800 & 2.20857800 & 1.39514400 \\
\hline $\mathrm{H}$ & -0.07582600 & 1.59402500 & -1.61312200 \\
\hline $\mathrm{C}$ & 1.21665700 & 0.63496900 & -0.21712300 \\
\hline $\mathrm{C}$ & 1.86998200 & -0.06039800 & -1.26820800 \\
\hline $\mathrm{C}$ & 1.71224500 & 0.50915900 & 1.10580800 \\
\hline $\mathrm{C}$ & 2.98050400 & -0.84879900 & -1.00645000 \\
\hline $\mathrm{H}$ & 1.49014300 & 0.03671900 & -2.28159400 \\
\hline
\end{tabular}




$\begin{array}{lrrr}\mathrm{C} & 2.82477500 & -0.28006100 & 1.35856100 \\ \mathrm{H} & 1.23059400 & 1.03325700 & 1.92364000 \\ \mathrm{C} & 3.45617200 & -0.95840500 & 0.30663800 \\ \mathrm{H} & 3.47991700 & -1.37582300 & -1.81311800 \\ \mathrm{H} & 3.20699500 & -0.37130600 & 2.37029500 \\ \mathrm{H} & 4.32567200 & -1.57599200 & 0.51341000\end{array}$

TS $_{\text {3-THF }}$

$\mathrm{E}(\mathrm{SMD} / \mathrm{B} 3 \mathrm{LYP} / \mathrm{BS} 1)=-774.980181753 \mathrm{au}$

$\mathrm{H}(\mathrm{SMD} / \mathrm{B} 3 \mathrm{LYP} / \mathrm{BS} 1)=-774.574914 \mathrm{au}$

$\mathrm{G}(\mathrm{SMD} / \mathrm{B} 3 \mathrm{LYP} / \mathrm{BS} 1)=-774.642228 \mathrm{au}$

$\mathrm{E}(\mathrm{SMD} / \mathrm{M} 06 / \mathrm{BS} 2 / / \mathrm{SMD} / \mathrm{B} 3 \mathrm{LYP} / \mathrm{BS} 1)=-774.691705484 \mathrm{au}$

C $\quad-0.99864600 \quad-2.15624100 \quad-1.05774000$

$\begin{array}{llll}\mathrm{O} & -0.80132300 & -1.65454700 & 0.27666600\end{array}$

$\begin{array}{llll}\text { C } & 0.99404300 & -0.66292800 & 0.17730800\end{array}$

C $\quad 1.17145300 \quad-1.00980400 \quad-1.26791600$

C $\quad 0.40960400-2.31270200 \quad-1.59546000$

$\mathrm{H} \quad-1.54163500 \quad-3.10676500 \quad-1.00390500$

$\mathrm{H} \quad-1.57918600 \quad-1.44023000 \quad-1.65043400$

$\begin{array}{llll}\mathrm{H} & 0.51477600 & 0.25102700 & 0.49017000\end{array}$

$\mathrm{H} \quad 1.34281700 \quad-1.33312100 \quad 0.94954200$

$\mathrm{H} \quad 0.81381300 \quad-0.18958200 \quad-1.89713100$

$\mathrm{H} \quad 2.23478200 \quad-1.16695200 \quad-1.47261600$

$\mathrm{H} \quad 0.40463800 \quad-2.48681000 \quad-2.67536400$

$\mathrm{H} \quad 0.90361500 \quad-3.16401800 \quad-1.11396200$

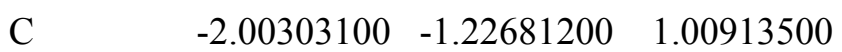

$\begin{array}{llll}\mathrm{C} & -1.61008600 & -1.15248100 & 2.47777300\end{array}$

$\mathrm{H} \quad-2.49246900 \quad-0.91628600 \quad 3.08117700$

$\mathrm{H} \quad-1.21122300 \quad-2.11666100 \quad 2.80818700$

$\mathrm{H} \quad-0.85531700 \quad-0.38219000 \quad 2.66489100$

$\mathrm{H} \quad-2.71688600 \quad-2.04761900 \quad 0.87263200$

$\begin{array}{llll}\mathrm{C} & -2.61118300 & 0.03998400 & 0.43192900\end{array}$

$\begin{array}{llll}\text { C } & -3.71914200 & -0.05634100 & -0.42297500\end{array}$

$\begin{array}{llll}\mathrm{C} & -2.10095000 & 1.31458200 & 0.72666400\end{array}$

$\begin{array}{llll}\text { C } & -4.29871000 & 1.08652400 & -0.97921700\end{array}$

$\mathrm{H} \quad-4.13749400 \quad-1.03462800 \quad-0.64750200$

$\begin{array}{llll}\mathrm{C} & -2.67601900 & 2.45799800 & 0.16990700\end{array}$

$\mathrm{H} \quad-1.26068100 \quad 1.42337100 \quad 1.40681600$

C $\quad-3.77557200 \quad 2.34717500 \quad-0.68593200$

$\mathrm{H} \quad-5.15865400 \quad 0.99091700 \quad-1.63657700$

$\mathrm{H} \quad-2.27057300 \quad 3.43690200 \quad 0.41155500$

$\mathrm{H} \quad-4.22512700 \quad 3.23863800 \quad-1.11486000$

$\begin{array}{llll}\mathrm{C} & 2.99083200 & 1.63618600 & -0.21609000\end{array}$

$\begin{array}{llll}\mathrm{O} & 2.75567500 & 0.39944600 & 0.53013500\end{array}$

$\begin{array}{llll}\mathrm{C} & 3.98840800 & -0.36744800 & 0.62357400\end{array}$

$\begin{array}{llll}\mathrm{C} & 5.09471800 & 0.64604300 & 0.35403800\end{array}$

$\begin{array}{lllll}\mathrm{C} & 4.44140300 & 1.56195500 & -0.69351200\end{array}$

$\mathrm{H} \quad 2.26375500 \quad 1.69805700 \quad-1.03020900$

$\begin{array}{llll}\mathrm{H} & 2.82096100 & 2.46455500 & 0.47947200\end{array}$

H $\quad 4.01723200 \quad-0.81738900 \quad 1.61914500$

$\mathrm{H} \quad 3.97928600 \quad-1.16362700 \quad-0.13266800$ 


$\begin{array}{lrrr}\mathrm{H} & 5.32762100 & 1.20603900 & 1.26650600 \\ \mathrm{H} & 6.01050300 & 0.16737000 & -0.00383000 \\ \mathrm{H} & 4.90269800 & 2.55180900 & -0.74684500 \\ \mathrm{H} & 4.49519100 & 1.10386200 & -1.68752100\end{array}$

\section{5_THF}

$\mathrm{E}(\mathrm{SMD} / \mathrm{B} 3 \mathrm{LYP} / \mathrm{BS} 1)=-542.546199652 \mathrm{au}$

$\mathrm{H}(\mathrm{SMD} / \mathrm{B} 3 \mathrm{LYP} / \mathrm{BS} 1)=-542.265508 \mathrm{au}$

$\mathrm{G}(\mathrm{SMD} / \mathrm{B} 3 \mathrm{LYP} / \mathrm{BS} 1)=-542.316258 \mathrm{au}$

$\mathrm{E}(\mathrm{SMD} / \mathrm{M} 06 / \mathrm{BS} 2 / / \mathrm{SMD} / \mathrm{B} 3 \mathrm{LYP} / \mathrm{BS} 1)=-542.329202067 \mathrm{au}$

C $\quad-1.88117800 \quad-0.84803600 \quad-1.03434400$

$\begin{array}{lllll}\mathrm{O} & -1.50507500 & 0.42164800 & -0.33894200\end{array}$

$\begin{array}{llll}\text { C } & -1.86749700 & 0.34700100 & 1.12280600\end{array}$

$\begin{array}{llll}\text { C } & -2.47434700 & -1.04121300 & 1.27086400\end{array}$

C $\quad-2.97913000 \quad-1.38626100 \quad-0.14091600$

$\mathrm{H} \quad-2.18399500 \quad-0.55635200 \quad-2.03972900$

$\mathrm{H} \quad-0.99630400 \quad-1.48682100 \quad-1.05720400$

$\begin{array}{llll}\mathrm{H} & -0.95600000 & 0.50412200 & 1.69569700\end{array}$

$\mathrm{H} \quad-2.57473800 \quad 1.16132600 \quad 1.27744600$

$\mathrm{H} \quad \begin{array}{llll}-1.70918500 & -1.75623300 & 1.58787600\end{array}$

$\mathrm{H} \quad \begin{array}{llll}-3.27624800 & -1.03573900 & 2.01320800\end{array}$

$\mathrm{H} \quad-3.10746700 \quad-2.46156600 \quad-0.28808300$

$\mathrm{H} \quad \begin{array}{llll}-3.92862500 & -0.88545000 & -0.35468500\end{array}$

$\begin{array}{llll}\text { C } & -0.22234700 & 1.15837500 & -0.80234600\end{array}$

$\begin{array}{llll}\text { C } & -0.40240700 & 2.60379500 & -0.38876500\end{array}$

$\mathrm{H} \quad \begin{array}{llll}\mathrm{H} & 0.43433200 & 3.18033200 & -0.79688200\end{array}$

$\mathrm{H} \quad-1.33207100 \quad 3.00595800 \quad-0.80149900$

$\mathrm{H} \quad-0.40693100 \quad 2.73732100 \quad 0.69616300$

$\mathrm{H} \quad-0.33049200 \quad 1.05798900 \quad-1.88336500$

$\begin{array}{llll}\mathrm{C} & 1.01270700 & 0.43531400 & -0.33905800\end{array}$

$\begin{array}{llll}\mathrm{C} & 1.59998300 & -0.50825700 & -1.19856500\end{array}$

$\begin{array}{lllll}\mathrm{C} & & 1.61283300 & 0.68440500 & 0.90698300\end{array}$

$\begin{array}{llll}\mathrm{C} & 2.74904900 & -1.20154200 & -0.81802000\end{array}$

$\mathrm{H} \quad 1.16143700 \quad-0.68384900 \quad-2.17783100$

$\begin{array}{llll}\mathrm{C} & 2.76001700 & -0.01228000 & 1.28806800\end{array}$

$\begin{array}{llll}\mathrm{H} & 1.20004800 & 1.43033300 & 1.57941900\end{array}$

$\begin{array}{llll}\text { C } & 3.32780900 & -0.95740400 & 0.42938200\end{array}$

$\mathrm{H} \quad 3.19424800 \quad-1.92331200 \quad-1.49672800$

$\mathrm{H} \quad 3.21558400 \quad 0.18945500 \quad 2.25332500$

$\mathrm{H} \quad 4.22406200 \quad-1.49407900 \quad 0.72787200$

\section{3_THF}

$\mathrm{E}(\mathrm{SMD} / \mathrm{B} 3 \mathrm{LYP} / \mathrm{BS} 1)=-775.009540537 \mathrm{au}$

$\mathrm{H}(\mathrm{SMD} / \mathrm{B} 3 \mathrm{LYP} / \mathrm{BS} 1)=-774.601975 \mathrm{au}$

$\mathrm{G}(\mathrm{SMD} / \mathrm{B} 3 \mathrm{LYP} / \mathrm{BS} 1)=-774.670424 \mathrm{au}$

$\mathrm{E}(\mathrm{SMD} / \mathrm{M} 06 / \mathrm{BS} 2 / / \mathrm{SMD} / \mathrm{B} 3 \mathrm{LYP} / \mathrm{BS} 1)=-774.721439091 \mathrm{au}$

$\begin{array}{lrrr}\mathrm{C} & 0.88019100 & 1.95276200 & -0.65782800 \\ \mathrm{O} & 0.84317800 & 1.25432100 & 0.58519800 \\ \mathrm{C} & -2.01300200 & 0.68737400 & 0.02982400 \\ \mathrm{C} & -1.57271400 & 1.35680800 & -1.25857000 \\ \mathrm{C} & -0.51668000 & 2.45208300 & -1.00893900\end{array}$




$\begin{array}{lrrr}\mathrm{H} & 1.26496100 & 1.30048600 & -1.45585000 \\ \mathrm{H} & -1.21616100 & 0.10786100 & 0.48976700 \\ \mathrm{H} & -1.17534300 & 0.59930600 & -1.94517300 \\ \mathrm{H} & -2.44071500 & 1.81799200 & -1.74396000 \\ \mathrm{H} & -0.86563200 & 3.13594000 & -0.22412500 \\ \mathrm{C} & 2.13023000 & 0.98651900 & 1.15177800 \\ \mathrm{C} & 1.88253500 & 0.50491100 & 2.58106800 \\ \mathrm{H} & 2.83401800 & 0.33174000 & 3.09453800 \\ \mathrm{H} & 1.31480300 & 1.25656500 & 3.14070500 \\ \mathrm{H} & 1.31751100 & -0.43433000 & 2.58191800 \\ \mathrm{H} & 2.69690900 & 1.93020500 & 1.18856800 \\ \mathrm{C} & 2.93713700 & -0.02140900 & 0.34163900 \\ \mathrm{C} & 4.30728800 & 0.17681500 & 0.13042000 \\ \mathrm{C} & 2.33642100 & -1.17912800 & -0.17310100 \\ \mathrm{C} & 5.06680400 & -0.76514300 & -0.56870800 \\ \mathrm{H} & 4.78383400 & 1.07529700 & 0.51729000 \\ \mathrm{C} & 3.09032300 & -2.11872000 & -0.87841200 \\ \mathrm{H} & 1.27167400 & -1.33994900 & -0.02538300 \\ \mathrm{C} & 4.45951000 & -1.91583700 & -1.07618200 \\ \mathrm{H} & 6.12943100 & -0.59555100 & -0.72275000 \\ \mathrm{H} & 2.61002000 & -3.01011100 & -1.27449300 \\ \mathrm{H} & 5.04662600 & -2.64773000 & -1.62472200 \\ \mathrm{H} & 1.56199400 & 2.81377700 & -0.57884300 \\ \mathrm{H} & -0.42661600 & 3.04601900 & -1.92622200 \\ \mathrm{H} & -2.43688300 & 1.38493400 & 0.75800800 \\ \mathrm{C} & -3.22127400 & -1.44332200 & 0.71681300 \\ \mathrm{C} & -4.45048400 & 0.20035200 & -0.65757000 \\ \mathrm{C} & -4.61962500 & -1.94639300 & 0.43191300 \\ \mathrm{H} & -3.10971300 & -1.01164200 & 1.71458800 \\ \mathrm{H} & -2.40726100 & -2.13112800 & 0.49012000 \\ \mathrm{C} & -5.40632900 & -0.65141100 & 0.16372000 \\ \mathrm{H} & -4.51350700 & 0.03258600 & -1.73233800 \\ \mathrm{H} & -4.46359400 & 1.26526200 & -0.42601600 \\ \mathrm{H} & -4.62108600 & -2.59883800 & -0.44694900 \\ \mathrm{H} & -5.01218900 & -2.50494000 & 1.28529600 \\ \mathrm{H} & -6.33380100 & -0.83074100 & -0.38551000 \\ \mathrm{H} & -5.64955200 & -0.14985500 & 1.10533800 \\ \mathrm{O} & -3.08089300 & -0.31691200 & -0.26782200\end{array}$

\section{0( $\left.\mathrm{H}_{2} \mathrm{O}\right)$}

$\mathrm{E}(\mathrm{SMD} / \mathrm{B} 3 \mathrm{LYP} / \mathrm{BS} 1)=-3365.81933026 \mathrm{au}$

$\mathrm{H}(\mathrm{SMD} / \mathrm{B} 3 \mathrm{LYP} / \mathrm{BS} 1)=-3365.573238 \mathrm{au}$

$\mathrm{G}(\mathrm{SMD} / \mathrm{B} 3 \mathrm{LYP} / \mathrm{BS} 1)=-3365.682985 \mathrm{au}$

$\mathrm{E}(\mathrm{SMD} / \mathrm{M} 06 / \mathrm{BS} 2 / / \mathrm{SMD} / \mathrm{B} 3 \mathrm{LYP} / \mathrm{BS} 1)=-3366.04076397 \mathrm{au}$

$\begin{array}{llll}\mathrm{C} & 1.72846500 & 3.38856900 & -1.16969900 \\ \mathrm{H} & 2.69615000 & 3.82802600 & -0.88082000 \\ \mathrm{H} & 1.22580600 & 4.05253200 & -1.87385700 \\ \mathrm{H} & 1.94480000 & 2.41238800 & -1.61103700 \\ \mathrm{C} & 0.94285200 & 3.23949900 & 0.06652300 \\ \mathrm{O} & 0.00533900 & 4.07219000 & 0.29247000 \\ \mathrm{C} & 1.23942200 & 2.18055800 & 1.06330000\end{array}$




$\begin{array}{lrrr}\text { C } & 0.69458600 & 2.40667000 & 2.46173900 \\ \text { O } & -0.22710200 & 3.21530700 & 2.62943600 \\ \text { C } & 1.30903700 & 1.64348900 & 3.58566600 \\ \text { H } & 2.35780300 & 1.95086000 & 3.69158000 \\ \text { H } & 0.76830500 & 1.83643200 & 4.51366900 \\ \text { H } & 1.31133000 & 0.57178300 & 3.35664000 \\ \text { H } & 2.29682600 & 1.91935200 & 1.05163900 \\ \text { H } & -0.36869600 & 3.89849600 & 1.24362700 \\ \mathrm{Au} & 0.37008100 & 0.35811500 & 0.36886000 \\ \text { O } & -0.42078700 & -1.53623200 & -0.28169700 \\ \text { S } & -0.61977900 & -2.72486200 & 0.68058500 \\ \text { O } & -0.47736300 & -3.99074300 & -0.04689300 \\ \text { O } & 0.08400800 & -2.56320400 & 1.96069500 \\ \text { C } & -2.42945100 & -2.57848800 & 1.09469700 \\ \text { F } & -3.16240100 & -2.67161900 & -0.01455400 \\ \text { F } & -2.76649400 & -3.55923500 & 1.93383000 \\ \text { F } & -2.66141400 & -1.39903600 & 1.68271500 \\ \text { O } & -3.02668800 & 2.90582100 & -0.72160200 \\ \text { S } & -2.14516900 & 1.79091700 & -1.06803300 \\ \text { O } & -1.17913700 & 1.92918000 & -2.16187500 \\ \text { O } & -1.47200200 & 1.27868600 & 0.25600000 \\ \text { C } & -3.28293200 & 0.38207500 & -1.53722100 \\ \text { F } & -4.10798700 & 0.82440900 & -2.48554600 \\ \text { F } & -2.57444400 & -0.64082700 & -2.00690200 \\ \text { F } & -3.98525700 & 0.00004600 & -0.47386600 \\ \text { O } & 3.80736400 & 0.77034200 & -0.65227600 \\ \text { S } & 3.42993800 & -0.58124500 & -0.23240100 \\ \text { O } & 4.42791100 & -1.45093100 & 0.38951300 \\ \text { O } & 2.16108500 & -0.61007700 & 0.68494400 \\ \text { C } & 2.85117700 & -1.45867100 & -1.77761500 \\ \text { F } & 3.89349400 & -1.60491700 & -2.59191100 \\ \text { F } & 2.35586500 & -2.65185900 & -1.45940200 \\ \text { F } & 1.90991400 & -0.73061100 & -2.38745900\end{array}$

\begin{tabular}{|c|c|c|c|}
\hline \multicolumn{4}{|c|}{$2\left(\mathrm{H}_{2} \mathrm{O}\right)$} \\
\hline \multicolumn{4}{|c|}{$\mathrm{E}(\mathrm{SMD} / \mathrm{B} 3 \mathrm{LYP} / \mathrm{BS} 1)=-309.642627438 \mathrm{au}$} \\
\hline \multicolumn{4}{|c|}{$\mathrm{H}(\mathrm{SMD} / \mathrm{B} 3 \mathrm{LYP} / \mathrm{BS} 1)=-309.501049 \mathrm{au}$} \\
\hline \multicolumn{4}{|c|}{ G (SMD/B3LYP/BS1) = -309.53959 au } \\
\hline \multicolumn{4}{|c|}{$\mathrm{E}(\mathrm{SMD} / \mathrm{M} 06 / \mathrm{BS} 2 / / \mathrm{SMD} / \mathrm{B} 3 \mathrm{LYP} / \mathrm{BS} 1)=-309.502665$} \\
\hline $\mathrm{C}$ & -0.68558900 & -1.77652700 & 0.00000000 \\
\hline $\mathrm{C}$ & -1.00952600 & -0.42162900 & 0.00000000 \\
\hline $\mathrm{C}$ & 0.00000000 & 0.56103900 & 0.00000000 \\
\hline $\mathrm{C}$ & 1.34040300 & 0.13377800 & 0.00000000 \\
\hline $\mathrm{C}$ & 1.66577000 & -1.22359400 & 0.00000000 \\
\hline $\mathrm{C}$ & 0.65330100 & -2.18542900 & 0.00000000 \\
\hline $\mathrm{H}$ & -1.48000900 & -2.51818100 & 0.00000000 \\
\hline $\mathrm{H}$ & -2.05439900 & -0.12457000 & 0.00000000 \\
\hline $\mathrm{H}$ & 2.13255500 & 0.87896200 & 0.00000000 \\
\hline $\mathrm{H}$ & 2.70886200 & -1.52820000 & 0.00000000 \\
\hline $\mathrm{H}$ & 0.90164900 & -3.24312900 & 0.00000000 \\
\hline $\mathrm{C}$ & -0.28324500 & 2.00688600 & 0.00000000 \\
\hline
\end{tabular}




$\begin{array}{lrrr}\mathrm{H} & 0.60440800 & 2.63952200 & 0.00000000 \\ \mathrm{C} & -1.48519900 & 2.60010200 & 0.00000000 \\ \mathrm{H} & -2.41984000 & 2.04443600 & 0.00000000 \\ \mathrm{H} & -1.56871800 & 3.68339800 & 0.00000000\end{array}$

\section{$9\left(\mathrm{H}_{2} \mathrm{O}\right)$}

$\mathrm{E}(\mathrm{SMD} / \mathrm{B} 3 \mathrm{LYP} / \mathrm{BS} 1)=-345.799186949 \mathrm{au}$

$\mathrm{H}(\mathrm{SMD} / \mathrm{B} 3 \mathrm{LYP} / \mathrm{BS} 1)=-345.667507 \mathrm{au}$

$\mathrm{G}(\mathrm{SMD} / \mathrm{B} 3 \mathrm{LYP} / \mathrm{BS} 1)=-345.708906 \mathrm{au}$

$\mathrm{E}(\mathrm{SMD} / \mathrm{M} 06 / \mathrm{BS} 2 / / \mathrm{SMD} / \mathrm{B} 3 \mathrm{LYP} / \mathrm{BS} 1)=-345.720495343 \mathrm{au}$

$\begin{array}{lrrr}\mathrm{C} & 2.54428800 & -0.76640900 & -0.00000700 \\ \mathrm{H} & 2.59630500 & -1.41676300 & 0.88162600 \\ \mathrm{H} & 3.39577700 & -0.08218900 & 0.00003100 \\ \mathrm{H} & 2.59635600 & -1.41673500 & -0.88165700 \\ \mathrm{C} & 1.24895100 & 0.00609600 & -0.00003100 \\ \mathrm{O} & 1.27372300 & 1.26657000 & 0.00010500 \\ \mathrm{C} & 0.01081900 & -0.72868700 & -0.00003300 \\ \mathrm{C} & -1.20210800 & -0.08679300 & -0.00001200 \\ \mathrm{C} & -2.52253200 & -0.78305200 & 0.00002300 \\ \mathrm{H} & -3.10127000 & -0.48503500 & -0.88271600 \\ \mathrm{H} & -2.39952300 & -1.86785500 & 0.00005200 \\ \mathrm{H} & -3.10124900 & -0.48498400 & 0.88275900 \\ \mathrm{O} & -1.29301200 & 1.25003000 & -0.00005100 \\ \mathrm{H} & -0.34304000 & 1.58561300 & -0.00013200 \\ \mathrm{H} & 0.03444200 & -1.81178600 & -0.00003700\end{array}$

\section{$3\left(\mathrm{H}_{2} \mathrm{O}\right)$}

$\mathrm{E}(\mathrm{SMD} / \mathrm{B} 3 \mathrm{LYP} / \mathrm{BS} 1)=-386.08614071 \mathrm{au}$

$\mathrm{H}(\mathrm{SMD} / \mathrm{B} 3 \mathrm{LYP} / \mathrm{BS} 1)=-385.915202 \mathrm{au}$

$\mathrm{G}(\mathrm{SMD} / \mathrm{B} 3 \mathrm{LYP} / \mathrm{BS} 1)=-385.958000 \mathrm{au}$

$\mathrm{E}(\mathrm{SMD} / \mathrm{M} 06 / \mathrm{BS} 2 / / \mathrm{SMD} / \mathrm{B} 3 \mathrm{LYP} / \mathrm{BS} 1)=-385.955231304 \mathrm{au}$

$\begin{array}{lllll}\mathrm{C} & -1.88677200 & 1.25609500 & -0.18213200\end{array}$

$\begin{array}{lllll}\text { C } & & -0.49529700 & 1.18945400 & -0.04949000\end{array}$

$\begin{array}{llll}\text { C } & 0.14576100 & -0.03713800 & 0.16303400\end{array}$

$\begin{array}{llll}\text { C } & -0.64187600 & -1.19804800 & 0.24022400\end{array}$

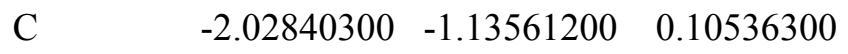

$\begin{array}{llll}\mathrm{C} & -2.65763900 & 0.09556000 & -0.10750600\end{array}$

$\mathrm{H} \quad-2.36426600 \quad 2.21847500 \quad-0.34650700$

$\mathrm{H} \quad \begin{array}{llll}\mathrm{H} & 0.08395100 & 2.10495300 & -0.11219100\end{array}$

$\mathrm{H} \quad-0.16001800 \quad-2.15778100 \quad 0.40525200$

$\mathrm{H} \quad-2.61858300 \quad-2.04612900 \quad 0.16771600$

$\mathrm{H} \quad \begin{array}{llll}\mathrm{H} & -3.73802400 & 0.14743100 & -0.21187700\end{array}$

$\begin{array}{llll}\text { C } & 1.65386700 & -0.16622000 & 0.32965300\end{array}$

$\begin{array}{llll}\mathrm{C} & 2.44951100 & 1.11959900 & 0.12489000\end{array}$

$\begin{array}{llll}\mathrm{H} & 3.51799800 & 0.90526700 & 0.24231300\end{array}$

$\begin{array}{lllll}\mathrm{H} & 2.28905600 & 1.52779800 & -0.87909400\end{array}$

$\begin{array}{llll}\mathrm{H} & 2.17887200 & 1.88224900 & 0.86313000\end{array}$

$\mathrm{H} \quad \begin{array}{llll}\mathrm{H} & 1.84685500 & -0.53154000 & 1.35011000\end{array}$

$\begin{array}{llll}\mathrm{O} & 2.08868300 & -1.17837900 & -0.60351000\end{array}$

$\mathrm{H} \quad 3.01977700 \quad-1.36584200 \quad-0.39498300$ 


\begin{tabular}{|c|c|c|c|}
\hline \multicolumn{4}{|c|}{$22\left(\mathrm{H}_{2} \mathrm{O}\right)$} \\
\hline \multicolumn{4}{|c|}{$\mathrm{E}(\mathrm{SMD} / \mathrm{B} 3 \mathrm{LYP} / \mathrm{BS} 1)=-3675.47079905 \mathrm{au}$} \\
\hline \multicolumn{4}{|c|}{ H (SMD/B3LYP/BS1) = -3675.08199 au } \\
\hline \multicolumn{4}{|c|}{ G (SMD/B3LYP/BS1) = -3675.216021 au } \\
\hline \multicolumn{4}{|c|}{$\mathrm{E}(\mathrm{SMD} / \mathrm{M} 06 / \mathrm{BS} 2 / / \mathrm{SMD} / \mathrm{B} 3 \mathrm{LYP} / \mathrm{BS} 1)=-3675.54583198 \mathrm{au}$} \\
\hline $\mathrm{C}$ & 1.10291900 & -3.13558000 & -1.00259400 \\
\hline $\mathrm{H}$ & 0.90875900 & -4.15485600 & -0.64296400 \\
\hline $\mathrm{H}$ & 1.99513400 & -3.13562800 & -1.63136800 \\
\hline $\mathrm{H}$ & 0.22625500 & -2.82535700 & -1.57825500 \\
\hline $\mathrm{C}$ & 1.30701700 & -2.24216300 & 0.18614600 \\
\hline $\mathrm{O}$ & 2.39750700 & -1.77792800 & 0.49461900 \\
\hline $\mathrm{C}$ & 0.09378900 & -1.97740300 & 1.07466000 \\
\hline $\mathrm{C}$ & 0.45207400 & -1.61456500 & 2.51170500 \\
\hline $\mathrm{O}$ & 0.29891800 & -0.48331800 & 2.95171500 \\
\hline $\mathrm{C}$ & 0.96374400 & -2.75234800 & 3.35304200 \\
\hline $\mathrm{H}$ & 1.79686500 & -3.26219100 & 2.85778200 \\
\hline $\mathrm{H}$ & 1.27882800 & -2.37692700 & 4.32942900 \\
\hline $\mathrm{H}$ & 0.16023200 & -3.48900700 & 3.48167500 \\
\hline $\mathrm{H}$ & -0.58994300 & -2.82874700 & 1.05174100 \\
\hline $\mathrm{Au}$ & -1.09429400 & -0.42137400 & 0.31365800 \\
\hline $\mathrm{O}$ & -2.41825100 & 1.28275700 & -0.29724200 \\
\hline S & -3.35494600 & 2.04248600 & 0.63518800 \\
\hline $\mathrm{O}$ & -4.21373900 & 2.96620300 & -0.12131800 \\
\hline $\mathrm{O}$ & -3.99533600 & 1.19915500 & 1.65911100 \\
\hline $\mathrm{C}$ & -2.18906200 & 3.13080000 & 1.59307500 \\
\hline $\mathrm{F}$ & -1.52948400 & 3.94563500 & 0.76683300 \\
\hline $\mathrm{F}$ & -2.88052300 & 3.86170000 & 2.47164000 \\
\hline $\mathrm{F}$ & -1.30141300 & 2.37768400 & 2.26019500 \\
\hline $\mathrm{O}$ & 2.71352900 & 1.49866100 & -0.83373500 \\
\hline $\mathrm{S}$ & 1.37934000 & 1.01065500 & -1.19569800 \\
\hline $\mathrm{O}$ & 1.24786600 & -0.11268700 & -2.12918800 \\
\hline $\mathrm{O}$ & 0.54979900 & 0.82931500 & 0.10967700 \\
\hline $\mathrm{C}$ & 0.53695500 & 2.45111900 & -2.03362200 \\
\hline $\mathrm{F}$ & 1.31652800 & 2.86337200 & -3.03438900 \\
\hline $\mathrm{F}$ & -0.64148500 & 2.06570900 & -2.52417700 \\
\hline $\mathrm{F}$ & 0.35975100 & 3.44601600 & -1.16712600 \\
\hline $\mathrm{O}$ & -2.31889400 & -3.59363600 & -0.80402000 \\
\hline S & -3.33637600 & -2.67849700 & -0.27860100 \\
\hline $\mathrm{O}$ & -4.53571200 & -3.22841400 & 0.35549900 \\
\hline $\mathrm{O}$ & -2.75553800 & -1.59090100 & 0.67485400 \\
\hline $\mathrm{C}$ & -3.93516800 & -1.69059200 & -1.74673100 \\
\hline $\mathrm{F}$ & -4.60188700 & -2.50918500 & -2.55897100 \\
\hline $\mathrm{F}$ & -4.73948000 & -0.71010000 & -1.34058900 \\
\hline $\mathrm{F}$ & -2.89289800 & -1.17035500 & -2.40322100 \\
\hline $\mathrm{C}$ & 5.77261500 & 0.38646400 & 0.39369600 \\
\hline $\mathrm{C}$ & 7.09645100 & 0.03964500 & 0.12400700 \\
\hline $\mathrm{C}$ & 7.98335500 & -0.47546400 & 1.12117000 \\
\hline $\mathrm{C}$ & 7.57145800 & 0.20731400 & -1.21586800 \\
\hline $\mathrm{C}$ & 9.27932100 & -0.80564200 & 0.78048000 \\
\hline $\mathrm{H}$ & 7.63723900 & -0.60466000 & 2.14021300 \\
\hline $\mathrm{C}$ & 8.87144500 & -0.12807800 & -1.54138400 \\
\hline
\end{tabular}




$\begin{array}{cccc}\mathrm{H} & 6.89209600 & 0.60007900 & -1.96599700 \\ \mathrm{C} & 9.72030300 & -0.63322200 & -0.54443400 \\ \mathrm{H} & 9.96008200 & -1.19730700 & 1.52851100 \\ \mathrm{H} & 9.23589800 & -0.00418500 & -2.55529300 \\ \mathrm{H} & 10.74238500 & -0.89691500 & -0.80032200 \\ \mathrm{H} & 5.19411500 & 0.76850200 & -0.44670800 \\ \mathrm{C} & 5.04474400 & 0.30012100 & 1.66002300 \\ \mathrm{H} & 4.62579000 & 1.29407200 & 1.88253600 \\ \mathrm{H} & 4.16051300 & -0.33672600 & 1.49600200 \\ \mathrm{H} & 5.62180100 & -0.06628600 & 2.50805500\end{array}$

\section{3( $\left.\mathrm{H}_{2} \mathrm{O}\right)$}

$\mathrm{E}(\mathrm{SMD} / \mathrm{B} 3 \mathrm{LYP} / \mathrm{BS} 1)=-310.070370951 \mathrm{au}$

$\mathrm{H}(\mathrm{SMD} / \mathrm{B} 3 \mathrm{LYP} / \mathrm{BS} 1)=-309.917547 \mathrm{au}$

$\mathrm{G}(\mathrm{SMD} / \mathrm{B} 3 \mathrm{LYP} / \mathrm{BS} 1)=-309.955693 \mathrm{au}$

$\mathrm{E}(\mathrm{SMD} / \mathrm{M} 06 / \mathrm{BS} 2 / / \mathrm{SMD} / \mathrm{B} 3 \mathrm{LYP} / \mathrm{BS} 1)=-309.92184157 \mathrm{au}$

$\begin{array}{llll}\mathrm{C} & 1.33646100 & 1.37852400 & -0.00022700\end{array}$

$\begin{array}{llll}\text { C } & -0.01280100 & 1.09483600 & -0.00050600\end{array}$

$\begin{array}{llll}\text { C } & -0.45215200 & -0.26727800 & -0.00026800\end{array}$

$\begin{array}{llll}\mathrm{C} & 0.51896900 & -1.31824600 & 0.00012400\end{array}$

$\begin{array}{llll}\mathrm{C} & 1.86787900 & -1.01804200 & 0.00036000\end{array}$

$\begin{array}{llll}\mathrm{C} & 2.27189600 & 0.32554400 & 0.00019700\end{array}$

$\mathrm{H} \quad 1.68228000 \quad 2.40640700 \quad-0.00037100$

$\mathrm{H} \quad-0.74447600 \quad 1.89549100 \quad-0.00089200$

$\begin{array}{llll}\mathrm{H} & 0.17707900 & -2.34848600 & 0.00028300\end{array}$

$\mathrm{H} \quad 2.60941300 \quad-1.80948800 \quad 0.00068300$

$\begin{array}{llll}\mathrm{H} & 3.33214500 & 0.56098600 & 0.00038100\end{array}$

$\begin{array}{llll}\mathrm{C} & -1.80076800 & -0.61794600 & -0.00017000\end{array}$

$\begin{array}{lllll}\mathrm{H} & & -2.01790900 & -1.68516700 & 0.00003700\end{array}$

$\begin{array}{lllll}\mathrm{C} & -2.95093900 & 0.30329600 & 0.00013900\end{array}$

$\mathrm{H} \quad-3.89773300 \quad-0.23654100 \quad-0.00284400$

$\mathrm{H} \quad-2.90748000 \quad 0.96349300 \quad 0.87835100$

$\mathrm{H} \quad-2.90458000 \quad 0.96917000 \quad-0.87351700$

\begin{tabular}{|c|c|c|c|}
\hline \multicolumn{4}{|c|}{$24\left(\mathrm{H}_{2}\right.$} \\
\hline \multicolumn{4}{|c|}{$\mathrm{E}(\mathrm{SMD} / \mathrm{B} 3 \mathrm{LYP} / \mathrm{BS} 1)=-3365.39484464 \mathrm{au}$} \\
\hline \multicolumn{4}{|c|}{ H (SMD/B3LYP/BS1) = -3365.161082 au } \\
\hline \multicolumn{4}{|c|}{$\mathrm{G}(\mathrm{SMD} / \mathrm{B} 3 \mathrm{LYP} / \mathrm{BS} 1)=-3365.273955 \mathrm{au}$} \\
\hline \multicolumn{4}{|c|}{$\mathrm{E}(\mathrm{SMD} / \mathrm{M} 06 / \mathrm{BS} 2 / / \mathrm{SMD} / \mathrm{B} 3 \mathrm{LYP} / \mathrm{BS} 1)=-3365.6195^{\circ}$} \\
\hline $\mathrm{C}$ & 1.75707100 & 3.62110100 & -0.77084600 \\
\hline $\mathrm{H}$ & 2.76827500 & 3.86162700 & -0.41652800 \\
\hline $\mathrm{H}$ & 1.34958700 & 4.47399500 & -1.31661000 \\
\hline $\mathrm{H}$ & 1.84550700 & 2.75102800 & -1.42793600 \\
\hline $\mathrm{C}$ & 0.89696500 & 3.30727400 & 0.41926100 \\
\hline $\mathrm{O}$ & 0.00479300 & 4.04439800 & 0.81905500 \\
\hline $\mathrm{C}$ & 1.22536000 & 2.03484400 & 1.19616200 \\
\hline $\mathrm{C}$ & 0.78143000 & 2.08846900 & 2.65472300 \\
\hline $\mathrm{O}$ & -0.19779100 & 1.47451300 & 3.05650200 \\
\hline $\mathrm{C}$ & 1.64984100 & 2.92123800 & 3.55742200 \\
\hline $\mathrm{H}$ & 1.81828200 & 3.91645000 & 3.13176800 \\
\hline $\mathrm{H}$ & 1.18685900 & 3.00539400 & 4.54333500 \\
\hline
\end{tabular}




$\begin{array}{lccc}\mathrm{H} & 2.63044500 & 2.43641000 & 3.64978600 \\ \mathrm{H} & 2.28810000 & 1.79660100 & 1.11778100 \\ \mathrm{Au} & 0.31552900 & 0.33884300 & 0.34791200 \\ \mathrm{O} & -0.65397000 & -1.60300900 & -0.26562400 \\ \mathrm{~S} & -0.89637300 & -2.74374700 & 0.71554400 \\ \mathrm{O} & -1.23065900 & -3.98935700 & 0.00814400 \\ \mathrm{O} & 0.09656700 & -2.82196400 & 1.80143800 \\ \mathrm{C} & -2.46850600 & -2.22451100 & 1.56454600 \\ \mathrm{~F} & -3.46362500 & -2.12278300 & 0.68072900 \\ \mathrm{~F} & -2.79557200 & -3.12949000 & 2.49109600 \\ \mathrm{~F} & -2.29499300 & -1.03603100 & 2.16244300 \\ \mathrm{O} & -3.23912700 & 2.74085300 & -0.83333300 \\ \mathrm{~S} & -2.04452200 & 1.96519100 & -1.17464600 \\ \mathrm{O} & -1.00111200 & 2.57387900 & -2.00517400 \\ \mathrm{O} & -1.50108100 & 1.32109100 & 0.13763600 \\ \mathrm{C} & -2.65061900 & 0.49777800 & -2.15710200 \\ \mathrm{~F} & -3.37862900 & 0.94920500 & -3.17936400 \\ \mathrm{~F} & -1.60762700 & -0.18868100 & -2.62980900 \\ \mathrm{~F} & -3.39990600 & -0.29309400 & -1.39195000 \\ \mathrm{O} & 3.58774000 & 0.60935700 & -0.92732500 \\ \mathrm{~S} & 3.28280200 & -0.70407000 & -0.35154100 \\ \mathrm{O} & 4.35959000 & -1.49051100 & 0.25260600 \\ \mathrm{O} & 2.09249700 & -0.67615400 & 0.65526100 \\ \mathrm{C} & 2.62228800 & -1.73548500 & -1.76278600 \\ \mathrm{~F} & 3.62340900 & -1.97970200 & -2.60711800 \\ \mathrm{~F} & 2.13775600 & -2.88584300 & -1.29845900 \\ \mathrm{~F} & 1.65619500 & -1.07103100 & -2.40513600\end{array}$

\begin{tabular}{|c|c|c|c|}
\hline \multicolumn{4}{|c|}{$25\left(\mathrm{H}_{2} \mathrm{O}\right)$} \\
\hline \multicolumn{4}{|c|}{$\mathrm{E}(\mathrm{SMD} / \mathrm{B} 3 \mathrm{LYP} / \mathrm{BS} 1)=-655.895947912 \mathrm{au}$} \\
\hline \multicolumn{4}{|c|}{$\mathrm{H}(\mathrm{SMD} / \mathrm{B} 3 \mathrm{LYP} / \mathrm{BS} 1)=-655.606515 \mathrm{au}$} \\
\hline \multicolumn{4}{|c|}{$\mathrm{G}(\mathrm{SMD} / \mathrm{B} 3 \mathrm{LYP} / \mathrm{BS} 1)=-655.665001 \mathrm{au}$} \\
\hline \multicolumn{4}{|c|}{$\mathrm{E}(\mathrm{SMD} / \mathrm{M} 06 / \mathrm{BS} 2 / / \mathrm{SMD} / \mathrm{B} 3 \mathrm{LYP} / \mathrm{BS} 1)=-655.681561998 \mathrm{au}$} \\
\hline $\mathrm{C}$ & 3.42391000 & -1.26865300 & 0.90359500 \\
\hline $\mathrm{H}$ & 3.63699100 & -0.77134000 & 1.86061400 \\
\hline $\mathrm{H}$ & 4.36212700 & -1.54653300 & 0.41960600 \\
\hline $\mathrm{C}$ & 2.66492900 & -0.30454300 & 0.05456300 \\
\hline $\mathrm{O}$ & 3.16755500 & 0.27008800 & -0.91422600 \\
\hline $\mathrm{C}$ & 1.20649200 & 0.00112100 & 0.41767300 \\
\hline $\mathrm{C}$ & 0.94668000 & 1.47029600 & 0.32774900 \\
\hline $\mathrm{O}$ & 1.47559400 & 2.14032200 & -0.61994800 \\
\hline $\mathrm{C}$ & 0.10596400 & 2.18817500 & 1.29637300 \\
\hline $\mathrm{H}$ & -0.14514200 & 3.18942100 & 0.94403300 \\
\hline $\mathrm{H}$ & -0.78636300 & 1.60386200 & 1.54037600 \\
\hline $\mathrm{H}$ & 0.69349300 & 2.25923900 & 2.22564200 \\
\hline $\mathrm{H}$ & 0.96338600 & -0.35620900 & 1.41973200 \\
\hline $\mathrm{H}$ & 2.15249400 & 1.54784700 & -1.10804900 \\
\hline $\mathrm{C}$ & 0.24927400 & -0.73882300 & -0.64068400 \\
\hline $\mathrm{H}$ & 0.48443000 & -0.28531600 & -1.60898200 \\
\hline $\mathrm{C}$ & -1.21724800 & -0.48279600 & -0.33091000 \\
\hline $\mathrm{C}$ & -1.98400100 & 0.31358400 & -1.19282600 \\
\hline
\end{tabular}




$\begin{array}{lrrr}\mathrm{C} & -1.83280200 & -1.03656100 & 0.80305600 \\ \mathrm{C} & -3.33704900 & 0.54889300 & -0.93331000 \\ \mathrm{H} & -1.51916900 & 0.74907500 & -2.07384400 \\ \mathrm{C} & -3.18316200 & -0.79908400 & 1.06518400 \\ \mathrm{H} & -1.25584800 & -1.65428800 & 1.48633900 \\ \mathrm{C} & -3.94009700 & -0.00621800 & 0.19695800 \\ \mathrm{H} & -3.91670700 & 1.16531400 & -1.61491000 \\ \mathrm{H} & -3.64480500 & -1.23558900 & 1.94662500 \\ \mathrm{H} & -4.99143200 & 0.17618500 & 0.40133900 \\ \mathrm{C} & 0.57428900 & -2.23805300 & -0.71195200 \\ \mathrm{H} & -0.12726000 & -2.71510900 & -1.40399400 \\ \mathrm{H} & 1.58620900 & -2.41477400 & -1.09019600 \\ \mathrm{H} & 0.47619800 & -2.72507000 & 0.26325700 \\ \mathrm{H} & 2.82312600 & -2.15403200 & 1.13298200\end{array}$

\begin{tabular}{|c|c|c|c|}
\hline & & & \\
\hline & $\mathrm{LYP} / \mathrm{BS} 1)=-$ & 655.87611336 & $7 \mathrm{au}$ \\
\hline & $\mathrm{LYP} / \mathrm{BS} 1)=$ & $655.589093 \mathrm{au}$ & \\
\hline $\mathrm{G}($ & $\mathrm{LYP} / \mathrm{BS} 1)=$ & $655.648852 \mathrm{au}$ & \\
\hline $\mathrm{E}($ & $6 / \mathrm{BS} 2 / / \mathrm{SMD} /$ & B3LYP/BS1) = & $=-655.656616$ \\
\hline $\mathrm{C}$ & 2.53626600 & -2.32853200 & 0.68547500 \\
\hline $\mathrm{H}$ & 3.61716200 & -2.22555700 & 0.52754500 \\
\hline $\mathrm{H}$ & 2.23253700 & -3.35495000 & 0.46981600 \\
\hline $\mathrm{C}$ & 1.80191500 & -1.36771800 & -0.20616100 \\
\hline $\mathrm{O}$ & 1.03387500 & -1.78722400 & -1.10349300 \\
\hline $\mathrm{C}$ & 1.99679200 & 0.05816500 & 0.00285300 \\
\hline $\mathrm{C}$ & 1.52895500 & 0.98302900 & -0.92863100 \\
\hline $\mathrm{O}$ & 0.71282100 & 0.61818000 & -1.89888500 \\
\hline $\mathrm{C}$ & 1.87360200 & 2.43044900 & -0.91239400 \\
\hline $\mathrm{H}$ & 0.95590700 & 3.02996800 & -0.88182100 \\
\hline $\mathrm{H}$ & 2.50909400 & 2.68227700 & -0.06263500 \\
\hline $\mathrm{H}$ & 2.39589800 & 2.68470100 & -1.84308600 \\
\hline $\mathrm{H}$ & 2.72078400 & 0.38733100 & 0.73762400 \\
\hline $\mathrm{H}$ & 0.60686000 & -0.38581200 & -1.82644000 \\
\hline $\mathrm{C}$ & 0.05495900 & 0.40074500 & 1.59248800 \\
\hline $\mathrm{H}$ & 0.51191900 & -0.45709600 & 2.07934300 \\
\hline $\mathrm{C}$ & -1.09328300 & 0.12651500 & 0.80174200 \\
\hline $\mathrm{C}$ & -1.51347000 & -1.22558500 & 0.66307700 \\
\hline $\mathrm{C}$ & -1.85611700 & 1.15239200 & 0.17834400 \\
\hline $\mathrm{C}$ & -2.66800400 & -1.53367100 & -0.04127600 \\
\hline $\mathrm{H}$ & -0.93166100 & -2.00938300 & 1.13794900 \\
\hline $\mathrm{C}$ & -3.00432200 & 0.83203600 & -0.52750200 \\
\hline $\mathrm{H}$ & -1.54313000 & 2.18705000 & 0.26037100 \\
\hline $\mathrm{C}$ & -3.41150700 & -0.50656400 & -0.63535800 \\
\hline $\mathrm{H}$ & -2.99331200 & -2.56490500 & -0.13159600 \\
\hline $\mathrm{H}$ & -3.58913200 & 1.61575400 & -0.99800500 \\
\hline $\mathrm{H}$ & -4.31432900 & -0.74835100 & -1.18867800 \\
\hline $\mathrm{C}$ & 0.42342100 & 1.72157200 & 2.15410200 \\
\hline $\mathrm{H}$ & -0.15577700 & 1.81994200 & 3.08836600 \\
\hline $\mathrm{H}$ & 1.47876500 & 1.75724600 & 2.43210600 \\
\hline $\mathrm{H}$ & 0.16721800 & 2.57111800 & 1.51865600 \\
\hline
\end{tabular}




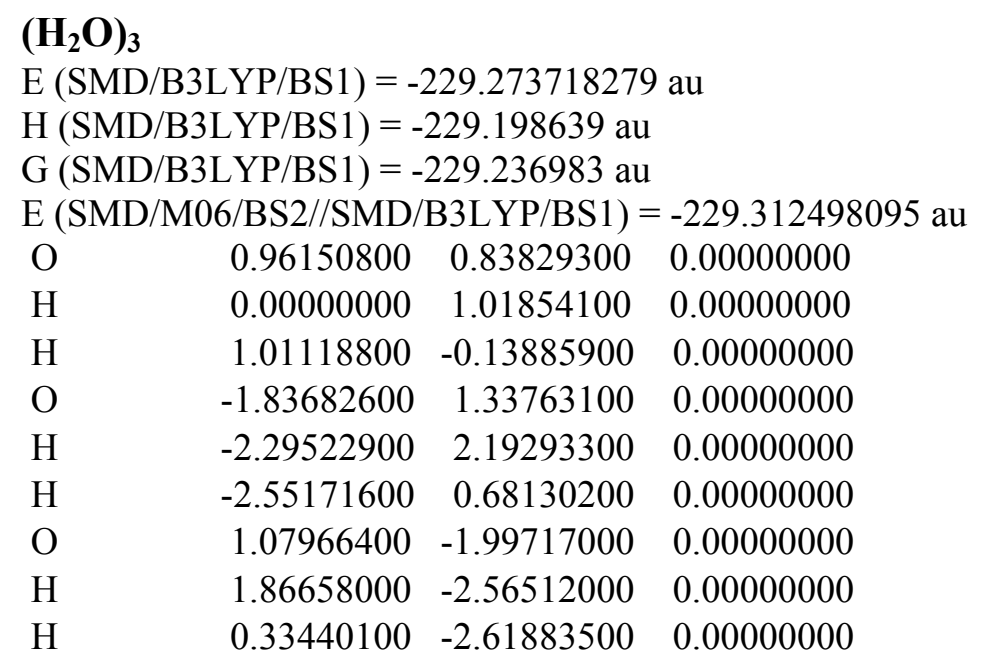

\section{$25 \mathrm{H}_{2} \mathrm{O}$}

$\mathrm{E}(\mathrm{SMD} / \mathrm{B} 3 \mathrm{LYP} / \mathrm{BS} 1)=-539.397858799 \mathrm{au}$

$\mathrm{H}(\mathrm{SMD} / \mathrm{B} 3 \mathrm{LYP} / \mathrm{BS} 1)=-539.160101 \mathrm{au}$

$\mathrm{G}(\mathrm{SMD} / \mathrm{B} 3 \mathrm{LYP} / \mathrm{BS} 1)=-539.215787 \mathrm{au}$

$\mathrm{E}(\mathrm{SMD} / \mathrm{M} 06 / \mathrm{BS} 2 / / \mathrm{SMD} / \mathrm{B} 3 \mathrm{LYP} / \mathrm{BS} 1)=-539.27651744 \mathrm{au}$

$\begin{array}{llll}\mathrm{C} & 2.59450800 & -0.56101400 & -1.18687800\end{array}$

$\begin{array}{llll}\mathrm{C} & 1.23357100 & -0.76910600 & -0.96236600\end{array}$

$\begin{array}{llll}\mathrm{C} & 0.64319200 & -0.35206600 & 0.24020400\end{array}$

$\begin{array}{llll}\mathrm{C} & 1.43453400 & 0.27950800 & 1.20808900\end{array}$

$\begin{array}{llll}\mathrm{C} & 2.80002800 & 0.47967300 & 0.98664500\end{array}$

C $\quad 3.38142100 \quad 0.06056400 \quad-0.21158200$

$\mathrm{H} \quad 3.04212800 \quad-0.88474700 \quad-2.12238500$

$\mathrm{H} \quad \begin{array}{llll}0.62788500 & -1.24988000 & -1.72545000\end{array}$

$\begin{array}{llll}\mathrm{H} & 0.98096000 & 0.60776700 & 2.13992700\end{array}$

$\mathrm{H} \quad 3.40461200 \quad 0.96548200 \quad 1.74731600$

$\mathrm{H} \quad 4.44180200 \quad 0.21864800 \quad-0.38766800$

$\begin{array}{llll}\mathrm{C} & -0.82617100 & -0.57812300 & 0.50640100\end{array}$

$\begin{array}{llll}\mathrm{C} & -1.27017800 & -2.03023400 & 0.45020100\end{array}$

$\mathrm{H} \quad-2.34641700 \quad-2.11240800 \quad 0.63472500$

$\mathrm{H} \quad-1.03926000 \quad-2.47861700 \quad-0.52090200$

$\mathrm{H} \quad \begin{array}{llll}-0.74463500 & -2.59240700 & 1.22828000\end{array}$

$\mathrm{H} \quad-1.10423400 \quad-0.12928200 \quad 1.46294500$

$\begin{array}{lllll}\mathrm{O} & -1.59651900 & 0.15893900 & -0.54274200\end{array}$

$\mathrm{H} \quad-1.35378600 \quad 1.17450200 \quad-0.50499700$

$\mathrm{H} \quad-2.62442000 \quad 0.07489200 \quad-0.36964600$

$\begin{array}{llll}\mathrm{O} & -4.11256300 & -0.01049000 & -0.13564500\end{array}$

$\mathrm{H} \quad \begin{array}{llll}\mathrm{H} & -4.27006600 & 0.39321700 & 0.73714500\end{array}$

$\begin{array}{llll}\mathrm{O} & -0.92659700 & 2.62234400 & -0.41814600\end{array}$

$\mathrm{H} \quad \begin{array}{rrrr}-1.51356500 & 3.03968000 & 0.23816500\end{array}$

$\mathrm{H} \quad-4.30131200 \quad-0.95639200 \quad 0.00340100$

$\begin{array}{llll}\mathrm{H} & -0.05967900 & 2.58798900 & 0.02712300\end{array}$

2 (optimized by SMD/B3LYP-D3/BS1)

E $($ SMD/B3LYP-D3/BS1 $)=-309.661862989$ au 


$\begin{array}{lrrl}\text { H }(\mathrm{SMD} / \mathrm{B} 3 \mathrm{LYP}-\mathrm{D} 3 / \mathrm{BS} 1)=-309.520313 \mathrm{au} \\ \mathrm{G}(\mathrm{SMD} / \mathrm{B} 3 \mathrm{~L} \text { YP-D3/BS1) }=-309.558979 \mathrm{au} \\ \mathrm{E}(\mathrm{SMD} / \mathrm{M} \text { 06/BS2//SMD/B3LYP-D3/BS1 })=-309.512849456 \mathrm{au} \\ \mathrm{C} & -0.68487900 & -1.77702000 & 0.00000000 \\ \mathrm{C} & -1.00892800 & -0.42215300 & 0.00000000 \\ \mathrm{C} & 0.00000000 & 0.56145400 & 0.00000000 \\ \mathrm{C} & 1.34068400 & 0.13372800 & 0.00000000 \\ \mathrm{C} & 1.66635900 & -1.22365400 & 0.00000000 \\ \mathrm{C} & 0.65414300 & -2.18585300 & 0.00000000 \\ \mathrm{H} & -1.47939400 & -2.51901000 & 0.00000000 \\ \mathrm{H} & -2.05408800 & -0.12602200 & 0.00000000 \\ \mathrm{H} & 2.13412900 & 0.87794200 & 0.00000000 \\ \mathrm{H} & 2.70981100 & -1.52811800 & 0.00000000 \\ \mathrm{H} & 0.90279500 & -3.24379400 & 0.00000000 \\ \mathrm{C} & -0.28455400 & 2.00758400 & 0.00000000 \\ \mathrm{H} & 0.60205400 & 2.64239600 & 0.00000000 \\ \mathrm{C} & -1.48662600 & 2.60060100 & 0.00000000 \\ \mathrm{H} & -2.42105400 & 2.04439300 & 0.00000000 \\ \mathrm{H} & -1.57144600 & 3.68409400 & 0.00000000\end{array}$

\begin{tabular}{|c|c|c|c|}
\hline \multicolumn{4}{|c|}{3 (optimized by SMD/B3LYP-D3/BS1) } \\
\hline \multicolumn{4}{|c|}{$\mathrm{E}(\mathrm{SMD} / \mathrm{B} 3 \mathrm{LYP}-\mathrm{D} 3 / \mathrm{BS} 1)=-655.504684781 \mathrm{au}$} \\
\hline \multicolumn{4}{|c|}{$\mathrm{H}(\mathrm{SMD} / \mathrm{B} 3 \mathrm{~L} Y \mathrm{P}-\mathrm{D} 3 / \mathrm{BS} 1)=-655.227293 \mathrm{au}$} \\
\hline \multicolumn{4}{|c|}{$\mathrm{G}(\mathrm{SMD} / \mathrm{B} 3 \mathrm{LYP}-\mathrm{D} 3 / \mathrm{BS} 1)=-655.287482 \mathrm{au}$} \\
\hline \multicolumn{4}{|c|}{$\mathrm{E}(\mathrm{SMD} / \mathrm{M} 06 / \mathrm{BS} 2 / / \mathrm{SMD} / \mathrm{B} 3 \mathrm{LYP}-\mathrm{D} 3 / \mathrm{BS} 1)=-655.263090721 \mathrm{au}$} \\
\hline $\mathrm{C}$ & -2.95360700 & -1.90344000 & -0.65922700 \\
\hline $\mathrm{H}$ & -2.59465000 & -2.59861500 & 0.11026500 \\
\hline $\mathrm{H}$ & -2.46704800 & -2.18064500 & -1.60091000 \\
\hline $\mathrm{H}$ & -4.03783900 & -2.00778000 & -0.74750300 \\
\hline $\mathrm{C}$ & -2.61202000 & -0.48421200 & -0.26185800 \\
\hline $\mathrm{O}$ & -3.46235900 & 0.29072700 & 0.14601600 \\
\hline $\mathrm{C}$ & -1.14265400 & -0.05568300 & -0.39681100 \\
\hline $\mathrm{H}$ & -0.88482300 & -0.20688400 & -1.45480000 \\
\hline $\mathrm{C}$ & -1.03715400 & 1.44605600 & -0.10105300 \\
\hline $\mathrm{C}$ & -1.37010000 & 2.36105300 & -1.25683900 \\
\hline $\mathrm{H}$ & -2.31119900 & 2.06018300 & -1.73082300 \\
\hline $\mathrm{H}$ & -1.43553000 & 3.39677500 & -0.91474900 \\
\hline $\mathrm{H}$ & -0.58010600 & 2.27748200 & -2.01579100 \\
\hline $\mathrm{O}$ & -0.69918800 & 1.88027800 & 0.98618000 \\
\hline $\mathrm{C}$ & 3.93326100 & 0.11451200 & -0.42482600 \\
\hline $\mathrm{C}$ & 3.31470900 & 0.60576800 & 0.72727900 \\
\hline $\mathrm{C}$ & 1.99251100 & 0.26150800 & 1.02252500 \\
\hline $\mathrm{C}$ & 1.26523200 & -0.58502800 & 0.17390500 \\
\hline $\mathrm{C}$ & 1.89976700 & -1.07297000 & -0.97955000 \\
\hline $\mathrm{C}$ & 3.21859600 & -0.72844300 & -1.28085600 \\
\hline $\mathrm{H}$ & 4.96157000 & 0.38322300 & -0.65252000 \\
\hline $\mathrm{H}$ & 3.86064800 & 1.26214500 & 1.40049300 \\
\hline $\mathrm{H}$ & 1.52373500 & 0.66423700 & 1.91320800 \\
\hline $\mathrm{H}$ & 1.35132400 & -1.73495800 & -1.64734400 \\
\hline $\mathrm{H}$ & 3.68885300 & -1.12249700 & -2.17840900 \\
\hline $\mathrm{C}$ & -0.18649900 & -0.97147300 & 0.43755000 \\
\hline
\end{tabular}




$\begin{array}{lrrr}\mathrm{H} & -0.31458500 & -1.97670800 & 0.01774300 \\ \mathrm{C} & -0.56631000 & -1.04699400 & 1.92434700 \\ \mathrm{H} & -0.55432600 & -0.06356300 & 2.39562300 \\ \mathrm{H} & -1.57544300 & -1.45933700 & 2.04087200 \\ \mathrm{H} & 0.12740100 & -1.70503100 & 2.45956200\end{array}$

9 (optimized by SMD/B3LYP-D3/BS1)

$\mathrm{E}(\mathrm{SMD} / \mathrm{B} 3 \mathrm{LYP}-\mathrm{D} 3 / \mathrm{BS} 1)=-345.810374986 \mathrm{au}$

$\mathrm{H}(\mathrm{SMD} / \mathrm{B} 3 \mathrm{LYP}-\mathrm{D} 3 / \mathrm{BS} 1)=-345.678867 \mathrm{au}$

$\mathrm{G}(\mathrm{SMD} / \mathrm{B} 3 \mathrm{LYP}-\mathrm{D} 3 / \mathrm{BS} 1)=-345.722242 \mathrm{au}$

$\mathrm{E}(\mathrm{SMD} / \mathrm{M} 06 / \mathrm{BS} 2 / / \mathrm{SMD} / \mathrm{B} 3 \mathrm{LYP}-\mathrm{D} 3 / \mathrm{BS} 1)=-345.721455792 \mathrm{au}$

$\begin{array}{llll}\mathrm{C} & 1.94653200 & 0.99241300 & 0.35660800\end{array}$

$\mathrm{H} \quad 1.28340400 \quad 1.80141900 \quad 0.02932300$

$\mathrm{H} \quad 2.91862600 \quad 1.07432300 \quad-0.13586100$

$\begin{array}{llll}\mathrm{H} & 2.06862700 & 1.09266300 & 1.44291600\end{array}$

$\begin{array}{llll}\mathrm{C} & 1.30051700 & -0.33087900 & 0.03081300\end{array}$

$\begin{array}{llll}\mathrm{O} & 1.83206300 & -1.16600200 & -0.68187400\end{array}$

$\begin{array}{llll}\mathrm{C} & -0.07619100 & -0.59638400 & 0.63154400\end{array}$

$\mathrm{H} \quad-0.29373800 \quad-1.66678700 \quad 0.56927700$

$\mathrm{H} \quad-0.08619500 \quad-0.31255200 \quad 1.69417600$

$\begin{array}{llll}\mathrm{C} & -1.20738100 & 0.20044000 & -0.02957400\end{array}$

$\begin{array}{llll}\mathrm{O} & -1.02030700 & 1.27446200 & -0.57571200\end{array}$

$\begin{array}{llll}\mathrm{C} & -2.57450400 & -0.43621800 & 0.05246400\end{array}$

$\mathrm{H} \quad-3.34019200 \quad 0.26128400 \quad-0.29569700$

$\mathrm{H} \quad-2.58819100-1.33820900 \quad-0.57378600$

$\mathrm{H} \quad-2.79022500 \quad-0.75604800 \quad 1.07921200$

10 (optimized by SMD/B3LYP-D3/BS1)

E $($ SMD/B3LYP-D3/BS1) $=-3365.87710885$ au

$\mathrm{H}(\mathrm{SMD} / \mathrm{B} 3 \mathrm{LYP}-\mathrm{D} 3 / \mathrm{BS} 1)=-3365.630113 \mathrm{au}$

$\mathrm{G}(\mathrm{SMD} / \mathrm{B} 3 \mathrm{LYP}-\mathrm{D} 3 / \mathrm{BS} 1)=-3365.735169 \mathrm{au}$

$\mathrm{E}(\mathrm{SMD} / \mathrm{M} 06 / \mathrm{BS} 2 / / \mathrm{SMD} / \mathrm{B} 3 \mathrm{LYP}-\mathrm{D} 3 / \mathrm{BS} 1)=-3366.02242479 \mathrm{au}$

$\begin{array}{llll}\mathrm{C} & 1.66632900 & 3.53455700 & -1.04978200\end{array}$

$\mathrm{H} \quad 2.56876500 \quad 4.06131600 \quad-0.70081300$

$\mathrm{H} \quad 1.11919800 \quad 4.16751800 \quad-1.74923600$

$\mathrm{H} \quad 2.01012400 \quad 2.60870900 \quad-1.51827100$

$\begin{array}{llll}\mathrm{C} & 0.85518400 & 3.23532800 & 0.14047000\end{array}$

$\begin{array}{llll}\mathrm{O} & -0.19545600 & 3.92818000 & 0.35015300\end{array}$

$\begin{array}{llll}\mathrm{C} & 1.23065400 & 2.17661400 & 1.09958300\end{array}$

$\begin{array}{llll}\mathrm{C} & 0.63910400 & 2.30460100 & 2.48792100\end{array}$

$\begin{array}{llll}\mathrm{O} & -0.39644300 & 2.96228400 & 2.64446400\end{array}$

$\begin{array}{llll}\mathrm{C} & 1.33970400 & 1.61695700 & 3.61136700\end{array}$

$\begin{array}{llll}\mathrm{H} & 2.30049900 & 2.12125000 & 3.78390300\end{array}$

$\begin{array}{llll}\mathrm{H} & 0.73269300 & 1.65558100 & 4.51747700\end{array}$

$\begin{array}{llll}\mathrm{H} & 1.56868000 & 0.57853300 & 3.34527900\end{array}$

$\begin{array}{llll}\mathrm{H} & 2.29932400 & 1.96853700 & 1.08175800\end{array}$

$\mathrm{H} \quad-0.58842000 \quad 3.64934700 \quad 1.26857100$

$\mathrm{Au} \quad 0.39217400 \quad 0.34745300 \quad 0.38778700$

$\begin{array}{llll}\mathrm{O} & -0.37280900 & -1.50817300 & -0.24557800\end{array}$

S $\quad \begin{array}{llll}-0.60479000 & -2.64231800 & 0.79822200\end{array}$

$\begin{array}{llll}\mathrm{O} & -0.49186200 & -3.94277600 & 0.14263600\end{array}$ 


$\begin{array}{llcc}\mathrm{O} & 0.08946200 & -2.38754900 & 2.06360800 \\ \mathrm{C} & -2.41487900 & -2.39286700 & 1.16589900 \\ \mathrm{~F} & -3.13818300 & -2.56940300 & 0.05978200 \\ \mathrm{~F} & -2.80128400 & -3.26948400 & 2.09529900 \\ \mathrm{~F} & -2.60571200 & -1.14925400 & 1.62764200 \\ \mathrm{O} & -2.89154500 & 2.85943000 & -0.90599300 \\ \mathrm{~S} & -1.99344600 & 1.73760000 & -1.15502900 \\ \mathrm{O} & -0.91542100 & 1.83455300 & -2.14234200 \\ \mathrm{O} & -1.43742300 & 1.24596300 & 0.24323300 \\ \mathrm{C} & -3.08976300 & 0.31239800 & -1.67556200 \\ \mathrm{~F} & -3.81652900 & 0.72006600 & -2.71672100 \\ \mathrm{~F} & -2.34935400 & -0.73305700 & -2.03074400 \\ \mathrm{~F} & -3.89371300 & -0.01704100 & -0.66719000 \\ \mathrm{O} & 3.46042000 & 0.82676600 & -0.95512300 \\ \mathrm{~S} & 3.33419300 & -0.51037500 & -0.37066300 \\ \mathrm{O} & 4.49544800 & -1.19373200 & 0.18518700 \\ \mathrm{O} & 2.18743200 & -0.57133600 & 0.70806200 \\ \mathrm{C} & 2.64720300 & -1.60252100 & -1.72604100 \\ \mathrm{~F} & 3.59588900 & -1.78344200 & -2.64276800 \\ \mathrm{~F} & 2.27437500 & -2.77373200 & -1.21988000 \\ \mathrm{~F} & 1.59411500 & -0.99769800 & -2.28918400\end{array}$

TS $_{10-22}$ (optimized by SMD/B3LYP-D3/BS1)

$\mathrm{E}(\mathrm{SMD} / \mathrm{B} 3 \mathrm{LYP}-\mathrm{D} 3 / \mathrm{BS} 1)=-3675.53932768 \mathrm{au}$

$\mathrm{H}(\mathrm{SMD} / \mathrm{B} 3 \mathrm{LYP}-\mathrm{D} 3 / \mathrm{BS} 1)=-3675.153885 \mathrm{au}$

G (SMD/B3LYP-D3/BS1) $=-3675.280493$ au

$\mathrm{E}(\mathrm{SMD} / \mathrm{M} 06 / \mathrm{BS} 2 / / \mathrm{SMD} / \mathrm{B} 3 \mathrm{LYP}-\mathrm{D} 3 / \mathrm{BS} 1)=-3675.51900685 \mathrm{au}$

$\begin{array}{lrrr}\mathrm{C} & -0.81558800 & 2.03338500 & 3.61482800 \\ \mathrm{H} & -1.77688600 & 2.52718400 & 3.45539100 \\ \mathrm{H} & 0.01788700 & 2.67458700 & 3.32292000 \\ \mathrm{H} & -0.69986800 & 1.83000700 & 4.69112200 \\ \mathrm{C} & -0.77433000 & 0.72256600 & 2.90550800 \\ \mathrm{O} & -1.85932600 & 0.14977100 & 2.67372500 \\ \mathrm{C} & 0.55915400 & 0.13499700 & 2.56709100 \\ \mathrm{C} & 0.82498600 & -1.25119100 & 3.16585800 \\ \mathrm{O} & -0.08992500 & -1.99230300 & 3.46933100 \\ \mathrm{C} & 2.27599100 & -1.56223200 & 3.41855700 \\ \mathrm{H} & 2.91766700 & -1.17500100 & 2.62208200 \\ \mathrm{H} & 2.57396400 & -1.05655900 & 4.34742300 \\ \mathrm{H} & 2.41593800 & -2.63807800 & 3.53335500 \\ \mathrm{H} & 1.35721100 & 0.83417400 & 2.82034700 \\ \mathrm{C} & -5.85451200 & -0.81337800 & 1.27519900 \\ \mathrm{C} & -6.79714700 & -0.05130900 & 0.59132800 \\ \mathrm{C} & -6.51195200 & 0.41386400 & -0.69685800 \\ \mathrm{C} & -5.28853500 & 0.10516400 & -1.30643400 \\ \mathrm{C} & -4.34757900 & -0.66200000 & -0.63148900 \\ \mathrm{C} & -4.61087900 & -1.11858000 & 0.67937800 \\ \mathrm{H} & -6.06465600 & -1.16893400 & 2.28053000 \\ \mathrm{H} & -7.74882400 & 0.18332700 & 1.05882800 \\ \mathrm{H} & -7.24462600 & 1.01378400 & -1.22960200 \\ \mathrm{H} & -5.06765200 & 0.46578000 & -2.30650000\end{array}$




$\begin{array}{lrrr}\mathrm{H} & -3.41399100 & -0.91409200 & -1.11871100 \\ \mathrm{C} & -3.64405600 & -1.87292900 & 1.44719800 \\ \mathrm{H} & -4.01084400 & -2.26384800 & 2.39708900 \\ \mathrm{C} & -2.30011300 & -2.03508500 & 1.18308100 \\ \mathrm{H} & -1.88764300 & -1.77605000 & 0.20938100 \\ \mathrm{H} & -1.98639700 & -0.91025800 & 1.99549300 \\ \mathrm{H} & -1.74410900 & -2.76979100 & 1.76204600 \\ \mathrm{Au} & 0.66345400 & 0.06266000 & 0.47298900 \\ \mathrm{O} & 0.78101000 & 0.14212700 & -1.66035000 \\ \mathrm{~S} & -0.46824500 & -0.19321400 & -2.50146000 \\ \mathrm{O} & -0.15540500 & -0.13599000 & -3.92779600 \\ \mathrm{O} & -1.20366800 & -1.34892300 & -1.96937500 \\ \mathrm{C} & -1.57315200 & 1.27675300 & -2.16412800 \\ \mathrm{~F} & -0.88642600 & 2.41485100 & -2.25267000 \\ \mathrm{~F} & -2.57224200 & 1.29091200 & -3.05133300 \\ \mathrm{~F} & -2.09626300 & 1.17413900 & -0.92857300 \\ \mathrm{O} & 0.72577200 & 4.46942000 & 0.59941300 \\ \mathrm{~S} & 1.37429500 & 3.16603000 & 0.68669500 \\ \mathrm{O} & 2.22392700 & 2.83389500 & 1.83310000 \\ \mathrm{O} & 0.26935200 & 2.07611100 & 0.43003100 \\ \mathrm{C} & 2.47452100 & 3.00740400 & -0.81720800 \\ \mathrm{~F} & 3.36834300 & 3.99580300 & -0.77931800 \\ \mathrm{~F} & 3.11031800 & 1.83210400 & -0.78006100 \\ \mathrm{~F} & 1.75356200 & 3.09336900 & -1.92931100 \\ \mathrm{O} & 3.37392200 & -1.54379300 & -0.12658400 \\ \mathrm{~S} & 2.35179500 & -2.57588000 & 0.06002300 \\ \mathrm{O} & 2.62376500 & -3.74954400 & 0.88548400 \\ \mathrm{O} & 0.97169200 & -1.96265700 & 0.51154100 \\ \mathrm{C} & 1.91542100 & -3.25103600 & -1.63760000 \\ \mathrm{~F} & 2.80927600 & -4.19385400 & -1.94334200 \\ \mathrm{~F} & 0.69535400 & -3.78170200 & -1.61131300 \\ \mathrm{~F} & 1.96720300 & -2.28346700 & -2.55134900\end{array}$

22 (optimized by SMD/B3LYP-D3/BS1)

E $($ SMD/B3LYP-D3/BS1) $=-3675.55459 \mathrm{au}$

$\mathrm{H}(\mathrm{SMD} / \mathrm{B} 3 \mathrm{LYP}-\mathrm{D} 3 / \mathrm{BS} 1)=-3675.16349 \mathrm{au}$

$\mathrm{G}(\mathrm{SMD} / \mathrm{B} 3 \mathrm{LYP}-\mathrm{D} 3 / \mathrm{BS} 1)=-3675.293233 \mathrm{au}$

$\mathrm{E}(\mathrm{SMD} / \mathrm{M} 06 / \mathrm{BS} 2 / / \mathrm{SMD} / \mathrm{B} 3 \mathrm{LYP}-\mathrm{D} 3 / \mathrm{BS} 1)=-3675.54104637 \mathrm{au}$

$\begin{array}{llll}\mathrm{C} & 0.14326400 & -3.51211700 & 1.98991800\end{array}$

$\mathrm{H} \quad 0.56200700 \quad-4.48665900 \quad 1.70195200$

$\mathrm{H} \quad-0.56952300 \quad-3.64195500 \quad 2.80520900$

$\begin{array}{llll}\mathrm{H} & 0.97854200 & -2.88107800 & 2.30788400\end{array}$

$\begin{array}{llll}\mathrm{C} & -0.53357800 & -2.91743700 & 0.78780400\end{array}$

$\begin{array}{llll}\mathrm{O} & -1.74639400 & -2.83760600 & 0.66035300\end{array}$

C $\quad 0.39014300 \quad-2.47497600 \quad-0.34122200$

$\begin{array}{llll}\mathrm{C} & -0.21740500 & -2.68178000 & -1.72433300\end{array}$

$\begin{array}{llll}\mathrm{O} & -0.57853300 & -1.74849400 & -2.42162000\end{array}$

C $\quad-0.31561600 \quad-4.12028900 \quad-2.16513000$

$\mathrm{H} \quad-0.82860500 \quad-4.72625400 \quad-1.40927600$

$\mathrm{H} \quad-0.84752100 \quad-4.18024500 \quad-3.11774400$

$\mathrm{H} \quad 0.69638100 \quad-4.52954000 \quad-2.28088700$ 


$\begin{array}{lccc}\mathrm{H} & 1.35281400 & -2.98418400 & -0.26320500 \\ \mathrm{Au} & 0.90588700 & -0.45117600 & -0.17588500 \\ \mathrm{O} & 1.46603600 & 1.66163800 & -0.13667800 \\ \mathrm{~S} & 1.53178500 & 2.53493400 & -1.39594100 \\ \mathrm{O} & 1.94542200 & 3.89768400 & -1.05026000 \\ \mathrm{O} & 2.15236600 & 1.86976100 & -2.54616500 \\ \mathrm{C} & -0.26913700 & 2.66405300 & -1.86330700 \\ \mathrm{~F} & -1.00212700 & 3.13725600 & -0.85076000 \\ \mathrm{~F} & -0.40670700 & 3.47588800 & -2.91687600 \\ \mathrm{~F} & -0.73961600 & 1.44596600 & -2.19798400 \\ \mathrm{O} & -2.58504000 & 0.64079100 & 2.30240200 \\ \mathrm{~S} & -1.17594800 & 0.27615200 & 2.13320200 \\ \mathrm{O} & -0.59746900 & -0.81078300 & 2.92117700 \\ \mathrm{O} & -0.91140400 & 0.13047700 & 0.59865700 \\ \mathrm{C} & -0.19540400 & 1.79200800 & 2.62102400 \\ \mathrm{~F} & -0.55272200 & 2.12301600 & 3.86613900 \\ \mathrm{~F} & 1.10867700 & 1.50797300 & 2.60201800 \\ \mathrm{~F} & -0.45555000 & 2.80688500 & 1.80221500 \\ \mathrm{O} & 3.48273900 & -2.39869600 & 0.78673600 \\ \mathrm{~S} & 3.88093500 & -1.47318000 & -0.27813500 \\ \mathrm{O} & 4.93904200 & -1.83699700 & -1.21534000 \\ \mathrm{O} & 2.65605600 & -0.95527800 & -1.10839000 \\ \mathrm{C} & 4.45191600 & 0.08059800 & 0.59298100 \\ \mathrm{~F} & 5.62763400 & -0.16836900 & 1.17323500 \\ \mathrm{~F} & 4.58523100 & 1.07636500 & -0.27995100 \\ \mathrm{~F} & 3.56220200 & 0.42266500 & 1.53196700 \\ \mathrm{C} & -4.22879600 & -0.09922400 & -0.37146300 \\ \mathrm{C} & -5.62566000 & -0.15717300 & -0.35187200 \\ \mathrm{C} & -6.38770800 & -0.80505800 & -1.37255200 \\ \mathrm{C} & -6.30513800 & 0.45490000 & 0.74754800 \\ \mathrm{C} & -7.76575500 & -0.83246000 & -1.28724900 \\ \mathrm{H} & -5.88305900 & -1.27280300 & -2.21028300 \\ \mathrm{C} & -7.68563900 & 0.41887300 & 0.82008900 \\ \mathrm{H} & -5.72006800 & 0.94127300 & 1.52280200 \\ \mathrm{C} & -8.41085000 & -0.22255200 & -0.19570900 \\ \mathrm{H} & -8.35314600 & -1.32080500 & -2.05776300 \\ \mathrm{H} & -8.20591900 & 0.88045800 & 1.65260700 \\ \mathrm{H} & -9.49546900 & -0.24967400 & -0.13893100 \\ \mathrm{H} & -3.75559900 & 0.41825700 & 0.46225300 \\ \mathrm{C} & -3.30703000 & -0.68195400 & -1.33701600 \\ \mathrm{H} & -2.54763900 & 0.06111800 & -1.60975800 \\ \mathrm{H} & -2.72670500 & -1.44680400 & -0.78749700 \\ \mathrm{H} & -3.76132900 & -1.12596600 & -2.22255500\end{array}$

23 (optimized by SMD/B3LYP-D3/BS1)

$\mathrm{E}(\mathrm{SMD} / \mathrm{B} 3 \mathrm{LYP}-\mathrm{D} 3 / \mathrm{BS} 1)=-310.082760853 \mathrm{au}$

$\mathrm{H}(\mathrm{SMD} / \mathrm{B} 3 \mathrm{~L} Y \mathrm{P}-\mathrm{D} 3 / \mathrm{BS} 1)=-309.929866 \mathrm{au}$

G (SMD/B3LYP-D3/BS1) $=-309.968014 \mathrm{au}$

$\mathrm{E}(\mathrm{SMD} / \mathrm{M} 06 / \mathrm{BS} 2 / / \mathrm{SMD} / \mathrm{B} 3 \mathrm{LYP}-\mathrm{D} 3 / \mathrm{BS} 1)=-309.924168865 \mathrm{au}$

$\begin{array}{lrrr}\mathrm{C} & 1.33318600 & 1.38027200 & -0.00023400 \\ \mathrm{C} & -0.01524300 & 1.09338100 & -0.00059600\end{array}$ 


$\begin{array}{lrrc}\mathrm{C} & -0.45300300 & -0.27084900 & -0.00036400 \\ \mathrm{C} & 0.52263600 & -1.31984900 & 0.00008800 \\ \mathrm{C} & 1.87065900 & -1.01615300 & 0.00043200 \\ \mathrm{C} & 2.27161300 & 0.32906900 & 0.00027600 \\ \mathrm{H} & 1.67664900 & 2.40917500 & -0.00036800 \\ \mathrm{H} & -0.74660500 & 1.89459100 & -0.00100200 \\ \mathrm{H} & 0.18722900 & -2.35259400 & 0.00025300 \\ \mathrm{H} & 2.61431300 & -1.80596400 & 0.00083100 \\ \mathrm{H} & 3.33160800 & 0.56703600 & 0.00054100 \\ \mathrm{C} & -1.80131700 & -0.62098300 & -0.00024300 \\ \mathrm{H} & -2.02278800 & -1.68798100 & -0.00008000 \\ \mathrm{C} & -2.95030700 & 0.30406800 & 0.00021800 \\ \mathrm{H} & -3.90000900 & -0.23150500 & -0.00263600 \\ \mathrm{H} & -2.90618200 & 0.96389400 & 0.87906400 \\ \mathrm{H} & -2.90356200 & 0.96961000 & -0.87406400\end{array}$

24 (optimized by SMD/B3LYP-D3/BS1)

$\mathrm{E}(\mathrm{SMD} / \mathrm{B} 3 \mathrm{LYP}-\mathrm{D} 3 / \mathrm{BS} 1)=-3365.4461268 \mathrm{au}$

$\mathrm{H}(\mathrm{SMD} / \mathrm{B} 3 \mathrm{LYP}-\mathrm{D} 3 / \mathrm{BS} 1)=-3365.211364 \mathrm{au}$

$\mathrm{G}(\mathrm{SMD} / \mathrm{B} 3 \mathrm{LYP}-\mathrm{D} 3 / \mathrm{BS} 1)=-3365.321305 \mathrm{au}$

$\mathrm{E}(\mathrm{SMD} / \mathrm{M} 06 / \mathrm{BS} 2 / / \mathrm{SMD} / \mathrm{B} 3 \mathrm{LYP}-\mathrm{D} 3 / \mathrm{BS} 1)=-3365.60015643 \mathrm{au}$

$\begin{array}{llll}\mathrm{C} & 2.62675700 & 2.80568200 & -1.01914600\end{array}$

$\mathrm{H} \quad 3.68374300 \quad 2.74639100 \quad-0.72686000$

$\mathrm{H} \quad 2.46489400 \quad 3.68249500 \quad-1.64803800$

$\mathrm{H} \quad 2.39521200 \quad 1.89082900 \quad-1.56954500$

$\begin{array}{llll}\mathrm{C} & 1.78795900 & 2.90493700 & 0.22571000\end{array}$

$\begin{array}{llll}\mathrm{O} & 1.20581300 & 3.92078800 & 0.56782300\end{array}$

$\begin{array}{llll}\text { C } & 1.74257300 & 1.66374800 & 1.11496600\end{array}$

$\begin{array}{llll}\mathrm{C} & 1.36268800 & 1.93686500 & 2.56585600\end{array}$

$\begin{array}{llll}\mathrm{O} & 0.38712300 & 1.41814900 & 3.08382800\end{array}$

$\begin{array}{llll}\mathrm{C} & 2.28919000 & 2.85923600 & 3.31788400\end{array}$

$\mathrm{H} \quad 2.13032700 \quad 3.88384400 \quad 2.96246700$

$\begin{array}{llll}\mathrm{H} & 2.07202600 & 2.80510700 & 4.38756900\end{array}$

$\mathrm{H} \quad 3.33786600 \quad 2.60147600 \quad 3.12904600$

$\begin{array}{llll}\mathrm{H} & 2.69823300 & 1.13331600 & 1.07209500\end{array}$

$\mathrm{Au} \quad 0.39373500 \quad 0.23285800 \quad 0.38850200$

$\begin{array}{llll}\mathrm{O} & -1.00948700 & -1.34462900 & -0.22490900\end{array}$

S $\quad \begin{array}{llll}\text { S } & -1.53253900 & -2.33159100 & 0.82672400\end{array}$

$\begin{array}{llll}\mathrm{O} & -2.01320900 & -3.56645600 & 0.19910100\end{array}$

$\begin{array}{llll}\text { O } & -0.67588900 & -2.42301300 & 2.01666400\end{array}$

$\begin{array}{llll}\text { C } & -3.05852700 & -1.45205900 & 1.43177700\end{array}$

F $\quad-3.97758300 \quad-1.36969900 \quad 0.46410400$

F $\quad-3.58075500 \quad-2.12260000 \quad 2.46723400$

F $\quad \begin{array}{llll}-2.74173200 & -0.21279400 & 1.83725500\end{array}$

$\begin{array}{lllll}\mathrm{O} & -2.33057200 & 3.43538200 & -0.92729900\end{array}$

S $\quad-1.38789300 \quad 2.34878900 \quad-1.18249700$

$\begin{array}{lllll}\mathrm{O} & -0.19164600 & 2.57513800 & -1.99493000\end{array}$

$\begin{array}{llll}\mathrm{O} & -1.07982100 & 1.64643400 & 0.18047200\end{array}$

C $\quad-2.34514500 \quad 1.04434100 \quad-2.11659600$

F $\quad-2.92812300 \quad 1.62751600 \quad-3.17034500$

F $\quad-1.51078700 \quad 0.09645700 \quad-2.55203500$ 


$\begin{array}{lrrr}\text { F } & -3.28169500 & 0.49981300 & -1.34186300 \\ \text { O } & 3.49709700 & -0.31435200 & -0.91928400 \\ \text { S } & 2.93041600 & -1.52079000 & -0.31003500 \\ \text { O } & 3.81019300 & -2.54331200 & 0.24990700 \\ \text { O } & 1.82300600 & -1.19952900 & 0.74472000 \\ \text { C } & 1.95250300 & -2.35805500 & -1.66557300 \\ \text { F } & 2.80847800 & -2.87663500 & -2.54971300 \\ \text { F } & 1.19695800 & -3.32862800 & -1.15499000 \\ \text { F } & 1.17346600 & -1.46306500 & -2.28669600\end{array}$

25 (optimized by SMD/B3LYP-D3/BS1)

$\mathrm{E}(\mathrm{SMD} / \mathrm{B} 3 \mathrm{LYP}-\mathrm{D} 3 / \mathrm{BS} 1)=-655.930778002 \mathrm{au}$

$\mathrm{H}(\mathrm{SMD} / \mathrm{B} 3 \mathrm{LYP}-\mathrm{D} 3 / \mathrm{BS} 1)=-655.641597 \mathrm{au}$

$\mathrm{G}(\mathrm{SMD} / \mathrm{B} 3 \mathrm{LYP}-\mathrm{D} 3 / \mathrm{BS} 1)=-655.700684 \mathrm{au}$

$\mathrm{E}(\mathrm{SMD} / \mathrm{M} 06 / \mathrm{BS} 2 / / \mathrm{SMD} / \mathrm{B} 3 \mathrm{LYP}-\mathrm{D} 3 / \mathrm{BS} 1)=-655.683409128 \mathrm{au}$

C $\quad 3.43641000 \quad-1.25223600 \quad 0.86508500$

$\mathrm{H} \quad 3.65940200 \quad-0.79504700 \quad 1.84032700$

$\mathrm{H} \quad 4.37111000 \quad-1.50568100 \quad 0.36113800$

$\begin{array}{llll}\mathrm{C} & 2.66937800 & -0.25189400 & 0.07001100\end{array}$

$\begin{array}{llll}\mathrm{O} & 3.17015100 & 0.39058600 & -0.85721800\end{array}$

$\begin{array}{llll}\mathrm{C} & 1.20360000 & 0.00218600 & 0.42827700\end{array}$

$\begin{array}{llll}\mathrm{C} & 0.88284900 & 1.45864700 & 0.35035900\end{array}$

$\begin{array}{llll}\mathrm{O} & 1.42804000 & 2.16443400 & -0.56036500\end{array}$

$\begin{array}{llll}\text { C } & -0.04628900 & 2.11605600 & 1.27882700\end{array}$

$\mathrm{H} \quad-0.36085000 \quad 3.09307900 \quad 0.90856300$

$\mathrm{H} \quad-0.89955300 \quad 1.46574200 \quad 1.49373300$

$\begin{array}{llll}\mathrm{H} & 0.50020100 & 2.24110900 & 2.22820600\end{array}$

$\mathrm{H} \quad 0.95543500 \quad-0.38425200 \quad 1.41874000$

$\mathrm{H} \quad 2.16898900 \quad 1.59627400 \quad-1.00763000$

C $\quad 0.27714000 \quad-0.75029000-0.65505000$

$\mathrm{H} \quad 0.51981500 \quad-0.29096200 \quad-1.61939100$

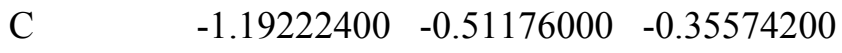

$\begin{array}{lllll}\mathrm{C} & & -1.95130600 & 0.31079900 & -1.19865600\end{array}$

$\begin{array}{llll}\text { C } & -1.80686900 & -1.08237000 & 0.76979400\end{array}$

$\begin{array}{llll}\mathrm{C} & -3.30025100 & 0.55593900 & -0.92859400\end{array}$

$\mathrm{H} \quad-1.48369300 \quad 0.76270300 \quad-2.07028700$

$\begin{array}{llll}\text { C } & -3.15340900 & -0.83538900 & 1.04217800\end{array}$

$\mathrm{H} \quad \begin{array}{llll}\mathrm{H} & -1.23203500 & -1.71821200 & 1.43827700\end{array}$

$\begin{array}{llll}\text { C } & -3.90402400 & -0.01545200 & 0.19319800\end{array}$

$\mathrm{H} \quad \begin{array}{llll}\mathrm{C} & -3.87579500 & 1.19266400 & -1.59489200\end{array}$

$\mathrm{H} \quad \begin{array}{llll}\mathrm{H} & -3.61751100 & -1.28487200 & 1.91582200\end{array}$

$\begin{array}{llll}\mathrm{H} & -4.95242300 & 0.17475600 & 0.40563900\end{array}$

C $\quad 0.63335500 \quad-2.24118700 \quad-0.72302300$

$\mathrm{H} \quad-0.05874100 \quad-2.73227500 \quad-1.41439500$

$\mathrm{H} \quad \begin{array}{llll}\mathrm{H} & 1.64939000 & -2.39859500 & -1.09866000\end{array}$

$\mathrm{H} \quad 0.54102900 \quad-2.72706000 \quad 0.25310500$

$\mathrm{H} \quad 2.83954800 \quad-2.14782700 \quad 1.06238600$

TS $_{23-25}$ (optimized by SMD/B3LYP-D3/BS1)

$\mathrm{E}(\mathrm{SMD} / \mathrm{B} 3 \mathrm{LYP}-\mathrm{D} 3 / \mathrm{BS} 1)=-655.914141154 \mathrm{au}$

$\mathrm{H}(\mathrm{SMD} / \mathrm{B} 3 \mathrm{LYP}-\mathrm{D} 3 / \mathrm{BS} 1)=-655.627651 \mathrm{au}$ 


$\begin{array}{lrrr}\text { G }(\mathrm{SMD} / \mathrm{B} 3 \mathrm{LYP}-\mathrm{D} 3 / \mathrm{BS} 1)=-655.684653 \mathrm{au} \\ \mathrm{E}(\mathrm{SMD} / \mathrm{M} 06 / \mathrm{BS} 2 / / \mathrm{SMD} / \mathrm{B} 3 \mathrm{LYP}-\mathrm{D} 3 / \mathrm{BS} 1)=-655.660118086 \mathrm{au} \\ \mathrm{C} & -2.36807500 & 2.49511300 & 0.59006200 \\ \mathrm{H} & -1.91022300 & 3.47621300 & 0.44672100 \\ \mathrm{H} & -2.35638600 & 2.22611500 & 1.65305100 \\ \mathrm{C} & -1.64475800 & 1.46134100 & -0.22764100 \\ \mathrm{O} & -0.74588000 & 1.79579100 & -1.02918100 \\ \mathrm{C} & -2.00224500 & 0.06136200 & -0.05294000 \\ \mathrm{C} & -1.52631000 & -0.90349300 & -0.93745500 \\ \mathrm{O} & -0.57793300 & -0.62343600 & -1.80508400 \\ \mathrm{C} & -2.00211300 & -2.31393100 & -0.96877200 \\ \mathrm{H} & -1.16362400 & -2.99447400 & -0.77652700 \\ \mathrm{H} & -2.78958800 & -2.49016600 & -0.23438800 \\ \mathrm{H} & -2.38021300 & -2.54299700 & -1.97228300 \\ \mathrm{H} & -2.81419700 & -0.19973300 & 0.61425400 \\ \mathrm{H} & -0.38002900 & 0.36634500 & -1.70788500 \\ \mathrm{C} & -0.11793800 & -0.49293900 & 1.61957200 \\ \mathrm{H} & -0.55807100 & 0.34599600 & 2.15299600 \\ \mathrm{C} & 1.03098400 & -0.20837700 & 0.84605300 \\ \mathrm{C} & 1.48559400 & 1.13957600 & 0.77034900 \\ \mathrm{C} & 1.74869200 & -1.21660600 & 0.14076700 \\ \mathrm{C} & 2.62942600 & 1.45672600 & 0.05475800 \\ \mathrm{H} & 0.93514300 & 1.91229400 & 1.29747900 \\ \mathrm{C} & 2.88693100 & -0.88711600 & -0.57462700 \\ \mathrm{H} & 1.40764700 & -2.24476400 & 0.16627400 \\ \mathrm{C} & 3.32828200 & 0.44512600 & -0.61601500 \\ \mathrm{H} & 2.97795800 & 2.48332300 & 0.00974400 \\ \mathrm{H} & 3.43585300 & -1.65699200 & -1.10732300 \\ \mathrm{H} & 4.22214600 & 0.69483100 & -1.18066000 \\ \mathrm{C} & -0.55687800 & -1.83355600 & 2.07473200 \\ \mathrm{H} & -0.05527500 & -2.00699100 & 3.04206700 \\ \mathrm{H} & -1.63168400 & -1.85400200 & 2.26993800 \\ \mathrm{H} & -0.27792200 & -2.64946500 & 1.40636700 \\ \mathrm{H} & -3.42058200 & 2.53627700 & 0.28124500 \\ & & & \end{array}$

TS $_{\text {25-23 }}$ (optimized by SMD/B3LYP-D3/BS1)

$\mathrm{E}(\mathrm{SMD} / \mathrm{B} 3 \mathrm{LYP}-\mathrm{D} 3 / \mathrm{BS} 1)=-965.669559466 \mathrm{au}$

$\mathrm{H}(\mathrm{SMD} / \mathrm{B} 3 \mathrm{LYP}-\mathrm{D} 3 / \mathrm{BS} 1)=-965.242204 \mathrm{au}$

G (SMD/B3LYP-D3/BS1) $=-965.316237$ au

$\mathrm{E}(\mathrm{SMD} / \mathrm{M} 06 / \mathrm{BS} 2 / / \mathrm{SMD} / \mathrm{B} 3 \mathrm{LYP}-\mathrm{D} 3 / \mathrm{BS} 1)=-965.191092425 \mathrm{au}$

$\begin{array}{llrr}\mathrm{C} & -2.72929800 & 0.56462900 & -2.41929100 \\ \mathrm{H} & -3.72483900 & 0.10583200 & -2.50409200 \\ \mathrm{H} & -2.25080700 & 0.59465800 & -3.39966300 \\ \mathrm{H} & -2.87901300 & 1.57777600 & -2.03005800 \\ \mathrm{C} & -1.91470800 & -0.23885300 & -1.44968900 \\ \mathrm{O} & -0.91753500 & -0.86285900 & -1.80268400 \\ \mathrm{C} & -2.36198400 & -0.23321300 & 0.00906400 \\ \mathrm{C} & -2.86934800 & -1.62180800 & 0.44055200 \\ \mathrm{O} & -2.36107700 & -2.65018200 & 0.02034300 \\ \mathrm{C} & -4.06039300 & -1.65588400 & 1.36512700 \\ \mathrm{H} & -4.16537800 & -0.75142900 & 1.96624800\end{array}$




$\begin{array}{lrrr}\mathrm{H} & -4.95208200 & -1.72794300 & 0.72477200 \\ \mathrm{H} & -4.02224300 & -2.54729700 & 1.99535700 \\ \mathrm{H} & -3.19118600 & 0.47193100 & 0.12745800 \\ \mathrm{C} & 3.71647300 & -0.97492800 & -0.94757100 \\ \mathrm{C} & 4.49996600 & -0.02694500 & -0.30554700 \\ \mathrm{C} & 4.25042200 & 0.27800900 & 1.04043500 \\ \mathrm{C} & 3.21978300 & -0.36205200 & 1.75143200 \\ \mathrm{C} & 2.42488400 & -1.30194600 & 1.11852500 \\ \mathrm{C} & 2.65746300 & -1.62500200 & -0.25062100 \\ \mathrm{H} & 3.88972000 & -1.21800600 & -1.99225000 \\ \mathrm{H} & 5.29736500 & 0.47867500 & -0.84029600 \\ \mathrm{H} & 4.86271400 & 1.02156100 & 1.54270900 \\ \mathrm{H} & 3.04518500 & -0.11286100 & 2.79295700 \\ \mathrm{H} & 1.62376200 & -1.78480200 & 1.66473900 \\ \mathrm{C} & 1.83368000 & -2.51257200 & -0.98074500 \\ \mathrm{H} & 2.12970800 & -2.68760600 & -2.01544900 \\ \mathrm{C} & 0.57437500 & -3.06785600 & -0.58106600 \\ \mathrm{H} & 0.35791500 & -3.11899800 & 0.48423200 \\ \mathrm{H} & -0.10719500 & -2.21436800 & -0.99622200 \\ \mathrm{H} & 0.26397800 & -3.95143800 & -1.14054100 \\ \mathrm{C} & -1.17558300 & 0.24822100 & 0.91849600 \\ \mathrm{H} & -0.37558500 & -0.48821900 & 0.81245000 \\ \mathrm{C} & -0.62960300 & 1.57427300 & 0.40807500 \\ \mathrm{C} & 0.60726600 & 1.61291600 & -0.25258000 \\ \mathrm{C} & -1.33992400 & 2.77236800 & 0.58224100 \\ \mathrm{C} & 1.12306800 & 2.81987500 & -0.73566700 \\ \mathrm{H} & 1.18109800 & 0.69360400 & -0.35011100 \\ \mathrm{C} & -0.82757700 & 3.98074000 & 0.10102000 \\ \mathrm{H} & -2.30087300 & 2.76237100 & 1.09038900 \\ \mathrm{C} & 0.40442400 & 4.00771400 & -0.56394300 \\ \mathrm{H} & 2.08517900 & 2.83119000 & -1.24094500 \\ \mathrm{H} & -1.38828500 & 4.90030500 & 0.24661900 \\ \mathrm{H} & 0.80217800 & 4.94743700 & -0.93704100 \\ \mathrm{C} & -1.56695400 & 0.31529000 & 2.40314900 \\ \mathrm{H} & -2.48008300 & 0.89820600 & 2.55800400 \\ \mathrm{H} & -1.72634400 & -0.68644400 & 2.81634400 \\ \mathrm{H} & -0.76458900 & 0.79233800 & 2.97474700\end{array}$

2 (optimized by SMD/B3LYP-D3/BS3)

E $($ SMD/B3LYP-D3/ BS3 $)=-309.688154516$ au $\mathrm{H}(\mathrm{SMD} / \mathrm{B} 3 \mathrm{LYP}-\mathrm{D} 3 / \mathrm{BS} 3)=-309.547165 \mathrm{au}$

$\mathrm{G}(\mathrm{SMD} / \mathrm{B} 3 \mathrm{LYP}-\mathrm{D} 3 / \mathrm{BS} 3)=-309.585756 \mathrm{au}$

$\mathrm{E}(\mathrm{SMD} / \mathrm{M} 06 / \mathrm{BS} 2 / / \mathrm{SMD} / \mathrm{B} 3 \mathrm{LYP}-\mathrm{D} 3 / \mathrm{BS} 3)=-309.512523034 \mathrm{au}$

$\begin{array}{lrrr}\mathrm{C} & -0.68618100 & -1.77877200 & 0.00000000 \\ \mathrm{C} & -1.01040100 & -0.42216600 & 0.00000000 \\ \mathrm{C} & 0.00000000 & 0.56177500 & 0.00000000 \\ \mathrm{C} & 1.34190700 & 0.13392300 & 0.00000000 \\ \mathrm{C} & 1.66786500 & -1.22545500 & 0.00000000 \\ \mathrm{C} & 0.65445300 & -2.18868100 & 0.00000000 \\ \mathrm{H} & -1.48058100 & -2.52055500 & 0.00000000 \\ \mathrm{H} & -2.05557100 & -0.12712900 & 0.00000000\end{array}$




$\begin{array}{lrrr}\mathrm{H} & 2.13573800 & 0.87741300 & 0.00000000 \\ \mathrm{H} & 2.71103600 & -1.53003100 & 0.00000000 \\ \mathrm{H} & 0.90279400 & -3.24646600 & 0.00000000 \\ \mathrm{C} & -0.28375000 & 2.00947700 & 0.00000000 \\ \mathrm{H} & 0.60248000 & 2.64442700 & 0.00000000 \\ \mathrm{C} & -1.48802300 & 2.60435700 & 0.00000000 \\ \mathrm{H} & -2.42193300 & 2.04774600 & 0.00000000 \\ \mathrm{H} & -1.56918100 & 3.68783900 & 0.00000000\end{array}$

3 (optimized by SMD/B3LYP-D3/BS3)

$\mathrm{E}(\mathrm{SMD} / \mathrm{B} 3 \mathrm{LYP}-\mathrm{D} 3 / \mathrm{BS} 3)=-655.556166983 \mathrm{au}$

$\mathrm{H}(\mathrm{SMD} / \mathrm{B} 3 \mathrm{LYP}-\mathrm{D} 3 / \mathrm{BS} 3)=-655.280251 \mathrm{au}$

$\mathrm{G}(\mathrm{SMD} / \mathrm{B} 3 \mathrm{LYP}-\mathrm{D} 3 / \mathrm{BS} 3)=-655.340254 \mathrm{au}$

$\mathrm{E}(\mathrm{SMD} / \mathrm{M} 06 / \mathrm{BS} 2 / / \mathrm{SMD} / \mathrm{B} 3 \mathrm{LYP}-\mathrm{D} 3 / \mathrm{BS} 3)=-655.262842089 \mathrm{au}$

C $\quad-2.94544800 \quad-1.89156000 \quad-0.69008800$

$\mathrm{H} \quad-2.64362400 \quad-2.58036700 \quad 0.10863100$

$\mathrm{H} \quad-2.39954000 \quad-2.17879200 \quad-1.59421200$

$\mathrm{H} \quad-4.02191400 \quad-1.98833900 \quad-0.84727300$

$\begin{array}{llll}\mathrm{C} & -2.61705900 & -0.47725900 & -0.27444800\end{array}$

$\begin{array}{llll}\mathrm{O} & -3.48140400 & 0.28885400 & 0.13075600\end{array}$

C $\quad-1.14897200 \quad-0.04075600 \quad-0.38609000$

$\mathrm{H} \quad-0.89016500 \quad-0.17747000 \quad-1.44550600$

C $\quad-1.03702600 \quad 1.45779000 \quad-0.07987800$

C $\quad-1.30609800 \quad 2.38381800 \quad-1.23942000$

$\mathrm{H} \quad \begin{array}{llll}\mathrm{H} & -2.20286000 & 2.07396500 & -1.78682400\end{array}$

$\mathrm{H} \quad-1.41092700 \quad 3.41361900 \quad-0.89144500$

$\mathrm{H} \quad \begin{array}{llll}-0.46085200 & 2.31927900 & -1.93802300\end{array}$

$\begin{array}{lllll}\mathrm{O} & -0.73488100 & 1.87834800 & 1.02646200\end{array}$

$\begin{array}{lllll}\text { C } & 3.93122800 & 0.11067800 & -0.42693900\end{array}$

$\begin{array}{llll}\text { C } & 3.32559600 & 0.56553000 & 0.74864600\end{array}$

$\begin{array}{llll}\mathrm{C} & 2.00148900 & 0.22033500 & 1.04318600\end{array}$

$\begin{array}{llll}\mathrm{C} & 1.25918100 & -0.58888000 & 0.17015000\end{array}$

C $\quad \begin{array}{llll}\text { C } & 1.88090200 & -1.04195000 & -1.00596800\end{array}$

C $\quad 3.20167100 \quad-0.69753400 \quad-1.30657400$

$\mathrm{H} \quad 4.95951900 \quad 0.37915300 \quad-0.65386300$

$\mathrm{H} \quad 3.88208800 \quad 1.19318500 \quad 1.43998800$

$\begin{array}{llll}\mathrm{H} & 1.54575700 & 0.59409000 & 1.95320500\end{array}$

$\mathrm{H} \quad 1.32236200 \quad-1.67546700 \quad-1.69241100$

$\mathrm{H} \quad 3.66114500 \quad-1.06383500 \quad-2.22104700$

$\begin{array}{llll}\mathrm{C} & -0.19382200 & -0.97383800 & 0.43323600\end{array}$

$\begin{array}{llll}\mathrm{H} & -0.32486800 & -1.96961500 & -0.00578400\end{array}$

$\begin{array}{llll}\mathrm{C} & -0.56666100 & -1.08005700 & 1.91979000\end{array}$

$\begin{array}{llll}\mathrm{H} & -0.52744100 & -0.11289600 & 2.42223700\end{array}$

$\mathrm{H} \quad-1.58347400 \quad-1.47196300 \quad 2.03263700$

$\mathrm{H} \quad 0.11518600 \quad-1.77006800 \quad 2.42833100$

9 (optimized by SMD/B3LYP-D3/BS3)

$\mathrm{E}(\mathrm{SMD} / \mathrm{B} 3 \mathrm{LYP}-\mathrm{D} 3 / \mathrm{BS} 3)=-345.846570384 \mathrm{au}$

$\mathrm{H}(\mathrm{SMD} / \mathrm{B} 3 \mathrm{LYP}-\mathrm{D} 3 / \mathrm{BS} 3)=-345.71642 \mathrm{au}$

$\mathrm{G}(\mathrm{SMD} / \mathrm{B} 3 \mathrm{LYP}-\mathrm{D} 3 / \mathrm{BS} 3)=-345.75559 \mathrm{au}$

$\mathrm{E}(\mathrm{SMD} / \mathrm{M} 06 / \mathrm{BS} 2 / / \mathrm{SMD} / \mathrm{B} 3 \mathrm{LYP}-\mathrm{D} 3 / \mathrm{BS} 3)=-345.724575699 \mathrm{au}$ 


$\begin{array}{lrrr}\mathrm{C} & 2.54842900 & -0.75882800 & -0.00000300 \\ \mathrm{H} & 2.60359800 & -1.40805100 & 0.88207600 \\ \mathrm{H} & 3.39364700 & -0.06746900 & -0.00003600 \\ \mathrm{H} & 2.60356700 & -1.40810100 & -0.88204600 \\ \mathrm{C} & 1.24767400 & 0.00231500 & 0.00000400 \\ \mathrm{O} & 1.25645300 & 1.26330700 & 0.00001700 \\ \mathrm{C} & 0.01064600 & -0.73771500 & -0.00000200 \\ \mathrm{C} & -1.20217200 & -0.08802400 & -0.00000200 \\ \mathrm{C} & -2.52874200 & -0.77211700 & 0.00000400 \\ \mathrm{H} & -3.10187200 & -0.46604500 & -0.88321600 \\ \mathrm{H} & -2.41899200 & -1.85833200 & 0.00001900 \\ \mathrm{H} & -3.10187800 & -0.46602000 & 0.88321100 \\ \mathrm{O} & -1.27501800 & 1.24318300 & -0.00001400 \\ \mathrm{H} & -0.31806200 & 1.56930400 & -0.00003300 \\ \mathrm{H} & 0.03350800 & -1.82099300 & -0.00000200\end{array}$

TS $_{\text {10-22 }}$ (optimized by SMD/B3LYP-D3/BS3)

$\mathrm{E}(\mathrm{SMD} / \mathrm{B} 3 \mathrm{LYP}-\mathrm{D} 3 / \mathrm{BS} 3)=-3675.67625828 \mathrm{au}$

$\mathrm{H}(\mathrm{SMD} / \mathrm{B} 3 \mathrm{LYP}-\mathrm{D} 3 / \mathrm{BS} 3)=-3675.293775 \mathrm{au}$

$\mathrm{G}(\mathrm{SMD} / \mathrm{B} 3 \mathrm{LYP}-\mathrm{D} 3 / \mathrm{BS} 3)=-3675.421592 \mathrm{au}$

$\mathrm{E}(\mathrm{SMD} / \mathrm{M} 06 / \mathrm{BS} 2 / / \mathrm{SMD} / \mathrm{B} 3 \mathrm{LYP}-\mathrm{D} 3 / \mathrm{BS} 3)=-3675.51917726 \mathrm{au}$

$\mathrm{E}(\mathrm{SMD} / \mathrm{M} 06-\mathrm{D} 3 / \mathrm{BS} 2 / / \mathrm{SMD} / \mathrm{B} 3 \mathrm{LYP}-\mathrm{D} 3 / \mathrm{BS} 3)=-3675.53716316 \mathrm{au}$

$\begin{array}{lrrr}\mathrm{C} & -0.78608400 & -4.02845200 & 1.77746300 \\ \mathrm{H} & -0.06268100 & -4.84019300 & 1.62620000 \\ \mathrm{H} & -1.79536300 & -4.42777700 & 1.88321800 \\ \mathrm{H} & -0.48159000 & -3.49750400 & 2.68623800 \\ \mathrm{C} & -0.70394000 & -3.09018200 & 0.62643000 \\ \mathrm{O} & -1.72656400 & -2.86919200 & -0.05258400 \\ \mathrm{C} & 0.65339300 & -2.54694100 & 0.28231900 \\ \mathrm{C} & 1.14596300 & -3.26393000 & -1.01182800 \\ \mathrm{O} & 0.51638500 & -3.14446600 & -2.04493400 \\ \mathrm{C} & 2.34329900 & -4.15357100 & -0.85882400 \\ \mathrm{H} & 3.10741100 & -3.71075400 & -0.21728600 \\ \mathrm{H} & 2.00580300 & -5.07560700 & -0.36444800 \\ \mathrm{H} & 2.74965700 & -4.40253800 & -1.84099500 \\ \mathrm{H} & 1.34550700 & -2.69071300 & 1.11341300 \\ \mathrm{C} & -5.74266600 & -1.51749800 & -1.69342100 \\ \mathrm{C} & -6.85080500 & -0.99992500 & -1.02547700 \\ \mathrm{C} & -6.68162400 & 0.03513100 & -0.09850200 \\ \mathrm{C} & -5.40327200 & 0.55213400 & 0.16064000 \\ \mathrm{C} & -4.29663300 & 0.04887400 & -0.51319200 \\ \mathrm{C} & -4.45205700 & -0.99465900 & -1.45455800 \\ \mathrm{H} & -5.86513500 & -2.32573600 & -2.40976400 \\ \mathrm{H} & -7.84017700 & -1.40266700 & -1.22054200 \\ \mathrm{H} & -7.54304200 & 0.43610300 & 0.42828600 \\ \mathrm{H} & -5.27451100 & 1.34456400 & 0.89150500 \\ \mathrm{H} & -3.31201800 & 0.44241900 & -0.29311800 \\ \mathrm{C} & -3.33748600 & -1.55713000 & -2.18890600 \\ \mathrm{H} & -3.58072700 & -2.42150200 & -2.80763100 \\ \mathrm{C} & -2.01212100 & -1.18609600 & -2.12079600\end{array}$




$\begin{array}{lccc}\mathrm{H} & -1.73260600 & -0.23379000 & -1.68104200 \\ \mathrm{H} & -1.80479600 & -2.08796600 & -1.04541800 \\ \mathrm{H} & -1.31507800 & -1.60217500 & -2.84225400 \\ \mathrm{Au} & 0.71031200 & -0.47934200 & -0.04083600 \\ \mathrm{O} & 0.88142500 & 1.66629800 & -0.35452600 \\ \mathrm{~S} & 0.50702900 & 2.34700100 & -1.68076400 \\ \mathrm{O} & 1.47587300 & 3.38103600 & -2.05311900 \\ \mathrm{O} & 0.04312500 & 1.39669800 & -2.70260300 \\ \mathrm{C} & -1.03321900 & 3.29643300 & -1.16910500 \\ \mathrm{~F} & -0.75044800 & 4.17362500 & -0.19587100 \\ \mathrm{~F} & -1.51794700 & 3.96763700 & -2.22693600 \\ \mathrm{~F} & -1.98031600 & 2.45180700 & -0.72913900 \\ \mathrm{O} & -2.75739200 & -0.02261100 & 2.65780600 \\ \mathrm{~S} & -1.32216500 & -0.08692400 & 2.40180400 \\ \mathrm{O} & -0.47691000 & -1.05954700 & 3.09845300 \\ \mathrm{O} & -1.10548700 & -0.13538600 & 0.85119500 \\ \mathrm{C} & -0.63246500 & 1.60616800 & 2.85699400 \\ \mathrm{~F} & -0.80180900 & 1.79411600 & 4.17242800 \\ \mathrm{~F} & 0.67200600 & 1.66112700 & 2.56850300 \\ \mathrm{~F} & -1.27831000 & 2.56375100 & 2.18774700 \\ \mathrm{O} & 3.81718900 & -1.61499500 & 0.82999100 \\ \mathrm{~S} & 3.86021700 & -0.82106100 & -0.40078800 \\ \mathrm{O} & 4.84534700 & -1.10788900 & -1.43916400 \\ \mathrm{O} & 2.45318600 & -0.72393600 & -1.09188000 \\ \mathrm{C} & 4.18435900 & 0.94931500 & 0.16170000 \\ \mathrm{~F} & 5.45084300 & 1.02607600 & 0.59320600 \\ \mathrm{~F} & 4.00856700 & 1.80192100 & -0.84692000 \\ \mathrm{~F} & 3.36032000 & 1.26144300 & 1.16820100 \\ & & & \end{array}$

22 (optimized by SMD/B3LYP-D3/BS3)

\begin{tabular}{|c|c|c|c|}
\hline \multicolumn{4}{|c|}{$\mathrm{E}(\mathrm{SMD} / \mathrm{B} 3 \mathrm{LYP}-\mathrm{D} 3 / \mathrm{BS} 3)=-3675.69582129 \mathrm{au}$} \\
\hline \multicolumn{4}{|c|}{$\mathrm{H}(\mathrm{SMD} / \mathrm{B} 3 \mathrm{LYP}-\mathrm{D} 3 / \mathrm{BS} 3)=-3675.307798 \mathrm{au}$} \\
\hline \multicolumn{4}{|c|}{$\mathrm{G}(\mathrm{SMD} / \mathrm{B} 3 \mathrm{LYP}-\mathrm{D} 3 / \mathrm{BS} 3)=-3675.43885 \mathrm{au}$} \\
\hline \multirow{2}{*}{\multicolumn{4}{|c|}{$\begin{array}{l}\text { E }(\mathrm{SMD} / \mathrm{M} 06 / \mathrm{BS} 2 / / \mathrm{SMD} / \mathrm{B} 3 \mathrm{LYP}-\mathrm{D} 3 / \mathrm{BS} 3)=-3675.54104637 \mathrm{au} \\
\mathrm{E}(\mathrm{SMD} / \mathrm{M} 06-\mathrm{D} 3 / \mathrm{BS} 2 / / \mathrm{SMD} / \mathrm{B} 3 \mathrm{LYP}-\mathrm{D} 3 / \mathrm{BS} 3)=-3675.5418837 \mathrm{au}\end{array}$}} \\
\hline & & & \\
\hline $\mathrm{C}$ & -0.36269400 & -2.95690000 & 2.36550900 \\
\hline $\mathrm{H}$ & -0.27534000 & -4.02131100 & 2.11164000 \\
\hline $\mathrm{H}$ & -0.79561700 & -2.84686000 & 3.36037700 \\
\hline $\mathrm{H}$ & 0.64714400 & -2.54038300 & 2.33438600 \\
\hline $\mathrm{C}$ & -1.24241300 & -2.30092500 & 1.34453700 \\
\hline $\mathrm{O}$ & -2.38655900 & -1.93121500 & 1.57730300 \\
\hline $\mathrm{C}$ & -0.67568800 & -2.14878000 & -0.06616800 \\
\hline $\mathrm{C}$ & -1.76207700 & -1.97008100 & -1.12165200 \\
\hline $\mathrm{O}$ & -2.03874800 & -0.87837300 & -1.59552700 \\
\hline $\mathrm{C}$ & -2.45155900 & -3.24002400 & -1.53910400 \\
\hline $\mathrm{H}$ & -2.69746300 & -3.86692600 & -0.67554700 \\
\hline $\mathrm{H}$ & -3.35191500 & -3.00382300 & -2.11010400 \\
\hline $\mathrm{H}$ & -1.75940100 & -3.81362900 & -2.16983800 \\
\hline $\mathrm{H}$ & -0.03246600 & -2.99907300 & -0.30572500 \\
\hline $\mathrm{Au}$ & 0.62943200 & -0.50916700 & -0.15046500 \\
\hline $\mathrm{O}$ & 2.00612000 & 1.19449900 & -0.36171700 \\
\hline
\end{tabular}




$\begin{array}{lccc}\text { S } & 2.21978600 & 1.88141000 & -1.71319400 \\ \text { O } & 3.59060300 & 2.38345300 & -1.85802000 \\ \text { O } & 1.60053400 & 1.15944500 & -2.83514700 \\ \text { C } & 1.16365300 & 3.42461100 & -1.52026700 \\ \text { F } & 1.60040500 & 4.18768600 & -0.50708300 \\ \text { F } & 1.21141700 & 4.15032900 & -2.65148700 \\ \text { F } & -0.11668700 & 3.08745200 & -1.28558400 \\ \text { O } & -2.23515800 & 1.29612100 & 2.61231900 \\ \text { S } & -0.84024500 & 1.02360000 & 2.26169600 \\ \text { O } & -0.02869000 & 0.11154200 & 3.06802500 \\ \text { O } & -0.77661600 & 0.70605700 & 0.73343300 \\ \text { C } & 0.01140100 & 2.69885800 & 2.36814100 \\ \text { F } & -0.08106400 & 3.13597700 & 3.63399200 \\ \text { F } & 1.29767200 & 2.59681500 & 2.03387600 \\ \text { F } & -0.59511400 & 3.57704900 & 1.56021200 \\ \text { O } & 2.25116800 & -3.50557700 & 0.50121200 \\ \text { S } & 2.93256300 & -2.70403000 & -0.51901900 \\ \text { O } & 3.70488400 & -3.35373800 & -1.57517200 \\ \text { O } & 1.99020800 & -1.64903700 & -1.18426700 \\ \text { C } & 4.15306100 & -1.61686000 & 0.42127400 \\ \text { F } & 5.10151300 & -2.39845700 & 0.95555700 \\ \text { F } & 4.72060300 & -0.73516100 & -0.40445500 \\ \text { F } & 3.52344600 & -0.96160300 & 1.40676700 \\ \text { C } & -3.87792300 & 0.67587700 & -0.14498500 \\ \text { C } & -5.07706200 & 0.04301600 & -0.49208900 \\ \text { C } & -5.98633100 & 0.57485000 & -1.45733700 \\ \text { C } & -5.38270200 & -1.19742900 & 0.15094300 \\ \text { C } & -7.14710700 & -0.11321600 & -1.75924900 \\ \text { H } & -5.76586000 & 1.51376300 & -1.95248500 \\ \text { C } & -6.54927400 & -1.87448300 & -0.16050000 \\ \text { H } & -4.68031100 & -1.59727500 & 0.87535900 \\ \text { C } & -7.42639400 & -1.33287800 & -1.11385400 \\ \text { H } & -7.84367400 & 0.28199400 & -2.49130000 \\ \text { H } & -6.78513300 & -2.81611600 & 0.32428500 \\ \text { H } & -8.34069900 & -1.86586500 & -1.36006800 \\ \text { H } & -3.26925100 & 0.17256400 & 0.60192900 \\ \text { C } & -3.33791600 & 1.93645600 & -0.64924500 \\ \text { H } & -3.18192700 & 2.60322600 & 0.21293200 \\ \text { H } & -2.32486600 & 1.73499200 & -1.02287800 \\ \text { H } & -3.93754800 & 2.43445600 & -1.40941100\end{array}$

23 (optimized by SMD/B3LYP-D3/BS3)

$\mathrm{E}(\mathrm{SMD} / \mathrm{B} 3 \mathrm{LYP}-\mathrm{D} 3 / \mathrm{BS} 3)=-310.102780161 \mathrm{au}$

$\mathrm{H}(\mathrm{SMD} / \mathrm{B} 3 \mathrm{LYP}-\mathrm{D} 3 / \mathrm{BS} 3)=-309.950695 \mathrm{au}$

G (SMD/B3LYP-D3/ BS3) $=-309.988894 \mathrm{au}$

$\mathrm{E}(\mathrm{SMD} / \mathrm{M} 06 / \mathrm{BS} 2 / / \mathrm{SMD} / \mathrm{B} 3 \mathrm{LYP}-\mathrm{D} 3 / \mathrm{BS} 3)=-309.924021714 \mathrm{au}$
$\begin{array}{llll}\mathrm{C} & 1.33614400 & 1.37993300 & -0.00023900\end{array}$
$\begin{array}{llll}\mathrm{C} & -0.01413000 & 1.09438500 & -0.00060900\end{array}$
$\begin{array}{llll}\text { C } & -0.45397300 & -0.26958300 & -0.00037100\end{array}$
$\begin{array}{llll}\mathrm{C} & 0.52099500 & -1.31984800 & 0.00009200\end{array}$
$\begin{array}{llll}\text { C } & 1.87110600 & -1.01765800 & 0.00044300\end{array}$ 


$\begin{array}{lrrc}\mathrm{C} & 2.27469400 & 0.32750800 & 0.00028500 \\ \mathrm{H} & 1.68042100 & 2.40863900 & -0.00037700 \\ \mathrm{H} & -0.74330200 & 1.89765400 & -0.00102900 \\ \mathrm{H} & 0.18504000 & -2.35257500 & 0.00026000 \\ \mathrm{H} & 2.61334600 & -1.80887500 & 0.00085000 \\ \mathrm{H} & 3.33502300 & 0.56428000 & 0.00055700 \\ \mathrm{C} & -1.80451600 & -0.61969900 & -0.00024900 \\ \mathrm{H} & -2.02500500 & -1.68730000 & -0.00008700 \\ \mathrm{C} & -2.95240100 & 0.30401700 & 0.00021400 \\ \mathrm{H} & -3.90167800 & -0.23106100 & -0.00263600 \\ \mathrm{H} & -2.90697800 & 0.96455700 & 0.87797600 \\ \mathrm{H} & -2.90437400 & 0.97034500 & -0.87291200\end{array}$

24 (optimized by SMD/B3LYP-D3/BS3)

$\mathrm{E}(\mathrm{SMD} / \mathrm{B} 3 \mathrm{LYP}-\mathrm{D} 3 / \mathrm{BS} 3)=-3365.566011 \mathrm{au}$

$\mathrm{H}(\mathrm{SMD} / \mathrm{B} 3 \mathrm{LYP}-\mathrm{D} 3 / \mathrm{BS} 3=-3365.33361 \mathrm{au}$

$\mathrm{G}(\mathrm{SMD} / \mathrm{B} 3 \mathrm{LYP}-\mathrm{D} 3 / \mathrm{BS} 3)=-3365.444776$ au

$\mathrm{E}(\mathrm{SMD} / \mathrm{M} 06 / \mathrm{BS} 2 / / \mathrm{SMD} / \mathrm{B} 3 \mathrm{LYP}-\mathrm{D} 3 / \mathrm{BS} 3)=-3365.59941209 \mathrm{au}$

$\begin{array}{llll}\mathrm{C} & 2.28207900 & 2.87866500 & -1.31330300\end{array}$

$\mathrm{H} \quad 3.35576200 \quad 2.99418700 \quad-1.11459400$

$\mathrm{H} \quad \begin{array}{llll}\mathrm{H} & 1.95386800 & 3.64513900 & -2.01616600\end{array}$

$\mathrm{H} \quad 2.14260800 \quad 1.88131900 \quad-1.73584200$

$\begin{array}{llll}\text { C } & 1.54934200 & 3.02897500 & -0.01100700\end{array}$

$\begin{array}{llll}\mathrm{O} & 0.93695800 & 4.03878800 & 0.30317200\end{array}$

$\begin{array}{llll}\mathrm{C} & 1.65884700 & 1.87118300 & 0.98335700\end{array}$

$\begin{array}{llll}\text { C } & 1.30284800 & 2.25047500 & 2.41843700\end{array}$

$\begin{array}{llll}\mathrm{O} & 0.30073900 & 1.82921800 & 2.97177300\end{array}$

$\begin{array}{llll}\mathrm{C} & 2.31048000 & 3.13606900 & 3.10541900\end{array}$

$\begin{array}{llll}\mathrm{H} & 2.56807000 & 3.99903700 & 2.48387400\end{array}$

$\begin{array}{llll}\mathrm{H} & 1.91219600 & 3.46897900 & 4.06638600\end{array}$

$\begin{array}{llll}\mathrm{H} & 3.23044000 & 2.56011800 & 3.26921500\end{array}$

$\mathrm{H} \quad 2.66522200 \quad 1.44468100 \quad 0.93922000$

$\begin{array}{llll}\mathrm{Au} & 0.44781100 & 0.26504700 & 0.39023000\end{array}$

$\begin{array}{llll}\mathrm{O} & -0.76702700 & -1.49126200 & -0.18176400\end{array}$

S $\quad-1.21462700 \quad-2.52784800 \quad 0.84921900$

$\begin{array}{llll}\mathrm{O} & -1.28627900 & -3.87612800 & 0.27185800\end{array}$

$\begin{array}{llll}\mathrm{O} & -0.58335000 & -2.35149500 & 2.16587500\end{array}$

$\begin{array}{llll}\text { C } & -3.00671000 & -2.03420100 & 1.12554100\end{array}$

F $\quad-3.71251600 \quad-2.12567100 \quad-0.01359500$

F $\quad-3.56680100 \quad-2.85084100 \quad 2.03724700$

F $\quad-3.08115400 \quad-0.77398300 \quad 1.58003000$

$\begin{array}{lllll}\mathrm{O} & -2.39417600 & 3.43171000 & -0.66498900\end{array}$

S $\quad \begin{array}{llll}-1.67735600 & 2.20902400 & -1.02329100\end{array}$

$\begin{array}{llll}\mathrm{O} & -0.65376400 & 2.22348200 & -2.07188100\end{array}$

$\begin{array}{llll}\mathrm{O} & -1.19801600 & 1.49521800 & 0.27853600\end{array}$

C $\quad-3.01493300 \quad 1.04336200 \quad-1.65332100$

F $\quad \begin{array}{llll}\text { C } & -3.59329400 & 1.60519400 & -2.72919500\end{array}$

F $\quad-2.49085200 \quad-0.13208800 \quad-2.00934600$

F $\quad \begin{array}{llll}-3.94826000 & 0.84887300 & -0.71422300\end{array}$

$\begin{array}{lllll}\mathrm{O} & 3.72983900 & 0.01862500 & -0.80974800\end{array}$

S $\quad 3.20350900 \quad-1.24102400 \quad-0.27665900$ 


$\begin{array}{llrr}\mathrm{O} & 4.10996100 & -2.23061300 & 0.30247400 \\ \mathrm{O} & 2.01484100 & -1.02873600 & 0.70857000 \\ \mathrm{C} & 2.37292200 & -2.09597500 & -1.73670000 \\ \mathrm{~F} & 3.31623600 & -2.44507300 & -2.62415800 \\ \mathrm{~F} & 1.72463500 & -3.18989200 & -1.33143900 \\ \mathrm{~F} & 1.50792500 & -1.26055500 & -2.32978200\end{array}$

25 (optimized by SMD/B3LYP-D3/BS3)

$\mathrm{E}(\mathrm{SMD} / \mathrm{B} 3 \mathrm{LYP}-\mathrm{D} 3 / \mathrm{BS} 3)=-655.97882322 \mathrm{au}$

$\mathrm{H}(\mathrm{SMD} / \mathrm{B} 3 \mathrm{LYP}-\mathrm{D} 3 / \mathrm{BS} 3)=-655.691238 \mathrm{au}$

$\mathrm{G}(\mathrm{SMD} / \mathrm{B} 3 \mathrm{LYP}-\mathrm{D} 3 / \mathrm{BS})=-655.74944 \mathrm{au}$

$\mathrm{E}(\mathrm{SMD} / \mathrm{M} 06 / \mathrm{BS} 2 / / \mathrm{SMD} / \mathrm{B} 3 \mathrm{LYP}-\mathrm{D} 3 / \mathrm{BS} 3)=-655.682837135 \mathrm{au}$

$\begin{array}{llll}\text { C } & 3.40059600 & -1.31595600 & 0.85442400\end{array}$

$\mathrm{H} \quad 3.68798700 \quad-0.84683900 \quad 1.80702400$

$\mathrm{H} \quad 4.30052800 \quad-1.62859900 \quad 0.32225900$

C $\quad 2.66111300 \quad-0.28728000 \quad 0.07507900$

$\begin{array}{llll}\mathrm{O} & 3.19070700 & 0.36421900 & -0.83583200\end{array}$

$\begin{array}{llll}\mathrm{C} & 1.20189900 & 0.00464000 & 0.42231600\end{array}$

$\begin{array}{llll}\mathrm{C} & 0.92581700 & 1.47294700 & 0.34134000\end{array}$

$\begin{array}{llll}\mathrm{O} & 1.52499000 & 2.15846000 & -0.54942600\end{array}$

$\begin{array}{llll}\mathrm{C} & -0.00600600 & 2.15462800 & 1.24701700\end{array}$

$\mathrm{H} \quad-0.23823000 \quad 3.16256800 \quad 0.90173900$

$\mathrm{H} \quad-0.90904000 \quad 1.55423700 \quad 1.39282900$

$\begin{array}{llll}\mathrm{H} & 0.49439000 & 2.20111700 & 2.22749000\end{array}$

$\mathrm{H} \quad \begin{array}{llll}0.94486600 & -0.37052200 & 1.41502700\end{array}$

$\begin{array}{llll}\mathrm{H} & 2.26536300 & 1.55394800 & -0.96814800\end{array}$

C $\quad 0.25865300 \quad-0.73974200 \quad-0.65601700$

$\mathrm{H} \quad 0.49484300 \quad-0.28313200 \quad-1.62302400$

C $\quad \begin{array}{llll}\text { C } & -1.20807900 & -0.49341500 & -0.34881500\end{array}$

$\begin{array}{lllll}\mathrm{C} & & -1.97366900 & 0.31569200 & -1.20051700\end{array}$

$\begin{array}{lllll}\mathrm{C} & & -1.81933800 & -1.05642300 & 0.78340400\end{array}$

$\begin{array}{lllll}\mathrm{C} & & -3.32559300 & 0.55581700 & -0.93159000\end{array}$

$\mathrm{H} \quad \begin{array}{llll}\mathrm{H} & -1.51065600 & 0.76094200 & -2.07788700\end{array}$

$\begin{array}{llll}\mathrm{C} & -3.16850900 & -0.81587000 & 1.05490700\end{array}$

$\mathrm{H} \quad \begin{array}{llll}\mathrm{H} & -1.24103000 & -1.68250100 & 1.45792900\end{array}$

$\begin{array}{llll}\mathrm{C} & -3.92592300 & -0.00799600 & 0.19790100\end{array}$

$\mathrm{H} \quad-3.90559500 \quad 1.18204700 \quad-1.60376300$

$\mathrm{H} \quad \begin{array}{llll}\mathrm{H} & -3.62888100 & -1.25971700 & 1.93326900\end{array}$

$\mathrm{H} \quad-4.97540200 \quad 0.17719600 \quad 0.40881800$

$\begin{array}{llll}\text { C } & 0.59864600 & -2.23535400 & -0.72803200\end{array}$

$\mathrm{H} \quad-0.10103600 \quad-2.71552300 \quad-1.41852000$

$\mathrm{H} \quad \begin{array}{llll}\mathrm{H} & 1.61090700 & -2.40440700 & -1.10714300\end{array}$

$\mathrm{H} \quad 0.50310800 \quad-2.72201400 \quad 0.24658100$

$\mathrm{H} \quad 2.76465600 \quad-2.17036000 \quad 1.09908700$

TS $_{\text {23-25 }}$ (optimized by SMD/B3LYP-D3/BS3)

$\mathrm{E}(\mathrm{SMD} / \mathrm{B} 3 \mathrm{LYP}-\mathrm{D} 3 / \mathrm{BS} 3)=-655.963942481 \mathrm{au}$

$\mathrm{H}(\mathrm{SMD} / \mathrm{B} 3 \mathrm{LYP}-\mathrm{D} 3 / \mathrm{BS} 3)=-655.677778 \mathrm{au}$

G (SMD/B3LYP-D3/ BS3) $=-655.736649 \mathrm{au}$

$\mathrm{E}(\mathrm{SMD} / \mathrm{M} 06 / \mathrm{BS} 2 / / \mathrm{SMD} / \mathrm{B} 3 \mathrm{LYP}-\mathrm{D} 3 / \mathrm{BS} 3)=-655.660117787 \mathrm{au}$

$\begin{array}{llll}\mathrm{C} & -2.52902600 & 2.31578800 & 0.62078900\end{array}$ 


$\begin{array}{lrrr}\mathrm{H} & -3.60193500 & 2.21295300 & 0.41592400 \\ \mathrm{H} & -2.21506300 & 3.34127800 & 0.41887800 \\ \mathrm{C} & -1.76426100 & 1.35554200 & -0.23934400 \\ \mathrm{O} & -0.96633800 & 1.76403600 & -1.11407800 \\ \mathrm{C} & -1.94521500 & -0.07465500 & -0.02350500 \\ \mathrm{C} & -1.43426200 & -0.98949800 & -0.95310500 \\ \mathrm{O} & -0.58798400 & -0.59626600 & -1.87316700 \\ \mathrm{C} & -1.75368100 & -2.43956700 & -0.95662800 \\ \mathrm{H} & -0.82480100 & -3.02113700 & -0.94488700 \\ \mathrm{H} & -2.37959800 & -2.71597700 & -0.10798200 \\ \mathrm{H} & -2.27780900 & -2.68433200 & -1.88875500 \\ \mathrm{H} & -2.70651600 & -0.40985400 & 0.66922100 \\ \mathrm{H} & -0.50768300 & 0.41607800 & -1.78160000 \\ \mathrm{C} & -0.16058500 & -0.38496200 & 1.57069700 \\ \mathrm{H} & -0.61297100 & 0.47309400 & 2.06265600 \\ \mathrm{C} & 1.02458400 & -0.11559700 & 0.82332500 \\ \mathrm{C} & 1.44881300 & 1.23356200 & 0.68279900 \\ \mathrm{C} & 1.79847000 & -1.14594200 & 0.22358500 \\ \mathrm{C} & 2.61883400 & 1.53663100 & -0.00216200 \\ \mathrm{H} & 0.85383300 & 2.02442300 & 1.12924500 \\ \mathrm{C} & 2.96369400 & -0.83324700 & -0.46119600 \\ \mathrm{H} & 1.47888100 & -2.17905500 & 0.30046000 \\ \mathrm{C} & 3.37478300 & 0.50483300 & -0.57359000 \\ \mathrm{H} & 2.94205800 & 2.56821900 & -0.09829600 \\ \mathrm{H} & 3.55450200 & -1.62218200 & -0.91583000 \\ \mathrm{H} & 4.28692300 & 0.74207200 & -1.11386400 \\ \mathrm{C} & -0.53341400 & -1.70644900 & 2.13701000 \\ \mathrm{H} & 0.03618500 & -1.80135600 & 3.07594400 \\ \mathrm{H} & -1.59265800 & -1.74678000 & 2.39758100 \\ \mathrm{H} & -0.26898600 & -2.55626800 & 1.50611400 \\ \mathrm{H} & -2.38218700 & 2.07804400 & 1.68110400\end{array}$

$\mathbf{T S}_{\text {25-23 }}$ (optimized by SMD/B3LYP-D3/BS3)

$\mathrm{E}(\mathrm{SMD} / \mathrm{B} 3 \mathrm{LYP}-\mathrm{D} 3 / \mathrm{BS} 3)=-965.669559466 \mathrm{au}$

$\mathrm{H}(\mathrm{SMD} / \mathrm{B} 3 \mathrm{LYP}-\mathrm{D} 3 / \mathrm{BS} 3)=-965.242204 \mathrm{au}$

$\mathrm{G}(\mathrm{SMD} / \mathrm{B} 3 \mathrm{LYP}-\mathrm{D} 3 / \mathrm{BS} 3)=-965.316237 \mathrm{au}$

$\mathrm{E}(\mathrm{SMD} / \mathrm{M} 06 / \mathrm{BS} 2 / / \mathrm{SMD} / \mathrm{B} 3 \mathrm{LYP}-\mathrm{D} 3 / \mathrm{BS} 3)=-965.191092425 \mathrm{au}$

$\mathrm{E}(\mathrm{SMD} / \mathrm{M} 06-\mathrm{D} 3 / \mathrm{BS} 2 / / \mathrm{SMD} / \mathrm{B} 3 \mathrm{LYP}-\mathrm{D} 3 / \mathrm{BS} 3)=-965.192092425 \mathrm{au}$

$\begin{array}{llrr}\mathrm{C} & -2.52902600 & 2.31578800 & 0.62078900 \\ \mathrm{H} & -3.60193500 & 2.21295300 & 0.41592400 \\ \mathrm{H} & -2.21506300 & 3.34127800 & 0.41887800 \\ \mathrm{C} & -1.76426100 & 1.35554200 & -0.23934400 \\ \mathrm{O} & -0.96633800 & 1.76403600 & -1.11407800 \\ \mathrm{C} & -1.94521500 & -0.07465500 & -0.02350500 \\ \mathrm{C} & -1.43426200 & -0.98949800 & -0.95310500 \\ \mathrm{O} & -0.58798400 & -0.59626600 & -1.87316700 \\ \mathrm{C} & -1.75368100 & -2.43956700 & -0.95662800 \\ \mathrm{H} & -0.82480100 & -3.02113700 & -0.94488700 \\ \mathrm{H} & -2.37959800 & -2.71597700 & -0.10798200 \\ \mathrm{H} & -2.27780900 & -2.68433200 & -1.88875500 \\ \mathrm{H} & -2.70651600 & -0.40985400 & 0.66922100\end{array}$




$\begin{array}{lrrr}\mathrm{H} & -0.50768300 & 0.41607800 & -1.78160000 \\ \mathrm{C} & -0.16058500 & -0.38496200 & 1.57069700 \\ \mathrm{H} & -0.61297100 & 0.47309400 & 2.06265600 \\ \mathrm{C} & 1.02458400 & -0.11559700 & 0.82332500 \\ \mathrm{C} & 1.44881300 & 1.23356200 & 0.68279900 \\ \mathrm{C} & 1.79847000 & -1.14594200 & 0.22358500 \\ \mathrm{C} & 2.61883400 & 1.53663100 & -0.00216200 \\ \mathrm{H} & 0.85383300 & 2.02442300 & 1.12924500 \\ \mathrm{C} & 2.96369400 & -0.83324700 & -0.46119600 \\ \mathrm{H} & 1.47888100 & -2.17905500 & 0.30046000 \\ \mathrm{C} & 3.37478300 & 0.50483300 & -0.57359000 \\ \mathrm{H} & 2.94205800 & 2.56821900 & -0.09829600 \\ \mathrm{H} & 3.55450200 & -1.62218200 & -0.91583000 \\ \mathrm{H} & 4.28692300 & 0.74207200 & -1.11386400 \\ \mathrm{C} & -0.53341400 & -1.70644900 & 2.13701000 \\ \mathrm{H} & 0.03618500 & -1.80135600 & 3.07594400 \\ \mathrm{H} & -1.59265800 & -1.74678000 & 2.39758100 \\ \mathrm{H} & -0.26898600 & -2.55626800 & 1.50611400 \\ \mathrm{H} & -2.38218700 & 2.07804400 & 1.68110400\end{array}$

18 (optimized by SMD/B3LYP-D3/BS1)

E $($ SMD/B3LYP-D3/BS1 $)=-3675.58104894$ au

$\mathrm{H}(\mathrm{SMD} / \mathrm{B} 3 \mathrm{LYP}-\mathrm{D} 3 / \mathrm{BS} 1)=-3675.187629 \mathrm{au}$

G (SMD/B3LYP-D3/BS1) $=-3675.309081$ au

$\mathrm{E}(\mathrm{SMD} / \mathrm{M} 06 / \mathrm{BS} 2 / / \mathrm{SMD} / \mathrm{B} 3 \mathrm{LYP}-\mathrm{D} 3 / \mathrm{BS} 1)=-3675.57176318 \mathrm{au}$

C $\quad 3.63242600 \quad 0.67751000 \quad-3.18358800$

$\mathrm{H} \quad 4.23481400 \quad 1.06807000 \quad-2.35667300$

$\mathrm{H} \quad 3.33593200 \quad 1.48142600 \quad-3.85973100$

$\mathrm{H} \quad 4.26402000 \quad-0.04680800 \quad-3.71587400$

C $\quad 2.42806400 \quad-0.03606600 \quad-2.67098800$

$\begin{array}{llll}\mathrm{O} & 1.29481800 & 0.19476000 & -3.09845900\end{array}$

C $\quad 2.62470200 \quad-1.09758500 \quad-1.58705700$

C $\quad 1.57848900 \quad-2.18137100 \quad-1.63124700$

C $\quad 1.84444000 \quad-3.51437800 \quad-1.06180000$

$\mathrm{H} \quad 0.91363700 \quad-4.04474500 \quad-0.85779700$

$\mathrm{H} \quad 2.41343100 \quad-4.06657200 \quad-1.82737600$

$\mathrm{H} \quad 2.47428100 \quad-3.45503500 \quad-0.17188900$

$\begin{array}{llll}\mathrm{O} & 0.46747500 & -2.02182100 & -2.22523800\end{array}$

$\mathrm{H} \quad 3.57550600 \quad-1.61536700 \quad-1.75097500$

$\begin{array}{llll}\text { C } & 2.75472800 & -0.45461700 & -0.11407800\end{array}$

$\begin{array}{llll}\mathrm{C} & 4.21479000 & -0.12071600 & 0.20424800\end{array}$

$\begin{array}{llll}\text { C } & 5.06445500 & -1.18084400 & 0.55709500\end{array}$

$\begin{array}{lllll}\mathrm{C} & 4.73200100 & 1.18161100 & 0.17761500\end{array}$

$\begin{array}{llll}\mathrm{C} & 6.40578500 & -0.94940700 & 0.85749100\end{array}$

$\mathrm{H} \quad 4.66852800 \quad-2.19331600 \quad 0.59613000$

$\begin{array}{llll}\mathrm{C} & 6.07639300 & 1.41390700 & 0.48525100\end{array}$

$\mathrm{H} \quad 4.10022500 \quad 2.02654800 \quad-0.07620200$

$\begin{array}{llll}\mathrm{C} & 6.91682100 & 0.35216200 & 0.82126300\end{array}$

$\mathrm{H} \quad \begin{array}{llll}\mathrm{H} & 7.04871300 & -1.78235200 & 1.12838400\end{array}$

$\begin{array}{llll}\mathrm{H} & 6.46097500 & 2.42966700 & 0.46157200\end{array}$

$\begin{array}{llll}\mathrm{H} & 7.96087600 & 0.53565200 & 1.05917400\end{array}$ 


$\begin{array}{lrrr}\mathrm{C} & 1.85293700 & 0.74106300 & 0.15677400 \\ \mathrm{H} & 2.00230200 & 1.57747300 & -0.52522200 \\ \mathrm{H} & 1.94532900 & 1.06796100 & 1.19379300 \\ \mathrm{H} & 2.48259000 & -1.24966600 & 0.57746800 \\ \mathrm{Au} & -0.18779800 & 0.38493600 & -0.01984600 \\ \mathrm{O} & -2.37254900 & 0.05115400 & -0.24687300 \\ \mathrm{~S} & -2.86166300 & -0.42486300 & -1.61456800 \\ \mathrm{O} & -4.16111100 & 0.14584700 & -1.97734800 \\ \mathrm{O} & -1.78942000 & -0.41986900 & -2.62986400 \\ \mathrm{C} & -3.19362900 & -2.22671300 & -1.27691000 \\ \mathrm{~F} & -4.09306500 & -2.35435600 & -0.29550800 \\ \mathrm{~F} & -3.67011000 & -2.80843800 & -2.38520600 \\ \mathrm{~F} & -2.06524500 & -2.85048600 & -0.91498600 \\ \mathrm{O} & 0.39274200 & -3.40886700 & 2.14785900 \\ \mathrm{~S} & 0.17862800 & -1.96501100 & 2.09576500 \\ \mathrm{O} & 1.06453500 & -1.05773000 & 2.82678700 \\ \mathrm{O} & 0.03416700 & -1.56066800 & 0.58711500 \\ \mathrm{C} & -1.54941100 & -1.65351400 & 2.73946900 \\ \mathrm{~F} & -1.62110100 & -2.10448200 & 3.99214000 \\ \mathrm{~F} & -1.80609700 & -0.34088200 & 2.72565500 \\ \mathrm{~F} & -2.43720100 & -2.29015400 & 1.97972200 \\ \mathrm{O} & 0.49686400 & 3.40506400 & 1.22798000 \\ \mathrm{~S} & -0.42610800 & 3.51654800 & 0.09636100 \\ \mathrm{O} & -0.41473800 & 4.70307000 & -0.75415000 \\ \mathrm{O} & -0.39383000 & 2.25219600 & -0.83118100 \\ \mathrm{C} & -2.14400900 & 3.40367500 & 0.82475700 \\ \mathrm{~F} & -2.38885400 & 4.51812800 & 1.51697700 \\ \mathrm{~F} & -3.04523000 & 3.27169100 & -0.14595400 \\ \mathrm{~F} & -2.21692200 & 2.35016100 & 1.64763800 \\ \mathrm{H} & 0.41012600 & -1.08371800 & -2.63504200 \\ & & & \end{array}$

19 (optimized by SMD/B3LYP-D3/BS1)

$\mathrm{E}(\mathrm{SMD} / \mathrm{B} 3 \mathrm{LYP}-\mathrm{D} 3 / \mathrm{BS} 1)=-3675.57426002 \mathrm{au}$

$\mathrm{H}(\mathrm{SMD} / \mathrm{B} 3 \mathrm{LYP}-\mathrm{D} 3 / \mathrm{BS} 1)=-3675.181393 \mathrm{au}$

G (SMD/B3LYP-D3/BS1) $=-3675.305912 \mathrm{au}$

$\mathrm{E}(\mathrm{SMD} / \mathrm{M} 06 / \mathrm{BS} 2 / / \mathrm{SMD} / \mathrm{B} 3 \mathrm{LYP}-\mathrm{D} 3 / \mathrm{BS} 1)=-3675.56894353 \mathrm{au}$

$\begin{array}{lrrr}\mathrm{C} & 4.61117200 & -3.23555400 & 0.21148700 \\ \mathrm{H} & 3.55127100 & -3.41248900 & 0.42972500 \\ \mathrm{H} & 5.22732000 & -3.47444500 & 1.08009000 \\ \mathrm{H} & 4.87852400 & -3.89123800 & -0.62828900 \\ \mathrm{C} & 4.83248700 & -1.82194200 & -0.20516400 \\ \mathrm{O} & 5.70440100 & -1.10615500 & 0.29396600 \\ \mathrm{C} & 3.90755400 & -1.23451400 & -1.27127000 \\ \mathrm{C} & 4.58208300 & -0.19901300 & -2.09587300 \\ \mathrm{C} & 4.24794700 & 0.02957300 & -3.51107300 \\ \mathrm{H} & 4.74038000 & -0.77303900 & -4.08359200 \\ \mathrm{H} & 3.17396900 & -0.07784800 & -3.68852400 \\ \mathrm{H} & 4.62018800 & 0.99602900 & -3.85582200 \\ \mathrm{O} & 5.48479100 & 0.52805300 & -1.56817800 \\ \mathrm{H} & 5.70910500 & 0.13250500 & -0.63414700 \\ \mathrm{H} & 3.50284400 & -2.01585300 & -1.91802700\end{array}$




\begin{tabular}{|c|c|c|c|}
\hline $\mathrm{C}$ & 2.62581100 & -0.60259600 & -0.47458000 \\
\hline $\mathrm{C}$ & 3.00346900 & 0.49149300 & 0.49653900 \\
\hline $\mathrm{C}$ & 3.10557900 & 0.17331500 & 1.85943600 \\
\hline $\mathrm{C}$ & 3.25406000 & 1.80877800 & 0.08580500 \\
\hline $\mathrm{C}$ & 3.45203400 & 1.15006700 & 2.79247000 \\
\hline $\mathrm{H}$ & 2.90563300 & -0.84391600 & 2.18759900 \\
\hline $\mathrm{C}$ & 3.60325600 & 2.78543000 & 1.01883800 \\
\hline $\mathrm{H}$ & 3.16449700 & 2.08466800 & -0.96067700 \\
\hline $\mathrm{C}$ & 3.70579300 & 2.45801400 & 2.37233600 \\
\hline $\mathrm{H}$ & 3.52138100 & 0.88994500 & 3.84501700 \\
\hline $\mathrm{H}$ & 3.77956300 & 3.80401800 & 0.68767700 \\
\hline $\mathrm{H}$ & 3.97401500 & 3.22134800 & 3.09746700 \\
\hline $\mathrm{C}$ & 1.54374400 & -0.25104800 & -1.48015800 \\
\hline $\mathrm{H}$ & 1.34918500 & -1.06143800 & -2.18710500 \\
\hline $\mathrm{H}$ & 1.68603000 & 0.69855900 & -1.99802900 \\
\hline $\mathrm{H}$ & 2.27863600 & -1.46772700 & 0.09151100 \\
\hline $\mathrm{Au}$ & -0.30890300 & -0.01680300 & -0.57574100 \\
\hline $\mathrm{O}$ & -2.37555700 & 0.23662300 & 0.19851000 \\
\hline S & -3.50239400 & 0.35022400 & -0.83331200 \\
\hline $\mathrm{O}$ & -4.54050500 & 1.30064200 & -0.42505000 \\
\hline $\mathrm{O}$ & -2.99303500 & 0.40944500 & -2.21558900 \\
\hline $\mathrm{C}$ & -4.31786900 & -1.31952500 & -0.69972400 \\
\hline $\mathrm{F}$ & -4.78783400 & -1.51248500 & 0.53774700 \\
\hline $\mathrm{F}$ & -5.34039500 & -1.37667000 & -1.56451300 \\
\hline $\mathrm{F}$ & -3.45174000 & -2.29673400 & -0.99469300 \\
\hline $\mathrm{O}$ & -0.82498400 & -4.36668300 & 0.16519200 \\
\hline S & -0.25105700 & -3.07195000 & 0.51870500 \\
\hline $\mathrm{O}$ & 1.15191600 & -3.00200700 & 0.95052400 \\
\hline $\mathrm{O}$ & -0.59153500 & -2.06113900 & -0.61533400 \\
\hline $\mathrm{C}$ & -1.21743300 & -2.43685600 & 1.98635300 \\
\hline $\mathrm{F}$ & -0.98793100 & -3.25147300 & 3.01833300 \\
\hline $\mathrm{F}$ & -0.80111800 & -1.20255900 & 2.30287900 \\
\hline $\mathrm{F}$ & -2.51823500 & -2.41414400 & 1.71659800 \\
\hline $\mathrm{O}$ & 0.13822400 & 2.23865300 & 1.73869000 \\
\hline S & -0.05736800 & 2.95864300 & 0.48083700 \\
\hline $\mathrm{O}$ & 0.73968400 & 4.14023800 & 0.15560200 \\
\hline $\mathrm{O}$ & -0.03842700 & 2.00534200 & -0.76812600 \\
\hline $\mathrm{C}$ & -1.84603700 & 3.56199000 & 0.49253400 \\
\hline $\mathrm{F}$ & -1.84940300 & 4.84188500 & 0.88032400 \\
\hline $\mathrm{F}$ & -2.37151400 & 3.47609900 & -0.73190700 \\
\hline $\mathrm{F}$ & -2.56824900 & 2.84695900 & 1.34740200 \\
\hline
\end{tabular}

TS $_{18-17}$ (optimized by SMD/B3LYP-D3/BS1)

$\mathrm{E}(\mathrm{SMD} / \mathrm{B} 3 \mathrm{LYP}-\mathrm{D} 3 / \mathrm{BS} 1)=-3675.50879215 \mathrm{au}$

$\mathrm{H}(\mathrm{SMD} / \mathrm{B} 3 \mathrm{LYP}-\mathrm{D} 3 / \mathrm{BS} 1)=-3675.122952 \mathrm{au}$

G (SMD/B3LYP-D3/BS1) $=-3675.245956 \mathrm{au}$

$\mathrm{E}(\mathrm{SMD} / \mathrm{M} 06 / \mathrm{BS} 2 / / \mathrm{SMD} / \mathrm{B} 3 \mathrm{LYP}-\mathrm{D} 3 / \mathrm{BS} 1)=-3675.49692627 \mathrm{au}$

$\begin{array}{llll}\mathrm{C} & 5.71001600 & -1.55149800 & 1.95174800 \\ \mathrm{H} & 6.15244700 & -0.74833600 & 1.34985800 \\ \mathrm{H} & 6.18531600 & -2.50626100 & 1.71769600 \\ \mathrm{H} & 5.87784600 & -1.29348300 & 3.00526200\end{array}$




\begin{tabular}{|c|c|c|c|}
\hline $\mathrm{C}$ & 4.23560200 & -1.62102800 & 1.67850500 \\
\hline $\mathrm{O}$ & 3.66472300 & -2.61825800 & 1.27788900 \\
\hline $\mathrm{C}$ & 3.42611700 & -0.31181500 & 1.91562000 \\
\hline $\mathrm{C}$ & 2.34779800 & -0.63167800 & 2.93103100 \\
\hline $\mathrm{C}$ & 2.67603600 & -0.54448800 & 4.38346700 \\
\hline $\mathrm{H}$ & 1.87760400 & -0.97407100 & 4.99111600 \\
\hline $\mathrm{H}$ & 3.62948300 & -1.04959600 & 4.58017600 \\
\hline $\mathrm{H}$ & 2.81432600 & 0.51414800 & 4.64252700 \\
\hline $\mathrm{O}$ & 1.22424000 & -0.98264800 & 2.54948000 \\
\hline $\mathrm{H}$ & 1.11629200 & -0.80203200 & 1.02508300 \\
\hline $\mathrm{H}$ & 4.09160500 & 0.44219000 & 2.34323600 \\
\hline $\mathrm{C}$ & 2.84389900 & 0.25248800 & 0.58604900 \\
\hline $\mathrm{C}$ & 3.93650600 & 0.58036300 & -0.42296700 \\
\hline $\mathrm{C}$ & 4.38064600 & 1.90537100 & -0.53395500 \\
\hline $\mathrm{C}$ & 4.52073800 & -0.40750800 & -1.23047200 \\
\hline $\mathrm{C}$ & 5.40401800 & 2.23663200 & -1.42257400 \\
\hline $\mathrm{H}$ & 3.91752600 & 2.67878400 & 0.07366900 \\
\hline $\mathrm{C}$ & 5.54095000 & -0.07310100 & -2.12346200 \\
\hline $\mathrm{H}$ & 4.18711800 & -1.43874200 & -1.16678900 \\
\hline $\mathrm{C}$ & 5.98691700 & 1.24755800 & -2.22004000 \\
\hline $\mathrm{H}$ & 5.73923000 & 3.26749400 & -1.49692700 \\
\hline $\mathrm{H}$ & 5.98504700 & -0.84658500 & -2.74385000 \\
\hline $\mathrm{H}$ & 6.78000600 & 1.50578900 & -2.91628800 \\
\hline $\mathrm{C}$ & 1.77421300 & -0.66537800 & -0.05184000 \\
\hline $\mathrm{H}$ & 2.01044400 & -1.73087800 & -0.11843400 \\
\hline $\mathrm{H}$ & 1.55424000 & -0.31456500 & -1.06200500 \\
\hline $\mathrm{H}$ & 2.35567500 & 1.19397400 & 0.85495100 \\
\hline $\mathrm{Au}$ & -0.49911600 & -0.24679500 & 0.12024000 \\
\hline $\mathrm{O}$ & -2.51542000 & 0.25090900 & -0.04850700 \\
\hline S & -3.50111800 & 0.01372900 & 1.14827300 \\
\hline $\mathrm{O}$ & -4.85246100 & 0.29717300 & 0.67274900 \\
\hline $\mathrm{O}$ & -3.20921400 & -1.20699500 & 1.89555700 \\
\hline $\mathrm{C}$ & -3.02726400 & 1.41567700 & 2.28841600 \\
\hline $\mathrm{F}$ & -3.11713300 & 2.58331300 & 1.65479700 \\
\hline $\mathrm{F}$ & -3.84357600 & 1.41222100 & 3.34238300 \\
\hline $\mathrm{F}$ & -1.76447400 & 1.23907700 & 2.71041900 \\
\hline $\mathrm{O}$ & 0.72893400 & 3.95295400 & -0.34958100 \\
\hline S & 0.32736600 & 2.65782600 & -0.88783200 \\
\hline $\mathrm{O}$ & 1.17585100 & 1.94586100 & -1.84376200 \\
\hline $\mathrm{O}$ & -0.07579300 & 1.74921700 & 0.33887200 \\
\hline $\mathrm{C}$ & -1.31976100 & 2.89601900 & -1.74287000 \\
\hline $\mathrm{F}$ & -1.16992300 & 3.81492700 & -2.69603400 \\
\hline $\mathrm{F}$ & -1.69865000 & 1.73909000 & -2.29469400 \\
\hline $\mathrm{F}$ & -2.23537200 & 3.29803900 & -0.86692400 \\
\hline $\mathrm{O}$ & 0.39725800 & -2.77173000 & -1.92637100 \\
\hline S & -0.80324200 & -3.10895200 & -1.16219600 \\
\hline $\mathrm{O}$ & -1.10149100 & -4.48911100 & -0.79988700 \\
\hline $\mathrm{O}$ & -0.93533700 & -2.24109900 & 0.14763800 \\
\hline $\mathrm{C}$ & -2.25148300 & -2.47754800 & -2.16612100 \\
\hline $\mathrm{F}$ & -2.39707600 & -3.24963400 & -3.24071500 \\
\hline $\mathrm{F}$ & -3.36178500 & -2.50653200 & -1.43276000 \\
\hline
\end{tabular}


TS $_{19-17}$ (optimized by SMD/B3LYP-D3/BS1)

$\mathrm{E}(\mathrm{SMD} / \mathrm{B} 3 \mathrm{LYP}-\mathrm{D} 3 / \mathrm{BS} 1)=-3675.49029914 \mathrm{au}$

$\mathrm{H}(\mathrm{SMD} / \mathrm{B} 3 \mathrm{LYP}-\mathrm{D} 3 / \mathrm{BS} 1)=-3675.102689 \mathrm{au}$

G (SMD/B3LYP-D3/BS1) $=-3675.223991$ au

$\mathrm{E}(\mathrm{SMD} / \mathrm{M} 06 / \mathrm{BS} 2 / / \mathrm{SMD} / \mathrm{B} 3 \mathrm{LYP}-\mathrm{D} 3 / \mathrm{BS} 1)=-3675.48063003 \mathrm{au}$

$\begin{array}{llll}\mathrm{C} & 2.58696500 & -0.98035200 & 0.76084100\end{array}$

$\begin{array}{llll}\mathrm{C} & 2.53495600 & -0.27189600 & 1.96932200\end{array}$

$\begin{array}{llll}\mathrm{C} & 2.41749200 & -2.37200100 & 0.78139700\end{array}$

C $\quad 2.32810400 \quad-0.94558000 \quad 3.17645900$

$\begin{array}{llll}\mathrm{H} & 2.65016800 & 0.80641500 & 1.96534700\end{array}$

C $\quad 2.21509400 \quad-3.04373900 \quad 1.98636500$

$\mathrm{H} \quad 2.43613800 \quad-2.93608500 \quad-0.14720200$

C $\quad 2.17418900 \quad-2.33238000 \quad 3.18837100$

$\mathrm{H} \quad 2.28843800 \quad-0.38156700 \quad 4.10448700$

$\mathrm{H} \quad 2.07239100 \quad-4.11986600 \quad 1.98262500$

$\mathrm{H} \quad 2.01318300 \quad-2.85659100 \quad 4.12634700$

C $\quad 2.83621500 \quad-0.24318600 \quad-0.55188800$

$\begin{array}{llll}\mathrm{H} & 2.96997600 & 0.81868500 & -0.34485300\end{array}$

C $\quad \begin{array}{llll}\mathrm{H} & 1.68564800 & -0.36794000 & -1.55164900\end{array}$

$\mathrm{H} \quad 1.29686500 \quad-1.36018400 \quad-1.77406300$

C $\quad 4.16216400 \quad-0.77628300 \quad-1.19698300$

$\mathrm{H} \quad 4.07343100 \quad-1.85736500 \quad-1.37625000$

$\begin{array}{llll}\mathrm{C} & 5.36998200 & -0.61408300 & -0.24475600\end{array}$

$\begin{array}{llll}\mathrm{C} & 5.60666700 & 0.74634900 & 0.36714400\end{array}$

$\mathrm{H} \quad 5.38287400 \quad 1.55390700 \quad-0.33861800$

$\begin{array}{llll}\mathrm{H} & 4.93994600 & 0.87254600 & 1.22875400\end{array}$

$\mathrm{H} \quad \begin{array}{llll}\mathrm{H} & 6.64004300 & 0.81602000 & 0.71400100\end{array}$

C $\quad 4.47674500 \quad-0.20849200 \quad-2.58372900$

C $\quad 5.90290600 \quad-0.20119900 \quad-3.04969400$

$\mathrm{H} \quad \begin{array}{llll}\mathrm{H} & 6.45874800 & 0.56241600 & -2.48947800\end{array}$

$\mathrm{H} \quad 6.37385700 \quad-1.16695400 \quad-2.83601700$

$\mathrm{H} \quad \begin{array}{llll}\mathrm{H} & .95036100 & 0.02522900 & -4.11610600\end{array}$

$\mathrm{H} \quad 2.18175000 \quad-0.05897700 \quad-2.56940600$

$\mathrm{H} \quad \begin{array}{llll}\mathrm{H} & 1.08443600 & 0.53850900 & -1.85540400\end{array}$

$\begin{array}{llll}\mathrm{O} & 3.58075500 & 0.18219500 & -3.33447600\end{array}$

O $\quad 6.09772000 \quad-1.56463400 \quad-0.03113500$

$\mathrm{Au} \quad-0.35842300 \quad 0.17628900 \quad-0.48207900$

$\begin{array}{llll}\text { O } & -2.20399800 & 0.39426700 & 0.48450900\end{array}$

S $\quad-3.38418600 \quad 1.28610300 \quad-0.04008500$

$\begin{array}{llll}\mathrm{O} & -4.56772000 & 0.92110100 & 0.73573100\end{array}$

$\begin{array}{llll}\mathrm{O} & -3.02846900 & 2.68856000 & -0.22674800\end{array}$

$\begin{array}{lllll}\mathrm{C} & -3.65838700 & 0.62260200 & -1.76992000\end{array}$

F $\quad-3.76607800 \quad-0.70660200 \quad-1.75905800$

F $\quad-4.77380100 \quad 1.15870600 \quad-2.26094500$

F $\quad \begin{array}{lllll}-2.62362200 & 0.96415900 & -2.55774100\end{array}$

$\begin{array}{llll}\mathrm{O} & -0.45767500 & -4.04780600 & 0.36180500\end{array}$

S $\quad-1.09326400 \quad-2.74953500 \quad 0.57252900$

$\begin{array}{llll}\mathrm{O} & -0.98109800 & -2.04059300 & 1.84257100\end{array}$

$\begin{array}{llll}\text { O } & -0.72791500 & -1.82949700 & -0.66226400\end{array}$ 


$\begin{array}{lccc}\text { C } & -2.91871000 & -3.06231800 & 0.26759200 \\ \text { F } & -3.26463400 & -4.14127400 & 0.97425600 \\ \text { F } & -3.65207000 & -2.02632500 & 0.65944600 \\ \text { F } & -3.12337900 & -3.29736200 & -1.03004300 \\ \text { O } & 2.09624100 & 2.66887300 & 0.50112200 \\ \text { S } & 0.71278000 & 3.13163000 & 0.37788400 \\ \text { O } & 0.43392700 & 4.51393800 & 0.00634300 \\ \text { O } & -0.14785700 & 2.21783200 & -0.56716500 \\ \text { C } & -0.05977200 & 2.84615300 & 2.05936000 \\ \text { F } & 0.64455000 & 3.53585200 & 2.95657600 \\ \text { F } & -1.32340500 & 3.25022900 & 2.06577500 \\ \text { F } & -0.00370600 & 1.54240000 & 2.36430300\end{array}$

TS $_{11-20}$ (optimized by SMD/B3LYP-D3/BS1)

$\mathrm{E}(\mathrm{SMD} / \mathrm{B} 3 \mathrm{LYP}-\mathrm{D} 3 / \mathrm{BS} 1)=-3675.51372678 \mathrm{au}$

$\mathrm{H}(\mathrm{SMD} / \mathrm{B} 3 \mathrm{LYP}-\mathrm{D} 3 / \mathrm{BS} 1)=-3675.128433 \mathrm{au}$

$\mathrm{G}(\mathrm{SMD} / \mathrm{B} 3 \mathrm{LYP}-\mathrm{D} 3 / \mathrm{BS} 1)=-3675.254426 \mathrm{au}$

$\mathrm{E}(\mathrm{SMD} / \mathrm{M} 06 / \mathrm{BS} 2 / / \mathrm{SMD} / \mathrm{B} 3 \mathrm{LYP}-\mathrm{D} 3 / \mathrm{BS} 1)=-3675.48839369 \mathrm{au}$

$\begin{array}{llll}\mathrm{C} & -2.96118800 & -2.03942300 & -1.76464300\end{array}$

$\mathrm{H} \quad-3.39952200 \quad-3.03716300 \quad-1.84325200$

$\mathrm{H} \quad-2.39885500 \quad-1.80871200 \quad-2.67692200$

$\mathrm{H} \quad-3.75732000 \quad-1.29423000 \quad-1.66585300$

C $\quad-2.03332600 \quad-1.92545300 \quad-0.59066900$

$\begin{array}{llll}\mathrm{O} & -1.68642600 & -0.66894300 & -0.36923200\end{array}$

C $\quad-1.62325200 \quad-3.03538000 \quad 0.11424600$

C $\quad-0.72676100 \quad-3.05779200 \quad 1.23093400$

$\begin{array}{llll}\mathrm{O} & -0.60049900 & -2.10525100 & 2.06384400\end{array}$

$\begin{array}{llll}\mathrm{C} & 0.12633400 & -4.27159400 & 1.44036000\end{array}$

$\mathrm{H} \quad 0.82804200 \quad-4.36077400 \quad 0.60184700$

$\mathrm{H} \quad 0.67831700 \quad-4.20072000 \quad 2.37941800$

$\mathrm{H} \quad-0.49873100 \quad-5.17283400 \quad 1.43790700$

$\mathrm{H} \quad-1.61139300 \quad-1.30526900 \quad 2.25537400$

$\begin{array}{llll}\mathrm{C} & -3.15295900 & 0.16164500 & 2.03642200\end{array}$

$\begin{array}{llll}\mathrm{C} & -2.59172900 & -0.69799800 & 2.96610500\end{array}$

$\mathrm{H} \quad-2.64803500 \quad 1.11340600 \quad 1.88533600$

$\mathrm{H} \quad-1.92792400 \quad-0.25116100 \quad 3.70849300$

$\mathrm{H} \quad-3.14508800 \quad-1.57536400 \quad 3.30167300$

$\begin{array}{llll}\text { C } & -4.27853100 & -0.07928500 & 1.16864200\end{array}$

$\begin{array}{llll}\text { C } & -4.62374400 & 0.92186500 & 0.23354200\end{array}$

$\begin{array}{llll}\text { C } & -5.02730700 & -1.27813500 & 1.19948600\end{array}$

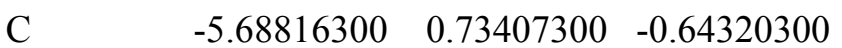

$\mathrm{H} \quad-4.05132500 \quad 1.84455900 \quad 0.20682200$

$\begin{array}{llll}\text { C } & -6.08441700 & -1.46297900 & 0.31829400\end{array}$

$\mathrm{H} \quad-4.77457000 \quad-2.06079400 \quad 1.90730100$

C $\quad-6.41750800 \quad-0.45819700 \quad-0.60214500$

$\mathrm{H} \quad-\quad-5.94607300 \quad 1.50991800 \quad-1.35753900$

$\mathrm{H} \quad-6.65515100 \quad-2.38651100 \quad 0.34264100$

$\mathrm{H} \quad-7.24658600 \quad-0.60901100 \quad-1.28790300$

$\mathrm{H} \quad-1.88785700 \quad-3.99984900 \quad-0.30527100$

$\begin{array}{llll}\mathrm{Au} & 0.16727200 & 0.04834100 & 0.00319900\end{array}$

$\begin{array}{llll}\mathrm{O} & 1.99708100 & 0.95545100 & 0.26429100\end{array}$ 


$\begin{array}{lccc}\text { S } & 2.52061900 & 1.25613000 & 1.71393200 \\ \text { O } & 3.63809300 & 2.18877800 & 1.60546500 \\ \text { O } & 1.43624400 & 1.49025100 & 2.66673100 \\ \text { C } & 3.24741500 & -0.40185500 & 2.17171600 \\ \text { F } & 4.12645500 & -0.78929100 & 1.24813300 \\ \text { F } & 3.85685300 & -0.29606100 & 3.35426600 \\ \text { F } & 2.26675100 & -1.30784300 & 2.25912800 \\ \text { O } & 1.85319300 & -3.57861200 & -1.74875400 \\ \text { S } & 1.36269600 & -2.20574500 & -1.83344600 \\ \text { O } & 0.24481000 & -1.85770700 & -2.70923300 \\ \text { O } & 1.14997800 & -1.70010700 & -0.35646000 \\ \text { C } & 2.79610100 & -1.13609600 & -2.37786400 \\ \text { F } & 3.20299400 & -1.54344400 & -3.57948700 \\ \text { F } & 2.38819800 & 0.13558800 & -2.45164600 \\ \text { F } & 3.79455800 & -1.23461400 & -1.50427200 \\ \text { O } & -1.49216400 & 2.08988600 & -1.91151200 \\ \text { S } & -1.22424700 & 2.77173300 & -0.64722300 \\ \text { O } & -2.20163000 & 3.69346200 & -0.07166800 \\ \text { O } & -0.78560600 & 1.78490600 & 0.49895900 \\ \text { C } & 0.35626900 & 3.74410300 & -0.90471000 \\ \text { F } & 0.06725800 & 4.85665600 & -1.57981800 \\ \text { F } & 0.89694600 & 4.05987100 & 0.27205600 \\ \text { F } & 1.21715200 & 3.00577900 & -1.60875100\end{array}$

20 (optimized by SMD/B3LYP-D3/BS1)

E $($ SMD/B3LYP-D3/BS1) $=-3675.52759291$ au

$\mathrm{H}(\mathrm{SMD} / \mathrm{B} 3 \mathrm{LYP}-\mathrm{D} 3 / \mathrm{BS} 1)=-3675.138848 \mathrm{au}$

G (SMD/B3LYP-D3/BS1) $=-3675.261334 \mathrm{au}$

$\mathrm{E}(\mathrm{SMD} / \mathrm{M} 06 / \mathrm{BS} 2 / / \mathrm{SMD} / \mathrm{B} 3 \mathrm{LYP}-\mathrm{D} 3 / \mathrm{BS} 1)=-3675.49991181 \mathrm{au}$

$\begin{array}{lrrr}\mathrm{C} & 1.72528700 & 3.17963200 & -2.06691100 \\ \mathrm{H} & 1.62831600 & 4.26033500 & -2.20203200 \\ \mathrm{H} & 1.19830300 & 2.66328700 & -2.87584600 \\ \mathrm{H} & 2.78446500 & 2.90296600 & -2.12673600 \\ \mathrm{C} & 1.16792600 & 2.72738800 & -0.74431900 \\ \mathrm{O} & 1.37966000 & 1.43737600 & -0.50516200 \\ \mathrm{C} & 0.55097100 & 3.57448800 & 0.12829300 \\ \mathrm{H} & 0.41630500 & 4.60468300 & -0.18581600 \\ \mathrm{C} & -0.00372800 & 3.19625300 & 1.42810300 \\ \mathrm{C} & -0.97184500 & 4.17434200 & 2.05550000 \\ \mathrm{H} & -1.88472700 & 4.20025600 & 1.44479300 \\ \mathrm{H} & -1.22630400 & 3.85681600 & 3.06951700 \\ \mathrm{H} & -0.56154900 & 5.19100900 & 2.07010300 \\ \mathrm{O} & 0.28502400 & 2.13525300 & 1.98670000 \\ \mathrm{C} & 5.25969400 & 2.10621400 & -0.13418000 \\ \mathrm{C} & 4.18279700 & 2.53471200 & 0.65979900 \\ \mathrm{C} & 3.44243300 & 1.61026900 & 1.37205100 \\ \mathrm{C} & 3.79130100 & 0.22597500 & 1.31053800 \\ \mathrm{C} & 4.89691600 & -0.18524900 & 0.49983100 \\ \mathrm{C} & 5.61884900 & 0.74952800 & -0.21556500 \\ \mathrm{H} & 5.82723200 & 2.84009300 & -0.69976000 \\ \mathrm{H} & 3.91632400 & 3.58582300 & 0.68981100\end{array}$




$\begin{array}{lccc}\mathrm{H} & 2.57275800 & 1.92213800 & 1.93643500 \\ \mathrm{H} & 5.15698000 & -1.23848600 & 0.45925600 \\ \mathrm{H} & 6.45395200 & 0.44386100 & -0.83686100 \\ \mathrm{C} & 3.06525100 & -0.75881800 & 1.98016900 \\ \mathrm{H} & 3.39060500 & -1.78672900 & 1.82272700 \\ \mathrm{C} & 1.93736000 & -0.59819300 & 2.89905200 \\ \mathrm{H} & 2.28486000 & -0.93141100 & 3.89357000 \\ \mathrm{H} & 1.51841700 & 0.40385700 & 2.97170800 \\ \mathrm{H} & 1.15381900 & -1.32041500 & 2.62870900 \\ \mathrm{Au} & -0.07280800 & 0.09911900 & -0.09320600 \\ \mathrm{O} & -1.49312500 & -1.41261300 & 0.16486900 \\ \mathrm{~S} & -1.74209600 & -2.13780400 & 1.51629700 \\ \mathrm{O} & -2.75503300 & -3.17114400 & 1.30789400 \\ \mathrm{O} & -0.51258800 & -2.45930800 & 2.24457000 \\ \mathrm{C} & -2.55827500 & -0.79115800 & 2.51622500 \\ \mathrm{~F} & -3.63943300 & -0.33104900 & 1.88622800 \\ \mathrm{~F} & -2.91115700 & -1.28308400 & 3.70685000 \\ \mathrm{~F} & -1.69413600 & 0.21820000 & 2.69850200 \\ \mathrm{O} & -3.17391400 & 3.03165200 & -1.10229300 \\ \mathrm{~S} & -2.28381400 & 1.92112700 & -1.43323100 \\ \mathrm{O} & -1.31611900 & 2.03917300 & -2.52423600 \\ \mathrm{O} & -1.63637400 & 1.42376000 & -0.09467900 \\ \mathrm{C} & -3.37105100 & 0.47070900 & -1.89081900 \\ \mathrm{~F} & -4.17414900 & 0.84005200 & -2.89177200 \\ \mathrm{~F} & -2.60313300 & -0.54615300 & -2.29528600 \\ \mathrm{~F} & -4.10445300 & 0.09180200 & -0.84674600 \\ \mathrm{O} & 2.27612400 & -1.04241900 & -2.26169700 \\ \mathrm{~S} & 2.12873500 & -1.94113400 & -1.11898000 \\ \mathrm{O} & 3.27181800 & -2.70568500 & -0.62191500 \\ \mathrm{O} & 1.44719900 & -1.26747300 & 0.12023300 \\ \mathrm{C} & 0.82816100 & -3.18722300 & -1.62068800 \\ \mathrm{~F} & 1.35635100 & -4.01628400 & -2.52358100 \\ \mathrm{~F} & 0.42055000 & -3.88466300 & -0.56014600 \\ \mathrm{~F} & -0.21452400 & -2.54940900 & -2.16276100\end{array}$

TS $_{\text {20-21 }}$ (optimized by SMD/B3LYP-D3/BS1)

$\mathrm{E}(\mathrm{SMD} / \mathrm{B} 3 \mathrm{LYP}-\mathrm{D} 3 / \mathrm{BS} 1)=-3675.51540939 \mathrm{au}$

$\mathrm{H}(\mathrm{SMD} / \mathrm{B} 3 \mathrm{LYP}-\mathrm{D} 3 / \mathrm{BS} 1)=-3675.126441 \mathrm{au}$

G $($ SMD/B3LYP-D3/BS1) $=-3675.248964$ au

$\mathrm{E}(\mathrm{SMD} / \mathrm{M} 06 / \mathrm{BS} 2 / / \mathrm{SMD} / \mathrm{B} 3 \mathrm{LYP}-\mathrm{D} 3 / \mathrm{BS} 1)=-3675.49850718 \mathrm{au}$

$\begin{array}{lrrr}\mathrm{C} & 1.72528700 & 3.17963200 & -2.06691100 \\ \mathrm{H} & 1.62831600 & 4.26033500 & -2.20203200 \\ \mathrm{H} & 1.19830300 & 2.66328700 & -2.87584600 \\ \mathrm{H} & 2.78446500 & 2.90296600 & -2.12673600 \\ \mathrm{C} & 1.16792600 & 2.72738800 & -0.74431900 \\ \mathrm{O} & 1.37966000 & 1.43737600 & -0.50516200 \\ \mathrm{C} & 0.55097100 & 3.57448800 & 0.12829300 \\ \mathrm{H} & 0.41630500 & 4.60468300 & -0.18581600 \\ \mathrm{C} & -0.00372800 & 3.19625300 & 1.42810300 \\ \mathrm{C} & -0.97184500 & 4.17434200 & 2.05550000 \\ \mathrm{H} & -1.88472700 & 4.20025600 & 1.44479300\end{array}$




\begin{tabular}{|c|c|c|c|}
\hline $\mathrm{H}$ & -1.22630400 & 3.85681600 & 3.06951700 \\
\hline $\mathrm{H}$ & -0.56154900 & 5.19100900 & 2.07010300 \\
\hline $\mathrm{O}$ & 0.28502400 & 2.13525300 & 1.98670000 \\
\hline $\mathrm{C}$ & 5.25969400 & 2.10621400 & -0.13418000 \\
\hline $\mathrm{C}$ & 4.18279700 & 2.53471200 & 0.65979900 \\
\hline $\mathrm{C}$ & 3.44243300 & 1.61026900 & 1.37205100 \\
\hline $\mathrm{C}$ & 3.79130100 & 0.22597500 & 1.31053800 \\
\hline $\mathrm{C}$ & 4.89691600 & -0.18524900 & 0.49983100 \\
\hline $\mathrm{C}$ & 5.61884900 & 0.74952800 & -0.21556500 \\
\hline $\mathrm{H}$ & 5.82723200 & 2.84009300 & -0.69976000 \\
\hline $\mathrm{H}$ & 3.91632400 & 3.58582300 & 0.68981100 \\
\hline $\mathrm{H}$ & 2.57275800 & 1.92213800 & 1.93643500 \\
\hline $\mathrm{H}$ & 5.15698000 & -1.23848600 & 0.45925600 \\
\hline $\mathrm{H}$ & 6.45395200 & 0.44386100 & -0.83686100 \\
\hline $\mathrm{C}$ & 3.06525100 & -0.75881800 & 1.98016900 \\
\hline $\mathrm{H}$ & 3.39060500 & -1.78672900 & 1.82272700 \\
\hline $\mathrm{C}$ & 1.93736000 & -0.59819300 & 2.89905200 \\
\hline $\mathrm{H}$ & 2.28486000 & -0.93141100 & 3.89357000 \\
\hline $\mathrm{H}$ & 1.51841700 & 0.40385700 & 2.97170800 \\
\hline $\mathrm{H}$ & 1.15381900 & -1.32041500 & 2.62870900 \\
\hline $\mathrm{Au}$ & -0.07280800 & 0.09911900 & -0.09320600 \\
\hline $\mathrm{O}$ & -1.49312500 & -1.41261300 & 0.16486900 \\
\hline S & -1.74209600 & -2.13780400 & 1.51629700 \\
\hline $\mathrm{O}$ & -2.75503300 & -3.17114400 & 1.30789400 \\
\hline $\mathrm{O}$ & -0.51258800 & -2.45930800 & 2.24457000 \\
\hline $\mathrm{C}$ & -2.55827500 & -0.79115800 & 2.51622500 \\
\hline $\mathrm{F}$ & -3.63943300 & -0.33104900 & 1.88622800 \\
\hline $\mathrm{F}$ & -2.91115700 & -1.28308400 & 3.70685000 \\
\hline $\mathrm{F}$ & -1.69413600 & 0.21820000 & 2.69850200 \\
\hline $\mathrm{O}$ & -3.17391400 & 3.03165200 & -1.10229300 \\
\hline $\mathrm{S}$ & -2.28381400 & 1.92112700 & -1.43323100 \\
\hline $\mathrm{O}$ & -1.31611900 & 2.03917300 & -2.52423600 \\
\hline $\mathrm{O}$ & -1.63637400 & 1.42376000 & -0.09467900 \\
\hline $\mathrm{C}$ & -3.37105100 & 0.47070900 & -1.89081900 \\
\hline $\mathrm{F}$ & -4.17414900 & 0.84005200 & -2.89177200 \\
\hline $\mathrm{F}$ & -2.60313300 & -0.54615300 & -2.29528600 \\
\hline $\mathrm{F}$ & -4.10445300 & 0.09180200 & -0.84674600 \\
\hline $\mathrm{O}$ & 2.27612400 & -1.04241900 & -2.26169700 \\
\hline S & 2.12873500 & -1.94113400 & -1.11898000 \\
\hline $\mathrm{O}$ & 3.27181800 & -2.70568500 & -0.62191500 \\
\hline $\mathrm{O}$ & 1.44719900 & -1.26747300 & 0.12023300 \\
\hline $\mathrm{C}$ & 0.82816100 & -3.18722300 & -1.62068800 \\
\hline $\mathrm{F}$ & 1.35635100 & -4.01628400 & -2.52358100 \\
\hline $\mathrm{F}$ & 0.42055000 & -3.88466300 & -0.56014600 \\
\hline $\mathrm{F}$ & -0.21452400 & -2.54940900 & -2.16276100 \\
\hline
\end{tabular}

TS $_{12-20}$ (optimized by SMD/B3LYP-D3/BS1)

$\mathrm{E}(\mathrm{SMD} / \mathrm{B} 3 \mathrm{LYP}-\mathrm{D} 3 / \mathrm{BS} 1)=-3675.49573626 \mathrm{au}$

$\mathrm{H}(\mathrm{SMD} / \mathrm{B} 3 \mathrm{LYP}-\mathrm{D} 3 / \mathrm{BS} 1)=-3675.111683 \mathrm{au}$

G $($ SMD/B3LYP-D3/BS1) $=-3675.238642 \mathrm{au}$

$\mathrm{E}(\mathrm{SMD} / \mathrm{M} 06 / \mathrm{BS} 2 / / \mathrm{SMD} / \mathrm{B} 3 \mathrm{LYP}-\mathrm{D} 3 / \mathrm{BS} 1)=-3675.47910305 \mathrm{au}$ 


\begin{tabular}{|c|c|c|c|}
\hline $\mathrm{C}$ & 0.56991400 & -0.81413500 & -3.16887700 \\
\hline $\mathrm{H}$ & 0.15977800 & 0.20389900 & -3.13753300 \\
\hline $\mathrm{H}$ & -0.27224500 & -1.51781900 & -3.12479800 \\
\hline $\mathrm{H}$ & 1.10755600 & -0.95580200 & -4.10721900 \\
\hline $\mathrm{C}$ & 1.45923500 & -1.04702700 & -1.98495400 \\
\hline $\mathrm{C}$ & 2.82554300 & -1.39013800 & -2.16878000 \\
\hline $\mathrm{H}$ & 3.37234700 & -0.06715700 & -1.92090600 \\
\hline $\mathrm{O}$ & 1.03289600 & -0.87510000 & -0.77875800 \\
\hline $\mathrm{C}$ & 7.94138000 & -0.07751300 & -0.30554600 \\
\hline $\mathrm{C}$ & 6.70304300 & 0.34350300 & -0.77461100 \\
\hline $\mathrm{C}$ & 5.63601600 & 0.56383800 & 0.12655700 \\
\hline $\mathrm{C}$ & 5.85629400 & 0.35433900 & 1.50633800 \\
\hline $\mathrm{C}$ & 7.10011400 & -0.05918900 & 1.97207000 \\
\hline $\mathrm{C}$ & 8.14367400 & -0.27819200 & 1.06702200 \\
\hline $\mathrm{H}$ & 8.75540200 & -0.24704000 & -1.00436300 \\
\hline $\mathrm{H}$ & 6.56031700 & 0.50078400 & -1.83907600 \\
\hline $\mathrm{H}$ & 5.03650500 & 0.51294300 & 2.20170700 \\
\hline $\mathrm{H}$ & 7.25684700 & -0.21711700 & 3.03504300 \\
\hline $\mathrm{H}$ & 9.11518000 & -0.60417500 & 1.42812200 \\
\hline $\mathrm{C}$ & 4.32153700 & 0.98234400 & -0.29853800 \\
\hline $\mathrm{H}$ & 3.58992400 & 1.11202200 & 0.49553300 \\
\hline $\mathrm{C}$ & 3.87705600 & 1.16850800 & -1.60219600 \\
\hline $\mathrm{H}$ & 4.59734100 & 1.27190300 & -2.41347600 \\
\hline $\mathrm{H}$ & 2.95634500 & 1.73739900 & -1.73260300 \\
\hline $\mathrm{H}$ & 3.09058300 & -1.64190800 & -3.19251500 \\
\hline $\mathrm{C}$ & 3.63270200 & -2.04129400 & -1.09592300 \\
\hline $\mathrm{O}$ & 3.47692600 & -1.76324400 & 0.08565500 \\
\hline $\mathrm{C}$ & 4.73124800 & -2.96776200 & -1.56842100 \\
\hline $\mathrm{H}$ & 4.34634400 & -3.71349700 & -2.27286100 \\
\hline $\mathrm{H}$ & 5.49287100 & -2.37891500 & -2.09733500 \\
\hline $\mathrm{H}$ & 5.19435700 & -3.45982000 & -0.71025500 \\
\hline $\mathrm{Au}$ & -0.79859000 & -0.11749900 & -0.34269800 \\
\hline $\mathrm{O}$ & -2.58580700 & 0.66703500 & 0.27893400 \\
\hline S & -3.57578100 & 1.31038000 & -0.76305500 \\
\hline $\mathrm{O}$ & -4.57297200 & 2.06701000 & -0.01436200 \\
\hline $\mathrm{O}$ & -2.88487100 & 1.89719100 & -1.90983200 \\
\hline $\mathrm{C}$ & -4.42747700 & -0.21543400 & -1.41974900 \\
\hline $\mathrm{F}$ & -4.95176900 & -0.91941200 & -0.41989600 \\
\hline $\mathrm{F}$ & -5.39040400 & 0.15540900 & -2.26417500 \\
\hline $\mathrm{F}$ & -3.53255100 & -0.96852400 & -2.07414900 \\
\hline $\mathrm{O}$ & -2.02366600 & -4.17734700 & 0.75279400 \\
\hline S & -1.37148100 & -2.90126600 & 1.02770300 \\
\hline $\mathrm{O}$ & 0.01342700 & -2.84074700 & 1.48305900 \\
\hline $\mathrm{O}$ & -1.64043700 & -1.97167100 & -0.22062000 \\
\hline $\mathrm{C}$ & -2.38539800 & -2.04307900 & 2.34543800 \\
\hline $\mathrm{F}$ & -2.41646200 & -2.81521600 & 3.43062000 \\
\hline $\mathrm{F}$ & -1.81118400 & -0.87139900 & 2.65121900 \\
\hline $\mathrm{F}$ & -3.62325300 & -1.83006600 & 1.90724000 \\
\hline $\mathrm{O}$ & 1.43223300 & 1.63333900 & 1.34908000 \\
\hline S & 0.75460800 & 2.52487400 & 0.40712900 \\
\hline $\mathrm{O}$ & 1.48435300 & 3.59947900 & -0.25897200 \\
\hline
\end{tabular}




$\begin{array}{llll}\mathrm{O} & -0.03896500 & 1.74191500 & -0.70696700 \\ \mathrm{C} & -0.64695400 & 3.31392700 & 1.36779500 \\ \mathrm{~F} & -0.14166800 & 4.27641400 & 2.13825800 \\ \mathrm{~F} & -1.54247300 & 3.83101300 & 0.52739200 \\ \mathrm{~F} & -1.23032900 & 2.38887600 & 2.13142700\end{array}$

TS $_{22}$ (optimized by SMD/B3LYP-D3/BS1)

$\mathrm{E}(\mathrm{SMD} / \mathrm{B} 3 \mathrm{LYP}-\mathrm{D} 3 / \mathrm{BS} 1)=-3675.48680716 \mathrm{au}$

$\mathrm{H}(\mathrm{SMD} / \mathrm{B} 3 \mathrm{LYP}-\mathrm{D} 3 / \mathrm{BS} 1)=-3675.096675 \mathrm{au}$

G (SMD/B3LYP-D3/BS1) $=-3675.221967$ au

$\mathrm{E}(\mathrm{SMD} / \mathrm{M} 06 / \mathrm{BS} 2 / / \mathrm{SMD} / \mathrm{B} 3 \mathrm{LYP}-\mathrm{D} 3 / \mathrm{BS} 1)=-3675.47118402 \mathrm{au}$

$\begin{array}{llll}\mathrm{C} & -1.97612800 & 1.06846800 & -2.45274500\end{array}$

$\mathrm{H} \quad-2.64492300 \quad 0.84890600 \quad-3.28887100$

$\mathrm{H} \quad-0.98864300 \quad 0.65735200 \quad-2.69024300$

$\mathrm{H} \quad-1.87000100 \quad 2.14568300 \quad-2.31152400$

C $\quad-2.48523300 \quad 0.37041500 \quad-1.20974900$

O $\quad-3.13563700 \quad-0.65286600 \quad-1.25408200$

$\begin{array}{llll}\mathrm{C} & -2.18565000 & 1.05304100 & 0.13920000\end{array}$

$\begin{array}{llll}\mathrm{C} & -1.96686300 & 0.25506500 & 1.43698600\end{array}$

$\begin{array}{llll}\mathrm{O} & -2.38436500 & -0.87916900 & 1.56624700\end{array}$

$\begin{array}{llll}\mathrm{C} & -1.31433100 & 1.00361100 & 2.58628700\end{array}$

$\mathrm{H} \quad-2.10185800 \quad 1.29324500 \quad 3.29360400$

$\mathrm{H} \quad-0.63164600 \quad 0.32594000 \quad 3.10590800$

$\begin{array}{llll}\mathrm{H} & -0.77746700 & 1.90056400 & 2.27079700\end{array}$

$\begin{array}{llll}\mathrm{H} & -1.57174500 & 1.94738900 & 0.06377100\end{array}$

$\begin{array}{llll}\mathrm{C} & -5.04634300 & 0.34480600 & 1.95655200\end{array}$

$\begin{array}{llll}\mathrm{C} & -6.07474400 & -0.55781200 & 2.15985800\end{array}$

$\begin{array}{llll}\mathrm{C} & -6.88243400 & -0.94569600 & 1.07796400\end{array}$

$\begin{array}{llll}\text { C } & -6.66248200 & -0.41684800 & -0.20624000\end{array}$

$\begin{array}{llll}\mathrm{C} & -5.63724100 & 0.48818600 & -0.41490400\end{array}$

$\begin{array}{lllll}\mathrm{C} & -4.81070000 & 0.90207200 & 0.66728300\end{array}$

$\mathrm{H} \quad-4.42315500 \quad 0.65593900 \quad 2.78841400$

$\mathrm{H} \quad-6.25551400 \quad-0.96684900 \quad 3.14863900$

$\mathrm{H} \quad-7.68235000 \quad-1.66371400 \quad 1.23363700$

$\mathrm{H} \quad-7.29184600 \quad-0.72678100 \quad-1.03423300$

$\mathrm{H} \quad-\quad-5.46463900 \quad 0.88335400 \quad-1.40895200$

$\begin{array}{llll}\mathrm{C} & -3.75211200 & 1.89599200 & 0.50546300\end{array}$

$\begin{array}{llll}\mathrm{H} & -3.44962800 & 2.31994300 & 1.46643200\end{array}$

$\begin{array}{llll}\mathrm{C} & -3.92398800 & 2.98937400 & -0.53539200\end{array}$

$\mathrm{H} \quad-4.14314100 \quad 2.61101800 \quad-1.53601600$

$\mathrm{H} \quad-4.76982400 \quad 3.61420800 \quad-0.22336200$

$\mathrm{H} \quad-3.03322700 \quad 3.62105800 \quad-0.58475900$

$\mathrm{Au} \quad 0.33686400 \quad 0.08263700 \quad-0.12271200$

$\begin{array}{llll}\mathrm{O} & 2.38402900 & -0.53565800 & 0.18869700\end{array}$

$\begin{array}{lllll}\mathrm{S} & 3.00281200 & -0.41323900 & 1.61491300\end{array}$

$\begin{array}{llll}\mathrm{O} & 4.43734700 & -0.68176900 & 1.50753800\end{array}$

$\begin{array}{lllll}\mathrm{O} & 2.52126400 & 0.75080700 & 2.35744100\end{array}$

$\begin{array}{llll}\mathrm{C} & 2.25026000 & -1.88985000 & 2.47488800\end{array}$

F $\quad \begin{array}{llll}\text { F } & 2.55395800 & -3.01096400 & 1.82433100\end{array}$

F $\quad 2.72099100 \quad-1.95242800 \quad 3.72177100$

F $\quad \begin{array}{llll}0.91541300 & -1.75348000 & 2.51740100\end{array}$ 


$\begin{array}{lccc}\mathrm{O} & -0.91211800 & -3.92620800 & -1.41549600 \\ \mathrm{~S} & -0.24972500 & -2.64401100 & -1.63581400 \\ \mathrm{O} & -0.64009400 & -1.77942700 & -2.74942600 \\ \mathrm{O} & -0.21116000 & -1.88450900 & -0.26428500 \\ \mathrm{C} & 1.55895400 & -3.01557600 & -1.92677300 \\ \mathrm{~F} & 1.65714500 & -3.87985400 & -2.93931000 \\ \mathrm{~F} & 2.19699200 & -1.88529100 & -2.24998600 \\ \mathrm{~F} & 2.10875000 & -3.54287900 & -0.83471100 \\ \mathrm{O} & 1.28693700 & 2.22454500 & -2.22186300 \\ \mathrm{~S} & 1.27365100 & 2.96038500 & -0.95462600 \\ \mathrm{O} & 0.64475500 & 4.27746000 & -0.87272100 \\ \mathrm{O} & 0.75221000 & 2.06865600 & 0.22382100 \\ \mathrm{C} & 3.05464400 & 3.18617900 & -0.43968500 \\ \mathrm{~F} & 3.63393100 & 4.04240600 & -1.28309100 \\ \mathrm{~F} & 3.10583700 & 3.67007100 & 0.79898400 \\ \mathrm{~F} & 3.68926200 & 2.01361900 & -0.49222100\end{array}$

18 (optimized by SMD/B3LYP-D3/BS3)

$\mathrm{E}(\mathrm{SMD} / \mathrm{B} 3 \mathrm{LYP}-\mathrm{D} 3 / \mathrm{BS} 3)=-3675.72141311 \mathrm{au}$

$\mathrm{H}(\mathrm{SMD} / \mathrm{B} 3 \mathrm{LYP}-\mathrm{D} 3 / \mathrm{BS} 3)=-3675.330756 \mathrm{au}$

$\mathrm{G}(\mathrm{SMD} / \mathrm{B} 3 \mathrm{LYP}-\mathrm{D} 3 / \mathrm{BS} 3)=-3675.453521 \mathrm{au}$

$\mathrm{E}(\mathrm{SMD} / \mathrm{M} 06 / \mathrm{BS} 2 / / \mathrm{SMD} / \mathrm{B} 3 \mathrm{LYP}-\mathrm{D} 3 / \mathrm{BS} 3)=-3675.57196642 \mathrm{au}$

E (SMD/M06-D3/BS2// SMD/B3LYP-D3/ BS3) $=-3675.59022893 \mathrm{au}$

$\begin{array}{llrr}\mathrm{C} & 3.53281400 & 1.15833700 & -2.96770100 \\ \mathrm{H} & 3.61862100 & 1.81607900 & -2.09402300 \\ \mathrm{H} & 3.32337800 & 1.76224700 & -3.85204200 \\ \mathrm{H} & 4.48609700 & 0.63056300 & -3.06384200 \\ \mathrm{C} & 2.41932300 & 0.19938900 & -2.73260600 \\ \mathrm{O} & 1.34497800 & 0.29874900 & -3.34061500 \\ \mathrm{C} & 2.62041000 & -0.93510700 & -1.72288300 \\ \mathrm{C} & 1.59910500 & -2.03789400 & -1.86286400 \\ \mathrm{C} & 1.84405200 & -3.38508300 & -1.31902400 \\ \mathrm{H} & 0.90413500 & -3.88744700 & -1.08668600 \\ \mathrm{H} & 2.34325700 & -3.94222800 & -2.12830600 \\ \mathrm{H} & 2.51890900 & -3.37394300 & -0.46286600 \\ \mathrm{O} & 0.53971300 & -1.85713600 & -2.53168600 \\ \mathrm{H} & 3.57799200 & -1.41953100 & -1.95215300 \\ \mathrm{C} & 2.75682900 & -0.45256700 & -0.19988900 \\ \mathrm{C} & 4.21816100 & -0.15137500 & 0.15669500 \\ \mathrm{C} & 5.07695700 & -1.24436600 & 0.36228700 \\ \mathrm{C} & 4.72593000 & 1.14553900 & 0.30941800 \\ \mathrm{C} & 6.41980100 & -1.04655700 & 0.68547700 \\ \mathrm{H} & 4.68902000 & -2.25639400 & 0.27085600 \\ \mathrm{C} & 6.07180600 & 1.34449700 & 0.63978800 \\ \mathrm{H} & 4.09039300 & 2.01424200 & 0.17656000 \\ \mathrm{C} & 6.92328700 & 0.25289400 & 0.82350900 \\ \mathrm{H} & 7.06870000 & -1.90413400 & 0.83959800 \\ \mathrm{H} & 6.44864400 & 2.35697100 & 0.75399100 \\ \mathrm{H} & 7.96725500 & 0.41018700 & 1.07931700 \\ \mathrm{C} & 1.85651600 & 0.70264400 & 0.21154000 \\ \mathrm{H} & 1.99164400 & 1.60682600 & -0.37990200\end{array}$




$\begin{array}{lrrr}\mathrm{H} & 1.95724800 & 0.91464100 & 1.27730900 \\ \mathrm{H} & 2.49421400 & -1.32144900 & 0.40101500 \\ \mathrm{Au} & -0.18895700 & 0.35827600 & 0.02854800 \\ \mathrm{O} & -2.39503100 & 0.01216800 & -0.19219000 \\ \mathrm{~S} & -2.93165700 & -0.35897800 & -1.56969300 \\ \mathrm{O} & -4.25285800 & 0.22070900 & -1.83925500 \\ \mathrm{O} & -1.90326700 & -0.28154200 & -2.62427000 \\ \mathrm{C} & -3.25707900 & -2.20019600 & -1.36787800 \\ \mathrm{~F} & -4.15829300 & -2.42169900 & -0.39712700 \\ \mathrm{~F} & -3.73782700 & -2.70460200 & -2.51936400 \\ \mathrm{~F} & -2.12542400 & -2.85183200 & -1.05838900 \\ \mathrm{O} & 0.44234200 & -3.54449100 & 2.00376800 \\ \mathrm{~S} & 0.24238500 & -2.09696300 & 2.02703200 \\ \mathrm{O} & 1.16772300 & -1.23257900 & 2.76216700 \\ \mathrm{O} & 0.02718000 & -1.61418600 & 0.55439400 \\ \mathrm{C} & -1.45921100 & -1.81920200 & 2.79146400 \\ \mathrm{~F} & -1.44986400 & -2.30158900 & 4.04075200 \\ \mathrm{~F} & -1.74003200 & -0.51051700 & 2.82921300 \\ \mathrm{~F} & -2.39307300 & -2.45362100 & 2.07974600 \\ \mathrm{O} & 0.50596400 & 3.41563900 & 1.29974200 \\ \mathrm{~S} & -0.42576900 & 3.52117400 & 0.17399000 \\ \mathrm{O} & -0.41889900 & 4.70452300 & -0.68367200 \\ \mathrm{O} & -0.41808800 & 2.25097500 & -0.73531100 \\ \mathrm{C} & -2.14980100 & 3.44027700 & 0.93072500 \\ \mathrm{~F} & -2.34898000 & 4.53647600 & 1.67647800 \\ \mathrm{~F} & -3.07780800 & 3.38739700 & -0.02874000 \\ \mathrm{~F} & -2.25682200 & 2.35653100 & 1.71063200 \\ \mathrm{H} & 0.57041900 & -0.89310300 & -2.94710000\end{array}$

19 (optimized by SMD/B3LYP-D3/BS3)

$\mathrm{E}(\mathrm{SMD} / \mathrm{B} 3 \mathrm{LYP}-\mathrm{D} 3 / \mathrm{BS} 3)=-3675.71680218 \mathrm{au}$

$\mathrm{H}(\mathrm{SMD} / \mathrm{B} 3 \mathrm{LYP}-\mathrm{D} 3 / \mathrm{BS} 3)=-3675.326849 \mathrm{au}$

$\mathrm{G}(\mathrm{SMD} / \mathrm{B} 3 \mathrm{LYP}-\mathrm{D} 3 / \mathrm{BS} 3)=-3675.451312 \mathrm{au}$

$\mathrm{E}(\mathrm{SMD} / \mathrm{M} 06 / \mathrm{BS} 2 / / \mathrm{SMD} / \mathrm{B} 3 \mathrm{LYP}-\mathrm{D} 3 / \mathrm{BS} 3)=-3675.56860773 \mathrm{au}$

$\mathrm{E}(\mathrm{SMD} / \mathrm{M} 06-\mathrm{D} 3 / \mathrm{BS} 2 / / \mathrm{SMD} / \mathrm{B} 3 \mathrm{LYP}-\mathrm{D} 3 / \mathrm{BS} 3)=-3675.58677949 \mathrm{au}$

$\begin{array}{llrr}\mathrm{C} & 4.41184300 & -3.40437100 & 0.36351900 \\ \mathrm{H} & 3.34202200 & -3.50314100 & 0.57329800 \\ \mathrm{H} & 5.00578100 & -3.60151300 & 1.25713800 \\ \mathrm{H} & 4.65467100 & -4.14512900 & -0.41120100 \\ \mathrm{C} & 4.73153700 & -2.06125500 & -0.18700900 \\ \mathrm{O} & 5.71931400 & -1.41002700 & 0.18001500 \\ \mathrm{C} & 3.79972500 & -1.46152300 & -1.23615100 \\ \mathrm{C} & 4.50935300 & -0.52562000 & -2.15763700 \\ \mathrm{C} & 4.08213700 & -0.30056100 & -3.54765800 \\ \mathrm{H} & 4.54863900 & -1.10538400 & -4.13950900 \\ \mathrm{H} & 3.00283000 & -0.40883100 & -3.66693600 \\ \mathrm{H} & 4.44206000 & 0.65970100 & -3.92131100 \\ \mathrm{O} & 5.53312600 & 0.09423400 & -1.73095900 \\ \mathrm{H} & 5.78268400 & -0.31978800 & -0.79258200 \\ \mathrm{H} & 3.31378300 & -2.24982500 & -1.81655900 \\ \mathrm{C} & 2.59918500 & -0.69883700 & -0.42770000\end{array}$




$\begin{array}{lccc}\mathrm{C} & 3.09858000 & 0.41387400 & 0.46761300 \\ \mathrm{C} & 3.23839300 & 0.15957000 & 1.84093500 \\ \mathrm{C} & 3.44440500 & 1.68035500 & -0.02646400 \\ \mathrm{C} & 3.71625200 & 1.14833700 & 2.70255200 \\ \mathrm{H} & 2.96735300 & -0.81649300 & 2.23520100 \\ \mathrm{C} & 3.92594400 & 2.66923700 & 0.83375100 \\ \mathrm{H} & 3.32880400 & 1.91167600 & -1.08190100 \\ \mathrm{C} & 4.06573100 & 2.40520600 & 2.19891700 \\ \mathrm{H} & 3.81066000 & 0.93850500 & 3.76417300 \\ \mathrm{H} & 4.17618100 & 3.64855200 & 0.43767700 \\ \mathrm{H} & 4.43452600 & 3.17791200 & 2.86755400 \\ \mathrm{C} & 1.51429600 & -0.32552800 & -1.42103300 \\ \mathrm{H} & 1.27098800 & -1.13863700 & -2.10839200 \\ \mathrm{H} & 1.67518200 & 0.61341000 & -1.95095200 \\ \mathrm{H} & 2.21569000 & -1.50414900 & 0.19983500 \\ \mathrm{Au} & -0.33823100 & -0.01362000 & -0.52440200 \\ \mathrm{O} & -2.45228300 & 0.28137400 & 0.20221600 \\ \mathrm{~S} & -3.54207600 & 0.50148400 & -0.84124400 \\ \mathrm{O} & -4.53167900 & 1.50605300 & -0.43250100 \\ \mathrm{O} & -3.00971800 & 0.56131900 & -2.21549100 \\ \mathrm{C} & -4.48784000 & -1.12286200 & -0.77917600 \\ \mathrm{~F} & -5.01183400 & -1.32683900 & 0.44048800 \\ \mathrm{~F} & -5.49481300 & -1.08847500 & -1.67240300 \\ \mathrm{~F} & -3.68746900 & -2.15598400 & -1.08319500 \\ \mathrm{O} & -0.95565100 & -4.37759100 & 0.13844000 \\ \mathrm{~S} & -0.41383000 & -3.08572800 & 0.55575800 \\ \mathrm{O} & 0.96504300 & -3.01042300 & 1.05684500 \\ \mathrm{O} & -0.71745300 & -2.04427200 & -0.55487300 \\ \mathrm{C} & -1.47228500 & -2.52161600 & 2.01059600 \\ \mathrm{~F} & -1.27634600 & -3.37439200 & 3.02725900 \\ \mathrm{~F} & -1.10751100 & -1.29251800 & 2.39923900 \\ \mathrm{~F} & -2.76436800 & -2.52031400 & 1.68333200 \\ \mathrm{O} & 0.50928400 & 2.38036300 & 1.67696300 \\ \mathrm{~S} & 0.20535500 & 3.02376800 & 0.39958900 \\ \mathrm{O} & 1.03529500 & 4.11596100 & -0.10975500 \\ \mathrm{O} & -0.04133800 & 2.00603000 & -0.76176400 \\ \mathrm{C} & -1.50684500 & 3.79266500 & 0.60860500 \\ \mathrm{~F} & -1.37266500 & 4.94919600 & 1.27967500 \\ \mathrm{~F} & -2.05557400 & 4.04711700 & -0.58663800 \\ \mathrm{~F} & -2.30684100 & 2.98222700 & 1.30130100\end{array}$

TS $_{\text {18-17 }}$ (optimized by SMD/B3LYP-D3/BS3)

$\mathrm{E}(\mathrm{SMD} / \mathrm{B} 3 \mathrm{LYP}-\mathrm{D} 3 / \mathrm{BS} 3)=-3675.64606867 \mathrm{au}$

$\mathrm{H}(\mathrm{SMD} / \mathrm{B} 3 \mathrm{LYP}-\mathrm{D} 3 / \mathrm{BS} 3)=-3675.261478 \mathrm{au}$

$\mathrm{G}(\mathrm{SMD} / \mathrm{B} 3 \mathrm{LYP}-\mathrm{D} 3 / \mathrm{BS} 3)=-3675.384904 \mathrm{au}$

$\mathrm{E}(\mathrm{SMD} / \mathrm{M} 06 / \mathrm{BS} 2 / / \mathrm{SMD} / \mathrm{B} 3 \mathrm{LYP}-\mathrm{D} 3 / \mathrm{BS} 3)=-3675.49623 \mathrm{au}$

$\mathrm{E}(\mathrm{SMD} / \mathrm{M} 06-\mathrm{D} 3 / \mathrm{BS} 2 / / \mathrm{SMD} / \mathrm{B} 3 \mathrm{LYP}-\mathrm{D} 3 / \mathrm{BS} 3)=-3675.5129764 \mathrm{au}$

$\begin{array}{llll}\mathrm{C} & -5.76825600 & 1.20835800 & 2.27974100 \\ \mathrm{H} & -6.26099600 & 0.57189100 & 1.53473100 \\ \mathrm{H} & -6.22432600 & 2.19967500 & 2.29019300 \\ \mathrm{H} & -5.89747800 & 0.71783400 & 3.25227500\end{array}$




$\begin{array}{lccc}\mathrm{C} & -4.30811700 & 1.30135300 & 1.95205700 \\ \mathrm{O} & -3.73483900 & 2.34962600 & 1.70507100 \\ \mathrm{C} & -3.52174500 & -0.03571100 & 1.91521600 \\ \mathrm{C} & -2.44153900 & 0.02925600 & 2.99052700 \\ \mathrm{C} & -2.81532900 & -0.36973400 & 4.38307700 \\ \mathrm{H} & -2.04660700 & -0.06284400 & 5.09381800 \\ \mathrm{H} & -3.78890700 & 0.05600900 & 4.65088200 \\ \mathrm{H} & -2.92356200 & -1.46249000 & 4.4051100 \\ \mathrm{O} & -1.30528400 & 0.40455800 & 2.70571500 \\ \mathrm{H} & -1.13258200 & 0.55164500 & 0.95412100 \\ \mathrm{H} & -4.20718900 & -0.84431100 & 2.18312500 \\ \mathrm{C} & -2.95596200 & -0.35163000 & 0.50135100 \\ \mathrm{C} & -4.05925100 & -0.47010600 & -0.54485500 \\ \mathrm{C} & -4.52849400 & -1.74590000 & -0.89294100 \\ \mathrm{C} & -4.63124300 & 0.66052900 & -1.15210500 \\ \mathrm{C} & -5.57176600 & -1.89009600 & -1.80965300 \\ \mathrm{H} & -4.07664000 & -2.62529900 & -0.44242800 \\ \mathrm{C} & -5.67027500 & 0.51416800 & -2.07482900 \\ \mathrm{H} & -4.27713700 & 1.65688100 & -0.90762200 \\ \mathrm{C} & -6.14555900 & -0.75960900 & -2.40386700 \\ \mathrm{H} & -5.93060900 & -2.88331800 & -2.06459800 \\ \mathrm{H} & -6.10637200 & 1.39608800 & -2.53529500 \\ \mathrm{H} & -6.95305000 & -0.87171900 & -3.12187600 \\ \mathrm{C} & -1.88540000 & 0.63873300 & -0.00012600 \\ \mathrm{H} & -2.10070400 & 1.70191300 & 0.12572800 \\ \mathrm{H} & -1.63244000 & 0.43711000 & -1.04017000 \\ \mathrm{~F} & -2.49497500 & -1.33896600 & 0.58921200 \\ \mathrm{H} & 0.48763700 & 0.24368200 & 0.16920300 \\ \mathrm{O} & 2.32872700 & 2.90011600 & -1.03029500 \\ \mathrm{Au} & & & \end{array}$


TS $_{\text {19-17 }}$ (optimized by SMD/B3LYP-D3/BS3)

$\mathrm{E}(\mathrm{SMD} / \mathrm{B} 3 \mathrm{LYP}-\mathrm{D} 3 / \mathrm{BS} 3)=-3675.63159143 \mathrm{au}$

$\mathrm{H}(\mathrm{SMD} / \mathrm{B} 3 \mathrm{LYP}-\mathrm{D} 3 / \mathrm{BS} 3)=-3675.248287 \mathrm{au}$

$\mathrm{G}(\mathrm{SMD} / \mathrm{B} 3 \mathrm{LYP}-\mathrm{D} 3 / \mathrm{BS} 3)=-3675.369218 \mathrm{au}$

$\mathrm{E}(\mathrm{SMD} / \mathrm{M} 06 / \mathrm{BS} 2 / / \mathrm{SMD} / \mathrm{B} 3 \mathrm{LYP}-\mathrm{D} 3 / \mathrm{BS} 3)=-3675.48070278 \mathrm{au}$

$\mathrm{E}(\mathrm{SMD} / \mathrm{M} 06-\mathrm{D} 3 / \mathrm{BS} 2 / / \mathrm{SMD} / \mathrm{B} 3 \mathrm{LYP}-\mathrm{D} 3 / \mathrm{BS} 3)=-3675.49882247 \mathrm{au}$

$\begin{array}{llll}\mathrm{C} & -5.76825600 & 1.20835800 & 2.27974100\end{array}$

$\mathrm{H} \quad-6.26099600 \quad 0.57189100 \quad 1.53473100$

$\mathrm{H} \quad-6.22432600 \quad 2.19967500 \quad 2.29019300$

$\mathrm{H} \quad \begin{array}{rrrr}\mathrm{H} & -5.89747800 & 0.71783400 & 3.25227500\end{array}$

C $\quad-4.30811700 \quad 1.30135300 \quad 1.95205700$

$\begin{array}{llll}\mathrm{O} & -3.73483900 & 2.34962600 & 1.70507100\end{array}$

C $\quad-3.52174500 \quad-0.03571100 \quad 1.91521600$

$\begin{array}{llll}\text { C } & -2.44153900 & 0.02925600 & 2.99052700\end{array}$

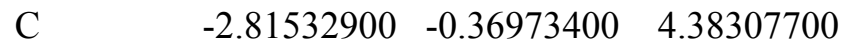

$\mathrm{H} \quad-2.04660700 \quad-0.06284400 \quad 5.09381800$

$\mathrm{H} \quad \begin{array}{llll}-3.78890700 & 0.05600900 & 4.65088200\end{array}$

$\mathrm{H} \quad-2.92356200 \quad-1.46249000 \quad 4.40511100$

$\begin{array}{llll}\mathrm{O} & -1.30528400 & 0.40455800 & 2.70571500\end{array}$

$\mathrm{H} \quad-1.13258200 \quad 0.55164500 \quad 0.95412100$

$\mathrm{H} \quad-4.20718900 \quad-0.84431100 \quad 2.18312500$

$\begin{array}{llll}\mathrm{C} & -2.95596200 & -0.35163000 & 0.50135100\end{array}$

$\begin{array}{llll}\text { C } & -4.05925100 & -0.47010600 & -0.54485500\end{array}$

$\begin{array}{llll}\text { C } & -4.52849400 & -1.74590000 & -0.89294100\end{array}$

$\begin{array}{lllll}\text { C } & -4.63124300 & 0.66052900 & -1.15210500\end{array}$

C $\quad-5.57176600 \quad-1.89009600 \quad-1.80965300$

$\mathrm{H} \quad-4.07664000 \quad-2.62529900 \quad-0.44242800$

$\begin{array}{llll}\text { C } & -5.67027500 & 0.51416800 & -2.07482900\end{array}$

$\mathrm{H} \quad-4.27713700 \quad 1.65688100 \quad-0.90762200$

C $\quad-6.14555900 \quad-0.75960900 \quad-2.40386700$

$\mathrm{H} \quad-5.93060900 \quad-2.88331800 \quad-2.06459800$

$\mathrm{H} \quad-6.10637200 \quad 1.39608800 \quad-2.53529500$

$\mathrm{H} \quad-6.95305000 \quad-0.87171900 \quad-3.12187600$

C $\quad-1.88540000 \quad 0.63873300 \quad-0.00012600$

$\mathrm{H} \quad-2.10070400 \quad 1.70191300 \quad 0.12572800$

$\mathrm{H} \quad-1.63244000 \quad 0.43711000 \quad-1.04017000$

$\mathrm{H} \quad-2.49497500 \quad-1.33896600 \quad 0.58921200$

$\begin{array}{llll}\mathrm{Au} & 0.48763700 & 0.24368200 & 0.16920300\end{array}$

$\begin{array}{llll}\mathrm{O} & 2.52403400 & -0.11751200 & -0.08821000\end{array}$

S $\quad \begin{array}{llll}\text { S } & 3.54636700 & 0.03710200 & 1.09362400\end{array}$

$\begin{array}{lllll}\mathrm{O} & 4.88462000 & 0.14442800 & 0.51715900\end{array}$

$\begin{array}{lllll}\mathrm{O} & 3.08994400 & 0.96969200 & 2.12167700\end{array}$

$\begin{array}{llll}\mathrm{C} & 3.45572500 & -1.67037600 & 1.88098200\end{array}$

F $\quad 3.74840700 \quad-2.61666200 \quad 0.98478300$

F $\quad 4.33535600 \quad-1.72981600 \quad 2.88939000$

F $\quad 2.22250600 \quad-1.88176900 \quad 2.35962400$

O $\quad-0.64138700 \quad-3.97659700 \quad-0.49560000$

S $\quad-0.30607800 \quad-2.64533800 \quad-0.99288000$

O $\quad-1.23610500 \quad-1.90691500 \quad-1.84698300$ 


$\begin{array}{lccc}\mathrm{O} & 0.17662500 & -1.79044700 & 0.23615700 \\ \mathrm{C} & 1.27636500 & -2.82932300 & -1.99946700 \\ \mathrm{~F} & 1.01555500 & -3.59558200 & -3.06493800 \\ \mathrm{~F} & 1.68803800 & -1.62484800 & -2.41170500 \\ \mathrm{~F} & 2.23172500 & -3.39935400 & -1.26408800 \\ \mathrm{O} & -0.41963900 & 2.88617700 & -1.73142300 \\ \mathrm{~S} & 0.69395600 & 3.24694700 & -0.85458000 \\ \mathrm{O} & 0.83414900 & 4.60010900 & -0.32738800 \\ \mathrm{O} & 0.84558000 & 2.24984200 & 0.35549500 \\ \mathrm{C} & 2.26475800 & 2.88855100 & -1.83664000 \\ \mathrm{~F} & 2.40897300 & 3.82817000 & -2.77744600 \\ \mathrm{~F} & 3.32872700 & 2.90011600 & -1.03029500 \\ \mathrm{~F} & 2.16263300 & 1.68809600 & -2.42054700\end{array}$

20 (optimized by SMD/B3LYP-D3/BS3)

$\mathrm{E}(\mathrm{SMD} / \mathrm{B} 3 \mathrm{LYP}-\mathrm{D} 3 / \mathrm{BS} 3)=-3675.65592317 \mathrm{au}$

$\mathrm{H}(\mathrm{SMD} / \mathrm{B} 3 \mathrm{LYP}-\mathrm{D} 3 / \mathrm{BS} 3)=-3675.268445 \mathrm{au}$

G (SMD/B3LYP-D3/ BS3) $=-3675.402758$ au

$\mathrm{E}(\mathrm{SMD} / \mathrm{M} 06 / \mathrm{BS} 2 / / \mathrm{SMD} / \mathrm{B} 3 \mathrm{LYP}-\mathrm{D} 3 / \mathrm{BS} 3)=-3675.4972945 \mathrm{au}$

$\mathrm{E}(\mathrm{SMD} / \mathrm{M} 06-\mathrm{D} 3 / \mathrm{BS} 2 / / \mathrm{SMD} / \mathrm{B} 3 \mathrm{LYP}-\mathrm{D} 3 / \mathrm{BS} 3)=-3675.51378859 \mathrm{au}$

$\begin{array}{lrrr}\mathrm{C} & 0.37438800 & 1.95362800 & -3.24460300 \\ \mathrm{H} & 0.00126400 & 2.72558200 & -3.92100600 \\ \mathrm{H} & -0.26352500 & 1.06859700 & -3.33296300 \\ \mathrm{H} & 1.38567000 & 1.65603800 & -3.54005900 \\ \mathrm{C} & 0.38449500 & 2.42266800 & -1.81988300 \\ \mathrm{O} & 0.88861200 & 1.58643900 & -0.91353200 \\ \mathrm{C} & -0.06089100 & 3.66385000 & -1.44119100 \\ \mathrm{H} & -0.39254300 & 4.32997800 & -2.23251000 \\ \mathrm{C} & -0.21323900 & 4.16822000 & -0.06012800 \\ \mathrm{C} & -1.20924600 & 5.29361700 & 0.09732300 \\ \mathrm{H} & -2.20097800 & 4.95010300 & -0.22078100 \\ \mathrm{H} & -1.24748400 & 5.61897400 & 1.13877700 \\ \mathrm{H} & -0.93370800 & 6.13600500 & -0.54882400 \\ \mathrm{O} & 0.40288700 & 3.72058100 & 0.90863800 \\ \mathrm{C} & 7.13910900 & 1.64383900 & 1.18085200 \\ \mathrm{C} & 6.36208400 & 2.35617900 & 2.11509000 \\ \mathrm{C} & 5.01259100 & 2.08263600 & 2.23756200 \\ \mathrm{C} & 4.41451000 & 1.08061900 & 1.41316400 \\ \mathrm{C} & 5.22345500 & 0.36930900 & 0.47235000 \\ \mathrm{C} & 6.57447400 & 0.65350200 & 0.36192500 \\ \mathrm{H} & 8.19914200 & 1.86616700 & 1.09423800 \\ \mathrm{H} & 6.82626500 & 3.11530800 & 2.73607600 \\ \mathrm{H} & 4.40776900 & 2.62400200 & 2.95648300 \\ \mathrm{H} & 4.76004800 & -0.38764000 & -0.15434000 \\ \mathrm{H} & 7.19233500 & 0.11851000 & -0.35167200 \\ \mathrm{C} & 3.05185800 & 0.76269800 & 1.46885400 \\ \mathrm{H} & 2.72256900 & -0.02365100 & 0.79251300 \\ \mathrm{C} & 2.00818300 & 1.37896600 & 2.27258900 \\ \mathrm{H} & 2.35411200 & 2.00956000 & 3.09196700 \\ \mathrm{H} & 1.42794700 & 2.01315300 & 1.56772900 \\ \mathrm{H} & 1.29804000 & 0.61658700 & 2.61624300\end{array}$




$\begin{array}{lccc}\mathrm{Au} & -0.25446300 & 0.00674300 & -0.32705800 \\ \mathrm{O} & -1.32729700 & -1.65402700 & 0.43354000 \\ \mathrm{~S} & -1.00953300 & -2.19161000 & 1.85151500 \\ \mathrm{O} & -1.26865300 & -3.63012500 & 1.92057400 \\ \mathrm{O} & 0.23922200 & -1.64643500 & 2.39990800 \\ \mathrm{C} & -2.37286300 & -1.38426200 & 2.86461200 \\ \mathrm{~F} & -3.57820800 & -1.78723200 & 2.44479400 \\ \mathrm{~F} & -2.22616200 & -1.72480100 & 4.15482200 \\ \mathrm{~F} & -2.29342200 & -0.05022300 & 2.75643900 \\ \mathrm{O} & -3.52249300 & 2.87275200 & -0.83788800 \\ \mathrm{~S} & -2.99980000 & 1.51874500 & -1.02293000 \\ \mathrm{O} & -2.67504100 & 1.02681700 & -2.36329000 \\ \mathrm{O} & -1.85061000 & 1.27959100 & 0.00682800 \\ \mathrm{C} & -4.35004900 & 0.37981100 & -0.37407600 \\ \mathrm{~F} & -5.44915600 & 0.54935300 & -1.12492900 \\ \mathrm{~F} & -3.96105700 & -0.89549900 & -0.45009700 \\ \mathrm{~F} & -4.64045100 & 0.68107000 & 0.89688500 \\ \mathrm{O} & 1.27592700 & -1.19949300 & -2.98218200 \\ \mathrm{~S} & 1.99009500 & -1.72403100 & -1.81910200 \\ \mathrm{O} & 3.45391800 & -1.71214600 & -1.77980700 \\ \mathrm{O} & 1.43520300 & -1.17928600 & -0.46551500 \\ \mathrm{C} & 1.48488400 & -3.53048000 & -1.66261200 \\ \mathrm{~F} & 1.90512500 & -4.20143600 & -2.74429100 \\ \mathrm{~F} & 2.03604800 & -4.07240400 & -0.56977800 \\ \mathrm{~F} & 0.15368400 & -3.62169000 & -1.57664200\end{array}$

TS $_{11-20}$ (optimized by SMD/B3LYP-D3/BS3)

$\mathrm{E}(\mathrm{SMD} / \mathrm{B} 3 \mathrm{LYP}-\mathrm{D} 3 / \mathrm{BS} 3)=-3675.6456608 \mathrm{au}$

$\mathrm{H}(\mathrm{SMD} / \mathrm{B} 3 \mathrm{LYP}-\mathrm{D} 3 / \mathrm{BS} 3)=-3675.262584 \mathrm{au}$

$\mathrm{G}(\mathrm{SMD} / \mathrm{B} 3 \mathrm{LYP}-\mathrm{D} 3 / \mathrm{BS} 3)=-3675.389549 \mathrm{au}$

$\mathrm{E}(\mathrm{SMD} / \mathrm{M} 06 / \mathrm{BS} 2 / / \mathrm{SMD} / \mathrm{B} 3 \mathrm{LYP}-\mathrm{D} 3 / \mathrm{BS} 3)=-3675.48596484 \mathrm{au}$

$\mathrm{E}(\mathrm{SMD} / \mathrm{M} 06-\mathrm{D} 3 / \mathrm{BS} 2 / / \mathrm{SMD} / \mathrm{B} 3 \mathrm{LYP}-\mathrm{D} 3 / \mathrm{BS} 3)=-3675.5023272 \mathrm{au}$

$\begin{array}{lrrr}\mathrm{C} & -1.12209200 & -1.47012100 & -2.51222300 \\ \mathrm{H} & -1.47772500 & -2.14308700 & -3.29371100 \\ \mathrm{H} & -0.03214700 & -1.40558700 & -2.57034200 \\ \mathrm{H} & -1.53090100 & -0.47108800 & -2.69158600 \\ \mathrm{C} & -1.54359200 & -1.94887700 & -1.15719800 \\ \mathrm{O} & -1.10860900 & -1.32450900 & -0.08132400 \\ \mathrm{C} & -2.42715400 & -3.00098500 & -1.00564100 \\ \mathrm{C} & -2.81386400 & -3.62650800 & 0.22160200 \\ \mathrm{O} & -2.63483400 & -3.16105500 & 1.38618500 \\ \mathrm{C} & -3.43944400 & -4.99524400 & 0.12574600 \\ \mathrm{H} & -2.64209900 & -5.73623800 & -0.01668600 \\ \mathrm{H} & -3.97871800 & -5.23261200 & 1.04498300 \\ \mathrm{H} & -4.10950300 & -5.06441800 & -0.73665800 \\ \mathrm{H} & -2.57731300 & -1.82058000 & 1.70821300 \\ \mathrm{C} & -3.27018100 & 0.08239400 & 1.43039000 \\ \mathrm{C} & -2.68576700 & -0.73678100 & 2.39503500 \\ \mathrm{H} & -2.59600200 & 0.62151900 & 0.76957200 \\ \mathrm{H} & -1.64051600 & -0.53766300 & 2.63229600 \\ \mathrm{H} & -3.29204700 & -1.10050300 & 3.22451700\end{array}$




$\begin{array}{lccc}\mathrm{C} & -4.66364700 & 0.21901100 & 1.11402800 \\ \mathrm{C} & -5.01096800 & 1.01184900 & -0.00735100 \\ \mathrm{C} & -5.68955800 & -0.40669200 & 1.86475500 \\ \mathrm{C} & -6.34544000 & 1.17370800 & -0.36884600 \\ \mathrm{H} & -4.22392000 & 1.49366800 & -0.58096500 \\ \mathrm{C} & -7.01812600 & -0.24603900 & 1.49348400 \\ \mathrm{H} & -5.43937000 & -1.01144800 & 2.73037700 \\ \mathrm{C} & -7.34729500 & 0.54374200 & 0.37908000 \\ \mathrm{H} & -6.60648200 & 1.78460300 & -1.22756700 \\ \mathrm{H} & -7.80442000 & -0.72681300 & 2.06744700 \\ \mathrm{H} & -8.38944300 & 0.66822100 & 0.09832000 \\ \mathrm{H} & -2.78556800 & -3.45947600 & -1.91950600 \\ \mathrm{Au} & 0.50340600 & -0.12043700 & -0.04900100 \\ \mathrm{O} & 2.16937500 & 1.09604400 & 0.07295100 \\ \mathrm{~S} & 2.46281000 & 1.97516100 & 1.33532700 \\ \mathrm{O} & 3.53135400 & 2.91342900 & 1.00216300 \\ \mathrm{O} & 1.23974000 & 2.42915900 & 1.99728200 \\ \mathrm{C} & 3.23276200 & 0.71660100 & 2.50841900 \\ \mathrm{~F} & 4.23409100 & 0.06629800 & 1.90856700 \\ \mathrm{~F} & 3.70737800 & 1.36030300 & 3.58389400 \\ \mathrm{~F} & 2.30537100 & -0.16627000 & 2.90364400 \\ \mathrm{O} & 2.96561300 & -3.75900700 & 0.38057000 \\ \mathrm{~S} & 2.21163900 & -2.81332900 & -0.43701400 \\ \mathrm{O} & 1.17071800 & -3.27610000 & -1.35273600 \\ \mathrm{O} & 1.72385800 & -1.66083600 & 0.51000200 \\ \mathrm{C} & 3.47632600 & -1.91346500 & -1.50841900 \\ \mathrm{~F} & 4.04145700 & -2.79462300 & -2.34223200 \\ \mathrm{~F} & 2.86576600 & -0.95914100 & -2.22730900 \\ \mathrm{~F} & 4.41824400 & -1.35596200 & -0.74528000 \\ \mathrm{O} & -0.19949100 & 1.74509000 & -2.75140400 \\ \mathrm{~S} & -0.96480300 & 2.28967200 & -1.63032000 \\ \mathrm{O} & -2.38757200 & 2.59446900 & -1.78074300 \\ \mathrm{O} & -0.76819400 & 1.47268700 & -0.30621900 \\ \mathrm{C} & -0.14847500 & 3.92367300 & -1.16647600 \\ \mathrm{~F} & -0.35509000 & 4.79475300 & -2.16314000 \\ \mathrm{~F} & -0.68376900 & 4.40533100 & -0.04145700 \\ \mathrm{~F} & 1.16501800 & 3.75146600 & -0.99975400 \\ \mathrm{~F} & & & \end{array}$

$\mathbf{T S}_{\text {20-21 }}$ (optimized by SMD/B3LYP-D3/BS3)

$\mathrm{E}(\mathrm{SMD} / \mathrm{B} 3 \mathrm{LYY}-\mathrm{D} 3 / \mathrm{BS3})=-3675.6518611 \mathrm{au}$

H (SMD/B3LYP-D3/ BS3) $=-3675.264926$ au

G (SMD/B3LYP-D3/ BS3) $=-3675.388536$ au

E (SMD/M06/BS2// SMD/B3LYP-D3/ BS3) $=-3675.49757505$ au

E (SMD/M06-D3/BS2// SMD/B3LYP-D3/ BS3) $=-3675.51430824$ au

$\begin{array}{llll}\mathrm{C} & -1.72702000 & -1.49799500 & -1.57756100 \\ \mathrm{H} & -2.62362000 & -1.74159700 & -2.14830500 \\ \mathrm{H} & -1.01497000 & -2.32034900 & -1.70160000 \\ \mathrm{H} & -1.27448300 & -0.58523300 & -1.97243700 \\ \mathrm{C} & -2.03888500 & -1.35908000 & -0.11941200 \\ \mathrm{O} & -1.16490600 & -0.87700000 & 0.71437000 \\ \mathrm{C} & -3.29031800 & -1.76229200 & 0.37422400\end{array}$




\begin{tabular}{|c|c|c|c|}
\hline $\mathrm{H}$ & -3.95794300 & -2.19963100 & -0.35764500 \\
\hline $\mathrm{C}$ & -3.59699400 & -1.98339000 & 1.79316600 \\
\hline $\mathrm{C}$ & -4.88490100 & -2.72392200 & 2.09645200 \\
\hline $\mathrm{H}$ & -5.57255500 & -2.76031800 & 1.24940000 \\
\hline $\mathrm{H}$ & -4.61924600 & -3.75384000 & 2.36930500 \\
\hline $\mathrm{H}$ & -5.37433000 & -2.26889400 & 2.96176300 \\
\hline $\mathrm{O}$ & -2.85583000 & -1.61577600 & 2.70696500 \\
\hline $\mathrm{C}$ & -8.31082300 & -0.59198100 & -0.56093600 \\
\hline $\mathrm{C}$ & -7.90755900 & -0.30905900 & 0.75207800 \\
\hline $\mathrm{C}$ & -6.58331700 & 0.01862400 & 1.01953600 \\
\hline $\mathrm{C}$ & -5.63950800 & 0.07761400 & -0.03482000 \\
\hline $\mathrm{C}$ & -6.06366100 & -0.21194300 & -1.35682300 \\
\hline $\mathrm{C}$ & -7.38770600 & -0.54240300 & -1.61597800 \\
\hline $\mathrm{H}$ & -9.34638600 & -0.85118600 & -0.76220300 \\
\hline $\mathrm{H}$ & -8.62886300 & -0.35095100 & 1.56241700 \\
\hline $\mathrm{H}$ & -6.27632200 & 0.22375200 & 2.03884900 \\
\hline $\mathrm{H}$ & -5.33975900 & -0.16955600 & -2.16647900 \\
\hline $\mathrm{H}$ & -7.70699900 & -0.75865900 & -2.63079400 \\
\hline $\mathrm{C}$ & -4.26826200 & 0.44559300 & 0.17408700 \\
\hline $\mathrm{H}$ & -3.68323000 & 0.59221000 & -0.72803000 \\
\hline $\mathrm{C}$ & -3.72880300 & 1.08513900 & 1.39210100 \\
\hline $\mathrm{H}$ & -4.26415900 & 0.83254300 & 2.30685100 \\
\hline $\mathrm{H}$ & -2.66492000 & 0.87587400 & 1.52487600 \\
\hline $\mathrm{H}$ & -3.81813700 & 2.17173300 & 1.22876900 \\
\hline $\mathrm{Au}$ & 0.62633500 & -0.06428800 & 0.21525400 \\
\hline $\mathrm{O}$ & 2.48082900 & 0.76933700 & -0.12898200 \\
\hline S & 3.27826900 & 1.56450900 & 0.96477400 \\
\hline $\mathrm{O}$ & 4.32690900 & 2.32394000 & 0.28813500 \\
\hline $\mathrm{O}$ & 2.40765900 & 2.19592500 & 1.95336400 \\
\hline $\mathrm{C}$ & 4.16198600 & 0.18700000 & 1.90332800 \\
\hline $\mathrm{F}$ & 5.13755200 & -0.34446500 & 1.16222600 \\
\hline $\mathrm{F}$ & 4.69289200 & 0.70295400 & 3.01982000 \\
\hline $\mathrm{F}$ & 3.28697800 & -0.77121400 & 2.23961200 \\
\hline $\mathrm{O}$ & 1.51044300 & -3.94829100 & -1.41669000 \\
\hline S & 1.87406000 & -2.53336300 & -1.36555200 \\
\hline $\mathrm{O}$ & 1.55445300 & -1.64073600 & -2.48022400 \\
\hline $\mathrm{O}$ & 1.40456500 & -1.94922200 & 0.01713200 \\
\hline $\mathrm{C}$ & 3.75179200 & -2.50862300 & -1.17915700 \\
\hline $\mathrm{F}$ & 4.27564600 & -3.30273400 & -2.12280000 \\
\hline $\mathrm{F}$ & 4.21590500 & -1.26985400 & -1.34130400 \\
\hline $\mathrm{F}$ & 4.10315700 & -2.96775600 & 0.02579200 \\
\hline $\mathrm{O}$ & -1.73953000 & 1.75589200 & -1.40097700 \\
\hline S & -0.95301400 & 2.60899100 & -0.50547700 \\
\hline $\mathrm{O}$ & -1.57150900 & 3.75445400 & 0.15513500 \\
\hline $\mathrm{O}$ & -0.17914800 & 1.78722500 & 0.57839600 \\
\hline $\mathrm{C}$ & 0.45182400 & 3.29345700 & -1.55965900 \\
\hline $\mathrm{F}$ & -0.05365500 & 4.17182300 & -2.43371900 \\
\hline $\mathrm{F}$ & 1.35355200 & 3.90400700 & -0.78730100 \\
\hline $\mathrm{F}$ & 1.04106800 & 2.29438000 & -2.22638400 \\
\hline
\end{tabular}

$\mathbf{T S}_{\mathbf{1 2 - 2 0}}$ (optimized by SMD/B3LYP-D3/BS3) 


\begin{tabular}{|c|c|c|c|}
\hline \\
\hline \multicolumn{4}{|c|}{$\begin{array}{l}\mathrm{E}(\mathrm{SMD} / \mathrm{B} 3 L Y P-D 3 / \mathrm{BS} 3)=-3675.63184374 \mathrm{au} \\
\mathrm{H}(\mathrm{SMD} / \mathrm{B} 3 L Y P-D 3 / \mathrm{BS} 3)=-3675.249505 \mathrm{au}\end{array}$} \\
\hline \multicolumn{4}{|c|}{$\mathrm{G}(\mathrm{SMD} / \mathrm{B} 3 \mathrm{LYP}-\mathrm{D} 3 / \mathrm{BS} 3)=-3675.378816 \mathrm{au}$} \\
\hline \multicolumn{4}{|c|}{$\mathrm{E}(\mathrm{SMD} / \mathrm{M} 06 / \mathrm{BS} 2 / / \mathrm{SMD} / \mathrm{B} 3 \mathrm{LYP}-\mathrm{D} 3 / \mathrm{BS} 3)=-3675.47878106 \mathrm{au}$} \\
\hline \multicolumn{4}{|c|}{$\mathrm{E}(\mathrm{SMD} / \mathrm{M} 06-\mathrm{D} 3 / \mathrm{BS} 2 / / \mathrm{SMD} / \mathrm{B} 3 \mathrm{LYP}-\mathrm{D} 3 / \mathrm{BS} 3)=-3675.49520118 \mathrm{au}$} \\
\hline $\mathrm{C}$ & 0.57807600 & -1.42768100 & -2.83899900 \\
\hline $\mathrm{H}$ & 0.23102700 & -0.41602700 & -3.08288300 \\
\hline $\mathrm{H}$ & -0.29905400 & -2.03604100 & -2.58708800 \\
\hline $\mathrm{H}$ & 1.06986900 & -1.85868500 & -3.71093900 \\
\hline $\mathrm{C}$ & 1.49765800 & -1.37686600 & -1.65977500 \\
\hline $\mathrm{C}$ & 2.84227400 & -1.81931400 & -1.77358500 \\
\hline $\mathrm{H}$ & 3.42978900 & -0.49258300 & -1.85655600 \\
\hline $\mathrm{O}$ & 1.11818300 & -0.84878800 & -0.54206400 \\
\hline $\mathrm{C}$ & 8.04495100 & -0.20225800 & -0.54948000 \\
\hline $\mathrm{C}$ & 6.78448300 & 0.13038500 & -1.03461100 \\
\hline $\mathrm{C}$ & 5.76901800 & 0.56132600 & -0.14893600 \\
\hline $\mathrm{C}$ & 6.06255100 & 0.65371900 & 1.23079700 \\
\hline $\mathrm{C}$ & 7.32817800 & 0.32668100 & 1.71147300 \\
\hline $\mathrm{C}$ & 8.32027500 & -0.10498000 & 0.82297200 \\
\hline $\mathrm{H}$ & 8.81817000 & -0.53542600 & -1.23558100 \\
\hline $\mathrm{H}$ & 6.58495200 & 0.05545700 & -2.09875300 \\
\hline $\mathrm{H}$ & 5.28294700 & 0.97322800 & 1.91681900 \\
\hline $\mathrm{H}$ & 7.54109600 & 0.40006400 & 2.77376900 \\
\hline $\mathrm{H}$ & 9.30712600 & -0.36447600 & 1.19594800 \\
\hline $\mathrm{C}$ & 4.43440700 & 0.89859400 & -0.58905000 \\
\hline $\mathrm{H}$ & 3.74486800 & 1.21143600 & 0.19229300 \\
\hline $\mathrm{C}$ & 3.92535000 & 0.78440100 & -1.87619300 \\
\hline $\mathrm{H}$ & 4.60119100 & 0.68280000 & -2.72442500 \\
\hline $\mathrm{H}$ & 2.98646000 & 1.29479500 & -2.08844900 \\
\hline $\mathrm{H}$ & 3.06698100 & -2.32134900 & -2.71087600 \\
\hline $\mathrm{C}$ & 3.65265600 & -2.23666300 & -0.59470700 \\
\hline $\mathrm{O}$ & 3.50895300 & -1.71604700 & 0.50601600 \\
\hline $\mathrm{C}$ & 4.70890400 & -3.28229300 & -0.86512400 \\
\hline $\mathrm{H}$ & 4.22747600 & -4.23256400 & -1.12736100 \\
\hline $\mathrm{H}$ & 5.32670600 & -2.98556800 & -1.72075700 \\
\hline $\mathrm{H}$ & 5.33543700 & -3.41711300 & 0.01834700 \\
\hline $\mathrm{Au}$ & -0.73550200 & -0.06013100 & -0.31697300 \\
\hline $\mathrm{O}$ & -2.57191100 & 0.77984400 & 0.08942200 \\
\hline S & -3.45115900 & 1.36307700 & -1.07201900 \\
\hline $\mathrm{O}$ & -4.25508600 & 2.46486300 & -0.55132400 \\
\hline $\mathrm{O}$ & -2.69925600 & 1.50137600 & -2.32070000 \\
\hline $\mathrm{C}$ & -4.65278500 & -0.05929700 & -1.35152500 \\
\hline $\mathrm{F}$ & -5.34868100 & -0.30624300 & -0.23768000 \\
\hline $\mathrm{F}$ & -5.49977400 & 0.27594500 & -2.33477100 \\
\hline $\mathrm{F}$ & -3.98134300 & -1.16062300 & -1.70817900 \\
\hline $\mathrm{O}$ & -1.94688800 & -4.16863400 & 0.65743100 \\
\hline S & -1.44016500 & -2.85267200 & 1.03764500 \\
\hline $\mathrm{O}$ & -0.15997700 & -2.70226100 & 1.72266800 \\
\hline $\mathrm{O}$ & -1.56855500 & -1.92264200 & -0.22704500 \\
\hline $\mathrm{C}$ & -2.72945700 & -2.09239700 & 2.18145900 \\
\hline $\mathrm{F}$ & -2.81005000 & -2.82811000 & 3.29665500 \\
\hline
\end{tabular}




$\begin{array}{lrrc}\text { F } & -2.36266500 & -0.84475900 & 2.50107300 \\ \text { F } & -3.92301500 & -2.06314200 & 1.58328200 \\ \text { O } & 1.64156600 & 1.77916100 & 1.24956400 \\ \text { S } & 0.81168700 & 2.62991400 & 0.39886300 \\ \text { O } & 1.37651900 & 3.79624200 & -0.27504600 \\ \text { O } & 0.00309000 & 1.81463700 & -0.67504500 \\ \text { C } & -0.57003700 & 3.27211900 & 1.50872900 \\ \text { F } & -0.04721700 & 4.13174700 & 2.39215400 \\ \text { F } & -1.49813400 & 3.89623400 & 0.77929300 \\ \text { F } & -1.13226700 & 2.25144600 & 2.16637700\end{array}$

$\mathbf{T S}_{\mathbf{2 2}}$ (optimized by SMD/B3LYP-D3/BS3)

$\mathrm{E}(\mathrm{SMD} / \mathrm{B} 3 \mathrm{LYP}-\mathrm{D} 3 / \mathrm{BS} 3)=-3675.62090783 \mathrm{au}$

$\mathrm{H}(\mathrm{SMD} / \mathrm{B} 3 \mathrm{LYP}-\mathrm{D} 3 / \mathrm{BS} 3)=-3675.233395 \mathrm{au}$

$\mathrm{G}(\mathrm{SMD} / \mathrm{B} 3 \mathrm{LYP}-\mathrm{D} 3 / \mathrm{BS} 3)=-3675.36009 \mathrm{au}$

$\mathrm{E}(\mathrm{SMD} / \mathrm{M} 06 / \mathrm{BS} 2 / / \mathrm{SMD} / \mathrm{B} 3 \mathrm{LYP}-\mathrm{D} 3 / \mathrm{BS} 3)=-3675.47025537 \mathrm{au}$

$\mathrm{E}(\mathrm{SMD} / \mathrm{M} 06-\mathrm{D} 3 / \mathrm{BS} 2 / / \mathrm{SMD} / \mathrm{B} 3 \mathrm{~L} Y \mathrm{P}-\mathrm{D} 3 / \mathrm{BS} 3)=-3675.48788558 \mathrm{au}$

$\begin{array}{llll}\mathrm{C} & -2.14880100 & 0.80231800 & -2.37237200\end{array}$

$\mathrm{H} \quad-2.89906100 \quad 0.71230500 \quad-3.16192700$

$\mathrm{H} \quad-1.25600000 \quad 0.25313100 \quad-2.69302800$

$\mathrm{H} \quad-1.87971300 \quad 1.84627600 \quad-2.20980400$

C $\quad-2.66235700 \quad 0.13161000 \quad-1.11696000$

O $\quad-3.33397200 \quad-0.88047900 \quad-1.15935000$

$\begin{array}{llll}\mathrm{C} & -2.34391900 & 0.80682300 & 0.22328300\end{array}$

C $\quad-2.13074300 \quad-0.02207900 \quad 1.49393800$

$\begin{array}{llll}\mathrm{O} & -2.49126700 & -1.18166300 & 1.57410100\end{array}$

$\begin{array}{llll}\mathrm{C} & -1.52441800 & 0.70623400 & 2.67621200\end{array}$

$\mathrm{H} \quad-0.84844700 \quad 0.02604800 \quad 3.20088000$

$\begin{array}{llll}\mathrm{H} & -0.99367200 & 1.62028300 & 2.40005900\end{array}$

$\mathrm{H} \quad-2.33590700 \quad 0.96753400 \quad 3.36679300$

$\mathrm{H} \quad-1.70500000 \quad 1.68712500 \quad 0.16266800$

$\begin{array}{lllll}\mathrm{C} & -5.22623400 & 0.27178000 & 2.02073400\end{array}$

$\begin{array}{llll}\mathrm{C} & -6.29419200 & -0.57983800 & 2.23099300\end{array}$

$\begin{array}{llll}\text { C } & -7.12058100 & -0.93982100 & 1.14879600\end{array}$

$\begin{array}{lllll}\text { C } & -6.87916100 & -0.43141100 & -0.14283500\end{array}$

$\begin{array}{lllll}\text { C } & -5.81392900 & 0.42292000 & -0.35924900\end{array}$

$\begin{array}{lllll}\mathrm{C} & -4.97028300 & 0.81126000 & 0.72358200\end{array}$

$\mathrm{H} \quad-4.58903600 \quad 0.56534400 \quad 2.84854200$

$\mathrm{H} \quad-6.49525500 \quad-0.97247400 \quad 3.22225800$

$\mathrm{H} \quad-7.94958600 \quad-1.62261800 \quad 1.31013100$

$\mathrm{H} \quad-7.52176200 \quad-0.72222200 \quad-0.96731100$

$\mathrm{H} \quad \begin{array}{rrrr}-5.62093200 & 0.79867700 & -1.35677500\end{array}$

$\begin{array}{llll}\mathrm{C} & -3.88081800 & 1.76391700 & 0.55717900\end{array}$

$\mathrm{H} \quad-3.54884000 \quad 2.17046100 \quad 1.51504000$

$\begin{array}{llll}\mathrm{C} & -3.99349400 & 2.84627200 & -0.49776400\end{array}$

$\mathrm{H} \quad-4.21848900 \quad 2.46758700 \quad-1.49653400$

$\mathrm{H} \quad-4.81685700 \quad 3.50682100 \quad-0.20114800$

$\mathrm{H} \quad-3.07902700 \quad 3.44133700 \quad-0.54312400$

$\mathrm{Au} \quad 0.37332600 \quad 0.12973200 \quad-0.13833300$

$\begin{array}{llll}\mathrm{O} & 2.53154700 & -0.24785600 & -0.03002200\end{array}$

S $\quad 3.26157100 \quad-0.10727700 \quad 1.32994500$ 


$\begin{array}{lccc}\mathrm{O} & 4.70500800 & -0.01175300 & 1.09571400 \\ \mathrm{O} & 2.60120800 & 0.82999200 & 2.24237900 \\ \mathrm{C} & 2.98509400 & -1.80527300 & 2.09227800 \\ \mathrm{~F} & 3.54248400 & -2.75751700 & 1.33608400 \\ \mathrm{~F} & 3.53792500 & -1.83941700 & 3.31424400 \\ \mathrm{~F} & 1.67029100 & -2.04727300 & 2.20372100 \\ \mathrm{O} & -1.12304800 & -3.84857100 & -1.21921100 \\ \mathrm{~S} & -0.19490700 & -2.74511100 & -1.45548800 \\ \mathrm{O} & -0.30392500 & -1.92728500 & -2.66666400 \\ \mathrm{O} & -0.08181600 & -1.87948100 & -0.16254600 \\ \mathrm{C} & 1.50764100 & -3.54414500 & -1.52547000 \\ \mathrm{~F} & 1.55280600 & -4.37169600 & -2.58049800 \\ \mathrm{~F} & 2.45529500 & -2.61220600 & -1.65920000 \\ \mathrm{~F} & 1.73096800 & -4.24776100 & -0.40945600 \\ \mathrm{O} & 1.28271000 & 2.34952100 & -2.27941700 \\ \mathrm{~S} & 1.03595000 & 3.10091500 & -1.04690900 \\ \mathrm{O} & 0.19705400 & 4.30061800 & -1.06809000 \\ \mathrm{O} & 0.59471700 & 2.17161100 & 0.12599000 \\ \mathrm{C} & 2.71768500 & 3.67199300 & -0.42211000 \\ \mathrm{~F} & 3.19563200 & 4.59999400 & -1.26257000 \\ \mathrm{~F} & 2.59862700 & 4.20546100 & 0.79896100 \\ \mathrm{~F} & 3.56801000 & 2.64336200 & -0.37614000\end{array}$

$\mathrm{TS}_{18-17-\mathrm{H} 2 \mathrm{O}}$

$\mathrm{E}(\mathrm{SMD} / \mathrm{B} 3 \mathrm{LYP} / \mathrm{BS} 1)=-3751.83061742 \mathrm{au}$

$\mathrm{H}(\mathrm{SMD} / \mathrm{B} 3 \mathrm{LYP} / \mathrm{BS} 1)=-3751.416317 \mathrm{au}$

$\mathrm{G}(\mathrm{SMD} / \mathrm{B} 3 \mathrm{LYP} / \mathrm{BS} 1)=-3751.545906 \mathrm{au}$

$\mathrm{E}(\mathrm{SMD} / \mathrm{M} 06 / \mathrm{BS} 2 / / \mathrm{SMD} / \mathrm{B} 3 \mathrm{LYP} / \mathrm{BS} 1)=-3751.92723306 \mathrm{au}$

$\begin{array}{llll}\mathrm{C} & 5.32970100 & -1.08805100 & -3.05676900\end{array}$

$\mathrm{H} \quad 6.17717600 \quad-1.03785300 \quad-2.35986400$

$\mathrm{H} \quad 5.58212700 \quad-0.56067900 \quad-3.97895700$

$\mathrm{H} \quad 5.15054700 \quad-2.15030400 \quad-3.26465000$

$\begin{array}{llll}\mathrm{C} & 4.12283300 & -0.46479900 & -2.40871900\end{array}$

$\begin{array}{lllll}\mathrm{O} & 3.64342500 & 0.58476700 & -2.81563200\end{array}$

C $\quad 3.53666600 \quad-1.22747400 \quad-1.21068500$

$\begin{array}{llll}\mathrm{C} & 2.54814300 & -2.31569400 & -1.69053200\end{array}$

C $\quad 2.58454200 \quad-3.61957600 \quad-0.94825800$

$\mathrm{H} \quad 1.74546400 \quad-4.25223300 \quad-1.24435600$

$\mathrm{H} \quad 3.52975800 \quad-4.13106900 \quad-1.17543300$

$\mathrm{H} \quad 2.56579400 \quad-3.44839400 \quad 0.13400400$

$\begin{array}{llll}\mathrm{O} & 1.78414800 & -2.13599700 & -2.63373800\end{array}$

$\mathrm{H} \quad 1.21175600 \quad-0.55001500 \quad-3.04973300$

$\mathrm{H} \quad 4.36318700 \quad-1.77936800 \quad-0.75086000$

$\begin{array}{llll}\mathrm{C} & 2.94451800 & -0.37798200 & -0.03004200\end{array}$

$\begin{array}{lllll}\mathrm{C} & 4.05539000 & 0.33077300 & 0.75212200\end{array}$

$\begin{array}{llll}\mathrm{C} & 4.33923800 & -0.08840000 & 2.06207300\end{array}$

$\begin{array}{lllll}\mathrm{C} & 4.79816700 & 1.39375300 & 0.20675200\end{array}$

$\begin{array}{llll}\mathrm{C} & 5.35533600 & 0.52010700 & 2.79967500\end{array}$

$\begin{array}{llll}\mathrm{H} & 3.76346400 & -0.89579100 & 2.50562600\end{array}$

$\begin{array}{llll}\mathrm{C} & 5.81164200 & 2.00316400 & 0.94823700\end{array}$

$\mathrm{H} \quad 4.59031000 \quad 1.75165300 \quad-0.79597400$ 


$\begin{array}{lrrr}\mathrm{C} & 6.09556200 & 1.56815800 & 2.24488500 \\ \mathrm{H} & 5.56670300 & 0.17571000 & 3.80835300 \\ \mathrm{H} & 6.37690800 & 2.82130300 & 0.51042000 \\ \mathrm{H} & 6.88422300 & 2.04460000 & 2.82063800 \\ \mathrm{C} & 1.84174700 & 0.67760000 & -0.29605000 \\ \mathrm{H} & 2.17072800 & 1.59177800 & -0.81279300 \\ \mathrm{H} & 1.53337300 & 1.07655700 & 0.67740200 \\ \mathrm{H} & 2.52104400 & -1.11633000 & 0.65258700 \\ \mathrm{Au} & -0.45131300 & 0.22015900 & -0.19941900 \\ \mathrm{O} & -2.49268000 & -0.12304800 & 0.09039400 \\ \mathrm{~S} & -3.47690400 & -0.39155000 & -1.10150800 \\ \mathrm{O} & -4.83402000 & -0.33770400 & -0.56309500 \\ \mathrm{O} & -3.12933600 & 0.33963600 & -2.31685800 \\ \mathrm{C} & -3.13672500 & -2.18612200 & -1.48763700 \\ \mathrm{~F} & -3.33004500 & -2.94183700 & -0.40886100 \\ \mathrm{~F} & -3.96002600 & -2.57726000 & -2.46036600 \\ \mathrm{~F} & -1.86948000 & -2.32542300 & -1.90314000 \\ \mathrm{O} & 0.50058800 & -3.34858500 & 2.21855900 \\ \mathrm{~S} & 0.10640000 & -1.94736400 & 2.09691300 \\ \mathrm{O} & 0.91687800 & -0.89297000 & 2.70924400 \\ \mathrm{O} & -0.17028200 & -1.67746300 & 0.57139700 \\ \mathrm{C} & -1.58471000 & -1.80704400 & 2.88570500 \\ \mathrm{~F} & -1.48244500 & -2.23467500 & 4.14419400 \\ \mathrm{~F} & -1.97995700 & -0.53258800 & 2.88292500 \\ \mathrm{~F} & -2.46476200 & -2.55486800 & 2.22772700 \\ \mathrm{O} & 0.32900800 & 3.43322700 & 0.68613700 \\ \mathrm{~S} & -0.75138500 & 3.39992200 & -0.29936900 \\ \mathrm{O} & -0.88452400 & 4.45962800 & -1.29322400 \\ \mathrm{O} & -0.82274600 & 2.03202200 & -1.07887500 \\ \mathrm{C} & -2.34001000 & 3.38178200 & 0.68352300 \\ \mathrm{~F} & -2.49359100 & 4.57209000 & 1.26090100 \\ \mathrm{~F} & -3.37399400 & 3.13374300 & -0.11669300 \\ \mathrm{~F} & -2.26709600 & 2.43667600 & 1.62674500 \\ \mathrm{O} & 0.92862400 & 0.38691500 & -2.88318100 \\ \mathrm{H} & 1.21112300 & 0.42804700 & -1.39060700 \\ \mathrm{H} & 1.68792300 & 0.91633900 & -3.20390600 \\ & & & \\ & & & \end{array}$

\section{2'}

$\mathrm{E}(\mathrm{SMD} / \mathrm{B} 3 \mathrm{LYP} / \mathrm{BS} 1)=-1763.90963201 \mathrm{au}$

$\mathrm{H}(\mathrm{SMD} / \mathrm{B} 3 \mathrm{LYP} / \mathrm{BS} 1)=-1763.598493 \mathrm{au}$

$\mathrm{G}(\mathrm{SMD} / \mathrm{B} 3 \mathrm{LYP} / \mathrm{BS} 1)=-1763.688815 \mathrm{au}$

$\mathrm{E}(\mathrm{SMD} / \mathrm{M} 06 / \mathrm{BS} 2 / / \mathrm{SMD} / \mathrm{B} 3 \mathrm{LYP} / \mathrm{BS} 1)=-1763.81143435 \mathrm{au}$

$\begin{array}{llll}\mathrm{C} & 2.10123900 & 3.55424000 & -1.09480200 \\ \mathrm{H} & 2.99593100 & 3.79626500 & -0.51404200 \\ \mathrm{H} & 1.52406300 & 4.47264700 & -1.25738600 \\ \mathrm{H} & 2.41094700 & 3.19231600 & -2.08393600 \\ \mathrm{C} & 1.21491900 & 2.51430000 & -0.42274400 \\ \mathrm{O} & 0.13700100 & 2.24327600 & -1.04159700 \\ \mathrm{C} & 1.67593800 & 1.95010000 & 0.78870900 \\ \mathrm{H} & 2.63471800 & 2.31356300 & 1.14338300 \\ \mathrm{C} & 1.03315100 & 0.98958600 & 1.60725600\end{array}$




$\begin{array}{lrrr}\mathrm{C} & 1.71771900 & 0.59942000 & 2.90951700 \\ \mathrm{H} & 1.17661400 & 1.04895300 & 3.75333600 \\ \mathrm{H} & 1.67095100 & -0.48743600 & 3.03896600 \\ \mathrm{H} & 2.76044000 & 0.92672700 & 2.95913900 \\ \mathrm{O} & -0.08454100 & 0.42557100 & 1.38899800 \\ \mathrm{C} & 7.65135700 & -1.01582300 & 0.89451000 \\ \mathrm{C} & 6.88111000 & -2.19051300 & 0.81766300 \\ \mathrm{C} & 5.72160400 & -2.20352500 & 0.07021500 \\ \mathrm{C} & 5.30938900 & -1.02263200 & -0.62487500 \\ \mathrm{C} & 6.11352500 & 0.15971700 & -0.52970700 \\ \mathrm{C} & 7.27081900 & 0.15723400 & 0.22435800 \\ \mathrm{H} & 8.56222800 & -1.01835200 & 1.48660000 \\ \mathrm{H} & 7.20214600 & -3.08170200 & 1.34644500 \\ \mathrm{H} & 5.12461600 & -3.10595600 & 0.00907200 \\ \mathrm{H} & 5.79626600 & 1.05287600 & -1.05999400 \\ \mathrm{H} & 7.88301400 & 1.04972400 & 0.29818500 \\ \mathrm{C} & 4.14770200 & -0.96004200 & -1.39512400 \\ \mathrm{H} & 3.93579900 & 0.00071500 & -1.86288500 \\ \mathrm{C} & 3.16561200 & -2.01096500 & -1.67626600 \\ \mathrm{H} & 3.37612300 & -2.98638500 & -1.23731500 \\ \mathrm{H} & 2.17907000 & -1.65260800 & -1.33953600 \\ \mathrm{H} & 3.06280200 & -2.10056700 & -2.76912600 \\ \mathrm{O} & -3.33385300 & -0.03010800 & -1.28469500 \\ \mathrm{~S} & -4.01753000 & -1.22738100 & -0.66484100 \\ \mathrm{O} & -5.17579700 & -1.68465900 & -1.44902700 \\ \mathrm{O} & -3.08935900 & -2.25806500 & -0.16399100 \\ \mathrm{C} & -4.76745800 & -0.49995900 & 0.87438400 \\ \mathrm{~F} & -5.58560700 & 0.51642000 & 0.56667000 \\ \mathrm{~F} & -5.47114500 & -1.43133300 & 1.53249000 \\ \mathrm{~F} & -3.81223200 & -0.03202600 & 1.69949900 \\ \mathrm{Ag} & -1.43067600 & 0.74563400 & -0.41952000 \\ \mathrm{H} & & & \end{array}$

\section{$17^{\prime \prime}$}

$\mathrm{E}(\mathrm{SMD} / \mathrm{B} 3 \mathrm{LYP} / \mathrm{BS} 1)=-1406.90224659 \mathrm{au}$

$\mathrm{H}(\mathrm{SMD} / \mathrm{B} 3 \mathrm{LYP} / \mathrm{BS} 1)=-1406.719782 \mathrm{au}$

$\mathrm{G}(\mathrm{SMD} / \mathrm{B} 3 \mathrm{LYP} / \mathrm{BS} 1)=-1406.78806 \mathrm{au}$

$\mathrm{E}(\mathrm{SMD} / \mathrm{M} 06 / \mathrm{BS} 2 / / \mathrm{SMD} / \mathrm{B} 3 \mathrm{LYP} / \mathrm{BS} 1)=-1406.85318388 \mathrm{au}$

$\begin{array}{lrrr}\mathrm{Au} & 0.14814400 & -0.94859900 & -0.00071600 \\ \mathrm{O} & -1.56753100 & -0.34955400 & -1.09522400 \\ \mathrm{~S} & -2.92979100 & -0.11422200 & -0.41713600 \\ \mathrm{O} & -3.99200100 & -0.00354400 & -1.41953600 \\ \mathrm{O} & -3.15158300 & -0.96236900 & 0.76125800 \\ \mathrm{C} & -2.70382800 & 1.60703700 & 0.25807400 \\ \mathrm{~F} & -2.39826200 & 2.45796700 & -0.72633500 \\ \mathrm{~F} & -3.83028600 & 2.01138800 & 0.85209400 \\ \mathrm{~F} & -1.71007200 & 1.62532600 & 1.16298600 \\ \mathrm{C} & 1.80197000 & -2.04236200 & 0.87024900 \\ \mathrm{H} & 1.34490500 & -2.65478700 & 1.64419400 \\ \mathrm{H} & 2.30487200 & -2.58002200 & 0.06993400 \\ \mathrm{C} & 2.10147200 & -0.70668200 & 1.15386000 \\ \mathrm{H} & 1.75013200 & -0.31432800 & 2.10729800\end{array}$




$\begin{array}{lrrr}\mathrm{C} & 3.04379000 & 0.17560800 & 0.44227700 \\ \mathrm{C} & 3.72510100 & -0.20977200 & -0.72849800 \\ \mathrm{C} & 3.28663900 & 1.45154700 & 0.98218000 \\ \mathrm{C} & 4.62251700 & 0.66271400 & -1.33705300 \\ \mathrm{H} & 3.55470400 & -1.19046000 & -1.16283600 \\ \mathrm{C} & 4.18689300 & 2.32348600 & 0.37038200 \\ \mathrm{H} & 2.76687600 & 1.75325800 & 1.88833500 \\ \mathrm{C} & 4.85564800 & 1.93120300 & -0.79106900 \\ \mathrm{H} & 5.14438500 & 0.35512100 & -2.23886200 \\ \mathrm{H} & 4.36529100 & 3.30506200 & 0.80014000 \\ \mathrm{H} & 5.55741000 & 2.60798900 & -1.27055400\end{array}$

$18 "$

$\mathrm{E}(\mathrm{SMD} / \mathrm{B} 3 \mathrm{LYP} / \mathrm{BS} 1)=-1752.67401757 \mathrm{au}$

$\mathrm{H}(\mathrm{SMD} / \mathrm{B} 3 \mathrm{LYP} / \mathrm{BS} 1)=-1752.357308 \mathrm{au}$

$\mathrm{G}(\mathrm{SMD} / \mathrm{B} 3 \mathrm{LYP} / \mathrm{BS} 1)=-1752.44406 \mathrm{au}$

$\mathrm{E}(\mathrm{SMD} / \mathrm{M} 06 / \mathrm{BS} 2 / / \mathrm{SMD} / \mathrm{B} 3 \mathrm{LYP} / \mathrm{BS} 1)=-1752.55492016 \mathrm{au}$

$\begin{array}{llll}\mathrm{C} & 2.91330900 & 0.18385900 & 3.00374600\end{array}$

$\mathrm{H} \quad 3.67846500 \quad-0.31607300 \quad 2.40131600$

$\mathrm{H} \quad 2.60425700 \quad-0.44802600 \quad 3.83893700$

$\mathrm{H} \quad 3.36866900 \quad 1.10537400 \quad 3.39289900$

$\begin{array}{llll}\mathrm{C} & 1.72965400 & 0.55711900 & 2.17058200\end{array}$

$\begin{array}{llll}\mathrm{O} & 0.57556300 & 0.35221600 & 2.55331700\end{array}$

$\begin{array}{llll}\mathrm{C} & 1.97457800 & 1.24101000 & 0.82823200\end{array}$

$\begin{array}{llll}\text { C } & 0.85488400 & 2.13825200 & 0.42682000\end{array}$

$\begin{array}{llll}\text { C } & 1.08175400 & 3.31643600 & -0.43627000\end{array}$

$\mathrm{H} \quad \begin{array}{llll}\mathrm{H} & 0.16239800 & 3.61829800 & -0.94281400\end{array}$

$\mathrm{H} \quad \begin{array}{llll}1.40205000 & 4.13653500 & 0.22602900\end{array}$

$\mathrm{H} \quad \begin{array}{llll}1.89254600 & 3.14017400 & -1.14749100\end{array}$

$\begin{array}{lllll}\mathrm{O} & -0.33395900 & 1.93516600 & 0.82858800\end{array}$

$\begin{array}{llll}\mathrm{H} & 2.87124100 & 1.86218200 & 0.88892400\end{array}$

$\begin{array}{lllll}\mathrm{C} & 2.26008400 & 0.18203900 & -0.39117500\end{array}$

$\begin{array}{lllll}\mathrm{C} & 3.77490200 & 0.05404100 & -0.58244400\end{array}$

$\begin{array}{lllll}\mathrm{C} & 4.45371400 & 1.04638200 & -1.30894600\end{array}$

$\begin{array}{lllll}\mathrm{C} & 4.51520300 & -1.02849900 & -0.08582800\end{array}$

$\begin{array}{lllll}\mathrm{C} & 5.83051500 & 0.97068500 & -1.52123300\end{array}$

$\mathrm{H} \quad 3.89334700 \quad 1.88389700 \quad-1.71982000$

C $\quad 5.89549000 \quad-1.10689500 \quad-0.29657200$

$\mathrm{H} \quad 4.01772600 \quad-1.82609600 \quad 0.45658600$

C $\quad 6.55875700 \quad-0.10819000 \quad-1.01140600$

$\mathrm{H} \quad 6.33210700 \quad 1.74817300 \quad-2.09162300$

$\mathrm{H} \quad 6.44893000 \quad-1.95615600 \quad 0.09559300$

$\mathrm{H} \quad 7.63062800 \quad-0.17300500 \quad-1.17803600$

$\begin{array}{llll}\mathrm{C} & 1.51686600 & -1.15571700 & -0.25469300\end{array}$

$\mathrm{H} \quad 1.84212900 \quad-1.68978500 \quad 0.64708500$

$\mathrm{H} \quad 1.83318000 \quad-1.76849500 \quad-1.10933200$

$\mathrm{H} \quad 1.88770000 \quad 0.69098300 \quad-1.28589500$

$\mathrm{Au} \quad-0.53410700 \quad-1.15857800 \quad-0.26569600$

$\begin{array}{llll}\mathrm{O} & -2.72307800 & -1.30352500 & -0.25339100\end{array}$

S $\quad-3.56743800 \quad-0.26845800 \quad 0.47599700$

$\begin{array}{llll}\text { O } & -4.91050100 & -0.77268000 & 0.78873700\end{array}$ 


$\begin{array}{lllr}\mathrm{O} & -2.84436700 & 0.45809900 & 1.53492900 \\ \mathrm{C} & -3.83933700 & 0.99738100 & -0.86054800 \\ \mathrm{~F} & -4.48786800 & 0.45207100 & -1.89754500 \\ \mathrm{~F} & -4.56612400 & 2.01860700 & -0.38752500 \\ \mathrm{~F} & -2.66653500 & 1.48206700 & -1.30393400 \\ \mathrm{H} & -0.33863400 & 1.15869500 & 1.50369800\end{array}$

\section{$19^{\prime \prime}$}

$\mathrm{E}(\mathrm{SMD} / \mathrm{B} 3 \mathrm{LYP} / \mathrm{BS} 1)=-1752.67597171 \mathrm{au}$

$\mathrm{H}(\mathrm{SMD} / \mathrm{B} 3 \mathrm{LYP} / \mathrm{BS} 1)=-1752.359382 \mathrm{au}$

$\mathrm{G}(\mathrm{SMD} / \mathrm{B} 3 \mathrm{LYP} / \mathrm{BS} 1)=-1752.447056 \mathrm{au}$

$\mathrm{E}(\mathrm{SMD} / \mathrm{M} 06 / \mathrm{BS} 2 / / \mathrm{SMD} / \mathrm{B} 3 \mathrm{LYP} / \mathrm{BS} 1)=-1752.55581024 \mathrm{au}$

$\begin{array}{llll}\mathrm{C} & 3.91668300 & -2.31856200 & 2.46391000\end{array}$

$\mathrm{H} \quad 2.84262900 \quad-2.30981400 \quad 2.67997700$

$\mathrm{H} \quad 4.48574400 \quad-1.98103000 \quad 3.33227100$

$\mathrm{H} \quad 4.18679000 \quad-3.35767200 \quad 2.23001100$

C $\quad 4.22965200 \quad-1.47098100 \quad 1.27442400$

$\begin{array}{llll}\mathrm{O} & 5.16824500 & -0.66531800 & 1.27333100\end{array}$

$\begin{array}{llll}\text { C } & 3.35176000 & -1.60655800 & 0.04313300\end{array}$

C $\quad 4.09983500 \quad-1.35616000 \quad-1.20490500$

$\begin{array}{llll}\mathrm{C} & 3.78074900 & -2.02905600 & -2.47867400\end{array}$

$\mathrm{H} \quad 4.16268300 \quad-3.05934800 \quad-2.40592900$

$\mathrm{H} \quad 2.69860300 \quad-2.10727200 \quad-2.61906600$

$\mathrm{H} \quad 4.25946800 \quad-1.52879100 \quad-3.32256100$

O $\quad 5.07429500 \quad-0.52877800 \quad-1.20111400$

$\mathrm{H} \quad \begin{array}{llll}\mathrm{H} & 5.27424400 & -0.28378600 & -0.21117900\end{array}$

$\mathrm{H} \quad 2.87242700 \quad-2.58535400 \quad 0.00478400$

$\begin{array}{llll}\mathrm{C} & 2.05535700 & -0.54574300 & 0.21553200\end{array}$

$\begin{array}{llll}\mathrm{C} & 2.51117000 & 0.89763000 & 0.30257300\end{array}$

$\begin{array}{llll}\mathrm{C} & 2.67323100 & 1.49812400 & 1.56161700\end{array}$

$\begin{array}{llll}\mathrm{C} & 2.75692500 & 1.67408600 & -0.84225500\end{array}$

$\begin{array}{llll}\mathrm{C} & 3.05985600 & 2.83474700 & 1.67702400\end{array}$

$\begin{array}{llll}\mathrm{H} & 2.48239300 & 0.91736300 & 2.46093300\end{array}$

$\begin{array}{llll}\mathrm{C} & 3.14866400 & 3.00919700 & -0.72966600\end{array}$

$\mathrm{H} \quad 2.63058800 \quad 1.24223600 \quad-1.83125800$

$\begin{array}{llll}\mathrm{C} & 3.30079900 & 3.59447000 & 0.53020100\end{array}$

$\begin{array}{llll}\mathrm{H} & 3.17043500 & 3.28030300 & 2.66196700\end{array}$

$\mathrm{H} \quad 3.33056800 \quad 3.59297900 \quad-1.62803200$

$\mathrm{H} \quad 3.60198500 \quad 4.63497600 \quad 0.61644300$

C $\quad 0.97403200 \quad-0.84464700 \quad-0.82071500$

$\mathrm{H} \quad 0.79355800 \quad-1.92622700 \quad-0.86252900$

$\mathrm{H} \quad \begin{array}{llll}1.27613300 & -0.52227900 & -1.82318600\end{array}$

$\mathrm{H} \quad 1.69725500 \quad-0.85620000 \quad 1.19993700$

$\mathrm{Au} \quad-0.81750500 \quad 0.06406700 \quad-0.38250100$

$\begin{array}{lllll}\mathrm{O} & -2.70187000 & 1.06261500 & 0.10025900\end{array}$

S $\quad-4.05145400 \quad 0.53490100 \quad-0.36619900$

$\begin{array}{lllll}\mathrm{O} & -5.12506800 & 1.50807900 & -0.12583500\end{array}$

$\begin{array}{llll}\mathrm{O} & -4.01513100 & -0.14266600 & -1.67206400\end{array}$

$\begin{array}{llll}\text { C } & -4.37753300 & -0.82836900 & 0.85880000\end{array}$

F $\quad \begin{array}{llll}\text { C } & -4.33734200 & -0.35521300 & 2.11180300\end{array}$

F $\quad-5.58675700 \quad-1.36184400 \quad 0.64000200$ 
$\mathrm{TS}_{18 "-17 "}$

$\mathrm{E}(\mathrm{SMD} / \mathrm{B} 3 \mathrm{LYP} / \mathrm{BS} 1)=-1752.65314285 \mathrm{au}$

$\mathrm{H}(\mathrm{SMD} / \mathrm{B} 3 \mathrm{LYP} / \mathrm{BS} 1)=-1752.342731 \mathrm{au}$

$\mathrm{G}(\mathrm{SMD} / \mathrm{B} 3 \mathrm{LYP} / \mathrm{BS} 1)=-1752.431945 \mathrm{au}$

$\mathrm{E}(\mathrm{SMD} / \mathrm{M} 06 / \mathrm{BS} 2 / / \mathrm{SMD} / \mathrm{B} 3 \mathrm{LYP} / \mathrm{BS} 1)=-1752.53495805 \mathrm{au}$

$\begin{array}{llll}\mathrm{C} & -5.59465700 & 0.91707300 & -0.38324200\end{array}$

$\mathrm{H} \quad \begin{array}{rrrr}\mathrm{H} & -5.70822000 & -0.07639200 & 0.06448400\end{array}$

$\mathrm{H} \quad-6.15285100 \quad 0.97692300 \quad-1.32017100$

$\mathrm{H} \quad-6.00177900 \quad 1.64053900 \quad 0.33578400$

C $\quad-4.14080300 \quad 1.22213000 \quad-0.62459400$

$\begin{array}{llll}\mathrm{O} & -3.70360700 & 1.58605300 & -1.70001200\end{array}$

$\begin{array}{llll}\text { C } & -3.18212500 & 1.06992000 & 0.60060900\end{array}$

$\begin{array}{llll}\text { C } & -2.46542100 & 2.38449800 & 0.75841600\end{array}$

$\begin{array}{llll}\text { C } & -3.09718600 & 3.48425400 & 1.54188200\end{array}$

$\mathrm{H} \quad-2.56420000 \quad 4.42560700 \quad 1.39540800$

$\mathrm{H} \quad \begin{array}{llll}\mathrm{H} & -4.15385500 & 3.59132100 & 1.27101300\end{array}$

$\mathrm{H} \quad-3.06757400 \quad 3.20876800 \quad 2.60554100$

$\begin{array}{llll}\mathrm{O} & -1.35504400 & 2.56501700 & 0.22350600\end{array}$

$\mathrm{H} \quad \begin{array}{llll}0.80337800 & 1.44843300 & -0.24824400\end{array}$

$\begin{array}{llll}\mathrm{H} & -3.79679400 & 0.90816900 & 1.49070500\end{array}$

$\begin{array}{llll}\text { C } & -2.19425900 & -0.14390600 & 0.44887600\end{array}$

$\begin{array}{llll}\text { C } & -2.97859700 & -1.45251700 & 0.35803900\end{array}$

$\begin{array}{llll}\text { C } & -3.29568000 & -2.14339100 & 1.53748300\end{array}$

C $\quad-3.41283400 \quad-1.98437400 \quad-0.86630100$

$\begin{array}{llll}\text { C } & -4.02682200 & -3.33259000 & 1.49899900\end{array}$

$\begin{array}{llll}\mathrm{H} & -2.95873400 & -1.74899700 & 2.49383800\end{array}$

C $\quad-4.14473600 \quad-3.17413400 \quad-0.90684300$

$\mathrm{H} \quad \begin{array}{llll}\mathrm{H} & -3.17843300 & -1.47489700 & -1.79665900\end{array}$

$\begin{array}{llll}\mathrm{C} & -4.45517000 & -3.85170500 & 0.27457800\end{array}$

$\mathrm{H} \quad \begin{array}{llll}\mathrm{H} & -4.25498100 & -3.85547700 & 2.42417700\end{array}$

$\mathrm{H} \quad-\quad-4.46947400 \quad-3.57137700 \quad-1.86500400$

$\mathrm{H} \quad \begin{array}{llll}-5.02112100 & -4.77880100 & 0.24112300\end{array}$

C $\quad \begin{array}{llll}-1.16817300 & 0.02245700 & -0.70424700\end{array}$

$\mathrm{H} \quad-1.62406500 \quad 0.40061100 \quad-1.62164100$

$\mathrm{H} \quad-0.77990700 \quad-0.97569600 \quad-0.94518200$

$\mathrm{H} \quad \begin{array}{llll}-1.64332600 & -0.17577800 & 1.39485600\end{array}$

$\mathrm{Au} \quad 0.88802500 \quad 0.62809200 \quad-0.41701300$

$\begin{array}{lllll}\mathrm{O} & 2.99021000 & 0.95944600 & -0.32626200\end{array}$

S $\quad \begin{array}{llll}2.87696500 & 0.21396700 & 0.67966300\end{array}$

$\begin{array}{lllll}\mathrm{O} & 5.20623500 & 0.82649200 & 0.75961000\end{array}$

$\begin{array}{llll}\text { O } & 3.18620700 & -0.13560100 & 1.92915100\end{array}$

C $\quad 4.14095500 \quad-1.41013700 \quad-0.19215900$

F $\quad \begin{array}{llll}\text { C } & 4.69646100 & -1.21237000 & -1.39254200\end{array}$

F $\quad 4.94529900 \quad-2.19166100 \quad 0.53608600$

F $\quad 2.96871500 \quad-2.04512900 \quad-0.36437700$

$\mathrm{TS}_{17 "-18 "}$

$\mathrm{E}(\mathrm{SMD} / \mathrm{B} 3 \mathrm{LYP} / \mathrm{BS} 1)=-1752.66153214 \mathrm{au}$

$\mathrm{H}(\mathrm{SMD} / \mathrm{B} 3 \mathrm{LYP} / \mathrm{BS} 1)=-1752.346687 \mathrm{au}$ 


$\begin{array}{lccc}\mathrm{G}(\mathrm{SMD} / \mathrm{B} 3 \mathrm{LYP} / \mathrm{BS} 1)= & -1752.433662 \mathrm{au} \\ \mathrm{E}(\mathrm{SMD} / \mathrm{M} 06 / \mathrm{BS} 2 / / \mathrm{SMD} / \mathrm{B} 3 \mathrm{LYP} / \mathrm{BS} 1) & -1752.53721017 \mathrm{au} \\ \mathrm{C} & -2.70258700 & 0.10706100 & -3.02167700 \\ \mathrm{H} & -3.51006000 & -0.37047000 & -2.45697200 \\ \mathrm{H} & -2.28757500 & -0.58940400 & -3.75368900 \\ \mathrm{H} & -3.14500800 & 0.96308500 & -3.54882000 \\ \mathrm{C} & -1.62107500 & 0.60054800 & -2.10331100 \\ \mathrm{O} & -0.42333600 & 0.37026700 & -2.33655500 \\ \mathrm{C} & -2.01181800 & 1.39750100 & -0.92523800 \\ \mathrm{C} & -1.04305500 & 2.24791500 & -0.33838400 \\ \mathrm{C} & -1.39082100 & 3.38208300 & 0.56135900 \\ \mathrm{H} & -0.83200800 & 3.29939000 & 1.50109500 \\ \mathrm{H} & -1.08054200 & 4.32026100 & 0.08308000 \\ \mathrm{H} & -2.46182700 & 3.42330600 & 0.76420200 \\ \mathrm{O} & 0.22967100 & 2.06861200 & -0.52927100 \\ \mathrm{H} & -3.02375000 & 1.78058400 & -0.91221500 \\ \mathrm{C} & -2.33654100 & 0.02572900 & 0.73291300 \\ \mathrm{C} & -3.81077100 & -0.06079800 & 0.65559900 \\ \mathrm{C} & -4.57688300 & 0.89777700 & 1.35003900 \\ \mathrm{C} & -4.48332700 & -1.09224100 & -0.02917100 \\ \mathrm{C} & -5.96776900 & 0.82953100 & 1.36131000 \\ \mathrm{H} & -4.06967900 & 1.69201600 & 1.89212200 \\ \mathrm{C} & -5.87552500 & -1.15487400 & -0.02124200 \\ \mathrm{H} & -3.92101600 & -1.85265200 & -0.56077500 \\ \mathrm{C} & -6.62169600 & -0.19591300 & 0.67124700 \\ \mathrm{H} & -6.54104700 & 1.57168400 & 1.90968400 \\ \mathrm{H} & -6.37973000 & -1.95754600 & -0.55185900 \\ \mathrm{H} & -7.70672500 & -0.25121200 & 0.67765100 \\ \mathrm{C} & -1.48290900 & -1.17324400 & 0.66122300 \\ \mathrm{H} & -1.92883700 & -1.96804700 & 0.05847200 \\ \mathrm{H} & -1.43947600 & -1.52062700 & 1.71113400 \\ \mathrm{H} & -2.00884100 & 0.75122100 & 1.47394400 \\ \mathrm{Au} & 0.53625900 & -1.09406000 & 0.25920700 \\ \mathrm{O} & 2.67207100 & -1.26792300 & -0.12225500 \\ \mathrm{~S} & 3.51984400 & -0.12091300 & -0.65820000 \\ \mathrm{O} & 4.79567300 & -0.59795000 & -1.20518700 \\ \mathrm{O} & 2.76210400 & 0.86993700 & -1.43977400 \\ \mathrm{C} & 3.98255400 & 0.76600300 & 0.91111000 \\ \mathrm{~F} & 4.66747800 & -0.04822500 & 1.72428600 \\ \mathrm{~F} & 4.74224000 & 1.83216300 & 0.62904900 \\ \mathrm{~F} & 2.88495700 & 1.18731500 & 1.56233400 \\ \mathrm{H} & 0.36598400 & 1.32644100 & -1.20456500 \\ & & & \end{array}$

$\mathrm{TS}_{17 "-19 "}$

$\mathrm{E}(\mathrm{SMD} / \mathrm{B} 3 \mathrm{LYP} / \mathrm{BS} 1)=-1752.6734184 \mathrm{au}$

$\mathrm{H}(\mathrm{SMD} / \mathrm{B} 3 \mathrm{LYP} / \mathrm{BS} 1)=-1752.358008 \mathrm{au}$

$\mathrm{G}(\mathrm{SMD} / \mathrm{B} 3 \mathrm{LYP} / \mathrm{BS} 1)=-1752.446552 \mathrm{au}$

$\mathrm{E}(\mathrm{SMD} / \mathrm{M} 06 / \mathrm{BS} 2 / / \mathrm{SMD} / \mathrm{B} 3 \mathrm{LYP} / \mathrm{BS} 1)=-1752.55168651 \mathrm{au}$

C $\quad 4.75812600 \quad-1.34592700 \quad 2.46841000$

$\mathrm{H} \quad 3.74990400 \quad-1.47241600 \quad 2.87976200$

$\mathrm{H} \quad 5.35096000 \quad-0.69398600 \quad 3.11318800$ 


$\begin{array}{lrrr}\mathrm{H} & 5.21794300 & -2.34270500 & 2.43694500 \\ \mathrm{C} & 4.70202800 & -0.79041800 & 1.07717400 \\ \mathrm{O} & 5.41112400 & 0.17139000 & 0.73659600 \\ \mathrm{C} & 3.76922500 & -1.41347000 & 0.10282000 \\ \mathrm{C} & 4.11639100 & -1.26668000 & -1.27843900 \\ \mathrm{C} & 3.65889100 & -2.20166600 & -2.33377300 \\ \mathrm{H} & 4.39289800 & -3.01852600 & -2.40166000 \\ \mathrm{H} & 2.69616900 & -2.64929000 & -2.07586000 \\ \mathrm{H} & 3.61306800 & -1.70226000 & -3.30490900 \\ \mathrm{O} & 4.84803200 & -0.27178400 & -1.66552900 \\ \mathrm{H} & 5.18710300 & 0.19934300 & -0.81754700 \\ \mathrm{H} & 3.40550500 & -2.40005600 & 0.37370200 \\ \mathrm{C} & 2.06292500 & -0.45190900 & 0.33984500 \\ \mathrm{C} & 2.29464900 & 0.97290000 & -0.02609000 \\ \mathrm{C} & 2.67581900 & 1.88374900 & 0.97584000 \\ \mathrm{C} & 2.11904800 & 1.45014300 & -1.33828700 \\ \mathrm{C} & 2.85482900 & 3.23565900 & 0.68265900 \\ \mathrm{H} & 2.81388800 & 1.52955600 & 1.99432600 \\ \mathrm{C} & 2.30338600 & 2.79995500 & -1.63192700 \\ \mathrm{H} & 1.82718400 & 0.76832300 & -2.13144000 \\ \mathrm{C} & 2.66938300 & 3.69676400 & -0.62276300 \\ \mathrm{H} & 3.13729100 & 3.92622100 & 1.47226700 \\ \mathrm{H} & 2.15870200 & 3.15366200 & -2.64894800 \\ \mathrm{H} & 2.80879800 & 4.74915900 & -0.85442600 \\ \mathrm{C} & 0.99976500 & -1.24732300 & -0.32354700 \\ \mathrm{H} & 1.05311900 & -2.30330800 & -0.04018800 \\ \mathrm{H} & 0.99929200 & -1.16378400 & -1.41368500 \\ \mathrm{H} & 2.08704600 & -0.60500500 & 1.41748200 \\ \mathrm{Au} & -0.81637900 & -0.52984900 & 0.36483800 \\ \mathrm{O} & -2.72040200 & 0.14339900 & 1.16844800 \\ \mathrm{~S} & -3.74792200 & 0.88878900 & 0.32248700 \\ \mathrm{O} & -4.81034500 & 1.46591300 & 1.15551000 \\ \mathrm{O} & -3.16066900 & 1.72833400 & -0.73294300 \\ \mathrm{C} & -4.57279700 & -0.50670100 & -0.59243200 \\ \mathrm{~F} & -5.07143100 & -1.40406400 & 0.26781800 \\ \mathrm{~F} & -5.57182600 & -0.03198800 & -1.34715000 \\ \mathrm{~F} & -3.69564100 & -1.13350100 & -1.39562400\end{array}$

TS $_{19 "-17 "}$

$\mathrm{E}(\mathrm{SMD} / \mathrm{B} 3 \mathrm{LYP} / \mathrm{BS} 1)=-1752.63776576 \mathrm{au}$

$\mathrm{H}(\mathrm{SMD} / \mathrm{B} 3 \mathrm{LYP} / \mathrm{BS} 1)=-1752.326893 \mathrm{au}$

$\mathrm{G}(\mathrm{SMD} / \mathrm{B} 3 \mathrm{LYP} / \mathrm{BS} 1)=-1752.414247 \mathrm{au}$

$\mathrm{E}(\mathrm{SMD} / \mathrm{M} 06 / \mathrm{BS} 2 / / \mathrm{SMD} / \mathrm{B} 3 \mathrm{LYP} / \mathrm{BS} 1)=-1752.52487249 \mathrm{au}$

$\begin{array}{lllr}\mathrm{C} & -2.24166800 & 1.19301100 & -0.09629200 \\ \mathrm{C} & -2.08989500 & 2.13376000 & 0.93211500 \\ \mathrm{C} & -2.27085100 & 1.65009800 & -1.42307300 \\ \mathrm{C} & -1.95837500 & 3.49565400 & 0.64519800 \\ \mathrm{H} & -2.06281400 & 1.79737400 & 1.96599300 \\ \mathrm{C} & -2.14080100 & 3.00945600 & -1.71270000 \\ \mathrm{H} & -2.38716100 & 0.94235200 & -2.24075300 \\ \mathrm{C} & -1.98271900 & 3.93776500 & -0.67896000\end{array}$




$\begin{array}{lrrr}\mathrm{H} & -1.83897000 & 4.20780200 & 1.45751100 \\ \mathrm{H} & -2.16396300 & 3.34372100 & -2.74662500 \\ \mathrm{H} & -1.88242900 & 4.99589200 & -0.90503800 \\ \mathrm{C} & -2.35600000 & -0.29338300 & 0.23657200 \\ \mathrm{H} & -2.34700400 & -0.38826400 & 1.32673900 \\ \mathrm{C} & -1.16624300 & -1.14267400 & -0.28242500 \\ \mathrm{H} & -1.05733000 & -1.28749800 & -1.36277300 \\ \mathrm{C} & -3.74512100 & -0.83204500 & -0.26840500 \\ \mathrm{H} & -3.83765000 & -0.62385000 & -1.34581100 \\ \mathrm{C} & -4.95239500 & -0.11263100 & 0.38130800 \\ \mathrm{C} & -4.99555100 & -0.01216000 & 1.88844400 \\ \mathrm{H} & -4.63876800 & -0.92755600 & 2.37308100 \\ \mathrm{H} & -4.33888400 & 0.80510900 & 2.21267500 \\ \mathrm{H} & -6.01469600 & 0.21510600 & 2.20974200 \\ \mathrm{C} & -3.90178100 & -2.34278700 & -0.20417400 \\ \mathrm{C} & -5.24898800 & -2.97554400 & -0.15376100 \\ \mathrm{H} & -5.70368600 & -2.77909800 & 0.82702600 \\ \mathrm{H} & -5.90604700 & -2.52319000 & -0.90511200 \\ \mathrm{H} & -5.17146300 & -4.05313000 & -0.30860600 \\ \mathrm{H} & -1.86681000 & -2.29729800 & -0.28279800 \\ \mathrm{H} & -0.67220700 & -1.85122900 & 0.41230900 \\ \mathrm{O} & -2.89057100 & -3.07155700 & -0.25520800 \\ \mathrm{O} & -5.82857700 & 0.35046400 & -0.32484500 \\ \mathrm{Au} & 0.71325000 & -0.13713100 & -0.05369100 \\ \mathrm{O} & 2.55914700 & 0.94117000 & 0.09814000 \\ \mathrm{~S} & 3.78067900 & 0.34182700 & 0.79979000 \\ \mathrm{O} & 4.81162500 & 1.36116200 & 1.02338200 \\ \mathrm{O} & 3.44632300 & -0.56868700 & 1.90477800 \\ \mathrm{C} & 4.48010600 & -0.75944500 & -0.52945900 \\ \mathrm{~F} & 4.73219800 & -0.05317300 & -1.63780100 \\ \mathrm{~F} & 5.61874700 & -1.31839000 & -0.10313000 \\ \mathrm{~F} & 3.61465500 & -1.74032700 & -0.83895000\end{array}$

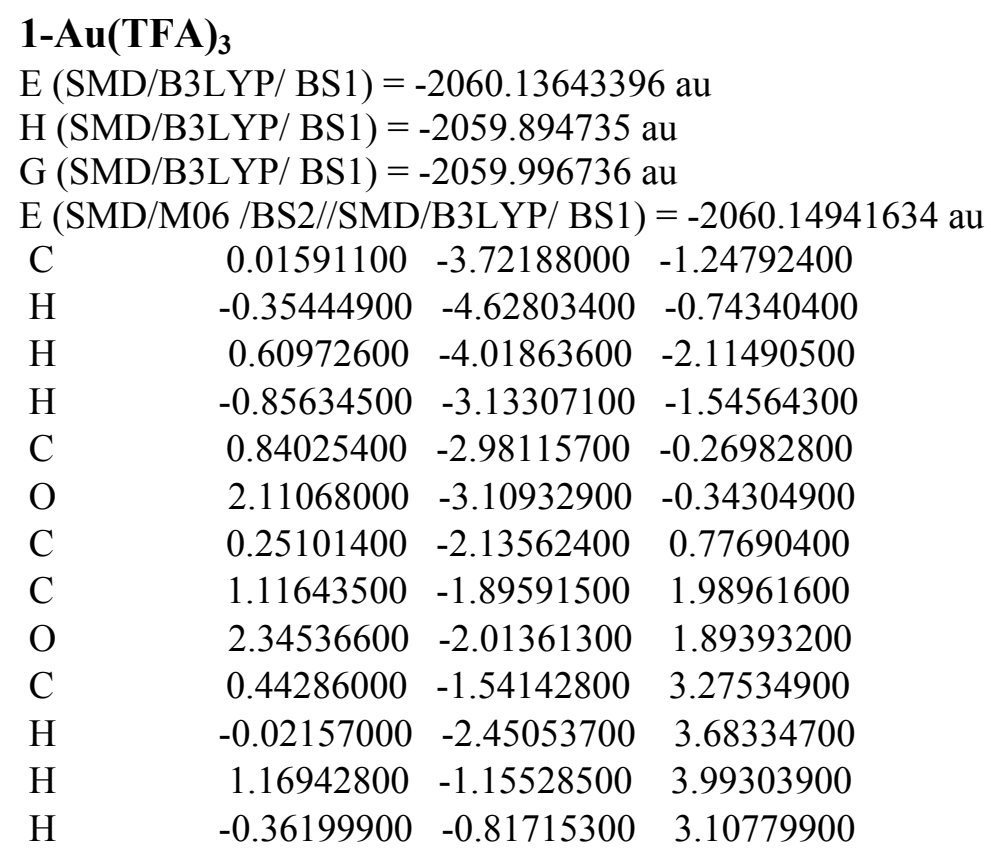




$\begin{array}{lrrr}\mathrm{H} & -0.77486700 & -2.41659800 & 1.01182400 \\ \mathrm{H} & 2.52608200 & -2.62493100 & 0.46970700 \\ \mathrm{Au} & -0.00281800 & -0.22033900 & -0.16693600 \\ \mathrm{O} & 1.98453400 & -0.19829600 & -0.61024700 \\ \mathrm{O} & -0.21644600 & 1.58487500 & -1.17948900 \\ \mathrm{O} & -1.99518500 & -0.29522300 & 0.24666000 \\ \mathrm{C} & -2.75796000 & -0.76343300 & -0.70474700 \\ \mathrm{C} & -0.48667500 & 2.77170400 & -0.70565400 \\ \mathrm{C} & 2.71673200 & 0.73617800 & -0.05571100 \\ \mathrm{O} & -2.43471600 & -1.22428500 & -1.78263900 \\ \mathrm{O} & -0.35187400 & 3.79604000 & -1.34671600 \\ \mathrm{O} & 2.39485700 & 1.57730800 & 0.75587600 \\ \mathrm{C} & -4.24557300 & -0.68041800 & -0.27518700 \\ \mathrm{C} & -1.11244100 & 2.89346600 & 0.71168600 \\ \mathrm{C} & 4.16869300 & 0.63874900 & -0.59229500 \\ \mathrm{~F} & -4.59741000 & 0.59040000 & -0.01569000 \\ \mathrm{~F} & -4.44920700 & -1.40840800 & 0.84167600 \\ \mathrm{~F} & -5.04523100 & -1.15447000 & -1.23590300 \\ \mathrm{~F} & -2.44283100 & 2.68231700 & 0.63191600 \\ \mathrm{~F} & -0.91358400 & 4.11793900 & 1.21135800 \\ \mathrm{~F} & -0.61548800 & 2.00568300 & 1.60659600 \\ \mathrm{~F} & 4.67179500 & -0.59316300 & -0.37649700 \\ \mathrm{~F} & 4.96067800 & 1.52850500 & 0.01313400 \\ \mathrm{~F} & 4.19721400 & 0.87450500 & -1.91677700\end{array}$

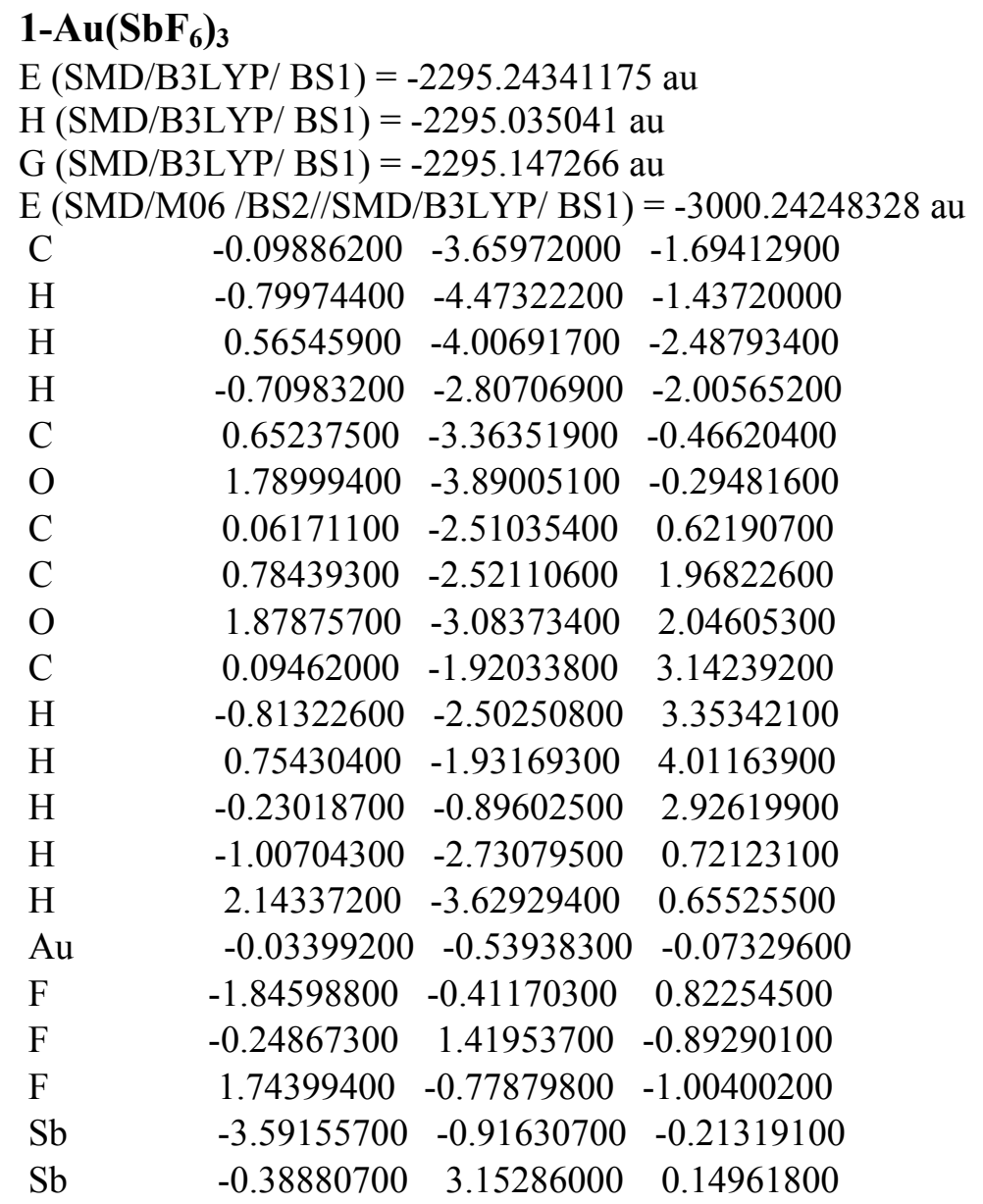




$\begin{array}{lrrr}\mathrm{Sb} & 3.64062400 & -0.19653300 & -0.25405800 \\ \mathrm{~F} & 3.81873000 & -2.05339000 & 0.01486400 \\ \mathrm{~F} & 2.78537700 & -0.12990200 & 1.43136500 \\ \mathrm{~F} & 5.33455100 & 0.27367100 & 0.36227300 \\ \mathrm{~F} & 4.09534700 & -0.43821300 & -2.05599500 \\ \mathrm{~F} & 3.02924900 & 1.53586200 & -0.62478900 \\ \mathrm{~F} & 0.78594200 & 2.33761300 & 1.39367400 \\ \mathrm{~F} & -0.50635200 & 4.76700600 & 1.09140000 \\ \mathrm{~F} & 1.07155400 & 3.71009500 & -0.89012700 \\ \mathrm{~F} & -1.58299200 & 3.65587300 & -1.21114600 \\ \mathrm{~F} & -1.80934200 & 2.35234800 & 1.09828900 \\ \mathrm{~F} & -3.72980200 & 0.89928800 & -0.64531900 \\ \mathrm{~F} & -4.37755300 & -0.63958200 & 1.46215600 \\ \mathrm{~F} & -2.38161200 & -1.11990400 & -1.66425100 \\ \mathrm{~F} & -5.11208200 & -1.47208900 & -1.13662300 \\ \mathrm{~F} & -3.09328800 & -2.66456700 & 0.31631700\end{array}$

\begin{tabular}{|c|c|c|c|}
\hline \multicolumn{4}{|c|}{ TS $_{1}-\mathbf{A u}(\mathrm{TFA})_{3}$} \\
\hline \multicolumn{4}{|c|}{$\mathrm{E}(\mathrm{SMD} / \mathrm{B} 3 \mathrm{LYP} / \mathrm{BS} 1)=-2369.7728034 \mathrm{au}$} \\
\hline \multicolumn{4}{|c|}{$\mathrm{H}(\mathrm{SMD} / \mathrm{B} 3 \mathrm{LYP} / \mathrm{BS} 1)=-2369.394331 \mathrm{au}$} \\
\hline \multicolumn{4}{|c|}{$\mathrm{G}(\mathrm{SMD} / \mathrm{B} 3 \mathrm{LYP} / \mathrm{BS} 1)=-2369.509106 \mathrm{au}$} \\
\hline \multicolumn{4}{|c|}{$\mathrm{E}(\mathrm{SMD} / \mathrm{M} 06 / \mathrm{BS} 2 / / \mathrm{SMD} / \mathrm{B} 3 \mathrm{LYP} / \mathrm{BS} 1)=-2369.6428$} \\
\hline $\mathrm{C}$ & 1.27666300 & 1.54312600 & -3.56526900 \\
\hline $\mathrm{H}$ & 0.67960700 & 1.76463900 & -4.46010000 \\
\hline $\mathrm{H}$ & 2.29689700 & 1.28373300 & -3.85491300 \\
\hline $\mathrm{H}$ & 1.27552400 & 2.44881400 & -2.94890300 \\
\hline $\mathrm{C}$ & 0.63678300 & 0.41059900 & -2.81220400 \\
\hline $\mathrm{O}$ & 1.25580100 & -0.65005000 & -2.67236900 \\
\hline $\mathrm{C}$ & -0.77586200 & 0.63098700 & -2.33643700 \\
\hline $\mathrm{C}$ & -1.77293500 & -0.42484700 & -2.82156400 \\
\hline $\mathrm{O}$ & -1.44134300 & -1.58040500 & -3.01649200 \\
\hline $\mathrm{C}$ & -3.16645100 & 0.07920300 & -3.11886200 \\
\hline $\mathrm{H}$ & -3.48474400 & 0.85510600 & -2.41660400 \\
\hline $\mathrm{H}$ & -3.16264000 & 0.52889900 & -4.12173500 \\
\hline $\mathrm{H}$ & -3.87314500 & -0.75402900 & -3.11199200 \\
\hline $\mathrm{H}$ & -1.10810300 & 1.63217500 & -2.62178000 \\
\hline $\mathrm{C}$ & 4.69679300 & -3.34841000 & -1.10907400 \\
\hline $\mathrm{C}$ & 5.84494100 & -3.07277000 & -0.38060300 \\
\hline $\mathrm{C}$ & 5.73552800 & -2.46058200 & 0.87401800 \\
\hline $\mathrm{C}$ & 4.47981400 & -2.12653100 & 1.40500400 \\
\hline $\mathrm{C}$ & 3.32734800 & -2.39909400 & 0.68572600 \\
\hline $\mathrm{C}$ & 3.41824800 & -3.01145300 & -0.59241700 \\
\hline $\mathrm{H}$ & 4.76455100 & -3.81832100 & -2.08623700 \\
\hline $\mathrm{H}$ & 6.82104900 & -3.32774900 & -0.78115700 \\
\hline $\mathrm{H}$ & 6.63353300 & -2.24281300 & 1.44547400 \\
\hline $\mathrm{H}$ & 4.41029100 & -1.65447400 & 2.37985200 \\
\hline $\mathrm{H}$ & 2.35985800 & -2.14464000 & 1.10309800 \\
\hline $\mathrm{C}$ & 2.27763900 & -3.30241000 & -1.39328000 \\
\hline $\mathrm{H}$ & 2.49117300 & -3.81968000 & -2.32942000 \\
\hline $\mathrm{C}$ & 0.93558700 & -2.91208100 & -1.18971100 \\
\hline $\mathrm{H}$ & 0.62617600 & -2.58725800 & -0.19709200 \\
\hline
\end{tabular}




$\begin{array}{lrrr}\mathrm{H} & 0.95205200 & -1.87190000 & -1.85543700 \\ \mathrm{H} & 0.19062700 & -3.48208400 & -1.74666000 \\ \mathrm{Au} & -0.83939900 & 0.79807200 & -0.23016400 \\ \mathrm{O} & 0.55160900 & 2.28236800 & -0.42365100 \\ \mathrm{O} & -1.00448800 & 1.31417100 & 1.82543700 \\ \mathrm{O} & -2.26442300 & -0.65533800 & -0.08720300 \\ \mathrm{C} & -3.47231400 & -0.27317900 & 0.22773500 \\ \mathrm{C} & -0.65576100 & 0.74082200 & 2.93011600 \\ \mathrm{C} & 1.66689400 & 2.13953800 & 0.24160500 \\ \mathrm{O} & -3.92735200 & 0.84978300 & 0.31681700 \\ \mathrm{O} & -0.75362400 & 1.23545500 & 4.04293300 \\ \mathrm{O} & 2.07803700 & 1.17742900 & 0.86092600 \\ \mathrm{C} & -4.36242500 & -1.52023600 & 0.47120000 \\ \mathrm{C} & -0.04634300 & -0.68550400 & 2.88775400 \\ \mathrm{C} & 2.49834300 & 3.44489800 & 0.14906100 \\ \mathrm{~F} & -3.82706800 & -2.31727800 & 1.41553700 \\ \mathrm{~F} & -4.48116500 & -2.24242900 & -0.66246300 \\ \mathrm{~F} & -5.58919600 & -1.16497600 & 0.86929600 \\ \mathrm{~F} & -0.64468500 & -1.47654300 & 3.79551000 \\ \mathrm{~F} & 1.26921300 & -0.64649000 & 3.18068500 \\ \mathrm{~F} & -0.17108600 & -1.29772200 & 1.68467000 \\ \mathrm{~F} & 2.72117500 & 3.78243600 & -1.13869100 \\ \mathrm{~F} & 3.68361300 & 3.30386300 & 0.75278400 \\ \mathrm{~F} & 1.84265700 & 4.46382200 & 0.73584900\end{array}$

\begin{tabular}{|c|c|c|c|}
\hline \multicolumn{4}{|c|}{$\mathbf{T S}_{1}-\mathbf{A u}\left(\mathrm{SbF}_{6}\right)_{3}$} \\
\hline \multicolumn{4}{|c|}{$\mathrm{E}(\mathrm{SMD} / \mathrm{B} 3 \mathrm{LYP} / \mathrm{BS} 1)=-2604.89100557 \mathrm{au}$} \\
\hline \multicolumn{4}{|c|}{$\mathrm{H}(\mathrm{SMD} / \mathrm{B} 3 \mathrm{LYP} / \mathrm{BS} 1)=-2604.543268 \mathrm{au}$} \\
\hline \multicolumn{4}{|c|}{$\mathrm{G}(\mathrm{SMD} / \mathrm{B} 3 \mathrm{LYP} / \mathrm{BS} 1)=-2604.67832 \mathrm{au}$} \\
\hline \multicolumn{4}{|c|}{$\mathrm{E}(\mathrm{SMD} / \mathrm{M} 06 / \mathrm{BS} 2 / / \mathrm{SMD} / \mathrm{B} 3 \mathrm{LYP} / \mathrm{BS} 1)=-3309.74856702 \mathrm{au}$} \\
\hline $\mathrm{C}$ & 2.03908500 & -1.94273200 & 3.51641100 \\
\hline $\mathrm{H}$ & 2.67766000 & -1.31889700 & 4.14424400 \\
\hline $\mathrm{H}$ & 2.62713200 & -2.52486300 & 2.80183200 \\
\hline $\mathrm{H}$ & 1.48808300 & -2.65950800 & 4.14337600 \\
\hline $\mathrm{C}$ & 1.04938300 & -1.09908300 & 2.78785300 \\
\hline $\mathrm{O}$ & 0.99796600 & 0.11707700 & 3.04818000 \\
\hline $\mathrm{C}$ & 0.09119200 & -1.85055500 & 1.88879200 \\
\hline $\mathrm{C}$ & -1.24962100 & -2.12260100 & 2.63218300 \\
\hline $\mathrm{O}$ & -1.62467300 & -1.32913900 & 3.47085200 \\
\hline $\mathrm{C}$ & -1.92997400 & -3.41507900 & 2.29275800 \\
\hline $\mathrm{H}$ & -2.16716900 & -3.44765400 & 1.22408900 \\
\hline $\mathrm{H}$ & -1.24937600 & -4.25004900 & 2.50288800 \\
\hline $\mathrm{H}$ & -2.84354100 & -3.51774800 & 2.87973200 \\
\hline $\mathrm{H}$ & 0.54726200 & -2.77962400 & 1.54154100 \\
\hline $\mathrm{C}$ & 1.50486000 & 4.68474000 & 3.54443000 \\
\hline $\mathrm{C}$ & 2.33814000 & 5.67806100 & 3.03991300 \\
\hline $\mathrm{C}$ & 2.35891400 & 5.93368300 & 1.66484900 \\
\hline $\mathrm{C}$ & 1.54079900 & 5.19993900 & 0.79504100 \\
\hline $\mathrm{C}$ & 0.70416600 & 4.21038400 & 1.29409000 \\
\hline $\mathrm{C}$ & 0.68135500 & 3.93055800 & 2.67931400 \\
\hline $\mathrm{H}$ & 1.48358700 & 4.47631200 & 4.61097500 \\
\hline
\end{tabular}




$\begin{array}{lccc}\mathrm{H} & 2.96996300 & 6.25150900 & 3.71167500 \\ \mathrm{H} & 3.00950700 & 6.70848700 & 1.26859200 \\ \mathrm{H} & 1.55498400 & 5.39928300 & -0.27188500 \\ \mathrm{H} & 0.06255400 & 3.66073300 & 0.61776600 \\ \mathrm{C} & -0.15750600 & 2.90367100 & 3.25633600 \\ \mathrm{H} & -0.14283800 & 2.86163800 & 4.34592600 \\ \mathrm{C} & -0.89534400 & 1.93996300 & 2.60254100 \\ \mathrm{H} & -1.06322700 & 2.00272700 & 1.53061800 \\ \mathrm{H} & 0.13363300 & 0.90505200 & 2.72208700 \\ \mathrm{H} & -1.63740500 & 1.38728800 & 3.17780300 \\ \mathrm{Au} & -0.21612700 & -0.93050000 & 0.03449000 \\ \mathrm{~F} & -1.98516200 & -0.11298300 & 0.63444400 \\ \mathrm{~F} & -0.37183800 & -0.04717000 & -1.97292600 \\ \mathrm{~F} & 1.35192500 & -1.98820700 & -0.72459000 \\ \mathrm{Sb} & -3.83096100 & -0.64703500 & -0.19568400 \\ \mathrm{Sb} & 0.12353300 & 1.88374400 & -2.12358700 \\ \mathrm{Sb} & 3.40425600 & -1.90077300 & -0.48398200 \\ \mathrm{~F} & -2.93409500 & -2.23801100 & -0.71775500 \\ \mathrm{~F} & -4.11824600 & -1.42199900 & 1.49203600 \\ \mathrm{~F} & -5.49805100 & -1.14080900 & -0.87585800 \\ \mathrm{~F} & -4.33139400 & 1.02894100 & 0.47868500 \\ \mathrm{~F} & -3.22415000 & 0.14566700 & -1.78590700 \\ \mathrm{~F} & 0.63345800 & 3.69051400 & -2.17167900 \\ \mathrm{~F} & 1.21434200 & 1.51519000 & -0.60344500 \\ \mathrm{~F} & -1.26097300 & 2.23569300 & -0.87281800 \\ \mathrm{~F} & -1.10235300 & 2.00632400 & -3.53785800 \\ \mathrm{~F} & 1.49819000 & 1.33671900 & -3.27713900 \\ \mathrm{~F} & 5.25298300 & -1.87324600 & -0.23150600 \\ \mathrm{~F} & 3.10187500 & -3.33817100 & 0.70627600 \\ \mathrm{~F} & 3.01270500 & -0.72637200 & 0.95222200 \\ \mathrm{~F} & 3.34198000 & -0.44987200 & -1.66833000 \\ \mathrm{~F} & 3.37946500 & -3.10967000 & -1.91600500\end{array}$

\begin{tabular}{|c|c|c|c|}
\hline \multicolumn{4}{|c|}{$\mathrm{TS}_{2}-\mathrm{Au}(\mathrm{TFA})_{3}$} \\
\hline \multicolumn{4}{|c|}{$\mathrm{E}(\mathrm{SMD} / \mathrm{B} 3 \mathrm{LYP} / \mathrm{BS} 1)=-2369.71962015 \mathrm{au}$} \\
\hline \multicolumn{4}{|c|}{$\mathrm{H}(\mathrm{SMD} / \mathrm{B} 3 \mathrm{LYP} / \mathrm{BS} 1)=-2369.335129 \mathrm{au}$} \\
\hline \multicolumn{4}{|c|}{$\mathrm{G}(\mathrm{SMD} / \mathrm{B} 3 \mathrm{LYP} / \mathrm{BS} 1)=-2369.454286 \mathrm{au}$} \\
\hline \multicolumn{4}{|c|}{$\mathrm{E}(\mathrm{SMD} / \mathrm{M} 06 / \mathrm{BS} 2 / / \mathrm{SMD} / \mathrm{B} 3 \mathrm{LYP} / \mathrm{BS} 1)=-2369.60106497 \mathrm{au}$} \\
\hline $\mathrm{C}$ & 1.49009600 & -1.12145400 & -2.45688800 \\
\hline $\mathrm{H}$ & 2.28079500 & -1.43969400 & -3.14419100 \\
\hline $\mathrm{H}$ & 0.84706900 & -0.41918900 & -3.00418400 \\
\hline $\mathrm{H}$ & 0.88411800 & -1.97914200 & -2.15902700 \\
\hline $\mathrm{C}$ & 2.11412100 & -0.37959900 & -1.28494400 \\
\hline $\mathrm{O}$ & 2.96523400 & 0.46886300 & -1.46421200 \\
\hline $\mathrm{C}$ & 1.68138700 & -0.77834500 & 0.12942400 \\
\hline $\mathrm{C}$ & 1.75787000 & 0.21599000 & 1.29702900 \\
\hline $\mathrm{O}$ & 2.39081100 & 1.25048700 & 1.22576400 \\
\hline $\mathrm{C}$ & 1.04915900 & -0.18624200 & 2.58223600 \\
\hline $\mathrm{H}$ & 0.37807500 & 0.62722400 & 2.87818800 \\
\hline $\mathrm{H}$ & 0.47807800 & -1.11404400 & 2.49738200 \\
\hline $\mathrm{H}$ & 1.79398200 & -0.29446100 & 3.37944700 \\
\hline
\end{tabular}




$\begin{array}{crrr}\mathrm{H} & 0.87244700 & -1.51151900 & 0.19783800 \\ \mathrm{C} & 4.78572400 & -0.82362200 & 1.75380200 \\ \mathrm{C} & 6.07941100 & -0.31907100 & 1.76960400 \\ \mathrm{C} & 6.88331600 & -0.42838500 & 0.62816300 \\ \mathrm{C} & 6.38997300 & -1.05392100 & -0.52561900 \\ \mathrm{C} & 5.09710100 & -1.55880700 & -0.54528000 \\ \mathrm{C} & 4.27046000 & -1.46297500 & 0.59948600 \\ \mathrm{H} & 4.16938600 & -0.75645200 & 2.64518100 \\ \mathrm{H} & 6.46591500 & 0.15688900 & 2.66563300 \\ \mathrm{H} & 7.89330800 & -0.02850400 & 0.63635600 \\ \mathrm{H} & 7.01806500 & -1.14051700 & -1.40709300 \\ \mathrm{H} & 4.72866700 & -2.04097600 & -1.44382400 \\ \mathrm{C} & 2.92026900 & -2.04847800 & 0.64507800 \\ \mathrm{H} & 2.57672200 & -2.16876200 & 1.67334500 \\ \mathrm{C} & 2.64552400 & -3.30705400 & -0.15955100 \\ \mathrm{H} & 2.86481600 & -3.20464200 & -1.22479700 \\ \mathrm{H} & 3.28944800 & -4.10245400 & 0.23669900 \\ \mathrm{H} & 1.60599700 & -3.62757200 & -0.04306600 \\ \mathrm{Au} & -0.76710300 & 0.19925200 & -0.32796100 \\ \mathrm{O} & -2.61961200 & 1.03420800 & -0.78697600 \\ \mathrm{O} & 0.06886800 & 1.97580900 & -0.83500300 \\ \mathrm{O} & -1.50968200 & -1.64977900 & 0.13127400 \\ \mathrm{C} & -2.00439200 & -2.35737500 & -0.85176100 \\ \mathrm{C} & -3.62474100 & 1.26209200 & 0.02798400 \\ \mathrm{C} & -0.15938600 & 2.99183100 & -0.04557800 \\ \mathrm{O} & -2.11517400 & -2.06440100 & -2.02405500 \\ \mathrm{O} & -4.54409600 & 1.98066800 & -0.30638800 \\ \mathrm{O} & -0.77406100 & 3.01666000 & 1.00120100 \\ \mathrm{C} & 0.47288900 & 4.27442800 & -0.64598200 \\ \mathrm{C} & -2.44325400 & -3.74626100 & -0.31836000 \\ \mathrm{C} & -3.71061100 & 0.52361200 & 1.38910800 \\ \mathrm{~F} & 1.79068400 & 4.11225300 & -0.85527500 \\ \mathrm{~F} & -0.10563300 & 4.56583000 & -1.82766100 \\ \mathrm{~F} & 0.29999600 & 5.31642200 & 0.17459200 \\ \mathrm{~F} & -2.53225800 & 0.48153300 & 2.04789700 \\ \mathrm{~F} & -4.11245000 & -0.74576800 & 1.18145800 \\ \mathrm{~F} & -4.60151800 & 1.12480300 & 2.18077300 \\ \mathrm{~F} & -2.99879900 & -4.47791600 & -1.28866700 \\ \mathrm{~F} & -1.36845900 & -4.41594400 & 0.15317300 \\ \mathrm{~F} & -3.32862400 & -3.62514400 & 0.68531700\end{array}$

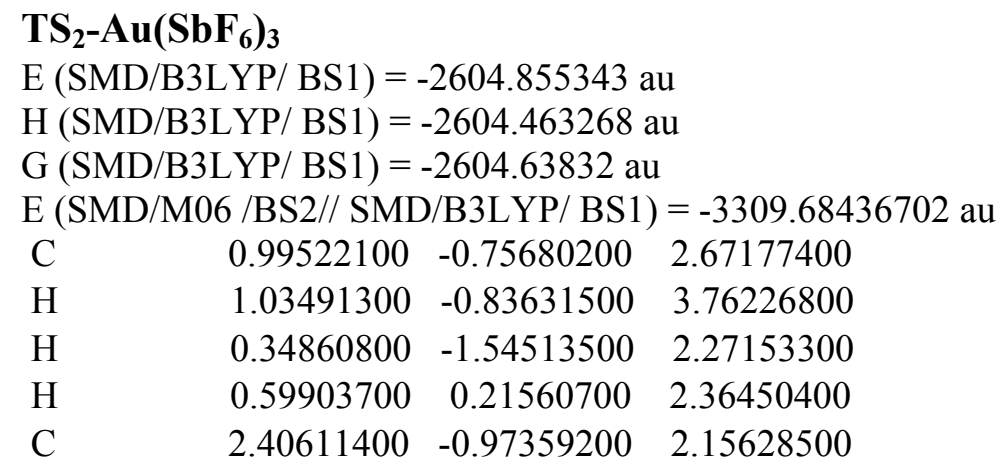




$\begin{array}{lrrr}\mathrm{O} & 3.27636800 & -1.49708800 & 2.81405800 \\ \mathrm{C} & 2.54993500 & -0.53761800 & 0.70903600 \\ \mathrm{C} & 2.54768700 & -1.62175800 & -0.36190900 \\ \mathrm{O} & 2.51814500 & -2.77717700 & 0.02671700 \\ \mathrm{C} & 2.50480300 & -1.21609400 & -1.80741500 \\ \mathrm{H} & 1.52412900 & -1.49991900 & -2.20961600 \\ \mathrm{H} & 2.65823200 & -0.14661300 & -1.95465400 \\ \mathrm{H} & 3.25582900 & -1.78799400 & -2.36245100 \\ \mathrm{H} & 2.18121700 & 0.45389700 & 0.43385600 \\ \mathrm{C} & 5.60355700 & -0.83493100 & -0.34829700 \\ \mathrm{C} & 6.43771500 & -1.91966100 & -0.28163400 \\ \mathrm{C} & 6.89132700 & -2.37077000 & 0.98178900 \\ \mathrm{C} & 6.51406600 & -1.73577900 & 2.17740400 \\ \mathrm{C} & 5.65837900 & -0.65627900 & 2.13928700 \\ \mathrm{C} & 5.18190300 & -0.15885600 & 0.86246000 \\ \mathrm{H} & 5.27251500 & -0.43764100 & -1.30267000 \\ \mathrm{H} & 6.76703000 & -2.42353700 & -1.18357000 \\ \mathrm{H} & 7.56009700 & -3.22578100 & 1.02563900 \\ \mathrm{H} & 6.88869800 & -2.10159300 & 3.12673100 \\ \mathrm{H} & 5.37760400 & -0.15162900 & 3.05371400 \\ \mathrm{C} & 4.52569300 & 1.07745000 & 0.71490300 \\ \mathrm{H} & 4.32693000 & 1.37496400 & -0.31508400 \\ \mathrm{C} & 4.22991900 & 2.08828500 & 1.73961500 \\ \mathrm{H} & 4.34208100 & 1.75662100 & 2.77188300 \\ \mathrm{H} & 4.93333100 & 2.91918800 & 1.54876000 \\ \mathrm{H} & 3.23731800 & 2.51809500 & 1.56967400 \\ \mathrm{Au} & -0.52575600 & -0.06658400 & -0.33048700 \\ \mathrm{~F} & 0.01951500 & 1.61843400 & -1.76932200 \\ \mathrm{~F} & -0.50939500 & -2.21016300 & 0.36919200 \\ \mathrm{~F} & -2.4716200 & 1.33062200 & -0.62884900 \\ \mathrm{~F} & 1.09955900 & 3.12952700 & -1.15732700 \\ \mathrm{Sb} & -3.45782900 & 1.38424000 & 1.13323800 \\ \mathrm{Sb} & -1.51485800 & -3.51985400 & -0.72661100 \\ \mathrm{Sb} & -2.48668900 & -3.93632800 & 0.82857700 \\ \mathrm{~F} & -2.75433700 & -2.14263300 & -1.12701100 \\ \mathrm{~F} & -2.41742100 & -4.75857400 & -1.80979100 \\ \mathrm{~F} & -0.16496200 & -4.74538900 & -0.26074900 \\ \mathrm{~F} & -0.43660700 & -2.89365300 & -2.16677100 \\ \mathrm{~F} & -2.02587600 & 2.50861700 & 1.66143200 \\ \mathrm{~F} & -4.49625700 & 2.86650400 & 0.61675500 \\ \mathrm{~F} & -4.16950600 & 1.38797100 & 2.87653600 \\ \mathrm{~F} & -4.75727700 & 0.15916800 & 0.53214400 \\ \mathrm{~F} & -2.32558300 & -0.09620400 & 1.62747900 \\ \mathrm{~F} & 1.07990800 & 3.82230200 & -2.89920600 \\ \mathrm{~F} & 2.63971300 & 2.46969900 & -0.44013700 \\ \mathrm{~F} & & 4.02564500 & -0.68318500\end{array}$

